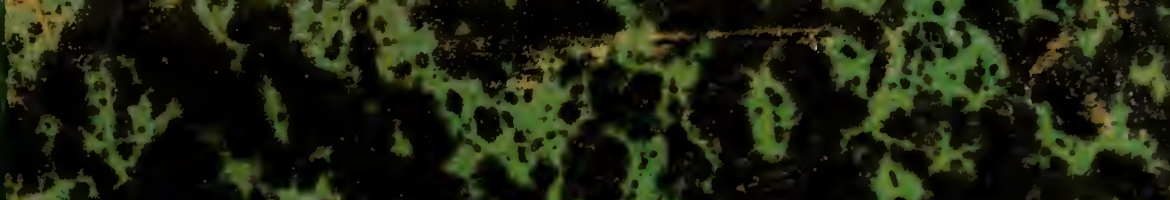

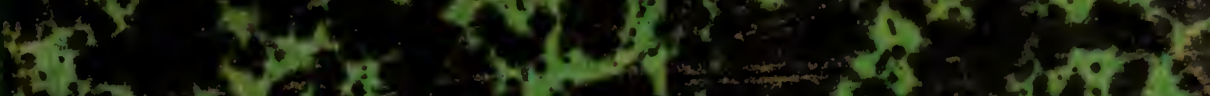

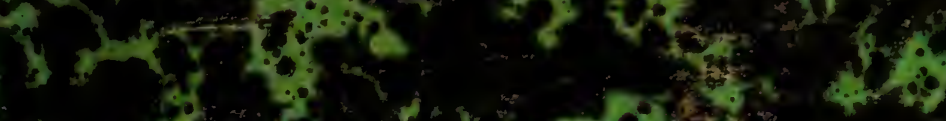

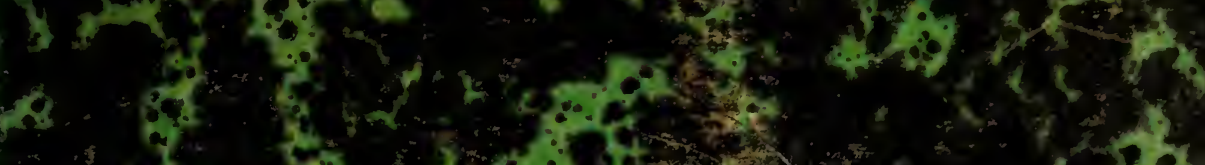
8

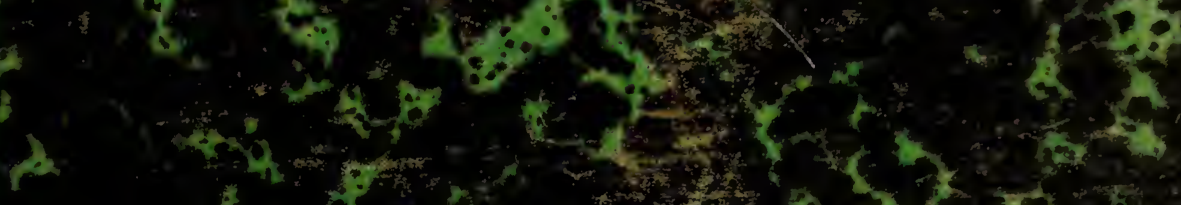

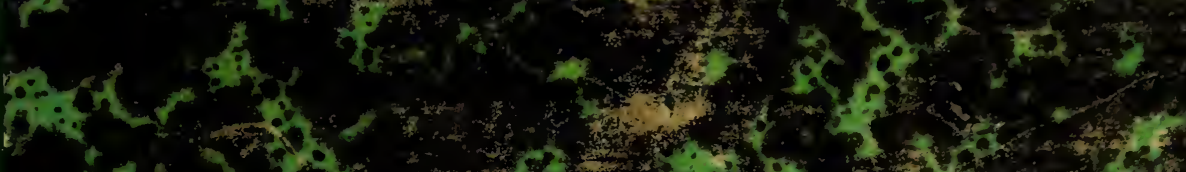

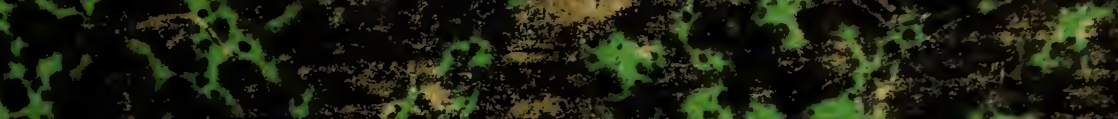

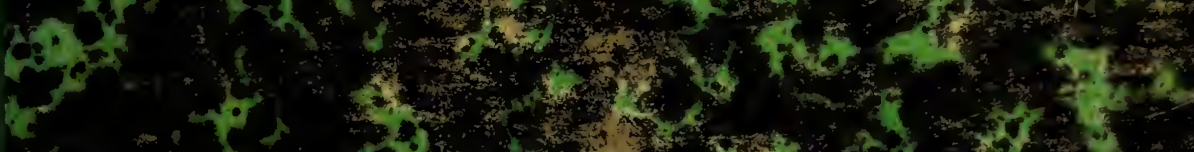

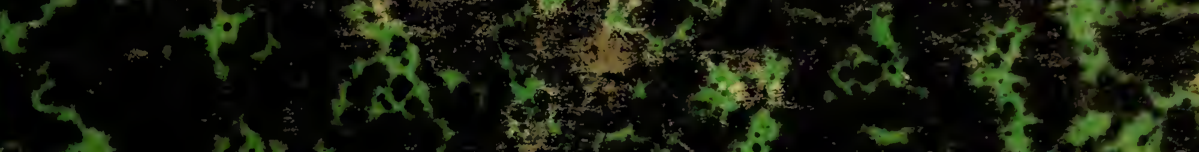

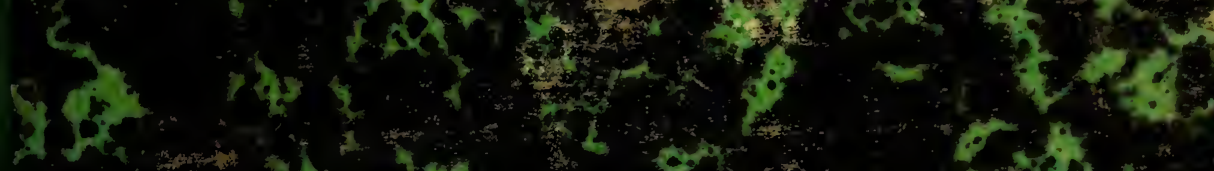

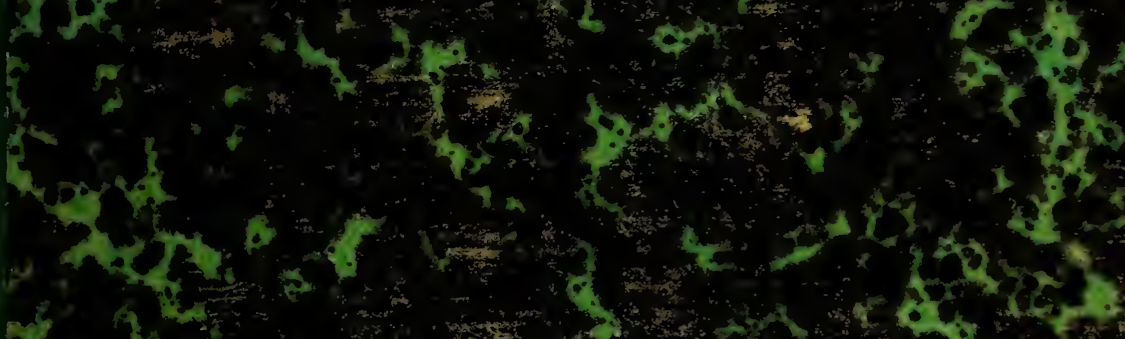

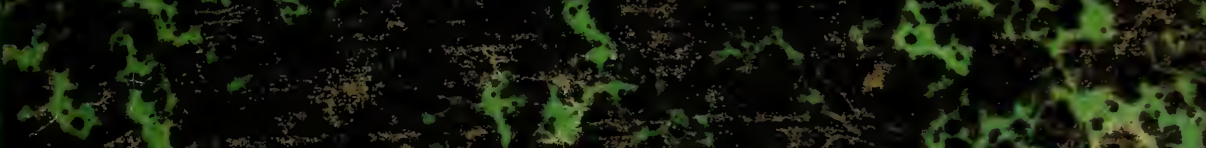

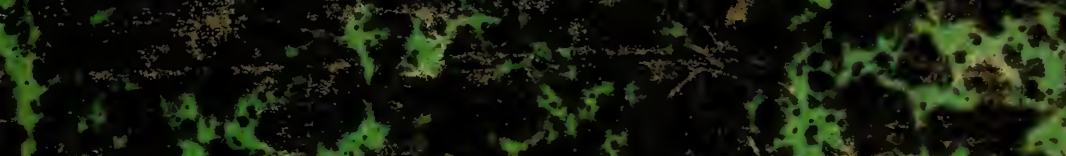
$\left(+x^{2}+x^{2}+1+20\right.$

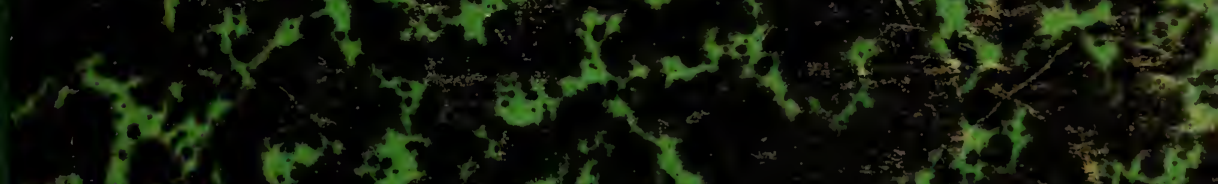

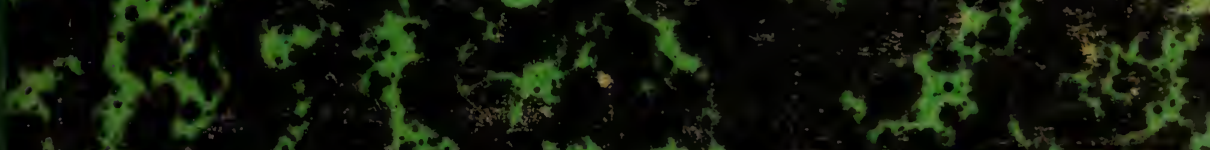

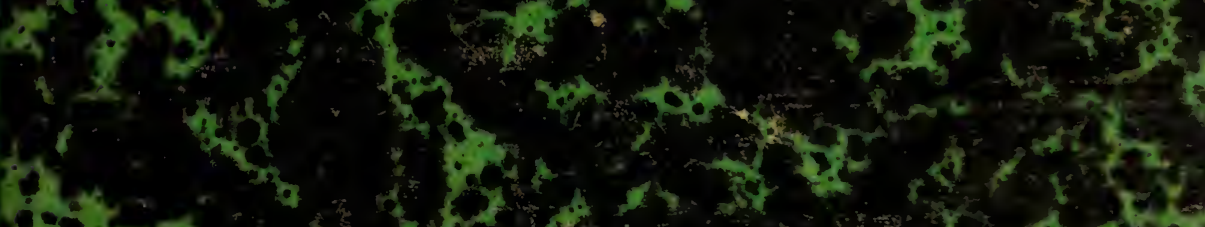




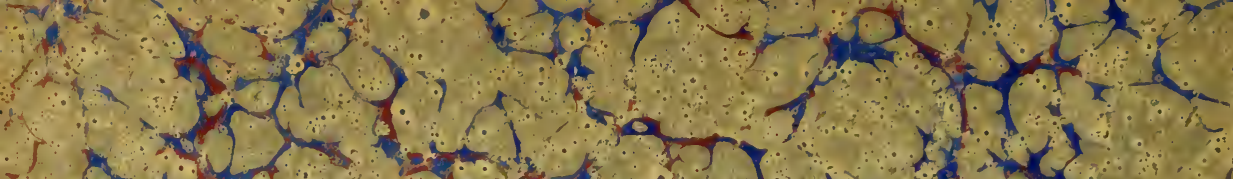

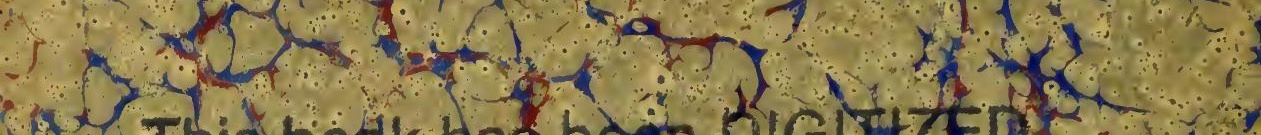

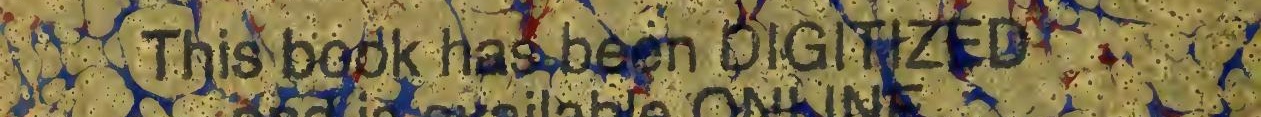

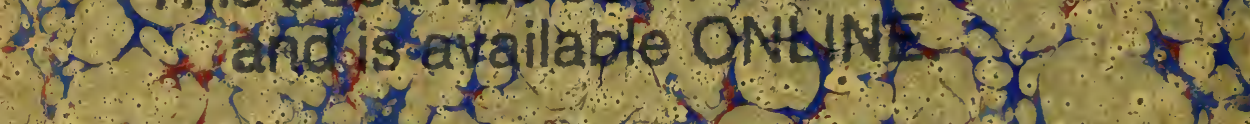

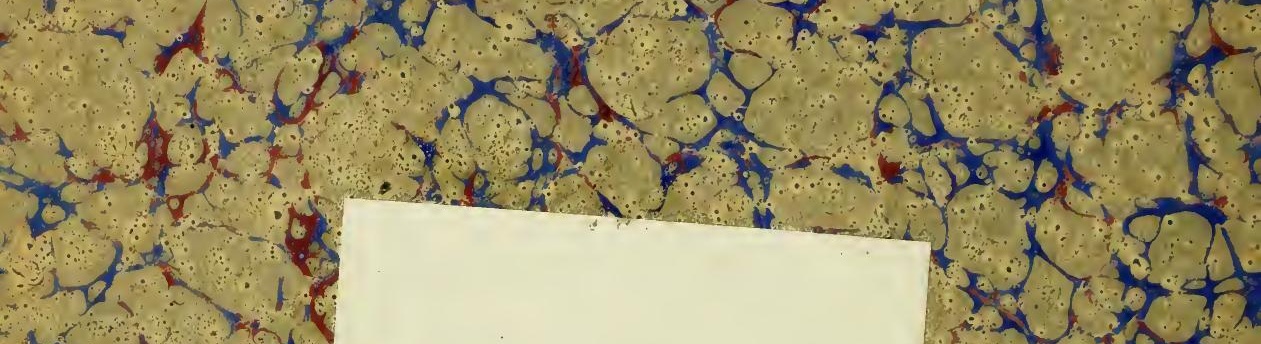
$x-1, x$

i.

$-2 x^{2}=$

$21+2+x^{x}$

$y^{3}-2+2$

(1)

$-3 h|c|$

LIBRARY

581.951

D $35 \mathrm{C}$

THE UNIVERSITY

OF ILLINOIS

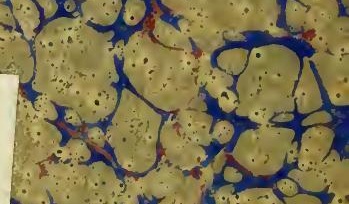

s. . . क

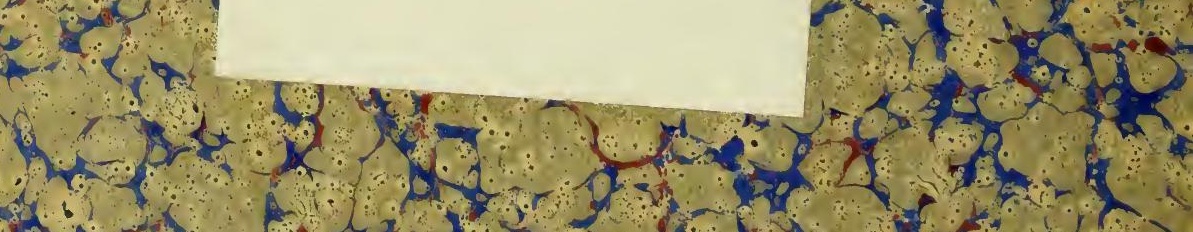

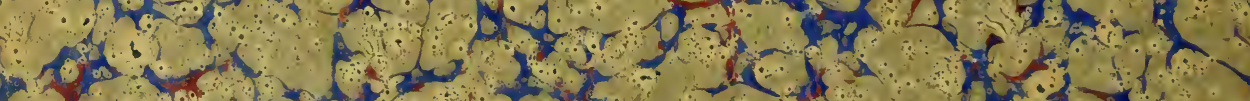

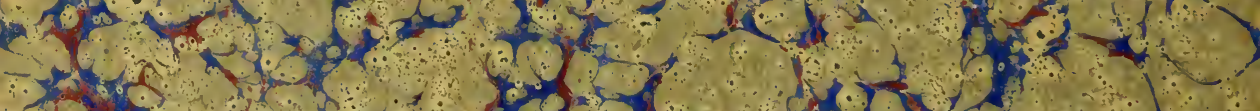

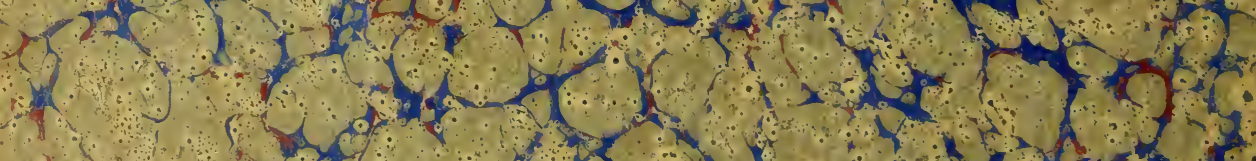

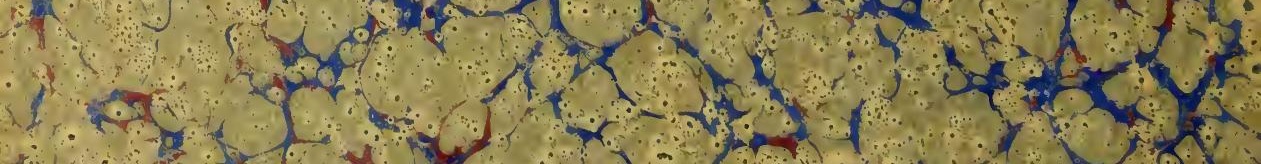

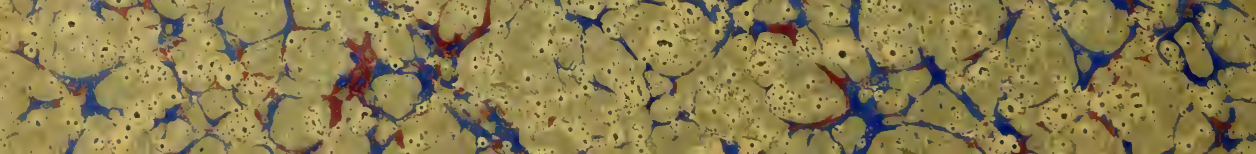


CONTRIBUTIONS

I LA

\section{FLORE DE LA CHINE}





\section{CONTRIBUTIONS}

A LA

\section{FLORE DE LA CHINE}

COMPRENANT

LES ALGUES MARINES RÉCOLTÉES EN CHINE

Et LES FLORULES DE SHANG-HAI, TCHÉ-FOU ET TIEN-TSIN

PAR

M. O. DEBEAUX

l'harmacien-major de première classe, attaché à l'expédition française en c'hine pendant les années 1860 à 1862

Chevalier de la Légion d'honneur

\section{Fascicules I-IV}

\section{PARIS}

F. SAVY, LIBRAIRE-ÉDITEUR

BOULEVARD SAINT-GERMAIN, $7 \%$ 

581.951

D) 350

3

in

jo

is

T

8

m

5

2)

3

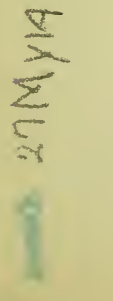


Digitized by the Internet Archive in 2014

https://archive.org/details/contributionslaf00debe 


\title{
CONTRIBUTIONS A LA FLORE DE LA CHINE
}

\author{
FASGIGULE I \\ ALGILS MARINES

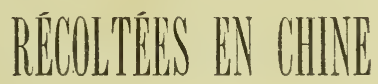

pendant l'expédition française de 1860-62

\author{
Par M. O. DEBEAUX, \\ PFARIMACIEN MAJOR DE $1^{\text {r }}$ CIASSE \\ CHETLLIER DE LA LÉGION D'HONKEUR,
}

Membre de la Soriété botanique de Franne,

Correspondant de la Société Linnéenne de Bordeaux et de plusieurs autres Sociétés savantes.

(Extrait des A'tes de la Société Linnéenne de Bordeaux, t. XXX, Ire liv., 1875.)

\section{PARIS}

F. SAVY, LIERAIRE-ÉITEUR,

24 - me hautefzulle - 24 



\section{ALGUES MARINES}

\section{RÉCOLTÉES EN CIIINE \\ PENDANT L'EXPÉDITHON FRANCSAISE DE 1860-1862;}

Par M. O. DEBEAUX,

Pharmacien-major de première classe.

Membre correspondant.

La publication, en 1870, du Catalogue des Algues japonaises du Musée botanique de Leyde, par M. Miquel (1), et celle plus récente encore des Alga japonica, par M. Suringar (2), ont mis en évidence les affinités de la flore sous-marine du littoral japonais, non-seulement avec celle des mers de Chine, mais encore de l'Océan Atlantique et de la Méditerranée. La liste des Algues japonaises publiée par M. Miquel, l'auteur de remarquables travaux sur la flore du Japon, ne comprend avec les Diatomacées que 77 espèces seulement. De ce nombre, 11 se retrouvent sur le littoral de la Chine, 12 vivent sur les côtes de la Méditerranée et de l'Océan Atlantique européen, et une seule au cap de Bonne-Espérance. Parmi les Diatomacées marines ou d'eau douce signalées au Japon, et au nombre de 30 environ, un tiers de ces espèces se retrouve à la fois dans une grande partie de l'Europe et de l'Amérique boréale.

Les mêmes affinités de géographie botanique paraissent exister à l'égard des Algues marines que j'ai recueillies pendant mon séjour en Chine, soit à l'île de Hong-Kong (mai 1860), soit sur les rivages de la presqu'île de Tché-fou, dans la province du Changtong (de juillet 1860 à mars 1861). Le nombre des espèces observées est malheureusement peu élevé, et ne permet de formuler,

(1) Catalogus musei botanici Lugduni-Batari, auct. Guill. Miquel.-La Haye. 1870 .

(2) Algæ japonicæ musei Lugduni-Batavi. Harlem, 1870. - Illustrations des Algues du Japon, 187 . 
sous le rapport de leur extension géographique, que les conclusions approximatives.

La végétation sous-marine des mers équatoriales et intertropi(ales (détroit de la Sonde, Singapoore et Hong-Kong) m'a paru être fort peu développée dans ces localités. Ainsi, dans la grande rade de Singapoore, située à 1 degré environ de latitude nord, ‘̀ l'entrée du détroit de la Sonde, je n'ai rencontré que deux espèces d'Algues Fucacées. Dans la baie de Victoria, au nord de l'île de Hong-Kong ( $22^{\circ} 9$ à $23^{\circ} 21$ de latitude N.) et sur les falaises de Kowlong, partie du continent chinois de la province du Kouan-tong, j'ai trouvé six espèces d'Algues seulement, appartenant à la même famille des Fucacées. Beaucoup plus au nord de la mer de Chine, et principalement dans les baies Ki-tsen-sôo et de Yan-taï, situées non loin du cap Tché-fou, à l'entrée du golfe de Pe-tchi-ly, et près du cap Chang-tong (370 40 lat. $\mathrm{N}$. et $118^{\circ} 30$ long. E.), mes récoltes phycologiques se sont élevées à 18 espèces en tout. C'est un bien minime résultat, si l'on veut, après huit mois de séjour et de recherches journalières dans cette partie du littoral de la Chine (1). Il y a donc lieu d'être surpris de la pauvreté numérique des Algues qui vivent dans les mers de l'extrême Orient; mais leur facies tout à fait européen mérite également de fixer l'attention de l'observateur. Il est facile, en effet, de reconnaître, au premier abord, l'identité de quelques Algues les plus répandues sur les rivages de la presqu'île de Tché-fou, avec les espèces analogues qui vivent dans la Méditerranée ou l'Océan Atlantique.

L'extension géographique ou A rea des Algues marines chinoises, offre les plus grands rapports avec celle des Algues japonaises du musée de Leyde. Ces rapports sont surtout frappants, en ce qui concerne les espèces des rivag'es du Chang-tong, du grolfe de Petchi-ly et de la mer de Corée. Comme exemple de leur grande extension géographique, je citerai les plantes suivantes: Padina paronia, Chordaria flagelliformis, Rhodymenia palmata, Dumontia filiformis, Grateloupia filicina, Gelidium corneum, Phycoșeris

(1) Le fait curieux de l'absence des Algues dans les mel's chaudes du globe, 'unce les deux zones tropicales, a été déjà observé. Le célèbre phycologiste Agardh :) démontré que le nombre spécifique des Algues décroit à mesure que'l'on avance vas l'équateur. et qu'il atteint son maximun mitre les $35^{\circ}$ et $45^{\circ}$ de latitude daus les deux hémisphères. 
australis, Enteromorpha compressa et $E$. intestinalis, Bryopsis plumosa et $B$. arbuscula, espèces qui se retrouvent en partie dans la mer du Japon, et qui abondent sur le littoral de la Méditerranée et de l'Océan Atlantique enropéen. Il en est d'autres, en petit nombre il est vrai, qui ont été observées aussi sur plusieurs points de l'Océan Indien et de l'Océan Pacifique, et dont l'A rea est par conséquent des plus étendus.

Qn pourrait établir une égale comparaison entre ces hydrophytes, dont l'habitat à des distances si éloignées les unes des autres a lieu de nous surprendre, avec une foule de plantes phanérogames qui sont assez répandues en Europe, et que l'on retrouve à l'état spontané dans les provinces centrales et boréales de la Chine.

Je signale comme étant remarquables par l'étendue de leur A rea géographique les plantes suivantes que j'ai récoltées soit à shang-haï, soit dans la presqu'île de T'ché-fou : Ranunculus sceleratus, Nasturtium sylvestre, Cardamine hirsuta, Sisymbrium. sophia, Thlaspi arvense, Lepidium ruderale, Abutilon Avicenna. Hypericum perforatum, Oxalis corniculata, Tribulus terrestris, Melilotus parviflora, Buplevrum falcatum, Daucus carota, Tripolium vulgare, Bidens bipinnata, Artemisia annua, Scorzonera austriaca, Sonchus oleraceus, Xanthium strumarium, Convolvulus arrensis, Physalis alkekengi, Linaria vulgaris, Mentha arvensis, Thymus serpillum, Brunella vulgaris, Verbena officinalis, Chenopodina, maritima, Polygonum bistorta et $P$. nodosum, Lemna minor, Acoru.s calamus, Cyperus fuscus, Imperata cylindrica, Phragmites communis, Eragrostis megastachya, Dactylis repens, Equisetum ramosum, Marsilea quadrifolia, etc.

J'ai déjà dit que le petit nombre des espèces d'Alg'ues qui vivent sur le littoral chinois, frappe d'abord l'observateur. Une foule de causes s'opposent, je crois, à la production de nombreux'Thalassiophytes dans les localités que j'ai explorées. Dans les régions intertropicales, la haute température de la mer (de $+24^{\circ} \grave{a}+32^{\circ}$ centig.), la composition granitique ou micaschisteuse des roches du littoral. ainsi que la formation de nombreux bancs madréporiques qui recouvrent le fond de la mer, doivent exercer une grande influence sur la végétation des plantes marines, et réduire considérablement, par leur action directe, le nombre des espèces qui peuvent. vivre dans ces parages. Les roches micaschisteuses qui entourent lîle de Hong-Kong dans le sud de la Chine, et les falaises abrup- 
tes du cap de T'ché-fou dans le nord, sont soumises sans cesse à la violence destructive des lames venant de la haute mer et des nombreux cyclones qui désolent cette partie de l'Océan asiatique oriental. Si on ajoute à ces causes dissolvantes des roches du littoral l'action d'un froid très-rigoureux dans le nord de la Chine, et la composition uniformément vaseuse du fond de la mer dans le golfe de Pe-lchi-ly, on pourrait expliquer avec quelques probabilités les circonstances qui tendent à diminuer de plus en plus le nombre des Algues vivant dans ces localités.

Les plantes marines jouent un très-grand rôle dans l'alimentation des Chinois et des Japonais, et elles trouvent aussi un emploi fréquent dans leur matière médicale. On importe à la Chine, de provenance japonaise, des quantités considérables de Laminaires (Laminaria japonica et $L$. debilis), qui servent à la confection des grelées dont les Chinois sont très-friands. Le Spharococcus lichenödes, Algue spéciale à l'Qcéan Indien intertropical, et nommé Agar-Agar par les habitants des îles de la Malaisie, sert aux mêmes usages que les Laminaires du Japon. On emploie aussi le Gelidium cartilagineum ou Tsai-Hôa des Chinois, Algue qui remplace souvent à la Chine, mais à moindres frais, les fameux nids de Salanganes, dont le prix encore très-élevé n'est accessible qu'aux privilégiés de la fortune.

La thérapeutique chinoise fait usage comme vermifuge d'un mélange de petites espèces de Thalassiophytes du littoral. Ce mélange vermifuge nommé Lou-jong-tsao dans la pharmacopée chinoise, c'est-à-dire la médecine des vers intestinaux, est l'analogue de notre mousse de Corse indigène. Je l'ai trouvé composé à Yantaï (ville située près de l'ancien camp français de Tché-fou) des espèces suivantes (1):

Chordaria flagelliformis Agardh

Laurencia thuyoïdes Kutz...

Rytiphlaa capensis Kutz...

sinensis 0. Debeaux.

Gelidium corneum Lamour

Grateloupia filicina $\mathrm{Ag}$.
Abondant.

Peu abondant.

id.

Abondant.

Peu abondant.

Abondant.

(1) Revue des mémoires de médecine et pharmacie militaires (octobre 1873) : Algues marines de Bastia (Corse), par O. Debeaux. 
L'Algue marine qui est si connue des navigateurs sous le nom de raisin des tropiques, le Sargassum bacciferum, est égalenent employée par les médecins chinois, soit comme vermifuge, soit dans le traitement du goître. Toutes les productions marines en général : crustacés, mollusques, échinodermes, annélides, zoophytes, etc., sont fort appréciées par les habitants de l'Empire du Vilieu, où leur emploi passe pour rendre l'homme plus vigoureux dans toutes les circonstances de la vie. (L. Soubeiran [1].)

Mes récoltes phycologiques faites sur le littoral chinois pendant les années 1860-1861 ne sont représentées que par 26 espèces appartenant à 10 familles et 18 genres. Les Fucacées, dont la texture est en général solide et résistante, représentent environ le tiers de mes récoltes. La détermination de ces Algues a été revue avec le plus grand soin par mon regretté ami feu René Lenormand (de Vire), et par M. Areschoug, le savant phycologiste suédois, pour qui l'étude des Algues exotiques n'offre aucune difficulté. M. Areschoug a reconnu, en outre, deux espèces nouvelles parmi les Algues chinoises soumises à son examen (Teste cl. R. Lenormand in litteris).

La liste des Algues qui va suivre présente donc tous les caractères de la plus grande exactitude, puisqu'elle s'appuie sur l'opinion de deux botanistes éminents, dont le nom fait, à juste titre, autorité dans la science. Je saisis cette occasion pour décrire, quoique bien tardivement, les deux Algrues reconnues nouvelles par M. Areschoug, et dont l'une, le Blossevillea Lenormandiana, porte depuis plusieurs années dans mon herbier le nom de l'excellent ami à qui je l'avais dédiée en 1867 , et que des circonstances indépendantes de ma volonté ne m'ont pas permis de livrer plus tôt à la publicité.

(1) La matière médicale des Chinois, par le Dr L. Soubeirau, et Dabry de Thiersant. Paris, 1873.

(1) Essai sur la pharmacie et la matière médicale des Chinois, par O. Debeaux. Paris, 1867. 
Fam. 1. FUCACÉES Agardh.

Gen. I. SARGASSUM Ag.

1. S. bacciferum Ag., Spec.alg. I, p. 6; Fucus natans Lin., Spec 1628; Hä̈-tzé des Chinois, qui veut dire graines ou fruit de la mer.

Habitat: Rade de Victoria (Hong-Kong), et sur les rivages du continent chinois à Kow-long (Kouan-tong); très-abondant sur la plage après les coups de mer.

Area geogr. : Océan ,Atlant.; Oc. Indien; le Pacifique; mers de Chine, dans les régions intertropicales.

2. S. carpophyllum J. Ag., Syst. alg., p. 304, nº 33.

Hab.: Rade de Victoria (Hong-Kong) et falaises de Kow-long (continent chinois).

Ar. geog.: Oc. Indien (rivages de Ceylan); Oc. austral (NouvelleHollande); mer de Chine (Areschoug et O. Debeaux); mer du Japon.

3. S. gracile J.Ag., Fuc. 310; Kutz., Spec. alg., p. 620; Miquel, Cat. mus. bot. Lugd.-Batav., p. 141.

$H a b$. : Mêmes lieux que les espèces précédentes.

Ar. geog. : Océan Indien; mers de Chine et du Japon.

4. S. polycystum Ag., Syst.alg., p.304; Kutz., Spec.alg., p. 611. $H a b$. : Rochers du littoral, dans la rade de Singapoore. Ar. geog. : Oc. Indien (Détroit de la Sonde).

\section{Gen. 2. BLOSSEVILLEA Decaisne.}

(Surgassum Ag. ex parte.)

5. Bl. Lenormandiana O. Debeaux, Mss. in Herb. (1867). Aff. S. heterophylli Ag.

«Blossevillea (Sect. Heterophyllæ) 1-2 pedalis, caule flexuoso, " subsimplici, nodoso, superne ramoso, subcorymboso, pennam " anserinam crasso, per totam longitudinem aculeis recurvis raris » que munito;ramulis inferioribus paucis (1-2), elongatis, flexuosis, " superioribus fructiferis linearibus, parum divaricatis ; foliis cau" linis breviter petiolatis, profunde inciso-dentatis (dentibus ple) rumque biserratis), 20 millim. circiter latis, 70 mill. longis, an- 
"gustissime lanceolatis, superioribus integerrimis, brevioribus, » linearibus que, vix sinuato-denticulatis; carpomatibus subra»cemosis, axillaribus, solitariis, teretibus, breviter pedicellatis, » bracteis filiformibus intermixtis; aerocystis subglobosis, mi» nutis, magnitudine pisi, breviter petiolatis, folio brevi non " coronatis. Color exsiccatione nigrescens. "

Hab. : Ad rupes subemersas maris sinensis, loco dicto Kowlong, provinciæ Kouan-tong, haud procul insulam Hong-Kong dictam, in limite maris, ubi rara occurrit. Fructiferam legi $20^{\circ}$ die mensis maii anni 1860.

Le Blossevillea Lenormandiana est voisin du $B$. heterophylla Kutz. (Sargassum heterophyllum Ag., Spec. I, p. 21). Il en diffère par ses stipes plus courts, sillonnés et noueux à la base, et non triquètres; par ses feuilles inférieures plus larges et plus allongées, doublement dentées; par les aérocysties des rameaux supérieurs moins nombreuses, et non couronnées par la dernière feuille.

Ar.geog. : Mer de Chine.

\section{Gen. 3. SPONGOCARPUS Kutzing.}

6. S. Horneri Kutz., Phyc.gen., p. 365, et Spec.alg., p. 631 ; Sargassum Horneri Ag., Spec. alg. I., p. 38; Miquel (loc. cit.), p. 141; Fucus Horneri Turner.

Hab. : Rade de Victoria (Hong-Kong); Kow-long (continent chinois), sur les roches micaschisteuses du littoral, à peu de profondeur.

Ar. geogr. : Mers de Chine, du Japon et de Corée.

\section{Gen. 4. HALOCHLOA Kutzing.}

7. H. patens Kutz., Phyc.gen., p. 366, et Spec.alg., 632; Sargassum patens Ag., Spec. I, p. 37: Fucus pilulifer var. major Turn. Tab. 65.

$H a b$. : Grande baie de Ki-tsen-sôo et rade de Yan-taï (presqu'île de Tché-fou, prov. du Chang-tong): sur les rochers submergés, à la limite de la basse mer.

Ar. géog. : Mers de Chine et dn Japou.

Gen. 5. MYAGROPミIS Kutz.

8. M. Thumbergii Kutz., Spec.alg.,p. 635: Cystoceira Thumbergii 
Ag., Spec. I, p. 81; Fucus Thumbergii Roth, Cat. bot., 3, 107. Hab. : Sur les rochers submergés de Yan-taï, au-dessous de la pointe du T'umulus (ancien camp de l'armée expérlitionnaire française), près du cap de Tché-fou.

Var. B. Swartzii Kutz. (loc. cit.); Cystoceira Srartzii dg.. Spec. I, p. 82; Cystophyllum Swartzii J. Ag., Syst., 233.

Hab. : Mêmes localités que le type.

Ar. geog. : Mers de Chine, du Japon et de la Corée.

Obs. : La famille des Laminariées produit un petit nombre d'espèces, dont l'usage, pour l'économie domestique, est pour ainsi dire universel dans toute la Chine. Les Chinois attribuent au Laminaria saccharina Lamour. (Fucus saccharinus Thumb. Flor. jap., 346 ; Loureiro, Flor. cochinch., p. 847) des propriétés nutritives et surtont aphrodisiaques. Cette espèce, nommée Haï-taï, Koûanpou, Hä̈-Hốan (P. Smith), Yan-tsaï, Chai-tai (Tatarinow) par les Chinois, et Kan-Hôa par les Japonais, croît principalement sur les côtes du Yé-so au Japon, où on y récolte trois variétés principales de cette espèce.

Le Laminaria saccharina, après avoir été lavé avec soin dans l'eau douce, est ensuite séché à l'air libre, puis incisé en petits fragments. Dans cet état, on en forme de grosses balles, qui sont expédiées dans les provinces du nord et du centre de la Chine. Cette Algue est surtout fort appréciée comme substance alimentaire. On en retire par décoction une gelée nommée Chin-chou, à laquelle on ajoute du sucre et divers aromates. Les Chinois ont la croyance que toutes les plantes marines gélatineuses ont la propriété de donner à l'homme plus de force et de vigueur; aussi se font-ils servir cette gelée dans leurs principaux repas.

On recueille également au Japon et sur les rivages du Changtong en Chine (Léon Soubeiran et Dabry) les Laminaria japonica, Suringar, et $L$. debilis Agardh., qui sont parfois substitués au L. saccharina et servent aux mêmes usages.

Fam. 2. DICTYOTÉES Lamouroux.

Gen. 6. PADINA Adanson.

9. P. pavonia Gaillon, Rés. class. thalass., p. 25; Zonaria pavonia Ag., Spec. I, p. 125: Dictyota pavonia Lamour. 
Hab. : Sur les rochers, dans les petites flaques d'ean de la rade de Yan-taï, à basse mer. Très-rare dans cette localité.

Ar. geog. : Médit. et Adriat.; mer Rouge; Océan Atlant. (côtes de France, d'Espagne et d'Angleterre); Océan Indien et mer de Chine.

\section{Fam. 3. CHORDARIÉES Agardh.}

\section{Gen. 7. CHORDARIA Ag.}

10. Gh. flagelliformis Ag., Spec. I, 366; Kutz., Spec.alg., p. 546: Gigartina flagelliformis Lamour.; Fucus fagelliformis Turn. Hab.: Sur les rochers submergés à la pointe du cap Tché-fou, et dans la baie de Ki-tsen-sôo, sur les roches exposées à l'action des lames.

Ar. geog. : Océan Atlant. et le Pacifique (îles Campbell); mer de Chine.

\section{Fam. 4. RHODOMÉLACÉES Agardh.}

\section{Gen. 8. RYTIPHLAA Ag.}

11. R. capensis Kutz., Phyc. gen., p. 448, et Spec. alg., p. 845.

$H a b$. : Très-abondant sur tous les rochers exposés aux lames venant de la pleine mer; à la pointe du tumulus (camp de Tchéfou); baie de Ki-tsen-sôo et les roches à l'entrée de la rade de Yan-taï.

Ar.geog.: Océan Atlant. (cap de Bonne-Espérance): mer de Chine.

12. R. sinensis O. Debeaux, Mss. in Herb. (1867).

$R$. sinensis 0. Deb. in Recueil de mémoires de médecine et pharmacie militaires "Algues marines de Bastia, " ${ }^{\circ}$ d'octobre 1873 (sine descriptione).

«R. sinensis, phycomate diffuso, cæspitoso, vage ramosissimo, »1-2 pollicari ; ramis spinuliformibus, bipinnatis, subdichotomis, 》apicibus ramorum lanceolatis uncinatis; pinnis pinnulis que จ erectis, filiformibus, setaceis. Color exsiccatione aterrimus. $C y s$ 》 tocarpia non vidi.»

$H a b$. : Ad rupes maritimas, in limite maris, propè promontorium dictum Tché-fou sinensium, ubi vulgatissima reperitur.

Ar. geog. : Mer de Chine. 
Cette espèce nouvelle (T'est. clar. Lenormand et Areschong) (croît en abondance sur tous les rochers du littoral, à la pointe du camp de Tché-fou, et non loin de la rade de Yan-taï (Prov. du Chang-tong). Le Ryliphlaa sinensis offre, sous des dimensions plus petites, le facies de l'Hypnæa musciformis, algue florilée dont l'aire d'extension est très-développé, mais que je n'ai pas rencontrée sur les rivages de la Chine.

Fam. 5. LAURENCIACEES Agardh.

\section{Gen. 9. LAURENCIA Lamour.}

\section{L. thuyoïdes Kutzing (Teste Areschoug !)}

«Phycomate cartilagineo, et roseo decoloranti lutescente, 2-3 "pollicari, erecto, tereti, gracillime pyramidato-ramoso; ramis » alternis, patentibus, filiformibus; ramulis abbreviatis, suboppo》 sitis, summis divaricatis, apice incrassatis, glomerulos carpo» cloniorum gerentibus: carpocloniis brevissimis apice globoso» clavatis. "

Hab. : Rochers submergés à la pointe du cap Tché-fou. Fructifié en novembre.

Ar.geog.: Mer de Chine.

Obs. : Je conserve à cette espèce le nom qui lui a été donné par M. Areschoug, et que ce phycologiste a écrit de sa propre main sur les spécimens qui lui ont été communiqués.

Gen. 10. CHAMPIA Lamour.

14. Ch. Kotschyana Endlich. et Dies, in Bot. Zeit., 1845, p. 269 : Kutz., Spec. alg., p. 861.

Cette rare espèce, dont je n'ai rencontré que trois spécimens, n'a été signalée jusqu'à présent que dans le golfe Persique. Sa petite taille (2 à 3 centimètres), sa couleur d'un pourpre clair, et ses ramifications peu nombreuses et surtout peu profondes, la distinguent suffisamment du Champia tasmanica Harvey.

Kutzing mentionne (loc. cit.) le Ch. Kotschyana parmi les species inquirenda, avec la diagnose suivante :

"Phycomate compresso, vage bipinnatim ramoso, ramis ramu"lisque abbreviatis, alternis, basi attenuatis, subclavatis ; articulis » diametro triplo brevioribus. Color in sićco sordide purpureus." 
Hab. : Sur les rochers, dans les flaques d'eau tranquille; rade de Yan-taï, au niveau de la basse mer.

Ar.geog. : Océan Indien (Golfe Persique, île Karek); mer de Chine.

Obs.: Le Spharococcus lichenö̈des Ag., Spec. I, p. 317; Gracilaria lichenoüdes Grév.; Plocaria candida L. Soubeiran sub Ché-hôa-tzé, in Mat. médic. chin., p. 86; Tsaï-Hôa (ex parte) 0. Debeaux, in E'ssai pharm. et mat.médic.chin., p. 106, est une Algue de l'Océan Indien intertropical, dont on importe à la Chine des quantités considérables pour la confection des gelées alimentaires. Les Malais donnent également à cette espèce le nom d'Agar-Agar, et les Chinois celui de Haï-tzé, qui veut dire Herbe de mer, Produit ou fruit de la mer.

Fam. 6. GÉLIDIÉES Kutz.

Gen. 11. GELIDIUM Lamour.

15. G. cartilagineum Gaillon, Rés. class. thalass., p. 15; Kutz., spec. alg., 763; Sphcrococcis cartilagineus var. setaceus L. Soubeiran, loc. cit., p. 87, sub Ché-Hôa-tsaï. - O. Debeaux, loc. cit. sub Tsaï-Höa, p. 106, ex parte.

$H a b$. : Sur les rochers et les rivages des mers intertropicales, à Hong-Kong, Amoy, etc., où cette Algue est recueillie par les indigènes, pour ses propriétés nutritives et antidyssentériques.

Ar. geog. : Océan Atlant. (cap de Bonne-Espérance); Iles Canaries; Océan Pacifique (côtes de la Californie); mers chaudes de la Chine.

16. G. corneum Lamouroux, Essai thal., p. 41; Miquel, Cat. mus. bot. Lugd.-Bat., p. 139; Spharococcus corneus Ag., Spec. I, p. 279.

Var. sericeum Kutz.(loc. cit.), p. 764; Ag., Spec.(loc.cit.); Fucus sericeus Gmelin, Tab. 15 (Teste Areschoug !).

$H a b .:$ Sur les rochers submergés un peu au-dessous du niveau de la mer; eaux peu profondes de la rade de Yan-tä̈; baie de Kitsen-sôo, non loin du promontoire de la presqu'île de Tché-fou.

Ar. geog. : Médit. et Adriat.; mers de Chine et du Japon.

Obs.: Le Gelidium corneum type se retrouve dans la mer du Japon. 
Fam. 7. RHODYMÉNIACÉES Harvey.

Gen. 12. RHODYMENIA Gréville.

17. Rh. palmata Lamour.; Gréville, Alg. Brit.: Delesseria palmata Lamour; Spherococcus palmatus Kutz.

Var. sinensis Debeaux, Mss. in Herb., 1867.

"Phycomate minutulo (vix 2 centim. alto), dichotomo, mem» branaceo; segmentis elongatis, angustissimis. Color exssica》 tione atro-purpurens. »

$H a b$. : Sur les rochers, dans les petites fiaques d'eau tranquille: grande rade de Yan-taï, près du promontoire de Tché-fou: rare dans cette localité.

Ar. geog. : Océan Atlant. (côtes de France et d'Angleterre); Océan Pacifique; mer de Chine.

Fam. 8. CRYPTONÉMIACÉES Hảrvey.

Gen. 13. DUMONTIA Lamour.

18. D. filiformis Gréville, Alg. Brit., p. 105; Kutz., Spec. alg., 719; Halymenia filiformis Ag., Spec. I, 214; Fucus contortus Gmel.; Fuc., 181.

Var. Tenuis Ag. (loc. cit.), 216; Kutz. (loc.cit.), 719.

Hab. : Roches micaschisteuses du cap Tché-fou; rade de Yan-taï et falaises de Ki-tsen-sôo; assez commune.

Ar.geog. : Océan Atlant. (côtes de France et d'Angleterre): cap de Bonne-Espérance; mer de Chine.

Gen. 14. GRATELOUPIA Agardh.

19. G. filicina Ag., Spec. I, 223; Kutz., Spec. alg., 730; Delesseria filicina Lamour.; Fucus filicinus Wulfen.

Hab. : Très-abondant sur toutes lesroches submergées, dans la rade de Yan-taï; cap de Tché-fou; baie de Ki-tsen-sôo, où cette espèce atteint les limites de la haute mer.

Ar. géog. : Médit. et Adriat.; Océan Atlant. (côtes de France et d'Angleterre); cap de Bonne Espérance; mer de Chine. 
20. Gr. conferta Zollinger, in Plante japonica exsiccalce no 2408 (T'este Areschoug); Grat.filicina var.conferla Kutzing, Spec. alg., p. 730 .

«Hæc species a Gr. filicinâ differt, phycomate humiliori, cæspi》 toso subfastigato, rigidiori; pinnis brevioribus, rigidis, angus》tissimis, lanceolatisve. »

$H a b$. : Mêmes localités que le Gr. filicina, mais beaucoup plus rare.

Ar. geog. : Océan Indien (Java); mer de Chine.

Fam. 9. SIPHONACÉES Gréville.

Gen. 15. CODIUM Agardh.

21. G. tomentosum Ag., Spec. alg. I, 451; Spongodium tomentosum Lamour.

$H a b . \cdot$ Sur les rochers submergés de la baie de Ki-tsen-sôo; rejeté sur la plage après les coups de mer.

Ar.geog. : Médit. et Adriat.; Océan Atlantique; cap de BonneEspérance; Océan Pacifique; Oc. Austral (Nouvelle-Hollande): mer de Chine.

Gen. 16. BRYOPSIS Lamour.

22. B. plumosa Huds.; J.Ag., Alg. Médit., p. 21 ; Kutz., Spec. alg., p. 493; Lejolis, Algues de Cherbourg; Harvey, Phyc. Brit., etc.

Hab. : Parois des roches submergées, dans les petites flaques d'eau de la rade de Yan-taï; roches au fond de la baie de Ki-tsensôo à basse mer.

Ar.géog.: Océan Atlant. (côtes de France et d'Angleterre); Médit. et Adriat.; mer de Chine.

23. B. arbuscula Agardh, Spec. I, 351; Lamour., Mém., p. 135; B. plumosa var. arbuscula J. Ag., Alg. Médit.; B. hypnoïdes Lamour. (loc. cit.), p. 136; Kutz., Spec. alg., 493.

$H a b$. : Les mêmes localités que l'espèce précédente, dont il ne diffère que par ses ramifications plus nombreuses et divergentes en tous sens.

Ar. geog. : Médit. et Adriat.; mer de Chine. 
Fam. 10. ULVACÉES Agardh.

(Enteromorphées Kutz. ex parte.)

Gen. 17. PHYCOSERIS Kutz.

24. Ph. australis Kutz., Phyc.gen., 298, et Spec.alg., 476; Miquel (loc. cit.), 141; Ulva latissima var. palmala Ag., Spec. I, 409. Hab.: Grande rade de Yan-taï, dans les flaques d'eau à basse mer; rejeté sur la plage de Ki-tsen-sôo, après les coups de mer. Doit se retrouver probablement sur les rivages de tout le golfe de Pe-tchi-ly.

Ar.geog.: Médit. et Adriat.; Océan Austral (Nouvelle-Hollande); mers de Chine et du Japon.

\section{Gen. 17. ENTEROMORPHA Link.}

25. E. intestinalis Link. Hor. phys. Berol., p. 5 ; Kutz, Spec.478; Miquel (loc. cit.), p. 129; Solenia intestinalis Ag., Syst., p. 185 ; Ulva intestinalis Lin.

Hab.: Grande rade de Yan-taï, sur les rochers et les flaques d'eau à basse mer.

Ar. geog. : Mers d'Europe; Oc. Atlant. (côte d'Amérique); mers de Chine et du Japon.

26. E. compressa Grév., Alg. Brit., 180; Miquel (loc. cit.), 139; Ulva compressa Lin.

Hab. : Même localité que l'espèce précédente.

Ar.geog. : Mers d'Europe; Océan Atlantique (côtes d'Amérique); Oc. Indien; Oc. Austral (Nouvelle-Hollande); Oc. Pacifique (Iles Sandwich et les Mariannes); mers de Chine et du Japon. 
TABLEAU indiquant par familles les principales affinités de géographie botanique des Algues récoltées en Chine pendant les années 1860-62.

\begin{tabular}{|c|c|c|c|c|c|c|}
\hline $\begin{array}{c}\text { DÉSIGNATION } \\
\text { des } \\
\text { FAMIILES }\end{array}$ & $\begin{array}{c}1 \\
\text { Spéciales } \\
\text { aux mersde } \\
\text { Chine }\end{array}$ & $\begin{array}{c}2 \\
\text { Mers } \\
\text { de Chine et } \\
\text { du Japon }\end{array}$ & $\begin{array}{c}3 \\
\text { Mers } \\
\text { de Chine et } \\
\text { du Japon, } \\
\text { Oc. Indien }\end{array}$ & $\begin{array}{c}4 \\
\text { Chine } \\
\text { et Japon } \\
\text { Oc.Atlant. } \\
\text { Méd., Adr. }\end{array}$ & $\begin{array}{c}5 \\
\text { Chine, } \\
\text { Oc. Indien, } \\
\text { Oc. Atl., } \\
\text { Pacifique }\end{array}$ & $\begin{array}{c}6 \\
\text { Chine, } \\
\text { J a p on, } \\
\text { Méditerr., } \\
\text { Adriatiq. }\end{array}$ \\
\hline Fucacees... .............. & 1 & 4 & 2 & " & 1 & $»$ \\
\hline Dictyotées..... & ” & " & $"$ & 1 & 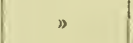 & ڤ \\
\hline Chordariées.......... & $"$ & ” & " & " & 1 & $»$ \\
\hline Rhodomélacées.......... & 1 & " & $"$ & $"$ & 1 & $n$ \\
\hline Laurenciacées............ & 1 & 》 & $"$ & $"$ & ] & " \\
\hline Gélidiées............. & " & $"$ & $"$ & $"$ & 1 & 1 \\
\hline Rhodyméniacées ........ & " & $"$ & $"$ & " & 1 & $»$ \\
\hline Cryptonémiacées... & $"$ & $"$ & 1 & 1 & 1 & " \\
\hline Siphonacées ..... & $"$ & 》 & " & z & ” & 1 \\
\hline Ulvacées.... & " & 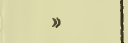 & 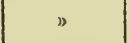 & 2 & $"$ & 1 \\
\hline Totaux... & 3 & 4 & 3 & 6 & 7 & 3 \\
\hline
\end{tabular}

\section{RÉGAPITULATION}

$1^{\circ}$ Alg'ues spéciales aux mers de Chine

$2^{\circ}$ Algues qui vivent à la fois sur le littoral de la Chine, du Japon et de la Corée.

$3^{\circ}$ Algues des mers de Chine et du Japon et qui se retrouvent dans quelques localités de l'Océan Indien

$4^{\circ}$ Algues des mers de Chine ou dn Japon, et qui vivent aussi dans l'Océan Atlantique, dans la Méditerranée et l'Adriatique...........................................................

$5^{\prime \prime}$ Algues de la mer de Chine, qui se retrouvent dans quelques localités de l'Océan Indien, de l'Océan Atlantique et du Pacifique

$6^{\circ}$ Algues des mers de Chine ou du Japon, qui se retrouvent dans la Méditerranée et l'Adriatique seulement............

Obserration : Je n'ai pas eu l'occasion de recueillir en Chine, soit des Algues confervacées, soit des Diatomacées marines ou d'eau 
douce, qui auraient pu servir aujourd'hui de moyen de comparaison, sous le rapport de leurs affinités de géographie botanique, avec les Algues de ces mêmes familles énumérées par M. Miquel, et qui font partie de l'herbier du musée de Leyde. C'est une lacune que je signale aux botanistes, mais qui ne peut infirmer de beaucoup les observations déjà faites sur les Algues du littoral chinois.

Perpignan, 25 avril 1874. 


\section{A D DENDA}

\section{Algues récoltées en Ghine en 1860 et 1861.}

Madame veuve René Lenormand ayant eu à disposer, au cours de l'anné 1878, quelques livres de botanique dans la bibliothèque spéciale dont la jouissance lui a été léguée par son mari, l'illustre et très-regretté phycologiste de Vire (Calvados), a trouvé placé sur un des rayons de cette bibliothèque, un petit carton renfermant quelques algues marines récoltées par moi dans le nord de la Chine, en 1860 et 1861. Ces divers échantillons, déjà examinés avant sa mort, par mon correspondant et ami R. Lenormand, devaient être soumis au visa de M. de Martens, lorsque la guerre franco-allemande a éclaté tout à coup en 1870, et a empêché l'envoi de mes algues au célèbre professeur de Berlin. $\mathbf{M}^{\mathrm{m}}$ Lenormand a pensé que ces spécimens pouvaient m'être encore de quelque utilité, et a eu l'obligeance de me les renvoyer en octobre 1878.

J'ai retrouvé dans ce fascicule, dont j'avais perdu entièrement le souvenir, depuis dix ans que je l'avais expédié à M. R. Lenormand, la plupart des algues marines que j'avais recueillies dans le nord de la Chine, et en plus deux espèces nouvelles pour la liste que j'en ai publiée, et qui portent à vingt-huit, au lieu de vingt-six, le nombre des algues récoltées sur le littoral chinois.

\section{Fam. I. FUCACÉES Agardh.}

4 (bis). Gystophora linearifolia 0. Debeaux (Spec. nova teste cl. R. Lenormand). - Octobre 1878.

"Frons gracilis, elegans, caule erecto flexuoso, in diametro 》 pennæ anserinæ crassitiem haud superante, 22-25 centim. alto, „ basi nudo, simplici, irregulariter nodoso, infra medium ramoso; - ramis 3-4 erecto-patulis, per totam longitudinem tuberculis $\downarrow$ raris parvisque munitis; ramu!is secundariis alternis, erecto- 
》patulis, superioribus fructiferis; foliis inferioribus deciduis » (itaque ignotis), eis ramorum ramulorumque anguste linearibus, »80-90 centim. elongatis, 1 millim. latis, parum subulatis, ramos » sæpe superantibus, omnibus integris ex axillis ramorum nas»centibus; aerocystiis minutis, solitariis, rarius geminis, ovato》 ellipticis, teretibus, apice arcuato-acuminatis, folio brevioribus, »pedunculatisque; pedunculo 4-6 millim. longo, gracili. Color » exsicatione nigrescens ».

$H a b$. : Sur les rochers submergés, granitiques ou micaschisteux de la rade de Ki-tsen-sôo et de Yan-taï, près du cap de Tchéfoû. - Fruct. septembre 1860.

Ar. géog. : Mer du Pé-tché-ly.

Obs. Cette nouvelle espèce du genre Cystophora, ne peut être rapprochée d'aucune des algues fucacées énumérées dans le premier fascicule de mes Contributions à la flore de la Chine. La tige inférieure rappelle, par ses nodosités irrégulières, celles du Blossevillea Lenormandiana, mais on ne saurait la confondre avec celui-ci. Le Cystophora linearifolia se distingue facilement des nombreuses espèces du genre Sargassum dont il a été démembré, par ses feuilles très-étroites, longuement linéaires, arquées-subulées, plus longues que les rameaux secondaires, et par ses aérocysties ovales-elleptiques courtement pétiolées, solitaires ou rarement géminées, arquées-acuminées au sommet, etc.

\section{Fam. 8. CRYPTONÉMIACÉES Harvey (Tribu des Gigartinées).}

20 (bis). Gymnogongrus (Oncotylus) japonicus Suringar Algæ japonicæ musei botanici Lugdun. batav. p. 36 (1870), tab. 24, fig. A (optima).

- Frons pumila, ramosissima, 4-5 centimet. alta, membranacea, » iterato dichotoma, flabellata, prolifera, laciniis gracilibus, » anguste-linearibus, compressis, superne paulum dilatatis, com» planatis, apicibus obtusis aut fuscatis, versus apices vix 1 mil»lim. latis; cystocarpiis in medio ramulorum superiorum sparsis, "subglobosis, in utraque pagina proeminentibus, frondisque »diametrum paulo excedentibus. Color in prole adulto exsica» tione fusco-purpurescens, in vivo intense purpureus. » 
Hab. : Abondant sur les rochers micaschisteux ou granitiques et découverts à marée basse, dans la baie de Yan-taï, près du cap de Tché-fou. - Fruct. septembre 1830.

Ar. géog. : Mers de la Chine et du Japon.

Obs. Cette espèce avait été rapportée avec doute par M. R. Lenormand, en 1868, au Gymnogongrus affinis Harvey. M. Suringar l'a décrite, deux ans plus tard, dans ses Algæ japonic $x$ sous le nom de $G$. japonicus, mais il n'a pu en décrire les cystocarpies dont tous les échantillons du musée de Leyde étaient dépourvus. Mes spécimens du Tché-foû sont munis de leurs fructifications, ce qui m’a donné lieu de compléter à leur égard la description de M. Suringar. Celles-ci se trouvent placées vers la base de la dichotomie des rameaux supérieurs, et au milieu du parenchyme de la fronde. Elles sont subglobuleuses et font un peu saillie sur les deux faces de la fronde dont elles dépassent même le diamètre (un millimètre au plus). La couleur de cette algue, qui est d'un pourpre vif dans la plante vivante, devient d'un brun-pourpré par la dessiccation. 



\section{CONTRIBUTIONS A LA FLORE DE LA CIIINE}

\section{FASGIGUIE II

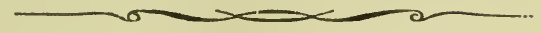 \\ FLORILLE DE SHANG-HAI (PROVINGE DE KIANG-SOU)}

COMPRENANT

l'énumération des plantes spoulanées ou généralement cultivées dans celle localité;

$$
\text { Par M. O. DEBEAUX. }
$$

PEAIIMACIEN MAJOR DE $1^{\text {ro }}$ CIASSE CHETALIER DE LA LÉGIOY D'HONERUR,

Membre de la Socièté botanique de France,

Correspandant de la Société Linnéenne de Bordeaux et de plusieurs autres Sociètés savantes.

(Extrait des Actes de la Société Linnéenne de Bordeaux, t. XXX, lre et 2e liv., 1875.)

\section{PARIS}
F. SAVY, LIBRAIRE-ÉDITEUR, RUE HAUTEFEUILLE, 24





\title{
FLORULE DE SHANG-HAÏ \\ (PROVINGE DE KIANG-SOU)
}

\author{
Par M. O. DEBEAUX, \\ Pharmacien-major de première classo, \\ Membre correspondant.
}

\section{AVANT-PROPOS}

Dans une notice déjà fort ancienne, et ayant pour titre : $S u r l a$ végétation de quelques points du littoral chinois (1), j'ai fait connaître en 1861 le résultat de mes explorations à Hong-Kong, A-moy, Shang-haï et Tché-fou, localités où j'ai séjourné en Chine pendant le cours de l'expédition militaire française de 1860 à 1862. Mes récoltes botaniques ne pouvant être nommées à cette époque, j'ai dû me borner dans la notice précitée à signaler les familles et les genres de plantes qui m’ont paru devoir caractériser la végétation de ces contrées.

Je m'étais proposé, aussitôt que mes exsiccata seraient mis en ordre et déterminés, d'en établir le catalogue par localités, et d'en faire ressortir leur distribution géographique, soit dans les diverses provinces de l'Empire chinois, soit dans les pays limitrophes de l'extrême Orient. Douze années se sont écoulées depuis cette époque, sans qu'il m'ait été possible d'étudier les plantes quej'avais rapportées de Chine en bon état de conservation, et ce n'est seulement qu'à la fin de l'année 1873 que j'ai pu les avoir nommées d'une manière exacte et rigoureuse.

J'ai hâte d'ajouter que, dans cette tâche laborieuse, j'ai été puissamment aidé par un botaniste des plus compétents, M. A.

(1) Recueil des Mémoires de médecine et pharmacie militaires, vol. $6^{\text {e }}$ (3^ série), p. 334, 1861. - Paris, Victor Rozier, éditeur. 
Franchet, à Cour-Cheverny, qui s'occupe depuis plusieurs années de l'étude spéciale de la flore du fapon. M. Franch t a revu, arec les soins les plus scrupuleux, les déterminations que j'avais déjà faites; il m’a signalé leš erreurs que j'ai commises parfois, faute d'ouvracres suffisants, et m'a inćiqué, en outre, les espèces qui ne sont signalées nulle part, et qui lui ont paru devoir être décrites comme nouvelles pour la flore de la Chine. M. Franchet a fait plus encore : afin d'avoir toute certitude à l'égard de quelques plantes douteuses ou litigieuses, il a eu l'extrême obligeance de les soumettre au visa de M. Maximowicz de Saint-Pétersbourg, l'auteur de remarquables travaux sur la végétation de l'Asie orientale, contrées dont il a été, pendant plusieurs années, l'intrépide explorateur.

Je ne saurais donc trop remercier mon honorable correspondant, M. Franchet, du concours actif qu'il m'a prêté en cette circonstance, et sans lequel je n'aurais jamais pu atteindre le but que je me suis proposé. Aussi je le prie de vouloir bien recevoir ici l'expression de ma plus vive reconnaissance.

Les localités du littoral chinois, oì j'ai herborisé à plusieurs reprises, de 1860 à 186:, sont les suivantes: Hong-Kong et Kowlong, dans le Sud de la Chine (Koûang-tong'); A-moy, dans le Fo-kien, dont je ne m'occuperai pas dans ce travail; Shang-hä̈ etWóo-sông, dans le Kiang-sou; Tchéfou et la partie montagneuse au-dessus de Yan-tä̈, dans le Chan-tong, et enfin Tien-tsin sur les rives du Peihô, dans le Pé-tché-ly. L'énumération des plantes observées dans chacune de ces trois régions : Shang-haï, Tché-fou et Tien-tsin prendra le titre de florule, nom qui indique plus par;iculièrement l'état de la végétation d'un point limité dans une vaste contrée dontl'ensemble de la flore n'est pas complètement connu.

Perpignan, ler septembre 1874. 


\section{Aspect général du territoire òe Shang-har et de sa végétation.}

La ville de Shang-haï, dont le port a été ouvert au commerce étranger en 1843, ne remonte pas à une haute antiquité. Cette citée fondée vers le vil ${ }^{e}$ siècle de notre ère, est située dans la province de Kiang-sou, par $31^{\circ} 20$ de latitude Nord, et $121^{\circ} 46$ de longitude Est, sur les rives du Whâng-pôo, à environ 15 kilomètres de l'embouchure de ce fleuve, dans le Hiang-tsé-kiang ou fleure bleu, et non loin du village de Wôo-sông.

La ville européenne qui touche la cité chinoise est divisée en trois parties appelées concessions : la concession américaine, la pre. mière en venant de Wôo-sông, qui est séparée de la concession anglaise par la rivière de Sou-tchéou; - la concession anglaise qui occupe le grand coude formé par le fleuve Whângr.jốo, et la concession française qui s'arrête aux murailles fortifiées de la ville chinoise.

Tout le pays qui entoure Shang-haï cst d'une platitude extrême, et aussi loin que la vue peut s'étendre, on n'aperçoit pas le moindre mouvement de terrain. Le sol, élastique comme tous ceux qui reposent sur l'eau, est un relai du fleure bleu, formé des sédiments amoncelés (Loam des Anglais) par ses eaux bourbeuses. D'immenses rivières, des canaux pleins d'une eau noire et fétide pendant l'été, des marécages et des tombeaux un peu partout, mais partout aussi d'adnirables cultures de riz, de coton et de diver:es plantes potagères, tel est l'aspect vrai du territoire de Shang.haï.

Entre Shang-haï et Wôo-sông, la plaine est également uniforme et marécageuse. Une chaussée assez bien entretenue par les résidants étrangers, et qui suit les sinuosités du Whâng-pòo, relie ces deux points. Il est même question aujourd'hui de les rapprocher davantage par l'établissement d'un chemin de fer. A l'époque de mon arrivée à Wôo-song, au commencement du mois de juin 1860, les Chinois avaient commencé la récolte de l'orge et d'une espèce de colza à graine oléagineuse (Brassica chinensis), et ils préparaient déjà le terrain de ces premières récoltes, pour y semer soit du coton, soit des haricots ou autres plantes potagères. Dans quelques terres, marécageuses pendant l'hiver, on récoltait au mois de juin la tève commune en fruits.mûrs, et par de nom- 
breuses irrigations, les agriculteurs chinois disposaient ces marécages à devenir de fertiles rizières.

Dans les jardins et autour des habitations rurales à WVô-sông et Shang-haï, on voit de nombreux arbres fruitiers, tels que abricotiers, jujubiers, péchers, poiriers, pommiers, pruniers, etc., ainsi que la plupart des plantes potagères de l'Europe. On admire surtout à Wôo-sông de magnifiques treilles de vignes, et à Shang-haï quelques spécimens d'arbres indigènes ou étrangers à la Chine: Magnolia yu-lan et M. purpurea, Eviobotrya japonica, Robinia pseudo-acacia, Punica granalum, Lagerstramia indica, Philadelphus coronarius, Diospyros schi-tze, Syringa vulgaris et $S$. persica, Olea fragrans, Salisburia adianthifolia, Chamarops Fortunei, etc.

Les haies des jardins et des propriétés dans la plaine de Shanghaï sont formées de plantations de bambous, dont les chaumes élevés et pressés les uns contre les autres servent à garantir les cultures de l'action des vents violents qui règnent dans ces localités. A Wôo-sông, un arbuste spécial à l'A sie-Mineure, le Fontanesia phillyreoüdes, et que je suis naturellement étonné de retrouver dans cette partie de la Cline, forme à lui seul, tant il y est abondant, toutes les haies et toutes les clôtures des propriétés rurales.

Quelques arbres conifères se font remarquer aussi par leurs formes particulières et leurs dimensions autour des pagodes et des nombreux tumulus dont le territoire de Shang-haï est couvert. Je cite parmi les plus intéressants, les Cuninghamia sinensis, Pinus Massoniana, Cupressus funebris, Cryplomeria japonica, Salisburia adianthifolia, etc.

Le botaniste voyageur Robert Fortune (1) a depuis longtemps fait connaitre le résultat de ses recherches dans la province du Kiang-sou, en 1842 et 1848 . Ce célèbre explorateur a eu pour but, non-seulement de parcourir les contrées où croissent les arbustes qui produisent le the (Thea sinensis Sims), et d'étudier sur place les divers modes de culture de cet arbrisseau, de la récolte des feuilles, de leur préparation, etc., mais encore de rechercher en Chine les végétaux dont l'introduction en Europe intéresserait

(1) Wanderings in China, by R. Fortune, London, 1847. 
l'agriculture et les horticulteurs. Robert Fortune (1) nous a donné également des renseignements très-précis sur les cultures de Shang-haï et sur les plantes d'ornement qu'il a vues dans les jardins de cette grande cité; mais il avoue que le territoire de cette contrée, à raison même des cultures variées dont il est couvert, ne pouvait lui offrir un champ d'exploration bien riche pour ses recherches le botanique.

La flore de Shang-lıaï ne me paraît pas en effet être des plus variées, et le nombre des plantes qui lui sont spéciales n'est pas considérable non plus dans un grand rayon autour de la ville chinoise et des concessions européennes. Mais toutes les plantes que l'on y recueille offrent au botaniste le plus grand intérêt, au point de vue le leur habitat et de leur extension géographique. La végétation de Shang-haï n'a pas de caractère particulier et de spécial à cette localité. On y remarque, à côté d'un petit nombre d'espèces propres à la Chine, une foule d'autres plantes que l'on retrouve un peu partout, dans les provinces limitruphes, en Corée, au Japon, dans les provinces boréales de l'Amour et de l'Ussuri, soit encore dans l'Asie intertropicale ou tempérée, les Indes orientales, les îles de l'Océanie et de l'Océan Indien, l'Afrique centrale et boréale, l'Europe australe et les deux Amériques.

Ce qui frappe au premier abord l'observateur qui parcourt les plaines de Shang-haï et de Wôo-sông, c'est le facies européen des plantes les plus vulgaires dans cette partie de la province du Kiang-sou. Quelques-unes sont si nettement caractérisées, qu'il est facile de les nommer sur place avec certitude. Je cite les suivantes qui m'ont le plus frappées lors de mes herborisations à Shanghaï et Wôo-sông : Cardamine hirsuta, Lepidium ruderale, Thlaspi arvense, Capsella bursa-pastoris, Melilotus parviflora, Oxalis corniculata, Myriophyllim spicalum, Daucus carota, Erigeron Iinifolius, Brunella vulgaris, Glechoma hederacea, Acorus calamus Sagittaria sagittifolia, Lemna minor, Spirodela arrhyza, Setaria glauca, Poiypogon littoralis, etc.

A part ces quelques espèces pour la plupart ubiquistes, et un petit nombre de spéciales à la Chine, le reste de la végétation se compose en grande partie (les $5 / 6^{\text {es }}$ environ) de plantes signalées,

(1) Two visits to the Thea coutries of China, by R. Fortune, London, 1853, in-8. 
dans l'empire japonais, par les botanistes voyageurs Thumberg (1), Von Siebold (2), et plus récemment encore par MM. Maximowicz (3), Miquel (4), Franchet et Savatier (5). J'insiste sur cette analogie de la végétation du littoral chinois avec celle du Japon, analogie vraiment remarquable, et que l'on ne peut expliquer que par les conditions de climatologie, de température, etc., de ces deux contrées voisines l'une de l'autre. Le Japon n'est, en effet, séparé de la Chine que par un grand espace de mer, que l'on franchit par bateaux à vapeur en 2 ou 3 jours, et dans lequel s'avance au nord, comme intermédiaire pour ainsi dire, la presqu'île de Corée. D'un autre côté, les rapports journaliers du commerce de Shang-haï avec le Japon ont facilité l'introduction à la Chine de plusieurs végétaux originaires de cette contrée, et qui ont pu s'y acclimater facilement.

Le climat de Shang-haï est très-variable, et les extrêmes de chaud et de froid y sont plus considérables que dans les provinces méridionales. Pendant les mois de juillet et aout, le thermomètre centigrade reste quelquefois plusieurs jours de suite à + $38^{\circ}$ cernt. L'hiver y paraît être aussi des plus rigoureux, et souvent le thermomètre descend à $-12^{\circ}$ centigrades. A l'exception de ces extrêmes de chaud et de froid, le climat de Shang-haï peut être comparé à celui de l'Espagne et de l'Italie centrale, du midi de la France, etc. La fertilité du sol n'a d'égale dans aucune autre partie de la Chine, et l'on peut dire, avec Robert Fortune, que cette contrée n'est qu'un immense jardin.

J'ai principalement exploré dans le territoire de Shang-haï les environs de cette vieille cité et des concessions européennes, les champs cultivés, les pâturages, les rizières, les canaux d'irrigation, les marais et les rives du fleuve Whàng-poo. Les anciens remparts en briques de Shang-haï, ainsi queles terrains incultes autour

(1) Thumberg, Flora Japonica, Lipsiæe, 1774, in-8..

(2) Siebold et Zuccarini, Flora Japonica, Lugduni-Batavorum, 1835-1844, in-fol.

(3) Maximowicz, Diagnoses plantarum novarum, in Mém. Acad. Scien.. St-Pétersbourg, Décades 1-14, 1870-1874.

(4) Miquel, Prolusio Florce Japonicce, Lugduni-Batav., in-fol., 1870.

(5) Franchet et Savatier, Enumeratio plantorum in Japonia sponte crescentium, 1 vol. in-8, 1874. 
des tombeaux dont ce pays est couvert, ontété l'objet de nombreuses recherches de ma part. En somme, mes récoltes botaniques commencées à Shang-haï et Wôo-sông en juin 1860, puis reprises en 1861 et terminées en avril 1862, représentent assez exactement la végétation de cette partie de la province de Kiang-sou.

Dans l'énumération qui va suivre, j'indique pour chaque espèce sa synonymie spéciale à l'Asie orientale, son habitat, sa station, son nom indigène chaque fois que j'ai pu me le procurer, ou en trouver la mention dans les livres spéciaux. La dispersion géographique de chaque plante dans les diverses régions du globe fait l'objet d'une observation particulière désignée du nom d'aire géographique, et placée immédiatement après l'habitat. Des diagnoses françaises ou latines, quelques notes et observations sont parfois ajoutées aux plantes qui m'ont paru les plus intéressantes, soit par leur rareté, soit par leur emploi dans l'économie domestique ou les arts industriels des Chinois.

Je n'ai rencontré dans mes nombreuses herborisations à Shanghaï qu'une espèce de graminée du genre Isachne, que je décris aujourd'hui comme nouvelle pour la flore de la Chine. Cette graminée (I. altissima Mihi) abonde le long des canaux d'irrigation entre Shang-haï et Wôo-sông, et j'ai lieu d'être étonné qu'elle n'ait pas été signalée plus tôt dans cette localité.

Il m'a été impossible, jusqu'à présent, de prendre connaissance des travaux qui ont été publiés par les botanistes anglais sur la végétation de la province de Kiang-sou. Ces publications faites par leurs auteurs, soit à Shang-haï même, soit dans les mémoires de la Socièté asiatique et chinoise de Calcutta, sont en général introuvables en France. Aussi ai-je l'espoir que la Florule de Shang-hai. dont j'essaie d'esquisser le tableau, rendra quelques services à tous ceuxqui s'intéressentaujourd'hui à la connaissance des plantes de l'extrême Orient. 


\section{RENONCULACÉES. Jussieu.}

1. Ranunculus ternatus Thumberg Flora Japonica, p. 241 ; Decandolle in Prodromus regni vegetabilis 1, p. 31; A. Gray Planta coll.jap. in exp. Perry (1859).

«Planta polymorpha, caulibus erectis vel repentibus, plus " minusve hirsuto-lanatis, omnibus semper trifidis. »

Habitat : Wôo-sông, Shang-haï et ailleurs : bords des fossés aquatiques; prairies des rives dụ Whâng-pôo, etc. - Juin.

Area géographique: Chine (Provinces de Kiang-sou, Tché-kiang, Chan-tong, Pé-tché-ly, etc.).- Japon.

Ols. : Par son état de polymorphisme le $R$. chinensis est à peine distinct du $R$. ternatus. D'après M. Maximowicz, le $R$. chinensis se reconnaîtrait surtout à ses carpelles largement marginés. Or, ce caractère se rencontre également sur les échantillons du R.ternatus récoltés à Wôo-sông. Cependant, il est à remarquer que dans les spécimens du $R$. chinensis provenant de la même localité, le bord des carpelles est encore plus large et beaucoup plus mince que dans le $R$. ternatus; les carpelles ont de plus une forme irrégulièrement ovale et comme gibbeuse. Ce caractère est suffisant, je crois, pour justifier la séparation de ces deux espèces.

2. R. chinensis Bunge in Enumeratio plantarum china borealis $\mathrm{n}^{\circ} 10$ (Bull. mém. sav.étran. mosc., vol.10, p. 76); Walpers Repert.botan. sys. 1, p. 39; Regel Tent. for. ussur., p. 8.

"Planta polymorpha, caulibus erectis, petiolisque hirsutissimis, "foliis longe petiolatis, trisectis, adpresse hispidis, segmentis bi» trisectis, lobis acute inciso-serratis, cuneatis. Carpella com》presso-plana, lateribus margine lato, l-nervio, prominente cir" cumvallatis, stylo recto abbreviato terminata."

Hab. : Wôo-sông et Shang-haï : fossés aquatiques, prairies marécageuses du Whâng-pôo, où cette espèce est moins répandue que la précédente. - Juin.

Ar.géog.: Chine (Kiang-sou,Pé-tché-ly); Prov. de l'Amour et de l'Ussuri (Maximowicz).

3. R. oryzetorum Bunge Enum.plant. chin. bor. n 9 (loc. cit.), p. 76, et tirage à part, p. 2 (1832); sinice TCHÉ-YUEN-TSAO, TCHÉTAN-TSaO O. Debeaux in Essai sur la phar'm. et la mat. médic. clin. (1867), sub Ranunculo scelerato. 
Cette espèce a tout à fait le port et le facies du $R$. sceleratus Lin., dont elle diffère par ses feuilles légèrement velues et non glabres, à divisions plus profondes, les supérieures entières et non trilobées, par son calice velu-pubescent, par son épi fructifère cylindrique et non oblong, et enfin par ses carpelles à bec plus prononcé, et offrant sur le dos, vers la moitié supérieure, une petite côte plus ou moins aiguë.

Hab. : Shang-haï, Wôo-sông et ailleurs : fossés aquatiques, canaux d'irrigation, rizières, marécages et prairies inondées. des rives du Whâng-pôo, etc. C. en juin.

Ar. géog.: Chine (Kiang-sou, Pé-tché-ly et probablement aussi le Chan-tong).

Obs. 1: M. le docteur Weber, médecin militaire attaché au corps expéditionnaire en Chine, a rencontré en mai 1860, dans les haies de Wôo-sông, une espèce de Clematis, nommée TI-CHI-LI par les habitants de cette localité. Cette clématite, qui, d'après les notes de M. Weber, a les fleurs assez grandes, paniculées et d'un hlanc jaunâtre, les tiges grimpantes, etc., paraît se rapprocher du Clematis paniculata Thumberg. N'ayant pas eu l'occasion de rencontrer cette plante dans les haies de Wôo-sông, ni d'en voir des échantillons d'herbier, je ne peux lui assigner aucune place dans cette florule, mais je la signale seulement à l'attention des botanistes-voyageurs.

Obs. 2: On rencontre en pleine terre dans tous les jardins de Shang-haï et de la province de Kiang-sou, les Pronia albiflora Pallas, sinice PÉ-Chô-ro, et P. Moutan Sims, ou Mou-TAN-HôA, qui sont, la dernière surtout, deux plantes favorites des horticulteurs et de presque tous les Chinois. Le Mou-tan-hôa, que les jardinier's du Céleste Empire obtiennent en fleurs presque toute l'année,croît spontanément dans les montagnes boisées du Hô-nan. Sa découverte remonte en Chine à plus de quatorze cents ans. Ce fut vers le milieu du septième siècle de notre ère que sa culture devint générale. Ses nombreuses et belles variétés acquirẹnt au Moutan-hôa une vogue extraordinaire.Les Chinois possèdent, à ce qu'on assure, plus de 240 variétés de cette plante, et ce nombre serait plus grand encore, si, par suite d'un préjugé bizarre, les pivoines panachées de diverses couleurs n'étaient pas exclues des jardin: (Robert Fortune). Cette aversion est basée sur un raisonnement des plus singuliers: "Les fleur's panachées, disent les horticul- 
" teurs chinois, sont des preuves de la faiblesse des plantes. Celles" ci ne sont donc pas belles, car rien n'est beau que ce qui est * dans l'ordre de la nature. »

MAGNOLIACÉES. Decandolle.

4. Magnolia Yulan Desfontaines Hist. arb. 2, p. 6; Dec. in Prod. 1, p. 81 ; sinice YU-LAN-HÔA, YU-LAN.

$H a b$. : Cette espèce est originaire des montagnes du Chen-si et du Tché-kiang. Elle est surtout remarquable par ses fleurs nombreuses et précoces. On la rencontre dans presque tous les jardins à Shang-haï et autour des habitations rurales. Le Yu-lan croît en p!eine terre et résiste aux hivers les plus rigoureux dans les provinces centrales et boréales de la Chine. Il est aujourd'hui parfaitement acclimaté en France, mème sous la latitude de Paris.

Ar.géog.: Chine (Chen-si, Tché-kiang, Hou-pé, Chan-tong, etc.). Obs.: Les Magnolia hypoleuca Siebold et Zuccarini, originaires du Japon, et le $M$. fuscata Andr. (sinice HaN-тsiN-HÔA), ce dernier provenant du Hô-nan et du Kiang-nan se rencontrent dans quelques jardins des résidants étrangers.

MÉNISPERMÉES. Juss.

5. Cocculus Thumbergii Dec. Syst. plant. 1, 521, et Prod. regn. veg. 1, 98; Menispermum orbiculatum Thumberg Flor. - jap., 194; Miquel Prol.jap., p. 198.

Varie à feuilles entières ou trilobées.

Hab. : Fissures des vieux remparts à Shang-haï, près de la porte du Sud. - Juin.

Ar. géog. : Chine (Kiang-sou, Chan-tong, etc.). - Japon.

BERBERIDÉES. Ventenat.

6. Nandina domestica 'Thumbg'. Diss. nov. gen. plant. 1, p. 14, et Fl. jap , 147; Dec. in Prod. 1, 109; sinice Tien-tchou, et Nan-TIN des Japonais.

$I I a b$. : Cultivé dans tous les jardins des provinces centrales et méridionales.

Ar.géog.: Chine. - Japon.

Obs.: Le Nandina domestica remplace à la Chine notre houx com- 
mun pour la décoration des jardins. C'est le Tien-tchou ou Bambou sacré. Vers le milieu du mois de janvier, on en coupe les branches, que l'on transporte dans les rues des villes et des hameaux; chacune de ces branches est couronnée par une grappe de baies rouges le l'effet le plus agréable. Les Chinois se servent de ces rameaux fructifères pour l'ornement des autels dans les temples bouddhiques et les maisons particulières.

\section{NÉLUMBIACÉES. Dec.}

7. Nelumbium speciosum Wild. Spec. plant. 2, 125; Dec. in Prod. 1, 113; Bunge Enum. chin. bor. n 19, p. 4; Wight et Arn. Ill. of Ind. bot., tab.9; Nelumbo nucifera Gærtn. Fruct., 1, 73; sinice Lien-hôA la fleur, LieN-TZÉ les graines, LieN-NÉôu les rhyzômes.

Hab. Shang-haï. - Marécages du Jardin à Thé dans l'intérieur de la ville, où il croît sans aucune culture; mares et terrains humides au nord de la concession française. Très-répandu et cultivé dans la province du Kiang-sou. - Août et septembre.

$A r$. géog. : Asie intertropicale et tempérée. Indes orientales, Cochinchine. - Kiang-sou à Shang-haï, Sou-tcheou et Nanking, etc.; Tché-kiang à Ning-po, Kiang-si, Kiang-nan, et jusque dans le Pé-tché-ly à Pé-king, où il résiste aux hivers les plus rigoureux. - Japon.

Obs. : Le Nelumbo dont on trouve aussi une variété à fleurs d'un beau jaune, est connu à la Chine dès la plus haute antiquité, et il y jouit de la plus grande renommée. Les docteurs de la secte Taô-tssé l'ont compris parmi les plantes qui entrent dans le breuvage de l'immortalité. On a longtemps supposé que le $N$. speciosum était le Lotus sacré des Grecs et des Egyptiens. On le cul tive en Chine sur une vaste échelle, pour ses rhyzômes que l'on considère comme l'une des meilleures racines alimentaires, et dont toutes les classes de la population font une consommation considérable.

\section{FUMARIACEES. DeC.}

8. Diclytra spectabilis Dec. Syst.plant. 2, 110, et Proa. 1, 126: Dicentra spectaóilis Niquel Prol. flor. jap. 200; Fumaria spectabilis Lin. Amcen. 7, 45\% 
$H a b$. : Shang-haï. - Décombres, lieux incultes et rocailles du Jardin à Thé; vieux remparts de la ville. - Subspont. dans tous les jardins. - Juin.

Ar. géog. : Sibérie, Chine (Provinces centrales et boréales). Japon.

9. Corydalis aurea Wild. Enum. hort. Berol. 740; Dec. Prod. 1, 128; Torr. et Gray Flor. of North Amer. 1, 68; Miquel Prol. jap. 201.

Var. parviflora Regel Plant. Radd. reisen 1, p. 146 (in nota).

Les botanistes russes ont réuni, sous le nom de Corydalis aurea, diverses formes de cette plante, que l'on serait tenté de considérer comme espèces distinctes. La forme parviflora ne diffère du type et surtout de la variété pallida (C.pallida Maxim. non Pers.) que par ses fleurs trois fois plus petites. Dans le $C$. aurea, les fleurs sont très-variables pour leur dimension (de 10 à 25 millim.). Leur couleur varie également du jaune d'or au jaune pâle. Les échantillons de cette Fumariacée, recueillis à Shang-haï par M.Savatier et par moi, ont des fleurs qui atteignent à peine 10 millimètres en longueur.

Hab.: Shang-haï. - Rocailles du Jardin à Thé, sur les vieux remparts près de la porte du Sud. - Avril.

Ar. géog.: Amer. boréale (Pensylvanie, Virginie, Canada). Chine (Kiang-sou). - Japon. - Kamtschatka.

10. C. Bungeana Turczaninow Decad.plant. nov. in Bull. Acad. scienc. mosc. xIv, p. 62; Maxim. Index Flor. Pekin. in Prim. flor. Amur. 468; Régel Plant. Radd. reisen 1, 143; C. racemosa Bunge Enum. Chin. bor., no 26, non Pers.

"Herba annua glauca, foliis eis Fumariæ similibus, bippinnati》sectis, lobis abbreviatis oblongis. Flores parvi, rosei, in apice " petalorum lateralium nigro maculati (Regel)."

$H a b$. : Shang-haï. - Vieilles murailles de la ville chinoise, sur les remparts près des portes du Sud et de l'Ouest. - Mars.

Ar. géog.: Chine (Kiang-sou, Pé-tché-ly, etc.).

11. G. Wilfordi Regel Plant. Radd.reisen in der suden von ostsiberien, 1, 148; Miquel Prol. flor.jap. 201; C. pallida Wilf. Plant. coreance exsic. non Maxim. nec Pers.

«Caulis 1-2 pedalis, foliis glaucis bipinnatisectis, segmentis »cuneato-obovatis, trifidis vel pinatifido incisis; floribus r'ace- 
" mosis, pallide ochroleucis, calcare apice inflato, obtusissimo " petala subæquante; bracteis linearibus, lanceolatis, integris, " pedicello brevioribus; siliquis anguste linearibus, torosis, 8-plo " longioribus quam latis. Semina opaca, atra, punctis minutissi" mis aspera (Regel). 》

C. Iutea proxima, habitu etiam $C$. capnoidis et $C$. aurea, sed " seminibus omnino diversa.

$H a b$. : Shang-haï, sur les rocailles du Jardin à Thé et les remparts de la ville. - Avril.

Ar. géog.: Chine (Kiang-sou). - Japon. - Corée. - Sibérie orientale.

\section{CRUCIFĖRES. Juss.}

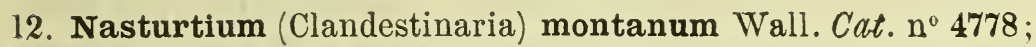
Bentham Flor. Hong-kongensis, p. 16; N. Madascariense Wight et Arn. Prod. flor. Ind. or. 1, p. 19.

Var. foliis glabris, Walpers Rep. bot. syst. 1, 126.

$H a b$.: Wôo-sông; prairies marécageuses des rives du Whângpôo, bord des canaux d'irrigation, des fossés aquatiques et des rizières. - Juin.

Ar.géog. : Asie intert. et temp., Indes orient. - Chine (Kiang-sou, Hong-Kong, Lôo-chôo, etc.).Iles de l'Océanie et de l'Océan Indien, Madagascar, Java, Philippines, etc. - Japon.

Obs. : Cette espèce, qui est répandue dans l'Asie centrale, la Malaisie et les îles de l'Océan Indien, ne paraît être pour M. Bentham qu'une forme du Nasturtium indicum Dec.

13. N. (Clandestinaria) microspermum Dec. Syst. 2, p. 199, et Prodrom. 1, p. 139; Bunge Enum. Chin. bor., p. 5, n² 29; Maximowicz Prim. flor. Amur. 42, var. A.

La forme de Chine se rapporte à la variété A du Primitice flor Amurensis, ainsi caractérisée par son auteur.

« N. gracile, divaricato-ramosum, foliis omnibus basi dilatatis, " inferioribus petiolatis, subfloralibus obovato-cuneatis, vel in" ciso-serratis. "

Hab.: Wôo-sông; champs et cultures des terres humides, près de la chaussée qui conduit à Shang-haï. - Juin.

Ar. géog.: Chine (Kiang-sou, Pé-tché-ly, etc.). - Prov. de l'Amour.

14. Gardamine hirsuta Lin. Spec. plant. ${ }_{4}^{915}$; Dec. Prod. 1, 
p. 152: Benth. Fl. IJong-Kong. 16; Torr. et Gray Fl. of north Amer. 1, 85.

$H a b$. : Cultures et tumulus à Shang-haï, etc. - Avril.

Ar. géog.: Eur. - Afriq. bor. - Amér. bor.: Pensylvanie, Virginie, etc.; - Asie, Tauride, Perse, Chine (Horg-Kong, Kiang-sou. etc.). - Japon.

15. Brassica chinensis Lin. Amon. 4, 280; Dec. Prod. 1, 215:

Maxim. Prim. fl. Amur. 47 ; sinice Pé-Tsaï, Yu-Tsaï.

Hab.: Cultivé en grand dans tout le territoire de Shang-haï et de Wôo-sông, la province de Kiang-sou, ainsi que dans tout le centre et le nord de la Chine. On retire de ses graines une huile douce fort estimée pour l'usage alimentaire. Le $P \dot{e}$-tsai se sème pendant l'hiver, ou au commencement du printemps, et arrive à matırité vers le milieu de mai. Le terrain qui a servi à sa culture est disposé de suite pour l'établissement des rizières ou autres cultures.

Ar. géog.: Chine (Kiang-sou, Tché-kiang, Chan-tong, etc.). Prov. de l'Amour. - Japon.

16. Capsella bursa pastoris Mænch Méth. 271; Dec. Prod. 1, 177; Benth. Fl. Hong-Kong. 16; Naxim. Prim. Al. Amur.46; Franch. et Sav. Enum.jap. $n^{0} 160$; sinice Tsé-YÉ.

Hab.: Chemins, champs et cultures à Shang-haï. - Avril.

Ar.géog.: Eur. - Amér. bor. - Afriq.bor. et aust. - Asie, Perse, Indes orient., Daoûrie, Sibérie, Chine (Hong-Kong, Kiang'sou, etc.). - Prov. de l'Amour. - Japon.

17. Thlaspi arvense Lin. Spec. 901; Dec. Prod. 1, 175; Ledebour, Flor. Altaica 3, 94; Franch. et Sav. Enum.jap. no 161.

Hab.: Champs de blé à Shang-haï et à Wôo-sông. - Avril-juin. Ar.géog.: Eur., Asie bor. etcent., Russie asiat., Baikal, Daoûrie. Chine (Kiang-sou). - Japon.

18. Lepidium incisum Roth Nov. cat. 1, 214; Dec. Prod. 1, 207:

L. micranthum Ledeb. Fl. Alt.3, p. 193; L. ruderale Bung'e Enum. Chin. bor. n 36, p. 6, non Lin. Regel Plant. Radd. 1. 269.

Le L. incisum que j'ai retrouvé dans le nord de la Chine, à Tientsin, sur les sables du Pei-ho, a été distribué par moi à quelques botanistes, sous le nom de $L$. ruderale L. La plante de Chine diffère sensiblement du L.ruderale, par la présence des pétales, par ses 
siliques ailées (très-étroitement) au sommet, par ses feuilles toutes dentées, inême les supérieures. Voici ce que m'écrivait en 1863 l'honorable président de la Société Linnéenne de Bordeaux, M. Charles Des Moulins, au sujet des échantillons chinois du $L$. incisum, que je lui avais communiqués:

"Cette plante ne diffère en rien du L. vuderale de France. "Comme lui, elle est diandre; son fruit et ses graines sont abso" lument pareils aux siens. Les pétales qui, d'après MM. Grenier. 》Godron et autres auteurs, avortent souvent, scnt ici presque " tous avortés et d'une excessive petitesse. Il n'y a que deux dif»férences un peu saillantes à signaler:

1 $1^{\circ}$ La plante chinoise est rameuse dès la base, et la plante 》 française n'est rameuse en général qu'au sommet de la tig'e;

" $2^{\circ}$ La pubescence de la plante de Chine est blanchâtre et grenue, tandis que la plante française est glabre, ou n'offre qu'une pubescence ordinaire.

"En somme, cette forme se rapproche beaucoup du Lepidium incisum Roth, dont les feuilles caulinaires sont moins étroites " que celles du $L$. ruderale; ce dernier a, d'un autre côté, les sili"cules tout à fait orbiculaires.» (Ch. Des Moulins, in litt., mars 1863).

Hab. : Shang-haï et Wôo-sông"; champs et cultures. - Mai-juin.

Ar. géog.: Asie cent., bor. et occid.; Russie austro-orient., Tauride, Caucase, Sôongarie, Altaï, Baikal. - Chine (Prov. cent. et boréales.)

19. Isatis indigotica Rob. Fortune, Mss. 1847; R. Fort. in Journ. of the hort. soc. of London, vol. 1, p. 269; sinice LAN-HOA, Tren-Ching. Affinis I. oblongate Dec.

$H a b .:$ Cultivé dans le territoire de Shang-haï.

Ar.géog.: Chine (Kouang-tong, Fo-kien, Koûang-si, Tché-kiang, Kiang-sou, Kiang-si, etc. - Ile Formose. (R. Fort. Wanderings in China, 1848.)

Obs.: Les Chinois cultivent en grand l'Isatis indigotica, dans le Kiang-sou et autres provinces centrales, afin d'en retirer une espèce d'indigo très-employé dans la teinture en bleu. On apporte, en grandes masses, la plante fraîche à Shang-haï, et sur tous les marchés des principales villes, pour la teinture des tissus de coton, dont toute la population agricole se sert pour se vêtir. Lcs 
feuilles de l'I. indigotica sont soumises aux mêmes préparations que celles des Indigofera, pour en extraire la couleur d'indigo. R. Fortune est très porté à croire que c'est la teinture de cette plante, qui sert à colorer les thés verts préparés dans le centre de la Chine, en vue de l'exportation.

On cultive à Shang-haï, à Wôo sông et dans toute la province, le Lô-pô (Raphanus sativus L.), dont les racines jouent un grand rôle daus l'alimentation et la cuisine chinoises.

\section{MALVACÉES. R. Brown.}

20. Abutilon Avicennæ Gærtn. Fruct. tab. 135; Sida abutilon Lin. Spec. 963; Dec. Prod. 1, 963.

$H a b$. : Champs cultivés, prairies à Shang-haï, etc. - Juin.

Ar. géog. : Eur. aust. - Afriq. bor. - Asie temp.,Sibérie, Indes orient., Chine (Kiang-sou, Chan-tong, etc.) - Japon.

Obs.: Les échantillons de l'Abutilon Avicenne que j'ai récoltés aux environs de Shang-haï et de Yan-taï dans le Chan-tong, ne diffèrent en rien de ceux provenant de l'Europe australe, de l'Algérie et du Japon.

21. Gossypium herbaceum Lin. Spec. 915; Dec. Prod. i, 456; Thumbg. Flor. jap. p. 271.

Var. indicum Lamark Dict. 2, 134; sinice MreN-HÔA.

$H a b$.: Cultivé dans tout le territoire de Shang-haï et la Chine centrale. - Août.

Ar.géog.: Asie intertrop. et temp.-Indes or., Chine(Kiang-sou, Tché-kiang, Pé-tché-ly, etc.). - Cochinch., Japon, Amér.bor., etc.

Obs. : Cette espèce produit à Shang-haï et dans le Kiang-sou une variété de coton qui sert à fabriquer les toiles dites Nan-kin. Les deux variétẻs blanche et jaune de coton sont un des éléments de la richesse du Kiang-sou et des provinces avoisinantes.La récolte des fruits capsulaires qui renferment le coton a lieu, depuis le mois d'aout jusqu'à la fin d'octobre, dans les plaines du Hiangtssé-kiang.

On trouve fréquemment cultivés dans les jardins : le FOU-SEN-HÔA (Althrea rosea Cav.), le Fou-yong-HÔA (Hibiscus mutabibis L.), le MoU-кiN-HÔA (Hibiscus syriacus L.), ainsi que l'Hibiscus rosa-sinensis L. qui porte le même nom indigène que l'Althrea rosea Cav. 
STERCULIACEES. R. Brown.

22. Sterculia platanifolia Lin. Suppl. 423; Dec. Prod. 1, 483; Benth. Fl. Hong-Kong. 36; Firmiana platanifolia R. Brown in Plant. Jav.rar. 235; sinice OU-Tong-CHû.

Hab. : Shang-haï, dans les concessions française et anglaise, ou il est fréquent autour des habitations, le long des chemins, etc.

Ar. géog.: Asie cent., Indes orient., Chine : Hong-Kong, Tchékiang, Kiang-sou, etc.) - Japon.

\section{TILIACÉES. Juss.}

23. Corchorus capsularis Lin. Spec. 746; Dec. Prod. 1, 505; Wight. et Arn. Prod. flor.penins. 1, 73; Benth. Fl. Hong-Kong. 40 ; sinice TSAN-LIN-MA.

$H a b$. : Shang-haï. Cultivé dans les terres humides l'hiver, et desséchées pendant l'été. Ses tiges produisent des fibres textiles, avec lesquelles on tisse des étoffes légères nommées Pô-LO-MA, TA-MA. - Juillet-septembre.

Ar.géog. : Asie intertrop.et temp.-Indes or., Chine(Hong-Kong, Tché-kiang, Kiang-sou, etc.). - Japon.

\section{OXALIDÉES. Dec.}

24. Oxalis corniculata Lin. Spec. 624; Dec. Prod. 1, 692; Benth. Fl.Hong-Kong. 56 ; A. Gray Plant.jap. exped. Perry,p. 309; sinice TSIEN-TSAÔ-TCHÉ.

$H a b .:$ Shang-haï; lieux incultes autour des tumulus; sur les vieux remparts de la ville. - Avril.

Ar. géog.: Eur., Afriq. bor.,Canaries, Ile Bourbon.-Asie temp. et intertrop., Cochinchine, Chine (Hong-Kong, Kiang-sou, etc.). - Japon. - Amér. bor., Antilles, Mexique, etc.

Obs.: La famille des Aurantiacées fournit aux horticulteurs plusieurs espèces, dont les fruits sont très-recherchés par les Chinois et les peuples orientaux en général pour l'usage domestique. Je citerai surtout les suivantes, que l'on trouve cultivées en serre tempérée par les jardiniers de Shang-haï.

Citrus japonica Thumbg., KıN-Kıû et KoûN-KoûA à Canton;

C. decumana Lin. dont les fruits sont nommés vulgairement 
Pamplemousses dans les Indes françaises, et Hian-yuex par les Chinois;

C. vulgaris Risso (C. bigaradia Duhamel) ou KAO-Kıû, fruits recherchés par leur saveur;

C. aurantium var. scabr $a$, dont les fruits nommés KıU-KôNG sont fort estimés, lorsqu'ils sont récoltés et desséchés avant leur matırité;

C. microcarpa Bunge ou le TsoûI-PI, dont les fruits très-petits, et de la grosseur à peine d'une petite noix, mûrissent dans les serres dès le mois de janvier, même sous le climat de Pé-king.

Quelques espèces d'Aurantiacées beaucoup plus rares se rencontrent aussi dans les jardins des résidants étrangers, entre autres le Clausena IVampiOliv.(Cookiapunctata Retz), qui produit des fruits noinmés Houang-PI, et fort estimés pour la préparation de conser: ves au sirop de sucre; le Sarcodactylis odorata Gærtn., qui crô̂t spontanément dans le Koûang-tong, le Fo-kien, etc., et dont le: les fruits nommés FAN-KIû, Fo-Cheoû-Kong, que les Chinois comparent à la main du Dieu Fo, se divisent vers la partie supérieure (par la séparation des carpelles) en cinq lanières allongées, irrégulièrement subcylindriques et acuminées à leur extrémité. Le Murraya exotica Lin.,originaire du sud de la Chine, est également cultivé dans quelques jardins, mais principalement dans le:provinces méridionales et l'Asie intertropicale.

\section{MÉLIACÉES. JusS.}

25. Melia azedarach Lin. Spec.550; Dec. Prod.1, 621; Thumbg. Fl. Japon. 180 ; sinice LIEN-TZÉ, TCHANG-MO-TZÉ.

$H a b$. : Arbre très-répandu à Shang-haï, dans les jardins, les haies, etc., en suivant la chaussée de WVô-sông.

Ar. géog.: Eur. aust.: Gibraltar, Malte ; - Afr. bor. : Algérie, Egypte; - Asie intertrop. et temp. : Syrie, Aden, Ceylan, Indes orient., Chine (Hong-Kong, Hou-pé, Kiang-sou, Tché-kiang, etc., jusqu'au $31^{\mathrm{e}}$ degré de latitude nord). - Japon. - Amér. bor.

\section{HIPPOCASTANÉES. DeC.}

26. Asculus turbinata Blume Rumphia 3, p. 195; Franch. et Sav. Enum.jap. no 355 ; E. pavia Thumb. jap. p. 154; di. hippocastanum L. ex parte; sinice Lô-LÔ-'TZÉ. 
Arbre distinct de l'E Esculus hippocastanum L. par ses fruits à hile beaucoup plus large, et par la couleur foncée de leur enveloppe.

Hab.: Shang-haï; bord des chemins, autour des habitations rurales, etc.

Ar. géog. : Chine (Hou-pé, Tché-kiang, Kiang-sou, etc.), Japon.

\section{AMPÉLIDÉES. JusS.}

27. Cissus pentaphylla Wild. Spec. plant. 1, 659; Dec. Prod. 1, 631; Vitis penlaphylla Thumbg. Flor. japon. 105; Cissus vitifolia Sieb. et Zuc. Fam. nat.jap. n 406.

Hab. : Wôo-sông et Shang-haï; bord des chemins, des haies, etc. - Juin.

Ar. géng.: Chine (Kiang-sou). - Japon.

Obs. : La vigne (Vitis vinifera) est cultivée à Wôo-sông et à Shang-haï, le Kiang-sou, le Chan-tong etle Pé-tché-ly, non pour en obtenir du vin ou autre liqueur alcoolique, mais pour les fruits (Pô̂-TAO-TZÉ) dont les Chinois sont très-friands. La vigne est connuc dès la plus haute antiquité. Il en est question dans les livres T'chéou-li, ouvrages attribués à Tchéou-kong, frère de l'empereur Woâ-Woûang, qui monta sur le trône l'an 1122 avant notre ère. Il est certain que la vigne était cultivée dans le Chen-si, le Chantong, le Hô-nan, le Hoû-kouang et le Pé-tché-ly plusieurs années avant l'ère chrétienne, et que les raisins servaient à cette époque à faire une boisson fermentée. Cette boisson fut interdite depuis par de nombreux édits. Les raisins ne sont plus employés actuellement à la préparation du vin, mais ils sont uniquement destinés à l'alimentation, soit à l'état frais ou séchés au soleil. Les raisins les plus renommés proviennent du Ha-mi, du Chen-si et du Pé-tché-ly.

\section{CÉLASTRINÉES. R. Brown.}

28. Evonymus Bungeanus Maxim. Prim. flor. Amur. 470 (in nota). Affinis $E$. Sieboldiani Blume.

«Suffrutex, gracilis, 10-16 decimet. altus, ramulis compressis "striatis, petiolisque glaberrimis, foliis herbaceis glaucis, gla» berrimis, ovato-ellipticis, ellipticisve, longe acuminatis, basi »subito in petiolum acuminato attenuatis, pedunculis in parte »-ramulorum inferiore congestis, numerosis, plurifloris, sepatis 
"rotundatis, staninibus stylum brevissimum superantibus, an-

» theris atropurpureis. Floret Junio (Maxim.).

$H a b$. : Dans les fissures des vieux remparts de la ville, près des portes du Sud et de l'Est. - Mai et juin.

Ar. géog.: Chine (Kiang-sou, Chan-tong, Pé-tché-ly, etc.!

\section{RHAMNÉES. JUSS.}

29. Zizyphus vulgaris. Lamark Illust. gen. tab. 185; Dec. Prodr. 2, 19; Bunge Enum. Chin. bor. p. 14, n 81 .

Var. A spinosus ( $\boldsymbol{Z}$. vulgaris Lam.).

"Aculeis geminis, altero longissimo recto, altero breviori re-

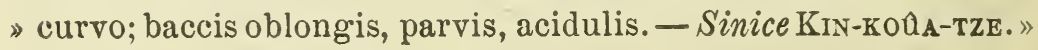
Var. B inermis ( $Z$. chinensis Lam.).

"Arboreus, inermis, baccis magnitudine Pruni, sapore dulci. 》Sinice KIN-TSAO-TZÉ. »

Hab. : Shang-haï et Wôo-sông. - La var. A subspont. dans les haies, les clôtures des propriétés, etc.; la var. B cultivée dans les jardins, où elle atteint les dimensions d'un arbre de moyenne grandeur.

Ar. géog.: Asie temp.: Asie-Min., Syrie, Indes orient., Cochinchine, Chine (Kiang-sou, Tché-kiang, Chan-tong, Pé-tché-ly,etc.). - Japon. - Afriq. bor.: Egypte, Algérie, Maroc, etc.

30. Rhamnus virgatus Roxburg Flor. ind. 2, 551; Dec. Prodr. 2, 24.

Var. apricus Maxim. Rhamn.orient.Asiat., $\mathrm{n}^{0}$ 15, tab. 1, fig.24-25. Rh. globosus Bunge Enum. Chin. bor. n॰ 83, p. 14 (ex parte).

Rh. chlorophorus Decaisne, in Comptes-rendus de l'Acad. des sciences, tom. XLIV (1857) ex parte.

La description et la figure du $R h$. chlorophorus publiées par M. Decaisne en 1858 (1), et la diagnose suivante du $R h$. globosus, conviennent en tout point au $R h$. virgatus var. apricus de Shanghaï, nom le plus ancien, et qui doit avoir la priorité.

* $\mathrm{Rb}$. erectus, ramis spinescentibus, patentibus, junioribus vil> losis; foliis obovato-oblongis, utrinque attenuatis, suboppositis, "serratis, subtus petiolisque pubescentibus; floribus confertis

(1) Notice sur le Lo-KAo ou vert de Chine, par M. Natalis Rondot, Lyon (1858), 1 vol. in-80. 
" dioïcis, feminis apetalis; stylo elongato, bifido; baccis globosis, » apice nudis. erectis, 2-3 spermis (Bunge).

"Baccis nigris, globosis, magnitudine Pisi minoris, nuculis - ovoideo-rotundatis, cylindraceis ve, dimidio inferiore sulcatis, "nitidis (Decaisne, loc. cit.):

La forme remarquable du sillon dorsal des nucules, dont M. Maximowicz a tiré un si bon parti pour la distinction des espèces orientales du genre Rhamnus, est exactement la même dans le $R h$. virgatus var. apricus et le $R h$. chlorophorus. Les échantillons du $R h$. virgatus provenant des vieux remparts de Shang-haï sont plus fortement spinescents que ceux du $R h$. chlorophorus; mais ainsi que le fait remarquer M. Decaisne (loc. cit., p. 51), les extrémités des rameaux du $R h$. chlorophorus et d'une autre espèce du Tché-kiang, le $R h$. utilis Decne, dont on retire aussi le Lo-KAo ou vert de Chine, sont spinescents ou inermes selon les localités.Le caractère tiré de la présence ou de l'absence des épines n'a donc qu'une faible valeur pour la détermination des espèces de ce genre.

M. Franchet, après avoir étudié avec soin les échantillons fructifères du Nerprun que j'ai récoltés à Shang-haï, les rapporte sans aucun doute au Rhamnus virgatus! var. apricus Maxim. (Rh. globosus Bunge ex parte); et il y a lieu de penser que cette espèce n'est autre que le $R h$. clorophorus Dne. (PÉ-PI-Lo-Chou des Chinois) (1), dont la dispersion en Chine paraît être considérable. M. N. Rondot a complètement oublié le $R h$. virgatus, dans l'énumération des espèces chinoises du genre Rhamnus (loc. cit.. p. 58), ou du moins il ne l'a connu que sous la forme globosus (Rh. globosus Bunge), qui n'en est qu'une variété. M. Decaisne a fait aussi le même oubli, car il ne compare son $R h$. chlorophorus qu'au $R h$. tinctorius de l'Europe australe.

D'un autre côté, le R. P. Hélot, missionnaire apostolique en Chine, dont les recherches ont contribué à faire connaître les Nerpruns qui produisent le Lo-KAO (2), assure que le PÉ-PI-LO-CHOU

(1) $\mathrm{P} \dot{E}-\mathrm{PI}-\mathrm{LO}-\mathrm{CHOU}$ signifie mot à mot blanche peau vert sarment.

(2) On the manufacture of the chinese green dye ealled Lo-kdo, in pharnaceut. journ. of London (1857).

Le ver't de Chine. Note du R. P. Hélot sur les procédés des fabriques d'A-zée dans le Tché-liang. (Etudes de théologie, philosophie, etc., 1856.) 
(Rh. chlorophorus) croît sans culture sur les montagnes du Chantong, où cet arbuste est connu sous le nom de Likv-LO-CHOu ou Saule-vert sarment.

L'assertion du R. P. Hélot est d'autant plus digne de foi, qu'elie est corroborée par mes propres recherches. J'ai en effet recueilli en 1860 , dans la partie montagneuse du Chan-tong qui avoisine l'ancien camp français du Tché-fou, le Rh.virgatus var. apricus, absolument identique à la forme de Shang-haï.

$H a b$. : Lieux incultes, au pied des vieux remparts de Shanghaï, où cette forme vit spontanément. - Juin et juillet.

Ar. géog. : Asie cent. et temp.; le type (Rh.virgatus), Himalaya, Nériaul, Nilgherries, dans les Indes orientales; la variété apricus, Chine, dans les provinces centrales et boréales, TchéKiang, Kiang-sou, Chan-tong', Pé-tché-ly, etc.

Obs. : Le Rh. chlorophorus, qui serait la même espèce cultivée que le $R h$. virgatus var. apricus, est cultivé pour l'usage tinctorial

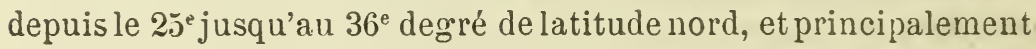
du $30^{\mathrm{a}}$ au $31^{\mathrm{e}}$ degré. Il est naturalisé dans le parc du consulat français à Shang-haï depuis l'année 1852, époque où il y fut planté par M. de Montigny, alors ministre de France en Chine. M. de Montigny le fit connaître au botaniste R. Fortune, lors de son deuxième voyage dans la province de Kiang-sou. La priorité de la découverte du $R h$. chlorophorus appartient incontestablement à M. de Montigny, qui avant son départ de Chine, en 1852, en adressa plusieurs pieds vivants au Jardin des Plantes de Paris.

\section{LÉGUMINEUSES. JUSS.}

31. Sophora japonica Lin. Mant. 68; Dec. Prodr. 2, 95; Benth. Fl. Hong-Kong. 95; Thumbg. Fl.jap. 178; Styphonolobium japonicum Schott; sinice HOAI-HOA.

$H a b$. : Répandu à Shang-haï dans tous les jardins des indigènes et des résidants étrangers. Souvent cultivé pour ses fleurs, qui produisent une belle couleur jaune employée dans la teinture.

Ar. géog. : Chine (du $23^{\mathrm{e}}$ au $40^{\mathrm{e}}$ degré de latitude nord) et principalement dans les provinces du Koûang-tong, Konang-si, Fo-kien, Tché-kiang, Kiangr-sou, Ho-nan, Tssé-chûen, Chan-tong, Pé-tché-ly, etc. - Importé de la Chine au Japon, où il s'y est. acclimaté. 
32. Medicago Jupulina Lin. Spec. 1097; Dec. Prodr. 2, 173, Benth. Fl. Hong-Kong. 75; Bunge Enum.pl. Chin.bor. n 90. p. 15; Miquel Prol.jap. 231; A. Gray Plant.jap.exp. Perry, p. 310 .

Hab. : Wôo-sông. - Prairies des rives du Whang-pôo. Il y croìt en société avec le Vicia sativa var. angustifolia, le Daucus carota, etc. - Mai.

Ar. géog. : Eur.; - Afriq. bor.; - Amér. bor.; - Asie :- AsieMin., Chine (Hong-Kong, Kiang-sou, Pé-tché-ly., etc.); - Japon

Obs. : Le Medicago sativa Lin. est cultivé comme fourrage aे Wôo-sông, et dans quelques localités du Kiang-sou.

33. Melilotus parviflora Desfontaines Fllor. atlant.2, 192; Dec. Prodr. 2, $18 \%$.

Hab. : Shang-haï. - Marécages herbeux plantés de Nélumbo, au nord de la concession francaise. - Septembre.

Cette espèce est identique dans tous ses caractères avec le $M$. parviflora de l'Europe australe. La forme des feuilles, la gousse, le calice, les rapports des pétioles entre eux sont exactement semblables. L'épi florifère de la plante chinoise est seulement un peu plus lâche, et sous cette forme, elle est encore l'analog'ue du $M$. parviflora provenant des Canaries.

Ar.géog. : Eur. aust.; - Afriq. bor., Algérie, Canaries, - cal de Bonne-Espérance; - Asie : Asie-Min. Caucase, Chine (Kiangsou), où cette plante a été probablement introduite avec des graines étrangères.

34. Trifolium pratense Lin. Spec. 1082; var. sativum Seringe in

Dec. Prodr. 2, 195; Miquel Prol. jap. 231.

Hab. : Shang-haï. - Cultivé en plein champ comme plante alimentaire, et comme produisant un excellent engrais. Les jeune: poussez sont employées par les Chinois sous forme de salade ou en guise d'épinards.

Ar. géog. : Europe, Afrique, Amérique, Asie, Chine (Kiang sou, etc.). - Japon.

35. Astragalus lotoïdes Lamark Dict. 1, 316; Dec. Prodr. 2, 282; A. sinensis Lin. Mant. 103; Thumb. Fl.jap. 290.

Hab. : Wôo-sông. - Champs d'orgee et de blé; prairies du Whâng-pôo. - Juin.

Ar. géog. : Chine (Kiang-sou), - Japon. 
36. Vicia sativa. Lin. Spec. 1037.

Var. angustifolia Seringe in Déc. Prodr. 2, 361; V. angustifolia Roth Tent. flor.germ. 310.

$H a b$. : Wôo-sông. - Bords des champs de blé, etc. Probablement introduit avec les céréales provenant d'Europe. - Mai.

Ar.géog. : Eur.; - Afrique bor.; - Asie : Chine et Japon.

Obs.: Le Tsan-Tsao (Faba vulgaris Mænch) et le Siao-Kéou (Pisum sativum Lin.), connus à la Chine sous le règne de HangHien, de la dynastie des Hans, sont cultivés en grand dans les provinces de Tché-kiang, Kiang-sou, Chan-tong, etc.

On trouve fréquemment, dans les haies et les clôtures des jardins, le Wistaria sinensis Sieb. et Zuccar. nommé vulgairement Glycine de la Chine.

37. Phaseolus Ricciardianus Tenore in Mem.di Torino, XXXVIII, 173, tab. 3; Durieu in Cat. graines du jard. bot. de Bordeaux, année 1867 (ad calcem, p. 26); Walpers Repert. botan. syst. 1,778 .

* Caule volubili piloso; leguminibus teretibus glaberrimis; " semine cylindrico compresso, dorso carinato nitido, plerum que » rubro sanguineo; micropilo fimbria carunculæ tecto (Walp. » loc. cit.).

Var. B hysterinus; Phaseolus hysterinus Dur. in Cat. gr. jard. bot. Bord. (années 1866 et 1867).

La variété hysterinus ne diffère du type Ricciardianus que par ses tiges basses et non grimpantes, tandis qu'elles sont hautes et volubiles dans le $P h$. Ricciardianus. Le type et sa variété produisent des graines fort estimées en Chine pour l'alimentation publique. J'en ai rapporté des spécimens que M. Durieu a cultivés eu jardin botanique de Bordeaux, puis répandus dans les cultures maraîchères de la Gironde, sous le nom adopté aujourd'hui d' $H a$ ricot-lentille. Ce qui rend cette espèce précieuse, dit M. Durieu (loc. cit.), c'est son extrême fertilité. On en jugera par l'exemple suivant: une touffe de $P h$. Ricciardianus type, c'est-à-dire la forme grimpante, composée de 2 à 3 pieds seulement, a produit au jardin de Bordeaux un kilogramme environ de graines, qui a suffi à diverses expérimentations et à de larges distributions.

$H a b .:$ Cultivé en grand dans tout le territoire de Shang-haï, où la variété hysterinus est beaucoup plus répandue que le type. Juillet-aont. 
Ar. giog. : Chine (provinces centrales).

Obs. : Les espèces suivantes sont également cultivées pour l'uságe alimentaire :

Phaseolus minimus Roxburg; sinice TIEN-TÉou-TZÉ ;

Dolichos Soja L. (Suja hispida Mænch.); sin. HoNG-TÉou-TzÉ, etc.; Lablab vulgaris Savi (Dolichos lablab Lin.); sin. Pien-TÉou-tzé, etc. On trouve fréquemment autour des pagrodes et des habitations rurales l'Albizzia julibrissin Benth. in Hook. journ. of botan. 1, 527 (Acacia julibrissin Wild.).

\section{ROSACEES. JUSS.}

38. Rubus parvifolius Lin. Spec. 707; Sieb. et Zuc. Fl.jap. 1, 18; Dec. Prodr. 2, 564; Benth. Fl. Hong-Kong. p. 105; $R$. triphyllus Thumbg. Fl. jap. 215 ; sinice FoU-PAN-TZÉ.

Hab. : Shang-haï et Wôo-sông. - Haies, bord des chemins. -- Mai. Ar.géog. : Asie subtrop. et temp., Indes orient., Himalaya, Chine (Hong-Kong, Lôo-chôo, Chen-si, Tché-kiang, Kiang-sou, Chantong, etc.). - Japon.

39. Duchesnea fragarioïdes Smith in Trans. of Linn. Soc. Lond. 10, 373; Miquel Prol. jap. 225; A. Gray Plant. jap. exp. Perry, p. 310; D. fragiformis Don Prod. Al. nepal. 2, 35: Fragaria indica Andr. Rep.bot.tab. 475; Dec. Prodr. 2, 57]; sinice SoU-BI-TZÉ.

$H a b$. : Shang-haï et Wôo-sông. - Abonde dans les lieux incultes, autour des grands tumulus, etc. - Avril et juin.

Ar. géog. : Asie temp. : Indes orient., Népaul, Chine (Kiangsou, Tché-kiang, Pé-tché-ly, etc.). - Japon.

Obs. : Cette espèce produit des fruits semblables, pour la grosseur, aux fraises des quatre-saisons. Ces fraises, d'apparence trèsbelles et appétissantes, sont insipides et sans aucun arôme. Dans le Duchesnea fragarioïdes, les fleurs sont jaunâtres, et les pétales caducs de bonne heure. Le calice est étalé à la maturité, et les divisions du calicule extérieur ou les bractées sont trilobées au sommet.

40. Rosa moschata Mill. Dict. n 13 ; Dec. Prodr. 2, p. 598;

Benth. Fl. Hong-Kong. 106.

Var. microphylla Miquel, non Roxburg; $R$. Lucia Franch. et Rocheb. in Bull. soc. bot. Brux. X, 237; sinice YûE-LI-HÔA: 
«Fleurs nombreuses blanches, à odeur très-suave, styles sou» dés en une seule colonne velue, et aussi longue que les étami" nes, folioles très-petites ( $\mathrm{D}^{\mathrm{r}}$ Weber, in Notis). "

HaZ. : Wôo-sông, dans les haies des jardins, près des magasins à charbon de terre. Cette rose, fort rare en Chine, a été trouvée en pleine floraison, au mois de mai, par M. le $\mathrm{D}^{\mathrm{r}}$ Weber, médecin militaire attaché à l'armée expéditionnaire.

Ar. géog. : Asie subtrop. et temp., dans la région montagneuse : Indes orient., Népaul, Chine (Hong-Kong, Kiang-sou, etc.).-Japon .

41. R. multiflora Thumberg $F l$. jap. 214; Dec. Prodr. 2, 598: Benth. Fl. Hong-Kong. 107; Miquel Prol. jap. 227; sinice NAN-TSIAN-HÔA.

Hab. : Haies des jardins à Shang-haï, etc. - Mai.

Ar. géog. : Chiue (Prov. cent. et mérid.) : Hong-Kong, Koûangtong, Fo-kien, Kiang-sou, Chan-tong, Pé-tché-ly, etc. - Japon.

Obs. : Les roses suivantes sont fréquemment cultivées dans les jardins :

Rosa sinica Ait. Hort. kew. (R. nivea Dec.);

R. rugosa Thumberg, Fl. jap.; sinice NAN-TSIAN-HôA;

R. indica Lin., sin. TSIAN-TA-KOUNG-TZÉ, dont on mange les fruits confits dans le sirop de sucre, etc.

42. Eriobotrya japonica Lindley Trans. of the Linn. Soc. Lond.

13, 102; Dec. Prodr. 2, 631; Mespilus japonica Thumberg Fl.jap. 206; Cratagus bibas Lour. Flor. Coch. 1, 391 ; sinice PI-PA, PI-Po, PI-Po-TZÉ; vulgo Bibas.

Hab. : Shang-haï, Wôo-sông; partout dans les jardins, les cultures, d'où les fruits sont apportés sur le marché des villes dès les premiers jours de juin.

Ar. géog. : Asie intertrop. et temp. : Cochinchine (Loureiro).Chine : Hong-Kong, Koûang-tong, Fo-kien, Tché-kiang, Kiangsou, Chan-si, etc. - Japon.

\section{GRANATÉES. Don.}

43. Punica granatum Lin. Spec. 676; var. A rubrum Dec. in Prodr. 3, p. 3; Bunge Enum.pl. Chin. bor.p. 28; Thumb. Fl. jap. 199; sinice TZÉ-TIEN-PI, N'GAN-TZÉ-LIEOU, HAN-LIEOU-PI.

Hab. : Shang-haï, Wôo-sông et ailleurs; cultivé dans les jardins et subspont. autour des pagodes, etc. 
Ar. géog. : Afriq. bor., d'où il a émigré dans l'Eur. australe, l'Asie occid., etc. Introduit du Caboul en Chine par Chan-Hien, de la dynastie des Hans. Se retrouve à Hong-Kong, le Tché-kiang; le Chan-tong, le Pé-tché-ly, etc. - Japon.

\section{ONAGRARIÉES. JusS.}

44. Trapa bicornis Lin. fil. Suppl. 128; Dec. Prodr. 3, 64; T. chinensis Lour. Fl. Cochinch. 1, 109; sinice LING, LING-Kıoa. "Foliis integris quadratis, seu subdentatis; fructibus brunneo$»$ fuscis, bicornibus, cornubus oppositis, crassis, obtusis, apice "recurvatis. "

Hab.: Province du Kiang-sou, dans le fleuve Whâng-pôo, en remontant ce cours d'eau à Kiang-sing-fou. Le lac Tai-hôo où il croît en quantités telles, qu'il y gêne la navigation. Les fruits sont apportés sur le marché de Shang-haï pour servir à l'alimentation.

Ar.géog. : Chine (Koûang-tong, Tché-kiang, Hoû-pé, Kiangsou, etc.). - Cultivé dans quelques localités.

\section{HALORAGÉES. R. Brown.}

45. Myriophyllum spicatum Lin. Spec. 1409; Dec. Prodr. 3, 68; Bunge Enum.pl.chin. bor. $\mathrm{n}^{\circ}$ 168; Maxim. in Bull. acad. scienc. St-Pétersbg. tom. $9^{\mathrm{e}}$ (Decas XV); Franch. et Savat. Enum.jap. n 639 ; sinice CHouI-TSAo, Fou-CHOUI-TSA0̂.

$H a i b$. : Marécages et rizières à Shang-haï; canaux d'irrigation autour de la ville. - Avril.

Ar. géog. : Eur., - Afriq. bor. - Amér. bor.; - Asie temp. . Chine (Kiang-sou, Pé-tché-ly, etc.). - Japon.

\section{LYTHRARIÉES. JusS.}

46. Lagerstræmia indica Lin. Spec. 764; Dec. Prodr. 3, 73; Miquel Prol.jap. 148; Franch. et Savat. Enum. jap. n 648 : sinice TSA-CHÔU-MOUI-HÔA.

Hab. : Shang-haï, Wôo-sông et ailleurs. - Répandu dans les jardins, autour des pagodes, des habitations rurales, etc., où il est cultivé, quoi qu'il soit d'origine indigène.

Ar.géog.: Asie subtrop. et temp. : Indes or., Cochinchine. Chine (Kiang-sou, Tché-kiang, etc.). - Japon. 
Obs. : La famille des Cucurbitacées fournit plusieurs espèces, que l'on cultive partout en Chine, pour servir à l'alimentation ou à quelques usages domestiques. Je citerai principalement les suivantes :

Trichosanthes anguina Lin., sinice NAN-KOUAA-TZÉ;

Cucurbita pepo L. ou TIEN-KOQA-TZÉ;

Cucumis melo L. et ses variétés;

Cucumis satirus L. TsIAN-KoûA-TZÉ;

Lagenaria vulgaris et ses variétés, Ho-LO-TzF́;

Momordica balsamina L. Koû-KoûA, etc.

\section{CRASSULACÉES. Dec.}

47. Sedum subtile Miquel in Prol. flor. jap., p. 88 (1867); Savatier Plant. japon. exsicc., n ${ }^{0} 443$; Franchet et Savatier Enum. plant. in japon. cresc., p. 161 (1873).

Petite espèce annuelle de 6 à 8 centim. de hauteur, caractérisée surtout d'après Niquel par ses tiges grêles, ses feuilles cunéiformes à la base, à limbe orbiculaire-elliptique, ses súpales verdâtres et ses pétales rougeâtres striés.

Hą. : Shang-haï et Wôo-sông. - Prairies humides des rives du Whâng’pôo. - Juin.

Ar. géog. : Chine (Kiang-sou). - Japon.

48. Penthorum sinense Pursh Fl. bor. Amer. 1, 326 (in Nota): Dec. Prodr. 3, 414.

$H a b$. : Shang-haï. - Bord des prairies et des fossés aquatiques, au nord de la concession anglaise. - Juillet.

Ar. géog. : Chine (Kiang-sou, etc.).

\section{OMBELLIFĖRES. Juss.}

49. Daucus carota Lin. Spec. 348; Dec. Prodr. 4, 211; Thumb. Fl.jap. 187; sinice HoNG-LO-PÔ.

Hab.: Wôo-sôngঃ. - Prairies humides sur les rives du Whângpôo. - Juin.

Ar.géog. : Eur., - Afr. bor., - Amér. bor.; - Asie : Asie-Min., Tauride, Caucase, Cochinchine. - Chine (Kiang-sou, à l'état spontané . - Cultivé dans toutes les prorinces de l'Empire. - Japon.

On cultive aussi, pour servir à la préparation des aliments, le Kin-TSAI (Apium graveoleus Lin.); le CHUI-TSAI (Peiroselinum sati- 
vum Hoffm.); le NiN-TSIN (Sium sisarum var. ninsi Dec.); le KonErKIANG (Faniculum vulgare Gærtn.); le Tzé-LÔ ou Hong-Yu-TZÉ (Coriandrum sativum L.), etc.

\section{CAPRIFOLIACÉES. A. Richard.}

50. Sambucus nigra Lin. Spec. 385; Dec. Prodr.4, 323; Thumbg. Fl. jap. 126 ; sinice TCHOU-FOU-HÔA.

Hah.: Haies des propriétés rurales à Shang-haï, Wôo-sông et ailleurs. - Mai.

Ar.géog. : Eur., - Afr. bor., - Amér.bor.; - Asie : Sibérie, Caucase, Chine (Kiang-sou, Chan-tong, etc.). - Japon.

51. Lonicera confusa Dec. Prodr. regn. veg. 4, 333; L. japonica, Andr. Rep.bot. Tab., 583, non Thumberg; sinice JIN-TONG-HOA Hab.: Wôo-sông. - Haies des jardins. - Juin.

Ar. géog. : Asie cent. et intertrop. : Népaul, - Chine (Hong-Kong, Kiang-sou, Tché-kiang, etc.). - Japon.

52. L. chinensis Wats. Dendr. Brit. Tab. 117; Dec. Prodr. 4 , 333; Bungాe Enum. pl. chin. bor. 195, p. 33; L. japonica Thumbg. Fl. jap. 85; Sieb. et Zucc. Syn. fam. natur. jap., 46: A. Gray Plant. jap. exp. Perry p. 313; sinice KINYEN-HÔA, c'est-à-dire fleur d'or et d'argent.

Hab.: Shang-haï et Wôo-song. - Dans les haies, les clôtures des jardins, etc. - Juin.

Ar. géog.: Asie cent. : Népaul, Chine (Kiang-sou, Tché-kiang, (Chan-tong, Pé-tché-ly, etc.). - Japon.

Obs.: J'ai rencontré dans quelques jardins à Wôo-sông le Lonicera brachypoda Dec. in Prodr. 4, 375 (L. Alexuosa Thumbg.), espèce que j'ai retrouvée à l'état spontané à A-moy dans le Fo-kien, et à Tché-fou dans le Chan-tong.

\section{RUBIACÉES. JusS.}

53. Pæderia fotida Lin. Mant.52; Dec. Prodr. 4, 471; Thumb.

Fl.jap. 107; Kæmpfer Icon. tab. 9; Benth. Fl. Hong-Kong. 162 . $H a b$. : Shang-haï. - Sur les vieux remparts de la ville. - Juin. Ar. géog.:A sie intertrop. et temp. : Indes or.,-Aunboine, Ceylan. - Moluques, Timor, Ile Maurice, - Chine (Hong-Kong, Chu-san, Lôo-chôo, Kiang-sou, etc.). — Japon. 
54. Galium miltorhyzum Hance Decades plant.nov. chin. bor. in The. Journ. of the Linn. Soc. (1872); G. triflorum Michaux, Fl. Tor. Amer. 1, p. 80, var. angustifolium 0. Debeaux, in Herb. olim. (1862).

Dans sa monographie des espèces asiatiques du genre Galium, 11. Maximowicz considère le $G$. miltorhyzum, du centre et du nord de la Chine comme étant synonyme du G. tiachyspermum Asa Gray, de l'Amérique boréale et du Japon, auquel le savant monographe réunit le G.gracile Bunge des environs de Pé-king. D'après des échantillons authentiques de ces diverses espèces que possède M. Franchet,les Galium miltorhyzum, G. trachyspermum et G.gracile doivent être maintenus comme étant parfaitement distincts entre eux. (A Franchet in litteris.)

Le Galium trachyspermum A. Gray, du Japon, à corolle glabre et à fruits brièvement pédicellés, est certainement distinct $d u G$. triflorum var. Yokoscense Franchet et Savatier, G. pogonanthum des mêmes auteurs, lequel a une corolle très-velue, et les fruits longuement pédicellés. Le G. miltorhyzum Hance (G. trifioruin var. angustifolium 0 . Deb.) est, de son côté, très-distinct du $G$. gracile par ses petits fruits portés sur des pédicelles étalés, presque réfractés à la maturité. Par ce dernier caractère, il diffère à peine du G. triflorum Mich. de l'Amérique boréale, et ce n'est que par les aiguillons des feuilles, lesquels sont réfractés en dessous. que l'on en sépare facilement la plante chinoise. Dans notre ancienne variété du G. triflorum, les feuilles sont en outre trèsétroites, et presque linéaires pour ainsi dire.

$H a b$. : Shang-haï; sur les vieilles murailles et les remparts de la ville, près de la porte du Sud. - Juin.

Ar. géog.: Amér. bor. - Chine (Kiang-sou, le Pé-tché-ly). Japon.

Obs.: On cultive dans une foule de jardins quelques espèces dı g'enre Gardenia, soit pour la beauté de leurs fleurs, soit à cause des propriétés tinctoriales de leurs fruits capsulaires. Les plus répandues sont: le Chang-PÉ-HôA (Gardenia florida Lin.), plante qui croît spontanément sur les falaises de l'île de Hong-Kong, du Konang-tong, etc.,et dont les fruits nommés KiN-Tzí ou fruits d’or, sont employés pour la teinture en jaune; le HôANG-TChÉ-TzÉ(Gardenia radicans Thumbg.) originaire du Japon, etc. 


\section{COMPOSEEES. Adanson.}

Sect. I. CORYMBIFĖRES. Vaill.

55. Boltonia indica Benth. Flor. Hong-Kong. 174; Asteromaa indica Blume Bijdraj, p. 901; Dec. in Prodr. 5, 302; Aster indicus Lin. Spec. 1230; Thumb. Flor.jap. 316.

"Plante annuelle de 60 à 100 centimètres de hauteur, à tiges dressées, glabres, à feuilles alternes, ovales, oblongues, sessiles, entières, celles des rameaux inférieurs dentées quelquefois, les supérieures obovées; capitules très-petits solitaires à l'extrémité des rameaux. »

Hab. : Shang-haï. - Dans les concessions européennes, les terres incultes, les jardins, les prairies, etc. C'est une plante que l'on voit partout au mois de septembre, et qui avec l'Artemisia Thumbergiana sont caractéristiques de la végétation de Shang-haï.

Ar. géog. : Asie intertrop. et temp. : Indes orientales, Chine (Hong-Kong, Koûang-tong à Canton où il constitue l'Hisutsua cantoniensis Dec., Iles Lôo-chôo, Chu-san, le Kiang-sou, le TchéKiang, etc.). - Japon. - Iles de la Sonde, Java (Boltonia Javanensis Garc.).

56. Erigeron linifolius Willd. Spec. 3, 1955; Benth. Fl. HongKong. 175; Conyza ambigua, Dec. Prodr.5, 381, et Flor. Franc. suppl., p. 468.

$H a b$. : Très abondant à Shang-haï, dans les rues, au bord des chemins et sur les remparts de la ville chinoise. - Juillet-août.

Ar. géog. : Eur. aust. (France mérid., Espagne, Italie, Sicile, Grèce) - Afriq. bor., - Canaries,-Asie intertrop. et temp., Chine à Hong-Kong, le Kiang-sou, et probablement ailleurs.

57. Inula (Bubonium) chinensis Ruprecht Mss; Maxim. Prim. Flor. Amur. 140; Inula salicina Bunge Enum. pl. chin. bor. n²15, p. 39, nọn Lin.

Hab. : Shang-haï. - Prairies humides des rives du whâng-pôo et du Séou-tchou, dans la concession anglaise, etc. - Août.

Ar. géog.: Chine (provinces cent. et bor.), Kiang-sou, Pétché-ly. - Prov. de l'Amour et de l'Ussuri. - Japon.

58. Eclipta alba Miquel Flor.ned. Ind. 2, 65; Benth. Fl. HongKong. 181; E. erecta Willd. Spec. 3, 286; Dec. Prodr. 5, 490. 
Hab. : Shang-haï et Wô-song, dans les champs cultivís. Abondant surtout dans les plantations de coton - Juin.

Ar. géog. : Région intertrop. des deux mondes : Afriq. cent. et aust. (Egypte, Sénégal, Owarre, cap de Bonne-Espér., Port-natal). - Amér. bor., cent. et mérid. 'Surinam, Cuba, St-Domingue, La Trinité, Floride, Virginie, Nile-Grenade, Brésil). - Asie: Indes orient., Cochinchine, Chine (Hong-Kong, Kiang-sou, Chan-tong, etc.). - Iles de l'Océan Indien (Maurice, Bourbon) et de la Sonde (Java). - Les Moluques. - Japon.

59. Siegesbeckia orientalis Lin. Spec. 1269; Benth. Fl. Hong Kong. 182; Maxim. Prim. Fl. A mur. 151; Dec. Prodr. 5, 495; sinice KI-KIEN.

Hab. : Shang-haï. - Champs cult. et souvent échappé des jardins. - Juillet.

Ar.géog.: Régions intertrop. et temp. des deux mondes: - Amér. mérid. (Chili, Pérou, etc.). - Océanie, $\mathrm{N}^{\mathrm{lle}}$-Zélande, Iles de la Société; - Australie; - Iles de l'Océan Indien (Maurice, etc.). Asie : Indes orientales, Chine (Hong-Kong, Iles Lôo-chôo, Chu-san. Prov. de Kiang-sou, Tssé-tchuen, Hô-nan, etc. - Japon.

60. Chrysanthemum indicum Lin. Spec. 1253; Benth. Fl. HongKong. 181; Pyrethrum indicum Cass. Dict. 41, p. 149 non Roxburg; Chrysanthenum japonicum Thumb. Fl. jap. 321: sinice TA-KIOU-HÔA, HOANG-KIOU-HÔA, etc.

Hab. : Shang-haï. - Bord des chemins, des fossés, etc., dans les concessions française et anglaise, sur les remparts de lia ville. - Septembre à décembre.

Ar.géog.: Asie intertrop. et temp.: Indes orient, Chine(Rivages: maritimes depuis Hong-Kong jusque dans le Pé-tché-ly). - Japon.

Obs. : Dans ces diverses stations chinoises, le Chrysanthemum indicum ne varie par aucun de ses caractères. Les spécimens de cette plante provenant des ravins du Pic Victoria à Hong-Kong (500 mèt. d'altıtude), ceux des prairies humides de Shang-haï, et les falaises micaschisteuses du cap de Tché-fou dans le Chan-tong*, sont absolıment identiques entre eux, et à ceux l'origine japo. naise. On ne peut confondre cette espèce des plus communes avec le Pyreltrum sinense Sabin. in Trans. hort. soc. Lond. 4, 330: Dec. in Prod. 5, p. 62, plante généralement cultivée dans tous les jardins chinois. 
Le $C h$. indicum que jaai récolté à l'étut spontané, est une plante vivace, sous-frutescente dès la base, à rameaux étalés on couchés (échantillons des falaises de Tché-fou), et pubescents an sommet. à feuilles épaisses et glabres, à capitules floraux très petits, et toujours de couleur jaune-clair. Cultivée dans les jardins, cette espèce produit plusieurs variations à fleurs pleines ou demipleines, et de couleurs variées, qui sont loin de valoir celles que l'on obtient du Pyrethrum sinense. Celui-ci constitue la plante favorite, la fleur préférée des Chinois. On la voit partout, chez le riche comme chez le pauvre, dans toutes les habitations et les jardins. M. R. Fortune a observé qu'd̀ Shang-haï et à Ning-po, les chrysanthèmes sont en général mieux soignés que dans le sud de la Chine à Canton, le mode de culture étant à peu près le même. Les beaux résultats obtenus par les horticulteurs du Kiang-sou doivent être attribués en partie à l'influence du climat natal, le $C h r$. sinense étant originaire des provinces du centre et du nord de la Chine.

61. Arthernisia Thumbergiana Max. Mélang. biol. in Mém. acad. sc. St-Pétersbourg, vol. 8, p.528; 1. apiacea Hanre, in Decad. plant. nov. chin. bor. (Journ. of the Linn. Soc. Lond., 1872).

$H a b$. : Cette rare espèce, signalée seulementau Japon, se trouve partout à Shang-haï, dans les terres incultes ou marécagenses, les prairies, les jardins, etc., de la concession française. - Septembrr et octobre.

Ar. géog. : Chine (Shang-haï). - Japon (Iles de Nippon et de Kiou-siou).

Obs. : Espèce voisine de l’A. annua L., mais bien distincte par ses capitules trois fois plus gros, ses feuilles moins divisées, à rachis pectiné et non entier.

62. Gnaphalium pallidum. Lam. Dict. 2, 750; Walr. Cat. $\mathrm{n}^{\circ} 2953$;

Dec. Prodr. 6, 230; G. orixense Roxburg Fl. ind. 3, 425.

Hab. : Wôo-sông, champs d'orge et prairies humides. - Juin.

Ar. géog. : Asie cent. et subtrop. : Népaul, Indes orient., Chine (Kiang-sou). - Iles de l'Océan Indien, Maurice, Bourbon, Madagascar.

Obs. : Les espèces suivantes sont cultivées comme plantes d'ornement dans tous les jardins à Shangr-haï, Wôo-sông et ailleurs.

- Tagetes patula et T. erecla L., TSIN-TSAN-HÔA: 
- Calendula officinalis L., KIN-TSIEN-HOA:

- Callistephus sinensis Cass. (Aster sinensis L.), P'̈-KING-HOA ои reine desfleurs;

- Helianthus annuus L., KoûEI-KoûAN-HÔA ;

- Artemisia dracunculus L., Tsin-KaO, cultivé pour l'usage culinaire, etc.

Sect. II. CYNAROCÉPIIAL ÉES. Vaill.

63. Aplotaxis Bungei Dec. in Prodr. 6, 539; Cirsium lyiatum, Bunge Enum.pl. chin. Bor. no 203, p. 36.

Hab.: Wôo-sông. - Prairies des rives du Whâng-pôo. - Juin. Ar.géog. : Chine (Kiang-sou, Pé-tché-ly, etc.). - Japon.

Obs. : Les échantillons de l'A plotaxis Bungei que j'ai récoltés à Wôo-sông, sont identiques avec ceux quej'ai reçus de provenance japonaise, et que M. Miquel rapporte à tort, je crois, à l'A. multicaulis Dec., espèce propre au Népaul.

64. Saussurea (Theodora) glomerata Poiret Enc. méth. suppl. 5. 71 ; Dec. in Prodr. 6, 537; Ledeb. Fl. alt. 4, 21.

Hab. : Shang-haï. - Prairies des rives du Whâng-pôo, et fossés aquatiques qui bordent la chaussée de Wôo-sông, dans la concession américaine. - Septembre à décembre.

Ar. géog.: Asie bor., Sibérie, Altaï, Chine (Kiang-sou, Pétché-ly, etc.).

65. Girsium sejetum Bunge Enum. pl. chin. bor. n 202, p. 36:

Dec. Prodr. 6, 643; sinice Yun-TChU, TA-KY et Sia0-KY.

"Caule erecto simplici, striato, floccoso : foliis sessilibus, oblon"gis, serrulato et spinoso-ciliatis, junioribus utrinque araneosis, > demum glabris; capitulis terminalibus subsolitariis, involucri squamis glabris, exterioribus ovatis, subapiculatis, internis " lanceolatis, inermibus, pappo subæqualibus. Flores pallide"purpurascentes.»

Var : forma A., foliis integris;

» forma B., foliis lyrato-partitis.

Hab. : Wôo-sông, dans les champs de blé, les jardins et les cultures, où cette espèce paraît remplacer en Chine le Cirsium arvense Scop. (Serratula arvensis L.), qui dans des stations analo. gues est si abondant en Europe et dans l'Amérique boréale. Juin et juillet. 
Ar. géog. : Prov. cent. et bor. de la Chine (Kiang-sou, Chantong, Pè-tché-ly à Tien-tsin et Pé-king).

Obs. : Le Carthamus tinctorius lin., sinice HoAng-HOA, et HongLAN-HÔA, introduit du Turkestan en Chine, par Chang-Hien, est cultivé dans les jardins du Kiang-sou, et en grandes cultures dans le Hô-nan, le Tssé-chuen, etc., pour ses fleurs qui sont très employées dans la teinture des étoffes.

Sect. III. CHICORACÉES. Juss.

66. Sonchus brachyotus Dec. Prodr. 7, 186; Ledeb. F'l. Ross. 2, 818; Maxim. Prim. flor. Amur. 180 ; S. uliginosus Turezan. Add. emend.Baik. Dahur.p. 169, non M. Bieb.; Led. Fl. Att.4, 142. Affinis $S$. maritimi L.

$H a b$. : Shang-haï, Wôo-sông et ailleurs. - Prairies humides du Whâng-pôo; bords des canaux d'irrigation, des rizières, etc. Août et septembre.

Ar.géog.: Asie bor.:Sibérie,Altaï, Chine (Kiang-sou, Chan-tong. Pé-tché-ly, etc.) - Provinces de l'Amour.

67. Taraxacum officinale Wigg. Prim. Hols. 56 (1780); Ledeb. Fl. Alt. 2, 812; Maxim. Prim. A. Amur. 179; T. dens-leonis Desfont. Flor. Atlant. 2, 228; Turczan. Fl. Baih.-Dahur. 2, 152; Benth. Fl. Hong-Kong. 192; sinice Pô-Kou-Tsao.

Hab.: Shang-haï et Wôo-sông. - Prairies, jardins et cultures. Mars-avril.

Ar. géog.: Eur.,-Afriq. bor. - Asie occ., cent. et bor. : Sibérie, Altaï, Baikal, Daoûrie, Chine (Hong-Kong, Kiang-sou, Pé-tchély, etc.) - Prov. de l'Amour. - Japon.

68. Ixeris debilis Asa Gray Bot. jap. 393, in Mém. Amèr. acad. soc.; Benth. Fl. Hong-Kong. 198; Youngia pygmaa. Ledeb. in Fl. Ross. et Prananthes polymorpha ejusdem in Flor. Altaic. 4, 144; Pr. debilis Thumbg. Fl. jap. 300; Youngia debilis Dec. Prod. \%, 194.

Hab.: Wôo-sông. - Bord des champs, des fossés aquatiques, et jardins des terres humides. - Juin.

Ar.géog.: Asie bor.: Sibérie, Altaï, etc. - Chine (Hong-Kong", Kiang-sou, etc.). - Japon.

69. Youngia humilis Dec. Prodr. 7, 194; Prananthes humitis Thumb. Fl. jap. 303. 
Hab.: Sur les vieux remparts de Shanch-haï, près de la porte du Sud. - Mai.

Ar. géog.: Chine (Kiangr-sou). - Japon.

\section{ÉBÉNACÉES. Ventenat.}

70. Diospyros schi-tzé Bunge Enum. pl. chin.bor. no 237, p. 92: D. costata Carrière in Rev. Torlic. $1870 ;$ D. kaki Dec. Prodr. 8, 229 (ex parte) non Lin., nec Thumb.: sinice Tzé-Tzr. TCHÉ-TZÉ, TCHI-TZÉ.

«D. arborea, ramis pedunculis calicibusque basi tomentosis: - foliis obovato-oblongis, 15-18 centimet. longis, 5-6 cent. latis, » acutis, supra pubescentibus, subtus villosis; floribus solitariis, 》axillaribus 4-plo majoribus quam in D. loto; Bacca maxima, » depressa, rubro-aurantiaca, subtus 4-sulcata, plerum que asper* ma, magnitudine mali majoris (Bunge).

Hab. : Shang-haï, Wôo-sông et la province de Kiang-sou, où il est très-répandu et cultivé sous le nom de Tché-tzé, nom qui s'applique également en Chine à toutes les espèces du genre Diospyros.

Ar. géog.: Asie intertrop. et temp.: Indes orient., Cochinchine. - Chine (Kiang-sou, Tché-kiang, Pé-tché-ly, etc.).

Obs. : Le Diospyros schi-tzé que j'ai rencontré fréquemment dans les cultures de Shang-haï, porte des fruits de la grosseur d'une pomme ordinaire, de couleur rouge orangée, arrondis au sommet, un peu aplatis à la face inférieure, et marqués en dessus de quatre sillons à peu près perpendiculaires entre eux. Ces fruits ont le plus souvent les graines avortées, et sont fort recherchés des Chinois pour leur goont délicieux.

Dans la Revue horticole (années 1870, 1871 et 1872), M. Carrière s'efforce de démontrer que son Diospyros costata qu'il a vait d'ahorel appelé $D$. kaki est bien différent du $D$. schi-tzée, et à ce sujet il donne des renseignements très-précis, qu'il tient d'une personne qui a longtemps résidé en Chine. Cette personne a vu beaucoup de ces gros fruits (ceux du Diospyros costata, l'espèce nouvelle de M. Carrière) aux environs de Shang-haï, où l'arbre qui les produit atteint la taille de nos beaux pommiers. Ce Diospyros costata n'est autre que le $D$. schi-tzé, bien différent du $D$. kutki, lequel a les feuilles plus minces, glabres, d'une forme différente et de moitié plus petites. J'ai lieu de penser que M. Carrière a décrit, 
sous le nom de $D$. costata, une forme à feuilles plus arrondies du D. schí-tzé. Je dois faire remarquer, contrairement à l'opinion de Decandolle qui réunit le D. schi-tzé au D. kaki (Prodr. 8, 229), que le $D$. kaki est une espèce propre au Japon, non cultivée par les Chinois, et dont les fruits sont beaucoup plus petits. Je n'ai vu à Shang-haï que le $D$. schi-tzé, à l'exclusion de toutes les autres. espèces.

\section{OLEACEES. Lindley.}

71. Fontanesia phillyræoïdes Labillardière, in Decad. plant. Syrice 1, p. 9; Lamark Ill.gen. Tab. 22; Dec. Prodr. 8, 287. Var. sinensis Debeaux in Herb. (1862); forma foliis magis elongatis lanceolatis que.

"Frutex 2-3 met. altus, Ligustri facie, ramis oppositis; folia " opposita, breve petiolata, elongata, lanceolata, integerrima, gla» briuscula; flores albi, inodori. Samara obovata, utrinque emar" ginata, compressa, margine membranacea, anguste 2-3 alata. " (Dec.)

La forme sinensis ne diffère du type que par ses feuilles $d u$ double plus allongées et longuement lancéolées.

Hą. : Wôo-sông. - Partout, dans les haies, où il sert à former des clôtures, et des abris contre la violence des vents qui régnent iruns cette contrée. - Fl. en mai ; fruct. en juillet.

Ar. géog. : Le type, Asie-Mineure (Syrie). - La variété, Chine (Kiang-sou).

Ous. : Cette plante des plus intéressantes, et sur l'identité de laquelle je ne possède aucun doute avec l'espèce de Syrie, est excessivement abondante dans les haies et les clòtures des propriétés à Woo-sông. Le type (Fontanesia phillyraoïles; est un sous-arbrisseau, qui paraît être spécial à l'Asie-Mineure, et dont la présence dans le Kiang'-sou a lieu certainement de m'étonner, beaucoup. Dans la plante de Wô-sông, les feuilles sont plus allongées que dans celle de Syrie; mais ce caractere, qui est constant en Chine, est probablement du à la nature tourbeuse du sol et au climat humide de cette contrée, et ne me paraît pas suffisant pour constituer une espèce distincte.

Le Fontanesia phillyraoüdes est tellement abondant à Wôo-sông, qu'il serait difficile de savoir si cette espèce est réellement indigène ou bien impórtée et acclimatée dans cette localité. J'ai lieu 
de penser cependant qu'elle a été importée dans le territoire de Wôo-sông, de même que le bambou, qui est également acclimaté dans le Kiang-sou, et qui provient évidemment des provinces méridionales de la Chine ou de l'Asie subtropicale.

La variété sinensis du Fontanesia phillayroüdes est extrêmement voisine du F. Fortunei Carrière in Rev. Hort. (1859). Cette espèce, d'une rusticité à toute épreuve, a été introduite eu 1854 dans les cultures du Jardin des Plantes à Paris par M. de Montigny, alor's conșul de France à Shang-haï. La description du r. Fortunei de M. Carrière est de tout point conforme à l'espèce de Wô-sông, à l'exception toutefois de l'époque de la floraison. Les fleurs du F. Fortunei, dit M. Carrière, qui paraissent en seplembre et octobre, sont pédicellées, axillaires, et disposées en grappes terminales spiciformes. Or, dans la plante de Wôo-sông, la floraison a lieu au commencement de mai, et le fructification est déjà avancée dès les premiers jours de juin. Le Fontanesia Fortunei cultivé au Jardin des Plantes de Bordeaux, mais dans des conditions climatologiques différentes de celles de Wôo-sông, ne commence à fleurir que dans la dernière quinzaine de septembre, et ne paraît pas devoir prospérer beaucoup à l'école botanique du jardin de cette ville. Il y aurait donc deux espèces de Fontanesia (lans les régions moyennes et froides de la Chine, le $F$. phillyraoïdes var. sinensis, et le $\vec{F}$. Fortunei introduiten France en 1854 par M. de Montigny.

72. Osmanthus fragrans Loureiro Fl. Cochinch. 1, p. 35; Dec. Prodr. 8, 291; Sieb. et Zucc. Fl. jap. fam. nat. 2, 43; Olea fragrans Thumb. Fl.jap., p.18; sinice KoûEI-HÔA, LAN-HOA. $H a b$. : Très-répandu à Shang-haï, dans les jardins, et autour des habitations rurales.

Ar.géog. : Asie intertrop.: Cochinchine, Chine (Hong-Kong, Koûan-tong, Fo-kien, Koûang-si, Yun-nan, Tché-kiang, Kiangsou, etc.). - Japon.

Obs. : Cet arbre est souvent cultivé pour ses fleurs à odeur forte et suave, qui servent à aromatiser une variété de thé noir nommée Lan-tcha.

On cultive aussi dans les jardins plusieurs espèces arborescentes, soit comme plantes d'ornement, soit à cause de l'odeur suave de leurs fleurs. J'ai remarqué entre autres les Syringa vul- 
garis et S.persica L., le Forsytlia suspensa Wahl ou LIEN-HÔA, le Jasminum sambac Ait. ou Mo-Ll-HOA, originaire des Indes orientales, le Jasminum officinale L. ou Yè-si-min, mot indigène qui paraît venir de celui de Jasmin, etc.

\section{GENTIANÉES. JUSS.}

73. Gentiana squarrosa Ledeb. in Act. Petrop. 5; 527; Bunge Enum. pl. chin. bor. no 252, p. 52; Dec. Prodr. 9, 107; Griseb. Monog. gent. 273; A. Gray Plant. jap. exp Perry, p. 317; G.aquatica var. Fræl. Gent.p. 80 ; sinice KI-KAN-Kıô. «Caule ramossissimo-piloso-glandıloso, foliis spathulatis mar» gine scabriusculis, apice recurvo-aristatis, imis rosulatis rotun»datis, calycis dentibus ovatis apice recurvis, plicis bifidis 》lobis acutis vix brevioribus, corollæ tubo ventricoso, capsula " cuneta basi acuta, apice truncata (Grisebach). »

Hab. : Lieux humides et incultes autour des tombeaux, à Shanghaï, Zi-ka-wé. - Avril.

Ar.géog.: Asie occ. et orient. : Caucase, Altaï, Chine (Kiang-sou, le Pé-tché-ly, etc.). - Japon.

\section{BIGNONIACEES. R. Brown.}

74. Catalpa Bungei C. A. Meyer Diss. (1837); Dec. Prodr. 9, 226 ; sinice Ko-TSIEN.

Hab. : Shang-haï, où cet arbre est planté le long des chemins, autour des pagodes, dans les parcs et les jardins. - Mai.

Ar. géog. : Chine (Kiang-sou, Pé-tché-ly, etc.). — Japon.

75. Sesamum indicum Dec. Plant. rar. Genève, p. 18.

Var. forma foliis indivisis; S. orientale L. spec. 883; sinice TCHÉ-MA, CHI-MA-TZÉ.

$H a b$. : Cultivé dans tout le territoire de Shang-haï. Ses graines produisent une huile oléagineuse journellement employée dans l'économie domestique. - Juillet.

Ar. géog. : Asie cent., occ. et or. : Indes orient., Iles de l'Océan indien, Java, etc.; - Chine(Tché-kiang, Kiang-sou, Chantong, Pé-tché-ly, etc.). - Japon. - Afriq. bor. : Egypte. - Amér. intertrop.

Obs. : On rencontre fréquemment dans les jardins de Shang-haï 
le Tecoma grandiflora Delaun. (Bignonia grandiflora Thumbgr. et B. sinensis Lam.); sinice LIEN-TSAÔ, TSI-KOÛEI-KOUA.

\section{CONVOLVULACÉES. JUSS.}

76. Ipomæa reptans Poiret Enc. suppl. 3, p. 460; Dec. Prodr. 9, 349; Convolrulus reptans Lin. Spec. 225; Ipomea aquatica Forsk. Descript. p. 44; sinice Pô-TING, Pô-TSAI, TSAN-LIEÔU-TZÉ. $H a b$. : Cultivé en grand à Shang-haï, dans les terres humides au pied des remparts de la ville, etc. Au printemps, on en apporte les feuilles sur le marché, et on s'en sert dans la cuisine chinoise en guise d'épinards. - Juin.

Ar. géog. : Afriq. cent. : Nubie, Sénégal; Asie, Arabie, Amboine, Népaul, Indes orient. - Chine (Kiang-sou, Tché-kiang, etc.).Iles de l'Océan Indien : Madagascar. - Iles de la Sonde : Java. Timor, etc.

77. Batatas edulis Choisy Convolvul. orient. p. 53; Dec. in Prodr. 9, 338; Sieb. et Zucc. Fam. nat.jap. 2, p. 34; Convolvulus batatas Lin. Amæn.; C. edulis Thumb. Fl.jap. p. 84 ; sinice HoûANG-CHÉ, HoûANG-TSAI.

$H a b$. : Cultivé sur une vaste échelle dans tout le territoire de shang-haï pour ses rlızzomes alimentaires.

Ar.géog. : Régions intertropicales des deux mondes. - Afrique cent., Amér. cent. - Dans toutel'Océanie. - Asie intertrop.et temp. : Indes orient. - Cochinchine, - Chine. - Japon.

Obs. : Le Quamoclit vulgaris Chois. (TSIEN-HôA des Chinois) et le pharbitis nil Chois. (TSIEN-NEÔU-TZÉ), se rencontrent parfois dans les haies, les décombres, mais échappés des jardins.

\section{BORRAGINÉES. JusS.}

78. Erithrychium pedunculare Alph. Dec. in Prodr. 10, 128; Maxim. Prim. A. Amur. 203; Ledeb. Fl. Altaï.3, 15; Myosotis peduncularis Bunge Enum.pl. chin. bor. n 265 (ex parte). Hab. : Wôo-sông et Shang-haï; - Bord des fossés aquatiques et des canaux d'irrigation. - Mai et juin.

Ar. géog. : Asie bor., Mongolie, Daourie, Astrakan, Chine Kiangr-sou, Pé-tché-ly). - Prov de l'Amour. - Japon. 
SOLANÉES. Juss.

79. Lycium sinense Lam. Dict. 3, p. 509 ; Dunald in Dic. Prodr. 13, p.510; Bunge Enum. chin. bor. n² 276; Benth. Fl. Hong-Kong. 245; sinice KôU-KI-TzÉ.

Hab. : Haies, lieux incultes et vieux remparts à Shang-haï, etc. - Juillet.

Ar. géog. : Eur. aust. - Afriq. bor. - Asie subtrop. et temp. : Chine (Hong-Kong, Tché-kiang, Kiang-sou, Chan-si, Chan-tongr, Pé-tché-ly, etc.). - Japon.

Obs. : Plusieur's espèces de Solanées sont cultivées à Shanğ-hä pour l'usage alimentaire, entr'autres les Capsicum annuum I. et $C$. longum Lin. (Lo-TSIAO et TsiN.tsiao), le Solanum tuberosum I. (Hô-LAN-CHOU); le S. melongena L. (KIA-Tzí); le Lycopersicum esculcntum Mill. (TAX-KIA-TZÉ), etc.

\section{SCROPHULARIÉES. JusS.}

80. Mazus rugosus Lour. Flor. cochinch. 38.5; Dec. Prod. X, 375; A. Gray Plant. jap. Perry exped. p. 316; Miq. Prol. th. jap. 48; Mazus bicolor Benth. in Walhr. cat. $\mathrm{n}^{\circ} 3913$; Maxim. Prim. fl. Amur. 295; Lindernia japonica Thumb. Fl. jup. 253. Hab. : Shang-haï, Wôo-sông, etc. - Lieux marécageux, bord des rizières, etc.- Avril et mai.

Ar. géog. :.Asie bor., subtrop. et centr. : Himalaya bor. et occ., Indes or. (depuis le Caboul jusqu'en Birmanic). - Iles de la Sonde, Java._Cochinchine.- Chine (Kiang-sou, Chan-tong, Pé-tché-ly, etc.) - Prov. de l'Amour et de l'Ussuri. - Japon.

81. Veronica anagallis Lin. Spec. 16; Dec. Prodt. X, p. 187; Thumb. Fl. jap. p. 20; Miquel Prol. A.jup. 52; A. Gray Plant.jap. Perry exped. p. 361:

IIab. : Wôo-sông. - Fossés aquatiques et prairies marécudgreuses. - Juin.

Ar.géog. : Eur. - Amér. bor. - Asie occ. et or. : Asie-Mineure, Arménie, Anatolie, Perse, Indes or., Chine (Kiang-sou). - Japon. - Afrique bor. et aust. : Égypte, Algérie, cap de Bonne-Espérance.

OROBANCHÉES. L. C. Rich.

8.2. Eginetia indica Roxburg Plant. coromand. p. 63: Benth. in 
Dec. Prodr. Xl, p. 43, et Fl.Hong-Kong. p. 257; Wight et Arn. Flor. Ind. orient. Icon. tab. 895; Miquel Fl.ind. bat.2, 712, et Prol. jap. 287; Orobanche aginetia Lin. Spec. 383.

Hab. : Shang-haï. - Parasite sur les racines du Tamarix chinensis et autres arbustes plantés autour des tombeaux. - Juin.

Ar. géog. : Asie cent., subtrop. et temp. : Népaul, Silhet, Circars, Tavoy, etc. - Iles Philippines. - Chine (Hong-Kong, archipel de Chu-san, Kiang-sou). - Japon (Kiu-siu, Nippon).

\section{LABIÉES. JusS.}

83. Galamintha (Clinopodium) repens Bentham in Dec. Prodr. 12, p. 233; Clinopodium repens Wall. var. A in Plant. asiat. rar. 1, 66; Melissa repens Benth. Labiat. 39:3; sinice Р0̂-ноे. Affinis C. arvensis Benth.

Hal. : Wôo-sông: - bord des fossés aquatiques, près les magaasius à charbon de terre. - Juin.

Ar. géog. : Asie bor. et cent.: Indes orient., Népaul. Assam. Chine (Kiang-sou .

84. Salvia plebeia Brown Prodr. 501; Benth. in Dec Prodr. 12, p. 356; et Fl. Hong-Kong. p. 277; Bunge E'num. pl. chin. bor. p. 50, no 283; Miquel Prol. jap. 40 : sinice KIX-KaI.

"Species fragrantissima, habitu Melissa, floribus minimis. "dense verticillato-paniculatis, pallide ceruleis. "

Hab. : Wôo-sông; prairies inondées, bord des fossés aquatiques. - Juin.

Ar.géog. : Asie cent, intertrop. et temp. : Caboul, Indes orientales, Cachemyr, Silhet, Afghanistan, Ceylin, - Chine, Hong-Kong. Kiang-sou, Chan-tong, Pé-tché-ly, etc.) - Japon (Réggion montag. près de Nangasaki). - Iles de la Malaisie, - Philippines, - Australie, etc.

85) Nepeta glechoma Bentham Labiat. mon. p. 485; Fl. HongKong. 277; et in Dec. Prodr. 12, p. 391; Miquel Prol.jup. p. 41: A. Gray in Plant. jap. exp. Perry, 316 et Bot. jap. p. 402: Glechoma hederacea Lin. Spec. 89; Maxim. J'rim. th. A mur. 218: Ledeb. Fl. alt.3, 379; sinice N'Go-P0̂. Тсны-тsiso. Val. hirsuta Waldst. et Kit, Plant. rar. hungar. 2. 12t: Benth. (or. cit.). 
$H a b$. : Shang-haï et Wôo-sông. - Bord des chemins, au pied des haies. - Avril-nai. La variété hirsuta se trouve seule dans ces localités.

Ar. géog. : Toute l'Europe. - Russie d'Asie, Sibérie, - Chine (Hong-Kong, Kiang-sou, etc). - Prov. de l'Amour, - Japon, Amér. bor.

86. Brunella vulgaris Lin. Spec. 837; Benth. in Dec. Prodr. 12, p. 10, var. B vulgaris; A. Gray in Plant. jap. Perry: sinice YÔ-KÔU-TSAO.

Hab. : Wôo-sông. Prairies des rives du Whâng-pôo; bord des fossés aquatiques, etc. - Juin.

$A r$ géog. : Régions temp. de l'hémisphère boréal. Eur. - Afriq. bor., - Amér. bor., - Asie bor. : Tauride, Caucase, Altaï, Perse, Indes orientales, Cachemyr, Himalaya, Chine (Kiang-sou). Japon.

Obs. : La plante de Chine est plus robuste que celle d'Europe. Ses tiges, hautes de 30 à 40 centimètres, sont simples ou un peu rameuses au sommet; l'épi floral allongé et très-compacte porte des fleurs à corolles grandes, tantôt glabres et tantôt velues: ou pubescentes. Les échantillons du B. vulgaris, récoltés au Japon par le $\mathrm{D}^{\mathrm{r}}$ Savatier, sont identiques à cenx des prairies de Wôosông.

87. Leonurus sibiricus Lin. Spec. 818; Benth. in Dec. Prodr. 12, p. 501, et F'l. Hong-Kong. 278; Maxim. Prim. Al. Amur. 221 : Miquel Prol.jap.p. 45 ; L. tataricus Burm. Fl. Indes orient. 127; Stachys artemisia Lour. Fl. Cochinch. 365; sinice KÉ-KoveI, Y'́-MOU-X'GaI.

Hab.: Shang-haï et Wôo-sông. Très-commun dans les fossés aquatiques, les canaux d'irrigation, etc. - Nai et juin.

Ar. géog. : Régions intertropicales des deux mondes.- Afrique cent. : Séuégambie. - Amér. cent. et méric. : Jamaïque, Cuba, Saint-Thomas, Trinité, Brésil, Rio-Janeiro, Sainte-Catherine. Asie: Indes orientales, Bengale, Silhet, Assam, Cochinchine, Chine (Hong-Kong, Koûang-tong, Fo-kien ì Amoy, Tché-kiang, Kiang-sou, Chan-tong, Pé-tché-ly). — Mongolie, Daoûrie, Sibérie. - Prov. de l'Amour et de l'Ussuri. - Japon. - Iles de la Sonde et de la Malaisie, Java, Timor. - Philippines.

Obs. : L'Ocymum basilicum L. (HIANG-TSAI-TSAO), le Mentha pipe- 
rita L. (Pô-hô), l'Esholtsia cristata L. (KIAN-Jot-TSAO) et autres labiées sont cultivés dans tous les jardins de Shang-haï.

VERBÉNACEES. JusS.

88. Verbena officinalis Lin. Spec. 29; Dec. Prodr. XI, p. 547 : et Fl. Hong-Kong. p: 268; Thumbg. Fl. jap. p. 22; Niquel Prol.jap. 29; A. Gray Plant.jap. exp. Perry. p. 316; sinice Ma-PIEN-TSAO.

Hab. : Shang-haï et Wôo-sông.- - Bord des chemins et deschamps cult. - Mai et juin.

Ar.géog. : Régions intertrop. et temp. des deux mondes : Eur. - Afriq. bor., cent. et aust. : Algérie, Egypte, Abyssinic, cap Vert, cap de Bonne-Fspérance-C Canaries.-Amér. bor., cent. et mérid. : Mexique, Géorgie, Cuba, Brésil. - Asie : Indes orient. - Chine (Hong-Kong, Kiang-sou, Tché-kiang, Chan-tong, etc.)Japon. -- Australie.

89. Glerodendron trichotomum Thumberg Flor. jap. p. 256: Dec. Prodr. XI, 668 : Banks Icon. Kæmpf. Tab. 22; Persoon Syn.plant.2, 145: Miq. Prol. fl.jap. p. 31.

Magnifique plante vivace de 10 à 14 décimètres de hauteur, à feuilles glabres, les supérieures largement ovales entières, les inférieures subtrilobées, à fleurs dispostés en une panicule terminale très-ample, trichotome, à corolles tubuleuses d'un blanc légèrement rosé.

Hab. : Shang-haï.-Bord des fossés autour des vieux remparts de la ville, et au nord de la concession française. - Juillet et août.

Ar.géog. : Chine (Kiang-sou). - Japon.

Obs. : Le Clerodendron tricholomum, qui est également une plante spéciale à la flore du Japon, est une de ces rares espèces. qui donnent à la végétation de Shang-haï un caractère particulier. L'effet qu'il produit dans les fossés aquatiques pendant les mois d'aont et septembre, à l'époque de la floraison, est dig'ne l'ètre signalé aux horticulteurs. C'est, à mon avis, l'une des plus belles plantes indigrènes du territoire de shang-haï.

ACANTHACÉES. JUSS.

90. Rostellularia procumbens Nées ab Esemb. in Will. Planl. 
rar. asial. 3, 101 : Dec. Prodr. XI, 1, 391 : Miquel Prol.jap. 57; Justicia japonica Thumbg. Fl.japon., p. 20 : J. hivtclla Wall. Cal. $\mathrm{n}^{\circ} 2448$.

Var. A in Dec. (loc. cit.).

«Herbacea, caule erecto, 10-15 centim. alto, geniculato, hispido : » foliis setoso-pilosis, oblongo-lanceolatis, breviter petiolatis, te》nuissime sinuato-dentatis; spicis subtetragonis; corolla rosea. "Planta speciminibus indicis simillima. "

Hab. : Shang-haï. - Champs humides au milieu des plantations re coton et de Corchorus capsularis. - Juillet.

$A r . g e ́ o g .:$ s sie cent., intertrop. et temp. : Himalaya, Coromandel. - Chine (Hong-Kong", Kiang-sou). - Japon (Nippon). Tles de la Malaisie, Timor.

\section{PRIMULACÉES. Vent.}

91. Lysimachia candida Lindley, in Journ. of the hort. sor. Lond. 1. p. 300.

Fspèce voisine du $L$. pentapetala Bunge (Apochoris pentapetala Duby), dont elle diffère par sa taille deux fois plus petite, ses tiges plus grêles, et par ses fleurs disposées en ombelle serréc, etc. Le Lysimachia pentapetala paraît être spécial à la Chine boréale, tandis que le $L$. candida n'a été trouvé jusqu'à présent que dans les provinces centrales.

Hab. : Shang-haï, - Prairies humides autour des concessions irançaise et anglaise. - Mai.

Ar. géog. : Chine Kiang-sou à Shang-haï, T'ché-kiang ì Ning-po).

92. Androsace saxifragæfolia Bunge Enum. plant. chin. bor. no 297, p. 35: Duby in Dec. Prodi. 8, p. 53; sinice Yu-TsINTSAO.

Plante très-voisine de l'Androsace rotundifolia Hardw. par son involucre à pédicelles allongés, divariqués, et les folioles extérieures, ovales, aiguës, ciliées, réfléchies, les intérieures presque linéaires: mais la corolle est bien celle de l'A. suxifragafolia de Bunge. L'auteur précité attribue à son espèce une sonche bisannuelle, tandis qu'elle est annuelle dans tous les échantillons récoltés à Shang-haï.

Hab. : Liemx incultes, autour des tumulus à Shang-haï.-Avríl. 
Ar. ge̊og. : Chine (Kiang-sou, Pé-tché-ly, etc.)

Obs. : On cultive dans les serres et les maisons particulières le primula sinensis Lindley, qui varie à fleurs blanches, pourpres ou rosées, et fleurit pendant toute la durée de l'hiver.

\section{PLANTAGINÉES. JusS.}

93. Plantago major Lin. Spec. 163; Decaisne in Dec. Prodr. XII, 698; Ledeb. Flor. Altai. 4, p. 476: Bunge Enum. pl. chin. bor. no 304; Benth. Fl. Hong-Kong. p. 280: Maxim. f. Amur. 221: Regel Tent.f. ussur. p. 120; Miquel Prol.jap. p. 282; sinice LIEU-KIN.

Hab. : Shang-haï et Wôo-sông. - Bord des chemins, des rues et sur les vieux remparts de la ville. - Juin.

Ar. géog. : Eur. - Afriq. bor. - Amér. bor. - Asie : Chine (Hong-Kong, Kiang'-sou, Chan-tong, Pé-tché-ly, etc.). - Prov. de l'Amour et de l'Ussuri. - Japon.

\section{AMARANTHACÉES. Juss.}

94. Amaranthus spinosus Lin. Spec. 1407 ; Moq. in Dec. Prorl. XIII, p. 260; Benth. Fl. Hong-Kong. 284; sinice KIA-YENTSAI.

Hab. : Shang-haï. - Borl des rues, des chemins, décombres. cultures et jardins dans la concession francaise. - Septembre.

Ar. géog. : Régions intertrop. et tem. des deux mondes : Amér. cent., Hispaniola, Vera-Cruz, Vexique, Porto-Ricco; - Pensylvanie. - Asie : Ceylan, Indes orien., Chine (Hong-Kong, Tchékiang, Kiang-sou, Chan-tong, etc.). Iles de la Sonde, Timor. Java; Iles Philippines.

95. Euxolus viridis Moquin in Dec. Prodr.XIII, 273; Miquel $P_{r}$ ol. fl. jap. p. 64; Amaranthus viridis Lin. Spec. 1405; Benth. Fl. Hong-Kong. 284.

$H a b$. : Shang-haï. - Champs cult., jardins, bord des chemins, etc. - Septembre.

Ar. géog. : Rég'ions intertrop. et temp. des deux mondes : Eur. aust. - Afriq. bor. et cent. : Algérie, Egypte, Abyssinie, Canaries, Madère, Acores -Amér. cent. et mérid. : Jamaïque, Brésil. - Iles de l'Océan austral : Taïti, Marquises. - Asie : Chine (Hong-Kong, Kiang-sou). - Japon. 
96. Achyranthes aspera Lin. Spec. 295: Moq. in Lec. Prodr. XII, 314: Benth. Fl. Hong-Kong. 285.

Var. indica Moq. (loc. cit.): Achyranthes obtusifolia Lam. Dict. 1, p. 545 .

Hab. : Shang-hä. - Champs de coton, jardins et cultures, dans les concessions française et anglaise. - Juin.

$A \uparrow$.géog. : Asie intertrop. et temp. : Indes orient., ceylan, Chine (Hong-Kong, Kiang-sou, etc.) - Japon. - Iles de l'Océmn Indien, Maurice, Bourbon, et de l'Océan austral (Iles de la Société).

Ols. : Plusieur's espèces d'Amaranthacées sont cultivées par les: Chinois, soit comme plantes d'ornement, soit pour l'usage alimentaire. Je citerai les suivantes : Celosia cristata Lin. (KrKoûANG-HOA) et $C$. argentea Lin. (Tsoun-TsAN-TzÉ): les Amaranthus paniculatus Lin., A. caudatus et $A$. polygamus qui sont regardés comme plantes potagères à la Chine, l'A. tricolor Lin. (Yun-HIEN . qui orne les jardins pendant l'automne, le Gomphrena globosa. Lin., etc.

\section{CHÉNOPODÉES Vent.}

97. Ghenopodina maritima. Moq. in Dec. Prodr. XIII, p. 161: Schoberia maritima C. A. Mey. in Ledeb. Fl. altaic. 1, p. 400: Bunge. Enum.pl. Chin. bor. n० 311: Miquel Prol. $f$. jap. p. 126; Suada maritima Ledeb. Fl. ross. 3, p. 786.

Hab. : Wôo-sông. - Prairies du Whâng-pôo et fossés aquatiques saumâtres. - Septembre.

$A r^{2}$ géog. : Fur. bor., cent. et aust. dans les régrions maritimes, Danemark, Belgique, France, Espagne, Corse, Italie, Moldavie, Dalmatie, Grèce, Turquie, Russie caucasique. - Asie bor. Sibérie, Baikal, à Irtysch .- Chine (Kiang-sou, Chan-tong, Pé-tchély à Pé-king). - Japon. - Afriq. bor., Algérie. - Sainte-Hélène dans l'Océan atlantique.

98. Chenopodium album Lin. Spec. 119 ; Noq. in Dec. Prodr.

13, p. 70: Benth. Fl. Hong-Kong. 282: Miquel Prol. jap. p. 126 ; sinice HIEN-TSI.

Var. commune Mioq. (loc. cit.).

Hab. : Wôo-sông - Bord des champs cult., jardins, etc. Juin.

Ar. géog. : Régions intertrop. et temp. des deux mondes : Eur. 
- Afriq. bor. : Algérie, Egypte, Canaries. - Anér. : Mexique, Louisiane, Cuba. - Asie : Arabie, Indes orient., Cachemyr, Pondichéry, Chine (Hong-Kong, Kiang-sou, Pé-tché-ly). - Japon. Iles Philippines.

\section{POLYGONÉES. Juss.}

99. Polygonum aviculare Lin. Spec. 519 ; Meisin. in Tee: Prodr. 14, p. 97 ; Maxim. Prim. A. Amur. 229: Bunge Énum. pl. chin. Jor. $11^{0} 325$ : sinice TCHÉ-JOU-TSAO.

Var. A segetum Meissn. (loc. cit.).

Hnb. : Shang-haï, Wôo-sông, et ailleurs, clans les champs cult.. les jardins, etc. - Juin.

Ar.géog. : Régrions temp. des deux mondes : Eur. - Afriq. bor. ct aust. : Algérie, Abyssinie, cap de Bonne-Espérance, etc. Amér. bor. et mérid.: Mexique, Chili. - Asie bor. et cent. : Caboul, Indes orient., Sibérie, Chine (Kiang-sou, Pé-tché-ly). Pror. de l'Amour. - Japon. - Océanie : Nouvelle-Zélande, Australie.

100. P. nadosum Pers. Syn. plant. 1, p. 440: Meissn. in Dec. Prodr. 14.p.118: Maxim. Prim. fl. Amur. 230: Niquel Prot. jap. 300: sinice Pien-tchou, Kollei-LIAo.

II!b. : Shang-haï.- Lieux humides, au bord des eaux. - Juin. Ar. géog. : Répandu dons les deux mondes : Eur.- Afriq. bor.. cont. et aust.: Algérie. Mozambique, cap de Bonne-Espér. Amér. bor. et mérid. : Mexique, Chili. - Asie : Sibérie, Caucase. Perse, Syrie, Indes orient., Chine(Kiang-sou). -Prov, de l'A mon" et de l'Ussuri. - Japon. - Australie.

101. P. orientale Lin. Spec.519; Meissn. in Dec. Proltr. 1t, p. 123; Benth. Fl. Hong-Kong. 288; Sieb. et Zucenr. Plant. acon. syn. p. 18; sinice TA-TSIN-YÉ.

Var. pilosum Meissn. (loc. cit.), $P$. pilosum Roxburg.

Hab. : Shang-haï. Rocailles et lieux incultes du Jardin à thé, où cette espèce se reproduit sans aucune culture.

$\alpha r$. géog. : Afriq. aust. - Asie intertrop. et temp. : Indes orient., Ceylan, Chine (Hong-Kong, Chu-san, Tché-kiang, Kiangsou, etc.) - - Japon.- Nouvelle-Hollande, Iles de la Sonde, Java.

Obs. : Les Polygonum barbatum Lin (Ma-Liao), P. chinense Lin. (TSIA-TSIN', et $P$. tinclorium. Lour. 'T.1-TSIN-IÈ) sont fréquemment cultivés dans les jardins. Le $P$. tinctorium est l'objet d'une culture 
spéciale, dans les provinces méridionales, pour en extraire une sorte de matière tinctoriale, analog'ue à l'indigo provenant des Indigofera.

\section{EUPHORBIACÉES. JusS.}

102. Euphorbia humifusa Wild. Enum. p7. hoi\% Berol. suppl. 1). 13: Boissier in Dec. Prodr. 15, p. 30: Miquel Prol. jup). 288: E. chamesyce Ledeb. Il. altä̈.; Bunge Enum.pl.chin. bor. non Lin.; E. thymifolia Thumbg. Fl.jap.106: E. confusa Blume Mss.; sinice TA-ky, TI-KIN.

Hah. : Shang-haï et Wôo-sông : champs cultivés. - JuiuJuillet.

Ar. géog. : Asie bor. : Sibérie, Oural, Altaï, Baikal, Mongolie : ('hine (Kiang-sou, N'gan-hôei, Chan-tong, Pé-tché-ly, etc., Archip. de Chu-san). - Corée. - Japon.

Obs. : Cette espèce a tout ì fait le port de $l^{\prime} \boldsymbol{E}$. chamresyce I... mais lès feuilles sont plus larges et beaucoup plus allongées. $\mathrm{II}$. Boissier pense (Prodr. loc. cit.) que l'E. polygonisperma Gren. et (iodr. Fl. de France, 3, p. 75, indiquée comme originaire de luri ('orse', ne serait autre que l' $E$. humifusa, mais de provenance adventive dans cette localité.

103. E. esuia Lin. Spec. 660.

Var. cyparissiödes Boiss. in Nec. Prodr. 15, p. 161 ; E. cyparissias Ledeb. Fl. Altaica, 1, p. 180, var B : Glauca, pumila, glabra vel pubesceris, foliis linearibus (Boiss.).

Hab.: Woô-sông. - Prairies des rives du Whâng-pôo. - Mai.

$4 r$. géog.: Eur. orient. et aust. : Moldavie, Russie austr. - Asie bor.: Transcauc. Sôongarie, Sibérie, Altaï, Baikal, İầurie. Mongolie; Chine (Kiang-sou, Pé-tché-ly, Chan-tong, etc.).

104. Ricinus communis Lin. ed. $2^{\mathrm{e}}, 1430$; Nuller in Dec. Prodr. 16, p. 1016 ; Benth. Fl.Hong-Kong.301; Bunge Enum. pl. chin. bor. no 333; Miquel Prol. fl. jap. 291: sinice TA-Ma, Pi-Ma-TZÉ.

Hab.: Cultivé partout, ainsi que les variétés inermis, megalos. permus, microcarpus, speciosus Mull. (loc cit.). pour les graines dont on retire une huile qui est alimentaire dans une grande partie de la Chine. La variété spcciosus, qui est sous-frutescenie et atteint plus de 3 mètres de hauteur, est surtont cultivée dans li province de Hou-pé. 
Ar. glog.: Régrions intertrop. et temp. des deux mondes: Eur. : ust. - Afriq. hor. : Algérie, Egypte, Naroc. Nubie, tbyssinie. etc. - Asie : Arabie, Bengale, Angola, Malabar, Indes orient., Népaul, Malacca. - Chine (Hong-Kong, Kiang-sou, Tché-kiang, Hou-pé,Pé-tché-ly, Chan-tong, etc.).— Japon. — Iles de la Malaisie et de la Sonde, Java. - Amér. cent. et aust. : Mexique, La Trinité. Antilles, Bahia, Brésil, Chili, etc.

105. Excrecaria sebifera Nuller in Dec. Prodr. 15, p. 1201: Croton sebiferum Lin. Spec. 1425: Stillingin sebifera Mich. F'. bor. amerr. 213: Benth. Fl. Hong-Kong. 302: Miquel $F l$. Ned. Ind. 693: S. sinensis Baillon in Etud. sur les Euphorb.; Sapium sebiferum Roxb. Fl. ind. 3, p. 693 ; Miquel Prol. jap. 290; sinice Hin-TZÉ, Ho-TIEN-TZÉ.

IIab.: Très-répandu à Shang-haï, dans toutes les plaines dı seôu-tchou et du Whâng-pôo. Souvent cultivé pour ses graines qui renferment une huile fixe, qui se solidifie à l'air, et qui est employée pour la fabrication des chandelles. Fl. juillet; fr. octobre et novembre.

Ar.géog.: Chine cent. et mérid., d'où cet arbre s'est prcpagé dans l'Asie intertrop. et les parties chaudes des deux Amériques (Hong-Kong, Fo-kien, Tché-kiang, Kiang-sou, Chan-tong, etc.): Iles Formose et de Chu-san. - Philippines. - Cochinchine. - Ile Haurice, etc. - Japon.

\section{CANNABINÉES. E. Meyer.}

106. Cannabis satva Lin. Spec. 1457: Dec. in Prodr. 16, p. 30; C. indica Lam. Dict. 1, 695 ; Niquel Prol. jap. 65; C. chinensis Delille Index sem. hort. Monsp. (1849): sinice HôMA-GIN, TCHOU-TSAO.

"Var. A. vulgaris, caule 3-6 pedali, foliis oppositis, vel sæpius alternis;

"Var. B chinensis, caule 8-12 pedali, ramis diffusis, foliis alters nis, segmentis anguste lanceolatis ut in speciminibus indicis. * raro latioribus."

Hab. : Shang-haï, Wôo-sông et ailleurs. Cultivé dans tout le territoire dès la plus haute antiquité pour les fibres textiles de ses tiges et les propriétés enivrantes de ses feuilles fraîches. Septembre. 
Ar.géog.: Asie cent. et bor.: Sôongarie, Kirghiz, Irtysch, Altuï. Transbaikalie: - Indes orientales, Birmanie, Chine (Tché,kiang. Kiang-sou, Chan-tong, Pé-tché-ly).—Prov. de l'Amour. - Japou.

\section{URTICÉES. JUSS.}

107. Bæhmeria nivea. Hook. et Arn. Bot. roy. Beck. 214: Weddel in Dec. Prodr. 16, p. 206; Miquel Prol.jap. 295: Urtica nivea Lin. Spec. 1398: sinice TCHOn-MA, Pô-MA-TzÉ.

$H a b$. :Shang-haï. - Rocailles et lieux incultes du Jardin ì thé ; rieux remparts de la ville chinoise, etc.; souvent cultivé pour ses fibres Tsia-Pô, qui servent à fabriquer des étoffes fines et résistantes.

Ar.géog.: Originaire de la Chine centrale, d'où cette espèce $\therefore$ 'est propagée par la culture dans les provinces boréales et méri. dionales, en Corée, au Japon, aux Indes orientales, aux îles Philippines, aux Malonines, etc. Le Bahmeria nivea est cultivé en grand en Chine, dans les provinces de Kiang-si, Tché-kiang. Kiang-sou, Tssé-chuên, Kouei-tchou, Konâng-si, Koûang-tong, Fo-kien, Chan-tong, Pé-tché-ly, dans l'archipel de Chu-san, à Formose, etc. Cette espèce a été importée en Europe, et sa culture paraît devoir réussir, principalement dans la région méditerranéenne, en France, en Espagne et en Algérie.

\section{MORACÉES. Lindley.}

108. 3oussonetia papyrifera Ventenat $T a b l$. du règne qégétal, 3, p. 547: Bunge Enum.pl.chin.bor.n 342 : Morus papyrifera Lin. Spec. 1899; Thumb. Fl.jap. 72; Miquel Prol.jap. 130 :

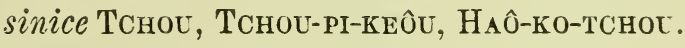

Hab.: Shang-haï; - Parois des remparts de la ville, près de la porte du Sud; cultures, jardins, bord des chemins, etc.

Ar. géog. : Chine méridionale d'où il s'est propagé dans l'Asie centrale, l'Europe australe et l'Afrique boréale. Répandu dans toute la Chine (Kouâng-tong, Fo-kien, Tché-kiang', Kiangr-sou, Chan-tong, Pé-tché-ly, etc.). Chu-san, Formose; - Iles de la Malaisie. Java, Timor: - Iles de l'Océan austral, Taïti. - Japon.

Obs. : L'écorce des jeunes branches de cet arbre sert à fabr jquer du papier très-fin et résistant. Ce papier est journellement employé dans le commerce chinois pour envelopper des objets de 
prix. On peut y tracer au pinceau les signes de l'écriture ordi naire, ou y appliquer des caractères d'imprimerie.

109. Mcrus alba Lin. Spec. 1398: Dec. Prorlr. 17, p. 238; Thumber. Fl. jap. 71 : Sieb. et '/nce. Syn. plant. cecon. 27: Miquel Prol. A.jap. 129.

Var. A rulgaris Burean in Dec. Prodr. 17, j, 238: M. alba Lam. l)ict. encycl. 4, 373: Roxb. Fil. ind. 3. 594: sinice TCHaxg, 'T'Chang-pí-Tz'.

Var. B latifolia Bur. (loc.cit., p. 244: M. latifolia Lam. Encycl. 4, 381 : M. multicantis Perrotet Ann. soc. Lin. Par. (1824), p. 129: Miquel Prol.jap. 130: M. Latarica Desfont. Cal. Kort. Par. p. 347 (1829: M. sinensis Lord. in Cat. '1836); sinice I.OU-KI-TCHANG.

Hab. : Cultivé partout à Shang-haï. La variété à tiges multicaules ou à basses tiges est la plus estimée dans les districts de la soie.

Ar. géog. : Asie intertrop. et temp. : Chine, Japon. - Acclimaté dàns l'Europe australe, l'Afrique boréale, la Guyane française, aux îles Philippines, l'île Bourbon, etc.

\section{SALICINEES. Rich.}

110. Salix Babylonica Lin. Spec. 1473; Ledeb. Fl. Ross. 3. p. 599 ; Bungాe Enum. pl. chin. bor. n³46, p. 61 : Anders. in Dec. Prodr. 16, p. 213 ; sinice Trâ̂-LIEou, Lreô̂-PI.

"Var. A typica Anders. (loc. cit.). Ramis erectiusculis, rubicun- dis, foliis subintegris, aut angustis, aut latioribus, subtus pal" lide viridibus. Planta feminea rarissima occurit. »

ILab. : Shang-haï; - Bord des champs, des fossés aquatiques: cà et là dans la concession trançaise, etc.

Ar. géog.: Asie occ. et bor.: Kurdistan, Mésopotamie, Perse, Cancase, Chine (Kiang-sou, Chan-tong, Pé-tché-ly). - Acclimaté dans toute l'Europe et les régrions subtropicales des deux mondes.

111. S. viminalis Lin. Spec. 1448; Ledeb. Fl. Altai. 4, p. 265: Maxim. Prim. fl. Amur. 242: Anders. in Dec. Protr. 16. p. 264 ; sinice LrEot-PI.

Hab. : Wôo-sông. - Bords des canaux d’irrigation: cultivé souvent dans les terres marécageuses.

Ar. géog. : Eur. -Amér.bor., Asie bor. - Sibérie, Russiensiat. 
Kamstchatka, Sôongarie, Altaï, Mongolie.-Chine(Kiang-son, Chan-tong). - Prov. de l'Amour.

Obs. : Le Salix alba Lin. ou P'́-Yang des Chinois est rarement cultivé dans la plaine de Shang-haï. Ses feuilles servent quelquefois, à falsifier le thé destiné à l'exportation.

\section{CONIFERES. JusS.}

112. Pinus Massoniana Lambert Pin. (ér. $\left.2^{\circ}\right)$, p. 16: Parlatore in Dec. Prodr. XVI, 389; Sieb. et Zucc. Fl.jap. $\{a b .113,114$ : Miquel Prol.jap. p. 330; p. sinensis Lamb. Pin. (éd. $3^{\mathrm{e}}$; p. 47: Carrière Traité des Conifères, 331: Benth. Fl. HongKong. 337: P. sylvestris Thumbg. Fl. jap. 247. non Lin.: sinice $\mathrm{T}_{\mathrm{A}-\mathrm{SONG}}$, TsONG-TCHON.

Hab. : Shang-haï. -.- Cà̀ et là dans la plaine, autour des pagodes et des tombeaux.

Ar. géog. : Chine (Hong-Kong, Koùang-tong, Tché-kiang. Kiang-sou, Chan-tong, etc.) - Formose, Chu-san. - Japon.

113. Cunninghamia siners:s R. Brown in Rich. Conif. p. 80: Parlat. in Dec. Prodr. XVI, 433 ; Sieb. et Zucc. F'l. jap. 2, p.7, tab. 103 : Benth. Fl.Hong-Kong. 337; Miquel Prol.jap. 331; Pinus lanceolaia Lamb. Pin. tab. 34: sinice СнаN-мôt, Chan-SONG.

Lab.: Répandu à Shang-haï autour des temples bouddhiques, des tombeaux, etc., en individus souvent isolés. - Cultivé dans les jarcs et les jardins pour son poit ornemental.

Ar. géog.: Chine (Prov. cent. et mérid.-Hong-Kong, Konang"tong, Tché-kiang, Kiang-sou, (han-tong, Iles Lôo-chòo,etc.). Japon.

114. Gryptomeria japonica Don in Linn. lrens. Soc. 18, p. 166: Sieb. et Zucc. Fl.jap. 2, p. 43, tab. 124; Brong'n. in Ann. scien. nal. (2e série), vul. XII, z31: Miquel. Prol. jap. 331: Parlat. in Dec. Prodr. 16, p. 438: Cupressus japonica Lin. suppl.421; Thumbg. Fl.jap. 265.

Hab. : shang-haï.- Individus isolés autour des tombeaux, dans les jardins, etc.

Ar.gćog. : Chine (Tché-kiang, Kiang-sou). - Japon (Yokoama, Hakodadé, Nippon, etc.. dans la régr:on montagneuse de 200 à 400 mètres alt. 
115. Cupressus funebris Endlicher Conif. p. 58; R. Fortune in

Twov isits to the Tea countries (1853); Parlat. in Dec. Prodr.

16, p. 471; C. pendula Lamb. Pin. (ed. 2), p. 154; Miquel Prol.jap. p. 332: sinice Tsian-TSoung.

IIab. : Shang-haï. - Planté fréquemment autour des pagodes, des tombeaux, etc.

Ar. géog.: Asie cent. et bor.: Thibet, Boutan, Sikkim, Mandchourie, Chine (Tché-kiang, Kiang-sou, etc.). Cult. au Japon.

116. Biota orientalis Endlicher Conif. 47; Parlat. in Dec. Prodr.

16, p. 461; Miquel Prodr. jap. 331; Thuya orientalis Liu. Śpec. 1422; Sieb. et Zuce. Fl. japon. 2, tab. 118; sirice Ṕchor, Pé-tsong, Pé-Fang.

Hab. : Shang-haï. - Haies, jardins et cultures autour de la ville et les habitations rurales. - Mai.

Ar.géog. : Chine (Prov. cent. et boréales'. - Japon. - Acclim. llaus toute l'Asie te.up., l'Océanie, l'Eur. aust.

117. Gingko biloba Lin. Mant. 2, 313; Thumbg. F'l. jap. 35.8: Salisburia adianthifolia Smith in Trans. soc. Linn. 3, 350: Sieb. et Zuce. Fl. japon. 2, tab. 136: Bunge Ënum. pl.chinbor. pl. 62. 1" 251: Miquel Prol. jap.; sinice PÉ-кoûo, (isК $\hat{0}-\mathrm{TZḰ}$.

Hab. : Shang-haï. - Répandu autour des tombeaux, dans les jardins, les parcs, etc.

Ar. géog.: Chine (Prov. du centre et du Nord), Tché-kiang. Kiang-sou, Pé-tché-ly, etc. - Japon.

Obs.: Le Gingko biloba est connu en Chine dès la plus haute antiquité. Bunge rapporte qu'il a rencontré à Pé-king, près du temple Tan-tsé-ssy, un individu de cette espèce, qui arait près de 40 pieds de circonférence, et dont l'histoire remontait à la dỵastie des Wounans 1122 à 897 arant notre ère'.

SAURURÉES. Cl. Rich.

118. Houttuynia cordata Thumberg Fl. jap. p. 23t; Benth. Fl. Hong-Kong. 334: Dec. Prodr. 16 (1 ${ }^{\text {rr }}$ part.), 2:38: Miquel Prol.jap. 303: Polypora corhinchinensis Lour. Fl. coch. 1, 76: sinice TSI-TSAN.

Hab. : Shang-haï. - Bord des fossés aquatiques, autour de l'hippodrume de la concession anglaise, etc. - Arril-mai. 
Ar géog.: Asie cent.: Himalaya, Khasia, Népaul, Siam, Cochinchine (Hong-Kong, Kiang·sou, lles Lôo-chôo, Formose). - Japon (Yedo, Nangasaki, etc.).

\section{PALMIERS. Lin.}

119. Ghamæerops Fortunei Hooker in Botan. magaz. $n^{\circ} 5221$; Ch. excelsa Thumb. Fl.jap. 130; J. Gay in Bull. soc. bot. de France, 8, p. 410. (ex parte); Kæmpf. Amanexol. V, 898; Truchycarpus Fortunei Herm. Wendland in Bull. soc. bot. de France, 8, 429; sinice T'song-Lleô, Poâ-Kouei.

M. J. (ray a publié en 1861, dans le bulletin de la Société bota . nique de France, une notice des plusintéressantes sur la patrie du Chamarops Fortunei (Ch. excelsa, Thumb. ex parte), ses conditions climatériques, son acclimatation dans l'Europe occidentale, se: usages économiques et sa dispersion géographique.

Il résulte du travail de M.J. Gay que le Chamarops de la Chine, qui a été regardé longtemps coinme identique avec celui du Japon et auquel Thumberg a dunné le nom d'excelsa dans sa flora japonica. diffère sensiblement de ce dernier, et qu'il doit caractériser une espèce distincte. M. Hooker l'a décrite et figurée dans le Botanical magazine sous le nom de Ch. Fortunei, qui ruppelle celui du célèbre voyageur $R$. Fortune. Dans un post-scriptum faisant suite à la notice de M. Gay, M. Hermand Wendland pense que les Chamarops de l'Indo-Chine, ne peuvent être classés dans le même grenre que celui qui renferme le Chamcerops humilis de la région inéditerranéenne, et il propose le genre Trachicarmus pour les Chumerops de l'Asie orientale.

Le nouveau genre Trachicarpus comprend quatre espèces échelonnéessur une zone qui, partant de l'Himalaya, aboutit au Japon en traversant la Chine, et ne dépassant pas la ligne tropicale de l'hémisphère boréal. Ces quatre espèces sont:

1" Trachycarpus excelsus Wendland (Chamerops excelsa T'humberg) exp parte du Japon :

2o T. Martianus Wendl. (Chainreiops Martiane Wallr.) du répaul et de l'Himalaya ;

$3^{\circ} T$. Fortunei Wend. (Chamaiops Fortunei Hook.), cultivé à Chu-san et dans les provinces limitrophes de la Chine moyenue:

$4^{\circ} T$. khasianus Wend. (Chamciops khasiana Griff.) du district de Khasia, dans l'Inde septentrionale. 
Robert Fortune a fait connaître le premier, dans ses relations de voyages en Chine en 1847 et 1853, la patrie du Chamcrops Fortunei, au port si élégant et gracieux à la fois. Ce savant explor:iteur l'a vu cultivé, sur les flancs des montagnes de l'île Chu-san. et sur le continent voisin dans des stations analogues de la prorince de Tché-kiang. Il l'a vu égralement dans les provinces centrales de Kiang-si, Kiang-sou, Kiang-nan, où il prospère d'une manière admirable, et où il atteint de grandes proportions.

Pendant mon séjour à Tché-fou dans le Chan-tong, et à Tientsin dans le Pé-tché-ly, je n'ai rencontré nulle partle $C h$. Furturiei. Je n'en ai vu que deux pieds rabougris dans un jardin de 'Tchéfou, et encore ont-ils péri par suite du froid très-rigoureux de l'hiver de l'année 1860 ( 15 à $16^{\circ}$ au-dessous de zéro). Le Chamcerops Fortunei n'est donc point originaire du nord et du sud de la Chine. ainsi que le fait ressortir l'illustre auteur de la notice précitée. Il est parfaitement établi qu'il provient des provinces moyennes, toutes riveraines du fleuve Yang-tsé-kiang, y coinpris l'archipel de Chu-san, d'où il s'est probablement étendu jusqu'à la frontière du Thibet vers le nord, etau Japon dans le sud où il est également acclimaté aujourd'hui.

Hab. : Shang-haï. - Planté dans une foule de jardins et dans le. parcs des concessions française et anglaise. Répandu à Ningr-plo (T'ché-kiang), d'où j'ai reçu des graines mures, qui on t parfaitement germé au Jardin botanique de Bordeaux en 1862, et ont prorhuit dopuis cette époque des sujets très-vigroureux.

Ar. géog. Chine (Prov. centrales', 'l'ché-kiang, Kiang-sun. Kiang-si, Kiang-nan, Chu-san. - Japon.

Obs. : Le Ch. Fortunei paraît d'evoir s'acclimater facilenent dans la région occidentale de la France, sur le littoral océanien. A Bordeaux, à Cherbourg et ailleurs, il a résisté en pleine terre aux froids rigoureux de ces douze dernières années. Je fais remarquer qu'à shang-haï, où ce palmier vit, pour ainsi dire. in loco malali, il y atteint des proportions considérables, et qu'il résiste chaque année à des froids de -10 et -12 centigrades.

\section{HYDROCHARIDÉES. Dec.}

120. Hydrocharis asiatica Miquel Flor. Ind. Bat. 3, p. 239'(186il) et Prol.,A.jap. 160: sinice Honng-PING-Lig:. 
«Planta sinensis Hydrocharide morsus rance simillima, radice 》stolonifera, foliis cordato-rotunlatis, 3-nerviis, quam in specim. » japonicis minoribus, 30-10 millim. longis latisque, petiolis gra》 cilibus, 60-80 millim. elongatis; pedunculis femineis robustis, » striatis, solitariis; stylis sex in duobus stigmatibus profunde » partitis; sepalis viridibus, petalis albido-pallescentibus, punc"tulatis: spatha mascula breve pedunculata, sæpius $1-4$ flora, "pedunculis gracilibus, filiformilibus, sepalis petalisque punctu"latis. " (Miquel.)

Hab. : Shang-haï. - Viares et rizières, autour de la concession irancraise. -- Juillet.

Ai. géog. : Asie? - Chine (Kiang'-sou). - Japon. - Cult. i Java où il a été importé probablement du .Japon (Miquel).

\section{ALIMACÉES. JUSS.}

121. Sagittaria sagittifolia Lin. Spec. 993; Thumbs. Fl. jap. 248; Regel Tent. fl. ussur. 140; Miquel Prol. jap. p. 70; sinice Tś́-Koû.

Forma longiloba Miquel (7or cit.); folia minora angustiorave, quam in planta europeana, lobis basilaribus magis elongatis, acuminatisque.

Hab. : shang-haï et Woo-song. - Marais, rizières et canaux d'irrigation. - Juin.

Ar. géog. : Eur. - Afriq. bor. - Amér. bor. - Asie bor.: Russie asiat., Sibérie, Mongolie. - Chine (Prov. du centre et du nord). -- Japon. - Prov. de l'Ussuri.

\section{POTAMÉES. JUSS.}

122. Potamogeton crispus Lin. Spec. ed. 1, 126: Ledeb. F'7. oss. 4, p. 28; Miquel Prol. fi. jap. 325.

Hab. : Wôo-sông. - Canaux d'irrigation of fossés d'eau courante. -Juin.

Ar. géog. : Hur. - Afriq. bor.. - Amér. bor.. - Asie, Russie asiat., Sibérie, Asie-Min., Chine. - Jayon.

LEMNACEES. DUbY.

123. Spirodela polyrrhiza Schleid, in Ann. scienc. natur. 13 
1840), p. 13: Naxim. Prim. fl. Amur. 266; Ledeb. Fl. altai. t, p. 18: Temna polyrrhiza Sin. Spec.erl. 1. 970: sinice Tsat-ping, Choñt-pung.

11ab. : Shangr-haï. - Marécages autour de la concession anéricaine, avec le Lemna minor. - Août et septembre.

Ar. géog. : Eur. - Afriq. bor., Amér., Asie bor. : Sibérie, Altaï, Chine. - Prov de l'Amour. - Japon.

12t. Lemne mizor Lin. Spec. 970; Turezan. Fl. Baikal. Dahur. p. 164; Thumb. Fl. jap. 24; sinice Shu-y, Chour-prsg.

Hab. : Shanģ-haï. - Nares et rizières, etc. — Septembre.

Ar. géog. : Eur.-Afriq bor.-Amér. bor. - Asie bor. : Russie asiat., Sibérie, Daoûrie, Nongolie, Chine - Prov. re l'Amour. Japon .

\section{AROIDEEES. JUSS.}

125. Acorus calumus Lin. Spec. 462: Schott Prodr. aroid. 578: Kunth Enum. plant. omn. 3, p. 87; Benth. Fl. Hong-Kong. 380; Naxim. Prim. A. A mur. 266; sinice Tснак-ро̂, ТсhaxGPô.TZÉ.

Hab. : Fossés aquatiques depuis Shang-haï jusqu'à Wôo-sông: prairies marécageuses des rives du Whâng-pôo.- Juin et juillet. Ar. géog. : Eur. - Amér. bor. - Asie cent. et intertrop. : Indes orient, Siam, Cochinchine, Chine (Hong-Kong, Tché-kiang, Kiang-sou, Pé-tché-ly, etc.). - Japon. - Prov. de l'Amour.

\section{ORCHIDEEES. JusS.}

126 Cymbidium ensirolium Swartz; Pers. Syn. plant. 2, 507: Lindley Gen. et spec. Orchid. 162; Benth. Fl. Hong-Kong. 35\%: Miquel Prol. fl. jap. 137: Limodorum ensifotium Thumb. F'l. jap. 29, et icon. Decas 1, no 8; sinice Moû-HÔA, LAx-HÔA. «C. perenne, foliis ensiformibus, striatis, acutis; scay,is crectis »multifforis; bracteis ovario duplo brevioribus, sepalis petalis » que lineari-oblongis, acutis; labello indiviso, planiusculo, oblongo. »ubtuso, revoluto, lamellis continuis. Flores viride-lutei, renis » purpureis; floret Aprili-Maio.»

La figure des Icones de Thumberg convient parfaitement à notre plante, qui ne peut être confondue qu'avec une espèce voisine, le C. riphiifolium Lindley. La figure des Icones de Banks, 
tab. 3, représente les fleur's une lois plus grandes, mais il est probable que Kempfer aura grossi son dessin, ainsi qu'il l'a fait pour d'autres plantes. Dans le Cymbidium sinense Wild. les fleurs sont très-nombreuses, et le labelle d'mu rouge de sany, jaune au milieu. Le C. riphiifoliun a les fleurs d'un vert-pâle unicolore.

Hal. : Shang-haï: Subsp. sur les tumulus: cultivé dans un grand nombre de jardins. - Aviril et mai.

Ar. géog. : Chine (Hong-Kong, Tché-kiang, Kiang-sou). Japon.

AMARYLLIDÉES. R. Brown .

127. Narcissus tazetta Lin. Spec. ed. 1, 290; 'Thumb. F'l jap. 121 ; sinice CHOû-LIEN-HÔA.

Var. sinensis Ræm.; Miquel Prol. fl. jap. 72; Kunth Enum. V, p. 741, sub Hermione.

«Spatha lanceolata-oblonga, subventricosa, 6-9 flora; perigoni " tubo 3-4 pollicari, limbo tubum nunc aquante nunc breviore, » in sicco albido; corona lutea, staminibus breve exsertis. » (Miquel.).

Hab. : Shang-haï : Terres incultes autour des tombeaux, et sur les tumulus où il est subspont. - Mars.

Ar.géog. : Eur. aust. - Afriq. bor. - Asie occ. et orient. : Asie-Mineure. - Chine (Kiang-sou, Chan-tong). - Japon.

\section{ASPARAGINÉES. JusS.}

128. Asparagus lucidus Lindl. Bot. reg. $\mathrm{n}^{0} 36$ (1844); Benth. Fl. Hong-Kong. p. 371; Kunth Enum. plant. V, p. 71; Miquel Prol.jap. $315 ;$ A. Gray Bot.jap. 423 ; A. falcatus Thumb. Fl. jap. p. 139, non Lin. nec Burm. Zeil, p. 36, tab. 13.

Hab. : Shang-haï. - Lieux incultes, bord des canaux, autour des tumulus, etc. - Avril-mai.

Ar. géog. : Chine mérid. et cent. : Nacao et Canton dans le Kouâng-tong; Iles de Hong-Kong et de Lôo-chôo; Kiang-sou, et probablement tout le littoral de la Chine. - Japon (Kiu-siu). Corée.

Obs. : Dans sa flora japonica, Thumberg avait considéré cette espèce comme identique avec l'A. falcatus Lin., plante qui croît spécialement à Ceylan et dans les Indes orientales. L'Asparagus 
Iucidus se rapproche beaucoup de l'A. falcatus, et ne parait en être du reste qu'une réduction. Iindley attribue à tort à cette plante des feuilles opposées. Cela est vrai quelquefois pour les feuilles des tiges fleuries ; mais les feuilles des rameaux stériles sont souvent fasciculées par 3 et par 5 , et dans ce cas très-inéga les. Bentham a relevé ce fait depuis longtemps dans sa flore de l'île de Hong-Kong.

\section{LILIACÉES. JusS.}

129. Orithyia edulis Miquel in Prol. flor. jap. p. 322; L. Savatier Livve Kna-ni, Herb. no 22, sub SAx-CHIC, AwA-N-A Japonensium.

«Bulbus parvus, solitarius; scapus gracilis, glaber, uniflorus, "12-15 centimet. altus, diphyllus; folia linearia, elongata, cana" liculata, florem haud attengentia; flos erectus, parvus, subcam»panulatus; petala exteriora acuto-oblonga, lutescente-viridia. » interiora basi angustissima, acuminata, albescentia; staninc » supra basim dilatata. Floret Martio-Aprili. »

Hab. : Shang-haï. - Lieux incultes et sablonneux, principalement autour des tumulus. Rare.

Ar. géog. : Chine (Kiang-sou). - Japon (Jeso, Nippon).

Obs. : L'Orithyia edulis Miq. est à peine distinct (Franchet in litleris) de l'0. oxypetala Kunth Enum. 4, 22\%: Ornithogalum oxypetalum Ledebour in flor. alt., 2, 2\%). Je ne trouve d'autre différence (ex descriptione Ledebouriana) que dans la forme des feuilles, qui sont longuement linéaires dans l'espèce chinoise, et non oblongues-linéaires, et aussi dans la couleur de la corolle, qui est blanche à l'intérieur et verdâtre à l'extérieur. Celle-ci est jaune dans l'O. oxypetala. Le livre Kwa-wi (loc. cit.) attribue à l'Orithyia edulis du Japon des fleur's violettes, blanches ou rougeâtres.

Le genre Orithyia ou Orythia ex Miquel a été créé par Endlicher, aux dépens des genres Tutipa et Ornithogalum, pour trois espèces originaires de la sibérie altaïque et des provinces du Wolga. Ces espèces sont l'O. uniflora Swet (Ornithogatum uniflorum Lin.), l'O. oxypetala et l'O. biflora Kunth (Tulipa biflora Lin). L'Orithyia edutis Miq. de la Chine et du Japon forme le complément des espèces connues jusqu'à ce jour de ce genre si intéressant. Cette plante est abondante dans les champs du Nippon, et elle est récoltée par les Japonais, qui extraient de ses bulbes une fécule alimentaire. 
L'Orithyia edulis, qui rappelle par son port et sa forme le Leucoium vernum du nord de la France, fleurit dès le commerce ment du mois d'avril. Ses feuilles glabres, luisantes, longuement linéaires et creusées en gouttière, ses tiges grêles et uniflore: ses fleurs grandes blanches, un peu verdâtres à l'extérieur, la font remarquer facilement à une époque où la végétation est encore peu avancée à Shang-haï.

DIOSCORÉES. R. Brown.

130. Dioscorea sativa Lin. Spec. 1463; Benth. Fl. Hong-Kong. p. 368; Thumb. Fl.jap. 151; Miquel Prol.jap. 223; Kelmia bulbifera Kunth Enum. plant. V, 435; sinice Снô-YU, TSAN-Yu.

Hab. : Cultivé en grand à Shang-haï. - Ses tubercules forment la base de l'alimentation de la classe laborieuse.

Ar. géog. : Asie cent. intertrop. et temp. : Indes orient., Chine (Hong-Kong, Kouâng-tong, Fo-kien, Tché-kiang, Kianğsou, Chan-tong, Pé-tché-ly). - Japon.

Obs. : Le TAô-yU, Dioscorea batatas Decaisne in Rer.hort. (18533), p. 243 , dont les tubercules sont vulgairement nommés. Ignames de la Chine, est également cultivé dans le territoire de Shanghaï.

\section{COMMÉLINACÉES. Reichb.}

131. Commelina communis Lin. Spec. 60; Benth. Fl. HongKong. 376; Thumbg. Fl. jap. 35; Miquel Prol. jap. 30.C. polyama Roth Catal.bot.1, p.1; Naxim. Prim.fl.Amur. 290; Bunge Enum.pl. Chin. bor. n 376 ; C. Wildenowi Kimth Enum.pl. omn. 4, 37; sinice TSIA-TSAO, SHU-YU-TSAr.

Hab. : Shang-haï, Wôo-sông et ailleurs. - Abond. au bord des chemins, dans les haies de bambous, etc. - Juillet.

Ar.géog. : Amér. bor. - Asie cent. intertrop. et temp. : Incies orient., Cochinchine, Chine (Hong-Kong, Tché-kiang, Kiangsou, Chan-tong, Pé-tché-ly; - Arch. de Chu-san). - Japon (Kiu. siu, Nippon). - Prov. de l'Amour et rle l'Ussuri.

\section{CYPERACEES. JuSS.}

132. Cyperus rotundus Lin. Sysl. reget. 98: Roxburg F'l. Indic I, p. 201 ; Benth. Fl. Hong-Kong.p. 387; Thumbg. Fl. Japon. 
p. 36: Kunth Enum.pl. 5, p. 58; Niquel Prol. jap. 73: C. Iydra Mich. Fl.amer. bor. 1, 27; C. hexastachyos Roth Gram.: sinice TSAN-LINC, KIN-TFANG-SAN-LING, Hiô-TSAO.

Hab. : Shang-haï, Wôo-sông et ailleurs. - Prairies humides des rives du Whâng-pôo. Bord des canaux d'irrigation, des rizières, etc. - Juillet.

Ar. géog. : Régions maritim. tropic. et temp. des deux mondes ; Atriq. cent. : Egypte, Sénégal, Canaries, Guinée, cap de BonneEspér. - Asie : Asie-Min., A rabie, Indes orient., Singapore, Ceylan. - Chine marit. - Japon. - Iles de l'Océan Indien : Maurice, Bourbon, Java. - Iles Philippines, Australie. - Amér. bor. cent. et mérid. : Martinique, Guadeloupe, etc.

Obs. : D'après Kunth (loc. cit.), le Cyperus hydra de l'Amérique boréale, et le $C$. tetrastachys Desfont. in Fl. alt. 1, 445 (C. oliraris Targ-Toz.) de l'Europe australe, de la Corse et de l'Algérie, ne seraient que des synonymes du C. rotundus de Lin. Cette opinion n'est point partagée par MM. Grenier et Godron in Flor. de F'rance, 3,359 , en ce qui concerne le $C$. tetrastachys. Les deux éminents botanistes précités considèrent les $C$. rotundus et tetrastachys comme étant deux espèces parfaitement distinctes.

133. Eleocharis twberosa Schult. Mant. 2, p. 86; Kunth Enum. pl. 2, p. 153; Scirpus tuberosus Roxb. Fl. Ind. 1, 213: sinice OU-It, Po-tsai et Pú-tsat.

Hab. : Cultivé à Shang-haï, dans les terres humides ou narécageuses, autour de la ville et des concessions européennes. - Les Hyyzomes qui se vendent sur le marché de Shar:g-haï renferment une sorte de fécule nornmée $M a-t i$-fen, et qui sert pour l'alimenlation.

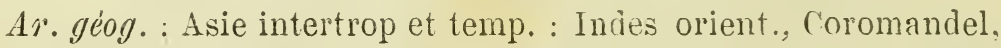
( hine (Kouâng-tong, Tché-kiang, Kiang-sou, etc.).

134. C. Pierotii. Miquel Prol. Flor.jap. p. 80.

"Culmus gracilis, 1-2 perlalis, lævis, inter spicas scaber, foliis "anguste lineavibus, abbreviatis, margine scabris superne grlabriusculis: spicis masculis 3 terminalibus, ferrugineis, parum »distantibus, ultima pedunculata, elongata, inferioribus subses》 silibus; femineis 2, remotis, brevissime pedunculatis, laxis, oblon》gis; bracteis culmum multo superantibus; squamis subellipti»cis, fusco ferrugineis. lanceolatis acuminatisve; utriculis fusco. 
" brunneis (in sicco), nervoso striolatis. elliptico-ovoïdeis, obsolete » trigonis, apice rostro brevi pallide-fusco terminatis: stigmatibus 》3, validis, longis, puberulis. »

Aftinis C. scabrifolice Steud. Syn. glum. p. 237, ex Miquel.

Hab. : Shang-haï et Wôo-sông. - Prairies marécagreuses des rives du Whâng-pôo. - Fl. et fr. 1-15 juin 1860.

$A r \cdot g \dot{o} o g$. : Chine (Kiang-sou). - Japon.

Obs. : Cette espèce est caractéríée par ses épis mâles (2-3) grèles. allongés et terminaux, par ses utricules fructifères allongées o voïdes-elliptiques, lég’èrement aplaties, d'un brun foncé, tinement dentelées à leur extrémité, munies de côtes saillantes, et formant un épi court et serré, par ses akènes bruns, orales allongés acuminés aux deux extrémités, finement pubescents; enfin par les bractées qui accompagnent les épis femelles, longuement linéai res, et dépassant les chaumes de 20 à 30 centimètres au moins.

135. C. pumila Thumb. Fi. jap. 39; A. Gray Plant. jap. exped. Perray in Mem. of Amér. soc. of sciences, vol. 11, 1. 325 (1859); Benth. Flor. Hong-Kong. 402; Kunth Enum. plant. 2, p. 517; C. littorea Labill. Plant. Nov.-Holl. 2, 69.

spicis 5, masculis 2, terminali cylindrica pedunculata, alteri » breviore, sessili, contiona, femineis 3 oblongis crassis, inferiori" bus exserte pedunculatis, longiuscule bracteatis; stigmatibus "3, longis; utriculis ovatis, rostratis, bifurcatis, nervatis, glabris, 》 lutescentibus, squama lanceolata vel orato-acuminata, cuspidata " purpurea vel pallida, margine hyalina, medio serrata brevioribus."

Hab. : Prairies humides des rives du Whâng-pôo, à shangh-haï et Wôo-sông. - Juin.

$A r^{\circ}$ géog. : Littoral de la Chine mérid. et cent. Hongr-hong. Kiang sou, etc.). - Japon. - Nouv.-Hollande.

Obs. : Le C.pumila, qui rappelle par sa forme et son facies, 1 . C. filiformis de nos contrées, n'avait èté signalé en chine, que sur less sables maritimes de l'île de Hong-Kong. Lees échantillons que j'ai récoltés à Shang-haï n'offient ancune différence avec ceux le provenance japonaise.

GRAMINELE. JUSS.

136. Oryza sativa Lin. S'pec. t75: 'Thumbg. Fl. japun. 147; Kunth Enum. planl. 1, p.7: Hiquel 7pol. jap. 160: sinice TAÔ, Mi, Y-TZÉ-GIN. 
Forma A aristata (O. precox Sieb. et B mutica Miq. loc. cit.).

Hah. : Cultivé dans tout le territoire de shang-haï, Woo-sông. etc.

L. qégg. : Régrion intertrop. et ternp. des deux mondes : Asie Siam, Camboge, Coehinchine. Chine mérid, et cent.) - Japon. -.- Lle de la Malaisie. - Amér. bor. - Afriq. or. - Eur. aust., etr.

137. Surrinan valuase Pers. Syn plant. 1, p. 101: - Naxim. Poiv. fl. Ammi. 331: Holcus soighum Lin. Spec. 1484: Kunth Enum.pl. 1, 5\$1: sinice Kaû-LLna.

Hal) : Cultivé en grand à shang-haï, Wûo-sông et aillenrs.

A $\imath^{\circ}$ grog : Asie cent. et temp. Indes orient., Chine (T'ché-kiang. Kiang sou, Chan-tong, ete.) - Pror, de l'Amour et le l'Ussuri. - Japon. - Eur. aust. - Afrig. bor. et cent.

Obs. : Cette plante, qui a été introduite en Chine sous la dynastie des Hans, cst cultivée dans plusieurs provinces pour ses graines qui sont alimentaires, et servent anssi à préparer une espece de boisson aløolique. Celle-ci est moins estinée que le Sar. chou, alcool provenant des graines de l'Oryza salixu.

138. Bechnamia erucepormis Host Gram. 3, tals. (j) Wnxim. Frim. A. thmer. 326: Kunth Enim.pl. 1, 27: "ure\%. Fl. Baik. 2, 295; Bunge Enmm.pht. chin. 7or. n' 395: Regat I'ent. A. ussur. 170: Niquel Prol. jap. 161: Phalaris enucrformis Lill. spec. 80.

Mab. : Shang-haï et Wôsong, bord des canaux d'irrigation. - Juin.

Ar. géog. : Eur. aust . - Italie, Dalmatie, Hongrie, Grèce, Turynie, Russie mérid. - Amér. bor. : Baie d'Hudson, Missouri. Asie bor. et occ. : Asie-Min., Sibérie, Altaï, Baikal. - Chine Kiang-sou, Pé-tché-ly, - Prov. de l'Amour et de l'Ussuri. Japon.

139. Setaria glauca P. Beauv. Agrost. 51: Kunth Innum. plant.

1, 149; Panicum glaucum. Lin. Spec. 83: Benth. Fl. Hong-

Kong.411: Regel Tent.ussur. 172: Niquel Prot.,t.jap. 163.

Hað. : Shang-haï et Wôo-song. - Champs, jardins et teries cultivées. - Juin.

$1 \gamma . g \dot{o} o g .:$ Régrion intertroll. et temp. des deux mondes. - Eur - Afriq. bor. et cent., Sénéganl. - Asie : Indes orient. - Chine 
(Hong-Kong, Kiang-sou, Pé-tché-ly). - Japon. - Amér. bor. - Nouvelle-Hollande. - Prov. de l'Ussuri.

140. S. italica Kunth Gram. 1, p. 46; Maxim. Prim. H. Amur. 33:30. Var. Jermanica, P. Beauv. Agrost. 51; Panicum germanicum. Roth Germ. 2, 71; Bunge linum. pl. chin. bor. no 399 ; Miquel Prol.jap. 163; sinice Siaû-mi, Ko-tźé, Liàa.

Hab. : Cultivé en grand dans la province de Kiang-sou et le nord de la Chine.

Ar.géog. : Eur. - Asie cent. et temp. : Ludes orient. - Chine (Kiang-sou, Chan-tong, Pé-tché-ly) - Prov. de l'Amour. - Japon. - Nouvelle-Hollande.

141. Panicum mi iaceum Lin. Spec. 86; Kinth Enum. planl. 1, 104: Maxim. Prin. Al. Amur. 330: Bunge Enum. pl. chin. bor. no 400: P. mitium Pers. Syn.plant. 1, 85; P. densepilosum stend. Syn. g7um. 72: Miquel Prol. jap. 163: sinice Сной-мг, Коे-ги́.

Hab. : Shang-haï. - Cultivé partout. - Ses graines sont alimentaires pour les animaux de basse-cour.

Ar. géog. : Eur. - Asie subtrop. et temp. : Indes orient. Chine 'Tché-kiang, Kiang'-sou, Chan-tong, Pé-tché ly . - Prov. de l'Amom et de l'Ussuri. - Japon.

142. Vr. chloa villosa Kunth Enum.plant. 1, 72 et Grom. 1, 203; Paspalum villosum Thumb. Hl.jap. 45; Maxim. Prim. $A$. Amu1. 327; Helopus villosus Nées ab Esemb. in Martius Fllor. Brasil. 2, 17; Miquel Prol. jap. 164.

"Herba gracilis, annua, caule simplici 1, 1/2 pedali, parce » folioso: spiculis tribus ovoüdo ellipticis, compressis, junioribus "viridibus villosulis, adultis glabrescentibus; glumis 2 æquali" bus, ovatis, acutiusculis, 5 nerviis, nervis 3 mediis; paleis carti"lagineis, ovatis, muticis, exteriore interiorem amplectente, "pallidis, punctulatis; pedicellis brevibus, inflatis, glabris, basi » (lense villosis: vaginis foliorum pubescentibus, ore barbatulis.» (Miquel.)

Hab. : Shang-haï. - Bord des fossés aquatiques, des canaux l'irrigation, etc. - Juillet.

Ar. géog. : Asie cent. et bor. : Chine!Kiang-sou, Chan-tong'. Prov. de l'Amour. - Japon. - Amér. mérid., Brésil. 
143. Isachne australis Brown Prodr. 1, 196, non Kunth: Benth. Fl.IIong-Kong. 414: Miquel Prol.jap. 164: Panicum atrorirens Trin. in Spreng. Syst.pl. 1, 318; Kunth Einum. 1, 127; P. batavicum Steud. Syn. gram. 96.

Hab. : Wôo-sông.-Bord des eaux, des canaux, etc. - Juin. Ar. géog. : Asie intertrop. et temp.: Ceylan, Intes orient.. Nalacea, Chine (Hong-Kong, Chu-san, Fo-kien, Kiang-sou). Japon. - Iles de la Sonde, Java.

144. I. altissima 0. Debeaux Msc. in Hert. 1862.

"Annua. Culmus erectus, gracilis, striatus, hispido-rillosus, "90-100 centimetr. et ultra altus. Panicula ampla, laxa. subef" fusa, ramoso-divaricata, ramis ramulisque elongatis, subver. » ticillatis, filiformibus, fiexuosis; ramulis nudis, unifloris. Fotia 》plana, lineari-lanceolatu, 6-8 milimet. lata, 25-30 centimet. " longa, superne glabra, ad paginam inferiorem pilis longe lanasti » induta, collo vaginæ marginibusque præsertim pilosiora. "Gluma 2, subæquales, concavæ, acutæ, muticæ; paleæe 2, » muticæ, nervosa, nitidæ, coriaceæ, inferior superiorem \&-10 "nerviam amplectens. Cariops glabra, lucida, fusco-brunnea. » complanata, paleis induratis inclusa."

Hab. : Shang-haï. - Bord des canaux d'irrigation, des fossés aquatiques, etc. - Juin.

Ar. géog. : Chine (Kiang-sou).

Obs. : Cette nouvelle espèce me paraît parfaitement caractérisée par ses feuilles longues, hérissées à la face inférieure, par sa panicule ample et diffuse, par les glumelles des fleurs hermaphrodites, glabres et luisantes. On ne peut la rapprocher que de l'Isachne australis, dont elle diffère par sa taille trois fois au moins plus élevée, par ses épillets du double plus grands et beaucoup plus aigus, etc.

145. Polypogon littoralis Smith Compend. 13: Kunth Enum. plant. 1, 233; Benth. Flor. Hong-Kong. 427: A. Gray Plant. jap. exped. Perry, 328; Miquel Prol. jap. 160.

Haz. : Shang-haï. - Bord des fossés aquatiques. - Juin.

Ar. géog.: Eur. aust., Afriq. bor., Asie cent., intertrop. et temp. : Asie-Min., Afghanistan, Jndes orient.-Chine(Hong-Kong, Kiang-sou). - Japon.

146. Phramites Roxburgii Nées ab Esemb. in Plant. Meyen. 173: 
Benth. Fl. Hong-Kon!.428; Miquel Flor. ind. Bat.3, 412 et

Prol. A. jap. 166 ; sinice OÛEI-Loú, Y-TSAO, TCHOÛ-TEN-TSAO. Hab. : Shang-haï et Wôo-sông. - Rives du Whâng-pôo.

Ar.géog. : Asie cent, intertrop. et temp. : Himalaya, Indes orient., Ceylan, Malacca, îles Philippines. - Chine (Hong-Kong, 'Tché-kiang, Kiang-sou, Pé-tché-ly). Arch. de Chu-san, etc. - Japon.

147. Bambusa arundinacea Wild. Spec. 2, 245; Kunth Enum. 1, 43; B. arundo Ruprecht Bamb. 53; Benth. Fl. HongKong, 435; Arundo bambos Lin. Spec. 120: sinice Tchôv, TCHA0-тCHÔU.

Hab. : Naturalisé dans toute la province de Kiang-sou, et très répandu autour de Shang-haï. Il y sert de clôture pour les propriétés rurales, et d'abri contre les vents. Les chaumes sont employés par les Chinois à une foule d'usages dans l'économie domestique.

A $\imath^{\circ}$ géog. : Asie cent., intertrop. et temp.: Indes orientales, Cochinchine, Siam; - Chine mérid. et cent. (Hong-Kong, Kouângtong,Fo-kien, Tché-kiang, Kiang-si, Kiang-nan, etc. Chu-san.Japon.

148. Rottboellia latifolia Steudel Syn. glum. (gram.) p. 361; R. foliosa Steudel (loc. cit.); Ischanum latifolium Miq. Prol. Л.jap. 179, non Kunth Enum. 1, 514.

"Var. A genuina, forma elatior, culmis erectis, 150-200 centim. 》) altis, foliis late-lanceolatis, 30-35 millim. latis, 40 centim. lon" gis : panicula $6-7$ spicis composita, spicis 20 centim. longis."

"Var. B angustifolia, forma gracilior culınis 50-60 cent. altis. 》gracilibus; foliis longe-lineari-angustatis, 4-5 millim. latis, " 25-30 cent. longis; panicula 2-3 spicis composita, spicis 10-12 cent. longis, plerumque geminis. »

Hal. : Shang-haï, Wôo-sông et ailleurs. - Bord des fossés aquatiques, des canaux d'irrigation, etc. - Juin.

A 2. géog.: Chine et Japon.

Obs. 1: La variété B angustifolia est tellement dissemblable, au premier abord, de la forme typique, que l'on serait tenté de la considérer comme une espèce distincte. Mais cette forme, qui paraît être spéciale aux terrains desséchés, se relie à la première par de nombreux intermédiaires, qui ne permettent pas de douter de leur identité spécifique. Cette variété, à feuilles étroites linéai- 
res, a été observée également au Japon, sur les rives du fleuve Bonja-Gawa, dans l'île de Kiu-siu.

Obs. 2: Le Y-YN-Gin (Coix Tacryma Lin.) est fréquemment cultivé dans les jardins et se trouve parfois dans les décombres, auprès des remparts de la ville.

Les espèces suivantes sont soumises à la grande culture dansle territoire de Shang-haï : Arena sativa Lin. ou LıN-Tong-MaÏ; Trilicum vulgare L. et ses variétés, Siso-Maï; Hordeum vulgare I. TA-MAï, plante connue à la Chiné depuis les temps les plus reculés, et qui a longrtemps servi à la nourriture de l'homme et des animaux; Hordeum hexastichum L. MAÏ et TA-MAÏ; Zea mays L. YU-MAï, etc.

FOUGËRES. Lin.

149. Pteris serrulata Lin. fil.; Hook. Spec. filic. 2, 203; Benth. Flor. Hong-Kong. p. 448; Miquel Prol. A. jap. 336; sinice Ché-Hou.

Hab. : Sur les vieux remparts de Shang-haï, près de la porte du Sud. - Mai et juin. - Très-abondant.

$A r^{\circ}$.géog.: Chine mérid. et cent. : Hong-Kong., Prov. du Kouâng. tong, Kiang-sou, etc. - Japon.

Obs.: Cette espèce dont les pinnes inférieures n'offrent qu'une seule ou très-rarement deux pinnules de chaque côté, ressemble en petit au Pteris cretica Lin., dont elle pourrait bien n'être qu'une variété, d'après Bentham (loc. cit.)

\section{MOUSSES. Lin.}

150. Barbula unguiculata Schimper Synops. musc. 167; Tortula unguiculata Hedw. Slirps. 1, tab. 23; Dec. et Duby Bot. gall. 2, 565; Miquel Prol. jap. 182.

Hab.: Sur les vieux remparts de Shang-haï, près de la porte du Sud. - Avril.

Ar.géog.: Eur. - Afriq. bor. - Asie temp.: Chine (Kiangsou, etc.). - Japon.

151. Funaria hygrometrica Hedw. Spec. 172; Schimp. Syn. musc. 323; Dec. et Duby Bot. gall. 2, 548; Miquel Prol.jap. 182.

Hab. : Shang-haï. - Sur les vieux murs, et à terre, dans les rues et les cours extérieures des maisons de la concession anglaise. - A vril. 
Ar. géog.: Eur. - Afriq. bor. - Amér. bor. - Asie bor. et cent. : Chine (Kiang-sou, etc.). - Prov. de l'Amour. - Japon.

152. Bryum argenteum Lin. Spec. 1586; Schimp. Syn. musc. 370; Dec. et Duby Bot.gall.2, 562; Miquel Prol.jap. 182.

Hab. : Sur les vieux remparts de Shang-haï. - Avril.

Ar. géog. : Eur. - Afr. bor. - Amériq. bor. - Asie bor. et cent. : Chine. - Japon.

Obs. : La famille des champignons produit quelques espèces que les Chinois du Kiang-sou récoltent avec soin pour l'usage alimentaire ou les besoins de la thérapeutique indigène. Je citerai principalement les suivantes :

Agaricus campestris L. (sinice PÉ-KIoù) que l'on trouve en abondance à l'état frais sur le marché de Shang-haï, et que l'on retroure ainsi à Tien-tsin, à Pé-king' et au Japon;

Auricutaria mesenterica Pers. (sin. Mo-x), d'origine indigène et alimentaire;

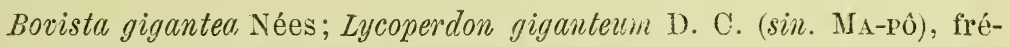
quent dans les prairies de Shang-haï;

Pachyma hoëlen Friès; P. coniferarun Horan.; P. cocos Friès ex clar. L. Soubeiran; (sin. Fou-LIN et PÉ-Fou-TIN), champignon hypogé qui croit sur les racines des Pinus Massoniana et Cunninghamia sinensis dans les provinces de Tché-kiang, Kiang-sou, Kouêi-tchou, 'Tssé-tchuen, Chan-tong, etc., et très-usité en médecine;

Pachyma tuber regium Friès (sin. TCHou-Liv), qui se récolterait d'après mes renseignements sur' les racines d'un Mimosa, ou d'un Liquidambar (L. Soubeiran), et avec lequel on fait des gàteaux très-estimés à Shang-hai;

Spharia sinensis Fr. (Sin. Hia-tSAO-TONG-CHONG ou rer-plante). Ce champignon qui se récolte dans les provinces limitrophes du Kiang-sou vit en parasite sur les chenilles d'un papillon du genre Hepiatus (L. Soub.), et jouit, comme réconfortant, d'une réputation presque aussi grande que celle des racines du fameux Gin-seng, de la famille des Araliacées, et qui constituent le remède par excellence des Chinois. 
TABLEAU indiquant par familles les principales affinités de géographie botanique des plantes de shang-haï.

\begin{tabular}{|c|c|c|c|c|c|c|c|c|c|c|c|c|}
\hline FIMILLES & 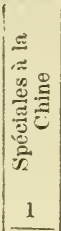 & 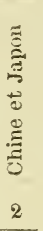 & 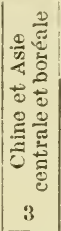 & 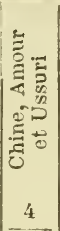 & 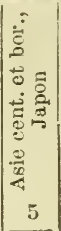 & 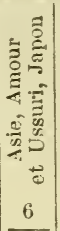 & 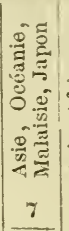 & 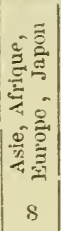 & 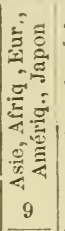 & 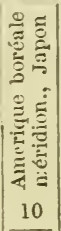 & 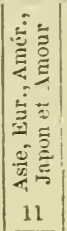 & $\frac{1}{3}$ \\
\hline Renonculacées...... & 2 & $\eta$ & b & 1 & ” & 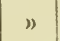 & $"$ & ") & n & ” & 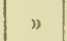 & 3 \\
\hline Magnoliacées. . . . . . . & 1 & ) & ) & $\eta$ & 》) & ” & " & " & 》) & 》) & $"$ & 1 \\
\hline Ménispermées ...... & " & 1 & $»$ & 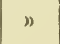 & " & 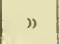 & ) & ) & ” & ” & 》) & 1 \\
\hline Berbéridées........ & " & 1 & ): & ” & n & ) & 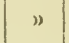 & $"$ & 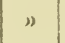 & ) & $n$ & 1 \\
\hline Nélumbiacées....... & ) & ") & $"$ & 》) & 1 & 》) & $"$ & " & 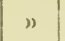 & " & ) & 1 \\
\hline Fumariacées........ & 1 & 1 & ” & 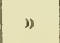 & 1 & $"$ & ) & $\eta$ & ) & 1 & 》) & 4 \\
\hline C'rucifères . . . . . . . . & 1 & 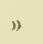 & 1 & 1 & ” & 1 & 1 & 1 & 2 & $”$ & ” & 8 \\
\hline Malvacées ......... & ” & 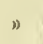 & ) & ” & ) & 》) & $n$ & 1 & 1 & 》 & ) & 2 \\
\hline Sterculiacées . . . . . . & ” & ) & ” & ) & 1 & 》) & ” & ” & $»$ & 》) & D & 1 \\
\hline Tiliacées... . . . . . . & $n$ & $\eta$ & " & ) & 1 & ) & 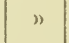 & b & ” & " & ” & 1 \\
\hline Oxalidées ......... & $”$ & 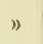 & ) & ") & ” & 》) & " & 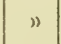 & 1 & $n$ & ” & 1 \\
\hline Méliacées. . . . . . . . & ) & ” & $n$ & 》) & $n$ & 》 & $"$ & ) & 1 & , & $”$ & 1 \\
\hline Hippocustanèes . . . . . & 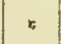 & 1 & ” & 》) & $»$ & 》) & $n$ & ) & ” & $》$ & 》 & 1 \\
\hline Ampélidées........ & ): & 1 & ” & $x$ & $》$ & 》 & ” & D & ) & ) & 》 & 1 \\
\hline Cèlastrinées . . . . . . & 1 & $\eta$ & $"$ & 》 & 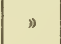 & , & " & ) & ) & $\eta$ & 》 & 1 \\
\hline Rhamnées........ & 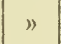 & $"$ & 1 & 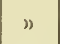 & 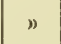 & $》$ & ) & 1 & ) & ) & $n$ & 2 \\
\hline Légumineuses....... . & 1 & 2 & ") & ” & ) & ” & ) & 2 & 2 & 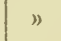 & ) & 7 \\
\hline Rosacées......... . & ) & 1 & ) & ” & 4 & 》 & $\eta$ & » & $n$ & » & n & 5 \\
\hline Granatées......... & $》$ & $n$ & ” & 》 & 》) & $》$ & ) & 1 & ” & 》 & 》 & 1 \\
\hline Onagrariées........ & 》) & 1 & 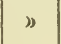 & ” & 》 & ” & $n$ & 》 & ") & ” & 》 & 1 \\
\hline Haloragées . . . . . . . & ” & 》 & ” & $n$ & 》 & $n$ & ” & 》) & 1 & $》$ & 》 & 1 \\
\hline Lythrariées....... . & $\eta$ & $\eta$ & 》 & $"$ & 1 & ”) & : & » & ) & 》 & 》 & 1 \\
\hline Crassulacées . . . . . . & $\mathbf{l}$ & 1 & ” & ) & ) & 》) & 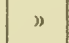 & ): & 》 & ” & ) & 2 \\
\hline Ombellifères . . . . . . & ) & $n$ & ” & ) & 》 & ” & ) & 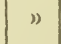 & 1 & ) & n & 1 \\
\hline Caprifoliacées........ & $n$ & ) & $"$ & 》 & 2 & 》) & 》 & 》) & J & ) & ) & 3 \\
\hline Rubiacees ........ & $"$ & $n$ & 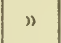 & $n$ & ” & ) & 1 & 》 & ) & 1 & $n$ & 2 \\
\hline Comp. : Corymbifères. . & D & 1 & " & $》$ & I & 1 & 2 & 1 & 2 & 》) & 》) & 8 \\
\hline - Cynarocéphales & 1 & 1 & 1 & $\eta$ & ) & ) & 》 & ) & ) & ) & $》$ & 3 \\
\hline - Chicoracées... & $n$ & 2 & ) & 1 & 》) & ” & ) & ) & 1 & 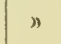 & ) & 4 \\
\hline Ebénacées ........ & $n$ & ” & 1 & ) & 》 & 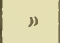 & 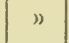 & ) & ) & ) & 》) & 1 \\
\hline Oléacées.......... & ") & $n$ & 1 & $n$ & 1 & 》) & ) & " & v & ) & ” & 2 \\
\hline Gentianées......... & 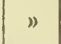 & ” & ") & ) & 1 & 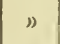 & ) & 》) & $》$ & ) & 》 & 1 \\
\hline Bignoniacées . . . . . . . & 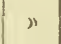 & 1 & $\eta$ & ) & 》 & 》 & $\eta$ & ) & $1(1)$ & ) & 》 & 2 \\
\hline Convolvulacées. ...... & ” & ) & 》) & 》) & ) & ” & $1(2)$ & ) & $1(1)$ & 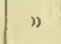 & ” & 2 \\
\hline Borraginées........ & $"$ & $\eta$ & 》 & 》 & 》 & 1 & $n$ & ” & $n$ & » & ) & 1 \\
\hline Solanées.......... & 》) & $"$ & $"$ & 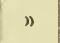 & $n$ & ” & ) & 1 & D) & ע & 》) & 1 \\
\hline Scrophulariées...... & $n$ & 》) & $n$ & $"$ & » & 1 & • & 》) & 1 & $\eta$ & $n$ & 2 \\
\hline
\end{tabular}




\begin{tabular}{|c|c|c|c|c|c|c|c|c|c|c|c|c|}
\hline FAIIILLE & 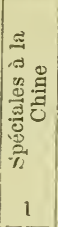 & 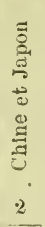 & 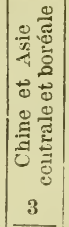 & 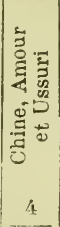 & 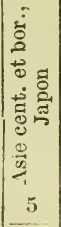 & 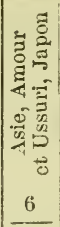 & 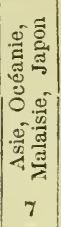 & 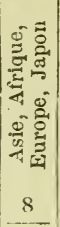 & 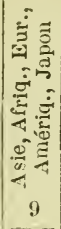 & 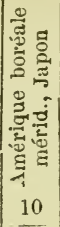 & 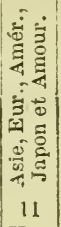 & 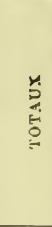 \\
\hline Orobanchées....... & D & $n$ & ) & $n$ & $n$ & " & 1 & ") & ) & 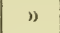 & $\omega$ & 1 \\
\hline Labiées . . . . . . . . . & ) & 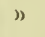 & 1 & ) & 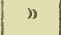 & $n$ & 1 & ) & 3 & $"$ & ") & 5 \\
\hline Verbénacées. . . . . . . & $n$ & 1 & ” & ) & $n$ & $"$ & " & 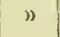 & 1 & ) & $n$ & 2 \\
\hline Acanthacées....... & ) & ") & $n$ & 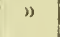 & ") & * & 1 & ) & $n$ & $n$ & v & 1 \\
\hline Primulacées. . . . . . . . & 2 & $\eta$ & $"$ & ) & $n$ & 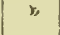 & $n$ & $n$ & $n$ & " & $n$ & 2 \\
\hline Plantaginées ....... & " & 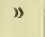 & ) & ) & ” & ;" & $n$ & ") & 1 & " & ) & 1 \\
\hline Amaranthacées ...... & ) & 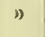 & ) & $"$ & $n$ & 1 & " & $n$ & 2 & ” & ” & 3 \\
\hline Chènopodées....... & $n$ & ) & 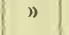 & ") & $"$ & ") & ) & l & 1 & $n$ & ") & 2 \\
\hline Polygonées . . . . . . . & $n$ & ) & 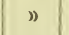 & ): & 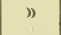 & ) & $1(2)$ & $n$ & 2 & $"$ & $n$ & 3 \\
\hline Euphorbiacees . . . . . . & ) & 1 & $1(3)$ & 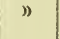 & 1 & ") & ” & ) & 1 & ” & ) & 4 \\
\hline Cannabinées......... & D & ) & ) & $n$ & ) & 1 & ) & $n$ & ) & ” & ) & 1 \\
\hline Lrticées ........... & $n$ & ") & $"$ & $"$ & ) & $n$ & 1 & $n$ & $n$ & $n$ & $n$ & 1 \\
\hline Moracées .......... . & ) & ) & 1 & 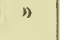 & ") & $n$ & 1 & $n$ & ) & ) & ) & 2 \\
\hline Salicinées. . . . . . . . & ) & " & 1 & ) & ) & $n$ & 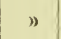 & ) & 1 & $n$ & ) & 2 \\
\hline Conifères.......... & ) & 5 & 1 & D) & ") & n) & " & $"$ & ) & ) & ") & 6 \\
\hline Saururées......... & $n$ & " & ) & ") & 1 & ) & $"$ & ) & ) & ) & 1) & 1 \\
\hline Palmiers . . . . . . . . & 1 & 》 & ) & " & ) & $"$ & ") & 》) & ) & ) & ) & 1 \\
\hline Hydrocharidées. . . . . . & ) & 1 & $n$ & bo & ") & ") & $"$ & $n$ & $\Rightarrow$ & ) & ) & 1 \\
\hline Alismacées . . . . . . . . & : & ) & ) & ) & ) & ") & ) & ) & 1 & ) & ) & 1 \\
\hline Potamées .......... & ) & ) & ): & $n$ & $n$ & $n$ & ) & ) & 1 & $"$ & ) & 1 \\
\hline Lemnacées... . . . . , & i) & ) & $n$ & )" & ) & " & )" & ) & 2 & ) & ) & 2 \\
\hline Aroïdées. . . . . . . . . . & ) & $"$ & ") & )) & ") & ") & ") & ) & l & $n$ & ) & 1 \\
\hline Orchidees ........ & " & 1 & " & ) & ) & ) & ) & ) & ) & ” & $n$ & 1 \\
\hline Amaryllidées. . . . . . . . & ") & ) & " & ) & $"$ & $\eta$ & 》 & 1 & 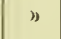 & ) & $n$ & 1 \\
\hline Asparaginées . . . . . . & $n$ & 1 & $n$ & ") & $n$ & ) & $n$ & $n$ & ") & 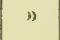 & 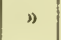 & 1 \\
\hline Liliacées. . . . . . . . . & ") & 1 & 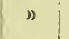 & ") & $n$ & " & ") & ) & " & ) & $n$ & 1 \\
\hline Dioscorées . . . . . . . & " &,$"$ & " & ) & 1 & " & $n$ & ); & $n$ & ) & ) & 1 \\
\hline Commélinacées . . . . . . & $n$ & ) & $n$ & 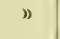 & ) & ) & $n$ & ") & 》) & 1 & $n$ & 1 \\
\hline Cypéracées.... & ) & 1 & 1 & ) & ) & : & 1 & ) & 1 & $n$ & $n$ & 4 \\
\hline frraminées ......... & 2 & ” & ") & ) & 1 & $n$ & 2 & 2 & 3 & ) & 3 & 13 \\
\hline Fougères.......... & 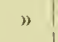 & 1 & " & ) & $n$ & ") & $n$ & ) & ) & " & ") & 1 \\
\hline Mousses. . . . & ) & ") & ") & D & ") & ) & $n$ & ") & 3 & ) & $n$ & 3 \\
\hline Totaux.... & 14 & 28 & 11 & 3 & 18 & 6 & 14 & 12 & 40 & 3 & 3 & $15 \%$ \\
\hline
\end{tabular}

(1) Moins l"Europe: Plantes non triuvees en Eurupe.

(2) Plus l'Afrique: Plantes qui se trouvent aussi en Afrique.

(3) Plus l'Europe: Plontes trouvées en Europe. 


\section{REGAPITULATION}

1" Spéciales a la Chine. - Plantes qui ne sont signalées jusqu'à présent que dans l'empire chinois et l'île de Hong-Kong ..............................................

20 Chine et JAPON. - Plantes spéciales à la fois à la Chine et au Japon..................................................

$3^{\circ}$ Chine et Asie boréale, Centrale et intertropicale. - Plantes qui se trouvent en Chine, et dans diverses régrions de l'Asis intertropicale ou tempérée

4" Chine, Axour et Ussuri. - Plantes qui se retrouvent dans les provinces boréales limitrophes de l'Amour et de l'Ussuri ..................................................

50 Aste bor., CENT. et Ixtertrop., Japox. - Plantes qui se retrouvent à la fois dans plusieur's régions de l'Asie et au Japon

$6^{\circ}$ Asie, Anour et Ussini, Japox. - Plantes dont la présence a été signalée en Asie, dans les provinces de l'Amour et au Japon.

70 Asik, OcÉAxiE, JApox. - Plantes dont l'aire de dispersion s'étend en Asie, au Japon, et dans les îles de la Malaisie, re l'Océan Indien et du Pacifique....................

$8^{\circ}$ Asie, kurope, Afrique et Japon. - Plantes qui se retrouvent à la fois dans diverses régions de l'Asie, de l'Europe, de l'Afrique et du Japon.............................

9" Asie, Europe, Afriquk, Avérique et Japon. - Plantes dont la présence a été constatée en Asie, Europe, Afrique, Amérique, au Japon, mais non dans les îles de l'Océanie et de l'Océan Indien..............................

$10^{\circ}$ A Mérique et Japox. - Plantes spéciales à la Chine et au Japon, et qui se retrouvent uniquement dans quelques parties de l'Amérique boréale.....

$11^{\circ}$ Asie, Europe, Avérique, Ayour et Japon. - Plantes dont la présence a été signalée en Asie, en Europe, en Amérique, dans les provinces de l'Amour et de l'Ussuri, et qui ne se trouvent pas en Afrique, ni dans les îles de l'Océrn Indier, de la Malaisie et du Pacifique........... 3 
Si nous considérons maintenant quel est le rapport géographique des plantes de Shang-haï, d'après leurs stations dans les diverses parties du globe, nous trouvons les indications suivantes d'habitat, classées selon leur importance numérique :

$\begin{array}{lllllll}\text { Japon ........... } & 124 & \text { espèces sur } 152 \text {, soit } & 5 / 6 \\ \text { Asie environ. }\end{array}$

Les affinités de géographie botanique des plantes observées dans le territoire de Shang-haï, ainsi que leur area, sont facilement démontrées par les chiffres qui précèdent. La flore de la Chine littorale paraît être liée étroitement avec celle du Japon, et nous trouvons en effet, dans cette dernière contrée, 124 espèces qui appartiennent à la flore de la Chine, soit les $5 / 6^{\mathrm{e}}$ environ du chiffre total.

Les rapports de la végétation chinoise avec la flore asiatique boréale, tempérée et intertropicale, ne sont pas moindres; car, si nous réunissons aux plantes signalées dans les diverses régions de l'Asie celles qui ont été observées également dans les provinces de l'Amour et de l'Ussuri, nous aurons à peu près le même chiffre que précédemment, soit 122 espèces, ou les $5 / 6^{\text {e }}$ environ des espèces de Shang-haï.

Viennent ensuite dans un ordre numérique presque égal les plantes que l'on retrouve en Afrique et en Europe. Celles-ci peuvent être considérées comme étant les ubiquistes, pour ainsi dire, de la flore de Shang-haï. L'Amérique boréale ou méridiouale, l'Océanie (Iles de l'Océan Indien, de la Malaisie et du Pacifique) n'occupent que le troisième rang par leurs affinités botaniques. Les provinces limitrophes de l'Amour et de l'Ussuri ne renferment qu'un cinquième environ de la totalité cles espèces de Shanghaï, tandis que la Chine à elle seule n'est représentée que par 14 spéciales, chiffre bien minime, eu égrard à l'étendue de cette vaste contrée. 
J'aurai bientôt l'occasion, en m'occupant de la flore du Tchéfou (Prov. du Chan-tong), de revenir sur cette importante question des affinités botaniques de la végétation du Kiang-sou, et de Shang-haï en particulier. Je tâcherai aussi d'expliquer les relations qui existent entre les plantes qui vivent spontanément dans eette localité, et celles des contrées avoisinantes de la Chine et de l'Asie boréale, centrale et orientale. 


\title{
CONTRIBUTIONS A LA FLORE DE LA CIIINE
}

FASCICUIE III

\section{FLORILE DI TCIIE-FOU (PROVINCE DE CHAN-TONG)}

\author{
Par M. O. DEBEAUX,

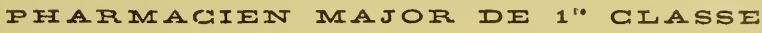 \\ CHPTALIRR DB LA LÉGIOT D'HONSEUR,
}

Membre de la Société botanique de France,

Correspondant de la Soriété Linnéenne de Bordeaux et de plusieurs autres So rétés savantes.

(Extrait des Artes de la Société Linnéenne de Bordeaux, t. XXXI, ze liv., 1876.)

\section{PARIS}

F. SAVY, LIBRAIRE-ÉDITEUR,

BOULEVARD SAINT-GERMAIN, iT 



\title{
FLORULE DU TCHÉ-FOU \\ (PROVINGE DE GHAN-TONG)
}

\author{
Par M. O. DEBEAUX,
}

Pharmacien-major de première classe,

\section{AVANT-PRUPOS}

La flore de la Chine est loin d'être connue, et à part quelques travaux publiés par MM. Bunge, Hance, Maximowicz, Turczaninow, etc., sur la végétation du territoire de Pé-king et du nord-ouest du Pé-tché-ly, ceux de MM. Bentham et de Hance relatifs à l'île de Hong-kong (1) et du docteur Cantor sur l'archipel de Chû-san (2), nous ne possédons encore aujourd'hui que fort peu de documents sur l'ensemble de la flore de l'empire chinois.

J'ai essayé, dans le $2^{\mathrm{e}}$ fascicule de mes Contributions à la flore de la Chine, d'esquisser le tableau de la végétation de Shang-haï et de Wôo-sông (province de Kiang-soû), et de faire ressortir les affinités de géographie botanique des plantes observées dans cette contrée. Comme suite naturelle à ces recherches, je vais m'occuper dans cette notice de la flore de la région montagneuse et littorale du Tché-foû (promontoire du cap Chan-tong), contrée qui, après Shang-haï, fut la deuxième occupée par l'armée française expéditionnaire en Chine.

Dans ma florule de Shang-haï, j'ai avancé cette opinion, «que " la flore de cette localité n'avait pas de caractère spécial et nette-

(1) Flora Hongkongensis, by G. Bentham; London, 1859, I vol. in-8 et supplément, auctore Hance.

(2) Flora and Fauna of Chusan, by Dr Cantor, in Annals and magazine of natural history; London, rol. IX (1842). 
" ment tranché; que, de plus, la vég’étation s'y trouvait représentée » par des espèces provenant de toutes les contrées limitrophes, et » qu'enfin le nombre des ubiquistes y était relativement considé» rable. » Je crois avoir trouvé, en partie, l'explication de ces faits remarquables dans l'examen d'une publication du $\mathrm{D}^{\mathrm{r}}$ Lamprey sur la géologie du Great-plain de la Chine (1). Dans ce travail, le $\mathrm{D}^{\mathrm{r}}$ Lamprey s'occupe de la formation et de l'étendue ou aréa du Great-plain (grande plaine), lequel repose entièrement sur le loam, ou dépôts successifs de sable et de limon, que les grands fleuves Hiang-tzé-kiang et Hoâng-hô, ainsi que leurs affluents, ont abandonné dans les vastes plaines de la Chine à lasuite des inondations. Le Great-plain embrasse dans son aréa la portion de la province du Pé-tché-ly arrosée par le Pei-hô, celle de la province de Chan-tong traversée par le canal impérial ou Yûen-kiang-hô des Chinois, et le fleuve Tâ-shing-hô, lequel se déverse dans le golfe de Pé-tché-ly. Il s'étend ensuite dans les provinces moyennes, le Kiang-soû et le Kiang-nan en entier, arrosées par le Hiang-tzé-kiang ou fils de l'Océan et le Hoâng-hô ou fleuve jaune; enfin nous le retrouvons dans les portions du Hô-nan, du Kiang-si et du Hoû-koûang, que traversent les nombreux affluents du Hiang-tzé-kiang, appelé aussi flewve bleu.

Le docteur Lamprey s'est assuré par quelques sondages pratiqués à Shang-haï et autres localités du Kiang-soû et du Pé-tché-ly, que le loam est constitué par des dépôts de sable et de limon régulièrement stratifiés. Ceux-ci renferment à une profondeur de 40 à 60 pieds (anglais) des̃ coguilles terrestres et d'eau douce, dont les analogues vivent actuellement dans ces mêmes contrées. De nombreux débris de végétaux (écorces, tig'es, racines) ont été trouvés à une profondeur de 140 à 180 pieds. Il est aussi à remarquer que les espèces de mollusques d'eau douce rencontrées dans les dépôts du loam à Shang-haï, se retrouvent à l'état vivant dans les cours d'eau du nord de la Chine. Je citerai parmi celles. ci, les IIydrobia striatella, Vivipara lecytoüdes, V. quadrata, Cyrena Largillerti, Unio Osbeckii, Anodonla gibba, etc., des dépôts du loam, et qui vivent encore aujourd'hui, soit dans le Wam-pôo à Shang-haï, soit dans le Pei-hô et le canal impérial à Tien-tsin.

(1) Notes on the geology of the great plain, by Dr Lamprey, in the journal of the nord-China branch of the Royal Asiatic society (1865). 
Enfin on a observé dans les sondagres des puits forés du Kiang-sou et à la profondeur de 12 à 13 mètres, des débris de coquilles marines appartenant aux genres Circe, Cardium, Solen, etc., dont les Chinois, voisins du littoral, font encore un grand usage pour leur alimentation.

Les dépôts successifs du loam ont eu pour conséquence naturelle, d'élever considérablement la surface du sol au-dessus du nireau de la mer, de rendre la végétation uniforme dans toutes les contrées inondées, et de la renouveler, pour ajnsi dire, après chaque inondation. Il est donc rationnel d'admettre, dans les provinces de la Chine comprises dans l'aréa du Great-plain, la présence d'une flore d'emprunt et à peu près identique. Je n'en excepte pas non plus le territoire du Chan-tong et du Kiang-soû, parcourus par le canal impérial, lequel relie Tien-tsin et Pé-king dans le Pé-tché-ly à Hang-tchéoû-foû dans le Tché-kiang. Les relations journalières du commerce qui s'établissent par la voie du canal impérial, entre le nord et le centre de la Chine, nous expliquent, en partie, la présence à Tien-tsin de quelques espèces : Ranunculus ternatus, Lepidium incisum, Potentilla supina, Xanthium strumarium, Eritrychium pedunculare, etc., qui sont répandues également beaucoup plus au sud, dans le Kiang-soû et le Chan-tong, à l'extrême limite du Great-plain.

Les provinces de la Chine qui possèdent une végétation endémique, c'est-à-dire qui leur soit spéciale, sont celles qui se trouvent situées en dehors de l'aréa du Great-plain, et par conséquent non soumises à l'influence des inondations. Ces provinces forment la région montagneuse de la Chine. Le Koûang-tong et le Fô-kien au sud, le Yûn-nan, le Koûei-tchoû, le Tssé-chûen et le Chen-si au centre, le Chan-tong au nord-est, et le Pé-tché-ly au nord-ouest, sont couverts de hautes montagnes, dont il serait fort intéressant de pouvoir connaître la végétation. Malheureusement, nous ne possédons presque pas de documents sur la flore de ces contrées. e nous ne pouvons que souhaiter aujourd'hui, de voir se réaliser l'entreprise d'une exploration scientifique, qui aurait pour mission de nous dévoiler les richesses de la faune et de la flore de ces régions qui nous sont inconnues. Déjà un intrépide. et savant missionnaire, M. l'abbé David, a exploré avec les plus heureux résultats une partie du Thibet oriental. Son exemple trouvera bientôt en Chine de nombreux imitateurs. 
La province du Chan-tong et celle du Pé-tché-ly qui lui est limitrophe ont été visitées, depuis longtemps déjà, par un botaniste bien connu, sir Georges Staunton, secrétaire de l'ambassade de lord Macartney en Chine pendant les années 1792 à 1794, et dont il nous a laissé une importante relation (1). On sait déjà également qu'après s'être rendue aux forts de Ta-koû situés à l'embouchure du Pei-hô, à bord des navires de S. M. Britannique le Lion et l'Indostan, et de Ta-koû à Tien-tsin et Pé-king sur des jonques chinoises, l'ambassade anglaise de lord Macartney prit, pour revenir à Chû-san, la seule voie de communication qui existait alors en Chine, et qui n'est autre que le $Y u n$-kiang-hô, ou le canal impérial. Autour des nombreuses villes et villages où l'ambassadeur Macartney fut obligé de séjourner dans sa marche de retour vers Hangtchéoû-foû, sir Staunton recueillait une foule de plantes dont il a publié des listes très-incomplètes. Plusieurs des espèces récoltées par ce botaniste ont été décrites dans le Prodromus de Decandolle, d'autres ne nous sont connues que par les noms Linnéens (les seuls en usage à cette époque), ou par le seul nom générique, lorsqu'il levenait impossible de leur assigner une place dans la nomenclature du Systema vegetabilium.

J'ai été assez heureux de pouvoir me procurer, en 186\%, quelque plantes récoltées par Staunton dans le Kiang-nan et le Chan-tong en 1793 et 1794, et provenant de l'herbier de feu Alphonse Maille. Je me suis assuré, par l'étude de cette intéressante collection, que plusieurs déterminations faites par Staunton, au cours de son voyage, sont des plus exactes, ainsi que les indications des noms génériques. Malgré leur mauvais état de conservation, ces exsiccata m'ont été des plus utiles, et j'ai cru devoir les signaler à la synonymie, chaque fois que la récolte en aura été faite sur les rives du canal impérial, dans la province du Chan-tong. Les plantes recueillies par sir Staunton, et les listes qu'il en a publiées dans le récit de son voyagre, ne donnent qu'une idée très-imparfaite de la végétation des contrées parcourues par l'ambassade anglaise: Mais on y trouve dejà l'influence du Great-plain dont je viens de

(1) Voyage de lord Macartney en Chine pendant les années 1792, 1793 et

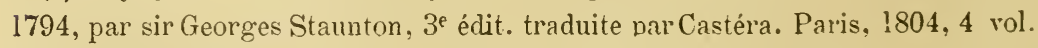
in $-8^{\circ}$ et atlas in $-4^{\circ}$. 
parler. Ces plantes n'ont aucun caractère local bien déterminé. Au point de vue de leur origine, elles paraissent provenir des contrées avoisinantes, et on les rencontre, en effet, dans les provinces centrales et maritimes de la Chine. Plusieurs des espèces signalées dans le Chan-tong sont généralement cultivées dans toute la Chine; d'autres sont ubiquistes et répandues dans l'Asie intertropicale et tempérée. Mais le plus grand nombre de Cypéracées, Graminées, etc., se retrouvent sur les sables des grands cours d'eau et du littoral de la Chine moyenne.

Ainsi que je l'ai déjà fait dans ma florule de Shang-haï, je mentionnerai, pour chaque espèce, sa synonymie exclusivement orientale, son habitat et son aréa géographique. J'ajouterai enfin quelques descriptions détaillées ou de simples diagnoses aux espèces et variétés nouvelles, et à celles qui offriront quelques particularités remarquables, ou dont les descriptions originales m'ont paru souvent douteuses et insuffisantes.

30 juin 1875. 


\section{$\mathbb{S}$ I. - Aspect physique du Tché-foa.}

Dès les premiers jours de juillet 1860 , je débarquais sur la plage du Tché-foû, point situé vers le milieu du promontoire du Chantong, à l'extrémité nord de la baie de Ki-tsen-sôo et à l'entrée de la petite rade de Yan-taï ( $37^{\circ} 40^{\prime}$ lat. N., $118^{\circ} 30^{\prime}$ long. E.). Un monticule peu élevé, entouré de falaises abruptes, et couronné par un fortin quadrangulaire, forme la pointe de Tché-foû. C'est sur le versant ouest de ce monticule, et sur la plage sablonneuse comprise entre la rade et la ville de Yan-taï et la baie de Ki-tsen-sôo que se trouve le campement du corps expéditionnaire en Chine. Le camp du Tché-foû est devenu permanent pendant la durée des opérations militaires sur Pé-king. Sa proximité de l'embonchure du Pei-hô, et la salubrité de son climat en toute saison, avaient fait choisir cette localité pour y installer un hôpital temporaire de 250 lits, et dont j'ai été appeléà remplir l'emploi de pharmacien en chef jusqu'à son entière évacuation, en février 1861.

Le Tché-foû, que les Anglais écrivent Ché-fôo, doit probablement ce nom au voisinage de Tang-tchéố-fôu, ville principale et cheflieu du district, dont fait partie la ville de Yan-taï, ainsi que le territoire que nous occupions. Cette contrée est limitée au Nord, à plusieurs lieues de Yan-taï, par les eaux du golfe de Pé-tché-ly, à l'Est et au Sud par la mer Jaune, et à l'Ouest par une chaîne de hautes montagnes, dont le nom indigène Chan-tong ou montagnes de l'Orient, sert à désigner la province qu'elles recouvrent, en effet, dans sa partie la plus orientale. Ce massif montagneux prend naissance à la pointe S.-E. du cap Chan-tong, suit la côte de l'Est à l'Ouest parallèlement au rivage, et s'incline ensuite vers le SudOuest, contournant la portion la plus septentrionale du promontoire jusqu'auprès de Lai-tchéoû-foû. A partir de ce point, cette chaîne prend la direction du Sud, et, formant un grand coude, vient se terminer à l'intérieur du promontoire près de la ville de Pin-toû-tchéoû, après avoir parcouru un développement de plus de 250 kilomètres. Unchaînon montagneux, qui ne paraît être qu'une ramification du massif prineipal et de peu d'étendue, s'avance vers la pointe Nord du promontoire, et se termine non loin de Tang-tchéoû-foû, à l'entrée du golfe de Pé-tché-ly, et en face de l'archipel de Mia-tâo. 
Il suffit de jeter les yeux sur une carte détaillée de cette contrée, pour avoir une idée précise sur le point de départ et de terminaison du soulèvement du Chan-torig. A l'exception du promontoire, les autres districts de cette province et les contrées avoisinantes, le Pé-tché-ly, le Hô-nan et le Kiang-sô̂ n'offrent partout qu'un sol plat et uniforme.

La pointe $\mathbf{N}$. de Tang-tchéoû-foû paraît devoir se relier par une suite de petits îlots ou groupes d'îles, qui émergent à la surface de la mer et à des distances assez rapprochées dans le détroit du Pétché-ly, à la pointe s. (pointe Charlotte), du Léa-tong (Mandchourie chinoise), qui fait face à cette partie du Chan-tong. Dans cette portion du détroit, qui donne accès de la mer Jaune dans les golfes de Pé-tché-ly et de Léa-tong, le fond de la mer se trouve par 10 à 18 brasses du côté de la Chine, et par 20 à 30 brasses en se rapprochant des côtes de la Mandchourie. C'est vers le milieu du cap de Léa-tong que se trouve la belle rade de Ta-lien-wân, où l'escadre anglaise se tenait à l'ancre, avant de combiner ses efforts avec ceux de la flotte française pour l'attaque des forts de Ta-koû.

Il ne peut exister de doute sur l'état primitif du golfe de Pétché-ly, qui n'a dû être qu'un grand lac ou mer intérieure, et se trouver séparé seulement de la mer Jaune que par un chaînon montagneux. Ce chaînon, commun à la fois aux systèmes du Chan-tong et du Léa-tong, devait partir de la pointe nord du Chantong et aboutir au cap S. du Léa-tong. Les nombreux îlots rocailleux, qui se trouvent encore entre ces deux points, indiquent évidemment la direction primitive de cet horizon montagneux aujourd'hui disparu.

Les causes physiques qui ont séparé, lors de la formation des continents actuels, l'Afrique de l'Espagne au détroit de Gibraltar, la Sicile de l'Italie, la Corse de la Sardaigne dans la Méditerranée, ont dû agir de la même manière pour opérer la séparation du Léatong d'avec le continent chinois, et, par suite, unir les eaux du Pé-tché-ly à celles de la mer Jaune. Je trouve dans la composition minéralogique des roches du Chan-tong, et celle des îlots rocailleux situés vers le milieu du détroit de Mia-tâo, et aussi dans l'identité des mollusques marins qui vivent dans les baies de Yantaï (Chine) et de Ta-lien-wân (Mandchourie), deux faits qui viennent corroborer mon opinion. Les falaises du Tché-foû et les roches du massif montagneux du Chan-tong sont formées unique- 
ment de micaschistes non stratifiés. Quelques rares affleurements granitiques se montrent parfois à la base ou sur les flancs des montagnes. La constitution physique des roches est identique dans les îles de Mia-tâo, et il y a lieı de penser que les falaises et le soulèvement montagneux du Léa-tong, ont la même structure et la même ancienneté ou origine que celles du Chan-tong. Les mollusques marins recueillis dans la baie de Ta-lien-wân par les naturalistes attachés à l'expédition anglaise en Chine se retrouvent en grande partie dans la rade de Yan-taï et la baie de Ki-tsen-sôo: ce qui est un indice, sinon une preuve, des relations zoologiques qui ont dû exister, et qui existent encore, entre ces deux points du littoral chinois.

La plaine du Tché-foû n'est point très-étendue. Elle comprend dans ses limites extrêmes les sables maritimes et les falaises de Ki-tsen-sôo (petit village situé en avant du camp français et adossé au pied des basses collines), les champs cultivés des environs de Yan-taï avec les nombreux tumulus qui les recouvrent, et enfin l'immense plage sablonneuse qui s'étend au nord entre cete ville et la mer. Yan-taï est loin de mériter en Chine le nom de ville: c'est un gros village peuplé d'environ 20,000 habitants, presque tous adonnés au commerce étranger. Il s'y fait un trafic énorme avec les jonques marchandes du Fô-kien, qui viennent apporter dans ce port les divers produits commerciaux du sud de la Chine de la Cochinchine, des îles de la Sonde, de Malacca, des IndesOrientales, etc., et en exportent en échange ceux du Chan-tong et des provinces voisines. La rade de Yan-taï, quoique peu sûre en hiver, à cause de la violence des vents de $\mathrm{N}$.-Est, possède en tout temps un nombre considérable de jonques, et qui occasiomment dans la ville une incroyable activité.

A peine a-t-on franchi la porte nord de Yall-taï, que l'on se trouve sur une plage sablonneuse large de 12 à 15 kilomètres, qui se relie d'un côté au cap rocailleux du Tché-fô, et se rattache de l'autre côté vers l'ouest aux basses collines, lesquelle: se terminent près diu village de Foû-chan-yen. Cette plage, d'abord plate et uniforme, s'élève successivement et forme des dunes de sable que le vent déplace sans cesse. Au milieu des dunes et de ce: monticules de sable, abrités des vents du Nord et de N.-Est. on rencontre plusieurs petites mares d'eau douce peu profondes, et aux bords desquelles apparaissent quelques plantes spéciales à 
ces localités. Souvent aussi, on rencontre sur la plage de Yan-tal des marécages assez rapprochés de la nner, qui les inonde parfois à haute marée, et l'eau qui en est toujours saumâtre ou salée, devient alors très-favorable au développement de quelques espèces halophytes.

Les basses collines qui sont profondément ravinées par les torrents, s'étendent depuis Ki-tsen-sôo jusqu'aux dunes de Foû-chanyen. Elles sont en général ondulées, de formation micaschisteuse près des falaises, et sablonneuses en s'avançant vers le golfe du Pé-tché-ly. Immédiatement situées à la base des hautes montagnes dont les crètes se dessinent à l'ouest, ces collines peuvent être considérées par leur faible altitude (250 à 350 mètres) comme étant intermédiaires entre la plaine et la région montagneuse supérieure. Sous le rapport de la végétation, je leur ai trouvé en général un caractère nettement délimité, et je les comprends dans la région montagneuse inférieure du Tché-foa.

Les hautes montagnes que j'ai eu l'occasion d'explorer plusieurs fois, se trouvent dans le voisinage immédiat du Yan-taï et de Ki-tsen-sôo. Celles-ci n'atteignent pas une altitude considérable, car les deux pics les plus élevés du Tché-foû ne dépassent pas 1,000 et 1,100 mètres d'altitude. Leur sommet est entièrement dénudé, soit par suite de la neige qui s'y accumule l'hiver et pendant plusieurs mois de l'année, soit par l'action des vents violents du N.-Est ou de la chaleur qui excluent toute végétation sur ce point. Les rares plantes qui se montrent dans cette haute région, ne se rencontrent seulement que dans les ravins ou les grandes fissure: des rochers abrités des vents du Nord. La région boisée occupe la partie moyenne de la chaîne montagneuse, c'est-à-dire entre 400 et 600 mètres d'altitude. Celle-ci serait la plus favorable à la végétation, mais le déboisement largement pratiqué par les indigènes tend à diminuer de plus en plus les espèces végétales propres à cette région.

Le climat du Tché-fou est très-froid en hiver, de décembre à mars, et excessivement chaud pendant les mois de juillet et aout. Le thermomètre centigrade s'élève alors jusqu'à +36 à $+38^{\circ}$ à l'ombre. Des pluies abondantes surviennent vers le milieu du mois d'aout, et indiquent le changement prochain de la mousson. Pendantl'hiver, la température s'abaisse jusqu'à-16 centig. au -dessous de zéro, et la moyenne de décembre et janvier est d'environ - $10^{\circ}$ 
centig. Cette basse température explique, en partie, pourquoi les végétaux ligneux ou vivaces de la Chine moyenne ne peuvent s'acclimater au Tché-foû, tandis qu'un petit nombre d'espèces annuelles de cette région ou plus mérìdionales encore, sont parfaitement acclimatées sur les sables maritimes du Chan-tong.

Je citerai comme exemple le fait survenu à deux pieds (de 5 à 6 ans) de Chamerops Fortunei, palmier qui prospère parfaitement à Shanghaï, où les hivers sont également très-rigoureux (de $-10^{\circ} \grave{a}-12^{\circ}$ centigrades), et qui ont été tués dans un jardin peu abrité du Tché-foû par un froid de $-16^{\circ}$ centig. pendant l'hiver de 1860 à 1861. Le Magnolia yulan, au contraire, qui appartient aux régions montagneuses et tempérées de la Chine moyenne, a résisté aux rigueurs de l'hiver de cette même année.

J'ai eu l'occasion de m'assurer dans une herborisation faite autour de Yan-taï à la fin d'avril 1861, pendant une relâche du transport de guerre la Nièvre, sur lequel j'étais embarqué pour me rendre à T'ien-tsin, que la végétation est nulle dans cette localité à cette époque de l'année. Je n'y ai pas rencontré, en effet, une seule plante en fleur, ou qui fut sur le point de le devenir. De plus, la température m'a paru être tout aussi rigoureuse que deux mois auparavant, lorsque nous avons abandonné le camp du Tché-fou. Les mois de juin et de juillet peuvent être considérés comme étant la saison printanière de cette contrée; les prairies sont à ce moment dans tout leur éclat, et partout la végétation se montre en pleine activité. Je dois faire remarquer en passant, que dans mes nombreuses excursions faites au commencement de juillet 1860, je n'ai rencontré aúcune espèce en fruits mûrs, ce qui aurait nécessairement indiqué, pour ces végétaux, une floraison antérieure d'a!ı moins quelques semaines.

Un fait intéressant à noter, et qui vient à l'appui de mon opinion sur le peu d'activité de la végétation du Tché-foû, d'avril à juillet, c'est la floraison de certaines espèces qui a lieu en Europe dans les premiers jours de mars et d'avril, et qui ne semontre sur le rivage du Chan-tong qu'à la fin de l'été ou au commencement de l'automne. Trois plantes printanières, même dans l'extrêmeOrient, m'ont surtout frappé par leur floraison attarlée. Ce sont le Pulsatilla chinensis que j'ai récolté en fleurs et fruits le 10 novembre 1860; le Viola Patrinii en fleurs et fruits le $1^{\text {er }}$ novembre, et le $V$. Gmeliniana en fruits mûrs le 7 août; enfin, j'ai rencontré en 
fleurs, vers le milieu d'octobre de la même année, le Narcissus tazetta var. chinensis, sur les terres incultes des tumulus près du villagre de Ki-tsen-sôo. Comme dernier exemple de floraison tardive, citons l'Imperata cylindrica var. Kanigii, que j'ai recueilli en fleurs, le 17 mai 1860, sur les sables de l'île de Hong-kong, et dont la floraison n'a lieu sur les rivages du Tché-fou que dans la deuxième quinzaine de septembre. Tout en tenant compte de la latitude de cette contrée, je ne saurais expliquer ces faits intéressants que par l'engourdissement prolongé de la végétation à la suite du froid rigoureux d'un long hiver, et aussi par l'orientation de ce pays, sans cesse exposé à l'action stupéfiante des vents du Nord et de Nord-Est.

A l'exception des mares d'eau douce situées au milieu des dunes de la presqu'île de Yan-taï, et des marécagees salés alimentés par la mer, il n'existe pendant l'été aucun cours d'eau, torrent ou rivière dans le territoire du Tché-foû. Les fontaines d'eal douce y sont inconnues, et les habitants n'ont d'autre eau potable que celle provenant des puits assez nombreux creusés autour des villages; et encore l'eau de ces puits est-elle fort désagréable au goût, à cause des quantités considérables de sels de soude et de magnésie qu'elle renferme. La sècheresse de cette contrée nous expliquera le nombre très-limitédes plantes ubiquistes qui ont pu s'acclimater sur un sol naturellement peu fertile, et celui plus restreint encore des espèces qui vivent au bord des marais ou dans les eaux courautes.

L'absence des roches calcaires, et la prédominance de la silice dans la plaine et la région montagneuse du Tché-foû, nous ont déjà fourni l'occasion de signaler aux naturalistes, la pauvreté de la faune malacologique de cette localité (1).

\section{\$ II. - Aperçu de la végétation du Tché-foû.}

Après huit mois de séjour au camp de Tché-fou (de juillet 1860 à mars 1861), et pendant lesquels je n'ai pas cessé un seul jour d'explorer dans un rayon assez étendu le pays où je résidais, je crois

(1) Notice sur la malacologie de quelques points du littoral chinois. Broch. in 80, 2 planches, in Journal de conchyliologie. Paris (1863). 
avoir réussi à réunir dans mon herbier, les végétaux dont se compose la flore de cette partie du littoral chinois. C'est donc le résultat de mes recherches personnelles que les botanistes trouveront dans cette florule, pour l'achèvement de laquelle M. A. Franchet a bien voulu m'aider, avec une extrême obligeance, de son savoir et de ses nombreuses observations.

Le botaniste qui, après avoir parcouru pendant les mois de mai et de juin les plaines marécageuses du Kiang-soû, arrive en juillet sur la presqu'île de Tché-foû, éprouvera un vif sentiment de curiosité en présence d'une végétation des plus variées, et dont il était loin de soupconner l'importance et la richesse. Les causes principales qui ont contribué à donner à la flore du Tché-foû, un caractère tout à fait local et original à la fois, doivent être attribuées, selon nous, à la diversité des stations botaniques, à la position et à la nature du sol, à l'influence de l'altitude, etc.

Trois régions botaniques naturelles, ayant chacune sa végétation spéciale, caractérisent la flore du promontoire du Chan-tong. Ces régions botaniques se trouvent parfaitement délimitées par l'altitude et la configuration même du sol dans cette partie du littoral chinois. Je les nommerai en suivant leur ordre d'altitude: $1^{\circ}$ la région littorale; $2^{\circ}$ la région des basses collines ou montagneuse inférieure (de 200 à 500 mètres d'altitude), et $3^{\circ}$ la région montagneuse supérieure (de 500 à 1,100 mètres environ).

\section{$1^{\circ}$ Région littorale.}

Celle-ci nes'éloigne pas sensiblement des rivag'es de la mer. Elle comprenddans son aréa les falaises micaschisteuses de la pointe et du cap de Tché-foû, celles de la baie de Ki-tsen-sôo, les sables maritimes formant les plag'es de Yan-taï et de Ki-tsen-sôo, les dunes de la grande baie de Foû-chan-yen, les prairies, les champs cultivés, les collines sablonneuses dont l'altitude ne dépasse pas 200 à 250 mètres, les marécag'es saumâtres qui avoisinent la mer, etc.

Sur les falaises et les roches micaschisteuses du littoral, on trouvera les espèces suivantes :

Silene aprica Turcz.

Ampelopsis serjanicefolia Bunge.

Rosa rugosa Thumbg.

Rubus parvifolius L.

Vicia rapunculus 0 . Deb.
Vicia unijuga A Braun.

Buplevrum scorzonercefolium Wild.

Rubia cordifolia L.

Calimeris altaïca Nées.

Bidens bipinnata L. 
Aster ageratoïdes Turez. Artemisia capillnris Thumb. - juponica Thumb.

Pyrethrum indicum Cass. Youngia son hifolia Max. Ectinops danuricus Fish. Adenophora coronopifolia Fish. Cynanchum pubescensBung. Orobanche ammophili Mey.
Lycium sineuse Mill.

Statire Franchetii O. Deb. Wikstroemia chinensis Meis. Securinega obovata Mull. Prordanthus dicholomus Led. Ophiopogon spicatus Gawl. Hemerocallis graminea Andr Eriochloa villosa Kunth. Selaginella mongolisa Rup.

Les sables de la plage du Tché-fou et les terres incultes voisines de la mer sont recouverts d'une végétation des plus intéressantes par le caractère dominant de certains genres, Phellopterus, Sophora, Lespedeza, Crotalaria, Cassia, Tournefortia, etc., et la beauté des fleurs de quelques végétaux herbacés. C'est là surtout que le botaniste récoltera les :

Dianthus Seguieri var. dentosus
Fish.
Hibiscus ternatus Car.
Abutilon Avicennce Gært.
Sophora galegö̈des Pall.
Crotalaria brevipes Champ.
Lespedeza tomentosa Sieb.
$\quad$ - trichocarpa Pers.
Cassia mimosoïdes L.
Potentilla viscosa Don.
Phellopterus littoralis A. Gray.
Stonocelium divaricatum Turc.
Artemisia mongolica Dec.
Chorisis repens Dec.
Xanthium indicum Roxb.
Vincotoxicum versirolor Bung.

Cuscuta chinensis Laru.

Tournefortia argusia Ræm.

Sculellaria scordiifolia Fish.

Vitex incisa Wild.

A maranthus sylvestris $\mathrm{L}$.

Salsola kali L.

Polygonum polymorphum Led.

Euphorbia esula L,

- humifusa Wild.

Asparagiıs Sieboldi Max.

Allium tenuissimum L.

Cyperus difformis L.

- rotundus L.

- iria L.

Carex macrocephala Wild.

Imperata Konigii P. B.

Après avoir franchi la porte Nord de Yan-taï, on rencontre de suite, à quelques mètres du rivage, de nombreux marais salés, le plus souvent inondés à la pleine mer. C'est là que croissent en abondance quelques plantes halophytes, que l'on ne saurait rencontrer ailleurs dans un grand rayon autour de Yan-taï :

Linum stelleroïdes Planch.

Sanguisorba tenuifolia Fish. - canadensis L.

Tripolium vulgare Nèes.

Artemisia indica Vild.

Sonchus brachyotus Dec.

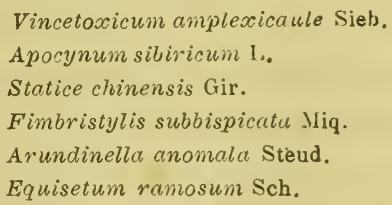

Equisetum ramosum Sch.

Sur les dunes et l'immense plag'e sablonneuse qui, de la rade de Yau-taï, s'étend jusqu'au rivage du Pé-tché-ly, et à la baie de Fô̂-chan-yen, on observe de juillet à octobre : 
Viola Palrinii Bunge.

- Gmeliniana Rom.

Lespedeza juncea Pers.

- striata var. Max.

Potentilla chinensis Ser.

Lythrum virgatum L.

Turczaninowia fastigiata Dec.

Boltonia pekinerisis Hance.

Lactuca amurensis Regel.

Asclepias sibirica Bunge.
Veronica spurir L.

Vitex ovata Thumbg.

Agriophyllum squarrosum Moq.

Corispermum Stauntoni Moq.

Salix purpurea L.

Typha angustifolia L.

Fimbristylis Buergeri Miq.

Ischomum barbatum Retz.

Phragmites communis var.

Marsilea quadrifolia L.

Les prairies sèches ou humides voisines du littoral possèdent, au mois de juillet, une flore des plus remarquables. Quelques graminées spéciales: Chloris caudata, Gymnotryx japonica, y sont tellement abondantes en compagnie de l'Eragrostis megastachya, qu'elles forment à elles seules la base du foin du Tché-foû. Au milieu de ces graminées, nous pourrons récolter quelques plantes caractéristiques des prairies maritimes du Chan-tong:

Hypericum perforatum var.

Melilotus graveolens Bunge.

Lespedeza juncea Pers.

Lathyrus paliustris L.

Potentilla paradoxa Nutt.

Sedum yantaiense O. Deb.

- pseudo-aizoon O. Deb

Galium pauciflorum Bung.

Eupatorium Kirilowii Turcz.

Inula japonica Thumb.

Senecio argunensis Turcz.

Scorzonera humilis var.

- pauciflora Jacq.

- macrocephala Turcz.
Mentha arvensis L. var.

Calamintha chinensis Benth.

Leonurus sibiricus L.

Salvia plebeia Brown.

Apochoris pentapetala Dub.

Lysimachia barystachys Bunge.

Euphorbia pekinensis Bois.

Sciila chinensis Benth.

Ischoemum Sieboldii Miq.

Gymnotryx japonica Kunth.

Chloris caudata Bunge.

Eragrostis megastachia Link.

- pilosa P. Beauv.

Etc., etc.

La végétation arborescente est loin d'être variée dans la plaine du Tché-foû. C'est à peine, si on trouve répandus ça et là les Quercus serrata et Pinus Massoniana, qui composent à eux deux, les parties boisées des basses collines et de la région montagneuse inférieure. Autour des pagodes et des tumulus disséminés dans la campagne, on remarque un petit nombre de conifères, dont l'habitat dans ces localités indique évidemment une origine étrangère. Je cite en passant les Biotia orientalis, Cunninghamia sinensis, Cupressus funebris, qui sont assez fréquents au Tché-fou, ainsi que l'Albizzia julibrissim de la tribu des mimosées.

Parmi les arbres indigènes cultivés puur servir à l'économie domestique, j'ai noté les Morus nigra et Excacaria sebifera. On 
cultive aussi, pour leurs fruits, quelques variétés d'amandier, de pécher, d'abricotier, de pommier et poirier. Les poires du Chantong passent pour être supérieures, par leur goût et leur arôme, ‘̀ celles qui proviennent des autres contrées de la Chine.

Un petit nombre d'arbustes et le sous-arbrisseaux se rencontrent fréquemment dans les terres incultes, les haies des jardins, autour des tumulus, etc. Ce sont les Eronymus verrucosus var. Tchefouensis, Zizyphus vulgaris, P'yrus prunifolia et $P$. Zelula folia, Lonicera confusa, Sambucus nigra, etc.

Enfin dans les champs sablonneux et les lieux vagues autour des villages, nous trouvons quelques espèces, que l'on peut avec raison appeler ubiquistes. Celles-ci s'acclimatent facilement dans toutes les parties du globe, où elles sont importées soit par les: échanges du commerce, soit avec les graines alimentaires ou servant à l'industrie (céréales, légumineuses, plantes tinctoriales et oléagineuses, etc.). Les plus fréquentes autour de Yan-taï et de Ki-tsen-sôo sont :

\footnotetext{
Sisymbrium sophia $\mathrm{L}$.

Malva sylvestris $\mathrm{L}$.

Portulaca oleracea $\mathrm{L}$.

Tribulus terrestris 1 .

Galium verum L.

Sonchus oleraceus L.

Linaria vulgaris Dc.
}

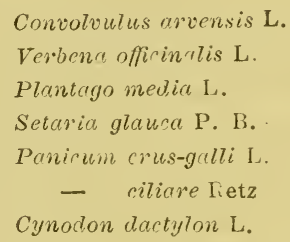

Le nombre des plantes endémiques de la région littorale, c'est-ìdire de celles qui sont spéciales à cette région ou aux provinces limitrophes seulement, est limité ì un dixième environ (26 esp.) dil chiffre total observé. Celui, au contraire, des plantes sporadiques ou qui proviennent de diverses régions du globe, mais qui ne sont pas ubiquistes, est beaucoup plus considérable dans les champs voisins de la mer. Les Cypéracées dominent surtout par le nombre des espèces qui croissent dans cette région : Carex macrocephala, Cyperus fuscus, difformis, rotundus, iria; Isolepis squarrosa; Fimbristylis complanata, subbispicata, Buergeri, etc.

Les cultures de la région qui nous occupe sont très-variées, malgré la nature siliceuse du sol. A côté de quelques végét:mx employés par l'industrie chinoise, j'en ai remarqué une fonle d'autres servant tous à l'alimentation des hommes et des animaux domestiques. Parmi les premiers, j'ai vu au Tché-foû de belles cultures de Sida titiafolia et de Cannabis indica (plantes textiles), 
et de Nicotiana tabacum, lequel produit dans la province du Chantong, le tabac le plus renommé de toute la Chine. Les plantes alimentaires les plus répandues dans les cultures sont les suivantes :

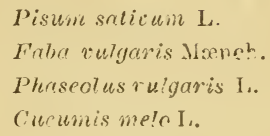

Sesamum oricritule $\mathrm{L}$ Batatas eduis Choisy. Capsicum longum I.

- annuum L.

Solanum tuberosunit.

- melongena I.

Lycopersicum esculcntum Mill.

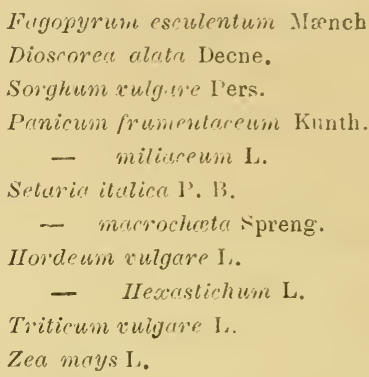

Il n'existe aucune culture de riz (Oryza sativa) dans le promontoire du Chan-tong, ni sur lelittoral du Tché-fou. Cette céréale est cultivée en grand dans la partie la plus occidentale.de la province et dans les terres marécageuses qui bordent le canal impérial.

$2^{\circ}$ Région des basses collines ou montagneuse inférieure.

Si, en suivant les crètes facilement accessibles des ravins, nous nous dirigeons vers le massif montagneux, nous ne tarderons pas à atteindre les premières collines ondulées qui s'étendent depuis la baie de Ki-tsen-sôo jusqu'à la plage de Foû-chan-yen. Ces collines, qui sont micaschisteuses vers le sud du Tché-foû et sablonneuses en se rapprochant du golfe de Pé-tché-ly, occupent, comme altitude, la partie moyenne entre la plaine et le sommet de la montagne. De nombreux bouquets de Pinus Massoniana donnent à ces collines un aspect tout à fait original, et que rend plus pittoresque encore la présence de quelques temples bouddhiques qui paraissent s'élever du milieu des bosquets de verdure.

C'est sur les pentes ondulées de cette région que se trouvent les belles cultures du Sida tiliafolia, et surtout du Sesamum orientale, dont les graines oléagineuses sont journellement employẻes dans la cuisine chinoise. Les plantations du zorgho commun (Sorghum vulgare Pers.) y sont également de toute beauté. La végétation spontanée offre le plus grand intérêt au botaniste qui parcuurt ces localités, au point de vue de l'aréa gesegraphique et 
de la rareté de quelques espèces propres à la régrion montag'neuse inférieure.

C'est là, en effet, que nous trouverons les Silene aprica, Prunus Bungei, Aster ageratö̈les, Leonlodon sibiricum, Picris japonica, Platycodon grandiflorus, Scutellaria lanceolaria, Sulvia milliorhyza, Andropogon schanan!lus, Anthistiria arguens, etc.

Les collines sablonneuses et les bois de pins nous offrent, près de la Pagode Newre, quelques espèces fort intéressantes : Polygala sibirica var., Indigofera macrostachya, Lespedeza bicolor, Cratagus pinnalifida, Potentilla discolor et Pot. flagellaris, Umbilicus fimbriatus, Grenia parviflora, Thymus serpillum var., Lilium tenuifolium, Commetina vulgaris, Pardanthus dichotomus, etc. L'altitude de 350 à 400 mètres m'a paru être la limite extrême de végétation des plantes caractéristiques de cette région.

\section{Région montagneuse supérieure.}

La chaîne de montagnes aui se dirigre parallèlement au rivage dans le promontoire du Chan-tong, atteint sa plus grande hauteur dans le district de Tché-fô, c'est-à-dire vers le milieu de son développement. L'ascension des points culminants du massif n'offre, en général, presque pas de difficultés, soit que l'on s'élève par l'arète des contreforts, soit encore en suivant les divers sentiers tracés par les indigènes pour traverser la montag'ne. Les bois de Quercus serrata, mêlé au Pinus Massoniana, apparaissent, aussitôt que l'on a quitté la régrion les basses collines, à une altitude de 450 à 500 mètres. Ces bois occupent les fiancs des ravins abrités et tendent à disparaître de jour en jour, par suite du déboisement et de la mise en culture du terrain qu'ils recouvraient. Dans les petites vallées de la montagne, le Pinus Massoniana atteint de grandes proportions; c'est alors un arbre superbe, dont la cime forme un vaste parasol, et à l'ombre duquel nous recueillons, eutre 500 et 600 mètres, les Hypericum ascyron, Polygonum bistorla, Polygonatum officinale, Selaginella involvens, etc. Le Polygonum bistorla est l'espèce dominante dans les bois de chênes et de pins, et elie n'offre aucune différence sensible avec sun analogue des Alpes et des Pyrénées.

Les bois disparaissent à l'altitude de 600 mètres, et la roche gr'anitique ou micaschisteuse apparait alors dans toute son aridité. 
Les pentes sont de pluśé plus difficiles à gravir, et ça et là nous recueillons dans les fissures des rochers une rare crucifère, le Dontostemum dentatus, puis le Gypsophila acutifolia var. sinensis. De belles touffes d'Umbilicus fimbriatus et de Pardanthus dichotomus recouvrent les roches dans cette haute région.

Parvenu au sommet des mamelons, que l'on atteint non sans peine, et dont l'altitude ne dépasse pas 1,000 à 1,100 mètres, nous trouvons, dans les anfractuosités des rochers, une forme glabre de l'Ampelopsis humulifolia. Auprès d'une pagode en ruines, qui couromne un des pics élevés du Tché-foû (1,000 mètres alt.), nous recueillons quelques rares espèces, entr'autres :

Clematis angustifolia Jacq.

Thalictrum hypoleusum Sieh.

Geranium nopalense L.

Indigofera marrostarluya Bunge

Patrinia scabiosafulia Link.

Artemisia sacrorum Ledeb.
Anrindria bellidiastrum Dc.

Cirsium thefouense sp. nov.

Plectranthus chinensis Bunge.

Andropogon schicnanthus $\mathrm{L}$.

Erianthus insignis sp. nov.

Asplenium nipponicum Mett.

Les crètes rocheuses au-dessus de Ki-tsen-sôo sont entièrement déboisées et des plus arides. Les quelques végétaux que l'on y rencontre ont un aspect rabougri qui contraste singulièrement avec la végétation des pics boisés que nous venons d'explorer.

\section{APpendice. - Végétation des falaises du cap Chan-tong.}

Il était pour moi de la plus grande importance de savoir si la flore d'un point éloigné du Tché-foù, sur le prolongement méridional du massif montagneux, étaitidentique aux deux extrémités de la chaîne. C'est ce qu'il m'a été possible de vérifier en partie, avec le concours actif et empressé du docteur Vesco, malacologiste distingué et chirurgien-major à bord du transport l'Européen. Le 18 septembre 1860, j’ai pu visiter, en compagnie de ce zélé naturaliste, et avec les embarcations montées de l'Européen mises à sa disposition, les falaises situées au-delà d'une grande rivière nommée

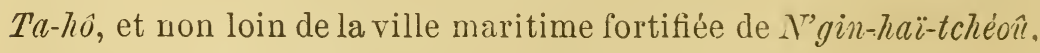
à environ vingt lieues au sud du camp de Tché-foû.

Ces falaises micaschisteuses, qui se terminent à la pointe du cap Chan-tong, suivent les sinuosités du rivage, et sont inabordables sur tout leur parcours. Les plantes que j'ai recueillies sur ces rochers maritimes sont identiques, pour la plupart, à celles des 


\section{FLORULE DU TCHE-FOU}

RENONCULACEES Juss.

Clematis Lin.

1. C. (Flammula) angustifolia Jacq. Collect. 1, p. 137, et Icon. plant. rar. tab. 104; Decand. Prodr. 1, p. 7; Ledeb. Flor. ross. 1, p. 2; Bunge Enum. plant. chin. bor. no 1; Regel in Plant. Radd. 1, p. 8 ; C. hexapetala Pallas Iter. 3, p. 226.

Var. Tchefouensis 0. Debeaux, Msc. et in Herb. (1860).

"Suffrutex perennis, bi-tripedalis, caulibus erectis sulcatis, » subglabris; foliis glabris tripinnatisectis, longe petiolatis, » subtus nervoso-reticulatis, segmentis petiolulatis, sæpius lanceo» latis acuminatisve integerrimis, medio bilobato profunde inciso; » inflorescentia terminali, corymboso-paniculata; floribus tricho» tomis folio plerumque longioribus; pedunculis multifloris apice » villosiusculis; sepalis 4-5, genitalia superantibıs, margine »revolutis, demum recurvatis, ovato-oblongis, dorso villosis » intus glabratis, pallide lutescentibus; staminibus in flore adulto » caudas æquantibus; carpellis sat numerosis (10-15), compressis, 》 ovato-oblongis, pubescentibus, caudis curtis albo-pilosis, gracil» lime recurvatis. »

Varietas nostra Tchefouensis Clematitis angustifolia, a forma typica differt, pedunculis terminalibus axillaribusque, $10-15$ floris, subpaniculatis, habitı humiliore, caulium glabrescentia, etc.

Habitat: Hautes montagnes du Tché-foû; point culminant de la montagne de Yan-taï (1,000 mètres d'altitude) autour d'une pagrode en ruines. Fleurs 20 juillet, fruits 18 septembre 1860 .

Area géographique : Le type : Daoûrie, Altaï, Monğolie près du fleuve Argun. - Prov. de l'Amour et de l'Ussuri. - Chine : nord du Pé-tché-ly. - La variété : Tehé-fou (Chan-tong').

Obs. : Le $C$. angustifotica est très-mal figuré par Pallas, sous le nom de $C$. hexapetala, et fort mal décrit par Decandolle et Ledebour qui lui attribuent des pédoncules uniflores, ce qui n'existe 
pas dans notre plante. Turczaninow (Catal. plant. Baik. no 1) lui assigne des pédoncules 1-3 flores subpaniculés. Decandalle avait mieux décrit cette espèce dans son S'ystema regni veget., en disant: Pedunculi terminales e dichotomia orti solitarii aut axillares. subpaniculati, (foliis) aquales aut paulo longiores. L'inflorescence dans la variété Tchefouensis est formée par une ample panicule terminale et axillaire. Chaque pédoncule porte de 10 à 15 fleurs, et les carpelles sont terminés par un appendice irès-court supérieurement et deux fois recourbé. Je la considère, en un mot, comme une forme floribonde du C. angustifolia.

Cette dernière espèce se distingue du $C$. orientalis L., de la section Flammula scandentes, par ses tiges droites non grimpantes, par ses feuilles coriaces, d'un vert sombre en dessous et nullement glauques et dentées au sommet, par ses filets staminaux glabres, par ses styles plus courts à la maturité, et enfin par les sépales non tomenteux sur les bords.

Elle diffère aussi du C. soongarica Bunge (Ind. semin. hort. Dorp.11, 1830) par ses feuilles à segments linéaires-lancéolés et non dentés, par le réseau nerveux-réticulé de leur face inférieure, et non simplement trinerviées, pạr son inflorescence axillaire terminale, et non disposée en cimes trichotomes, par la pubescence des sépales, des pédoncules, etc.

\section{THALICTRUM L.}

2. Th. minus Lin. Spec. 769; Franchet et Savatier Enum. plant. jap. $\mathrm{n}^{\circ} 13$.

Var. Hypoleucum in Miquel Prolusio flora japonica, p. 191; Th. hypoleucum Siebold et Zuccarini Flor. japon. p. 178, et fam. nat. japon. syn. $n^{\circ} 306$.

"Caulis striatus, erectus, glaber, foliis glabris, segmentis par»vis obovato-cuneatis, apice trilobatis, subtus glaucis; floribus » erectis laxe paniculatis; carpellis 4-Ј ellipticis, obliquis, sulcatis: » stigmatibus primum lato ovatis, subobliquis, dein convolutis 》 angustatis. »

Hab. : Région mont. supér. (de 1,000 à 1,100 mètres d'altitude), dans les fissures des rochers micaschisteux. - Fl. et fr. 6 septembre.

Ar.géog.: Le type : Eur.-Asie bor., Sibérie, Sôongarie, Russie 
asiat., Tauride, Caucuse, etc. - La variété hypoleucum: Chine (Tché-foû) et Japon.

Obs. : MM. Franchet et Savatier considérent la variété hypoleucum de Sieb. et Zucc. comme une forme à feuilles plus pâles et plus glauques en dessous du Thalictrum minus. Miquel paraît être du mème avis, quand il dit (loc. cit.) : «Folia juniora subtus glaucescentia. Le Th. minus est d'ailleurs une plante qui varie beaucoup pour la forme et la grandeur des folioles. Ia disposition de la panicule. lo nombre et la comue des carpelles sont également trèsrariables. Te tronve cependaut quelques caracteres asse\% stables lans la plante lu leché-fou et qui justifieut la distinction déjà faite par siebold et \%accariui da Th. hypolencum, sinon comme espèce lis gitime, mais comme une forme remarquable du Th. minus. CF lelnier, de provenance européenne, a les tiges lisses et flexueuses, les segments des feuilles dentés au sommet, et les carpelles allongés-elliptiques aux deux extrémités. Dans la plante chinvise, les tiges sont droites et striées, les folioles entières, et les carpelles oblongs léggèrement striés. Malgré ces quelques différences, j’adopte l'opinion de Miquel (Prolusio flor. jap. p. 191) qui considère le Th. hypoleucum comme une variété du Th. minus.

\section{ANEMONE L.}

3. A. (Pulsatilla) chinensis Bunge Enum. chin. 7or. $\mathrm{n}^{\mathrm{n}}$ 6, in Vém. Acar. Saint-Pelesby. 2, p. 76: Regel Tentam. flor. ussur. p. 15, tab. 2.

"Cautis erectus tomentosus, uniflorus, 12-18 centim. altus, petio» lis pedunculis que lense villosis; foliis ternatisectis, segmentis » late obovatis, supra pilosis, subtus villosis, lobis omnibus obovatis, 》 profunde incisis: stipulis lense lanatis: floribus magnis quam in >Pulsatilla rulgari, erectis rel suberectis ; sepalis sex, ovato»oblongis, subacutis, violaceis; carpellon num caudis 3.) millim. »longis, carpellis dense lanatis. »

Hab. : Sıbles maritimes de Yan-tï et ds Ki-tsen-sòo, principalement autour des tumulıs. - Fl. et fr. 10 novembre 1860.

Ar.géog.: Chine : Chan-tong et Pé-tché-ly, Mongolie chin. Pror. de l'Ussuri.

Ohs.: Je fais renary'ler ejmbien est rariable l'époque de la flo- 
raison de cette espèce. Bunge dit qu'elle fleurit en mars et arril, époque bien éloignée de la floraison de la plante de Yan-taï.

\section{VÉNISPERMÉES JUSS.}

Cocculus C. Bauh.

1. G. Thumbergii Dec. Prod. 1, p. 98; Franch. et Sav. Enum.jap. n' 82; Miqusel Prol. jap. p. 198; O. Debeaux Fr7or. Shang-ha. 11" 5: Menispermum orbiculatum Thumgb. Flor. jap. p. 194: sinice MOU-TONG.

forma trilobata, foliis presertim superioribus subtrilobatis. vel rarius subcordatis.

Hab. : Rochers du littural à la pointe de l'ché-foù, près du vieus fort; falaises de Ki-tsen-sôo, etc. - Juillet-août.

Ar.géog.: Chine et Japon.

Obs. : Le Nelumbium speciosum Wild. ne se rencontre pas à l'état spontané dans la région littorale du Chan-tong, mais il est excessivement commun dans l'intérieur de cette province sur les limites du Kiang-nan. Sir Georges Staunton, botaniste attaché à l'ambassade de lord Macartney en Chine (1793), signale le Nélumbo sur les rives du lac Wei-châng-hâi, lequel n'est séparé du canal impérial (Yûn-kiang-hồ, ou $Y u-h \hat{o}$ ) que par une chaussée assez élevée. Le Vélumbo est également très-abondant dans les terres marécageuses qui bordent le canal impérial, avant de pénétrer dans la province du Kiang-soû.

\section{CRUCIFERES TUSS. \\ 1) ONTOSTEMON And1\%.}

Ђ. D. dentatus Bunge Enum. chin. bor. p. 6, 11" 33, sub Andreoskia Ledeb. Flor. ross. 1, p. 175; Nrxim. Prim. amur. p. 45 : Regel Tent. flor. ussur. p. 22.

Var. A. typicus : D. eglandulosus C.A. Meyer, et D. oblongifolius Led. teste Maximowicz.

"Caulis sulcosus, pilis raris adspersus, foliis oblongis lanceola"tis, linearibusve, utrinqueattenuatis, denticulatis, hispido-cilia》tis, eglandulosis; petalis roseis, obovatis, sepala duplo superanti» bus; siliquis pelicellisque glabris; stylo crassitie fere stig"matis. » 
Hab. : Région montagneuse du Tché-fon, à 1,000 mètres d'altitude; fissures des roches micaschisteuses les plus escarpées. - Fl. et fr. 6 septembre.

Ar. géog. : Asie bor., Daoûrie près du fleuve Argun (Turczan.) - Chine bor. : Pé-tché-ly, montagnes de Pan-shan(Bunge), Pé-king (Maxim.); - Prov. de l'Amour et de l'Ussuri.

Obs. : Cette rare et remarquable crucifère de la tribu des Sisymbriées est très-variable dans sa taille, selon qu'elle croît dans les fissures des rochers ou dans les crevasses renfermant un peu de terre végétale. J'ai recueilli au Tché-foû des échantillons n'ayant pas plus de 10 centimètres de hauteur, et d'autres atteignant 40 centimètres environ.

\section{SISYMBRIUM L.}

6. S. sophia Lin. Spec. 122; Bunge Enum. chin. bor. p. 6, n 35 ; Ledeb. Flor. ross. 1, p. 180; Franch. et Savat. Erum. plant. jap. p. 37; Miquel Prol. for.jap. p. 6; Regel in Plant. Radd. 1, p. 213; sinice CHоûr-KIÉ.

Forma canescens, tota pilis curtis, densis, albo-tomentosis induta.

Hab. : Champs sablonneux non loin du littoral, à Yan-taï. - Fl. aout.

Ar. géog. : Eur. - Afriq. bor. - Asie; Russie arctique, Tauride, Caucase, Arménie, Sibérie, Altaï, Baikalie, Daoûrie; - Chine : Chan-tong, Pé-tché-ly à Pé-king, Mongolie, etc.; - Japon.

Obs. : On cultive en grand autour de Yan-taï et dans les plaines à l'intérieur dü Chan-tong le Pé-TSAI (Brassica chinensis Lin. Aman. IV, p. 280), dont les graines servent à la fabrication d'une huile fixe employée dans l'économie domestique. Le Lô-pô ou Lô-pé (Raphanus sativus Lin.) est fréquemment cultivé pour ses racines dont les nombreuses variétés servent à l'usagre alimentaire.

\section{VIOLARILES Dec.}

VIOLA I.

7. V. Patrinii Dec. Prodr. 1, p. 293; Regel Flor. uss. p. 28 et Plant. Radd. reisen 1, 1. 230; Ledeb. Flor. ross. 1, p. 245; Turczan. Flor. Baik. Dahur. 1, p. 181; Miquel Prol.jap.p. 8t; 
Franch. et Sav. Enum.jap. p. 4l; Maxim. Prim. amur. p. 48; Wight et Arnot Prod. flor.pen. ind. or. 1, p. 32; V. chinensis G. Don; sinice TsA-Hoâ.

Forma A typica : «Foliis e basi subcordatis vel rotundatis vel » cuneatis-oblongis. Variat magnitudine omnium partium, foliis »plus minus hirtellis, floribus parvis, appendicibus rotundatis » integris, vel vix repandis, etc. »

$H a b$. : Sables maritimes au-delà de Yan-taï, baie de Foû-chanyen, etc. - Fl. et fr. $1^{\text {er }}$ novembre.

Ar. géog. : Eur. : Russie movenne, Kasan; - Asie bor. : Sibé- rie altaïque et orientale, Baikalie, près d'Irkutsk, Daoûrie; Chine, Kiang-nan (Staunton), Chan-tong (O. Debeaux); - Prov. de l'Amour et de l'Ussuri; - Japon.

Obs. : Le Viola Patrinii, qui paraît être fort peu répandu en Chine, offre une singulière anomalie dans l'époque de sa floraison. M. Maximowicz l'a récolté en fleurs dans la province de l'Amour les $1^{\text {er }}$ et 10 juin 1855,20 juin 1856 , et en fruits le 29 août 1855 . Je l'ai recueilli en fleurs sur les sables du Tché-foû, le $1^{\text {er }}$ novembre 1860.

8. V. Gmeliniana Ræm. et Schult. Syst. veget. 1, 354; Ledeb. Flor. ross. 1, p. 256; Turcz. Flor. Baik. Dahur. 1, p. 181; Maxim. Prim. amur. p. 49; Regel Plant. Radd. reis. I, p. 232; Trautv. et Mey. in Middend. Ochotsk. ${ }^{\circ} 52 ; V$. Patrinii var. Gmeliniana Miquel Prol.jap. p. 84: Franch. et Savat. Enum. jap. p. 41.

Forma hispida: «Acaulis, radice perenni, subfusiformi, simplici; "foliis longe elongatis, lanceolatis, basi in petiolum breviorem » sensim attenuatis, crenulato-dentatis; sepalis acutis lanceolatisve, » appendicibus rotundatis, haud dentatis, calcare incurvato, sepa" lis breviore; stiputis elongatis linearibus."

$V$. Gmeliniance forma hispida (Ledeb. loc. cit.) a typica differt foliis petiolisque pilis hispidis, rigidis, plus minusve dense adspersis, calicibusque basi ciliatis.

$H a b$. : Sables maritimes de la grande baie de Yan-taï. - Fl. et fr. le 7 août 1860 .

Ar.géog. : Asie bor. : Sibérie, Baïkalie à Irkutsk, Sibérie orientale, Ochotsk, Transbaikalie, Daoûrie, Chine au Tché-fon: - Prov. de l'Amour; - Japon. 
Obs. : La variété hisnida du $V$. Gmeliniana n'avait pas encore été observée sur le territoire chinois. La plante de Yan-taï est surtout remarquable par ses feuilles longuement lancéolées, presque sagittées à la base et atténuées en pétiole. Miquel la considère comme une forme du V. Patrinii, à éperon plus allongé.

\section{POLYGALEES JusS.}

\section{POLYGiald I.}

9. P. sibirica lin. Spec. 987 (ex parte): I'. sibirica r. tenuifolia J.edeb. Flor. ross. 1, p. 269: Regel in Plant. Radd. reisen. ?. p. 276; p. tenuifolia Dec. Prodt. 1, p. 324: Bunge Ënum. chin. Zor. n 46 ; Maxim. Prim. amur. p. 51; Turcznan. Fllor. Baik. Dahur. 1, p. 197; Miquel Prol.jap. p. 148?; Franch. et Sav. E'num. jap. p. 44 ; sinice Y UEN-TCHÉ.

Var. Stricta O. Debeaux Mss. et in Herl. 1860). P. stricta O. Deb. olim.

"Perennis, humilis, cautibus erectis basi suffructicosis, ciliato》 hispidis, 2̌-30 centim. altis; foliis strictis, angustissime lineari» bus, mucronulatis, ad margines ciliatis, racemis axillaribus laxis, " paucifloris, ebracteatis; alis scariosis, transverse venosis, ovali》obcordatis; floribus carneis, capsula duplo longioribus; petiolis " inflexis sub flore crassioribus: ovario subsessili stipitato. Semina 》. nigra pyriformia, 2 millim. longa, 1 millim. lata, pilis strictis 》 curtis, albisque vestita. »

$H a b$. : Basses collines micaschisteuses ou sablonneuses au-dessus de Yan-taï, loco dicto : Pagode neuve. - Fl. et fr. 12 juillet.

Ar. géog.: Asie bor.: Sibérie altaïque et transbaikalienne, Daôurie. - Prov. de l'Amour et de l'Ussuri. - Chine : Hô-nan, Chan-si, Chan-tong au Tché-fô̂, Pé-tché-ly à Pé-king, etc.-Japon?

Obs. : Totre variété stricta du P. sibirica (P. tenuifolia Dec.) n'a pas encore été signalée, que je sache, par aucun botaniste dans l'Asie orientale. Decandolle (Prodr. 1, 324) assigne au P. tenuifolia des feuilles linéaires-mucronées et des tigres droites et glabres. Dans son étude sur le $P$. sibirica, Regel passe en revue toutes lelormes de cette espèce à laquelle il réunit le $P$. tenuifolia, qu'il considère comme une variété à tiges et à feuilles golabres ou un peu pubérulentes. Nais il est certain que Regel n'a eu aucune connaissance de la plante du T'hé-foû, qui est bien distincte par 
ses tiges ciliées-liispides, et ses feuilles rudes, ciliées sur les bords, et très-élroitement linéaires. Notre variété se rapproche beaucoup d'une forme de l'Àmour, que M. Maximowicz décrit ainsi : «Planta amurensis tenuissima, foliis linearibus, floribus paucissimis.» L'anteur précité ne fait aucume mention ni de sa glabrescence ni de sa villosité.

\section{SILENEESS Dec.}

Grypsophila I..

10. G. acutifolia Fischer in Cat. hort. Goren. p. 59 (1812); Dec. Prodr. 1, p. 53; Ledeb. Flor. ross. 1, p. 295.

Var. chinensis Regel in Planta Raddeana reisen in den von ost. siberien. (Bull. soc. imp. Mosc. 1864, p. 294.)

"Planta perennis, glabra, 1-2 pedalis, cautibus erectis, divari》 cato-ramosis; foliis carnosis, ovato-acutis, basi semi-amplexi»caulibus; inflorescentia ampla, late diffusa, cymis plurimis »composita, pelalis angustis albidis, calice duplo longioribus ; » capsulis glabris, minutis, subglobosis.»

Hab. : Région montagneuse du Tché-foû (800 à 1,000 mèt. d'alt.), dans les fissures des rochers micaschisteux. - Fl. et fr. 6 septembre 1860.

Ar. géog. : Asie bor., Sibérie aust.; —Chine : Chan-tong (Regel), le Tché-foû (O. Debeaux).

Obs. : La variété chinensis du G. acutifolia constituerait plutôt une bonne espèce qu'une simple variété. On peut la caractériser par ses feuilles charnues, ovales, et demi-embrassantes à la base, etc. (A. Franchet in litt.)

\section{DIANTHUS L.}

11. D. Seguieri Chaix in Villars Dauph. 3, p. 594; Fenzl in Ledeb. Flor. ross., 1, 277; D. Seguieri var. sylvaticus Koch Syn.fl.germ. p, 105; Regel Tent.fl.uss. p. 26.

Var. dentosus: D. dentosus Fish. in Dec. Prod. 1, 358; Turczan. Flor. Baik. Dahur. p. 197; Karel. et Kiril. Enum. Soong. 150; Franch. et Savat. Enum.jap. 187; Miquel Prol. jap.; D. Fisheri Spreng. Cat. hort. Hall. $(1810)$ : Bunge L'mum. chin. bor. no 49; sinice Kroû-HOà, Ti-sol̂-HOà. 
"Planta polymorpha, perennis, radice crassa, repente, caulibus „cæspitosis erectis, strictis, simplicibus; floribus 3 -10 in paniculam » confertam aggreg atis, breve pedunculatis, bracteis calicinis folia» ceis, ovalibus, acuminatis, mucronulatis, adpressis, margine > membranaceis, tubo calicis subconico, multistriato multo bre» vioribus; petalis inciso-dentatis imberbibus, roseis, unicoloribus » vel rarius purpureo-maculatis aut albidis. Folia lineari-lanceo" lata, glauca, margine scabra, subtus trinervia. "

$H a b$. : Sables maritimes depuis la pointe du Tché-foú jusqu'audelà de Ki-tsen-sôo. - Très-abond. en juillet et août.

Ar. géog. : Eur. bor. et or. : Russie arctique et moyenne, Oural, Moscou, Volhynie, Podolie, Kasan, Chersonèse, Wolga. - Asie bor., Prov. caspiennes, Caucase, Sibérie arctique, Altaï, Baïkal., Daoûrie, Mongolie, Sôongarie; Chine(Chan-tong et Pé-tché-ly). Prov. de l'Amour et de l'Ussuri. - Japon.

Obs. : M. Maximowicz adopte pour cette espèce, qui est trèsrépandue dans tout l'extrême Orient, le nom de $D$. dentosus Fish., lequel ne serait, d'après Ledebour, qu'une forme du $D$. Seguieri à écailles calicinales égalant la moitié du tube, ou plus courtes que lui. MM. Franchet et Savatier pensent (loc. cit.) que la plante orientale tient le milieu entre le $D$. Seguieri Vill. et le $D$. sylraticus Hoppe in Wild. Enum. 467, mais qu'elle se rapprocherait darantage de ce dernier par ses feuilles linéaires lancéolées, glauques, etc. La plante des sables du Tché-foû ne diffère en rien du $D$. dentosus Fish. dont j'ai reçu des échantillons récoltés en 1873 par un botaniste russe, M. G. Clerc, dans la chaîne montagneuse de l'Oural. Aussi serait-il plus rationnel peut-être de désigner la plante de Chine du nom de $D$. dentosus, ainsi que M. Maximowicz l'a déjà fait, pour la même espèce, dans son index florce Pekinensis.

\section{SILENE L.}

2. S. (Rupifraga) aprica Turczan. Cat. Baik. Dahur. n²21; Ledeb. Flor. ross. 1, p. 317; Fish. et Mey. Index semin. hort. Petrop. p. 38 (1835); Bunge Envm. chin. bor. no 50 ; Maxim. Prim. amur. p. 55 ; sinice HoûANG-PÔ-Lieô.

Hab. : Collines sablonneuses et micaschisteuses au-dessus de Yantaï et de Ki-tsen-sôo. - Fl. 10 juillet. 
$A r . g e ́ o g .:$ Asie bor. : Sibérie, Baikalie, Daourie.-Chine (Chantong au Tché-foû, Pé-tché-ly à Pé-king). — Prov. de l'Amour.

Obs. : Le Silene aprica se distingue de tous ses congénères du groupe Rupifraga, par sa pubescence presque tomenteuse, par ses tiges clroites de 35 à 45 centim. de hauteur, par ses feuilles linéaires oblongues, par son inflorescence terminale et son calice ovale-cylindrique pluristrié, à divisions allongées, acuminées, recourbées et de même longueur que les pétales; enfin par sa capsule elliptique égalant le calice, et ses graines dépourvues d'un sillon dorsal. Ce dernier caractère serait, toutefois, de mince valeur, selon M. Franchet, car on trouve souvent sur le même individu des graines offrant cette dépression dorsale, et d'autres qui ne l'ont pas.

\section{LINÉES Dec.}

\section{LINUM L.}

13. L. stelleroïdes Planchon in Hook. Lond. jou'n. of botany, vol. 7, p. 178; Franch. et Savat. Enum. plant. jap. p. 68, n² 276; Maxim. Ind. flor. Pekin. ad calcem Prim. flor. amur. 460 ; L. dahuricum Miquel Prol. flor. jap. p. 149, non Schultes Syst.6, 752.

Var. salsugineum O. Debeaux Mss. et in Herb.(1860).

"Planta annua, gracilis, subglabra, bi-tripedalis, caulibus erectis » simplicibus, minutissime striatis; foliis lineari-lanceolatis, acu" minatis, mucronulatis, subtus trinerviis, caule adpressis; inflo"rescentia paniculata, corymboso-subdichotoma, ramulis floriferis » laxe paucifloris; floribus intense violaceis, petalis calice 2-3 plo» majoribus, sepalis apice acuminatis, 3 exterioribus late-ovatis » supra medium glanduloso-ciliatis, interioribus 2 tenuioribus, » margine membranaceis, capsula subbrevioribus; capsula lævi" gata, ovoidea-subglobosa, apice mucronulata."

Hab. : Prairies saumâtres et inondées à haute mer, au nord de Yali-taï. - Fl. et fr. 14 septembre.

Ar. géog. : Chine : Tché-foû, Pé-tché-ly à Pé-king (1/rxim.). Japon : Owari et Nippon. - Corée.

Obs. : La variété salsugineum se distingue du type par son habitat dans les prairies saumatres et inondées, par ses fleurs grandes, d'un violet foncé et disposées en une panicule làche sub- 
dichotome, par ses tigres grêles élevées, atteignant parfois 1 mètre de hauteur. MM. Franchet et Savatier attribuent au L. stelleroüdes du Japon des fleurs violacées à pétales longs à peine de 5 inillimètres, tandis que dans notre plante ceux-ci atteignent de 15 à 20 millimètres. Ce dernier caractère serait conforme à la description qu' 'u donne Miquel dans son Prolusio "Pelala fere semipollicaria. Mais la station de la plante japonaise "in argillosis siccis (Franch. et Sav.), et in regionibus clirosis (Niquel) diffère notablement de celle du Tché-foû, et c'est ce qui m'a décidé à maintenir comme légitime, la variété salsugineum. Il ne nous paraît pas possible, ainsi que l'a fait Miquel (loc. cit.), de rapporter le $L$. stellerö̈des Planch. au $L$. dahuricum Schult., cette dernière espèce étant une plante vivace.

\section{MALVACÉES R. Brown.}

\section{MaLVi I.}

14. M. sylvestris Lin. Spec. 969; Dec. Prodr. 1, p. 432; Ledeb. Flor. ross. 1, p.435; Franch. et Sav. Enum.jap. p. 62, no 253: Miq. Prol.jap. p. 208.

Hab. : Talus extérieurs des fortifications de Ki-tsen-sôo, où il, paraît être subspontané dans cette localité. - Fl. juillet et août.

Ar. géog. : Eur. - Afriq. bor. - Amér. bor. - Asie bor. occ. et or. - Russie asiat., Tauride, Caucase, Arménie, Prov. Caspiennes, Sibérie ouralienne, Altaïque et Baikalienne, Sôongarie, Chine au Tché-foû. - Japon.

\section{HYBISCUS L.}

15. Y. (Trionum) ternatus Cavan. Dissert. 3, p. 172, tab. 64; Ledeb. Flor. ross. 1, p. 438; Fish. et Mey. Index semin. hort. Petrop. p. 10(1839); Regel, Flor.ussur. 29; Maxim. Prim. amur. p.61; Franch. et Sav. Enum.jap. p. 64, n² 264; Miq. Prol.jap. 207; H. trionum var. ternatus Dec. Pröodr. 1, p. 453: H. resicarius Besser Enum. IVolh. n 574.

Var. sinensis O. Debeaux Mss. et in Herb. 1860.

"Cautibus basi ramosi , quam in forma typica elatioribus, "pilosioribusque, foliis magis dissectis, floribus pallide-virenti" bus, seminibus atris, ut in $I I$. ternato echinulatis. " 
Hab. : Sables maritimes de la grande baie de Ki-tsen-sôo. - Fl. 9 aout, fr. 23 septembre 1860.

Ar. géog.: Le type : Eur., Russie aust., Podolie, Hongrie;-Afriq. aust., Cap de Bonne-Espérance;- Asie : Asie bor., Astrakan, Tauride, Caucase, Arménie - La variété : Chine(Chan-tong et Pé-tchély), Prov. de l'A mour et de l'Ussuri. - Japon.

Obs. : Notre variété sinensis ne diffère de la forme typique de l'H. ternatus que par ses tiges plus rameuses dès la base, et be:ucoup plus velues, par ses feuilles découpées en lan ères plus étroites et ses fleurs d'un vert-pâle. Les graines dans les deux formes sont plus ou moins hérissées à leur surface. Cecandolle (Prodr. 1,453 ) et Ledebour (Flor. ross.) attribuent à l' II. ternatus des graii nes glabres « semina glabra ». Cette assertion est en contradiction évidente avec les observations faites par M. Franchet et par mosur les graines d'H. ternatus provenant de l'Europe orientale, de la Chine et du Japon, et qui sont toutes échinulées. La plante chinoise se rapproche de l'H. armeniacus Bouché in Walp. Rep. bot. l, p. 302, par la villosité soyeuse des feuilles et de l'involucre, etc.; mais elle s'en éloigne par la forme des feuilles, la villosité des tiges et des pétioles, et surtout par ce caractere remarquable des graines qui sont hérissées de points tuberculeux.

\section{ABUTILON Gærtn.}

16. A. Avicennæ Gærtn. Fruct. et sem. plant. 2, p. 135; Ledeb. Flor. ross. I, 439; Franch. et Sav. Enum.jap. p. 63, n² 257; Regel Tent. flor. ussur. 30; O. Debeaux Flor. Shang-hä̈, n. 20; Sida abutilon Lin.; Pallas Iter. 1, p. 367 ; sinice Tsı-M-MA "Caule 1-2 pedali, floribus parvis, carpellis biaristatis, aristis » initio erectis, dein patentibus. »

Hab. : Sables maritimes et bord des champs voisins de la mer, à Yan-taï, Ki-tsen-sôo, etc. - Fl. et fr.jıillet-aout.

Ar. géog. : Eur. aust. et or. - Afriq. bor. - Asie bor., Astrakan, Wolga, Prov. casp., Kirghis, Caucase, Arménie. - Chine (Kiang-soû, Chan-tong). - Prov. de l'Ussuri. -Japon.

SIDA L.

17. S. tiliæf lia Fish. Cat.hort. Goren. (1818) ex parte; Dec. Prod. 1, 470; Bunge Enum. chin. bor. no 55; Maxim. Prim. flor. 
amur. 62; R. Fortune, 2e voyage horticole en Chine (traduction francaise, dans les Mémoires de la Société centrale d'Agricult. et d'Horticult., $2^{\mathrm{e}}$ partie, 1853); Abutilon Avicenne var. B tiliafolium Regel Flor. ussur.p. 30; sinice Siang-Ma, Tsing-Ma.

«Caule 2-3 pedali, floribus fere $1 / 2$ pollicem in diametro attin》gentibus, carpellis apice birostratis, rostris lanceolato-subulatis, » patentibus. » (Regel.)

Hab. : Bord des champs sablonneux à Yan-taï. Cultivé en grand sur les basses collines micaschist. au-dessus de Yan-taï, Ki-tsensôo, etc., pour les fibres textiles que l'on retire de ses tiges. Fl. et fr. aout.

Ar. géog.: Asie bor., Thibet, Chine (Chan-tong, Pé-tché-ly à Péking'). - Prov. de l'Amour et de l'Ussuri.

Obs. : Le Gossypium indicum Lin. var. herbaceum (G. herbaceum Dec.) est cultivé dans les plaines occidentales du Chan-tong, principalement dans les terres humides qui avoisinent le canal impérial, à Lin-tsin-tchoû, Tong-tchang-foû, Tsin-jin-tchoû, etc.

\section{TILIACÉES Jusş.}

Grewia L.

18. G. parviflora Bunge Enum.plant. Chin. bor. no 57, in Mém. acad. St-Pétesb. sav. étrang. vol. 3, p. 83; Miquel Prol. flor. jap. 206; R. Fortune Plant. chin. exsic. $n^{\circ} 15$.

"Suffrutex 1-1 1/2 met. altus, ramosus, caulibus rugulosis, ju » nioribus ramulisque puberulis; foliis ovatis, acutis, supra pu» bescentibus, subtus plus minusve stellato-tomentosis, canescen» tibus, trinerviis, ad nervos præsertim hirsutioribus, inæqualiter » serratis; floribus terminalibus axillaribusve, umbellatis, um» bella 2-6-flora; sepalis. linearibus trinerviis, dorso albo-tomen» tosis; pelalis albidis; baccis parvis, nigris, glabris, 2-4 lobis."

Hab. : Région moyenne boisée et basses collines micaschist. au bord des ravins, de 200 à 400 mètres d'altitude. - Fl. 12 juillet, fr. 6 septembre 1860 .

Ar. géog. : Chine : Chan-tong au Tché-foû, Pé-tché-ly à Péking. - Archipel de Corée. 


\section{HYPÉRICINÉESS Dec.}

\section{Hypericum L.}

19. H. ascyron Lin. Spec. 1, 102; Dec. Prodr. 1, 545; Pallas, Iter. 2, 559; Ledeb. Flor. ross. 1, 446: Turezan. Filor. Laik. dahur. 1, 150; Maxim. Prim. amù. p. 64: Reg'el T'ent. fl. ussur. p. 32; Franch. et Sav. Enium. jap. 55; Asciron sibiricum Lam. Enc. mílh. 4, p. 147 et ill. lab. 642.

Var. brevistylum Naxim. (loc. cit.) p. 65.

«Ab H.ascyron forma brevistyla differt, sepalis duplo longiori"bus, stylis usque ad basim liberis, orario sextuplo brevioribus, se» minibus minutulis, rufis, subcylindricis, seriatim punctulatis. »

$H a b$. : Région montagneuse moyenne et boisée du Tché-loû, entre 400 et 600 mètres d'altitude; bois de Pinus Massoniana et de Quercus serrata. - Fr. 6 septembre 1860.

Ar. géog. : Asie bor., Sibérie altaïque, baikalienne et australe, Daoûrie. - ('hine: Chan-tong (O. Deb.) et Pé tché-ly (Maxim.).Prov. de l'Amour et de l'Ussuri. - Corée. - Japon.

Obs. : M. Maximowicz signale la présence de l'H. ascyron, à la limite inférieure de la régrion montagneuse de Buraja dans l'Amour, et M. Maak dans les bois de la région mont. du Nippon au Japon.

20. H. perforatum Lin. Spec. 1, 104; Dec. Prod. 1, 539; Ledeb. Flor. ross. 1, 447, et Flor. alt. 3, 364; Karel. et Kiril. Enum pl. alt. p. 61; Zolling. Syst. verz. indish. archip. 1. 151 .

Var. conferliflorum O. Debeaux Mss. et in Herb. (1860).

«Floribus quam in forma typica duplo majoribus densisque, in » corymbum terminalem dispositis. »

$H a b$. : Prairies sablonneuses du littoral, à Yan-taï, près du camp de Tché-foû, etc. - Fl. 15 juillet.

Ar. géog.: Eur. occ. et or.: Russie, Esthonie, Livonie, Lithuanie, Wolhynie, Wolga, Oural, Ukraine, etc. - Afriq. bor. Asie bor., Caucase, Tauride, Prov. caspiennes, Arménie, Sibérie altaïque, Sôongarie, Himalaya, Thibet, Chine au Tché-fou, etc.

Obs.: L'Hypericum perforatum de la Chine semble devoir être distingué au premier coup d'œil de la plante d'Europe, par ses fleurs disposées en un corymbe terminal très-serré, et du double 
plus grandes, par la brièveté des sépales, etc. Cependant on trouve en France et autres contrées de l'Europe des individus analogues quant à la longueur des sépales, et ceux-ci varient beaucoup dans la même inflorescence.

L'habitat de cette plante dans les prairies du Tché-fô̂ constitue un fait intéressant de géographie botanique. L'Hypericum perforatum possède, en effet, une aire d'extension très-développée en Europe et dans l'Asie boréale et occidentale. Cet area s'étend d'Europe en Asie par les régions boréales, s'arrête au Thibet sur les confins de la Chine moyenne, et vient se terminer dans la partie la plus orientale du Chan-tong, après avoir traversé la Sibérie baikalienne, la Sôongarie, etc.

AMPÉLIDÉES• Kunth.

Vitis L.

21.V. (Ampelopsis) serjaniæfolia Bunge Enum. pl. chin. bor. $\mathrm{n}^{\circ} 70$; Walp. Repert. bot. syst. 1, 441; Vilis pentaphylla Thumb. Flor. jap. 105 (ex parte); Miquel Ann. mus. bot. Lugdun.batav. 1, p. 91; Cissus viticifolia Sieb. et Zuccar. Fam. nat.jap. 406, var pinnatifida; Franch. et Sav. Enum. jap. p. $85, \mathrm{n}^{\circ} 350$.

«Foliis palmatis quinque-lobatis, foliolis 2 lateralibus, obovato»trilobatis, 2 mediis ternatis pinnatisve, bi-trijugis, lobis pro» funde inciso-dentatis, utrinque glabris, margine serrulatis sca»bris; pedunculis subdichotomo-corymbosis; capsula biloculari, »4-ovolulata.»

Forma A humilis, caule 22 centim. alto, erecto, simplici, glabro, foliolis late inciso-dentatis.

Forma B elatior, caule flexuoso subscandente, ad apicem ramuloso, 40-50 centim. alto, foliolis triplo brevioribus quam in præcedente, crenulato-dentatis.

Hab. : Rochers micaschist. du littoral. - Pointe du Tché-fou; falaises de Ki-tsen-sôo.

$A r$.géog. : Mongolie australe.-Chine (Chan-tong et Pé-tché-ly). - Japon.

Obs. : Les formes humilis et elatior croissent ensemble dans les mêmes localités. Elles n'ont pas des caractères assez trauchés pour pouvoir être séparées comme espèces distinctes. 
22. V. (Ampelopsis) humulifolia Bunge Enum. pl. Chin. bor. $\mathrm{n}^{\circ}$ 69; Walp. Repert. bot. 1, 444; Cissus humulifolia Regel Tent. fl. ussur. var. typica p. 34. tab. 3.

Forma glabra 0. Debeaux in Herb. "Caulis humilis, glaber, » erectus, subsimplex; foliis late cordatis subtrilobatis, vel palmato" 3-5 fidis, pedunculis folia parum superantibus, dichc tomo-corym" bosis; floribus parvis; baccis globosis, 2-3 spermis, maturitate » atro-cæruleis, apice stylo brevissimo coronatis. »

Hab. : Sommet des plus hautes montagnes du Tché-foû, de 1,000 à 1,100 mètres d'altitude, dans les fissures des rochers. - Fr. 6 septembre 1860 .

Ar. géog. : Mongolie, Chine (Chan-tong et Pé-tché-ly). — Prov. de l'Ussuri.

Obs.: Cette espèce a été signalée dans la Mongolie, comme ayant les feuilles pubescentes ou hispides en dessous, tandis qu'elle a toujours les feuilles glabres dans le Tché-foû. Notre plante a de plus, les lobes des feuilles beaucoup plus larges, et leurs divisions moins profondes que dans les spécimens du nord de la Chine.

\section{GÉRANIACÉES Dec.}

Geranium L.

23. G. nepalense Swet. Geran. tab. 12; Dec. Prodr. 1, p. 643; Miq. Prol.flor. jap. p. 201; Franch. et Savat. Enum. jap. p. 69 ; G. affine Wight et Arn. Prod. 1, p. 133; G. Thumbergii Sieb. et Zuccar. Fam. nat.jap. p. 105; G. palustre Thumb. Flor. jap. 268, non Lin.

Hab. : Région montagneuse moyenne et boisée au-dessus de Yan-taï, de 400 à 800 mètres d'altitude. Bords des ravins près de la vieille pagode, etc.

Ar. géog. : Asie centrale, Népaul, Himalaya, Sikkim. - Chine (Chan-tong). - Japon.

\section{ERODIUM L'Hérit.}

24. E. Stephanianum Wild. Spec. 3, p. 625; Dec. Prod. 1, 645; Ledeb. Flor. ross. 1, 475, et flor. altai. 3, 221; Turczan. Cat. plant. baik. n²83; Bunge Enum. chin. bor. no 72; 
Maxim. Prim. amur. p. 71 ; Geranium multifidum Patrin. Ined. ex Dec.

$H a b$. Sables maritimes et falaises près du cap Chan-tong: sables de la rivière $T a-h \hat{o}$, près de la ville de N'gin-haï-tchéoû. - Fl. et fr. 18 septembre.

Ar.géog. : Sibérie altaïque et baikalienne, Daoûrie, désert de Kirghiz, Mongolie, Chine (Chan-tong et Pé-tché-ly). - Prov. de l'Amour.

\section{ZYGOPHYLLEES R. Brown.}

Tribulus Tournef.

25. T. terrestris Lin. Spec. 544; Pallas Iter., 1, p. 372; Ledeb. Flor. ross. 1, p. 486; Turczan. Cat. Baik. n ${ }^{0} 286$; Bunge Enum. chin. bor. n०76; Franch. et Savat. Enum.jap. n² 278; Miquel Prol. jap. p. 256; sinice KI-LY, KıÉ-LY-TZÉ.

Var. subinermis; forma hirsutior, carpellis subinermibus, tuberculatis.

Hab. : Sables maritimes du Tché-foû, et champs autour de Yantaï. - Fl. et fr. juillet à août.

Ar.géog. : Eur. aust. - Afriq. bor., Sénégal, Cap-Vert. - Asie bor. cent. et or.: Wolga, Désert caspique, pays des Kalmouks et des Cosaques, Taurile, Caucase, Arménie, Sibérie ouralienne, altaïque et transbaikalienne, Thibet, Mongolie. - Chine (Chantongr, Hônan, Pé-tché-ly). - Japon. - Iles de l'Océan indien, Manrice, etc.

Obs. : La plante des sables du Tché-foû est velue, soyeuse dans toutes ses parties; les carpelles sont également hérissés de poils courts et soyeur. Cette forme, qui est décrite dans le Prodromus sous le nom de subinermis, serait originaire du Thibet.

\section{CÉLASTRINÉES Bartl.}

Evonymus L.

26. E. verrucosus Scop. Fl. carn. $\left(E d .2^{\mathrm{e}}\right)$ p. 268 ex parte; Ruprecht in Plant. Maack. p. 538; E. pauciflorus Maxim. Prim. A. amur. p. 516; Regel Tent. fl. ussur. p. 41; sinice LÔU-TCHON.

Var. Tchefouensis O. Debeaux, Msc. et in Herb. 1860. 
"Frutex 4-5 pedalis, glaber, parce ramosus, ramis teretibus, » cinerascentibus, subverrucosis, glabris; foliis ellipticis-oblongis » vel subovato-oblongis, atrinque glabris, in petiolum breve atte»nuatis, apice obtusis, crassiusculis, coriaceis, serrulato-crenatis, » serraturis minutis; pedunculis ebracteatis, 3-10-floris, dichotomis, 》 ex axillis foliorum ramulorumque inferiorum nascentibus, folia 》s sepe superantibus; pedicellis lateralibus dichotomo-divaricatis »-centrali duplo longioribus; capsulis quadrilobis, obtusis, brevi"bus, lævibus, apteris; arillo miniato, exsiccatione rugoso»decolori. »

Hab. : Lieux incultes et sablonneux autour des tumulus; plaine de Yan-taï et de Ki-tsen-sôo. - Fr. 26 septembre 1860.

$A r . g \dot{o} o g .:$ Le type (E. verrucosus) : Eur. or., Autriche, Pannonie, Carniole, Lithuanie, Livonie, Kasan. - Asie bor., occ. et or., Tauride, Caucase, Arménie, Sibérie de l'Oural. - Prov. de l'Amour et de l'Ussuri. - La variété : le Tché-foû dans le Chan-tong.

Obs. : La variété Tchefouensis diffère de l'E. verrucosus (forma Amurensis), par sa taille plus élevée, ses rarneaux d'une couleur grise cendrée, par ses feuilles beaucoup plus larges, glabres et non pourvues de poils rares et courts en dessus et beaucoup plus longs en dessous, par l'absence des bractées, celles-ci étant caduques avant l'anthèse dans la plante du Tché-foû, par la présence d'un pédicelle central dans la panicule florale qui est composée de 7 à 10 fleurs, etc.

M. Franchet, qui avait d'abord rapporté cette espèce à l' $E$. Bungeanus Maxim., est revenu sur cette détermination, parce que, dans notre plante, les rameaux ne sont pas comprimés, et que les fleurs sont toutes disposées en panicules divariquées, ce qui n'a pas lieu dans I' $E$. Bungeanus.

Les descriptions de l'E. verrucosus de Ledebour et de M. Maximowicz conviennent en grande partie aux spécimens récoltés au Tché-foû. Ceux ci ont, en effet, les tiges à épiderme entièrement recouvert de glandes verruqueuses de couleur cendrée, les feuilles glabres, coriaces, finement crénelées et dentées au sommet, une inflorescence divariquée, et les pédoncules 3-10 flores; mais à cause des différences que j'ai déjà signalées, je n'hésite pas à regarder cette plante comme une variété remarquable et tout à fait locale de l'E. vern'ucosus. 
RHAMNÉES JusS.

Zizyphus Tourn.

27. Z. vulgaris Lam. Dict 3, 318; Ledeb. Fl. ross. 1, 501; Bunge Enum. chin. bor. n 44 ; Franch. et Sav. Enum. jap. n $35 \%$; Miquel Prol.jap. 318; Maxim. Rhrimn. asiat. orienl. n" 3.

Var. A. spinosus Bunge (loc. cit.), n' 81 ; O. Debeaux Flor. Shanghä̈, $11^{\circ} 29$; sinice Kin-Kouan-TzÉ, TsaÔ-TZÉ.

Hab. : La variété A spinosus se rencontre partout au Tché-foû, dans les lienx incultes, les haies, les clôtures de jardins des environs de Yan-taï et de Ki-tsen-sôo, etc. - La var. B inermis n'est point cultivée dans cette partie du Chan-tong. - Fl. 10 juillet, fr. octobre.

Ar. góog. : Asie temp., Asie min., Syrie, Perse, Prov. casp., Indes-Orient. - Cochinchine, Chine (Kiang-soû, Tché-kiang, Chan-tong, Pé-tché-ly), etc. - Mongolie. - Japon. - Importé probablement de l'Asie dans l'A frique boréale.

Obs. : Dans la var. spinosus, les drupes sont oblongues, petites, et d'une saveur acide.

\section{RHAMNUS L.}

28. Rh. virgatus Roxbg Flor. ind. 2, p. 551 ; Benth. Flor. Hongkong. 68; Rh. globosus Bunge Enum. chin. bor. p. 14; Maxim. Rhamn. asiat. orient. $\mathrm{n}^{\circ} 13 ;$ Rh. parvifolius Turczan. Enum. chin. bor. non Bunge; Rh. polymorphus Turcz. Flor. Baik. dahur. 1, p. 269; Rh. dahuricus Regel Tent. fl. ussur. p. 41.

Var. apricus Maxim. (loc. cit.), tab. 1, fig. 24; Rh. chlorophorus Dene. in Comptes-rendus de l'A cadémie des Sciences, vol. XLIV(1857) ex parte; O. Debeaux Flor. Shang-haï, n ${ }^{\circ} 30$; sinice ToN-Loû-chou.

' Forma Tchefouensis fruticosa, interdum nana, squarrosa, foli is » minoribus minutisve, ellipticis vel ovatis, utrinque acuminatis 》vel apice subito cuspidatis, serrulatis; floribus parcioribus, la» ciniis calicinis interdum acutis. »

Hab.: Lieux incultes, pierreux et micaschisteux des basses. collines du Tché-foû. Collines de Yan-taï et de Ki-tsen-sôo (200 à 250 mètr. d'alt.) - Fr. 21 septembre 1868.

Ar.géog. : Asie cent. et temp., Indes-Orientales. - Chine bor., 
cent. et moy., Hong-kong, Tché-kiang, Kiang-soa, Chan-tong, Pé-tché-ly, îles Lôo-chôo. - Mandchourie, Daoûrie. - Prov. de l'Amour et de l'Ussuri.

Obs. : Le Rhamnus virgatus var. apricus des collines du Tchéfoû se rapporte exactement à la description du $R h$. globosus Bunge, et dont l'auteur soupçonnait déjà l'identité avec le $R h$. virgatus de Roxbg. par cette remarque "an Rh.virgatus Roxbg.?» (Bunge loc.cit.). Notre plante ne diffère de celle de Shang-haï, que par son aspect plus rabougri et la forme en général plus petite de toutes ses parties, par ses rameaux moins longuement spinescents au sommet, et par ses feuilles pubescentes en dessous ainsi que les pétioles.

M. Maximowicz fait observer que cette espèce est très-polymorphe dans la même localité. Ainsi, dans les bois de la Mandchourie, ce botaniste a vu le Rh.virgatus atteignant 15 pieds de hauteur, tandis que dans les lieux rocailleux de la Daoûrie, la même espèce ne dépasse pas un mètre. La largeur des feuilles, la spinescence et la pubescence des rameaux sont également très-variables. Mais, malgré ces différences qui ne sont dues qu'à l'influence du sol et du climat, M. Maximowicz ne pense pas pouvoir séparer l'espèce chinoise de la plante des Indes-Orientales. Il ne distingue seulement que les deux variétés A sylvestris, des bois de la Mandchourie, que j'ai retrouvée sur les pentes du Pic Victoria à Hong-kong, et la var. B apricus des lieux rocailleux ou incultes du centre et du nord de la Chine.

\section{LÉGUMINEUSES JusS.}

SOPHORA L.

29. S. galegoïdes Pallas, Astrag.p. 118, tab. 88; S. favescens Ait. Hort. Kew. 2, 43; S. flavescens var. galegoïdes Dec. in Prodr. 2, p. 96; Ledeb. Fl. ross. 1, 716; Bunge Enum. chin.bor. $\mathrm{n}^{\circ} 89$ ex parte.

"Suffrutex valde insignis, perennis, 3-4 pedalis, basi pluricaulis, » caulibus erectis, simplicibus, tenuiter striatis, hirsuto-incanes»centibus; foliis imparipinnatis, 8-12 jugis, foliolis ovato-oblongis »basi truncatis, apice obcordatis, breve petiolulatis, margine » sinuato-dentatis, supra subglabris, subtus setoso-pilosis, pilis 
》adpressis densis, in sicco albescentibus; stipulis integris lineari» bus, villosis; racemo florifero terminali, floribus lilacinis, dense » spicatis, spicis 10-15 centim. longis, folia duplo superantibus; » calice corolla duplo breviore, bilabiato, obsolete 5 -dentato, »hispido-setoso, vexillo carina alisque paulo longiore, reflexo»unguiculato, apice elliptico-ovato; alis carinam superantibus, »anguste unguiculatis; carina in flore aperto subdeflexa, » biphylla, phyllis subconformibus.

»Capitulis fructiferis 2 decim. usque longis, junioribus erectis, » demum horizontaliter in petiolo communi incidentibus, puberu» lis, 4-angulatis, apteris, moniliformibus, compressis, in mucronem » bisulcatum, 1-3 cent. longum subito attenuatis; seminibus 2.4 " ovato-rotundatis, brunneo-fulvis, glabris, tenuiter punctulatis, » hilo 2 millim. longo, concolore, nigricante. »

Hab. : Lieux incultes et sablcnneux du littoral. - Trèsabondant sur les sables de la baie de Ki-tsen-sôo, autour des tumulus.-Fl. 15 juillet, fr. 14 septembre.

Ar. géog. : Asie bor., Daoûrie orient. à Nertschingsk et près du fleuve Arcrun. - Chine (Chan-tong et Pé-tché-ly). - Nongolie. Prov. de l'A mour et de l'Ussuri.

Obs.: Cette rare espèce, qui est surtout remarquable par la beauté de son épi floral, est pour ainsi dire caractéristique avec le genre Lespedeza, de la végétation litturale du Tché-foû. Elle se distingue du $S$. flarescens, auquel Decandolle et Ledebour l'ont réunie, par ses fleurs disposées en un long épi terminal, et toujours de couleur rose-lilas, par la villosité de toutes ses parties, par la forme singulière des légumes qui sont dans notre plante, quadrangulaires, comprimés sur les faces, aptères, irrégulièrement moniliformes, et longuement acuminés.

Le Sophora angustifolia Sieb. et Zucc. (Abhand. Bayer Acad. Ph. class. IV , 2, p. 118) paraît n'être qu'une variété à folioles trèsétroites du $S$.flavescens. Je considère cette variété comme une forme du $S$. flavescens plante spéciale au Japon, tandis que le $S . g a-$ legoïdes serait une autre forme à fleur's rosées de cette même espèce, mais particulièrè aux sables maritimes du Tché-foû. Le S.galegoüdes Pall. constitue une plante ornementale du plus gracieux effet, et mérite de fixer l'attention des horticulteurs. 


\section{CROTALARIA L.}

30. G. brevipes Champ. in Kew. journ. botan. IV, p. 44; Benth. Fl. Hong-kong. 74; Franch. et Savat. Enum pl. jap. 24; C. Oldhami Miquel Prol. fl.jap. $\mathrm{n}^{\circ} 231$.

Hab. : Sables maritimes de la grande baie de Ki-tsen-sôo. - Fl. juillet, fr. septembre.

Ar. géog.: Chine littorale à Hong-kong, Tché-foû, etc. - Japon.

Obs.: Le genre Crotalaria, dont on cunnaît un grand nombre d'espèces répandues dans les régions intertropicales des deux mondes, est représenté dans lîle de Hony-kong par cinq espèces, dont l'une, le $C$. brevipes, s'étend jusque sur les rivages du Chan-tong, au nord de la Chine, et de l'île Kiû-siû au Japon.

Le $C$. brevipes est une petite plante annuelle, à fleurs d'un beau bleu azuré. Ses divisions calicinales recouvertes extérieurement de poils longs, soyeux, serrés et de couleur roussâtre, ses feuilles longuement linéaires-larcéolées sembleraient le rapprocher, pour M. Bentham, du C. sessiliflora, espèce propre à l'Asie intertropicale et retrouvée à Can-ton. Mais notre plante s'en éloigne par sa taille plus courte, par ses feuilles étalées, plus petites et plus larges à la fois, marquées de points transparents, par ses fleurs sessiles, plus longues que les bractées, par les lobes du calice dépassant à peine l'étendard, par son légume noirâtre, oblong, glabre, et ses graines réniformes de couleur jaupe-pâle. Le $C$. brevipes ne varie nullement au Tché-foû. C'est une espèce parfaitement spontanée dans cette contrée, et non une forme accidentelle du $C$. sessiliflora.

\section{MELILOTUS L.}

31. M. graveolens Bunge Enum.plant. chin.bor. $\mathrm{n}^{\circ}$ 94, p. 16, in Mém. sav. étr. acad. St.-Petesb. II, p. 90.

"Planta herbacea, annua aut bisannua, 3-4 pedalis, caulibus » erectis, gläbris, ramosis, ramis longissimis, tenuiter striatis; "foliolis cuneato-oblongis, irregulariter arguteque serrato-denta" tis, apice truncatis, mucronulatis, mucrone 3-4 millim. longo, » erecto vel recurvato; floribus parvis, dense spicatis, flavis, cali》cis dentibus inæqualibus, tubo æquilongis, pedicellis recurvatis, » calice longioribus; stipulis integris, linearibus, longe setaceis; 
》leguminibus ovatis, 1-2 spermis, glabris, lacunoso-rugosis, nigri» cantibus, stylo 5-6 millim. longo, recurvato, apice coronatis. »Semina matura non vidi.»

Hab. : Prairies sablonneuses et marécageuses près de Yan-taï. - Fl. et fr. 4 septembre.

Ar. géog. : Chine (Chan-tong au Tché-foû, et Pé-tché-ly à Péking).

Obs. : Le M.graveolens, dont son auteur (loc. cit.) n'a donné qu'une description écourtée, paraît se rapprocher du $M$. suaveolens Ledeb. Index sem. hort. Dorpat.p. 5, 1824, et Flor. ross. 1, p. 536, avec lequel il est facile de le confondre. Il se distingue facilement de celui-ci, par ses rameaux plus allongés, par ses pédicelles recourbés, plus longs que le calice ou l'égalant à peine, par son étendard égalanı ou dépassant les ailes et non plus court que celles-ci, par ses feuilles mucronées à leur extrémité, etc. Cette espèce paraît être spéciale au nord de la Chine. Le $M$. suaveolens a une aire d'extension beaucoup plus développée, depuis la Sibérie altaïque et la Transbaikalie jusqu'aux provinces de l'Amour, à travers la Daoûrie, la Mongolie et l'extrême-nord du Pé-tché-ly.

\section{INDIGOFERA L.}

32. I. macrostachya Bunge Enum. chin. bor. no 95, p. $16 \mathrm{du}$ tirage à part; Ventenat Jardin de la Malmaison tab. 41; Dec. Prodr. 2, p. 226.

"Suffruticosus, caulibus erectis, 30-35 centim. altis, parce » ramosis, ramis gracilibus, caule adpressis, pubescentibus; " foliis 4-6 jugis, foliolis ovato-oblongis, obtusis, longe mucronu$\checkmark$ latis, supra pubescentibus, subtus venoso-reticulatis, racemis 》floriferis axillaribus, 8-10 floris, folio longioribus. Flores lilacini, »6-plo calicem superantes. Legumina non vidi. »

Hab. : Région montagneuse sup. (800 à 1,000 mètres d'alt.), dans les fissures des rochers micaschisteux. - Fl. 16 juillet.

J'ai trouvé une haie composée d'arbustes de cette espèce et d'origine cultivée, dans la région montagneuse inférieure, à 300 mèt. d'alt. près de la pagode Neuve.

Ar. géog.: Chine boréale: T'ché-foû (0. Debeaux), Pé-king (Bunge). 
GULDENS'TADTIA Fisher.

33. G. stenophylla Bunge Enum. chin. bor. $\mathrm{n}^{\circ} 107$, in Mém. sav. étrang. acad. St-Petest. 11, p. 92; Turczanin. Flor. Baik. dahur. 1, p. 333; Walp. Rep. bot. syst. 1, 684.

"Planta perennis, humilis, acaulis, villoso-canescens, radice » longa, extus fusco-brunnea, intus fibrosa, albida, digiti parvi » crassitie, foliis impari-pinnatis, vel lineari-oblongis, obtusis, " petiolisque utrinque canescentibus; inflorescentia subumbellata, "floribus parvis, subsessilibus, ad apicem pedunculi communis » valdeque elongati confertis. Legumina subcylindrica, unilocu"laria, seminibus reniformibus, parvis, scrobiculatisque.»

Hab. : Sables humides aux bords des ravins et des torrents (à sec pendant l'été) qui se dirigent vers la mer, à Ki-tsen-sôo. — Fl. et fr. 23 aout.

Ar. géog. : Chine bor. (Chan-tong au Tché-fou, Pé-tché-ly à Péking).

Obs. : Le genre Guldenstadtia a été créé par Fisher (Mém. soc. nat. Mosc. IV, p. 170) pour deux plantes, les $G$. monophylla et $G$. pauciflora, voisines du genre Astragalus, et qui croissent dans les régions montagneuses de la Sibérie altaïque, la Baïkalie, la Daoûrie, etc. Bunge (loc. cit.) en décrit deux autres espèces, les G. multiflora et notre $G$. stenoplcylla trouvées par lui aux environs de Pé-king. Enfin une cinquième espèce, le G. cuneata Royle (Ill. Himalay. plant. tab. 200), complète la monographie de ce genre si intéressant.

\section{LESPEDEZA Michaux.}

34. L. bicolor Turczan. in Bull. soc. nat. Mosc. 14, p. 60 (1840); Ledeb. Flor. ross. 1, 715; Maxim. Prim. A. amur. p. 86 et Syn. gener. Lespedezce p. 30 var. A typica; Regel Tent. $f$. ussur. 48; Franch. et Sav. Enum.jap. no424, p. 101; Miquel Prol.fl.jap. p. 235.

Hab.: Région montagneuse sup. du Tché-foû, de 1,000 à 1,100 mètres d'alt. dans les fissures des rochers les plus escarpés. - Fl. 6 septembre.

Ar.géog. : Chine bor. (Chan-tong, Pé-tché-ly). - Prov. de l'A mour et de l'Ussuri. - Japon.

Obs. : Petit sous-abrisseau de 30 à 50 centim. de hauteur, peu 
rameux ou seulement au sommet, à feuilles presque orbiculaires mucronulées. d'un vert-foncé en dessus, blanches-pubescentes en dessous, et à fleurs subsolitaires d'un violet pourpré. Identique avec les spécimens récoltés au Japon par M. Savatier.

35. L. tomentosa Sieb. in Schad.; Maxim. Syn.gen.Lesped. 30; Franch. et Savat. Enum.pil.jap. no 434; L. villosa Pers Syn. plant. 2, p. 318 ex parte; Desmodium tomentosum Dec. Prodr. 2, p. 337; Hedysarum tomenlosum Thumbg. Fl. jap. p. 286; Lespedeza hirta Miquel Prol. fl.jap. 357, non Lin. sub Hedysaro.

«Suffrutex perennis, 3-4 pedalis, caulibus erectis, hirsutis, to》mentosis, ad apices ramorum villosiusculis, parce ramosis, ramis 》 erectis, adpressis; foliis trifoliatis, coriaceis, ellipticis, folio medio » ovato-obtuso, mucronulato, alteris minoribus, suboralibus, supra 》glabris, subtus incanescentibus reticulato-venosis, ad nervos »hirsutioribus; inflorescentia longe spicata axillarisque, spicis "subpaniculatis, gracilibus, 5-plo folia superantibns. Flores fer»tiles semper apetales, in axillis inferioribus foliorum sessiles, et " quasi verticillum squamatum formantes; flores steriles, albidi, » vexillo alisque roseo-striatis, calice triplo longiores, calicinis 》 segmentis hirsutis, lineari-lanceolatis acuminastisve, legumine » longioribus. Legumen minutum, 2-3 millim. longum, ovatum, " margine compressum, villosiusculum, monospermum, semini»bus minutulis, 1 i/2 millim. latis, subovatis, fusco-brunneis, »versus hilum apice parum recurvatis.»

Hab.: Basses collines et sables du littoral à Yan-taï, Ki-tsen-sôo, etc. - Fl. 1-15 juillet, fr. 15 octobre.

Ar. géog. : Mandchourie, Corée. - Chine littor. au Tché-foû. Japon austral et moyen.

Obs.: Dans son Synopsis du genre Lespedeza, M. Maximowicz adopte pour cette espèce le nom déjà donné par Siebold dans ses exsiccata du Japon, réșervant celui de Linné (L. hirta sub Hedysaro) qui est le plus ancien, à la plante de l'Amérique boréale. MM. Franchet et Savatier (Enum. plant. jap.), à l'exemple de Miquel, n'adoptent pas cette manière de voir; ne trouvant aucune différence qui puisse légitimer la séparation de ces deux espèces. Aussi les réunissent-ils sous le nom le plus ancien de $L$. villosa Pers. in Enchir. botan. (1805). 
Je trouve cependant, une différence très-importante entre la plante chinoise et celle de l'Amérique boréale telle qu'elle est décrite par Decandolle (Prodr. 2, p. 359). C'est dans la disposition remarquable des fleurs (infertiles) en un long épi floral de 10-15 centimètres, et des fleurs apétales fertiles placées à l'aisselle des feuilles et quiforment, après la disparition des fleurs corollées, des verticilles superposés entourant presqu'entièrement la tige à la maturité. Ce sont précisément ces fleurs qui paraissent avortées, et qui forment un faisceau écailleux à l'aisselle des feuilles (Persoon), qui sont les fleurs fructifères du L. tomentosa. On aurait donc, dans le genre Lespedeza, des espèces hèrmaphrodiles, et d'autres qui paraissent monö̈ques par suite de l'avortement des fleurs axillaires, et de la stérilité-de celles des rameaux supérieurs.

M. Maximowicz a également observé cette particularité singulière de la présence des fleurs apétales fertiles du L. tomentosa placées en dessous des fleurs régulières et stériles. Il a ret ouvé ce caractère sur 17 espèces de Lespedeza, de la section Eulespedeza. Aussi. ce savant monographe a-t-il jugé né essaire de baser la diagnose de ce genre sur de nouveaux caractères. Celui dont nous parlons, est ainsi décrit par l'auteur :

Genus Lespedeza: "Flores apetali sub anthesi, interdum non »facile inventi, statu fructifero statim cognoscuntur vestigio » columnæ subnullo et stylo legıminis sæpissime hamato. » Occurrunt vulgo a racemorum inferiorum parte infima, vel in » axillis foliorum in racemis abbreviatis vel fasciculos collecti, "sed non in quovis individno. et autumno semper frequentiores, » ubi inveniuntur plantæ suos flores gerentes. »

36. I. trichocarpa Pers. Syn.pl. 2, p. 318; Ledeb. Flor. ross. 1, 318; Turcz. Cat. Baik. no 371; Dec. Prodr. 2, p. 349; Hedysarum trichotomum Wild. Spec. 3, p. 1194; Trifotium dahuricum Pallas Iter. 3, p. 321.

Forma A genuina: «Caulibus erectis, foliis breviter petiolatis, »foliolis 35 millim. longis, 12 millim. latis, ellipticis mucrunatis 》subtus adpresse pilosis supra, demum glabriusculis, reticulato»venosis, venis utrinque eximie conspicuis; racemis floriferis sub» umbellatis axillaribusque, subsessilibus; calice corolla bre^ viore; leguminibus ellipticis, orbiculatis, parum puberulis. »

Forma B ramosa : "Cautibus villoso-tomentosis, erectis, e basi 
"laxe ramosis; ramis late divaricatis; foliis quam in præcedenti »2-3-plo latioribus; floribus apice ramorum spicatim confertis. »

$H a b$. : Rochers micaschisteux du littoral; falaises de la pointe de Tché-foû, de Ki-tsen-sôo, etc. - Fl. 10-30 juillet.

Ar. géog. : Sibérie, Transbaikalie, Daoûrie, Mandəhourie, Mongolie. - Chine (Chan-tong et Pé-tché-ly à Pé-king).

37. L. floribunda Bunge Decas prima plant. nov. Mongholicochinensium, in Ann. scienc. natur. vol. 6, p. 59 (1835).

"Fruticosa, multicaulis, cautibus basi diffusis prostratisve, » parce ramosis; petiolis subelongatis; foliolis oblongis angustis, » retusis, mucronatis, supra tenuissime puberulis, subtus sericeo" strigulosis; racemis folium superantibus, 6-8 floris ; calicis laci» niis sericeis, lanceolatis, corolla dimidio brevioribus; leguminibus 》 ovatis, acutis, sericeis, calice longioribus. 》

Hab. : Sables maritimes et dunes sablonneuses près de Fouchan-yên, à 10 kilom. N. de Yan-taï.

Ar. géog. : Chine (Chan-tong et Pé-tché-ly).

Ols. : Cette espèce se distingue facilement du L. trichocarpa, par ses tiges nombreuses, couchées à la base et diffuses, par ses rameaux courts, par ses feuilles $2-3$ fois plus petites $(5$ millim. long. 3 millim. larg.), étroites, à pubescence plus courte, et à réseau veineux peu apparent, par ses rameaux florifères dépassant à peine les feuilles, par ses légumes ovales-aigus, un peu plus longs que le calice lequel n'excède pas la moitié de la corolle.

38. L. juncea Pers. Syn.plant. 2, 318; Dec. Prodr. 2, p. 348; Led. Fl. ross. 1, 714; Turczan. Flor. Baik. dahur. 1, 340; Bunge Decas prima plant. Monghol. chin. (loc. cit.) p. 10; Maxim. Prim. fl. amur. 86; Regel Tent. ussur. 49; Franch. et Savat. Enum.jap.432; L. argyraa Sieb. et Zucc. Abh.IV, 2, p. 120; L. sericea .IIquel Prol.jap.237 non Roxbg.; Hedy sarum sericeum Thumbg. Fl.jap. 287.

Hab. : Prairies sablonneuses et grandes dunes de Fou-chan-yên (Golfe de Pé-tché-ly). — Fl. 4 septembre.

Ar. géog.: Asie bor., Sibérie, Transbaikalie, Daourie, Mandchourie, Corée, Prov. de l'Amour et de l'Ussuri. - Chine (Chantong, Pé-tché-ly). - Indes-Orientales, Himalaya. - Japon. Iles de l'Océan indien et austral, Maurice, Java, Nouvelle-Hollande. 
39. L. striata Hook. et Arn. in Bot. voy. Beckey, 262; Benth. Flor. Hong-kong, 85; Miquel Prol. jap. 237; Franch. et Savat. Enum. jap. no 431, forma stipulacea; $L$. stipularea Maxim., Prim. A. amur. 85; Hedysarum striatum Thumbg. Fl. jup. 289.

Var. stipulacea Maxim.

«Herba annua, gracilis, caulibus parce ramo:is, ramis diffusis "prostratisve; foliis breviter petiolatis, petiolis alpresse pilosis, " foliolis obovatis, retusis, mucronatis, margine preterque co.tum 》 mediam dense ciliato-pilosis; stipuits petiolum æquantibus vel » superantibus, acutis, erectis, adpressis, fuscis, membranaceis, 》parallele venosis; floribus breve pelunculati., subsolitariis, "axillaribus; calicis laciniis obtusis; leguminilus sessilibus, "rotunlato-ellipticis, obtusis, tenuite: reticulatis, puberulis, »calice duplo longioribus. Semina conformia, levia, opaci, atro»brunnea."

Hab. : Sables maritimes de Ki-tsen-sôo: grantes dunes de Fouchan-yên. - Fl. 10 août. fr. 4 septembre.

Ar. géog : Asie littorale bor. et moy., Hanlchomrie, Corée. Sacchalien. - Chine (Chan-tongr, Pé-tché-ly, Hotıg-konğ). Japun, îles Bonin-Sima, etc.

Ols. : M. Maximowicz a décrit cette plante comıne es; ’̀̀ce nouvelle dans son Primitia.flora amurensis, sous le nom de $L$. stipulacei. L'éminent botaniste de Saint-Pétersbour'gr est revenu depuis sur cette opinion, dans sa monographie du genre Lespedeza, et il ne regarde plus aujourd'hui son ancien $L$. stipulacea, que comme une forme à fruits plus allongés du L. striata, auquel notre plante se rapporte en tout point.

\section{VICIA L.}

40. V. (Cracca) rapunculus O. Debeảux, Mss., et in Herb. (1860). "Herbacea, perennis, radice fusiformi ut in campanula rapun«culo, crassa, 36 centim. longa, extus sulcato-suberosa, ad collum 》2.3 stolones longissimos interdum radicantes emittente; caulibus " striatis subtetragonis, simplicibus, 50-75 centim. altis, erectis, in 》 axillis foliorum flexuoso-recurvatis, pilis albidis rarisque indu" tis ; foliis 45 jugis, apice cirrhosis, foliolis distantibus plerumque »alternis, mucronulatis, medianis obovatis, apice retusis vel 
»obcordatis, inferioribus ellipticis-lanceolatis, margine ciliatis, » utrinque pilis albidis adpressisque prosertim ad nervos obsitis; » stipulis integris, semisagittatis, pedunculis multifloris, foliolon» gioribus; vexillo carina alisque sepala triplo superantibus; calicis » laciniis acutis, subulatis; peliolis hirsuto-pilosis. Flores amæne " purpurei. »

Hab. : Ravins micaschisteux de la pointedu Tché-fou, au-dessous du petit fort chinois. - Fl. $1^{\text {er }}$ septembre.

Ar. géog.: Chine : le Chan-tong.

Obs. : Cette espèce nouvelle du groupe du $V$. cracca, se distingue au premier abord de ses congénères, par ses racines charnues, longues, fusiformes et pivotantes, émettant au collet des rejets stolonifères, d'où naissent ça et là de nouvelles racines semblables à celle de la tige mère. Nutre Vicia rapunculus paraît se rapprocher du $V$. japonica A. Gray, et $V$. amana Fisher, à côté desquels il convient de le placer. Il rappelle tout à fait par son port le $V$. japonica A. Gray ( $V$. pallida Turcz. non Hook.), mais il en diffère:

1. Par la nervation des feuilles. Dans le $V$.japonica, les nervures latérales se ramifient, et forment un réseau très-apparent dans lequel les nervilles sont presqu'aussi saillantes que les nervures principales. Chez le $V$.rapunculus, les nervures seules sont apparentes et leurs ramifications sont noyées, pour ainsi dire, dans le parenchyme.

$2^{\circ}$ Par la forme du calice, dont les dents sont plus allongées, et la division inférieure au moins aussi longue que le tube.

$3^{\circ}$ Par l'absence des bractéoles à la base des pédicelles, tandis que dans le $V$.japonica les bractéoles sont sétacées, et à peu près aussi longues que les pédicelles.

On distinguera aussi notre espèce du $V$. amana Fish. (in Dec. Prod. 2, p. 355), par ses racines napiformes, à rejets stolonifères émettant à des distances assez rapprochées, des racines d'abord aériennes et qui s'implantent ensuite dans le sol, par ses fleurs de beaucoup plus petites, par ses grappes moins fournies, ses folioles moins nombreuses, ses stipules entières et non dentées, par la villosite de toutes ses parties, son port, etc. Le $V$. rapunculus a des tig'es qui s'élèvent en zig-zag, et changent de direction, d'une aisselle de feuille à l'autre. Mais le caractère sur lequel j'insiste le plus, est celui que nous fournit la racine de cette curieuse espèce, dont les stolons, non florifères la première année, émettent des 
racines adventives napiformes, et dont aucun auteur n'a signalé encore la présence dans le genre Vicia.

41. V. unijuga Al. Braun Ind. semin. hort. Berol.p. 12 (1853); Orobus lathyrö̈des Lin. Spec. 1027; Dec. Prodr. 2, p. 377; Ledeb. Flor. ross. 1, p. 688; Maxim Prim. f. amur. 81; Turczan. Flor. Baik. dahur, 1, 351; Regrel Tent. ussur. 48.

Hab. : Rochers micaschisteux et falaises du cap de 'Tchéfoû; collines sablonneuses au-dessus de Yan-tiï. - Fl. 12 juillet, fr. 7 aoat.

Ar. géog. : Asie bor., Sibérie, Altaï, Transbaikalie, Daoûrie, Mongolie, Prov. de l'Ussuri. - Chine (Chan tong et Pé-tché ly).

Obs.: La Vicia unijuga A. Braun est l'ancien Orobus lathyrö̈des de Linné; son style velu, et l'æstivation condupliq'ée des folioles constituent un véritable Vicia. Cette espèce est très abondante au Tché-foû, et recouvre de ses fvilioles larges et luisantes, et de ses grappes de fleurs violacées, toutes les falaises des abords de Yan-taï.

\section{LATHYRUS I.}

42. L. palustris Lin. Spec. 1034; Ledeb. Flor. ross. 1, 686; Turcz. Flor. Baik. dahur. 349; Maxim. Frim. amur. 83; Regel Tent. ussur. 47; Niquel Prol. A.jup. 233 : Franch. et Savat. Enum.jap. n 444 ; L. viciaformis Rupr. Flor. ingr. 1, 285.

Forma genuina, omnin" glaberrima.

Hab.: Prairies humides dans la plaine de Ki-tsen-sôo. - Fl. 8 septembre.

Ar.géog. : Eur. bor., cent. et aust. - Asie bur., Sibíri altaïque et ouralienne, Baikalie, Davûrie, Kamstchatka.- Prov. de l'Amo ır et de l'Ussuri. - Chine (Chan-tong' et Pé-tché-ly). - Japon. Amér. bor. (Hook).

\section{CASSIA L.}

43. G. (Chamacrista) mimosoïdes Lin. Spec. 543; Dec. Prod. 2, 543; Benth. Fl. Hong-kong, 98; Miquel Prol.jap. 242, et Flor. ind. Batav. 1, 101; Franch. et Sav. Enum.jap n 472 ; C. Thumbergiana Blume Mss.; C. procumbens Thumbg. Flor. jap. 179 (ex-Miquel).

Forma A puberula Miquel in Herb. mus. Lugd. Batav. et in Des- 
cript.prol.fl.jap. «Annua, humilis, dense puberula, e basi ramosa, » caulibus prostratis, ramulisque diffusis; foliis 10-12 jugis, folio» lis minutis, linearibus, mucronulatis; pedicellis axillaribus » uni-bifloris, folio brevioribus; foliis sepalis leguminibusque » pubescentibus, caulibus pilosis, pilis flavidulis. »

Forma B elatior O. Debeaux in Herb. 1860. "Tota puberula, » caulibus erectis, 60 cent. et ultra altis, in parte superiore ramu» losis, ramis erectis adpressisque; foliis 20-30 jugis, petiolo com»muni recurvato-falcato, foliolis anguste lineari-lanceolatis, » mucronatisque. »

Hab. : La var. A puberulu, sur les sables les plus arides du littoral à Ki-tsen-sso; - la var. B elatior dans les pelouses des basses collines au-dessus de Yan-taï, de 200 à 300 mètres d'altitude. Fl. 12 juillet, fr. 24 août.

Ar.géog. : Afrique intertrop. - Asie intertrop., Indes-Orient., Ceylan; - Iles de la Sonde, Java. - Chine bor. et aust., Hongkong, Chan-tong, etc. - Japon.

\section{ALBIZZIA Durazzo.}

14. A. julibrissin Boiv. Encycl. du $19^{\mathrm{e}}$ siècle, 2, p. 32; Niquel Prol.jap. 243; Franch. et Sar. Enum jap. n" 478; Acacia julibrissin Wild. Spec. 4, 1065; Ledeb. lilor.ross, 1, 724; A. nemu Bunge Enum. pl. chin. bor. no 121 ; Mimosa arborea Thumbg. Fior. jap. 229; sinice Ho-koûAN.

Hab.: Basses collines micaschisteuses an-dessus de Yan-taï, autour des Pagodes et des vieux temples bouddhiques. - Fl. 11 juillet.

Ar.géog. : Asie intertrop. et temp., Asie min., Arménie, Perse, Inde:-Or., Népaul, etc. - Chine (Kiang-soû, Chan-tong, Pé-tchẻ-ly). - Japon.

Obs.: Quelques légumineuses sont cultivées par les Chinois du Chan-tong, entr'autres les Pisum sativum L. ou le HôAANG-TÉou, Phaseolus vulgaris L. var. (Tsian-TÉoU-TzÉ), FABA vulgaris Mænch. (Tsian-Tsâu), etc.

\section{AMYGDALÉES JusS.}

Prunus L.

45. P. (Cerasus) Bungei Walpers Repert. Zot. syst. 2, p. 9 ; Prunus 
humilis Bunge Enum.pl. chin. bor. n 133; Turczan. Enum. chin.bor. $\mathrm{n}^{\circ} 69$, non Moris!

Var. A glabrata Bunge (loc. cit.).

«Fruticulus humilis, 15-20 centim. altus, caule gracili, e basi » parce ramoso, ramulis erectis subglabris; foliis oblongo-lanceo》 latis, tenuissime serrato-dentatis, supra glabris, subtus rugosis, »glaucescentibus, nervosis, nervis prominulis; floribus solitariis, " pedunculis erectis folia subæquantibus; calicis laciniis campa»nulatis.

»Drupæ eis Cercusi avium. var. sylvestris magnitudine, rotundæ, » supra vix depressæ, atro-purpureæ, carne nucleo adherente, » sapore acidulo. »

$H a b$. : Collines micaschisteuses au bord des ravins, dans la région montag. infér., au-dessus de Ki-tsen-sôo. - Fl. avril (Bunge), fr. mûrs $1^{\text {er }}$ août $(0$. Debeaux).

Ar. géog. : Chine (Chan-tong au Tché-foû, Mongolie chin. au nord du Pé-tché-ly).

Obs. : L'amandier commun (HYN-Hô-GIN), l'abricotier (KIN-GIN), le péchคr (Tâo Gin-Tzḱ), etc., sont cultivés daus tous les jarc̈ins de Yan-taï, de Ki-tsen-sôo, etc.

\section{ROSACEEES JusS.}

\section{Spirka L.}

46. S. betulæfolia Pallas Flor. ross. 1, 33, tab. 16 ; Cambess. Mon. spiraa, in Ann. scien. nat. 1, p. 368, tab. 27; Ledeb. Flor. ross. 2, 27; Maxim. Prim. A. amur. 91; Regel Tent. flor. ussur. 54; Miquel Prol. jap., 221; Franch. et Sav. Enum. jap. $\mathrm{n}^{\circ} 495$; A. Gray Bot.jap. p. 386 ; S. chamadrifolia Cham. et Schlecht. in Linnea 2, p. 2; Hook. Flor. bor. amer, 1, p. 172.

«Suffrutex erectus, 50-75 centim. altus, caulibus striatis, simpli» cibus, subglabris; foliis ovato-oblongis, inæqualiter inciso-ser» ratis, brevissime petiolatis, supra glabris, subtus pubescentibus; 》 corymbis terminalibus hemisphæricis, subfastigiatis; calicibus 》 reflexis; capsulis erectis, elongatis, subtrigonis, apice mucro» nulatis, fusco-brunneis, glabriusculis.

»Folia eis Sp. betulafolia in provinciâ ussuriensi a cl. Regel » lectæ simillima, id est: ovato-acuta, duplicato-dentata, subtus 
》pubescentia. Bracteæ etiam pubescentes, panicula carpellaque »glabra. »

Forma A angustifolia: "Foliis augustis, 12 millim latis, 40 mil» lim. longris, supra medium profunde inciso-dentatis. »

Forma B latifolia : "Foliis latioribus, 25 millim. latis, 40 millim. »longis, breviter inciso-serratis.»

Hab. : R'grion montagr. supér., au bord des ravins, dans les fissures des rochers, de 900 à 1,000 mètres d'altitude. - Fr. 6 septemhre.

Ar. gúng.: A ie bor., Sibérie orient., Kamstıhatka, îles de Chann:so. - Chine ¿Chın-tong au Tché-foû). - Prov. de l'A mour et de l'Usisuri. - Japon. - Amér. bor.

Obs. : Cette espèce a lesp'us grands rapports avec le Spirca rubescens Bungre (Decad. plant. chin. no 11 ), plante qui, d'après sa description, a une inflorescence corymbiforme. Mais M. Maximowicz, qui a conn i cette plante mieux que son descripteur original, affirme que les pédicelles du $S$. pubescens sont en ombelle, ce qui ne saurait convenir à l'espèce du Tché-fô̂, que je considère aujourd'hui comme une forne appaurrie du S. betulafolia. La figure de Camis ssérlès convient aussi à notre espèce.

Decandolle, Ledəbour et Cambessédès la décrivent comme étant tout à fait glabre, mais les botanistes russes Middendorf, Regrel, etc, s'iccordent dans leurs diagnoses, et assurent qu'elle est pubescente, ce qui existe, en effet, dans tous les spécimens provenant de l'extrême Orient.

\section{RUBUS L.}

47. R. parvi folius Lin. Spec. 707; Maxim. in Mélang. biol. vol. 8, p. 392; Miquel Prol. fl. jap. 222; Franch. et Sav. Enum. jap. n ${ }^{\circ} 524 ; 0$. Debeaux Flor. Shang-haï, no $38 ; R$. Thumbergii Blume Bijdraj. p. 1, 109; R. purpureus Bunge, apud Miquel in Journ. bot. Néerl. 1, 121; sinice HıuEN-Tıâo-TZÉ.

Hab. : Rochers micaschisteux du littoral à la pointe du Tchéfoû; ravins des basses collines au-dessus de Yan-taï. - Fl. et fr. août 1860 .

Ar.géog. : Asie subtrop. et temp., Indes-Orient., Himalaya. Chine littorale, depuis Hung-kong jusqu'au 'Tché-foù. - Japon. 


\section{POTENTILLA L.}

48. P. viscosa Don. Catal. hort. Pesth. (1802); Dec. Prodr. 2, 581; Lehman Revis. potent. p. 57; Ledeb. Flor. russ. 2, p. 11, et Fl.altä̈ca 2, 238; Bunge Enum. plant. chin. bor. no 141 ; P. hispida Nestler Monog. pol. p. 30; sinice Fan-PÉTsâo.

Hab. : Sables du littoral à Ki-tsen-sôo; dunes de Fou-chan-yên; sables de la grande presqu'île de Yan-taï, etc. - Fl. septembre.

Ar.géog.: Asie bor., Sibérie ouralienne et altaïque, Baikalie, Daoûrie. - Chine (Chan-tong, Pé-tché-ly). - Arabie (Lehman).

Obs. : Le $P$. viscosa et le suivant sont tellement abondants à Yan-taï qu'ils forment, pour ainsi dire, des prairies naturelles sur les sables où croissent ces deux espèces.

49. P. chinensis Seringe in Dec. Prodr. 2, 581; Lehm. Rev. potent. p. 69, tab. 23; Maxim. Prim. fl. amur. 91; Franch. et Sav. Enum. jap. nu 533; P. exaltata Bunge Enum. chin. bor. no 142 ; sinice Foû-pÉ-Tsâo.

Hab. : Sables au bord de la mer; dunes de Fou-chan-yên; grande presqu'île de Yan-taï. - Fl. 4 septembre.

Ar.géog. : Chine (Chan-tong et Pé-tché-ly). — Prov. de l'Amour. - Japon.

50. P. discolor Bunge Enum. chin. bor. n 149, non Jaquemt. voy. p. 13; Lehm. Rev. potent. p. 35, tab. 12; Walp. Rep. bot. syst. 2, p. 38; Franch. et Sav. Enum. jap. n 335.

«Planta valde insirnis, radice perenni, caulibus flexuosis » decumbentibus, mediis erectis floccoso-tomentosis, basi villosio» ribus; foliis radicalibus pinnatis, foliolis 3-5 oblongis lanceola» tis, serrato-dentatis, supra atro-viridibus, subtus niveo-tomen»tosis, caulinis ternatis sessilibus; stipulis latis semi-amplexi》caulibus, trifidis; floribus dichotomo-paniculatis, petalis obovatis » sepala albo-tomentosa superantibus. »

Hab.: Région montag. infér. et basses collines micaschisteuses au-dessus de Yan-taï, près de la pagode neuve, 300 à 400 mètres d'altitude. - Fl. 10 juillet.

Ar. géog. : Chine bor. (Chan-tong et Pé-tché-ly). - Japon. 
51. P. flagellaris Wild. ex Spreng. Syst.veget. 2, p. 538; Lehm. Rev.pot.p. 187: Turczan. Flor. Baik. dahur. 1,379: Bunge Enum. chin. bor. no 151; Maxim. Prim. fl. amur. p. 97. Forma pilosior Lehım. (loc. cit).

«Heıbacea, perennis, pluricaulis, pilis albidis adpressis omnino " induta, caulibus filiformibus longe repentibus, interdum nodoso" angulatis; foliis quinatis supra puberulis, subtus ad nervos 》) præsertim pilosioribus nec glabris, foliolis ovato-ellipticis, » rofunde inciso dentatis: pedunculis axillaribus uıifloris, folio 》 æquilungis: relalis flavis obovatis, calice subæqualibus; stipulis » minntis, lanceulit s. integris, vel bifidis.»

Hab. : Barses collines sablonneuses ou micaschisteuses au-rlessus de Yall-taï, près de la pagode neure, de 300 à 400 mètres d'altiturle.-Fl. 10 juillet.

Ar.géog. : Asie bor., Sibérie, Altaï, Daoûrie. - Chine (Chantong et P'é-tuht'-ly,.- Prov. de l'Amour.

52. F. parad xa Nittal mss. iu Torr. et Gray Flor. of nor'sh amer. 1. p. $437 ; P$. paralora et supina Lehm. Rev. rot. p 194; $P$. supina Lin. (ex parte): Ledeb. Flor. ross. 2, p. 35; Turczan. Flor. Baik. duhur. 1, 1). 391; Bunge Enum. chin.bor. no 150; P. paradoxa Maxin. Prim. fl. amur. 97; Regel, Tent fl. ussur. 56.

"Herbaced, annua, pubescens, caulibus e basi ramosis, subdi》(hotomis, erectis; foliis pinnatis, foliolis 5-7 obovato-oblongis, » inciso-serratis, superioribus profunde incisis, tridentatis; stipu»lis ovatis, plerumque integris; pedunculis florigeris unifloris, 》 axillaribus, villosis, fructiferisque non deflexis ut in $P$. supina; » calicis laciniis æqualibus, oblongis, acutis; petalis obovatis, » integris, receptaculo villoso.

»Carpella ininutissima, basi rugosula, quasi gibberula. »

$H a b$. : Prairies humides et fossés aquatiques du littoral - Fl. et fr. 18 juillet.

Ar. géog. : Asie bor., Sibérie, Altaï, Baikalie, Daoûrie. - Chine (Chan-tong et Pé-tché-ly). - Prov. de l'Amour et de l'Ussuri.Amér. bor.

Obs.: Le Potentilla paradoxa paraît n'être pour les botanistes russes qu'une forme du $P$. supina spéciale à l'extrême Orient. J'ai peine à adopter cette manière de voir. Dans la plante chinoise, les 
carpelles sont munis à la base d'un gros bourrelet ridé, qui simule presque une gibbosité; de plus, les pédoncules fructifères ne sont pas défléchis comme daus le $P$. supina. Ce sont là des caractères qui se rapportent au $P$. paradoxa réuni à tort par plusieurs auteurs, au $P$. supina de Linné. M. Maximowicz a également observé la gibbosité dont nous parlons sur les graines du $P$. paradoxa prove nant de la Chine boréale ou de la Sibérie. Mais cette gibbosité, qui est un des caractères distinctifs de la plante américaine, est trèsvariable dans l'Asie orientale, selon l'âge ou l'habitat de cette espèce. Le $P$. paradoxa du Tché-loû et de la province de l'Amour a toujours les tiges droiles, élevées et non couchées. La plante entière est pubescente, et plus ou moins velue dans ces localités.

\section{AGRIMONIA L.}

53. A. viscidula Bunge Enum. chin. bor. n 152 ; Sieb. et Zuccar. Abh. IV. (loc. cit.) p. 125; Miquel Prol. fl. jap. p. 226; A. eupatoria Thumbg. Flor. jap. 195, non Lin.

«Tota valde pilosa, caulibus erectis. hirsuto-pilosis, pilis adpressis; foliis ovatis usque fere ad basim grosse dentatis, » supra puberulis, subtus molliter tomentoso-villosis, ad petiolos » præsertim hirsutioribus; racemis floriferis elongatis, basi laxis, »dein confertis; calicis fructiferi tubo ubconico, profunde sulcato, " demum glabriusculo, maturitate pendulo.»

A. viscidula medium tenet inter $A$. eupatoriam L. et $A$. pilosam Fish.

Hab. : Pelouses des basses collines; bord des champs, etc. - Fl. et fr. 10-25 juillet.

Ar. géog. : Chine bor. (Chan-tong et Pé-tché-ly).-Japon.

\section{ROSA L.}

54. R. rugosa Thumbg. Flor. jap. 213; Maxim. Prim. $A$. amur. 101; Bunge Enum. chin. bor. n ${ }^{\circ} 156$; Sieb. et Zuccar. Flor. jap. 1, 66, tab. 28; Miquel Prol. Al.jap. 227; Franch. et Sav. Enum. pl. jap. n 556; R. Regeliana André (olim); sinice NAN-TSING-HÔA.

Forma amurensis Maxim. (loc. cit.). 
"Suffrutex 2-3 pedalis, aculeatissimus, apice dense ramosus; » floribus saturate roseis, odoratisque, subcorymbosis. »

Hab. : Falaises de la pointe du Tché-foû, et roches micaschisteuses au-dessus de Ki-tsen-sôo.- Fl. 14 juillet, fr. 16 août 1860.

Ar.géog.: Chine (Chan-tong et Pé-tché-ly in hortis culta). Prov. de l'Amour. - Japon.

Obs. : Le Rosa rugosa, qui est la seule espèce de rosier que l'on trouve à l'état spontané au Tché-foû, se rapporte exactement à la forme dérrite par M. Maximowicz sous le rom d'amurensis. Snn inflorescence est souvent en pseudo-corymbe (par l'avortement des rameaux), et dans cet état, il a été décrit fort mal à propos par André, comme espèce distincte, du nom de $R$. Regletiana.

\section{SANGUISORBA L.}

55. S. canadensis Lin. Spec. 169 ; Dec. Prodr. 2, 594; Franch. et Sav. Enum.pl. jap. n 546, sub Poterio; Hook. Flor. bor. amer. 1, 198.

Var. latifulia Ledeb. Flor. ross. 2, p. 28; S. media. Lin. ex parte; Regel T'ent.fl.ussur. p. 55.

«Caule erecto-ramoso, 40-50 centim. alto, racemis floriferis » longe pedunculatis, spicis terminalibus elongatis, cylindricis, » erectis vel cernuis; staminibus longe exsertis, bracleis calici» busque glabris; foliis $3-5$ jugis, foliolis radicalibus petiolulatis, » late ovatis ellipticisve, apice mucronatis, basi obcordatis, grosse » serratis, glabris; stipulis nullis. »

Hab. : Marécages salés et prairies inondées à haute mer, dans la presqu'île de Yan-taï. - Fl. 4-10 septembre.

Ar. géog. : Asie bor. - Iles Sitcha et Unalaska. - Prov. de l'Ussuri. - Japon. - Chine (Chan-tong). - Amér. russe.

Obs. : Le $S$. canadensis, qui est rare dans les marais salés de Yan-taï, se distingue facilement del'espèce suivante ( $S$. tenuifolia) par ses feuilles ovales ou ovales-elliptiques, en cœur à la base, et bordées de grosses dents égales. Jen'ai à signaler qu'une différence de peu d'importance, entre la plante chinoise et celle d'origine japonaise décrite par MM. Franchet et Savatier. Dans celle-ci, les folioles seraient longuement pétiolulées, tandis qu'elles sont courtement pétiolées dans la plante du Tché-foû. 
56. S. tenuifolia Fisher in Hort. Gorenk ex Lin. Enum. hort. Berol. 1, 144; Ledeb. Flor. 1’oss. 2, 28; Maxim. Prim. A. amur. 94, var. A; Regel Tent. fl. ussur. 54; Turczan. Flor. Baik. dahur. 1, 403; Trautv. et Meyer Flor. Ochotsk, p. 35; Miquel Prol. A. jap. 226 ; Franch. et Sav. Enum. jap. n 515.

"Caule simplici-erecto, duplo longiore quam in præcedente, » supra medium plurispicato, 95-100 centim. alto; spicis breve * pedunculatis, cylindricis elongative, staminibus exsertis; foliis "7-9 jugris, foliolis glabris, anguste lanceolatis, aryute serratis, "basi late cuneatis, radicalibus petiolulatis, caulinis sessilibus; " stipulis parvis, ovatis, lanceolatis subulatisve. "

Hab. : Narais salés du littoral; dunes et mares de Fou-chanyên. - Fl. 4-10 septembre.

Ar. géog. : Asie bor., Sibérie, Daoûrie, Ochotsk, Kamtschatka. - Prov. de l'Amour et de l'Ussuri.- Chine (Chan-tong). - Japon.

\section{POMACÉES Lind.}

\section{Crategus L.}

57. G. pinnatifida Bunge Enum. chin. bor. no 157; Turczan. Enum. chin. bor. no 74; Maxim. Prim. A. amur. 101; Regel Tent. Al. ussur. 58; sinice SHâN-CHÉ-TZÉ.

"Frutex 1-1 1/2 met. altus, diffuse ramosus, inermis, foliis " late ovatis, profunde pinnatifidis, 3-7 lobatis, lobis oblongis, acu》tis, grosse serratis, supra glabris, subtus ad nervos pilosulis; " floribus corymbosis, pedunculis calicibusque basi villosis. 》 "Poma coccinea eis $C$. azaruli similia, verruculis albis notata, » sapore acidulo.»

$H a b$. : Région montag. inférieure; collines boisées, bord des ravins, etc., de 300 à 500 mètres d'altitude. - Fruits mûrs 2 soût 1860.

Ar. géog.: Chine (Chan-tong et Pé-tché-ly). — Prov. de l'Amour et de l'Ussuri.

Obs. : Les fruits de cette espèce sont recherchés par les indigènes du Chan-tong, et apportés sur le marché des villagres. On mange ces fruits confits dans le sirop de sucre. Ceux du Rosa rugosa et du $R$. indica cultivés dans les jardins (TSIAN-LA-KO ÛN-TZÉ) servent au même usage. 


\section{PYRUS L.}

58. P. malus Lin. Spec. 686; Pallas Iter. 1, 1. 16; Ledeb. Flor. ross. 2, p. 96; Bunge Enum. chin. bor. n 158; A. Gray in Plant.jap.exped. Perry, p. 311 ; in iquel Prol. fl.jap. 228.

Var. glabra; Pyrus acerba. Dec. Prodr. 2, p. 635: fructibus parvis, acerbis, foliis germinibusque glaberrimis.

$H a b$. : Haies des jardins et des propriétés rurales à Yan-taï et Ki-tsen-soô, etc. - Fr. mûrs 18 septembre.

Ar.géog. : Eur., Afriq. bor.-Asie bor. et occ, Russie arctique, Caucase, Arménie. - Chine (Chan-tong et Pé-tché-ly). - Japon.

59. P. (Malus) prunifolia Wild. Phys. 1, p. 8, et Spec. plant. 2, p. 1018; Ledeb. Flor. ross. 2, p. 97; Dec. Prodr. 2, p. 605; Malus hybrida Poiret Encyc. suppl. 4, p. 524.

$H a b$. : Bord de ravins dans la région montag. infér. et collines micaschisteuses, de 300 à 400 mètres d'altitude. - Fr. mûrs 20 juillet.

Ar.géog. : Asie bor., Sibérie.-Chine : Chan-tong (O. Debeaux), Pé-tché-ly (Maxim.).

Obs.: Le Pyrus prunifolia n'est point cultivé dans le Chan-tong, mais ses fruits glabres de la grosseur d'une cerise ordinaire, et d'un rouge vif à l'extérieur, sont fort recherchés par les enfants chinois, qui s'en font des colliers d'une durée éph émère. Les feuilles sont longuement ovales-elliptiques, acuininées, finement dentées, glabres en dessus, un peu velues en dessous, ainsi que les nervures et les pétioles. Les fruits sont couronnés par les divisions calcinales à leur maturité.

60. P. (Pyrophorum) communis Lin. Spec. 686; Ledeb. Flor. r'oss. 2, p. 94; Bunge Enum. chin. bor. no 159; Miquel Prol. A.jap. p. 228; Franch. et Sav. Enum. jap. $11^{\circ} 558$; sinice LITZÉ, KaOûO-TSANG.

Var. A Pyraster, Dec. Prodr. 2, 643.

«Frutex spinosus, foliis subrotundatis, coriaceis, acutis, argute » serratis, utrinque glabris. Poma nucis juglandis magnitudine, » rubro-coccinea, glabra, solitaria, subglobosa, juniora sapore » acido, maturitate dulcia, sed non edulia.»

Var. B sativa Dec. (loc. cit.). 
Ramis inermibus numerosæ varietates in hortis coluntur.

Hab. : La variété A dans les lieux incultes de la plaine et des plus basses colines près de Ki-tsen-sôo; la var. B dans tous les jardins.

Ar. géog. : Eur. - Afriq. bor. - Asie bor., occ. et or. - Russie arctique, Caucasse, Arménie, Sibérie, etc. - Chine (Kiang-soû, Chan tong, Pé-tché ly, etc.). - Japon.

Obs. : La forme spontanée et spinescente produitau Tché-foû des fruits aiğres avant leur entière maturité. Mais dès l'arrivée des premiers froids, au commencement de novembre, ces fruits devien. nent mous, et ont alors une saveur douceâtre. La varité $B$, cultivée, produit une sortede fruit très-renommé à la Chine, et connu du nom de Poire duChan-tong.

61. P. (Pyrophorum) betulæfolia Bunge Enum.plant. chin. bor. $\mathrm{n}^{\circ} 161$.

« Frutex 2-3 met. altus, foliis coriaceis, utrinque glabris, longe » petiolatis, ovato-rotundatis vel ovatis, apice acuminatis, argute »serrato-dentatis, acumine recurvato; petiolis pedunculis gem» misque tomentosis; floribus subcorymbosis.

»Fructus (Poma) minuti, pisi vulgaris magnitudine, subglabri, »luteoli, cum pedınculo petiolos æquantes, calicis laciniis »deciduis. »

Hab. : Bord des champs; haies des jardins et lieux incultes à Yan-taï. - Fr. 2 septembre.

Ar. géog. : Chine (Chan-tong, O. Debeaux, Pé-tché-ly à Pé-king, Bunge).

Obs. : Cette rare espèce de poirier, peu connue jusqu'd présent, est remarquable par la petitesse de ses fruits qui ne sont pas plus gros qu'un pois ordinaire.

62. P. cydonia Lin. Spec. 687; Thumbg. Fl. jap. 208; Miquel Prol. A. jap. 228; Franch. et Sav. Enum. jap. p. 138; Cydonia vulgaris Pers. Syn.plant. 2, 638; Ledeb. Flor. ross. 2, 101; C. chinensis Bunge Enum. chin. bor. $n^{\circ} 162$; sinice MAô-MouKoûA.

Hab. : Haies des jardins à Yan-taï et Ki-tsen-sôo.

Ar.géog. : Asie or. et occ., Tauride, Caucase, Arménie, Mingrelie - Chine (Chan-tong, Pé-tché-ly). - Japon. - Naturalisé dans l'Europe australe, et le nord de l'Afrique. 
Obs.: Les fruits (coings) de l'espèce chinoise sont beaucoup plus petits que ceux de l'Europe australe, mais leur forme est identique. En général, ils sont d'une couleur jaune-pâle, très-odorants, et d'un gont très-acerbe à leur maturité.

Je n'ai point rencontré dans les jardins du Tché-foû l'Eriobotrya japonica, qui est répandu et cultivé dans le Kiang-soû. Bunge dit qu'il l'a vu cultivé en serre chaude à Pé-king, mais qu'il n'y mûrit point ses fruits.

Le Grenadier commun, Punica granatum Lin., est acclimaté à Yan-taï autour des temples bouddhiques, des pagodes, etc.

\section{ONAGRARIÉES JusS.}

Trapa L.

63. T. natans Lin. Spec. 175 ; Ledeb. Fl. ross. 2, 114 ; Maxim. Prim. A. amur. 273: Regel Tent. A. ussur. 60; sinice Ling.

Hab. : Mares d'eau douce, au milieu des dunes de la baie de Fou-chan-yên.

Ar. géog. : Eur. - Asie bor., occ. et or., Russie arctique, Astrakan, Uralsk, Caucase, Sibérie altaïque, Baikal. - Prov. de l'Amour et de l'Ussuri. - Chine (Chan-tong' et Pé-tché-ly).

\section{L'YTHRARIÉES JusS.}

LYTHRUM

64. L. virgatum Lin. Spec. 642; Ledeb. F'lor. ross. 2, 128; Dec. Prodr. 3, 83; Karel. et Kiril. Enum.pl. altä̈. no 315; Miquel Prol. Al.jap. 149; Franch. et Sav. Enum.jap. 647; L. acuminatum Wild. Spec. 2, 866.

$H a b$. : Mares et marécages d'eau douce, au milieu des dunes de Fou-chan-yên. - Fl. 2-12 septembre.

Ar. géog. : Eur. cent. et or., Russie bor. et moy., Podolie, Chersonèse, pays des Cosaques, etc. - Asiebor., occ. et or., Volga, Astrakan, Caucase, Sibérie de l'Oural et altaïque, Daoûrie. Chine (Chan-tong O. Debeaux). - Japon. - Corée.

\section{CUCURBITACÉES Juss.}

Cucumis L.

65. C. melo Lin. Spec. 1436; Ledeb. Fl. ross. 2, 142; Bunge Enum. chin. bor. $\mathrm{n}^{\circ} 175$; Maxim. Prim. Al. amur. 111; Regel 
Tent.fl. ussur. 63; Miquel Prol. fl. jap. p. 12; sinice KIANGroûA.

«Fructus (Melo) sinensis, ei plantæ amurensis simillimus, par» vus, rotundato-ovatus, sulcatus, levis, pallide-viridis, carne viri» descente, sapore fere insipido. »

Hab. : Cultivé en grand à Yan-taï, et dans les plaines du Chantong de l'autre côté des montagnes.

Ar. géog. : Originaire de l'Asie. - Caucase. - Chine (Kiangsoû, Kiang-nan, Chan-tong, Pé-tché-ly, etc.). — Prov. de l'Amour et de l'Ussuri. - Japon.

66. G. citrullus Lin. Spec. 1435. sub Cucurbita; Seringe in Dec. Prodr. 3, 310; Bunge Enum.chin.bor. n 177; Maxim. Prin. fl. amur. 111; Regel Tent. fl. ussur. 64; Staunton Plant. Chantong. exsice (1793); sinice Tsi-koû.

Hab. : Cultivé dans toute la proviuce du Chan-tong.

Ar. géog. : Orig. de l'Asie. - Indes-Orientales. - Chine (Kiang-sou, Chan-tong, Pé-tché-ly).-Prov. de l'Amour et de l'Ussuri. - Japon.

\section{PORTULACÉES Juss.}

\section{Portulaca L.}

67. P. oleracea Lin. Spec. 638; Ledeb. Fl. ross. 2, 145; Benth. Fl. Hong-kong. 127; Bung'e Enum. fl. chin. bor. n 180; Maxim. Prim. fl: amur. 113; Regel Tent. fl. ussur. 69 ; sinice MATCHÉ-YEN.

Hab. : Champs sablonneux du littoral, autour de Yan-taï. Fl. août.

Ar. géog. : Eur. - Afriq. bor. - Asie : prov. Caspiennes, Caucase, Tauride, Arménie. - Chine (Hong-kong, Chan-tong, Pétché-ly). - Prov. de l'Amour et de l'Ussuri. - Japon. - Iles de la Sonde, Java. - Natur. dans l'Amér. bor., etc.

\section{CRASSULACÉES Dec.}

\section{UMBILICUS L.}

68. U. fimbriatus Turczan. in Bull. soc. nat. mosc. vol. XVII, 2, p. 241 ; Cotyledon fimbriatus Hance. in North china plants, p. 80 . 
Affinis $U$. malacophylli Dec. cujus habet formam staturamque, sed differt :

$1^{\circ}$ Foliis ad marginem laceratis, longe mucronatis, nec inermibus;

$2^{\circ}$ Pedunculis bi-quadrifloris nec simplicibus;

$3^{\circ}$ Inflorescentia spiciformi, apice dense pyramidata, floribus lacteis, corolla usque ad basim 5-partita.

$H a b$. : Sur les vieux murs et les toits en chaume de Yan-taï; région montagueuse infér. sur les roches micaschisteuses. Fl. 10 août 1860.

Ar. géog. : Mongolie chinoise; Chine, dans les prov. du Chantong et du Pé-tché-ly.

Obs.: L'Umbilicus fimbriatus se rapproche de l'U. malacophyllus dont il a tout à fait le port et la taille. Mais en l'examinant avec attention, on lui trouve des caractères de premier ordre, qui ne permettent pas de le confondre avec cette dernière espèce. Ainsi dans l' $U$. fimbriatus, toutes les feuilles sont munies à leur extrémité d'un mucron long de 5 à 6 millimètres au moins, triangulaire et spinescent, et sont lacérées sur les bords, ce qui n'existe pas dans l'U. malacophyllus dont les feuilles sont inermes. De plus, dans notre plante, les pédoucules sont tous bi-quadriflores, et non simples uniflores. Ceite rare espèce a été retrouvée aux environs de Pé-king par M. l'abbé David et le $\mathrm{D}^{\mathrm{r}}$ Wells Williams.

\section{SEDUM L.}

69. S. (Aizoon) pseudo-aizoon O. Debeaux Msc. in Herb. 1860.

"Radix repens, perennis, ad collum præsertim crassa, quasi » tuberosa, uni vel rarius bicaulis, caulibus simplicibus erectis, » firmis, cylindricis, 20-35 centim. altis, glabris; foliis anguste » lanceolatis, spathulatis, supra medium crenato-dentatis, ad basim » integris, utrinque glabris, suboppositis; foliis involucrantibus » nullis, vel cyma multo brevioribus; floribus luteis, in cymam 》 terminalem confertamque dispositis; carpellis ad tertiam partem » coalitis, ex inde patentibus. »

Hab. : Prairies sablonneuses du littoral; rivages de la baie de Ki-tsen-sôo. - Fl. 12 juillet.

Ar. géog. : Chine : Chan-tong (O. Debeaux).

Obs.: Notre Sedum pseudo-aizoon appartient au groupedu $S$. aizoon. 
Il offre quelques rapports avec le S. Selskianum Regel et Maak, et le S. Kamtschalicum Fish., par ces capsules soudées jusqu'au tiers et s'étalant ensuite. On peut aussi lui trouver quelques affinités avec les $S$. hybridum L. et $S$. Middendorfii Maxim. par ses cymes florables dépourvues de feuilles involucrales, ou n'en présentant du moins que de très-courtes et égalant à peine le tiers des rameaux. Mais dans ces dernières espèces, les capsules sont très-brièvement soudées à la base et étalées en étoile à la maturité.

La plante du Tché-foû a les feuilles semblables à celles du $S$. Selskianum (ex Icone Regeliana), avec cette difference que dans celui-ci, les feuilles, tiges et pétioles sont hérissés de poils blanchâtres, tandis que le $S$. pseudo-aizoon est tout à fait glabre, et a les fleurs du double plus grandes.

70. S. (Aizoon) yantaiense O. Debeaux Msc. in Hert. (1860).

"Radix perennis, multicaulis, plus duplo crassior quam in præ» cedente, subcylindrica, radicellis rectis nec repentibus, caulibus » 8-10, diffusis, prostratis, vel flexuoso-erectis, gracilibus, 7-10 cen»timet. longis, cymisque papillis numerosis plus minusve densis »obsitis; foliis brevibus, oblongo-spathulatis, supra medium » crenato-dentatis, utrinque glandulosis, glandulis brevibus, foliis » involucrantibus nullis; floribus aureis, in cymam terminalem » compositam depauperatamque dispositis.

»Affinis S. Selskiuni Regel, a quo differt : pubescentia glandu » losa, basi papillosa; caulibus diffusis, 6-plo brevioribus, e basi » cæspitosis nec simplicibus; foliis oblongis, ovalibus, spathulatis 》triplo-brevioribus; panicula laxa, pauciflora, nec conferta, etc.

»A S.pseudo-aizoon nostro recedit, caulibus brevioribus, diffusis, »nec rectis; foliis hispido-glandulosis nec glabris; panicula laxa, " pauciflora, etc. »

Hab. : Prairies sablonneuses du littoral, dans la plaine de Yantaï et de Ki-tsen-sôo. - Fl. 4-10 août.

Ar.géog. : Chine (Chan-tong' au 'Tché-fou, Yan-taï, etc.).

Obs. : Le $S$. yantaiense croît dans les mêmes localités quel'espèce précédente. Il fleurit un mois plus tard, et ne forme aucun hybride arec elle. Ces deux espèces sont bien tranchées dans l'ensemble de leurs caractères, et parmi les nombreux échantillons que j'en ai récoltés, ou que j'ai vus sur place, je n'ai point trouvé de formes intermédiaires qui puissent les rallier l'une à l'autre. 
Par les papilles glanduleuses qui recouvrent les tiges et les feuilles, notre $S$. yantaiense n'est pas sans analogie avec le $S$. $M$ c ximowiczii Regel in Gartenflora (1866), p. 196, tab. 513. Ce dernier, est très-robuste; il a les feuilles larges de plus de 20 millimètres, et ses tiges sont anguleuses. Ce dernier caractère le distingue surtout des autres espèces du groupe aizoon.

\section{OMBELLIFĖRES Juss.}

\section{Buplevrum L.}

71. B. scorzoneræfolium Wild. Enum. hort. Berol. 300 ; Turczan. Fl. Baik. dah. 1, p. 480; Maxim. Prim. Al.amur. p. 125; B. falcatum var. scorzonercfolium Ledeb. Fl. ross. 2, 266; Regel Tent. A. ussur. 69; Miquel Prol. Al. jap. 246; Franch. et Sav. sub B. falcato, Enum. A.jap. no 688.

«A Buplevro falcato differt, foliis oblongo-linearibus, involucro » monophyllo subnullo, umbellisque paucis triradiatis. »

Hab. : Roches micaschiteuses du littoral; falaises de Ki-tsensôo. - Fl. 1 ${ }^{\text {er }}$ août.

Ar.géog. : Asie bor., Sibérie altaïque et baikalienne, Daoûrie Mongolie. - Chine (Chan-tong, Pé-tché-ly). - Prov. de l'Amour et de l'Ussuri. - Japon.

\section{STENOCALLIUM Ledeb.}

72. St. divaricatum Ledeb. $F l$.ross. 2, p. 232 ; Turczan. Cat. pl. Baik. n 529, et Fl. Baik. dahur. 1, 494; Maxim. Prim. $A$. amur. 128; Regel Tent. $A$. ussur. 71.

$H a b$. : Sables maritimes sur les rivages de la rade de Yan-taï; falaises micaschisteuses de la pointe du Tché-foû. - Fl. 2-10 septembre.

Ar. géog. : Sibérie transbaik., Daoûrie, Mongolie. - Chine (Chan-tong, Pé-tché-ly). - Prov. de l'Amour et de l'Ussuri. Japon.

Obs. : M. Franchet m'a assuré que cette espèce avait été observée récemment au Japon, quoiqu'il n'en soit fait encore aucune mention dans les ouvrages publiés sur la flore de cette contrée. 
Le $S$. divaricalum, de la section des Pachypleura, abonde sur les sables du Tché-foû, et il est probable qu'il sera signalé dans d'autres localités du littoral.

\section{CZERNEVIA Turczan.}

73. G. lævigata Turczan. Fl. Baik. dahur. 1, 499; Ledeb. Fl. ross. 2, 29; Maxim. Prim. $f$. amur. 127; Regel Tent. $f$. ussur. 70.

«Radix napiformis, ad collum præsertim crassa, radicellis 2-3 di» varicatis, caule tripedali, simplici, erecto, striato, apice subsul» cato; foliis supra glabris, subtus pilis rariusculis indutis, demum » plerumque glabratis, inferioribus pinnatisectis, segmentis tri» partitis, superioribus integris, laciniis lanceolatis oblongisve, » argute et inæqualiter serrato-dentatis, serraturis acuminatis, » margine cartilagineis, intermediis minus dissectis; umbella mul» tiradiata, radiis interne pilosis ; umbellulis multifloris, pedicellis » longitudine variis, pilosis glabrisve; floribus albis. - Carpella »non vidi.»

Hab.: Réggion supér. montag. etravins boisés, de 600 à 1,000 mètres d'altitude. - Fl. 6 septembre.

Ar. géog. : Sibérie transbaik., Daoûrie. - Chine (Chan-tong). Prov. de l'Amour et de l'Ussuri.

Obs. : En l'absence de fruits mûrs, il seraitimpossible d'affirmer que l'ombellifère de la tribu des $A$ ngelicea, que je rapporte au $C z e{ }^{\prime-}$ navia lavigata, soit identique avec l'espèce de la Daoûrie, et décrite par Turczaninow. Cependant la description de Ledebour convient de tout point à notre plante, moins ce qui a rapport aux fruits que je n'ai pu observer. L'époque de la floraison est à peu près la même dans le Tché-foû ( 6 septembre) et dans la province de l'Amour (du 2 août au 2 septembre). Le caractère tiré des dentelures des feuilles qui sont comme cartilagineuses sur la marge et à leur sommet me paraît avoir une grande valeur. Ce caractère, auquel viennent s'adjoindre ceux fournis par la forme des racines, des tiges; des feuilles et des ombelles florales, m'a fait adopter pour la plante des montagnes du Tché-foû le nom de Czernavia lavigata. M. Franchet, qui a vu une échantillon incomplet de cette ombellifère provenant du Tché-foû, me dit qu'il lui paraît être très-voisin de la plante de Turczaninow. 


\section{PHELLOPTERUS Benth.}

74. P. littoralis F. Schmidt, in Amurl. und auf der inseln Sacha lin, p. 138; Franch. et Sav. Enum.pl.jap. n 698 ; Cymopterus littoralis A. Gray Bot.jap. 491. - Gleitnia littoralis F. Schmidt Flor. Sachalin. ined. in Miquel Prol. fl. jap. 294, et in Annal. mus. Lugd. batav. 3, p. 61.

"Caulis in parte subterranea penna anserina paulo crassior, » usque 4 pollicibus longus, foliis inferioribus compositis, segmen» tis trilobis vel tripartitis, petiolatis, terminali lateralibusque » pinnatisectis, lobis infimis passim ternatis, reliquis liberis nunc »subpetiolatis, supremis confluentibus, omnibus plus minusve " obovatis, obtusis vel rotundatis, argute calloso-serratis. »

Ha乙. : Sables maritimes de la baie de Ki-tsen-sôo. - Fl. $1^{\text {er }}$ août, fr. 30 août.

Ar. géog.: Chine (Tché-foû, O. Debeaux), îles Lôo-chôo. - Corée. - Sacchalien. - Japon (Yé-so, Nippon, Nangasaki).

Obs.: Le Phellopterus littoralis est une des plantes lesplus intéressantes de la flore du Tché-foû. On le reconnaîtra facilement à ses racines longues et pivotantes, à ses tiges florifères étalées sur le sable et recouvertes d'un tomentum laineux dense et roussâtre, à ses ombelles serrées, à ses fruits ovales-arrondis et munis de six côtes angulaires, entièrement enveloppées d'un duvet laineux, Cette espèce, découverte d'abord sur les sables d'Hakodaté (Yé-so) au Japon par Ch. Wright en 1855, a été observée par moi-même, au Tché-foû, en août 1860. Elle était retrouvée vers la même époque (septembre 1860) par Gleihn dans l'île Sachalin, et enfin le $\mathrm{D}^{\mathrm{r}}$ Savatier l'a récoltée en 1867 dans le Nippon au Japon.

Obs. : On cultive dans la province du Chan-tong, pour l'usage alimentaire, la carotte (Hô-LÔ-PÉ des Chinois), le persil commun (Hû-TSAï), le céléri KIN-TSAï), la coriandre (CHÉ-LO, HONG-YÉ-TZÉ), etc. 


\section{LORANTHACÉES DOn.}

\section{VISCUMI L.}

75. V. album Lin. Spec. 1451; Ledeb. F'. ross. 2, 380 ; Maxim. Prim. fl. amur. 134; Regel Tent. fl. ussur. p. 74; Miquel Cat. fl. jap. p. 99.

Hab. : Yan-taï, parasite sur les Pyrus malus et $P$. communis. Fr. 13 juillet.

Ar.géog. : Eur. - Afriq. bor. - Asie bor., Russie arctique, Caucase, Arménie, Sibérie ouralienne. - Prov. de l'Amour et de l'Ussuri. - Chine (Chan-tong). - Japon.

\section{CAPRIFOLIACÉES Dec.}

\section{SambuCUS Lin.}

76. S. racemosa Lin. Spec. 386; Ledeb. Flor. ross. 2, p. 383; Turczan. Flor. Baik. dahur.1, p. 518; Karel. et Kiril. Enum. plant. alt. no 402; Bunge Enum. chin. bor. no 93; Regel Tent. $A$. ussur. p. 74; Miquel Prol.jap. 153; A. Gray Bot. jap. 303; Franch. et Savat. Enum. jap. p. 198; Boiss. Flor. orient. 3, p. 3 ; sinice Tchoû-Foû-HôA.

Var. A glabra; foliis glabris vel subglabris (Miquel, loc. cit.).

$H a b$. L Les haies et les clôtures des jardins autour de Yan-taï, de Ki-tsen-sôo, si-nen-kô, etc. - Fl. juillet-août.

$A r . g e ́ o g$. : Eur.-Afriq. bor.-Amér. bor. et occ.-Asie bor. et or. -Sibérie de l’Oural, Altaïque, ${ }^{\prime}$ etc., Baikal, Daoûrie, Kamtschatka, iles Si-tcha.- Amour et Ussuri. - Chine (Chan-tong, Pé-tché-ly). - Japon (Kiôu-siôu, Nippon).

\section{LONICERA L.}

77. L. confusa Dec. Prodr. 4, 333; Don. Prodr. Al.nep. 446; Miquel Prol.fl.jap. 157; L. japonica Andr. Repert. tab.70; A. Gray Bot. jap. 170, non Thumbg. nec Benth.; sinice KIN-YENHÔA.

Hab. : Haies autour de Yan-taï. - Fl. 12 juillet. 
Ar. géog. : Asie subtrop. et temp., Indes-Orient., Népaul.Chine (Kiang-soû, Fo-kien, Chan-tong). — Japon.

Obs. : La plante du Tché-foû se rapporte exactement à la description du Prodromus. Cette espèce paraît être très-répandue sur le littoral de la Chine; mais dans l'extrême-nord, elle y est remplacée par le L. chinensis Wats. D'après Bentham (in Flor. Hongkong.), le synonyme cité par Decandolle (L. japonica Andr.) se rapporterait au $L$. macrantha de cet auteur. Decandolle décrit trop bien ces deux espèces, pour qu'il puisse y avoir quelques doutes sur elles.

Le $L$. confusa a les tiges pubescentes, les feuilles ovales arrondies à la base, aiguës au sommet, ciliées sur les bords, et pubescentes sur les deux faces, ainsi que les pétioles. Les fleurs sont axillaires, et la corolle à tube et divisions calicinales pubescents n'atteint pas 30 millimètres de longueur. Dans le $L$. macrantha Dec. les feuilles sont obcordées à la base, glabres en dessus, hispides-tomenteuses en dessous. La corolle atteint dans cette espèce plus de 60 millimètres en longueur. Le $L$. macrantha ne s'éloignerait pas de l'Asie subtropicale, et ne dépasserait pas l'île de Hong-kong et le Koûang-tong dans le sud de la Chine.

\section{RUBIACÉES Juss.}

\section{RUBIA L.}

78. R. cordifolia Lin. Mant. 197; Ledeb. Flor.ross. 2, 405; Bunge Enum. chin. bor. p. 35; Turczan. Fl. Baik. dah. 1, 528; Maxim. Prim. Al. amur. 139; Regel Tent. A. ussur. 76; Miquel Flor. ind. batav. 2, 337, et Prol. Al. jap. 275; R. cordata Thumbg. Fl.jap. $60 ;$ R. mungista Roxbg. Fl.ind. 374; sinice T'srâN-Tsâo.

Forma A genuina; foliis cordato-oblongis vel ovalibus, acuminatis, 15-20 millim. longis.

Forma B pratensis (Maxim.): foliis cordatis, acuminatis, elongatis, 35-40 millim. longis.

Hab. : Haies, bords des champs, etc. La forme B pratensis, dans les lieux herbeux du cap de Tché-foû. - Fl. 25 août.

Ar. géog. : Asie bor., moy. et subtrop. - Indes-Orient. — Sibérie transbaikal., Daoûrie, Mongolie. - Chine (Chan-tong et Pé- 
tché-ly). - Prov. de l'Amour et de l'Ussuri. - Japon. - Iles de la Sonde, Java.

\section{GALIUM L.}

79. G. verum Lin. Spec. 155; Ledeb. Flor. ross. 2, 401 ; Turczan. Fl. Baik. dahur. 1, 533; Maxim. Prim. A. amur. 141; Regel Tent. A. ussur. 77, et de Herder in. Plant. Raddean. reisen in der suden ostsiberien, III, 1, p. 33; Thumbg. Fl. jap. 58 ; Miquel Prol. fl.jap. 27\%.

Forma maritima : caulibus erectis ramosis, basi glabris, apice hispido-villosis, fructibus glabris.

Hab. : La plaine sablonneuse de Yan-taï, bord des champs, prairies, etc. - Fl. 10-12 juillet.

Ar. géog. : Eur. bor., cent. et aust., Russie arct., Wolga et Oural. - Afrique bor. - Asie bor., occ. et or., Caucase, Arménie, Sibérie, Altaï, Baikalie, Daoûrie, Mongolie. - Chine (Chan-tong, Pé-tchély). - Prov. de l'Amour et de l'Ussuri. - Japon. - Amér. bor.

80. G. (Euaparine) pauciflorum Bunge Enum. pl. chin. bor. no 199, in Mém. Sav. étr. Acad. St.-Pétesb. II, p. 109.

"Planta annua, humilis, e basi ramosa, racemis decumbentibus, 》 divaricatis erectisve, tetragonis, secus angulos aculeato-sca" bris, aculeis recurvatis; foliis quinque, sæpius sex, verticillatis, "spathulato-oblongis, basi attenuatis, spinuloso-cuspidatis, supra " hispidulis, subtus subglabris, margine spinosis; pedunculis 1-3 " axillaribus, folia vix æquantibus vel brevioribus, divaricatis, " glabris; corollis minutis, obtusis; fructibus parvis, didymis, hir" sutissimis, setis uncinatis instructis. »

Hab. : Prairies humides et sablonneuses du littoral; plaine de Yan-taï. - Fl. et fr. 15 juillet.

Ar. géog. : Chine boréale: Chan-tong (0. Debeaux), Pé-tché-ly (Bunge).

Obs. : Le Galium pauciflorum, dont on ne connaissait jusqu'd présent que la seule localité des environs de Pé-king indiquée par Bunge, est abondant dans les prairies du 'Tché-foû, et se retrouvera probablement ailleurs. Cette espèce est voisine du $G$. aparine $\mathrm{L}$. dont on le distinguera facilement, par sa taille toujours petite et ne dépassant pas un décimètre, par l'exiguité de toutes ses parties, par ses feuilles longuement spathulées-oblongues, par 
ses pédoncules courts, 1-2 flores, divariqués et non subpaniculés, par ses fleurs très-petites et ses fruits entièrement hérissés de poils recourbés au sommet. MM. Maximowicz, Regel, de Herder, Ruprecht et autres botanistes qui ont exploré les provinces de l'extrême-Orient voisines de la Chine, ne font aucune mention de la présence du Galium pauciflorum lans ces contrées. Celui-ci se trouverait donc exclusivement cantonné dans le Nord de la Chine, et principalement dans le Chan-tong et le Pé-tché-ly.

\section{VALÊRIANÉES Dec.}

Patrinia Juss.

81. P. scabiosæfolia Link. Enum. hort. Berol. 1, 131; Ledeb. Flor. ross. 2, 427; Bunge Plant. Monghol. chin. decas 1, p. 24; Turczan. Flor. Baik. dahur. 1, 537; Maxim. Prim. $A$. amur. 142; Regel Tent. A. ussur. 78; Miquel Prol. l. jap. 279; Fedia scabiosafolia Trev. in Act. soc. cur. nat. 13, 1, p. 165.

Hab. : Région montag. supér. du Tché-fô, dans les fissures des rochers micaschist. de 1,000 à 1,100 mèt. d'alt. - Fl. 6 septembre.

Ar. géog. : Asie bor., Daoûrie près du fleuve Argun, Mongolie. - Chine (Chan-tong, Pé-tché-ly). - Prov. de l'Amour et de l'Ussuri. - Japon.

\section{COMPOSÉES Ȧdanson.}

\section{§ I. - CORYMBIFE்RES Vaill.}

\section{Eupatoriun L.}

82. E. kirilowii Turczan. Enum. chin. bor. n ${ }^{\circ} 408$, in Bull. soc. nat. Mosc. X, 153; Maxim. Prim. Al. amur. 193; Reg'el et de Herder Plant. Raddeana reisen, etc. III, 2, p. 1.

«Planta annua, herbacea, 50-60 centim. alta, caule simplici, »erecto, sulcato, præsertim ad mediam partem valde piloso, pilis » crispatis, foliis oppositis, ovatis-lanceolatis, sessilibus, trisectis, » segmento medio multo majore, lanceolato, in petiolum attenuato » segmentis lateralibus parvis, lineari-lanceolatis, omnibus sub» tus trinerviis, grosse dentatis, margine hispido-ciliatis, præter 
》 costam mediam densius piloso-hispidis; inflorescentia terminali, » corymbosa, composita, capitulis numerosis, cylindricis, pauci"floris; involucri squamis paucis (8-12), laxis, imbricatis, ovato" oblongis, membranaceis, glabris, interioribus hyalinis.

»Flores violacei. - Akenia quadricostata, punctis minutissi" mis, glandulosis, cinerascentibus rarisque instructa (Max.). "

Hab. : Pelouses sablonneusesịdu littoral à Ki-tsen-sôo. - Fl. 19 août, fr. 10 septembre.

Ar. géog. : Mandchourie. - Chine (Chan-tong, Pé-tché-ly). Prov. de l'Amour et de l'Ussuri.

\section{ASTER L.}

83. A. ageratoïdes Turczan. Enum. plant. chin. bor. n 109; Maxim. Prim. Al.amur. p. 144 ; Regel Tent. Al. ussur. 80, et de Herder in Plant. Ruddean. III, 1, p. 19.

Var. adustus Maxim. (loc. cit.).

«Perennis, radice fibrosa, caulibus simplicibus, 4050 centim. » altis, sed in siccis humilioribus, strictis, scabris, in parte supe» riore hispido-pilosis, ramulosis, angulatis ; foliis ovatis, lanceo» latis, acuminatis, grosse dentatis, sessilibus, subtrinerviis, »venis reticulatis distinctissimis percursis, supra scaberrimis " margineque papillis albidis retrorsum aculeatis notatis, subtus » subglabris, ad venas tantum hispidulis; corymbis paucifloris, » terminalibus seu axillaribus; capitulis in diametro 1-1/2 centim. » seu duplo minoribus, radiis lilacinis; involucri squamis anguste " oblongis, triseriatim laxe imbricatis, adpressis, ciliatis, omnibus " apice fuscis; akeniis sericeo-villosis. 》

$H a b$. : Hautes montagnes du Tché-fon, dans les fissures des rochers de 800 à 1,000 mèt. d'altitude. - Fl. 6 septembre.

Ar. géog. : Chine (Chan-tong, Pé-tché-ly). - Prov. de l'Amour et de l'Ussuri.

\section{TRIPOLIUM Nées.}

84. T. vulgare Nées ab Esemb. Aster. p. 153; Ledeb. Flor. ross. 2, 477; Maxim. Prim. A. amur. 145; Miquel Prol. A.jap. p. 100; Aster tripolium Lin.; Turczan. Flor. Baik.dahur. 2,

- p. 12; Regel et de Herder in Plant. Radde. III, 1, p. 11. 
Forma salina Dec. Prodr. V, 253; Aster salinus Schrad. Hort. Gorenk. 1, tab. 3.

"Caule erecto, basi subsimplici; foliis inferioribus lanceolatis » aut lineari-lanceolatis, vix ciliatis, serratis, involucro colorato, »obtuso ; ligulis cyaneis, paulo angustioribus quam in forma "typica."

$H a b$. : Marais salés, dans la grande presqu'île de Yan-taï. — Fl. 4-20 septembre.

Ar. géog. : Eur. maritim. - Afriq. bor. - Asie bor., occ. et or. - Astrakan, mer Caspienne, Caucase, Arménie, Sibérie ouralienne, Altaï, Sôongarie, désert de Gobi, Daoûrie. - Chine (Chantong, Pé-tché-ly). — Prov. de l'Amour. - Japon.

Obs. : La forme des marais de Yan-taï n'est autre que la variété Salina de Decandolle. Ses tiges sont grêles, simples, pauciflores, de 20 à 30 centim. de hauteur, et feuillées seulement dans leur partie moyenne et supérieure. Les feuilles sont linéaires-lancéolées, trèsespacées, et les fleurs tantôt solitaires portées sur de courts petioles, et tantôt disposées en une panicule lâche de 4 à 5 fleurs. Le Tripolium vulgare varie beaucoup, même en Europe, selon sa station dans des lieux plus ou moins humides.

\section{TURCZANINOWIA Dec.}

85. T. fastigiata Dec. Prodr. 4, 558 ; Ledeb. Flor.ross. 2, 480 ; Turcz. Flor. Baik. dahur. 2, p. 16; Maxim. Prim. A. amur. 145; Regel Tent. ussur. 82, et de Herder in Plant. Raddea. III, 1, p. 17; Miquel Prol.jap. 101; Aster fastigiatus Fisher et Meyer in Mém. nat. Mosc. III, p. 74, non Lehm. nec. Ledeb.; Calimeris fastigiata Schultz Bipont. in Zollinger Cat.p. 126.

Hab. : Prairies sablonneuses du littoral et dunes de Foû-chanyên. - Fl. 4 septembre.

Ar. géog. : Asie bor., Daoûrie près du fleuve Argun. - Prov. de l'Amour et de l'Ussuri. - Chine (Chan tong. Pé-tché-ly). - Japon.

\section{CALIMERIS Nées.}

86. G. altaïca Nées Aster. p. 228; Ledeb. Flor. ross. 2, 482; Turcz. Flor. Baik. dahur. 2, 18; Karel. et Kiril. Plant. Sôoñgar. 
n ${ }^{\circ} 420 ;$ Aster altaicus Wild. Enum. hort. Berol. $881 ; A$.

Gmelinii, Tausch. in Flora 1, p. 78 (1839).

Var. scabra Lallem. in Ind. sem. hort. Petrop. (1841), p. 52; Regel et de Herder in Plant. Raddean. reisen III, 1, p. 98; Ledeb. Flor. ross. (loc. cit.).

"Caule simplici, supra medium ramoso, 30 centim. alto, hir» suto-piloso, pilis canescentibus adpressis densisque: foliis lineari»oblongis, basi attenuatis, utrinque pilosis, margine scabris, "serratis, setosis, setis longiusculis, recurvatis; involucri squa» mis pilosis, biseriatis, erectis, oblongo-lanceolatis, acuminatis, " margine membranaceis.

» Formæ diversæ hujus speciei occurunt in provinciis altaïcis. » Nonnullas varietates enumerat clar. Ledebour in Flora rossica, " præsentim var. A subincanam, B subviridem, C scabram, etc. » Hab. : Falaises micasehit. vers la pointe du cap Chan-tong, et non loin de la ville de $N^{\prime}$ gin-hä̈-tchéố. - Fl. 18 septembre 1860.

Ar.géog.: Asie bor., cent. et or., Himalaya, Sibérie altaïque et baikalienne, Sôongarie, désert de Kirghiz, Daoûrie, Mongolie. Chine (Chan-tong, Pé-tché-ly).

\section{BOLTONIA L'Hérit.}

87. B. pekinensis Benth. et Hook. Gen. plant. 2, p. 209; Asteromaa pekinensis Hance in Ann. sc. nat. (4 $4^{\mathrm{e}}$ série), vol. 14, p. 228; Boltonia indica var. canescens 0 . Debeaux in Herb. olim. "Forma ramosa; herbacea perennis, cautibus simplicibus, in » parte superiore plus minusve ramosis, undique cano-tomentosis » erectis, virgatis, 60-80 centim. altis, ramis laxis vel confertis; "foliis oblongo-linearibus, acuminatis, parum mucronulatis, inte"gerrimis, sessilibus, numerosioribusque; capitulis solitariis, ad » apices ramorum dispositis, corymbum laxum foliatumque effor» mantibus. Akenia eis $B$. indice similia. »

Hab. : Pelouses sablonneuses du littoral, et dunes de Foû-chanyên. - Fl. 4 septembre 1860.

Ar. géog. : Chine bor.: Chan-tong au Tché-foû (O. Debeaux), Pé-tché-ly à Pé-king (Swinhoë, octobre 1860).

Obs. : La plante du Tché-foû se rapporte exactement à la description du $B$. pekinensis de Hance (Symbole ad floram sinicam. loc. cit.), avec cette différence toutefois qu'elle est plus ou moins ra- 
meuse au sommet, tandis qu'elle a les tiges simples dans les échantillons de Pé-king. Ce caractère de la ramification des tiges est très-variable dans le genre Boltonia, ainsi que je l'ai observé à Shang-haï pour le $B$. indica. L'habitat et la nature du sol doivent d'ailleurs influer beaucoup sur la végétation du B. pekinensis. Ainsi. nous le trouvons indiqué par Hance autour de Pé-king " ad vias »; tandis qu'au Tché-foû, nous le rencontrons dans les sables humides du littoral, mais dans un état beaucoup plus vigoureux.

\section{B. Lautureana 0. Debeaux Msc. in Herb. 1860.}

"Caulis subsimplex vel apice parce ramosus, erectus, virgatus, 》70-80 centimet. altus, striato-sulcatus, ad angulos setoso-hispi»dus, basi subglaber, foliis coriaceis ovato-lanceolatis vel lineari" lanceolatis, superioribus integris, inferioribus grosse serrato》dentatis, fusco-brunneis, vel fusco-virentibus, omnibus margine " valde setoso-serratis, supra crebrissime papillosis, subtus gla" bris, præter costam mediam tantum hispidulis: serraturis » foliorum aculeatis, aculeis retrorsum decumbentibus : capitulis » terminalibus, solitariis, apice ramorum dispositis, 20 millim. in " diametro latis.

» Ligula cyaneæ, involucri squamis cartilagineis ovatis, obtu» sissimis. Akenia parva, plano-compressa, marginata, glabra, " margine scabra, pilis rufulis minutisque basi connexis coro"nata.»

Hab. : Prairies sablonneuses et humides du littoral de la presqu'île de Yan-taï; dunes de Foû-chan-yên. - Fl. 2-10 septembre 1860.

Ar.géog. : Chine bor., Chan-tong.

Obs. : Le B. Lautureana n'a de rapport qu'avec le B. pekinensis, dont il se distingue par ses tiges scabres presque simples, .ses feuilles coriaces ridées vers les bords, papilleuses en dessus, glabres en dessous et non blanches-tomenteuses sur les deux faces. Il s'en sépare surtout par les écailles de l'involucre qui sont cartilagineuses, ovales, très-obtuses. L'aigrette est beaucoup plus longue que dans les autres espèces de Boltonia, et formée de poils roux évidemment soudés à leur base, et égalant à peu près le tiers de l'akène.

J'ai dédié cette rare espèce à feu M. le comte d'Escayrac de Lauture chargé d'une mission scientifique en Chine à la suite 
de l'armée expéditionnaire, et avec lequel j'ai eu l'honneur de faire une excursion botanique pendant son séjour au Tché-foa.

\section{INULA L.}

89. I. (Bubonium) chinensis Ruprecht Mss. in Maxim. Prim. A. amur. 149; 0. Deb. Flor. Shang-hä̈, n ${ }^{\circ} 54 ;$ I. Bvitannica var. chinensis Regel Tent. fl. ussur. 89, et de Herder in Plant. Raddeanc, III, 2, p. 29; I. repanda Turczan. Enum. plant. chin. bor. $\mathrm{n}^{\circ} 154$; sinice Siuen-Foû-HÔA.

«Planta sat polymorpha, caule simplici, apice polycephalo, " foliisque hirto-pubescentibus, supra parcissime hirtis, subtus »albo-sericeis, inferioribus oblongo-lanceolatis, superioribus » lanceolatis, sessilibus, semiamplexicaulibus; involucri squamis » lanceolatis, vel lineari-lanceolatis, margine medioque dorso "pubescentibus, exterioribus discum æquantibus, squarrosis; " akeniis striato-sulcatis, pilosulis. »

Hab. : Pelouses saumâtres et inondées, au milieu des dunes dans la presqu'île de Yan-taï. - Fl. 23 août et 4 septembre.

Ar. géog. : Chine bor. et moy., Kiang-soû, Chan-tong, Pé-tchély. - Prov. de l'Amour et de l'Ussuri. - Japon.

90. I. (Bubonium) linariæfolia Turczan. Enum. plant. chin. bor. $\mathrm{n}^{\circ} 1141$ in Bull. nat. Mosc. XV, p. 154; Maxim. Prim. . amur. 150; Regel Tent. f. ussur. 85, et de Herder in Plant. Radd. III, 2, p. 30; I. japonica var linariafolia Turcz. ex Miq. Prol. jap. 103.

«Herba perennis, 1-2 pedalis, caulibus polycephalis, sæpe ramo》sis, ramis valde pubescentibus; foliis anguste lineari-lanceolatis "acuminatis, superne glabris seu paulo papillosis, subtus arach» noïdeo-villosis, minute denticulatis, margine plerumque revolu»to, inferioribus in petiolum longe attenuatis, semiamplexicauli"bus, mediis superioribusque sessilibus; involucri squamis viri»dibus, linearibus, glabris, subglandulosis, adpressis, exteriori» bus apice herbaceis, quam intimis brevioribus, subsquarrosisque; "akeniis pilosulis. "

Species peculiaris, sensu cl. Maximowiczii a præcedente differt : caulibus apice valde polycephalis, foliis lineari-lanceolatis, supra glabris, subtus molliter pubescentibus, basi anguste sessilibus; 
capitulis demum plus duplo brevioribıs, numerosissimis; foliolis involucri linearibus, glanduloso-pubescentibus, etc.

Hab. : Prairies sablonneuses du littoral, au cap de Tché-foa, à Yan-taï et Ki-tsen-sôo. - Fl. 8-14 juillet.

Ar. géog.: Chine bor. : Chan-tong, Pé-tché-ly (Kirilon). - Pruv. de l'Amour et de l'Ussuri. - Japon.

Obs.: MM. Regel et de Herder, in Planta Raddeana reisen (loc. cit.), considèrent les Inula chinensis et linariafotia comme deux variétés à peine distinctes de l'I. britannica. Pour M. Maximowicz, au contraire, ce sont deux espèces nettement caractérisées. D'un autre côté, Miquel in Prol. Al.jap. regarde l'I.linariafolia comme une forme à feuilles très-étroites de l'I. japonica Thumbg.

Il suffit d'examiner avec soin ces deux espèces affines, pour leur trouver des caractères qui semblent les rapprocher. Ainsi la grandeur ou la petitesse des feuilles, le nombre et la dimension des capitules, la pubescence plus ou moins prononcée de toutes les parties de la plante, ne seraient que des caractères de second ordre, si ces espèces vivaient ensemble et dans les mêmes localités; mais il n'en est pas ainsi au Tché-foû, où chaque plante a un habitat spécial, et dans chacune de ces stations l'époque de la floraison est différente. Ainsi, l'Inula chinensis ne se rencontre que dans les prairies saumâtres et inondées par la mer; sa floraison a lieu du 23 août au 4 septembre. L'I. linariafolia recherche, au contraire, les prairies sèches sablonneuses, et voisines de la mer. Celui-ci fleurit au commencement de juillet. Son port tout différent, ses tiges rameuses au sommet, ses capitules disposés en un corymbe serré, sa station botanique et l'époque de la floraison, suffisent pour le distinguer des $I$. chinensis et japonica.

\section{ECLIPTA L.}

91. E. marginata Hochst. et Steud. in Hohenak. Sched.; Boissier Flor. orient. 3, p. 249; E.prostrata Ledeb. Flor. ross. 2, p. 512 ; Lin. Mant. ex parte.

«Herbacea, annua, e basi opposite ramosa, ramis sæpius pros» tratis; foliis pilis brevibus adspersis obsitis, dentatis, oblongo» lanceolatis, utrinque attenuatis; pedunculis axillaribus, capitulo » 4-plo longioribus; phyllis oblongo-lanceolatis, ciliatis, strigosis, » flosculos duplo superantibus; paleis anguste linearibus, apice 
》 hirtis; acheniis læviusculis ad angulos albo-marginatis (Boiss.).» Hab. : Champs sabl. du Tché-foû, près du village de Si-nen-kô, etc. - Fl. et fr. août-septernbre.

Ar. géog. : Caucase à Lenkoran. - Perse (Prov. de Ghilan). Java (Zollinger). - Chine (Prov. du Chan-tong).

Obs. : M. Boissier caractérise l'E. marginata par ses akènes à peu près lisses, surtout à leur base, et bordés d'une membrane mince et blanchâtre, par ses folioles involucrales lancéolées aiguës, dépassant une fois les calathides.

L'Eclipta alba, qui abonde dans les champs cultivés autour de Shang-haï, diffère de l' $E$. marginata par ses akènes verruqueux sur le milieu des faces, dont les bords sont épaissis et non membraneux. Ses folioles involucrales sont ovales-arrondies au sommet et ne dépassent guère les capitules.

Malgré les excellents caractères tirés de l'akène, et à cause de la variabilité bien connue de l'E. alba, M. Boissier élève quelques doutes sur la valeur spécifique de l' $E$. marginata, qui pourrait bien n'être qu'une des nombreuses variétés de l' $E$. alba. Quoi qu'il en soit, l'E. marginata constitue une forme remarquable, qui paraît être fort peu répandue dans l'extrême-Orient. MM. Franchet et Savatier ne signalent point sa présence au Japon, où cette espèce est remplacée par la forme typique de l'E. alba.

\section{BIDENS L.}

92. B. pilosa Lin. Spec. 1166, non Thumbg.; Miquel Prol. fl.jap. 105; Benth. Flor. Hongkong. 183; Dec. Prodr. 5, 597; B. chinensis Wild.; Sieb. et Zucc. ex Miquel (loc. cit.); sinice KoûEI-TCHEN-Tsâo.

Forma dissecta, O. Deb. in Herb. 1860.

«Herba annua, caule elato, 60-80 centim. alto, tetragono; foliis » inferioribus impari-pinnatifidis, superioribus trisectis, segmentis 》 anguste lanceolatis, supra molliter hirto-pilosis, subtus præsertim 》 ad costam mediam puberulis; capitulis corymbum late panicu» lato-divaricatum efformantibus, radiatis; involucri squamis » lineari-lanceolatis, margine pubescentibus.

»Akenia linearia, elongata, striato-angulata, hirtella, nigri" cantia, 2-3 aristata, aristis subæquilongis, pallide luteis, setosis ;

》 setis retrorsum aculeatis, spinescentibus. 》 
Hab. : Champs sablonneux autour de Ki-tsen-sôo. - Fl. et fr. 28 septembre.

Ar. géog.: Amér. bor., Pensylvanie, Caroline. - Iles de l'Océan Atlantique, Saint-Vincent, Ténériffe. - Afrique cent., SierraLeone. - Iles de l'Océan Indien, Maurice, Java, Nouvelle-Zélande. - Chine (Hong-kong, Chan-tong, etc.). - Japon.

Obs. : La forme dissecta ne diffère du type, que par ses feuilles divisées en lanières plus étroites, et surtout par ses akènes linéaires un peu hérissés, munis au sommet de quatre arètes presqu'égales, et dont chacune est, ainsi que l'akène, bordée sur les côtés de 3 à 5 paires de soies raides, acuminées, et terminées en une pointe aiguë.

M. Bentham in Flora Honkongensi (loc. cit.) rapporte au Bidens pilosa les B. leucantha Wild., B. sundaïca Blume et B. Wallichii Dec.

93. B. bipinnata Lin. Spec. 1166; Dec. Prodr. 5, 603; Benth. Flor. Hongkong. 183; Miquel Prol. Al. jap. 105; Kernera bipinnata, Gren. Godr. Fl. de France, 2, 169; B. pilosa Thumbg. non Lin. ex parte.

Hab. : Collines micaschit. du littoral, au cap de Tché-foû; falaises de Ki-tsen-sôo, etc. - Fl. et fr. 2 septembre.

Ar. géog. : A mér. bor. - Afriq. cent., Sierra-Leone, Sénégal, cap Vert. - Eur. aust., naturalisé dans la France mérid., en Lombardie, au Tyrol, etc. - Asie or. : Chine (Hong-kong, Chan-tong). - Japon.

\section{CHRYSANTHEMUM L.}

94. Ch. indicum Lin. Spec. 1253; Benth. Hongkong. 180; O. Deb. $F l$. Shang-hä̈, n 60 ; Pyrethrum indicum Cass. Dict. 44, p. 109 non Roxbg. Miquel Prol. A. jap. 106; P. sinense Sieb. et Zucc.; A. Gray in Plant. jap. exped. Perry, 314, ex parte; sinice TA-KIÔU-HÔA, HoâNG-KIÔU-HÔA.

Hab. : Sur toutes les roches micasch. du cap de Tché-foû, les falaises de Ki-tsen-sôo, les basses collines, etc. - Fl. du 15 septembre au $1^{\text {er }}$ novembre.

Ar.géog. : Asie intertrop. et temp. - Indes-Orientales. Chine littorale (Hong-kong, Shang-haï, Tché-foa, etc). - Japon. 


\section{ARTEMISIA L.}

95. A. (Dracunculus) japonica Thumbg. Flor. japon. p. 310; Besser Diss. Dracunc. no 19; Dec. Prodr. 6, 100; Benth. Fl. Hongkong. 186; Miquel Prol.fl.jap. 107; Sieb. et Zucc. Fam. nat. jap. 2, 62 .

Var. rotundifolia O. Debeaux Mss. in Herb. 1860.

« Radix crassa, perennis, caulibus $1-2$ erectis, 20-30 centim. altis; " foliis ramorum sterilium bipinnatifidis, floraliumque inferiori»bus ovato-rotundatis, 3-5 sinuatis, apice vel ad medium grosse »dentatis, vel incisis, basi cuneatis, in petiolum attenuatis, cauli»nis trisectis, laciniis inciso-dentatis, lobo medio apice trifido, » superioribusque linearibus, supra glabris, subtus puberulis; » capitulis in paniculam spiciformem densamque dispositis, par» vis, subglobosis; involucri squamis inæqualibus, glabris, exti» Inis perspicue brevioribus, omnibus albido-scarioso-margina» tis, medio flavidis, centro fusculis. "

Hab. : Rochers micaschit. du littoral au cap de Tché-foû, falaises de Ki-tsen-sôo. - Fl. 23-30 août.

Ar. géog. : Chine (Hong-kong, Koûang-tong, Chan-tong). Japon.

Obs. : Notre variété rotundifolia de l'A. japonica ne diffère du type que par ses feuilles inférieures presqu'orbiculaires et pubescentes en dessous. Cette forme se retrouve également au Japon. (A. Franchet.)

96. A. (Abrotanum) sacrorum Ledebour Flor. altä̈. 4, 72, et Flor. ross. 2, 578; Turcz. Flor. Buik. dahur. 2, 49; Maxim. Prim. A. amur. 159; Regel Tent. fl.ussur. 88; Karel. et Kiril. Enum. pl. altaïc. n' 461; A. santolinafolia Turczan. Plant. Baik. exscic. (1830.)

Var. Minor Ledeb. (loc. cit.).

«Herbacea perennis, pluricaulis, odorem suaveolentem redolens, 》 caulibus 25-30 cent. altis, simplicibus, in parte inferiore nudis; " foliis supra læte-virentibus, subtus villosis incanescentibus, » inferioribus tripinnatisectis, superioribus bipinnatifidis, summis »linearibus; capitulis minutis, pendulinis, paniculam densam » spicatamque efformantibus. » 
$H a b$. : Haute région des montagnes, dans les fissures des rochers, de 900 à 1,000 mètres d'alt. - Fl. 6 septembre.

Ar.géog. : Asie bor., Sibérie, Altaï, Baikal., Sibérie-Orient., Daourie près de l'Argun. - Chine (Chan-tong) - Prov. de l'Amour et de l'Ussuri.

97. A. vulgaris Lin. $S p$. 1188, var. mongolica Dec. Prodr. 6, 113; A. mongolica Besser Diss. Abrotanis, 53; A. vulgaris var. tenuifolia Turczan.; Ledeb. Flor. ross. 2, 585; Regel et de Herder in Plant. Radd. III, p. 75; sinice N'GAI-YÉ.

Hab. : Sables maritimes autour des baies de Yan-taï et de Kitsen-sôo. - Fl. 12 août.

Ar. géog. : Asie bor., Sibérie altaïq. et Baikal. près de Nertschinsk, désert de Gobi, Daoûrie près du fleuve Argun, Sôongarie. - Chine (Chan-tong). - Prov. de l'Amour et de l'Ussuri.

98. A. indica Wild. Spec. 3, p. 1846; Besser Abrotan. p. 34; Dec. Prodr. 6, 114; Sieb. et Zuccar. Fam. nat. jap. 2, 62; A. vulgaris Miquel Prol. jap. 108; Bentham Fl. Hongkong. 187 ex parte.

Hab.: Prairies saumâtres de Yan-taï, et dunes marit. de Fouchan-yên - Fl. 4 septembre.

Ar. géog. : Asie cent., Indes-Orient., Népaul. - Chine (Hongkong, Chan-tung). - Japon.

Obs. : Cette espèce, que Bentham (loc. cit.) considère comme identique avec l'A. leptostachys Dec., est réunie par cet auteur à l'A. vulgaris, dont elle ne serait qu'une variété.

L'A. indica se distingue des diverses formes asiatiques de l'A. vulgaris par ses tiges droites, simples, non ramifiées au sommet, velues-tomenteuses, par ses feuilles à lobes largement trifides, blanches-tomenteuses en dessous, par ses capitules disposés en petites grappes spiciformes nombreuses, et formant un long épi grêle (25 à 30 cent.), non interrompu, par les écailles de l'involucre recouvertes d'un tomentum très-épais, etc.

99. A. integrifolia Lin. Spec. 1189; Besser Abrotan. n 36; Turczan. Fl. Baik. dahur. 2, 64; Maxim. Prim. Al. amur. 166;A. vulgaris var. integrifolia Ledeb. Flor. ross.2, 585; Regel et de Herder in Plant. Raddeane, III, 2, 75; sinice PÉ, N'GÄ̈. 
Forma longifolia : foliis superioribus integris, anguste lanceolatis, 8-10 centim. longis.

$H a b$. : Sables humides et saumâtres non loin de la ville de N'gin-haï-tchéoû, et de la rivière Ta-hô, près du cap Chantong. - Fl. 18 septembre 1860.

Ar. géog. : Asie bor., Sibérie arctique, Baikalie, Altaï, Daoûrie près du fleuve Argun, Mongolie.- Chine (Chan-tong, Pé-tché-ly). -Prov. de l'Amour et de l'Ussuri.

Obs. : La variété longifolia diffère de l'A. integrifolia, forma typica du nord de la Chine, par ses tiges du double plus petites, par sa panicule moins fournie, et surtout par ses feuilles florales longuement lancéolées. Les feuilles inférieures sont de beaucoup plus étroites, entières, rarement à 3-5 divisions linéaires-lancéolées, toutes d'un vert foncé en dessus, et blanches-tomenteuses en dessous.

100. A. annua Lin. Spec. 1187 ; Ledeb. Flor. ross. 2, 592; Benth. Flor. Hongkong. 187; Turczan. Flor. Baik. dahur. 256; Trautv. Enum. plant. Sôong. n 616 ; Regrel et de Herd. in Plant. Radd. III, 2, p. 85; Sieb. et Zucc. Fam. nat. jap. 2, 62; Miquel Prol. fl.jap. 109; sinice Tsâo-tsâo.

Hab. : Champs cultivés et sablonneux près de Yan-taï.-Fl. 23 août.

Ar. géog. : Asie bor. et temp., Sibérie orient.près du lac Baikal, Selinginsk, Irkutsk.- Mongolie.- Asie occ., Caucase, Karabagh, Arménie. - Eur. orient., où il est naturalisé, Constantinople, Croatie, France à Marseille, etc. - Chine (Chû-san, Hong-kong, Chan-tong, Pé-tché-ly). - Japon.

101. A. capillaris Thumbg. Flor. jap. 309; Kæmpfer Amcenit. exot. fasc. V, p. 897; A. capillaris var. arbuscula Miquel Prol. A. jap. 107; affinis A. scoparice Waldst. et Kitaib.

«Caulibus 15-25 centim. altis, erectis, basi subnudis, cæterum » densissime stricteque ramosis et floridis; foliis ramorum flori» dorum glaberrimis, sterilium dense undique villosis (Miquel). »

$H a b$. : Roches micaschist. à la pointe du Tché-foû; falaises de Ki-tsen-sôo. - Fl. 2 septembre.

Ar. géog. : Asie intertrop. et temp., Indes-Orient. (Hooker). Chine (Chan-tong). - Japon. 


\section{MYRIOGYNE Less.}

102. M. minuta Lessing in Linnea VI, p. 219 ; Benth. Flor. Hongkong. 186; Maxim. Prim.amur. 163; Regel Tent.ussur. 91, et de Herder in Plant. Radd. III, 2, 92; Miquel Prol. fl.jap. 109; Artemisia minima Thumbg. Flor. jap. 311; Cotula minuta Forst. Prodr. p. 30: Centipeda orbicularis Loureiro $F l$. cochinch.; Miquel $F l$. ind. bat. 2, 89.

Hab. : Champs sablonneux à Yan-taï, et dans l'intérieur de la province du Chan-tong (G. Staunton).

Ar. géog.: Asie intertrop. et temp., Indes-Orient., Ceylan, Cochinchine. - Chine (Hong-kong, Chû-san, Chan-tong). Sôongarie. - Prov. de l'Amour et de l'Ussuri. - Japon. - Iles de l'Océan indien et austral, Java, les Philippines, Nouvelle-Hollande, Nouvelle-Calédonie. - Amér. mérid. - Chili.

\section{LEONTOPODIUM R. Brown.}

103. L. sibiricum Cassini Dict. scienc. natur. XXV, p. 475; Dec. Prodr. 6, 276; Ledeb. Flor. ross. 2, 614; Karel. et Kiril. Enum. pl. Sôong. no 460; Turczan. Flor. Baik. dahur. 2, 81; Maxim. Prim. amur. 164; Gnaphatium leontopodium var. sibiricum Regel et de Herder in Plante Raddeance reisen, etc. III, 2, p. 105; G. leontopodium Bunge Enum.pl. chin. bor. $\mathrm{n}^{\circ} 210$, ex parte.

Var. A sibiricum genuinum de Herder (loc.cit.).

Hab. : Roches micaschisteuses des basses collines, et falaises de Ki-tsen-sôo. - Fl. 30 juillet.

Ar.géog. : Asie bor., Sibérie altaï., Baikal., Daoûrie. - Chine (Chan-tong et Pé-tché-ly). - Prov. de l'Amour.

O3s. : Cette plante, qui a été réunie par MM. Franchet et Savatier au Gnaphalium leontopodiö̈des Wild., n'avait été trouvée que dans la Sibérie, le nord de la Chine et la province de l'Amour. Sa découverte dans les montagnes du Tché-foú et de l'île de Kiôusiôu à Japon, étend considérablement l'aréa de cette rare espèce. MM. Franchet et Savatier font remarquer que la présence du Leontopodium sibiricum dans l'île de Kiôu-siôu, constitue un fait de géographie botanique, d'autant plus intéressant, qu'on ne lui 
connait aucune station intermédiaire au Japon, soit dans l'île de Yéso, soit dans celle du Nippon.

\section{SENECIO L.}

104. S. argunensis Turczaninow Flor. Baikal. dahur. 2, 91; Regel Tent. A. ussur. 92, et de Herder in Plant. Radd. III, 2, p. 120; S. Jacobea Lin. var. grandiflorus, Turczan. in Dec. Prodr. 6, p. 350; Ledeb. Flor. ross.2, 635.

«Planta annua, caule erecto, simplici, supra medium polyce" phalo, glabro; foliis omnibus glabris, subtus puberulis, pinna» tisectis, radicalibus basi attenuatis, longe petiolatis, subglabris, "pinnatipartitis, caulinis sessilibus laciniatis, laciniis lineari»oblongis vel linearibus, grosse dentatis.

"Capitula erecta, magnitudine eis S. Jacobac, pedunculata, in » corymbum laxum disposita, involucri squamis linearibus, mar"gine scariosis, ligulis discum duplo superantibus. Akenia omnia " glabra, pappo caduco piloso coronata. »

Hab. : Marécages saumâtres du littorāl; presqu'île de Yan-taï. - Fl. 4 septembre.

Ar. géog. : Asie bor., Sibérie altaïque et baikalienne, Daoûrie, Mongolie. - Chine (Chan-tong, et Pé-tché-ly à Pé-king). - Prov. de l'Amour et de l'Ussuri.

Obs. : Dans le $S$. argunensis des plaines du Tché-foû, les feuilles caulinaires supérieures ont les divisions bien plus étroites que dans la plante des rives de l'Argun en Daoûrie, mais toutes les deux ont les akènes entièrement glabres, ce qui est un des caractères distinctifs de cette espèce.

\section{§ II. - GYNAROCÉPHALẺES Vaill.}

\section{ECHINOPS Lin.}

105. E. Gmelini Ledebour Flor. altaïca 4, 45, et Flor. ross. 2, 653 non Turczaninow; Trautv. Dissert. de Echinope $\mathrm{n}^{0} 3$, p. 14 (1833); Maxim. Prim. amur. 167; E. dahuricus Turczan. Fl. Baik. dahur. 2, 101 ; Fisher Hort. Gorenk. 37; Dec. Prodr. 6, 523 . 
Var. angustifolius Dec. loc. cit.; Regel et de Herder in Plant. Radd. "Laciniis lacinulisque angustioribus. "

"Caule erecto, stricto, apice bi-tricephalo, basi pubescente" hirto, demum tomentoso; foliis pinnatifidis, irregulariter denta» tis, supra atro-virentibus subglabris, subtus densissime albo» tomentosis, spinulosis, laciniis omnibus spina terminatis; spinis " brevibus tenuibusque; squamis involucri setiformibus, elonga"tis, inferioribus glabris setaceo-acuminatis, margine ciliatis, " patulis; pappo setoso, setis linearibus vix ciliolatis, basi connatis. „Flores cærulei, odore suaveolentes. »

Hab. : Falaises micaschist. et basses collines rocailleuses au dessus de Ki-tsen-sôo. - Fl. 20 juillet.

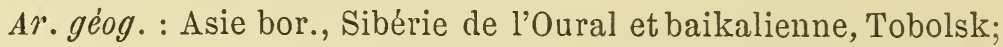
Daoûrie, Mongolie - Chine : Chan-tong (0. Debeaux). - Prov. de l'Amour.

\section{CIRSIUM Tourn.}

106. C. sejetum Bunge Enum.plant. chin. bor. no 202; 0. Deb. Flor. Shang-hai, no 65; Dec. in Prodr. 6. p. 643; sinice YûN-TCHû, TA-KY, Siâo-KY.

Hab. : Champs cultivés de la plaine du Tché-foû à Yan-taï, etc. Ar. géog. : Chine moy. et bor., Kiang-sou, Chan-tong, Pé-tché-ly.

107. G. (Corynotrichum) Tchefouense O. Debeaux, Msc. in Herb. 1860.

«Radix perennis, crassa, cautibus simplicibus, erectis, tricephalis, » 50-60 centim. altis, striato-sulcatis, basi subglabris, glandulosis, » apice arachnoïdeo-pilosis; fotiis semiamplexicaulibus, supra vi» rentibus glabris, subtus lævissime ad costam mediam puberulis, » angustis, sinuato-pinnatifidis, acuminatis, margine dentato-spi》 nosis: spinis inæquilongis subulatis, terminali atque eis lacinia》 1um longissimis; capitulis tribus subglobosis, solitariis, nudis, " erectis, longe pedunculatis; involucri squamis seriatim dense » spicatis, externis ciliato-glandulosis, lineari-lanceolatis, acumi» natis, dorso brunneis, intimis elongatis, subspathulatis, apice "scariosis, fimbriatis; setis pappi superne nudis, albidis, in clavu„ lam parum dilatatis. Flores disci purpurei. Akenia glabra." Hab. : Sommet des hautes montagnes du T'ché-foû, de 1,000 à 
1,100 mèt. d'altitude, dans les fissures des rochers.-Fl. 6 septembre 1860 .

Ar. géog. : Chine (Chan-tong).

Obs. : Notre C. Tchefouense appartient à la section Corynotrichum, laquelle se compose de trois espèces seulement, dont deux appartiennent à la flore du Népaul et une à l'A mérique boréale. Il se distingue par la glabrescence de toutes ses parties, par ses capitules au nombre de trois, portés chacun sur de longs pédoncules de 25 à 30 centim. de longueur, par les écailles intérieures de l'involucre dilatées en un long appendice lancéolé, scarieux frangé ou incisé sur les bords. Le caractère le plus remarquable est celui que fournissent les poils de l'aigrette, lesquels sont nus au sommet et dilatés en forme de petite massue.

\section{ANANDRIA Siegesb.}

108. A. bellidiastrum Dec. Prodr. 7, 40; Turczan. Flor. Baik. dahur. 2, p. 140; Maxim. Prim. A. amur. 175; Regel Tent. f. ussur. 96; Gerbera anandria Schultz Bipont. in Walp. Repert. bot. syst. 2, 792; Miquel Prol. jap. 118; Perdicium tomentosum Thumbg. Fl.jap. 316 (forma autumnalis).

Var. B autumnalis Turczan. in Dec. loc. cit.; Tussilago anandria Lin. Spec. 1813; Ledeb. Flor. altaïc. 4, p. 89.

Hab. : Sommet des hautes montagnes du Tché-foû, dans les fissures des rochers de 1,000 à 1,100 mèt. d'altitude; descend le long dess ravins dans la région mont. infér. à 400 mèt. d'alt.; falaises micaschit. de N'gin-haï-tchéou au cap Chan-tong. - Fl. 6-18 septembre 1860.

Ar. géog. : Asie bor. et or., Sibérie altaïque et baikalienne, Sibérie orientale près d'Ochostk, Daoûrie à Irkustk, Mongolie. Chine (Chan-tong et Pé-tché-ly). - Prov. de l'Amour et de l'Ussuri. - Japon (hautes montagnes du Nippon et de Fakonnat).

\section{§ III. - GHICORACÉES JUSS.}

SCORZONERA L.

109. S. austriaca Wild. Spec. 3, 1498; Dec. Prodr. 7, 120; Turczan. Fl. Baik. dahur. 2, 147 ; Maxim. Prim. $A$. amur. $17 \%$. 
Var. longifolia O. Debeaux Msc. in Herb. 1860; S. radiata Bunge. Enum. chin. bor. $\mathrm{n}^{0} 231$ non Fisher, nec Dec.; sinice Tien-MingTÔNG.

"Radix crassa, collo externo fibrillosa, foliis glabris, multi" nerviis, plus minusve elongatis latisque, margine undulatis, »12-20 centim. longis, 20-25 millim. latis, 4-5 plo flores superan»tibus; capituiis fere acaulibus, solitariis; squamis involucri » puberulis, elongatis, margine scariosis. »

$H a b$. : Prairies sablonneuses du littoral, plaine de Yan-taï. Fl. 12 juillet.

Ar.géog. : Le type : Eur. et Asie occid. - La variété: Asie bor., Sibérie altaïque, Baikal.,Daoûrie.-Chine (Chan-tong et Pé-tché-ly). - Prov. de l'Amour.

110. S. parviflora Jacq. Aust. 4, tab. 105; Ledeb. Flor. altaïca 4, 161; Dec. Prodr. 7, 121.

Forma elatior O. Debeaux Msc. in Herb. 1860 ; S. caricifolia Pallas Iter. 3, 131.

«Pluricaulis, cautibus 25-30 centim. altis, elongatis, erectis, " gracilibus, puberulis; foliis lineari-lanceolatis, glabris, omnibus 》 conformibus, superioribus flores 3-4 plo superantibus. »

Hab. : Pelouses sablonneuses du littoral; plaine de Yan-taï. Fl. 14 juillet.

Ar.géog. : Le type : Eur. or. et aust., France mérid., Hongrie, etc.- La variété : Asie bor., occ. et or., Sibérie, Altaï, Sôorgarie, désert de Khirghiz. - Chine, Chan-tong (O. Debeaux).

111. S. macrosperma Turczan. Flor. Baik. dahur. 2, 149; Ledeb. Flor. ross. 2, 795; Dec. Prodr. 7, 121; Maxim. Prim. fl. amur. 177; Regel Tent. fl. ussur. 97.

Forma angustifolia O. Debeaux Msc. in Herb. 1860.

«Hæc species ab omnibus hujus greneris valde distincta, akeniis 》 ultra semen-longe productis, et quasi rostratis, stylisque basi D persistente mucronulatis. »

Hab. : Pelouses sablonneuses du littoral, près de Yan-taï et de Ki-tsen-sôo. - Fl. 12 juillet.

Ar. géog.: Asie bor., Daoûrie. - Chine (Chan-tong au Tché-fou). - Prov. de l'Amour et de l'Ussuri.

Obs. : Le S. macrosperma, var. angustifolia du Tché-foû, est une 
simple forme à feuilles beaucoup plus étroites que celles du type décrit par Turczaninow. Cette rarissime espèce est caractérisée par le bec allongé de ses akènes, lequel n'est autre que la base persistante du style, particularité unique dans le genre Scorzonera.

MM. Bentham et Hooker n'ont point connu cette plante, qui n'a été trouvée jusqu'à présent qu'à Irkutsk et à Nerkintsk par Turczaninow, dans la province de l'Amour par M. Maximowicz, et dans celle de l'Ussuri par M. Regel.

\section{PICR IS L.}

112. P. japonica Thumbg. Flor. jap. 299; Dec. Prodr. 7, 131 ; Ledeb. Flor. ross. 2, 800; Turczan. Flor. Baik. dahur. in add. XLII; Maxim. Prim. fl. amur. 177; Sieb. et Zucc. Fam. nat.jap. 2,70; A. Gray in Plant. jap. exped. Perry, 314; Miquel Prol.jap. 120; P. dahurica Fisher in Dec. Prodr. 7, 129; P. kamtschatica Ledeb. Flor. altaïca 4, 159.

Hab. : Pelouses sablonneuses et prairies saumâtres autour de la rade de Yan-taï. - Bords des torrents, à sec pendant l'été, au-dessus de Ki-tsen-sôo. - Fl. 17 août.

Ar. géog. : Asie bor., Sibérie baikalienne, Daoûrie. - Chine (Chan-tong). -- Prov. de l'Amour et de l'Ussuri. - Kamtschatka. - Japon.

\section{LACTUCA L.}

113. L. amurensis Regel Index semin. hort. Petropol. p. 42 (1857), et Tent. flor. ussur. 97; Maxim. Prim. f. amur. p. 178.

«Planta herbacęa, annua, 2-3 pedalis, radice crassa, quasi napi"formi, collo fibrillosa, caulibus elatis, simplicibus, striatis, » lævigatis; foliis polymorphis, caulinis basi sagittatis, sessilibus, " grosse dentatis, margine setulosis ciliatis, nunc integris lineari》 lanceolatis, nunc pinnatifidis vel runcinatis-dentatis, lobis lineari» lanceolatis, acuminatis, inæqualiter dentatis, lobo terminali » integro acuminato.

»Flores in paniculam longe subspicatam dispositi, pedunculis »brevibus, 1-3-cephalis; involucri squamis ovatis-acuminatis; $\gg$ akeniis in rostrum subito attenuatis.» 
$H a b$. : Sables marécageux au milieu des grandes dunes de Fôuchan-yên, marais saumâtres de Yan-taï. - Fl. 2-10 septembre.

Ar. géog. : Chine : Chan-tong (O. Debeaux), Pé-tché-ly à Pé-king (Tatarinow). - Prov. de l'Amour et de l'Ussuri.

Obs. : Plante à feuilles polymorphes, vertes en dessus, glaucescentes en dessous, les inférieures et les caulinaires longues de 15 à 20 centim., les supérieures linéaires-lancéolées et toutes diversement sinuées-pinnatifides. La,racine est de la grosseur du doigt, fusiforme, striée et fibreuse au collet. Au Tché-foû, cette espèce ne se rencontre que dans les sables marécageux et voisins de la mer.

114. L. sonchifolia Benth. et Hook Gen. plant. 2, 55; Youngia sonchifolia Maxim Prim. amur. 180; Prananthes sonchifolia Bunge Enum. pl. chin. bor. no 226, non Wildenowii quæ Lactuca Heyneana Dec.

$H a b$. : Rochers micaschisteux des basses collines; falaises de Ki-tsen-sôo de 10 à 200 mètres d'altitude. - Fl. 15 juillet.

Ar. géog. : Prov. de l'Amour. - Chine : Chan-tong au Tché-foû, Pé-tché-ly à Pé-king où les jeunes pousses de cette espèce, nommées Koutaï, sont recherchées par les Chinois pour l'usage alimentaire. - Japon (A. Franch. et L. S'avatier).

Obs. : Le Lactuca sonchifolia Maxim. sub Youngia, qu'il ne faut pas confondre avec le Prenanthes sonchifolia de Wildenow, auquel Bunge avait rapporté ì plante de Pé-king, se distingue de celleci par les caractères suivants :

$1^{\circ}$ Par ses tiges peu élevées, de 30 à 40 centim. de hauteur, rameuses dès la base, à rameaux subdichotomes, divariqués et glabres;

$2^{\circ}$ Par ses feuilles roncinées, étroitement pinnatifides, à divisions dentées-aiguës, acuminées au sommet, les caulinaires embras. santes à divisions également dentées ou entières, et non hérissées ciliees sur les bords;

$3^{\circ}$ Par ses fleurs disposées en un corymbe largement dichotome, paniculé, à capitules petits, isolés, longueinent pédonculés, et formant une dichotomie régulière, et non disposées en glomérules géminés et brièvement pédonculés ;

$4^{\circ}$ Par ses akènes petits, noirs, linéaires-oblongs, glabres, striés, et dont la moitié supérieure se prolonge en un bec sensiblement acuminé et terminé, par une aigrette de même longueur que l'akène. 


\section{IXERIS Cassini.}

115. I. repens Dec. Prodr. 7, 157; Choirisis repens A. Gray Bot. jap. 397, et Plant. jap. exped. Perry, 315 ; Benth. Flor. Hongkong. 194; Pronanthes repens Lin. Amcen. acad. 2, 360; Lactuca repens Benth. et Hook. Gen. plant. 2, 526; sinice Yû-HOÛAN-LIEN.

Hab. : Plage maritime de Ki-tsen-sôo et de Yan-taï. - Fl. $1^{\text {er }}$ septembre.

Ar. géog. : Kamtschatka, îles Kouriles. - Chine (Hong-kong, Chan-tong, et probablement tout le littoral). - Japon.

\section{SONCHUS L.}

116. S. oleraceus Lin. Spec. 1, 116; Benth. Flor. Hongkong. 194 ; Roxbg. Fl. ind. 3, 462; Ledeb. Fl.altaïc. 4, 143; A. Gray Plant. jap. exped. Perry, 315; Miquel. Prol. A. jap. 123; sinice SHONG-TSAÏ.

Hab. : Champs cultivés à Yan-taï.

Ar. géog. : Eur. - Afriq. bor. et occ., St-Vincent. - Asie bor., occ. et or. - Indes-Orient., Sibérie altaïque. - Chine (Hong-kong, Chan-tong). - Japon. - Amér. bor.

Obs. : Cette plante, qui est identique à celle d'Europe, a été probablement introduite dans les cultures du 'Tché-fôu, où elle s'est acclimatée.

117. S. brachiotus Dec. Prodr. 7, p. 186; Ledeb. Flor. ross. 2, 818; Maxim. Prim. amur. 180; Turczan. Flor. Baik. dahur. 2, 169; 0. Debeaux Flor. Shang-haï, n $66 ;$ S. uliginosus Ledeb. Flor. altä̈. 4, 142, non M. Bieb.

Hab. : Marais saumâtres de la presqu'île de Yan-taï; dunes maritimes de Foû-chan-yên. - Fl. 4 septembre.

Ar. géog. : Asie bor., Sibérie, Altaï. - Chine (Kiang-soû, Chantong, Pé-tché-ly). — Prov. de l'Amour. - Japon.

\section{XANTHIUM L.}

118. X. strumarium Lin. Spec. 1400; Dec. Prodr. 5, 523; Benth. 
Fl. Hongkong. 181; Maxim. Prim. amur. 152; Turczan. Flor. Baik. dahur.2. 6I; Bunge Enum.pl. chin.bor. n 339;

Sieb. et Zucc. Fan. nat.jap. 2, 61 .

Var. indicum; X. indicum Roxbg. Cat. hort. calcut. 67 ; Wight Contrib. bot. of. ind. p. 17\%; sinice TSAN-EUL-TSÉ.

"Forma indica ( $X$. indicum Auct. asiat.) a $X$. strumario typico » recedit, fructibus basi ovalibus, longioribus, apice magis elon》gatis acuminatis; rostris uncinatis nec rectis; statura humiliore; 》 caulibus simplicibus, parce racemosis, foliis minoribus, etc.

Hab. : Sables maritimes sur la plage de Ki-tsen-sôo. — Fr. août et septembre.

Ar. géog. : La var. indica : Asie bor., occ. et or., Indes-Orient.Chine (Hong-kong, Chan-tong, Pé-tché-ly). - Prov. de l'Amour. - Japon.

\section{CAMPANULACEES Juss.}

Platycodon Alph. Dec.

119. P. grandiflorus Alph. Dec. Monogr. campanul.p. 125, et Prodr. 7, 442; Sieb. et Zuccar. Fam.nat.jap. 2, 55; Miquel Prol. fl. jap. 123; Ledeb. Flor. ross. 2, 150; Turczan. Fl. Baik. dahur. 2, 180; Maxim. Prim. amur. 84; R. Fortune Plant. chin. exsicc. $\mathrm{n}^{\circ} 149$; Campanula grandiflora Lin. fil . Supplem. 140.

Var.glaucus Sieb. et Zucc. loc. cit.; Campanula glauca Thumbg. Flor.jap. p. 88.

"Foliis caulinis magis approximatis aut subverticillatis, subtus » intense glaucis; floribus magnis, cæruleis, rarius albidis. »

Hab. : Basses collines micaschisteuses au bord des ravins, au-dessus de Ki-tsen-sôo (de 100 à 200 mètres d'altitude). - Fl. 14 août.

Ar.géog.: Asie bor., Sibérie, Altaï, Daoûrie, Mandchourie. Chine (Hong-kong, Tssé-chuen, Hou-pé, Ho-nan, Chan-s̀i, Chan. tong); cultivé dans les jardins à Pé-king et à Shang-haï. - Prov. de l'Amour et de l'Ussuri. - Japon.

Obs. : Le P. grandiflorus, par son long épi floral, à corolles d'un bleu clair, de 3 à 4 cent. de diamètre, par ses tiges simples, hautes de 60 centim. à 1 mètre, constitue une plante d'ornement du plus bel effet. 


\section{ADENOPHORA Fisher.}

120. A. coronipifolia Fisher Adumbr.gener. adenoph. p. 5 ; Dec. Prodr. 7, 493; Ledeb. Flor. ross. 2, 893; Turczan. Flor. Baik. duhur. 2, 189; Maxim. Prim. amur. 185; Campanula coronopifolia Ræm. et Schultes Syst.veget. 5, $15 \%$.

«Planta sat polymorpha, radice perenni, longa, crassa; cautibus » simplicibus striato-sulcatis, erectis flexuosisve; foliis alternis » radicalibus caulinisque petiolatis, floralibus tantum subsessili» bus, ovato-rotundatis, basi cordatis, crenato-dentatis, supra " glabris vel parce pilosis, subtus ad nervos præsertim hirto» setosis glabrisve, floribus nutantibus, nunc solitariis spicam » laxam efformantibus, nunc parce racemosis, in petiolo communi » 2-3 glomeratis; calicis lobis lineari-lanceolatis, integris, tertiam » partem corollæ haud superantibus, adpressis vel patentibus.

»Flores campanulati, azurei, stylo longitudine corollæ. »

Var. A latifolia Fisher loc. cit. : foliis latioribus, ovato-cordatis, anguste serratis, 45-50 millim. longis, 30-40 millim. latis.

Var. B angustifolia O. Debeaux in, Herb.: foliis radicalibus angustis, subintegris, inæqualiter serratis, 80 centim. longis, 10-15 millim. latis.

$H a b$. : Les deux formes A et B abondent sur les rochers micasch. au bord de la mer; pointe du Tché-foû, falaises de Ki-tsen-sôo, etc. - Fl. 16 août.

Ar. géog. : Asie bor., Sibérie altaïque, Daoûrie. - Chine (Chantong, Pé-tché-ly). - Prov, de l'Amour.

Obs. : Cette plante est très-polymorphe dans la même localité; on la trouve tantôt à feuilles larges, ovales, profondément cordées à la base, tantôt à feuilles étroites, longuement lancéolées. Son inflorescence est également variable. Dans quelques échantillons, les fleurs sont solitaires, presque sessiles, penchées et disposées en un long épi lâche et terminal; dans quelquẹs autres, celles-ci sont disposées en petits glomérules (de 2 à 3 ) et longuement pédonculées. Les divisions calicinales sont, ou courtes et appliquées sur la corolle, ou bien à divisions écartées et égalant au plus le tiers de celle-ci.

Les deux variétés latifolia et angustifolia de l'A. coronopifolia ont été aussi observées par M. Maximowicz dans la région de 
l'Amour. Miquel signale au Japon une autre espèce d'Adenophora (A. verticillata Fish.), qui aurait les deux formes à feuilles larges ovales obcordées, et à feuilles longuement linéaires-lancéolées.

\section{A. trachelioïdes Maximowicz Primitice flore amurensis} p. 180 (in nota).

"Radice perenni, crassa, caulibus simplicibus, apice ramulosis, * striatis, flexuoso-erectis, ad axillas foliorum angulatis, ramis » divaricatis; foliis alternis, læte virentibus, in petiolum longum » attenuatis, omnibus utrinque glabris, angustissimis, acuminatis, » apice falcatis, grosse crenato-dentatis, inferioribus 15 millim. » latis, 20 centim longis; floribus paucis (2-3), ad ramulos verticil» latis, erectis; calicis laciniis lanceolatis, angustis, integerri» mis, stellatim patentibus, dimidiam partem corollæ æquantibus, » capsulamque globosam plus duplo superantibus, stylis filiformi»bus, apice dilatatis, longe exsertis; corolla azurea, 2 centimet. » usque longa. »

Forma A cordatifolia 0. Debeaux in Herb. : foliis radicalibus angustis, superioribus ovatis, cordatis, acutis.

Forma B angustifolia : foliis omnibus angustissimis.

Hab. : Falaises micaschisteuses de la pointe de Tché-foû. Fl. 7-20 août.

Ar. géog. : Chine (Chan-tong au Tché-foû, Pé-tché-ly à Péking).

Obs.: Au milieu des nombreux spécimens d'A. coronopifolia, que j'ai récoltés au Tché-foû, il s'est rencontré quelques échantillons d'un facies tout différent, qu'il m'a été impossible de rapporter à cette espèce. La description de M. Maximowicz de son A. trachelioïdes observé aux environs de Pé-king, convient de tout point à la plante du Tché-foû. Ses rameaux divariqués, terminés au sornmet par des glomerules de 3 à 4 fleurs, à divisions calicinales toujours étalées en étoile, égralant presque la corolle, ses feuilles longuementacuminées-lancéolées ou ovales-lancéolées, arquées, son style dépassant de beaucoup la corolle dans toutes les fleurs que j'ai examinées, et enfin sa glabrescence, la distinguent suffisamment de l'A. coronopifolia. 
APOCYNÉES R. Brown.

APOCYNUM L.

122. A. sibiricum Pallas, in R. Brown. Asclepiad. 58 (1819); Ræm. et Schult. Syst. veget. 4, 415; Ledeb. F'lor. altaïc. 1, 235; Bunge Enum. pl. chin. bor. n० 243; A. venetum Lin. Spec. 311; Dec. Prodr. 8, 440, var. sibiricum; Miquel. Prol. $f$. jap. 62, ex parte.

Hab. : Prairies inondées et saumâtres près de la rade de Yantaï; dunes marécageuses de Foû-chan-yên. - Fl. 15 juillet, fr. 4 septembre.

Ar.géog. : Asie bor., occ. et or., Sibérie altaïque.-Chine(Chantong, Pé.tché-ly). - Astrakan. - Japon.

Obs. : L'Apocynum sibiricum du Tché-foû ne diffère de la plante décrite dans le Prodromus (loc. cit.), que par ses fruits géminés, longs de 170 millim. au lieu de 110 millimètres.

\section{ASCLÉPIADÉES R. Brown.}

Metaplexis R. Brown.

123. M. chinensis Decaisne in Dec. Prodr. 8,511; Maxim. Prim. f. amur. 97 ; Regel Tent. A. ussur. 104; Miquel Prol.jap. 58; Sieb. et Zucc. Fam. nat.jap. 37; M. Stauntoni Ræm. et Schultes Syst.veget. 6, p. 3; Urostelma chinensis Bunge Enum. chin. bor. n 247 ; R. Fortune Plant. chin. exsicc. no 87 .

Hab. : Haies et clôtures des jardins, autour de Ki-tsen-sôo. Fr. 23 août.

Ar. géog.: Chine (Chan-tong et Pé-tché-ly). - Prov. de l'Amour et de l'Ussuri. - Japon.

\section{VINCETOXICUM Mænch.}

124. V. versicolor Decaisne in Dec. Prodr. 8, 524; Cynanchum versicolor Bunge Enum. pl. chin. bor. n० 252.

Hab. : Sables maritimes, sur la plage de Yan-taï. - Fl. 4 septembre.

Ar. géog. : Chine boréale, Chan-tong, Pé-tché-ly. 
125. V. amplexicaule Sieb. et Zucc. Fam. nat.jap. 2, 38; Miquel prol. flor. jap. 58; Regel. Tent. A. ussur. 166; sinice TsarKIO.

«Planta perennis herbacea, glaurescens, caule erecto, basi sim» plici, apice ramuloso, puberulo; foliis caulinis densis, sessilibus, 》amplexicaulibus, basi cordatis, obovato-ellipticis, mucronula» tis vel brevissime cuspidatis, utrinque glabris, subtus venoso» reticulatis; cymis umbelliformibus pedunculatis, subdichotomis, » folia floralia subæquantibus; corollæ laciniis ovato-lanceolatis, 》) acutis, puberulis ciliolatisve; corolla patente, intus parum "puberula; corona staminea phyllis obtusis, carnosis, basi unitis, »dorso depressis: folliculis plerumque solitariis, inferne anguste » ovoïdeis, dein longe rostrato-acuminatis, 50 millimet. longis, » erectis, maturitate pendulinis. »

Hab. : Sables maritimes et marécageux de la presqu'île de Yantaï; dunes de Foû-chan-yên. - Fl. 4 septembre, fr. 2 octobre.

Ar. géog. : Chine (Chan-tong). - Prov. de l'Ussuri. - Japou.

Obs.: Cette plante se distingue de toutes les espèces asiatiques du genre Vincetoxicum, par ses feuilles coriaces glaucescentes, largement embrassantes à la base. Dans les échantillons de Yan-taï, les tiges sont pubescentes au sommet, tandis qu'elles sont glabres dans ceux de provenance japonaise.

126. V. sibiricum Decaisne in Dec. Prodr. 8, p. 525; Cynanchum sibiricum R. Brown Asclepiad. p. 48, no 15; Bunge Enum. pl. chin. bor. no 244; Ledeb. Flor. allaï. 1, 279; Asclepias sibirica Lin. Gmel. Flor. sibir. 4, 77; sinice TSAN-KIO.

Forma linearifolia 0. Debeaux. Msc. in Herb. (1860) : foliis omnibus anguste lineari-lanceolatis.

Hab. : Sables maritimes de la presqu'île de Yan-taï; dunes de Foû-chan-yên. - Fl. 14 juillet, fr. 23 août.

Ar.géog. : Asie bor., Sibérie arctique, Altaï, Baikal., Sôongarie, désert de Kirghiz, Daoûrie. - Chine (Chan-tong, Pé-tché-ly).

Obs. : Le Cynanchum sibiricum, qui paraît être spécial aux régions sibériennes, n'avait été trouvé jusqu'à présent en Chine que dans les montagnes au Nord de Pé-king. Cette espèce est abondante au Tché-fou, et ne diffère du type décrit par Ledebour, que par ses feuilles plus étroites et longuement linéaires-lancéolées. 
CYNANCHUM R. Brown.

127. G. pubescens Bunge Enum. plant.chin. bor. n² 248; Decaisne in Dec. Prodr. 8, p. 548.

«Herbaceum, volubile, radice crassa, repente, perenni, cautibus » incano-tomentosis, foliis oppositis cordatis, acuminatis, longe » pedunculatis, plus minusve pubescentibus; inflorescentia corym" bosa, pedunculo communi foliis breviore, apice diviso, duas » umbellulas lateralesque efformante, pedicellis calicibusque inca» nis, pubescentibus; corollæ duplicis laciniis seu gymnostegio " acutis, linearibus, externis decemfidis, lobis alternis ovatis, "acutis; internis quinquefidis brevioribus, filiformibus; folliculis » semper solitariis, fusco-brunneis, læviter striatis, puberulis, 80 " millim. longis, 8 millim. latis; seminibus apice comosis, ovato» ellipticis, complanatis, margine membranaceis revolutis, lævi" gatis, fuscescentibus. »

Hab. : Rochers micaschist. du littoral, au cap de Tché-foá; falaises de Ki-tsen-sôo et dunes de Foû-chan-yên - Fl. août, fr. 7 octobre.

Ar. géog. : Chine (Chan-tong et Pé-tché-ly).

Obs.: Cette espèce rappelle par son facies le $C$. acutum de l'Europe australe. Ni Bunge ni M. Decaisne ne paraissent avoir connu le fruit de cette plante, car ils en auraient fait mention dans leurs descriptions. C'est une lacune que j'ai pu remplir.

Le $C$.pubescens a une racine noueuse rampante, de la grosseur du doigt, et émettant des radicelles fibreuses à la face inférieure des nœuds; les tiges grêles et volubiles sont recouvertes d'un duvet fin et blanchâtre. Ses feuilles inférieures sont largement cordiformes à la base, acuminées, les supérieures beaucoup plus petites, mais de forme semblable. L'inflorescence est axillaire dans notre plante; chaque pédoncule commun se bifurque à son extrémité en deux pédicelles floraux écartés, divariqués, et supportant chacun une ombellule de 6 à 10 fleurs blanchâtres. Les follicules sont allongés, acuminés aux deux extrémités, solitaires dressés, d'une couleur fauve-roussâtre; enfin les graines sont aplaties, membraneuses, enroulées sur les bords, d'un brunmarron et entièrement glabres. On trouve communément le $C$. pubescens sur les rochers maritimes dı littoral du Tché-foû. 
CONVOLVULACEES JUSS.

Pharbitis Choisy.

128. Ph. nil Choisy Convolv. orient. p. 57 et in Dec. Prodr. 9, 343; Sieb. et Zuccar. Fam. nat. jap. 2, 24; Ipomea nil Roth. Catal. botan. 1, 36; Bunge Enum. chin. bor. n 258; I. triloba Thumbg. Flor. jap. 86; Plıarbitis triloba Miquel Prol. A.jap. 26; Convolvulus nil Lin. Spec. 219; sinice KIEN-NEÔUTZÉ.

Var. abbreviata Choisy (loc. cit.).

«Planta humilis, foliis cordatis, trilobatis, lobis acuminatis, » pedunculis brevissimis. »

Hab. : Rochers micaschisteux du littoral, falaises de la pointe du Tché-foû, haies autour de Ki-tsen-sôo. - Fl. et fr. 25 septembre.

Ar.géog.: Régions intertrop. des deux mondes. - Asie cent., Indes-orient., Cochinchine. - Java. - Chine (Kiang-soû, Chantong, Pé-tché-ly). - Japon, etc.

\section{CONVOLVULUS L.}

129. G. arvensis Lin. Spec. 218; Choisy in Dec. Prodr. 9, 406: Bunge Enum. chin. bor. n² 260; sinice PÉ-TSAN-LIÉoû.

Var. insignis O. Debeaux in Herb. 1860.

"Planta gracilis, annua, caulibus simplicibus, prostratis, » 60-80 centim. elongatis et ultra, puberulis; foliis crassiusculis, » breviter in petiolum attenuatis, basi hastato-sagittatis, glabris, » auriculis lanceolatis lobo intermedio elongato, apice elliptico»obtuso mucronulato; foribus (1-2) in axillis foliorum nascenti» bus, longe pedunculatis, peduuculo filiformi folia plus duplo » superante.

»Corolla rosea, ampla, calice plus triplo longior, sepalis ovato»rotundatis, margine scariosis.»

Hab. : Champs cultivés, bords des chemins à Yan-taï, etc. Fl. août.

Ar.géog. : Le type : Eur. - Afriq. bor. et cent., Égypte, Abyssynie, Maroc, Madère, îles de l'Océan indien, Maurice.-Asie bor., oce. et or. - Prov. caspiennes, sibérie, Daoûrie, Mongolie, Indes- 
orientales, Arabie. - Chine (Chan-tong, Pé-tché-ly). - Japon. Amér. bor. et aust., Mexique. - La Plata, etc.

Obs.: Notre variété insignis du $C$. arvensis est remarquable par ses feuilles hastées-sagittées, ses fleurs longuement pédonculées, par ses pédoncules naissant à l'aisselle des feuilles et dirigrées du même côté que celles-ci. Cette disposition des feuilles et des fleurs rend la tige entièrement nue d'un côté, celui qui touche le sol. Dans la plante du Tché-foû, les tiges sont couchées, rampantes et non volubiles.

\section{CALYSTEGIA R. Brown.}

130. G.dahurica Choisy in Dec.Prodr.9,433; Turczan. Flor. baik.dahur. 2, 287; Maxim. Prim. Al. amur. 200; Regel Tent. Al. ussur. 107; C. subvolubilis Ledeb. Flor. ross. 3, 94; sinice SrûEN-HOâ.

Var. pellita Choisy (loc. cit.); Conv. pellitus Ledeb. Fl. altaïca 1, 223; C. subvolubilis Bunge Enum. chin. bor. no 259.

«Caulibus volubilibus, dense villosis; foliis ovato-oblongis, basi 》 cordatis, apice mucronulatis, tomentosis ; pedunculis unifloris, 》 solitariis; corolla magna, rosea; sepalis villosis ciliatisque. »

Hab. : Rochers micaschit. et falaises du cap de Tché-fon, sables maritimes de l'ancien camp français près de Yan-taï. Fl. 12 juillet- 4 août.

Ar. géog. : Asie bor., Sibérie altaïque et baikalienne, Daoûrie à Irkutsk. - Prov. de l'Amour et de l'Ussuri. - Chine (Chan-tong. et Pé-tché-ly).

\section{CUSCUTA L.}

131. G. chinensis Lamk. Encycl. 2, 229; Choisy in Dec. Py'odrr. 9, 45\%; Engelm. Gen. Cuscute spec. no 27; Wight Icones 4, tab. 1373; Miquel. Prol. jap. 26; C. sulcata Roxbg. Flor. ind. 1, 447; Grammica aphylla Loureiro Flor. coch. 1, 212; sinice Tou-TZÉ-TZÉ.

Hab. : Sables maritimes du littoral, plage de Ki-tseu-sôo sur diverses plantes, Hitiscus ternatus, Tribulus terrestris, Cassia mimosoïdes, Crotalaria brevipes, etc. - Fl. août.

Ar. géog : Asie intertrop. et bor., Ceylan, Indes-orient., Silhet, Malabar, Bengale, Mongolie. - Chiue bor.: Chan-tong (O. De- 
beaux), Pé-tché-ly sur le Corispermum pungens (Staunton). Japon (Miquel).

Obs. : Le Batatas edulis Choisy (Convolvulus batatas Lin.), sinice Hoûang-TChé et Houangr-Tsaï, est cultivé en grand dans le Tchéfoû et toute la province du Chan-tong pour ses racines alimentaires.

\section{BORRAGINEEES JusS.}

TOURNEFORTIA L.

132. T. arguzia Ræm. et Schult. Syst.veg. 4, p. 540; Dec. Prodr. 9, p. 512; Bunge Enum. chin. bor. n ${ }^{\circ} 268$; Franch. et Savat. Enum.plant.jap. 1, p. 331; Boiss. Flor. orient. 4, p. 125; Messerschmidia arguzia Lin. fil. Suppl. 132; Heliotropium japonicum A. Gray, Bot.jap., p. 403; sinice TsôtTsâo.

Var. A latifolia : foliis subspathulatis, ovatis, acutis;

Var. B angustifolia Dec. (loc. cit.) : foliis omnibus lanceolatis.

$H a b$. : Sables maritimes du Tché-foû, plages de Ki-tsen-sôo et de la baie de Yan-taï. - Fl. juillet, fr. août-septembre.

$A r$. géog. : Europe austro-orient., Russie moyenne, Podolie, Caucase. - Astrakan. - Asie bor. et occ., Asie-min., Sibérie altaique sur les rives de l'Irtisch, Daoûrie près de l'Argun, Mongolie. Chine bor., Chan-tong et Pé-tché-ly. - Japon.

Obs. : Cette espèce se retrouve en abondance dans la province limitrophe du Pé-tché-ly, sur les rives du Pei-hô à Tien-tsin et autour de Pé-king. Elle varie beaucoup par la forme des feuilles, et la villosité plus ou moins dense de toutes ses parties. Son port varie également dans la même localité, car elle offre des tigres tantôt droites et tantôt diffuses et couchées sur le sol. Les deux formes $\mathrm{A}$ et $\mathrm{B}$, à feuilles larges ou étroitement lancéolées, se trouvent souvent ensemble sur les sables du Tché-foû.

BOTHRIOSPERMUM Fish. et Mey.

133. B. kusnetzowii Bunge Index sem. hort. Dorpat. (1840), p. 7; Dec. Prodr. 10, p. 116; B. chinense Fish. et Mey. Ind. sem. hort. Petrop. (1835), non Bunge.

«Planta annua, herbacea, patenti-hispida, seu setoso-pilosa, cau- 
》 libus simplicibus suberectis, basi ramosis vel in parte dimidia 》 superiore; racemis erectis subflexuosisve; foliis oblongis, supe》 rioribus lineari-oblongis, utrinque setosis, setis basi subtuber" culatis; calicibus fructiferis nutantibus, pedicello minoribus, » vel subæqualibus, laciniis lanceolatis conniventibus; nucutis » calice brevioribus, obovoïdeis, ellipsoïdeo-convexisve, minute " granulatis.

» Flores cærulei, calicem superantes, juniores ad apices ramo» rum condensati, inferne laxiores. »

Hab. : Bords des champs sablorıneux à Yan-taï. - Fl. et fr. 20 juillet.

Ar.géog. : Chine bor. (Dec.), Chan-tong au Tché-foû (O. Debeaux).

Obs.: Le B. kusnelzonii paraît très-voisin du $B$. secundum Maxim. in Prim. flor. amur. p. 202. On le distingue facilement de celui-ci par ses nucules beaucoup plus grandes, et surtout par le nombre des écailles situées à la gorge de la corolle. Ces écailles sont au nombre de dix dans le $B$. secundum, et de cinq seulement dans le $B$. kusnetzowii. Le $B$. chinense Bunge non Fish. et Mey. est une autre espèce affine, et spéciale au nord de la Chine.

\section{ECHINOSPERMUM Swartz.}

134. E. lappula Lehm. Asperifol. no 94; Dec. in Prodr. X, p. 136; Bunge Énum. chin. Zor. n²67; Boiss. Flor. orient. 4, p. 249; Franch. et Savat. Enum.jap. 1, p. 336; Myosotis lappula Lin. Hab. : Champs sablonneux antour de Yan-taï. - Fl. et fr. 15 juillet.

Ar. géog. : Fur. bor., cent. et aust. - Asie bor. et occ. - Arménie, Lazistan, Transcaucasie, Perse, Sibérie altaique, Mongolie. Chine (Chan-tong et Pé-tché-ly).- - Japon. - A mériq. bor., Canada, Orégon. - Afriq. bor.

Obs. : Nos spécimens récoltés au Tché-foû n'offrent aucune différence avec ceux provenant de l'Europe centrale. Le facies en est absolument le même.

SOLANEEES Juss.

LYcrim L.

135. L. chinense Mill. Dict. n" 5; Blume Bijdraj. p. 707; Dun. 
in Dec. Prodr. XIII, p. 510; Bunge Enum. chin. bor. no 276; O. Debeaux Flor. Shang-hä̈, no 79; Benth. Flor. Hongkong. p. 245; Sieb. et Zuccar. Abhand. bayer. Acad. phil. class. 4, p. 147; Miquel Prol.jap. p. 282; Franch. et Savat. Enum.jap. 1, p. 341; L.barbarum var. chinense Ait. Hort. kew. 1, p. 257 ; L. barbarum Thumbg. Flor. jap. 93 ; sinice Koû-KI-TZÉ, Koû-KI, Tr-Koû-PI.

«Suffrutex humilis, parce ramulosus, in axillis foliorum spi»nescens, spinis brevibus; ramis pallide-albidis, acute angulatis, » nunc diffusis prostratis, nunc virgatis pendulisque; foliis ternis 》vix petiolatis, basi attenuatis, late ovatis, acutis; calicibus 》 subscariosis, 4-5 fidis, laciniis sæpe dentatis; corolla calice duplo »longiore, flavo-viridescente; staminibus exsertis; baccis ovato》oblongis, rubro-coccineis. »

Hab. : Rochers micaschisteux du littoral, à la pointe de Tchéfoû et au-dessous du fort chinois. - Fl. et fr. juillet-août.

Ar.géog. : Chine (Hong-kong, Koûang-tong, Kiang-sou, Chansi, Chan-tong, Pé-tché-ly). - Japon (Nangasaki, Yokohama). Cochinch. - Natur. dans l'Eur. aust., France mérid. à Montpellier, Perpignan, etc.

\section{NICOTIANA Tourn.}

136. N. tabacum Lin. Spec. 258; Dun. in Dec. Prodr. XIII, p. 557; Regel Tent. flor. ussur.p. 110 ; Miquel Flor. ind. batav. 3, p. 560, et Prol. Al. japon. 281.

Var. attenuatum Dun. (loc. cit.) : foliis lanceolatis, acutis, basi attenuatis, inferioribus latioribus.

Hab. : Cultivé en grand dans la province du Chan-tong.

$A r . g e ́ o g .:$ Origin. de l'Amér. aust. et cent, d'où il a été importé en Europe, en Asie, en Afrique, dans les îles de la Malaisie, aux Philippines et au Japon.

Obs.: Le tabac a été introduit à la Chine vers la fin du $16^{\mathrm{e}}$ siècle, et son usage en fut interdit par les empereurs de la dynastie des Mings. On le cultive aujourd'hui à l'île Formose, et dans les provinces méridionales et centrales de la Chine, ainsi que dans le Chan-tong. Le tabac récolté dans cette dernière province est très-estimé des Chinois, et il s'en fait un trafic considérable dans le petit port de Yan-taï. 
On cultive aussi les Nicotiana rustica L. et $N$. chinensis Fisher. Cette dernière espèce nommée YÉ-YÊN et YÊN-Tsâo est beaucoup moins répandue dans les cultures que le $N$. tabacum. Miquel (Prolusio flore japonica, p. 281) signale le $N$. chinensis comme étant spontané dans l'île de Kiû-siû au Japon.

Plusieurs autres solanées alimentaires sont cultivées au Tchéfoû. Je citerai principalement les suivantes : Solanum tuberosum L. (sinice Hô-LAN-CHôu et YANG-CHôu) ; S. melongena L. (sinice KIA-TZÉ) ; les Capsicum annưm et C. longum L. (sinice Lô-TsIâo; très-fréquemment employés comme condiment dans la cuisine chinoise, etc.

\section{SCROPHULARIEEES R. Brown.}

LINARIA Juss.

137. L. vulgaris Mill. Dict. $\mathrm{n}^{\circ} 1$; Chavannes Monog. Antirnh. p. 131; Maxim. Prim. fl. amur. 205 ; Reg.el Tent. fl.ussur. 110; Turczan. Flor. baik.-dahur. 2, p. 328.

Var. chinensis Bunge Ined. in Herb. Dec. « Foliis anguste linearilanceolatis, lanceolatisve. »

Hab. : Champs cultivés du littoral, fossés humides autour de Yan-taï. - Fl. et fr. septembre.

Ar. géog. : Europe, Asie bor., Russie arct., Sibérie altaiq. et baikal., Daoûrie, Tartares Tongoûs, Mongolie. - Chine. - Prov. du Chan-tong (O. Debeaux) et du Pé-tché-ly vers les limites de la Mongolie et près de la grande muraille. - Prov. de l'Amour et de l'Ussuri. - Amér. bor. à Boston.

Obs. : Le L. vulgaris, dont la station au 'Tché-foû me paraît être très-intéressante, n'a pas varié dans cette localité. Les nombreux spécimens que j'ai récoltés à Yan-taï ont tous les feuilles étroites linéaires-lancéolées, et les tiges simples ou peu rameuses au sommet. Il est à prěsumer que cette plante a été introduite avec les céréales dans cette partie du Chan-tong.

\section{MAZUS Lour.}

138. M. rugosus Loureiro Flor. coch. p. 385 ; Dec. in Prodr. X, p. $375 ;$ O. Deb. Flor'. Shang-haï, n 80 ; Franch. et Savat. Enum.jap. 1, p. 344; Lindernia japonica Thumbg. 
Hab. : Champs sablonneux autour de Yan-taï, surtout dans les terres semées de blé noir (Fagopyrum esculentum). - Fl. et fr. 1-15 octobre.

Ar. géog. : Asie subtrop., cent. et bor., Indes-orient., Birmanie, Himalaya. - Iles de la Míalaisie. - Cochinch. - Chine littor., Kiang-sô̂, Chan-tong, Pé-tché-ly. - Japon. - Prov. de l'Amour et de l'Ussuri.

\section{VERONICA L.}

139. V. spuria Lin. Spec. 12; Ledeb. Flor. ross. 3, p. 251 ; Turczan. F'lor. baik.dahur. 2, p. 339; Maxim. Prim. amur. 207; $V$. paniculata Lin. Spec. 18 (pro parte); Miquel Prol. jap. p. 51 ; Franch. et Savat. Enum. jap. 1, p. 348; G. Staunton Plant. chin. exsicc. (1794).

Forma A angustifolia Fisher in Cat. horti. Gorin.: Dec. in Prodr. X, p. 465 .

«Herbacea, perennis, radice fasciculata; caulibus simplicibus, »vel superne parum ramulusis, 50-85 centim. altis, foliisque »adpresse puberulis, plerumque oppositis, lanceolatis, acutis, » irregulariter crenato-serratis, superioribus linearibus, apice »dentatis, basi angustatis; racemis longe paniculatis, panicula » conferta, pedicellis calice longioribus. »

Forma B latifolia O. Deb. in Herb. (1860).

«Foliis inferioribus 3-plo latioribus quam in præcedente, »serraturis minus profundis, margine ciliato-dentatis. »

Hab. : Collines sablonneuses au nord de Si-nen-kôo; pelouses maritimes sur la plage de Fô̂-chan-yên. -- Fl. 7 août.

$A r$. géog. : Eur. austro-orient., France orient., Italie sup., Hongrie.-Asie bor., Sibérie altaïque, Baïkalie, Daoûrie, Mongolie. Chine bor. (Chan-tong et Pé-tché-ly). - Prov. de l'Amour. Japon.

\section{SIPHONOS'IEGIA Benth.}

140. S. chinensis Benthain Scrophul. indic. p. 51, et in Dec. Prodr. X, p. 538; Houk. et Arn. Bot. voy. Beech. p. 203, tab. 44; Bunge in Bull. soc. acad. St-Pétest. VII, p. 278; Maxim. Prim. amur. 208; Regel Tent. A. ussur. 112; Sieb. et Zuc. Fam. nat. jap. 2, p. 21; Miquel Prol. jap. 53; Franch. et Savat. Enum.jap. 1, p. 350 ; sinice LiEoû-KI-Noû, Loû-Tsi-noû. 
«Annua, herbacea, erecta, 30-45 cent. alta, caulibus rigidis » puberulis, simplicibus, racemo primario paniculam amplam effor》mante; foliis alternis, apice caulium oppositis, pinnatisectis; »calice auguste tubuloso, sulcato, 10-costato, laciniis lanceola» tis acutis, integris; corolla lutea, labio superiore incurvato, » inferiore trifido, palato triplicato.

» Flores sessiles, vel breve pedunculati, inferiores alterni, supe» riores oppositi. »

Hab. : Collines micaschist. du littoral à Ki-tsen-sôo; falaises du cap de Tché-fou. - Fl. 10 août.

Ar. géog. : Chine bor etaust. (Macâo, Chan-tong, Pé-tché-ly). Prov. de l'Amour et de l'Ussuri. - Japon.

\section{OROBANCHÉES L. C. Rich.}

OROBanche L.

141. O. ammophila C. A. Meyer in Ledeb. Icon. plant. flor. ross. et alt. illust. tab. 389; Ledeb. Flor. ross. 3, p. 320, et Flor. alt. 2, p. 454; Miquel Prol.jap. 287; Franch. et Sav. Enum.jap. 1, p. 353.

Hab. : Sur les rochers micasch. à la pointe de Tché-foû et autour du vieux fort, parasite sur les racines de l'Artemisia capillaris. Fl. 2 septembre.

Ar. géog. : Asie bor. et or., Sibérie altaïque près du fleuve Irtysch, Sôongarie à Irkutsch, désert de Kirghyz. - Chine (Chantong et Pé-tché-ly). - Province de l'Amour et de l'Ussuri. Corée. - Japon.

Obs. : Dans l'O. ammophila du Tché-foû, les bractées sont un peu plus courtes que dans la plante de Sibérie et elles atteignent à peine le milieu dẹ la corolle. Sa taille est moins élevée (8 à 10 centim.) et sa villosité moins dense. L'épi florifère est un peu velulaineux dans tous nos échantillons.

BIGNONIACEEES R. Brown.

Sesamulu L.

142. S. indicum Dec. Plant. rar. Genèv. p. 18, tab. 5, et in Prodr. IX, p. 250 ; Miquel Prol.jap. 286. 
Forma subindivisa Dec. (loc. cit.); S. orientale Lin. Spec. 883 ; 0. Deb. Flor. Shang-hä̈, no 75 ; sinice Hoû-мâ, Tché-мA-тzé.

$H a b$. : Cultivé en grand sur les collines sablon. au-dessus de Si-nen-kôo, de Ki-tsen-sôo et de Yan-taï, ainsi que dans presque toute la province du Chan-tong.

Ar. géog. : Asie cent., or. et occ., Indes-orientales. - Java. Chine (Tché-kiang, Kiang-soû, Kiang-nan, Chan-tong, Pé-tchély, etc.). - Japon (Kiû-siû). - Afriq. or., occ. et subtrop. Égypte, Sénégal, Bénin, Fernando-Pô, etc. - Amér. intratrop

\section{LABIÉES Juss. \\ Plectranthus L'Hérit.}

143. P. (Isodon) Pekinensis Maxim. Prim. A. amur. p. 213.

«Herbaceus, radice bisannua? vel potius perenni; caulibus » erectis simplicibus, aut superne ramulosis, tenuiter puberulis, »40-50 centim. altis, racemis subfastigiatis, inflorescentiam 》 corymboso-paniculatam efformantibus: foliistriangulari-ovatis, " acutis, inferioribus caulinisque longe acuminatis, in petiolum " medio alatum subito attenuatis, reticulato-venosis, ad venas »utrinque puberulis, grosse crenato-dentatis, serraturis obtusis, » superioribus subglabris; bracteis pedunculos æquantibus vel " paulo superantibus, ovato-acutis, integris; cymis 3-5 floris, " pedunculis ramulos subæquantibus; calicis laciniis 5, incano»pubescentibus, pedicello brevioribus; corolle tubo basi deflexo, » superne gibbo inflato calice duplo longiore, labio superiore » quadrilobato, quam inferior ovali-ellipticum recurvatumque bre»viore; staminibus exsertis; calice fructifero deflexo, aperte » campanulato, subbilabiato, obsolete striato; nuculis ovato-rotun»datis, obtusis. Flores roseo-purpurei (Maxim.).

Forma A floribunda : caulibus ad medium ramosis; calicibus pedicellisque dense incanescentibus;

Forma B paniculata : caulibus simplicibus, inflorescentia paniculata, brevi ; calicibus pedicellisque subglabris vel puberulis.

$H a b$. : Régrion mont. du Tché-foû (900 à 1,000$)$ mètres d'alt. dans les fissures des roches granit. ou micasch. - Fl. et fr. 16 septembre.

$A r$ géog. : Chine bor., le Chan-tong ( 0 . Deb.), Pé-tché-ly à Péking (Maxim.). 
Obs. 1 : Cette rare espèce, dont je dois le nom à l'obligeance de M. A. Franchet, convient de tout point à la description de M. Maximowicz dans sa Flora amurensis. Toutefois, j'ai observé au Tché-foû deux formes bien distinctes qui doivent être rapportées à la même plante: l'une (forma floribunda) à tiges rameuses au sommet, et supportant une belle touffe de fleurs subpaniculées d'un rose pourpré, à calices et pétioles couverts d'un duvet blanctomenteux ; l'autre (forma paniculata) à tiges simples et terminées par une panicule florale courte, composée de verticilles rapprochés de 2 à 4 fleurs naissant à l'aisselle des feuilles, et à calices simplement pubérulents.

Obs. 2: Il est à présumer qu'une autre espèce de Plectranthus, le $P$. amethystö̈des Berıth., et dont je possède un exemplaire récolté par Georges Staunton en 1793 dans le Kiang-nan, sur les limites du Chan-tong, se rencontrera dans la partie occidentale de cette dernière province. En voici une courte diagnose :

P. amethystö̈des Benth. Monog. labiat. p. 45 et in Dec. Prodr. XII, p. 61 .

«Herbaceus, caule erecto pedali, superne ramoso, pubescente ; 》foliis distantibus, ovato-rotundatis, crenatis, puberulis, longe » angustatis, petiolo communi cuneato-dilatato; floribus laxe » paniculatis, bracteis ovatis; calicibus fructiferis cæruleis, erec" tis, campanulatis, subglobosis, laciniis brevibus ovatis, acutis, "venosis. An perennis?"

\section{MENTHA L.}

144. M. arvensis Lin. Spec. 806, var. vulgaris Benth. in Dec. Prodr. XIII, p. 178, et Flor. Hongkong. 276; Maxim. Prim. fl. amur. 215; Regel Tent. $f$. ussur. 114 ; Miquel Prol. $f$. japon. 37 ; sinice Po-нô.

Forma chinensis O. Deb. in Herb.; Mentha arvensis var. vulgaris Benth. et Miq. (loc. cit.) pro parte.

"Planta chinensis præsertim notanda radice repente stoloni» fera, caulibus simplicibus, 20-50 centim. altis, erectis (nunquam » prostratis), rarius ad medium ramosis, ramis patentibus, foliis "que plus minus hirsuto-puberulis, foliis utrinque pubescentibus » supra subglabris, longe petiolatis, basi attenuatis, 4-5 centim. " longis, 10-15 millim. latis, oblongis vel anguste ellipticis, acutis 》serratisque, verticillis inferioribus pedunculatis, cæteris sessili- 
» bus, floribus pedicellatis, pedicellis calicibusque hirtellis, seu " laxe puberulis. »

$H a b$. : Prairies humides du littoral à Yan-taï et sur la plage de Ki-tsen-sôo. - Fl. 19 aon̂t.

Ar.géog. : Eur. - Asie bor., cent.et or., Sibérie, Altaï. - Indes. orient. - Ceylan. - Iles Philipp. - Chine bor. et aust. (Hongkong, Chan-tong, Pé-tché-ly). — Prov. de l'Amour et de l'Ussuri. - Japon. - Iles de l'Océan atlant. (Madère). - Amér. bor.

\section{THYMUS L.}

145. Th. serpillum Lin. Spec. 825; Ledeb. Flor. ross. 3, p. 345 ; Turczan. Flor. baik.dahur. 2, p. 399; Maxim. Prim. amur. 217; Regel Tent.f.ussur. p. 116 ; Trautv. et Mey. Flor. Ochotsk.72; A. Gray Bot. jap. 402; Miquel Prol. jap. 38 ; Th. angustifolius Bunge Enum. chin. bor. no 290.

Var. vulgaris Benth. in Dec. Prod. XII, p. 201 ; Franch. et Sav. Enum.jap. 1, p. 367.

«Planta speciminibus indicis simillima, caulibus prostratis, » stolones longissimos efformantibus, dense hirtellis, foliis oblon》go-lanceolatis, basi ciliatis, infra venosis, venis 3 prominulis, » calicis laciniis ciliatis. »

Hab.: Les basses collines micasch. ou sablonn. au-dessus du village de Si-nen-kôo, près de la pagode newve (250 à 350 mèt. d'alt.) - Fl. 12 juillet.

Ar.géog. : Eur. bor., cent. et aust. - Régions caucasiques et caspiennes, - Asie bor., cent. et or. - Himalaya, Sibérie alt., Daoûrie, Mongolie. - Chine (Chan-tong et Pé-tché-ly). — Prov. de l'Amour, de l'Ussuri et de l'Ochotsk; Ajan. - Japon. - Afriq. bor. et subtrop. - Abyssinie. - Amér. bor.

Obs. : Le Thymus serpillum des collines du 'T'ché-foû est de tout point semblable aux échantillons provenant du Japon. Miquel fait toutefois remarquer (loc. cit.) qu'il existe une légère différence entre la plante du Japon et celle de l'Amour, différence qui lui paraît insuffisante pour constituer une espèce nouvelle. Dans tous les échantillons du Th. serpillum de Chine et du Japon, les glandules des feuilles sont déprimées, et forment une petite cavité à la surface du parenchyme, tandis que ces mêmes glandules sont saillantes dans la plante de l'Amour et de l'Ussuri. 
CALAMINTHA Mænch.

146. C. (clinopodium) chinensis Benth. in Dec. Prodr. XIII, p. 233; Maxim. Prim. amur. 217; Franch. et Sav. Enum.jap. 1, p. 369 ; Rob. Fortune Plant. chin. exsicc. A, n $90 ;$ Melissa repens var. Hook. et Arn. Bot. voy. Beechey, 268 ; Calamintha clinopodium var. chinensis Miquel Prol. $A$. jap. 39.

«Herbacea, annua, caulibus erectis simplicibus, vel rarius basi 》 binis, 45-50 centim. altis, pilis albido-fuscis, retrorsum adpressis 》 præsertim ad angulos instructis; foliis ovato-oblongis petiolatis, " grosse crenato-serratis, basi cuneatis, 30-40 millim. longis, 20 " mill. latis, valde puberulis, pube rufescente adpressaque ; verti» cillastris pedunculatis, multifloris, bracteis subulatis calicem » æquantibus.»

Hab. : Prairies sablonn. et humides du littoral ; bords des torrents qui se déversent dans la mer, près de Ki-tsen-sôo. - Fl. 13 août.

Ar. géog. : Chine bor. (Chan-tong et Pé-tché-ly). - Iles Lôo-chôo. - Prov. de l'Amour. - Japon.

Obs. : Espèce bien différente du $C$. clinopodium par ses feuilles plus fortement dentées, par ses bractées plus courtes et ses fleurs de moitié plus petites (A. Franchet).

\section{SALVIA L.}

147. S. miltiorhyza Bunge Enum. pl. chir. bor. $\mathrm{n}^{0} 281$; Benth. in Dec. Prodr. XIII, p. 277 ; sinice TAN-SEng, TAN-SAN.

«Planta tchefouensis humilis, radice miniata, perenni; caule » herbaceo, simplici, erecto, 20 centim. alto, hirsuto-piloso; foliis » amplis pinnatisectis, segmentis 3 , petiolatis ovatis, cuneatis, ul» timo majore, margine grosse crenato-dentatis, utrinque pubes» centibus, petiolis hirsutis ; verticillis multifloris, in spicam ter" minalem laxamque dispositis; calice campanulato, glanduloso »villoso, tribolo, lobis cuspidatis ; bracteis lanceolatis, pedicellis " brevioribus; corolla magna, cærulea, calicem triplo superante 》 extus subvillosa; staminibus styloque longee exsertis. Semina »non vidi. » 
Hab. : Collines micasch. au-dessus de Ki-tsen-sôo et de Si-nenkôo. - Fl. 20 juillet.

Ar.géog. : Chine bor. et cent. (Chan-si, Chan-tong et Pé-ché-ly).

Obs. : La plante du Tché-foû ne diffère de la forme typique décrite par Bunge, que par ses tiges hautes de 20 à 30 centim. au plus, et non de 75 cent. à un mètre de hauteur. Le S. miltiorhyza est une fort belle plante, à fleurs grandes d'un bleu foncé, et très-connue des médecins chinois. Ceux-ci attribuent des propriétés toniques et reconstituantes à ses racines de couleur rougefoncée, et qu'ils considèrent comme étant l'une des cinq quintessences. Je dois ajouter que ces propriétés dues probablement à la couleur rouge de la racine sont purement imaginaires.

148. S. plebeia R. Brown Prodr. p. 501; Benth. in Dec. Prodr. XII, p. 356, et Flor. Hongkong. 277; O. Deb. Flor. Shang-hai, no 84; Miquel Prol.jap. 40; Franch. et Savat. Enum.jap.1, p. $372 ;$ S. minutiflora Bunge Enum. chin. bor. n 285 ; sinice TCHoûI-TZÉ, KIN-Kâ.

$H a b$. : Les prairies humides au bord de la mer; fossés autour de Yan-taï. - Fl. 16 juillet.

Ar. géog. : Asie cent. et subtrop., Indes-orient., Ceylan.-Chine (Hong-kong, Fô-kien, Kiang-soû, Chan-tong, Pé-ché-ly). - Iles Philipp. - Nouv.-Hollande. - Japon (Nippon et Kioû-sioû).

\section{SCUTELLARIA L.}

149. S. (Stachymacris) macrantha Fisher in Reichbch. Icon. bot. V, tab. 488; Benth. in Dec. Prodr. XII, p. 424; Maxim. Prim. amur. 218; Turczan. Fl. baik.-dahur. 2, p. 413; Franch. et Savat. Enum. jap. 1, p. 377; S. grandiflora Adams ex Bunge in Enum. chin. bor. n² 293, non Sims.; S. baikalensis Georgi in Ledeb. Flor. ross. 3, p. 397; $S$. lanceolaria Miquel Prol.jap. 43.

Vur. pubescens O. Debx. in Herb. (1860).

«Herbacea, basi fruticosa, radice crassa perenni; caulibus erec» tis, simplicibus, superne ramulosis, pube albo-rufescente valde » instructis, 50-60 cent. altis; foliis oppositis, breve petiolatis 》 sessilibusve, anguste lanceolatis, acutis, integris, supra atro» virentibus, hispidis, subtus fusco-pubescentibus, margine cilio» latis, floralibus caulinisque conformibus; floribus apice ramo- 
» rum aggregatis, oppositis, unilateralibus; calicibus villoso» pubescentibus, pedicellos æquantibus, lobis obtusis.

»Corolla cærulea ampla, 30 millim. longa, extus puberula; tubo » longiusculo superne dilatato ; lobis lateralibus parvis, lobo me» dio convexo, galeam subæquante. Antheræ albo-villosæ. Semina » atra, minutissima, subreniformia, granulosa, dorso unisulcata. " Hab. : Basses collines micaschist. et région montagneuse boisée, au-dessus de Ki-tsen-sôo et de Si-nen-kôo (200 à 450 mèt. d'alt.) Fl. 13 aout, fr. 6 septembre.

Ar.géog. : Asie bor. et or., Sibérie alt., Daourie à Nertschinsk près du fleuve Argun. - Mongolie. - Chine (Chan-tong et Pé-tchély près de la grande muraille). - Prov. de l'Amour et de l'Ussuri. Japon (Nippon).

Obs. : Notre variété pubescens diffère de la forme typique (ex descript. Benthamiana) par la villosité plus dense de toutes ses parties, et par les tiges et les feuilles couvertes d'un duvet fin, serré et roussâtre, et non subglabres. Mais Turczaninow et Ledebour, qui ont mieux connu cette plante, lui atribuent des tiges pubescentes. La description du $S$. lanceolaria par Miquel dans son Prolusio lui convient exactement, à l'exception toutefois de la glabrescence des tiges, des rameaux et de la face supérieure des feuilles qui a été observée dans la plante du Japon. Ce caractère spécial de la villosité du $S$. macrantha dans le Tché-foû ne me paraît pas suffisant pour constituer une espèce distincte. A mon avis, ce n'est qu'une forme locale, et susceptible de variation selon son habitat.

Le $S$. macrantha est une plante vraiment remarquable par ses épis floraux denses, terminaux, et par ses fleurs grandes d'un bleufoncé. M. A. Franchet m'a assuré (in litteris) qu'il n'existe aucune différence appréciable avec l'espèce du Japon, et celle qui est cultivée au Jardin du Muséum de Paris, laquelle provient de graines envoyées par le botaniste Fisher. Le nom de $S$. lanceolaria de Miquel fait évidemment double emploi avec celui de $S$. macrantha de Fisher qui est plus ancien, et qui doit avoir la priorité dans la synonymie.

150. S. scordifolia Fisher Index semin. hort. Petrop. p. 62; Benth. in Dec. Prodr. XII, p. 425; Ledeb. Flor. ross. 3, p. 398; Maxim. Prim. amur. 219; Miquel Prol. jap. 42: Franch. et Savat. Enum.jap. 1, p. 377. 
Forma pubescens Miq. (loc. cit.).

»Caulibus, foliis, calicibus pedicellisque valde puberulis; pilis " albidis, densis adpressisque. "

$H a b .:$ Sables maritimes sur la plage de Ki-tsen-sôo et de Yan-taï. - Fl. 30 juillet.

Ar.géog. : Asie bor., Sibérie alt., Baikulie à Irkutsk et Selinginks, Daoûrie, Mongolie, Kamtschatka. - Chine (Chan-tong et Pé-tché-ly). - Prov. de l’Amour. - Japon.

\section{LEONURUS L.}

151. L. sibiricus Lin. Spec. 818; Benth. in Dec. Prodr. XII, p. 501, et Flor. Hongkong., 278; 0. Deb. Flor. Shang-hä̈, no 87; Franch. et Savat. Enum. 1, p. 380; sinice TchongKOÛEI-TZÉ, YÉ-MOÛ-N'GAI, KÉ-Kô̂EI.

$H a b$. : Prairies et fossés aquatiques dans toute la plaine du Tché-foû. - Fl. et fr. août et septembre.

Ar.géog. : Régions intertrop. et temp. des deux mondes : Afriq. cent. - Amér. cent. - Asie bor., subtrop. et or. - Cochinch. Chine bor., cent. et aust. - Mongolie, Daoûrie, Amour et Ussuri. - Japon, Malaisie, îles Philipp., Java, etc. - Afriq. occ., Sẻnég’al, Guinée.

Obs. : Le L. sibiricus est très-répandu dans toute la Chine, et varie beaucoup selon ses diverses stations. On le rencontre à feuilles plus ou moirss laciniées, subglabres ou pubescentes, ou blanches-tomenteuses en dessous, à verticilles plus ou moins multiflores, lâches ou serrés. Les arètes qui terminent les divisions du calice varient également quant à la longueur de l'acumen, lequel dépasse souvent le tube de la corolle, ou l'égale parfois à peine.

\section{VERBÉNACÉES JusS.}

\section{VERBENA}

152. V. officinalis Lin. Spec. 29; 0. Deb. Flor. Shang-hä, n 88 ; Miquel Prol.jap. 29; Franch. et Sav. Enum.jap. 1. p. 217; G. Staunton Plant. chin. exsicc. (1793); sinice MA-PIENTsâo.

$H a b$. : Champs sablonn. et cult. à Yan-taï. - Fl. juillet-aoat. 
Ar.géog. : Eur.-Afriq. bor., occ. et aust. - Amér. bor. et cent. - Asie or. et subtrop. - Indes-orient. - Chine : Amoy, Shang-haï et Tché-foû $(O . D e \succeq$.$) . - Japon.$

\section{VITEX L.}

153. v. ovata Thumbg. Fl. jap. 25\%; Benth. Fl. Hongkong. 273; Sieb. et Zuce. Fam. nat.jap. 2, p. 28; Miquel Prol. jap. 30; Hook. et Arn. Bot. voy. Beechey, p. 206, tab. 17; $V$. repens Blanco Fl. de las filipp. 513; V. trifolia Lin. Suppl. 293; Dec. Prod. XI, p. 683, var. unifoliata Franch. et Sav. Enum.jap. 1, p. 260.

Hab. : Sables marit. du Tché-foû; dunes de Foû-chan-yên; falaises micasch. de N'gin-haï-tchéoû au cap Chan-tong. - Fl. et fr. 4-18 septembre.

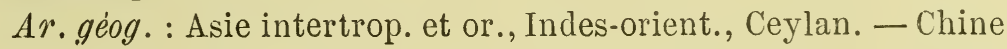
bor. et aust. (Hong-kong, Koûang-tong, Chan-tong). - Iles de l'Océan indien, Maurice, Seychelles. - Iles Philipp. - Nouv.Hollande. - Japon (Kioû-sioû).

Obs.: Le Vitex ovata de Thumberg qui est considéré dans le Prodromus comme une simple variété unifoliata du $V$. trifolia L., constitue, à mon avis, une espèce parfaitement distincte en Chine et au Japon. Celle-ci est nettement caractérisée par ses tiges rampantes couchées sur le sol, à rameaux courts et redressés, par ses feuilles toujours simples, obovales-elliptiques, entières, cunéiformes à la base et atténuées en un court pétiole, vertes pubescentes en dessus, d'un blanc tomenteux en dessous, par ses fleurs disposées en une panicule terminale courte, de 4 à 6 fleurs seulement, etc.

154. V. incisa Lamk. Dict. 2, p. 605; Dec. Prodr. XI, p. 684; Bunge Enum. chin. bor. no 295; V. negundo Lin. ex Benth. Flor. Hongkong., et $V$. bicolor Wild. Spec. 273, pro parte; sinice Min-King, HoûAng-Kin, Tsin-Tsâo.

Hab. : Sables maritimes et terres incultes, dans la plaine de Yan-taï; sur les nombreux tumulus autour de Ki-tsen-sôo. Fl. 1-15 août.

Ar. géog. Chine bor., cent. et aust. : Hong-kong, Koûang-tong, Tché-kiang, Kiang-si (abbé Darid), Chan-si, Hô-nan, Chan-tong, Pé-tché-ly, etc. - Mongolie. - Asie subtrop ex Cl. Bentham. 
Obs.: Cette remarquable espèce paraît être répandue dans les provinces boréales et centrales de la Chine. Elle est endémique au Tché-fou, et en si grande abondance, qu'elle caractérise pour ainsi dire la végétation littorale de cette contrée.

\section{PRIMULACÉES Vent.}

LYSIMACHIA L.

155. L. (Ephemerum) barystachys Bunge Enum. chin. Gor. no 298 ; Duby in Dec. Prodr. VIII, p. 62; Turczan. Enum. chin. bor. n 165; Maxim. Prim. amur. 193; Regel Tent. A. ussur. 103, tab. 9, fig. 1-3 (optima); Miquel. Prol.jap. 284: Franch. et Savat. Enum.jap. 1, p. 301.

«Herbacea, annua, cautibus erectis, simplicibus, 30-40 centim. 》altis, undique foliosis; foliis pedunculisque hispido-puberulis: » foliis lineari-oblongis vel oblongro-lanceolatis, basi attenuatis, » utrinque puberulis, subtus pallidioribus; inflorescentia termi»nali, spiciformi; spica densa, in anthesim nutante; bracteis » lineari-subulatis, puberulis, pedicellos superantibus; corolla 》 albida, laciniis oblongo-linearibus, obtusis, erectis; staminibus » brevibus inclusis, filamentis pubescentibus, basi dilatatis. »

Hab. : Les basses collines micasch. et les pelouses sablonn. dans la région mont. infér. au-dessus de Si-nen-kôo (200 à 350 mèt. d'alt.). - Fl. 13 août-6 septembre.

Ar.géog.: Chine bor. (Chan-tong, Pé-tché-ly). - Mongolie. Prov. de l'Amour et de l'Ussuri. - Japon.

Ols. : Plante bien caractérisée par son inflorescence en épi court dense, terminal et recourbé-penché vers son milieu pendant la floraison. Son aire de dispersion s'étend depuis le nord de la Chine jusqu'au Japon, par une ligne qui passerait à travers les provinces de l'Amour, de l'Ussuri et du Chan-tong.

\section{APOCHORIS Duby.}

156. A. pentapetala Duby in Dec. Prodr. VIII, p. 67; Lysimachia pentapetala Bunge Enum. chin. bor. 11. 299.

"Herbacea, annua, caulibus gracilibus, erectis, subglabris, » apice puberulis, 40-50 centim. altis, laxe ramosis; foliis sparsis 
» lineari-lanceolatis, acutis, in petiolum attenuatis, inpunctatis, » supra glabris virentibus (in sicco pallidioribus); corolla penta" petala, alba, minuta, petalis distinctis; capsula subglobosa cali»cem multo superante, stylo maturitate persistente coronata; " seminibus atris, parvis, subglobosis, ruguloso-pluricostatis. " Ha乙. : Les prairies sabl. du littoral, à Yan-taï, Ki-tsen-sôo, etc. - Fl. 13 août, fr. 6 septembre.

Ar. géog. : Chine bor. (Chan-tong et Pé-tché-ly. - Mongolie. - Chine.

Obs. : Le genre Apochoris, limité à cette seule espèce, est nettement caractérisé par ses pétales séparés et entièrement distincts entr'eux. Dans la diagnose de ce genre, Duby assure (loc. cit.) que les capsules renferment 5-7 graines membraneuses-ailées, les ailes étant au nombre de trois. Cela existe en effet, dans les capsules dont les graines sont avortées et stériles. Mais dans les capsules fructifères fertiles, il n'y a qu'une senle graine subglobuleuse, à 4.5 côtes fortement rugueuses à leur surface.

\section{PLUMBAGINÉES Juss.}

\section{Statice L.}

157. S. sinensis de Girard in Ann. scienc. nat., série 3, vol. 2, p. 329; Boiss. in Dec. Prodr. XII, p. 642; Benth. Fl. Hongkong. 281 ; S. Fortunei Lindl. in Bot. regist. (1845), tab. 63; R. Fortune Plant. chin. exsicc. $\mathrm{n}^{\circ} 130$.

Hab. : Marais salés et pâturages saumâtres du littoral, au nord de Yan-taï. - Fl. 14 septembre.

Ar. géog.: Chine marit., Hong-kong, Fo-kien à Amoy, Tchékiang, Chan-tong, etc.

158. S. Franchetii O. Debeaux Mss. et in Herb. (1873).

Icon. nostra, tab. 1.

«Herbacea, radice crassa, perenni; caulibus erectis, 30-50 cen» tim. altis, glabris, striatis, in parte superiore ramosis, ramis 》 erectis, laxe dichotomis, corymbum amplum efformantibus, ra»mulis inferioribus vel ad dichotomias erectis, minutis curvulis, » aut apice furcatis sterilibusque; foliis radicalibus obovatis-spa» thulatis, in petiolum breve attenuatis, margine undulatis, apice 》 obcordatis, caulinis semiamplexicaulibus, ovato-lanceolatis; spi- 
》culis bifloris in spicas breves subscorpiö̈deas congestis; bracteis »virentibus, late membranaceo-marginatis, inferiore minima obo»vata, apiculata, inferiore fere 4-plo longiore oblonga; calicis »tubo piloso, limbo albido, amplo, calice æquilongo, obtuse »5-lobato, ad costas 10-plicato; corolla aurea.

"Species pulcherrima et valde distinctissima, habitu $S$. bico" loris, sed differt caulibus cylindricis, glabris, striatis nec angulo» sis, ramisque glabris, teretibus, subcylindricis nec ancipitibus. »

Hab. : Rochers micaschisteux du littoral, à la pointe du Tchéfoû. - Falaises au-dessus de Ki-tsen-sôo et au cap Chan-tong près de N'gin-haï-tchéoû. - Fl. 10-30 juillet 1860.

Ar. géog. : Chine bor., Chan-tong (O. Deb.); Nandchourie (Swinhoё).

Obs.: Notre Statice Franchetii, l'une des plantes les plus remarquables dı 'Tché-foû, et que je me fais un devoir de dédier à M. A. Franchet, l'un des auteurs de l'Enumeratio plantarum japonie, comme témoig'nağe de ma vive reconnaissance, croît abondamment sur les falaises du Chan-tong. M. E. Boissier, de Genève, à qui je l'ai communiqué pour avoir son avis, trouve cette espèce fort distincte du S.chinensis, et surtout du S. bicolor avec lequel il serait facile de la confondre, au premier abord.

Le savant monographe du genre Statice, dans le Prodromus de de Candolle, attribue (Proar. XII, p. 642) au S. bicolor des tiges anguleuses et pour ainsi dire $\grave{a}$ deux faces. Bunge ne mentionne pas cette particularité dans sa description; il dit seulement "ramis interne latere planiusculis.» M. Hance assure, au contraire, que les spécimens du S. Jicolor récoltés sur les rivag’es de Tâ-lienwhân en Mandchourie par M. Swinhoë offrent des tiges arrondies dans toute leur étendue, et ceux-ci doivent se rapporter évidemment à notre $S$. Franchetii et non au $S$. bicolor.

\section{PLANTAGINLES JUSE.}

Plantago L.

159. P. asiatica Lin. Spec. 163 ; Ledeb. Flor. ross. 2, p. 479, et Flor. alt. 1, p. 143 ; Franch. et Sav. Enum.jap. 1, p. 384; P. major var. asiatica Decne in Dec. Prodr. XIII, p. 294; Miquel Prol.jap. 283 ; P. exaltata Bunge Enum. chin. bor. $n^{0} 305$, non Hornem. ex Regel: sinice Tché-tsian tsaô, Lioì-Lô̂, Lioû-Kia. 
Hab. : Champs sablonneux autour de Yan-taï. - Juillet.

Ar.giog. : Asic subtrop., bor. et or., Sibérie alt., Baikal, Mongolie. - Chine (Macâo, Chan-tong, Pé-tché-ly). — Prov. de l'A mour et de l'Ussuri. - Japon. - Iles de l'Océan indien, Maurice, Java. - Amér. bor. et aust., Bahia, etc.

Obs. : Le $P$. asiatica est considéré par Miquel et M. Decaisne comme une simple variété du $P$. major. Il est facile de le distinguer de celui-ci par ses proportions plus grêles, par ses feuilles plus petites, étalées-redressées, et atténuées en un long pétiole, par son épi grêle, lâche à la base, par ses bractées deux fois plus courtes que le calice, celui-ci égalan` ou dépassant à peine la corolle à lobes aigus acuminés, et enfin par ses capsules fructifères plus petites et arrondies, ses graines moins nombreuses, etc.

160. P. media Lin. Spec. 163 ; Decne in Dec. Prodr. XIII, p. 697;

Ledeb. Frlor. alt. 1, p. J.46; A. Gray Plant. jap. Perry exped. 316 ; sinice KI-KIA.

Hab. : Champs sablon, cultures, décombres autour de Yan-taï. - Fl. 14 juillet.

Ar.géog.: Toute l'Europe. - Asie bor. et or., Sibérie alt. Chine (Chan-tong). - Japon à Hakodadé.

\section{CHÉNOPODÉES Vent.}

\section{ATRIPLEX Gærtu.}

161. A. littoralis Lin. Spec. 1494; Moq. in Dec. Prodr. XIII, 2, p. 97 ; Ledeb. Flor. ross. 3, p. 697, et F'lor. all. 4, p. 311 ; Turcz. Flor.baik.-dahur. 2, p. 24; Bunge Enum.chin. bor. no 315; Maxim. Prim. amur. 223; Miquel Prol. jap. 126; Franch. et Sar. Enum.jap. 1, p. $38 \%$.

Hab. : Fossés et sables inondés par la mer, dans la baie de Yantaï. - Septembre.

Ar. géog. : Eur. litt. - Asie bor., Sibérie alt. sur les rives de l'Irtysch, Baikalie, Daoûrie. — Chinebo: (Chan-tong, Pé-tché-ly). - Prov. de l'Amour. - Japon (Decima). - Afriq. bur.

AGRIOPHYLLUM M. Bieb.

162. A. squarrosum Moq. in Dec. Prodr. XIII, 2, p. 139; Corispermum squarrosum Lin. Spec. non Bieb.; C. pungens Vahl Enum. 1, p. 17; Agriophyllum arenarium M. Bieb. Flor. taur.-cauc. 3, p. 6, ex Moq.! 
Hal. : Collines sablonneuses et dunes marécageuses au nord de la baie de Yan-taï; plage de Foû-chan-yên. — Septembre.

Ar. géog. : Eur. orient., Russie aust. sur les sables du Wolga (M. Bieb.). - Asie bor., Sôongarie sur les rives du fleuve Lepsa (Karel. et Kiril.). - Chine au Tché-fô̂ (O. Debeaux).

\section{CORISPERMUM Juss.}

163. G. Stauntoni Moq. Chenop. enum. monog. p. 104, et in Dec. Prodr. XIIT, 2, p. 140; Staunton Plant. chin. exsicc. (1793) sub Corispermo..........

Hab. : Sables maritimes sur la plage de Ki-tsen-sôo. - Fl. juillet.

Ar. géog. : Chine : Prov. du Chan-tong (G. Staunton!), le Tchéfoû (O. Debeaux).

\section{CHENOPODINA Moq.}

164. Gh. maritima Moq. in Dec. Prodr. XIII, 2, p. 161, var. A vulgaris; Schoberia maritima C. A. Neyer in Ledeb. Flor. alt. 1, p. 400; Bunge Enum. chin. bor. n 311; Franch. et Savat. Enum.jap. 1, p. 388; Suceda maritima var. vulgaris Moq. Chen. enum. p. 128; Ledeb. Flor. ross. 3, p. 186; Chenopodium maritimum Lin.

Hab. : Fossés saumâtres ou inondés par la mer, et marais salés au nord de Yan-taï. - Août.

Ar.géog. : Eur. marit. - Asie bor., or. et occ., Sibérie alt., Baikal. - Chine litt., Kiang-soû, Chan-tong, Pé-tché-ly. - Japon . - Afriq. bor. et or. - Amér. bor.

\section{SALSOLA Gærtı.}

165. S. kali Lin. Spec. 322; Moq. in Dec. Prodr. XIII, 2, p. 187 ; Ledeb. Flor. ross. 3, p. 747, et Fl. alt. 1, p. 392; Turczan. Flor. baik.-dahur. 2, p. 45; Maxim. Prim. amur. 227.

Var. A vuigaris Auct. omn.

«Glabra, diffusa, ramosa, ramis inferioribus erectis vel patenti» bus, ramo primario 30-40 centim. alto, adscendente-erecto; flo»ribus axillaribus pentandris, solitariis; sepalis acuminatis, » margine membranaceis, nervosis, roseo-coloratis, alas patentes » xquantibus. » 
Var. B spicata O. Debx. in Hert. 1860.

"Humilior, glabra, atro-viridis, e basi ramosa, ramulis omnibus » prostratis seu prostrato-erectis, 10-15 centim. elongatis, spicam » gracilem apice densam atque sensim clavatam efformantibus.» Hab. : Sables maritimes, sur la plage de Yan-taï et de Ki-tsensôo. - Fl. 10-30 août.

Ar.géog. : Eur. marit. - Afriq. bor. - Amér. bor. - Asie bor. et or., Sibérie altaïque, Baikal. - Chine : le Chan-tong (O. Debx.). - Prov. de l'Amour.

Obs.: La forme vulgaris du S. kali n'offre aucune différence avec la plante de l'Europe maritime. Il n'en est pas de même de notre variété spicata, que l'on serait tenté de prendre au premier abord pour une espèce distincte, tant son habitus et son facies diffèrent de la forme typique.

Cette variété spicata est une petite plante rameuse à la base, et à rameaux couchés-redressés qui ne dépassent pas 8 à 15 centimètres en longueur. L'épi floral commence à la naissance même des rameaux. D'abord grêle et lâche à la base, il devient plus dense et plus fourni vers le sommet, et se termine en forme de massue. Tous les autres caractères sont ceux du $S$. kali, dont la plante chinoise n'est qu'une forme remarquable. Sa floraison est d'environ trois semaines plus tardive que dans la variété vulgaris, et dans les mêmes localités.

On cultive dans tous les jardins pour l'usage alimentaire le Spinacia oleracea L., sinice Pô-LING et Pô-TSAÏ, ainsi que diverses variétés de Betteraves Beta vulgaris L., sinice Tâ-sonG-Lô-Pôu.

\section{AMARANTHACÉES R. Brown.}

Cfilosia L.

166. C. argentea Moq. in Dec. Pror. XIII, 2, p. 212 ; Benth. Flor. Hongkong. 284; Thumbg. Flor. jap. 106 ; Sieb. et Zuc. Fam. nat. jap. 2, p. 85 ; Miq. Prol. jap. 63; Franch. et Sav. Enum. jap. 1, p. 389 ; sinice T'SIN-TSIANG, T'sin-HIônG-TzĹ. Var. A vera Moq. (loc. cit.); C. argentea Lin. Spec. 296 ; folits inlerioribus caulinisque lineari-lanceolatis.

Var. B margaritacea Noq. (loc. cit.); C. margaritacea Lin. : Miquel Prol.jay. 63; foliis oratis vel ellipticis, acutis, basi cuneatis.

Ilal. : Champs d'orge et de panic (Panicum miliaceum), dans la 
grande presqu'île sablonneuse, au nord de Yan-taï. - Fl. 4-10 septembre.

Ar.géog. : Régions intertrop. des deux-mondes; Asie: Indesorient., Malabar, Ava, Népaul. - Cochinch. - Chine, Hong-kong, Ciran-tong. - Japon. - Iles de la Malaisie, Java, Timor, etc. Afriq. subtrop., Kigypte, Nubie, Nigritie, Saint-Thomas. - Amér . cent. et aust., Maryland, Havane, Bahia, etc.

Obs. : Les variétés vera et margarilacea ne sont que des formes à feuilles plus ou moins larges de la même espèce, et elles doivent,être réunies, ainsi que Moquin et Miquel l'ont déjà fait avec raison.

\section{AMARANTHUS Tourn.}

16\%. A. caudatus Lin. Sspec. 1400 ; Moq. in Dec. Prodr. XIII, 2, p. 255; Wild. Monog. amarant. 36; Maxim. Prim. amur. 227; Miquel Prol.jap. 64; Franch. et Savat. Enum.jap. 1, p. 390.

Var. albiflorus Moq. (loc. cit.); G. Staunton, Plant. chin. exsicc. (1793); sinice MA-TCHÉ-YêN.

Hab. : Cultivé dans les jardins comme plante alimentaire, et subspontané autour des habitations.

Ar. géog.: Régions intertrop. des deux-mondes. - Asie : Indesorient., Ceylan, Népaul, Perse, Mésopotamie. - Chine (Chan-tong, Kiang-nan). - Japon. - Afriq. subtrop, Abyssinie, Sénégal, Sierra-Leone. - Amér. tropic.

Obs. : D'après les observations de A. Braun et de Bouché, l'A. caudatus est caractérisé par des graines blanches entourées d'un bourrelet obtus et nettement circonscrit.

168. A. paniculatus Moq. in Dec. Prodr. XIII, 2, p. 257; Ledeb. Flor. ros.s. 3, p. 856; Regrel Tent. Al. ussur. 123.

Var. A sanguineus Moq. (loc. cit.); A. sanguineus Lin. Spec.; Wild. Mon. amar. 31.

«Spicis lateralibus gracilibus, laxifloris, apice nutantibus, flo» ribus purpureis. »

Var. B strictus Moq. (loc. cit.); A. strictus Wild. Monog. umarant. 27; G. Staunt. Plant. chin. exsicc.

«Spicis longioribus, gracilibus, laxifloris, rigidiusculis, caule " adpressis; floribus viridibus. 》

Hab. : Les deux variétés qui sont cultivées dans les jardins, 
se trourent fréquemment subspontanées autour des habitations.

Ar.géog.: Asie subtrop., Indes-orient.; - Chine (Chan-tong, Kiang-nan. - Prov. de l'Ussuri. - Afrique occid., Nigritie. Amér. bor.

169. A. spinosus Lin. Spec. 1407; Noq. in Dec. Prodr. XIII, p. 260; Wild. Mon. amarant. 38; Beuth. Flor. Hongkong. 284; 0. Debx. Flor. Shang-haï, no 94; G. Staunton, Plant. chin.exsic. (1793); sinice KIA-YÊN-TSAÏ.

Hab. : Champs et cultures dans la plaine du Tché-foû. - Fl. septembre.

Ar. géog. : Régions intertrop. des deux-mondes; Asie : Ceylan, Indes-orient; - Chine bor., cent. et aust. - Iles de la Malaisie, Java. - Afriq. occ., Ile Bona-vista (Hook.), Sierra-Leone, Fernando-Pô, Sénégal. - Amér. bor. et cent.

170. A. blitum Lin. Spec.1405; Moq. in Dec. Prodr. XIII, 2, p. 263; Bunge Enum. chin.bor. no 30\%; Maxim. Prim. amur. 227; Regel Tent. f.ussur. 125.

Var. sylvestris Moq.(loc.cit.); A. sylvestris Desfont. Cat. hort. par. (1804), no 44; A. prostratus Maxim. et Regel.

Hab. : Champs sablonneux du littoral à Yan-taï, Ki-tsen-sôo, camp du Tché-foû. - Fr. 15 octobre.

$d r$.géog. : Eur. or. et cent., Russie aust., Astrakan.-Afriq. bor., Égypte. - Canaries. - Asie: Arabie, Indes orient. - Chine: Hong-kong (de Filippi), Chan-tong (O. Debx.); Pé-tché-ly (Maxim. et Bunge). - Prov. de l'Amour et de l'Ussuri.

Obs. : On cultive comme plantes d'ornement dans quelques jardins les Celosia cristata L., sinice KI-KoûAN-HÔA, le Gomphrena globosa, les Amaranthus speciosus sims., A. melancholicus L. et autres espèces de ce dernier grenre.

\section{POLYGONEES Juss.}

Polygonum L.

171. P. aviculare Lin. Sspec. 519; Meissn. in Dec. Prodr. XIV, p. 97; Ledeb. Flor. ross. 3, p. 531 ; Turczan. Flor. baik.dahur. 3, p. 70; Trautv. et Meyer F'lor. Ochotsk. 76; I.axim. Prim. amur. 229; Regel Tent. ussur. 127; Bung'e Enum. chin. 325; O. Debx. Flor. Shang-hai, no 99; Franch. et Sav. 
Enum.jap. 1, p. 394 ; Miq. Prol. jap. 299 ; sinice T'cнÉCHoûI-Tsâo.

Forma A erecta Ledeb., Meissn. et Miquel.

Forma B diffusa Meissn. et Miquel (loc. cit.).

Hab. : Champs et cultures dans toute la plaine du 'l'ché-foû, à Yan-taï, Ki-tsen-sôo, etc.

Ar. géog. : Eur. - Asie bor., occ. et or. - Chine (Kiang-soû, Chan-tong, Pé-tché-ly). - Japon. - Iles de l'Océanie. - Afriq. bor. et aust. - Amér. bor.

172. P. bistorta Lin. Spec. 1506; Meissn. in Dec. Prodr. XIV, p. 12.5; Turczan. Fl. baik.dahur.; Trautv. et Ney. Fl. Ochotst. 75; Regrel Tent. ussur. 124; Miquel Prol.jap. 300; Franch. et Savat. Enum. jap. 1, p. 397; sinice Kı̂uıNTsAn, Tsâo-HÔ-TCHú.

Var. angustifotium Meiss. (loc. cit.); P. bistorta var. alpinum Turczan. Mss. in Herb. Dec.; P. bistortö̈des Boiss. Diagn. plant. nov. 5, p. 40 ; Miquel (loc. cit.).

"Caulibus glabris simplicibus, erectis, 60-75 cent. altis, unispi» catis; foliis inferioribus oblongo-lanceolatis, basi obcordatis, » longe petiolatis; petiolis 20 centim. longis in parte superiore 》) sensim alatis, limbum duplo superantibus; foliis caulinis lanceo" latis linearibusve; spica valde elongata, ovato-oblonga, utrin» que attenuata. »

Hab. : Région moy. boisée du 'Tché-foû, de 400 à 600 mèt. d'alt, dans les bois de chênes et de pins. - Fl. 6 septembre.

Ar. géog. : Eur., Caucase. - Asie bor., cent. et or., Sibérie alt., Baikalie, Daoûrie, Mongolie, Himalaya, Asie min. - Chine : Chantong, Pé-tché-ly. - Amour et Ussuri. — Ochotsk, Kamtschatka. Japon (Kioû-sioû, Nippon). - Amér. bor.

Obs. : La variété angustifolium, la seule que j'ai rencontrée dans les montagnes du Tché-foû, se distingue facilement du type, par ses fetilles inférieures ovales-lancéolées, à pétioles 2 à $21 / 2$ fois plus longs que le limbe, les supérieures lancéolées-aig’uës ou linéaires, par son épi cylindrique-allongé atténué des deux côtés. Cette forme est également répandue dans les montagnes du Japon.

173. P. polymorphum Ledeb. Flor. ross. 3, p. 524: Meissn. in Dec. Prodr. XIV, p. 139.

Var. arenarium O. Debx. in Her?. (1860). 
"Caulibus flexuoso-erectis, simplicibus vel e basi ramulosis, » brevibus, cum spica 20 centim. altis, glabris; foliis ovato-lan» ceolatis, acutis, in petiolum breve attenuatis, 5-6 centim. longis, »6-10 millim. latis utrinque glabris, viridibus, crassiusculis; » spica terminali valde elongata, simplici, laxa, circiter 8 centim. »longa; bracteis bifloris membranaceis; akenio breviter exserto.» Hað. : Sables maritimes sur la plage de Ki-tsen-sôo. — Fl. 10 août.

Ar. géog. : Chine bor., Chan-tong et Pé-tché-ly. Le type : Eur., Asie bor. et cent., Amér. bor.

Obs. : La plante des sables de Ki-tsen-sôo offre quelques ressemblances avec le Polygonum salignum Wild. (P.polymorphum var. salignum Meissn. in Dec. Prodr. (loc. cit.). Elle en diffère par la pubescence de toutes ses parties, et surtout par son épi terminal grêle, allongé, et parfois interrompu à la base.

\section{FAGOPYRUM Tourn.}

174. F. esculentum Mænch Method. 290; Meissn. in Dec. Prodr. XIV, p. 143; Ledeb. Flor. ross. 3, p. 517; Maxim. Prim. amur. 236; Regel Tent. usur.; Franch. et Savat. Enum. jap. 1, p. 415; Polygonum fagopyrum Lin. Thumbg. Flor. jap. 169 ; sinice Kiâ̂-MEI, CHoûr-Fô-TZÉ.

$H a b$. : Cultivé en grand dans les terres lègères de la plaine et des collines sablonn. du Tché-foû.

Ar.géog.: Origin. de l'Asie centrale, Népaul et Assam, d'où il s'est propagé dans la Sibérie altaïque, la Mongolie, la Chine et le Japon, en Europe et dans l'Amérique boréale.

\section{THYMÉLÉES}

Wikstroemia Endl.

175. W. chinensis Meissn. in Dec. Prodr. XI, p. $513 ; \mathrm{W}$. indica C. A. Meyer (olim).

"Suffrutex humilis, cautibus erectis cinerascentibus, nigro » punctulatis, apice ramosis, 30-40 centim. altis, inferne glabris, »superne incano-tomentosis; foliis oblongo-lanceolatis, acutis, » breve petiolatis, supra atro-viridibus, subtus pallide ferrugineis, »venosis, præsertim ad costas adpresse-ciliatis; pedunculis flori» feris terminalibus, sessilibus, solitariis, calicibusque albo-pilosis; 
"lobis calicinis obtusis, tubo angusto-subcylindrico triplo brevio. » ribus. Flores dilute violacei, demum lilacini. »

IIab. : Rochers micasch. à la pointe du T'ché-foû et près du vieux fort du camp francais; collines au-dessus de Ki-tsen-sôo. - Fl. 15-30 septembre.

Ar.géog. : Chine boréale; le Chan-tong.

Obs. : Le $W$. chinensis, dont on ne connaissait jas encore la station exacte en Chine, croît sur la falaises herbeuses de la presqu'île de Tché-foû, et sur les basses collines micachist. dans la région montag. infér. de cette contrée. Ce petit arbuste produit à la fin de septembre une panicule terminale de 6 à 8 fleurs, d'un bleu violacé etqui mériteraient par łeur élégance de fixer l'attention des horticulteurs. Son acclimatation serait d'autant plus facile en France, que le $W$. chinensis résiste dans le nord de la Chine à des froids de $-12^{\circ}$ à $-16^{\circ}$ centigrades.

\section{EUPHORBIACÉES JusS.}

\section{EtPHORBIA.}

176. E. humifusa Wild. Enum. hort. berol. suppl. p. 13; Boiss. in Dec. Prodr. XV, p. 30; Bunge Enum. chin. bor. no 329; 0.Debx. Flor. Shang-hä̈, $1^{\circ} 10^{2} ;$; E. pseudo-chamesyce Fisch. et Mey.; E. polygonisperma Gren. et Godr. Flor. franc. ex Boissier; sinice TA-KI, TI-KIN.

Hab.: Abonde sur les sables maritimes de la baie de Ki-tsen-sôo. - Fl. et fr. juillet et août.

Ar.géog. : Asie bor., Sibérie de l'Oural, Altaï, Baikalie, Mongolie, - Chine bor. et moy. - Corée. - Japon. - Sables de la Corse?

177. E. pekinensis Boiss. Cent. Euphorb. p. 31, et in Dec. Prodr. $\mathrm{XV}, \mathrm{p} .121$.

Hab. : Prairies marécageuses et fossés saumâtres, autour de Yan-taï - Fl. et fr. 1U-15 juillet.

Ar.géog. : Chine bor., Chan-tong, Pé-tché-ly.

Obs. : Plante élevée de 35 à 40 centim. droite; tigres simples ou rameuses au sommet couvertes de poils laineux et crépus; feuilles ovales-lancéolées, acuminées, sessiles, glabres, grlaucescentes en dessus, pubescentes en dessous sur la côte médiane seulement; ombelle à 5 rayons bi-trifides; involucre campanulé, à lobes ovales- 
tronqués; styles bifides, écartés; glabres et épaissis à leur extrémité; capsules globuleuses, couvertes de verrues coniques.

Cette espèce a le port et l'aspect de l'E. palustris L., et offre beausoup d'affinités avec l'E. lucorum Rupr. On la distingue de celle-ci par ses tiges droites, ses feuilles entières à peu près glabrescentes en dessous, par ses capsules globuleuses, et surtout par la forme des verrues qui sont cylindriques-coniques dans l'E. pekinensis, et non triangulaires comme dans l'E. Tuiorum.

178. E. esula Lin. Spec. 660; Maxim. Prim. amur. 238, var. cyparissiödes Boiss. in Dec. Prodr. XV, p. 161; 0. Debx. Fl. Shang-hä̈, $\mathrm{n}^{\circ} 103 ;$ E. cyparissias var. B, Ledeb. Fl. alt. 4, p. 180, et Turczan. Cat.pl. baik. p. 16.

"Planta in ditione Tché-fố dicta herbacea, circiter 20 centim. »alta, caulibus floriferis glaucis, apice ramosis; floribus dense » aggregatis; foliis brevibus anguste linearibus (20 mill. longis, " 2 millim. latis, utrinque glaberrimis. »

Hab. : Champs et cultures du littoral à Yan-taï, Ki-tsen-soô, etc. - Fl. 15 juillet.

Ar.géog.: Eur. or.et aust. - A sie bor., Sibérie altaïque, Baikalie, Sôongarie, Daoûrie, Mongolie, — Chine bor. et moy. - Amour et Ussuri. - La forme typique se retrouve dans l'A mérique boréale.

179. E. lunulata Bunge Enum. plant. chin. bor. n ${ }^{\circ} 330$; Boiss. in Dec. Prodr. XV, p. 162 ; sinice Minô-yen-Tsaô, 'TchÉ-tí́.

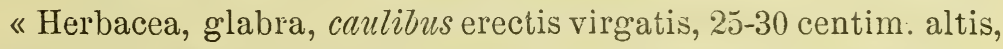
» in parte superiore dense ramosis, foliis caulinis sparsis, laxis, » anguste lanceolatis, acutis, apice subspathulatis, basi attenuatis 》fere sessilibus, glabris, integris, foliis umbellaribus dilatatis, »obcordatis, suborbiculatis, bi-trilobis, lobis inæqualibus; appen»dicibus involucri lunulatis ; capsulis glabris, lrevibus, trisulca" tis, rugulosis; seminibus ovatis. »

Hab. Sables maritimes sur la plagre de Ki-tsen-sôo. - Fl. 14 juillet.

Ar.géog. : Chine bor. : Chan-tong et Pé-tché-ly.

Obs. : Cette espèce se disting'ue nettement de l'E. esulc par les feuilles qui accompagnent les rayons de l'ombelle obcordées ou réniformes, bi-trilobées et non entières. 


\section{EXCFCARIA L.}

180. E. sebifera Mull. in Dec. Prodtr. XV, p. 1210; 0. Debx. Fl. Shang-hä̈, no 105; Slillingia sebifer'a Mich.; Benth. Fl. Ilongkong. 302; Sapium sebiferum Roxbg. Fl. ind. 3, p. 693; Miq. Prol. jap. 290; Croton sebiferum Lin.; sinice Kiû-TZÉ, Pi-MA-TZÉ, Ho-TIEN-TZÉ.

$H a b$. : Champs et cultures dans la plaine, et sur les basses collines du Tché-foû. - Fr. octobre.

Ar.géog.: Asie intertrop. et temp. - Indes-orient. - Chine (Hong-kong, Koûang-tong , F ô-kien, Kiang-si, Kiang-nan, Kiangsoû, T'ché-kiang, Chan-tong, etc.). - Chû-san et Formose. - Cochinch. - Les Philipp. - Japon. - Amér. bor.

\section{ACALYPHA .}

181. A. pauciflora Hornem. Hort. hafn. p. 240 (1815); Bunge Enum. chin. bor. 355; Maxim. Prim. amur. 240; Regel Tent.fl.ussur. 128; Miquel Prol.jap. 291; A. gemina Mull. in Dec. Prodr. XV, p. 866, var. A genuina; A. virgata Thumbg. Fl. jap. 268 non Lin.; A. clinensis Roxbg. Fl. ind. 3, p. 677 .

"Annua, herbacea, humilis, caulibus erectis, petiolisque dense » hirtellis ; ramulis patenti-divaricatis; foliis ovatis vel elliptico» oblongis, crenato-dentatis, supra glabris atro-virentibus, subtus » pallidioribus, ad nervos parce ciliatis.

»Ad varietatem umbrosam Maxim. et Regel (loc. cit.) ex carac» teribus supra notis valde refert. »

Hab. : Champs sablonn. à Yan-taï et Si-nen-kôo, au milieu des cultures de Cannabis indica et de Fragopyrum esculentum, etc. Fl. et fr. 28 septembre.

Ar.géog.: Asie intertrup. et temp., Indes-orient. - Chine (Hong-kong, Koûang-tong, Chan-tong, Pé-tché-ly); - Amour et Ussuri. - Japon. - Afriq. occid., Nigritie.

\section{PHYLLANTHUS Swartz.}

182. Ph. puberus Mull. in Dec. Prodr. XV, p. 307.

Var. impubes Mull. (loc. cit.); Agyneia impubes Lin. Mant. 296; G. Staunt. Plant. chin. exsicc. (1793). 
«Suffrutex, ramulis glabris, foliis ovatis ellipticis, junioribus » pubescentibus demum glabratis, subtus glaucis. »

Hab. : La province du Chan-tong (G. Staunt.). Fréquemment cultivé dans les jardins.

Ar. géog. : Chine moy. et aust. (Hong-kong, Koûang-tong, Kiang-nan, Chan-tong, etc.).

Obs. : Je possède deux spécimens du Phillanthus niruri L. recueillis parG. Staunton, dans la partie la plus occidentale du Chantong qui avoisine le canal impérial. Peut-être trouvera-t-on cette espèce dans la région montagneuse du Tché-foû. La forme de Chine est intermédiaire entre les var. A genuina et var. B javanica du Ph. niruri. Mull. in Dec. Prodr. Elle est caractérisée par ses racines annuelles, courtes, se ramifiant au collet, à radicelles divariquées traçantes, la médiane bifurquée; par ses tiges ligneuses à la base, de 15 à 18 centim. de hauteur, peu rameuses, à rameaux étalés glabres, par ses feuilles ovales-elliptiques acuminées, et glabres des deux côtés, par son calice fructifère plus grand que dans la forme typique. Le $P h$. nirur $i$ se retrouve dans le Sud de la Chine à Hong-kong et au Japon.

\section{SECURINEGA Juss.}

183. S. obovata Mull. in Dec Prodr. XV, p. 449 ; S. abyssinica Rich. Tent. abyss. 2, p. 256; Xylophylla obovata Wild. Enum. berol. 329 ; Cicca pentandra Blanco Fl. de las filip. 486; Phyllanthus virosus Miquel Filor. ind. bat. 1, p.356, et Baillon Etud. Euphort. tab. 26; Fluggea microcarpa Blume Bijdr. 580 .

Hab. : Rochers micasch. et falaises près de N'gin-haï-tchéoû; sables de la rivière 'Tâ-hô, près du cap Chan-tong. - Fr. 18 septembre.

Ar. géog. : Région intertrop. de l'Asie et de l'Afrique. - Indesorient. - Chine au cap Chan-tong (O.Debx.). - Iles Philip., Timor, Java, Australie, Maurice, Madagascar, Les Comores - Abyssinie, Benguela, Nigritie, Guinée, Sénégambie, etc.

Obs. : Cette remarquable espèce, qui a été classée dans des genres divers selon sa provenance, a été rangée définitivement par Müller (loc. cit.) dans le genre Securinega. Son area est des plus étendus, et comprend dans ses extrêmes limites au sud, les rivages océa- 
niques de l'Asie et de l'Afrique intertropicales. Elle ne parâ̂t pas dépasser au nord les falaises du promontoire du Chan-tong.

\section{CANNABINLES E. Mey.}

CANNABIS Tourn.

184. C. sativa Lin. Spec. 1457; Alph. Dec. in Prodi. XVI, p. 30; O. Deb. Fl. Shang-hä̈, n 106; Miq. Prol. jap. 65, var. angustifolia; C. indica Lam. Dict. 1, p. 695; C. chinensis Delile Ind. sem. hort. monsp. (1819); sinice MA; japonice AsA.

Forma A vulgaris Dec. (loc. cit.).

Hab. : Cultivé en grand dans toute la plaine du 'Tché-foû, et au cap Chan-tong.

Ar. géog. : Asie bor. et cent., Sibérie altaïque, Baikalie, Sôongagarie, Kirghyz, Nongolie, Chine bor. et moy. — Prov. de l'Amour et de l'Ussuri. - Indes-orient. - Acclim. dans l'Eur. aust., l'Afriq. bor. et l'Amér. bor.

\section{MORACÉES Lindl.}

Morus L.

185. M. alba Lin. Spec.1398; Bureau in Dec. Prodr. XVII, p. 231 ; O. Deb. Fl. Shang-hä̈, no 109.

Var. A vulgaris Bur. (loc.cit.); M. alba Lam. Dict. 4, p. 373; Roxbg. Fl. ind. 3, p. 594; sinice TChang, TChang-PÉ-TZÉ.

Hab. : Cultivé dans les basses collines au-dessus de Si-nen-kôo (région littor.).

Ar.géog. : Asie intertrop. et temp.-Chine bor., cent.-Japon.Natur. en Eur., dans l'Afriq. bor., l'Amér. bor. et les îles Philippines.

Obs. : Le Morus nigra Lin. a été observé par G. Staunton dans les cultures de la région occidentale duChan-tong, et dans la province du Kiang-nan. Au Tché-foù, qui n'est point un district produisant la soie, le $M$. alba est peu répandu, et seulement comme arbre d'agrẻment.

\section{CUPULIFÈRES Rich.}

\section{Quercus L.}

186. Q. serrata Thumbg. Fl.jap. 176; Alph. Dec. in Prodr. XVI, 
p. 50 non Roxbg.; Sieb. et Zucc. Fam. nat.jap. 2, p. 102;

G. Staunt. Plant. chin. exsicc. (1793); sinice Tsin-KaN-TZÉ, Fô-LI-TZÉ.

"Arbor mediæ magnitudinis, foliis autumno deciduis, juniori» bus lanceolatis, longe acuminatis, dense costatis, superne pu» bescentibus, adultis elongatis-oblongis, mucronato-serratis, » utrinque glaberrimis; amentis masculis gracilibus, fere filifor» mibus; floribus pentandris, filamentis basi monadelphis; » antheris imberbibus. »

Hab. : La plaine et la région montagn. moy. et infér. du Tchéfoû, jusqu'à 500 mètres d'altitude. - Fr. 15 septembre.

Ar. géog.: Chine bor., le Chan-tong (O. Debx.), Kiang-nan (G. Staunt.). - Mandchourie méridion. à Tâ-lien-whân. Japon.

Obs. : Cette espèce de chêne quej'avais confondue avec le Q. castanerfolia de C. A. Mey. dans mon Essai sur la pharmacie et la matière médicale des Chinois, p. 92 (1867), est très-voisine du Q.chinensis Bunge Enum. n 347 , par son port, son facies général, et surtout par la forme des feuilles qui ressemblent assez exactement à celles du Q. castaneafolia. Mais on reconnaît le $Q$. serrata à ses feuilles glabres des deux côtés, et non blanches pubescentes en dessous, ainsi qu'à la forme des cupules.

Celles-ci sont hémisphériques, un peu aplaties dans le Q. serrata. Ses écailles sont variables quant à leur forme, les inférieures recourbées et penchées en dehors, les moyennes écartées divergentes, les supérieures à sommet recourbé et appliqué sur le fruit. Le fruit (gland) est petit et ellipsoïde. Dans le Q.chinensis, les fruits n'arrivent à maturité que la deuxième année. Les cupules sont subglobuleuses, à écailles lancéolées recourbées et dépassant de beaucoup le fruit qui est presque sphérique.

Le Quercus serrata est un bel arbre de moyenne grandeur, propre à la région montagneuse moyenne et inférieure du promontoire du Chan-tong. On le rencontre disséminé dans la plaine de Yan-taï, formant çà et là quelques bouquets sur les tumulus, ou isolé autour des tombeaux et des temples bouddhiques. 


\section{CASTANEA Tourn.}

187. G. vulgaris Lamk. Dict. 1, 708; Alph. Dec. in Prodr. XVI, p. 114; C. vesca Gærtn. Fruct. tab. 3; Bunge Enum. chin. bor., Fagus castanea Lin. Spec. an Thumbg?; sinice PANLI-LI, LIEN-TZÉ.

$H a b$. : Répandu çà et là au pied des basses collines, mais peu abondant. - Fl. juin, fr. mûrs en octobre.

4r. géog. : Eur. mérid. - Asie bor., or. et occ. - Chine bor. et moy. - Japon. - Afriq bor - Amér. bor.

Obs. : Les fruits de l'espèce chinoise sont beaucoup plus petits que ceux du $C$. vulgaris de l'Europe méridionale. Ce caractère me paraît insuffisant pour distinguer spécificuement le châtaignier qui croît dans le nord dé la Chine.

\section{SALICINÉES Rich.}

\section{SALIX L.}

188. S. babylonica Lin. Spec. 1473; Anders. in Dec. Prodr XVI, p. 212; Ledeb. Fl. ross. 3, p. 599 ; Bunge Enum. chin. bor. no 346; O. Deb. Fl. Shang-hä̈, no 110 ; Miquel Prol. fl.jap 213 ; sinice TIâ̂-LIÉou, LiÉou-PI.

Hab. : Cultures autour des habitations dans la plaine de Yantaï et de Ki-tsen-sôo.

dr. géog. : Asie bor., occ. et or.-Chine bor. et moy., Kiang-sou, Kiang-nan, Chan-tong, Pé-tché-ly. - Mongolie. - Japon. Natur. en Eur., Afriq., Amér., etc.

189. S. triandra Lin. Spec. 1442; Anders. in Dec. Prodr. XVI, p. 202; S. amygdalina Lin. (pro parte); Maxim. Prim.amur. 242; Regel Tent. ussur. 131; Ledeb. Flor. altai. IV, p. 258; sinice Hiang-LiÉoû.

Forma submaritima O. Debx. in Herb.

«Suffrutex 3-4 pedalis, caulibus erectis virgatis, parce ramosis, » ramis erectis caule adpressis, glabris; foliis lanceolatis vel » anguste lineari-lanceolatis, acuminatis, argute serratis, utrinque " glaberrimis, subtus glaucescentibus. »

Hab. : Mares d'eau saumâtre au milieu des dunes de Foû-chanyên. - Fl. et fr....; feuilles seulement le 4 septembre 1860. 
Ar. géog. : Le type : Eur.-Asie bor. et occ. - Régions caspienues, Sibérie alt. sur les rives de l'Irtysch; Baikalie. - Prov. de l'Amour et de l'Ussuri.

Obs. : Je n'ai pu observer les fleurs mâles et femelles du Salix des sables maritimes du Tché-foû, qui, par son port et ses rameaux feuillés, rappelle le fascies du $S$. triandra du midi de la France. Mes spécimens du Chan-tong comparés à ceux du S. triandra de la collection des Saules desséchés de la Suisse par Seringe ( $2^{\mathrm{e}}$ cahier, $\mathrm{n}^{\mathrm{os}} 1,2$ et 3 ) ne me laissent aucun doute sur l'identité de la plante chinoise, qui paraît d’ailleurs être assez répandue dans l'extrême-Orient.

CONIFÈRES Juss.

(Gymnospermées Lindl. ex parte.)

PinUs L.

190. P. Massoniana. Lamb. Pin. (ed. 2e), p. 16; Parlat. in Dec. Prodr. XVI, p. 389 ; Hook. et Arn. Bot. voy. Beech. 271 ; Sieb. et Zucc. Fl. jap. tab. 112-114; 0. Deb. Fl. Shanghä̈, $\mathrm{n}^{0} 112$; P. sinensis Lamb. Pin. (ed 3e), p. 47; Benth. Flor. Hongkong. 337; sinice TA-sông, Sông, Tsông-Tchoû.

Hab. : Région montagn. moy. et infér. du Tché-foû, jusqu'à 600 mètres d'alt. Répandu au cap Chan-tong et dans la plaine de Yan-taï autour des tumulus. - Fr août et septembre,

Ar. géog. : Chine litt., bor., cent. et aust. de Hong-kong à Péking. - Se retrouve dans le Kiang-si, Kiang-nan, Tssé-tchûen, Kiang-soû, Chan-si, etc. - Japon.

\section{CUNNINGHAMIA R. Brown.}

191. G. sinensis R. Brown in Rich. Conif. p. 80, tab. 18 ; 0. Debx. Fl. Shang-haï, n 113 ; Sieb. et Zucc. Flor.jap. 2, p. 7, tab. 103; Belis jaculifolia Salisb. in Trans. soc. Lin. Lond. 8, p. 315 ; sinice CHAN, CHAN-MOÛ.

$H a b$. : N'existe qu'en individus isolés autour des pagodes et des tombeaux, dans la plaine de Ki-tsen-sôo et de Yan-taï.

Ar. géog. : Chine bor., cent. et aust. - Iles Lôo-chôo. — Japon.

BIOTIA Endlich.

192. B. orientalis Endl. Conif.p. 47 ; Bunge Enum. chin. bor. 
$n^{\circ} 352$; 0. Debx. Flor. Shang-haï, no 114; Sieb et Zucc. Fl. jap. 2, tab. 118; Miquel Prol. jap. 241; Thuja orientalis Lin.; sinice PÉ-song, PÉ-Chân, PÉ-Fang.

Hab. : Mêmes localités que l'espèce précédente, mais plus abondant. - Fr. septembre.

Ar. géog.: Chine bor. et cent. (Kiang-sou, Kiang-si, Kiang-nan, Hô-nan, Chan-tong, Pé-tché-ly, etc.). — Japon.— Nat. rans l'Asie temp, les îles du Pacifique, etc., et l'Eur aust.

Obs. : J'ai observé, une seule fois, la présence de la variété pendula du B. orientalis (Thuya pendula Lamb.; Sieb. et Zucc., etc., sinice CHI-CHAN) dans une cour intérieure de la pagode de Ki-tsensôo. Cet habitat dénote, sans aucun doute, l'origine étrangère de la var. pendula dans cette localité.

\section{ZOSTÉRACÉES Ad. Juss.}

\section{ZOSTERA L.}

193. Z. marina. Lin. Spec. 1374; Kunth Exum. plant. 3, p. 116; Ledeb. Flor. ross. IV, p. 20 ; Maxim. Prim. amur. 446 ; Thumbg. Fl.jap. 238 ; sinice HaI-TSAï.

Ha乙. : Rade de Yan-taï et baie de Ki-tsen-sôo, sur les rocher's à basse mer.

Ar. géog. : Toutes les mers du globe : Médit., Oc. atl. bor. et aust.; mer Rouge, Oc. ind., mers de Chine, du Japon, d'Ochotsk, etc.

Obs. : Le Posidonia caulini Kœn. (Caulinia oceanica Dec., Zostera oceanica L.) se rencontrera probablement dans les havres abrités du cap Chan-tong. Je l'ai récolté dans la rade de Singapoore en 1860. Cette espèce est également indiquée sur les rivages de l'Australie.

\section{TYPHACÉES Juss.}

\section{TYPHA L.}

194. T.angustifolia Lin. Spec. 1377; Kunth. Enum.plant. 3, p. 91 ; Ledeb. Fl. alt. IV, p. 249 ; Bunge Enum. chin. bor. n 378 (pro parte); sinice HiânG-PôU, PôU-HÔUang.

$H a b$. : Marécages d'eau douce ou saumâtre au milieu des dunes de Foû-chan-yên, au nord de Yan-taï. - Fl. 4 septembre.

Ar. géog. : Eur., Caucase; - Asie bor. et cent., Indes-orient., Sibérie alt. - Chine (Kiang-sou, Chan-tong, Pé-tché-ly). - Ti- 
mor. - Afriq. bor. et occ., Égypte, Algérie. - Canaries. - Amér. bor. et aust.

\section{IRIDÉES R. Brown.}

Pardanthus Ker.

195. P. chinensis Ker. in Karn. ann. bot. 1, p. 217; Spreng. Syst. veg. 1, p. 166 ; Bent. Fl. Hongkong. 365; Miq. Prol. jap. 306; P. dichotomus Ledeb. Flor. ross. IV, p. 106 ; Turczan. Flor. baik.-dahur. 2, p. 199; Maxim. Prim. amur. 271; Iris dichotoma Bunge Enum. chin. bor. n 357 ; sinice СнÉ-HôAN, CHÉ-KIAN-HÔA.

Hab. : Collines micaschist. au-dessus de Si-nen-kôo; - Région mont. moy. et inf. jusqu'à 600 mètres d'alt., dans les fissures des rochers et sur les bords des ravins. - Fl. 30 juillet.

Ar.géog. : Asie mont. bor., cent. et subtrop.; Himalaya, Thibet, Sibérie baikalienne, Daoûrie, Mongolie. - Chine (Hong-kông, Chan-tong, Pé-tché-ly, île Formose). - Prov. de l'Amour. Japon. - Java (Zollinger). - Introd. dans l'Amér. bor.

Obs.: Je dois faire remarquer combien la station de cette plante dans la Chine boréale (montagnes du Chan-tong, de Péking et Grande muraille) diffère de celle du Japon et de la Chine australe. Miquel dit (loc. cit.) que le Pardanthus chinensis se trouve au Japon " in paludosis » dans la province de Nippon et autour de Nangasaki. M. Bentham assigne une station analogue à la plante de Hong-kông et de l'île Formose. Je n'ai pu trouver d'ailleurs aucune différence entre les échantillons de la région montagneuse du Tchẻ-foû, et ceux provenant des marécages maritimes du Japon et qui m'ont été communiqués par M. A. Franchet.

\section{AMARYLLIDÉES R. Brown.}

NARCISSUS L.

196. N. tazetta Lin. Spec. (ed. 1), 290; Kunth Enum. pl. V, p. 741, sub Hermione; Thumbg. Fl.jap. 121 ; var. sinensis Miq. Prol.jap. 72; 0. Debx. Flor. Shang-haï, n 127 ; sinice CHÔUI-TSIEN-HÔA; CHÔU-LIEN-HÔA.

$H a b$. : Terres incultes sur les tumulus, dans la plaine de Yantaï et de Ki-tsen-sôo. - Fl. 15 octobre.

Ar. géog. : La variété sinensis: Chine bor. et Japon - Le type : Eur. aust., et Asie occid., Afriq. bor., etc. 


\section{ASPARAGINEES R. Brown.}

Asparagus L.

197. A. Sieboldi Maxim. Prim. amur. 287; A. Schoberiödei Kunth Enum.pl. V, p. 70; Miq. Prol.jap. 315; A. Wrighti A. Gray Bot.jap. $403 ;$ A. micranthus Sieb. et Zucc. Plant. jap. exsicc. in sched. Teste Maximowicz; A. parviflorus Turczan. Flor. baik.-dahur. 3, p. 226, et Regel Tent. ussur. p. 153

"Planta dioïca, glabra vel apice scabriuscula, 60-75 centim. " alta, erecta, ramosa, ramis subfastigiatis patentibus, caulibusque " angulatis; squamis foliaceis hyalinis, cladodiis secus ramulos » ternis binisve, fasciculatis, linearibus, acute mucronatis, trique» tris, subfalcatis, patentibus, margine scabriusculis; perigonio " masculo campanulato, basi rotundato, abbreviato, albido; laci. "niis oblongis obtusiusculis; pedunculis ad caules ramosque "primarios geminatis, deflexis, recurvatis, sub bacca articulatis; "baccis globosis, rubro-coccineis, pisi majoris magnitudine."

Hab. : Collines micasch. et sablonn. au-dessus de Si-nen-kôo ; falaises de Ki-tsen-sôo et de la pointe de Tché-foû. - Fl. 10 juilet, fr. 6 septembre 1860 .

Ar.géog. : Chine bor., Chan-tong et Pé-tché-ly. - Province de l'Amour et de l'Ussuri. - Japon.

Obs.: L'A. Sieboldii Maxim. ne serait pour M. Regel qu'un synonyme de l'A. parviflorus Turcz., espèce propre aux déserts de la Daoûrie traversés par le fleuve Argun. M. Maximowicz fait tou tefois observer, que d'après la courte diagnose de Turczaninow, l'A. parviflorus serait hermaphrodite et qu'il aurait les feuilles verticillées par quatre. Ces caractères n'existent pas dans l'A. Sie boldi du Tché-foû, qui est une plante dioïque, et dont les feuilles sont verticillées par trois ou par deux, même sur les rameaux stériles.

\section{POLYGONATUM TOUrn.}

198. P. officinale All. Fl. pedem. 1, p. 131 ; Ledeb. Fl. ross. IV, p. 123; Turczan. Fl. baik.-dahur. 3, p. 203; Maxim. Prim. amur. 274; Regel Tent. ussur. 149; Niq. Prol. jap. 312; $P$. vulgare Desf. Ann. mus. IX, p. 49; A. Gray Bot. jap. 413; 
Convallaria polygonatum Thumbg. Fl. jap. 142 pro parte; sinice HôUEI-JONG.

"Affine P. japonici Morr. et Decne. videtur, sed differt caule » simplici, sulcato, compresso, ancipiti, statura humiliore (20-30 » centim.), foliis glaberrimis, ovatis ellipticisve, junioribus ovatis »acutis. »

Hab. : Région montag. moy. dars les bois de pins (Pinus Massoniana), et de chênes (Quercus serrata), de 400 à 600 mèt. d'altitude. - Fr. 6 septembre.

Ar.géog. : Eur. montag.; - Asie bor. et or., Sibérie altaïque, Sôongarie près de l'Irtysch, Désert de Kirghyz, Daoûrie. - Chine (Chan-tong et Pé-tché-ly). - Prov. de l'Amour et de l'Ussuri. Japon.

Obs. : Les rhyzômes de cette plante sont recherchés avec soin par les indigènes du Chan-tong, qui les apportent sur le marché des villages comme denrée alimentaire. ('es rhyzômes se mangent après avoir été confits au vinaigre, ou dans le sirop de sucre.

\section{OPHIOPOGONÉES Kunth.}

(Aspidistrées Endl.)

OpHiopogon Gawl.

199. O. spicatus Gawl. in Curtis Bot. reg., tab. 593; Kunth Enum.plant. V, p. 299; Benth. Fl. Hongkong. 371; Miq. Prol.jap. 141 ; Maxim. Ophiop. spec. in Bull. acad. sc. StPétersb. XV, p. 83, var. A communis; Convallaria spicata Thumbg. Fl. jap. $14^{\mathrm{e}}$; Liriope spicata Lour. Fl. coch. 1, p. 200 ; sinice MI-MÔUN-TONG, MI-MEN-TONG.

Hab. : Falaises micaschist. à la pointe du Tché-fô et près du vieux fort chinois, dans les fissures des rochers. - Fl. 7 août, fr. 15 septembre.

Ar.géog. : Asie intertrop. et temp. - Cochinch. - Chine marit. bor. et aust. (Hong-kông, Kouâng-tong, Tché-kiang, Chan-tong, îles Lôo-chôo). - Japon. - Iles Philipp., etc.

Obs. : Cette espèce répand à l'état sec une odeur douce aromatique, qui rappelle celle du Faham (Angracum fragrans L.), orchidée spéciale aux îles de l'Océan indien. Les animaux herbivores recherchent beaucoup l'Ophiopogon, et il devient très-difficile d'en trouver une touffe qui ne soit broutée, excepté toutefois dans 
les anfractuosités des rochers les plus escarpés. Les médecins chinois emploient les rhyzômes de cette plante comme toniques et fortifiants.

\section{LILIACÉES JUSS.}

I.ILIUM L.

200. L. tenuifolium Fish. Ind. plant. hort. Gorenk. (1812), p. 8 Ledeb. Fl. ross. IV, p. 158; Maxim. Prim. amur. 280, Turczan. Fl. baik.dahur. 3, p. 212 ; Bunge Enum. chin. bor. $\mathrm{n}^{\circ} 371$; sinice CHAN-TAN-HÔA.

«Caule humili (20-30 cent.), apice bifloro, erecto-striato, foliis » caulinis inferioribus sparsis, anguste-linearibus, adpressis, mar" gine subrevolutis, floralibus subduplo latioribus; corolla erecta, " flava, unicolore.»

Hab. : Les hautes montagnes (500 à 800 mèt. d'alt.), dans les fissures des rochers. - Fl. 10 juillet.

Ar. géog. : Asie bor., Daoûrie, Mongolie. - Chine (Chan-tong, Pé-t-ché-ly). - Amour et Ussuri.

Obs. : Dans la plante du Tché-fou, les tiges sont biflores, et les corolles dressées. Tous les autres caractères se rapportent exactement au $L$. tenuifolium, auquel les botanistes russes assignent une tige uniflore et des fleurs penchées.

\section{SCILLA L.}

201. S. chinensis Benth. Flor. Hongkong. 373; Barnardia scilloïdes Lindl. Bot. reg. tab. 1029, et Bot. magaz, tab. 3788; Maxim. Ind. A. pekin. in Prim. amur. 478; Kunth Enum.1V, p. 337; Hook. et Arn. Bot. voy. Beech. p. 28.

«Bulbo tunicato ovato, scapo 1 (rarius 2), gracili, tereti, erecto "(40-50 centim.), folia parum superante; foliis anguste lineari» bus, striatis, canaliculatis; racemo florigero spicato, spica 10" 12 centim. longa, subcylindrica, apice acuta; pedicellis erectis. » maturitate patentibus, corolla longioribus; floribus lilacinis. »

$H a b$. : Les prairies sablonn. du littoral à Yan-taï. - Basses collines au-dessus de Ki-tsen-sôo, etc. - Fl. 7 août.

Ar. géog.: Chine bor. et aust. (Hong-kông, Kouâng-tong’ à Macâo, Chan-tong, Pé-tché-ly, îles Lôo-chôo).

Obs. : Espèce très-voisinedu Barnardia japonica Ræm. et Schult., dont elle diffère par ses feuilles linéaires canaliculées, atteignant 
presque la base de l'épi floral, celıi-ci grêle, cylindrique, acuminé au sommet, et par ses pédicelles écartés-divariqués après l'anthèse.

\section{ALLIUM L.}

202. A. (Porrum) Bouddhæ O. Debeaux Mss. et in Herb. 1860; sinice KIÄ̈-PÉ.

«A. læte-virens, bulbo tunicato, ovoïdeo-oblongo, extus parce " proligero; scapo nudo, tereti, erecto, striato, 40-50 centim. alto, » inferne foliato, folia parum superante; foliis omnibus crassitie » pennæ anserinæ, glabris, fistulosis, leviter striatis, erecto" patentibus, dein prostratis, 35-40 cent. longis; spatha hyalino" membranacea, bivalvi, pedicellis duplo breviore; valvis breve »acuminatis; umbella capsulifera multiflora, lata, subglobosa; » floribus albidis, perigonii laciniis parvis, ovato-lanceolatis, acu» tis; staminibus styloque perigonio brevioribus rel subæqualibus: » antheris flavidis.

»Planta insignis, omnino saporem Allii porri præstans, Allium » que sativum subredolens. 》

Hab.: Cultivé en grand dans les jardins du Tché-foû. - Fl. 15-30 août.

Ar. géog.: Chine bor., Chan-tong et Pé-tché-ly à Tien-tsin (Doct. Larivière).

Obs. : Je ne connais point l'origine de cette espèce d'ail, qui est cultivée dans tous les jardins du nord de la Chine, et qui, à l'état frais, est usité comme condiment dans la cuisine indigène. J'ai lieu de croire cependant que la culture de cet ail doit être fort ancienne dans ces contrées.

Il est aussi d'usage, à l'occasion des repas offerts aux idoles de Bouddhâ dans les pagodes, par suite des ex-voto ou des fêtes locales, de mêler plusieurs coupes remplies de cet ail (incisé en menus fragments), aux mets préparés pour la circonstance : ce qui doit flatter agréablement la divinité, et la rendre plus favorable aux souhaits des assistants.

L'ail de Bouddhâ se distingue de toutes les espèces du groupe Porrum, par ses feuilles longuement fistuleuses dressées-écartées, par ses tiges droites cylindriques, non ancipitées, mais seulement striées, par sa spathe hyaline membraneuse bivalve, par ses fleurs blanches disposées en un corymbe large et arrondi, par ses anthères de couleur jaune pâle, etc. 
203. A. (Rhyziridion) tenuissimum Lin. Spec. 433; Kunth Enum. IV, p. 428; Ledeb. Flor. alt. 2, p. 23, et Icon. ad fl. ross. tab. 358 .

Hab.: Sables maritimes sur la plage de Ki-tsen-sôo. - Fl. 1-15 septembre.

Ar. géog. : Asie bor., Sibérie altaïque. - Chine, Prov. du Chantong (O. Debx.).

Obs. : L'A. tenuissimum est caractérisé par son bulbe très-petit entouré d'un réseau fibreux épais, par ses feuilles glabres, filifor mes canaliculées et atteignant les deux tiers environ du scape; celui-ci dressé, grêle, glabre et subcylindriqne, feuillé à la base seulement; par son ombelle pauciflore et bulbifère, à pédicelles écartés, par les divisions du périgone ovales, très-obtuses, de même longueur que les étamines, ou les dépassant à peine. Son aire d'extension ne paraîtpas être très développée dans l'Asie orientale, car l'A. tenuissimum n'est signalé seulement qu'aux deux extrémités d'une ligne, qui partant de la Sibérie altaïque s'arrèterait aux rivages du Chan-tong.

204. A. (Rhyzividion) Tchefouense O. Debeaux Mss. et in Herb. 1860.

«A. bulbo parvo, globoso-ovoïdeo, fibroso, tunicato; scapo " unico, glabro, recto, striato-compresso, apice ancipiti, quam in "A. tenuissimo robustiore, hasi foliato; foliis planis, nervosis, » linearibus, vix canaliculatis, scapi dimidiam partem attingenti» bus; spatha univalvi, hyalino membranacea, apice acuminata, » pedicellis breviore; umbella capsulifera, pauciflora; pedicellis » sub anthesi rectis, inæqualibus; perigonio campanulato; sepalis " lanceolatis acutis, albidis; staminitus perigonio brevioribus; » capsula subglobosa, triloculari; seminibus atris, compressis rugo» sisque. »

Hab. : Falaises micasch. de la pointe du Tché-foû, et rochers autour du vieux fort de cette localité. - Fl. 2-16 août 1860 .

Ar. géog. : Chine bor. (le Chan-tong).

Obs.: Notre A. Tchefouense vient se placer près des $A$. tenuissimum et $A$. Thumbergii dont il est voisin, et avec lesquels il ne saurait être confondu. Ses tiges ancipitées au sommet, comprimées, deux fois plus robustes que dans l'espèce précédente, les fleurs à sépales lancéolées, aigus, le font reconnaître au premier 
coup d'œil. Sa floraison est plus précoce d'un mois environ que celle de l'A. tenuissimum, ses feuilles sont aussi beaucoup plus larges, et atteignent à peine en longueur la moitié inférieure du scape. L'A. Tchefouense se distingue de l'A. Thumbergii par sa spathe univalve et son ombelle toujours capsulifère.

205. A. (Rhyziridion) Thumbergii Don. Monog. gen. Allii, 83; Kunth Enum. IV, p. 15̆4; A. Gray Plant. jap. Perry exped. 322; Miq. Prol. jap. 318; A. odorum Thumbg. Flor.jap. 132, non Lin.; sinice Kreoû.

«A. gracile, minutum, bulbo parvo globoso-ovoïdeo, solitario, 》 tunicis albidis membranaceis; scapo 25-35 centim. alto, tereti » erecto, striatulo, apice parum attenuato, usque ad tertiam partem » e basi foliato, vaginibus foliorum scariosis membranaceis ; foliis " anguste linearibus, planis, acuminatis, scapo multo brevioribus, 》 canaliculatis, dorso perspicue costato-nervatis; spatha bivalvi, 》hyalino-membranacea, pedicellis breviore, valvis seu phyllis » rostrato-acurninatis; umbella pauciflora, subglobosa, pericellis » bulbiliferis abbreviatis; bulbilis (4-5), tunica rosea sacciformi » indutis, pedicellis fructiferis 10-12 in eadem umbella bulbilis " longioribus, circiter 15-20 millim. longis, sæpe arcuatis; perigo» nii laciniis albidis, liberis, oblongo-lanceolatis, acutiusculis, » exterioribus parum latioribus magisque carinatis, apice dorsoque » rubellis; staminibus exsertis; ovario triquetro, sessili : stylo te»nui, exserto.

»Affine Allii chinensis Don. Monogr. p. 83, sed caracteribus 》 supra descriptis valde distinctum. »

Hab. : Sables maritimes, sur les plages de Yan-taï et de Ki-tsen-sôo. Ar.géog. : Chine: Chan-tong (O. Debeaux), Pé-tché-ly (Maxim.) - Japon (Thumbg.) - Cochinch.?

Obs. : Nous devons à Miquel (vide Prol. loc. cit.) une très-exacte description de l'A. Thumbergii, qui paraît être très-répandu sur tout le littoral de l'empire japonais. Cette espèce se distingue des précédentes, et de l'A. chinense qui ne croît pas̀ au Tché-foû, par son ombelle bulbifère, à bulbilles entourées d'une tunique membraneuse sacciforme et d'un rouge violacé, par les divisions du périgone teintées de rose sur le dos et vers le sommet, par les feuilles presque filiformes, planes, plurinerviées sur la face inférieure, et atteignant le tiers à peine des tiges florifères, etc. 
On cultive en grand dans les jardins du Tché-foa, plusieurs espèces du genre Allium généralement usitées dans la cuisine chinoise. Ce sont l'ognon commun Allium cepa L., sinice Tsôung; l'échalotte $A$. ascalonicum var. sinense Lour., sin. Hiaï; l'ail cultivé A. sativum L., sin. TA-SonANG, dont l'introduction en Chine est due aux Tartares Mongols; l'ail des marais A. uliginosum L., sin. Kieou, petite plante à tiges hautes de 10 à 15 centim. au plus, et qui remplace la ciboule comme condiment dans l'art culinaire, etc.

\section{HEMEROCALLIS L.}

206. H. graminea Andr. Bot. rep. tab. 244; Ledeb. Fll. ross. IV, p. 194; Turczan. Fl. baik.-dahur. 3, p. 225; Bunge Enum. chin. bor. $\mathrm{n}^{0}$ 360; Maxim. Prim. amur. 285; Regel Tent. l. ussur. 152; Miq. Prol. jap. 316 ; sinice HiûEn-Tsâo, HrûENHôA.

Forma elatior Maxim. (loc. cit.).

«Scapo foliis plerumque longiore, floribus longe pedunculatis, » perigonii tubo sæpius elongato, basi subcrassiore. »

Hab. : Les falaises et les collines micaschist. au-dessus de Ki-tsen-sôo et de Si-nen-kôo. - Fr. 1-10 août.

Ar. géog. : Asie bor. et or., Sibérie alt., Baikalie, Daoûrie, Mongolie. - Chine bor. (Chan-tong et Pé-tché-ly). - Amour et Ussuri. - Japon (Nippon, Kiû-siû).

\section{DIOSCORÉES R. Brown.}

\section{Dioscorea L.}

207. D. sativa Lin. Spec. 1463; Benth. Fl. Hongkong. 368; 0. Debx. Frl. Shang-här, no 130; Thumbg. Flor. jap. 151;D. bulbifera Wight Icon. tab. 878 non Lin.; Kelmia bulbifera Kunth Enum. V, p. 435 ; sinice CHou-Yû, T'sâN-rû.

Hab. : Cultivé dans la plaine et sur les basses collines du Tchéfoû, principalement dans les terrains sablonneux.

Ar. géog. : A sie cent. et or., Indes orient. - Chine bor., moy. e aust. - Japon. - Iles de la Malaisie.

COMMELYNACEES Reichb.

Commelya L.

208. G. communis Lin. Spec. 60; Benth. Fl. Hongkong. 376: 0. 
Debx. Fl. Shang-hä̈, n $131 ;$ C. polygama Roth. Catal.bot. 1, p. 1; Maxim. Prim. amur. 290; Bunge Enum. chin.bor. $\mathrm{n}^{\circ} 376$; C. IVildenowii Kunth Enum. IV, p. 37 ; sinice HIâTSI-TSAÔ, SHû-Yû-TSAÔ.

Hab. : Les grands ravins à l'ouest de Yan-taï, dans la région montag. infér., jusqu'à 200 mèt. d'alt. - Fl. 6 septembre.

$A r . g e ́ o g .:$ Asie intertrop. et or. - Indes-orient., Cochinch. Chine bor., moy. et aust., Archipel de Chû-san. - Prov. de l'Amour et de l'Ussuri. - Japon. - Afriq. occ., Nigritie, Amér. bor.

\section{CYPÉRACÉES JUSS.}

\section{Cxperus L.}

209. G. (Galilea) sinensis O. Debeaux Mss. et in Herb. (1860).

Icon. nost. tab. II.

«Humilis, annuus, radice fibrosa; culmis simplicibus vel basi 2-3 » nis, minutis, trigonis, teretibus, basi foliatis, erectis rigidis, 10 » 12 centim. altis: foliis radicalibus umbellam inferiorem superanti» bus, anguste linearibus, acuminatis, canaliculatis, dorso parum » carinatis, curvulis, margine scabriusculis; spiculis capituli » inferni fasciculato-congestis, polystachyis, basi involucratis; » involucri phyllis 4-6, inæqualibus, capitulo superiore multo bre* vioribus, basi dilitatis, linearibus, acuminatis, margine scabris; " capitulis superioribus 2, longe pedicellatis, subsphæricis; spi》culis 4-6 floris, ovato-oblongis, compressis, involucri phyllis » minutis, linearibus, spiculas paulo superantibus; squamis 12»nerviis, nervis valde præminentibus, membranaceis, in medio " vel ad margines irregulariter rubro-sanguineo maculatis, cæte» rum pallide fusculis; stylo apice trifido, exserto, basi parum » incrassato; akenio ovato elleptico, apicato, convexo plano, punc" tulato, scabro, squama triplo breviore. "

Hab : Sables maritimes sur les plages de Yan-taï et de Ki-tsensôo. - Fl. et fr. septembre.

Ar. géog. : Chine bor. et or.

Obs. : Notre Cyperus sinensis de la section Galilea vient se placer à côté du C. agyptiacus (Schanus mucronatus) L. dont il paraît n'être qu'une forme appauvrie. Mais en l'examinant avec attention, on voit qu'il en diffère, outre ses proportions très-réduites, par ses racines annuelles, fibreuses, non radicantes ni stolonifères, par' ses 
feuilles recourbées, molles, celles de l'involucre inférieur atteignant à peine les $2 / 3$ des pédicelles florifères supérieurs, par ses écailles fortement nerviées, et marquées très-irrégulièrement de taches d'un rouge sanguin. Le Cyperus rubicundus Vahl, originaire des Canaries, et dont notre espèce offre le port, a les fleurs des épillets disposées d'une manière beaucoup plus régulière. Ce dernier est d'ailleurs vivace, à souches crspiteuses, gazonnautes, et ne saurait être confondu avec le $C$. sinensis qui est annuel.

210. G. (Pycreus) sanguinolentus Vahl Enum. 2, p. 35̆l, Nées ab Esemb. in Wight Contr. bot. of india, 75; Miquel Prol. jap. 72; C. eragrostis Steudel Syn. glum. II, p. 5 ; Benth. F'l. Hongkong. 385, an Wahl?, G. Staunt. Plant. chin. exsicc. (1793).

«Planta Chinæ borealis humilis gracilisque, radice annua, fibro» sa; culmis trigonis erectis, 15-20 centim. altis, sulitariis vel » rarius basi 2-3-nis; foliis anguste linearibus, planis, apice » marginibusque scabris, culmo brevioribus vel eum æquantibus; » umbella (anthela) composita, 4-10 radiata, in capitulum subglo» bosum contracta; spiculis omnibus fasciculatis, ovato-oblongis, »10-12 floris; squamis (glumis exter.) carinatis, late ovatis, obtu》 siusculis, apice contractis, dorso trinerviis, membranaceis, pal» lide ferrugineis, marginibus fusco-sanguineis, carina viridi; » involucro triphyllo, phyllis patentibus inæqualibus, umbella "multo longioribus, ad margines apices que tantum subscabris, " phyllo breviore, basi scabrido-serrulato. "

Hab. : Les sables de la rivière Ta-hô inondés à marée pleine, près de la ville de N'gin-haï-tchéoû, et non loin du cap Chantong. - Fl. et fr. 18 septembre 1860.

Ar. géog. : Asie subtrop. et temp.; Indes-orient. Himalaya, Bengale, Singapôore; Chine bor., moy. et aust.; Hong-kông, Chantong et Kiang-nan (G. Staunt.); Ile Maurice. - Afriq. bor. et aust., Tanger, Cap de Bonne Espér.

Obs. : Plante très-voisine du C. eragrostis Wahl, de l'Asie subtropicale, de l'Afrique bor. et aust., et même du midi de l'Espag'ne, dont elle diffère : par ses tiges dressées et non couchées à la base, par ses feuilles étroitement-linéaires, et non largement linéaires, par ses épillets de 10-12.fleurs, ovales, et non de 16-24 fl., linéairesaigus, par ses écailles d'un rouge-pâle, à bords teintés de rouge fer- 
rugineux et trinerviées, et non simplement ponctuées de petites taches d'un rouge pâle, et 5-nerviées.

Je n'ai point rencontré cette rare cypéracée ailleurs que sur les sables saumâtres de la rivière Ta-hô (Grande rivière), au cap Chan-tong.

211. C. (Eucyperus) subfuscus O. Debeaux Mss.; C.fuscus var. chinensis 0 . Debx. olim.

Icon. nostra, tab. III, fig. 2.

"Humilis, radice fibrosa, annua; culmis 1-3 gracilibus, erectis, » 6 - 12 centim. altis, trigonis, striatis, glabriusculis, basi foliatis; » involucro triphyllo, phyllis anguste linearibus, subpatentibus, » inæquilongis, anthelam 2-3-plo superantibus; foliis linearibus, » culmis multo brevioribus, e vagina membranacea fulvo-striata, » apice mucronulata nascentibus; umbella simplici vel sæpius 》 composita, radiis 5-9 valde inæqualibus, ad apices culmorum » fasciculatim dispositis; spiculis lanceolatis, compressis, 20-30 » floris, flosculis parum distantibus alternisque; squamis flosculi » fertilis ovato-lanceolatis, emarginatis, in media parte (ad margi»nem) flexuoso-sinuatis, apice subrotundatis mucronulatisque, » fusco-brunneis, exsiccatione atro-ferrugineis, carinato-navicu» laribus, trinerviis, nervis parum distinctis, mucrone incurvato; » squamis flosculi sterilis obovato-lanceolatis, emarginatis, 3-5 » nerviis (nervis valde distinctis viridescentibus), apice mucro» nulatis, mucrone deflexo erecto; akenio obovoïdeo, subtrigono, » læviter punctulato, castaneo-fusco, squamis parum breviore; 》 staminibus 2.

»Affinis $C$. fusci videtur, sed valde ab eo differt, culmis gracili» bus, subsolitariis nec cæspitosis, foliis lineari-filiformibus, squa» mis distincte 3-5 nerviis nec uninerviis, akeniis obovoïdeis 》 angulatis, angulis maturitate obtusissimis punctulatisque. ”

Hab. : Sables maritimes et champs du littoral à Yan-taï, Kitsen-sôo, etc. - Fl. 22 septembre 1860.

Ar. géog. : Chine bor., Chan-tong.

Obs. : Le C. subfuscus quej'avais pris d'abord, et d'après l'avis de M. A. Franchet, pour une variété remarquable du vulgaire $C$. fuscus L., me paraît devoir constituer une espèce distincte de celui-ci. La plante chinoise diffère surtout du $C$. fuscus :

10 Par ses écailles d'un brun clair, noircissant par la dessiccation 
distinctement triverviées, ou même 3 -nerviées et non uninerviées, avec une largre bande verte, à sommet plus obtus incombant, et non plus ou moins étalé comme dans le $C$. fuscus du midi de l'Europe;

2. Par soll rachis bordé d'une aile plus étroite;

$3^{\circ}$ Par ses achaines obovés au sommet, subtrigônes, et dont les angles sont très-obtus à la maturité, et couverts de ponctuations plus apparentes et scabres.

Dans notre $C$. subfuscus, les écailles fertiles sont largement ovales, arrondies vers le sommet et même un peu émarginées, d'un brun ferrugineux sur les côtés, avec la marge plus pâle, et embrassant par leur base la moitié du rachis. Ces écailles enveloppent entièrement l'achaine, et uffrent vers la partie médiane du dos 3 à 5 nervures vertes, celle du milieu plus robuste n'atteignant pas le sommet de l'écaille, ou se prolongeant en un mucron extrêmement court, calleux et accombant. Dans les glumes des fleurs stériles, ce mucron est défléchi en dehors, puis redressé. Le caractère le plus important qui justifie la séparation de notre plante du $C$. fuscus, se trouve dans la forme remarquable des écailles, qui embrassent par leur base la moitié au moins du rachis.

212. G. (E'ucyperus) difformis Lin. Spec. 67; Steud. Syn. glum, 2, p. 23; Ledeb. Fl. ross. 1V, p. 242; Roxbg. Fl. ind. 1, p. 195; Wight in Contr. bot. of Ind. p. 88; Naxim. Prim. amur. 297; Benth. Fl. Hongkong. 385; Miq.Prol. jap. 73; Forma A. genuina G. Staunt. Plant. chin. exsicc. (1793).

«Culmo erecto, elongato, 35- 10 centim. alto, simplici vel basi » pluricauli, subcrespitoso, umbella 4-5 spicis composita.»

Forma B. humilis O. Debx..in Herb. :

"Culmo erecto, humili, 6-10 centim. alto, simplici, umbella 1-2 » spicis globosis composita. »

Hab. : La forme genuina dans les plaines occidentales du Chantong, sur lessives du Canal impérial (G. Staunton); la forme $h u$ milis, sur les sables de la plage de Ki-tsen-sôo, où elle est abondante. - Fl. et fr. 28 septembre.

Ar.géog. : Eur. aust., Italie, Grèce. - Asie intertrop., bor. et or., Indes-orient.- - Chine (Hong-kông, Fô-kien, Kiang-nan, Chantong). - Amour et Ussuri. - Japon. - Iles de la Malaisie, Timor, Java, etc. - Les Philipp. - Australie. - Ile Maurice. - 
Afriq. bor., or. et aust., Égypte, Guinée, Sierra-Leone, Cap de Bonne-Esp.

Obs. : La variété humitis, la seule que l'on trouve en abondance sur les sables maritimes du Tché-foû, est conforme aux spécimens provenant de la province de l'Amour. Ceux-ci ont 6 à 10 centim. de hauteur, et leur ombelle est le plus souvent composée d'un épillet (rarement deux), subsphérique, et d'un brun trèsfoncé.

213. G. (Eucyperus) iria Lin. Spec.67; Steud. Syn. glum. 2, p. 23; Wight Contr. bot. of India, 87; Roxbg. Flor. ind. 1, p. 204; Benth. Flor. Hongkon. 386; Miquel Prol. jap. 73; G. Staunt. Plant. chin. exsicc. (1793); C. panicü̈des Lamk. Ill. gen. 1, 145.

« Culmo triquetro, 35-40 cent. alto, foliis planis linearibus, gla» bris, caule multo brevioribns, margine scabris; involucro tri» phyllo, phyllis spiculas duplo superantibus; umbella radiis spi» catis composita, spiculis imbricatis, oblongo-linearibus, pallidis, » compressis, 10-20 floris; squamis obovatis, obtusis, submucronu » latis, 3-5 nerviis, emarginatis, carinatis, caryopsim (akenium) » æquantibus; akenio trigono, castaneo, nitido, apice mucronu» lato. »

Hab. : Champs sablonneux et prairies marécageuses du littoral au N.-O de Yan-taï. - Fl. et fr. d'août à septembre.

Ar.géog.: Asie intertrop. et temp., Indes-orient., Himalaya, Népaul, Mysore, Ceylan. - Chine (Hong-kông, Koûang-tong à Macâo, Kiang-nan, Chan-tong, Pé-tché-ly). — Japon. - Iles Philipp. - Amér. bor.

214. G. (Eucyperus) rotundus Lin. syst. 98; Kunth Enum. 2. p. 58, et Steud. Syn. glum. 2, p. 32 (ex parte); Roxbg; Flor. ind. 1, p. 201; Benth. Fl. Hongkong. 387; 0. Debx. F'l. Shang-hä̈, n 132; Miquel Prol. jap. 75; C. hexastachyus Rottb. Gram. 28 ex Wight Cont. bot. of Ind. 90; C. tuberosus Roxbg. ex Walh.; sinice Tsan-LIN, Hiô-Tsâo.

"Planta chinensis quam in speciminibus indicis multo robus» tior, erecta, 40-50 centim. alta, glaberrima; anthela decompo» sita, involucro triphyllo umbellam superante, spiculis fusco» castaneis, nitidis, lineari-lanceolatis. 
Hab. : Les sables maritimes de Yan-taï et les grandes dunes des Foû-chan-yên. - Fl. et fr. 4-14 septembre.

Ar. géog. : Régions intertrop. et temp. des deux mondes, Afriq. occ. et or. Sierra-Leone ( $\mathrm{Vog} C \mathrm{l})$. - Asie intertrop., Indes-orient., Ceylan, Singapôore. - Chine (Hong-kông, Ki iang-soû, Chantong). - Japon. - Iles Philipp. - Java. - Australie, Iles Maurice et Bourbon. - Amér. bor., cent. et aust., — Martinique et Guadeloupe, etc.

\section{KILLINGIA Rottb.}

215. K. monocephala Lin. Sípec. 104; Kunth Enum. 2, p. 129; Steud. Syn. glum. 2, p. 67; Roxbg. Fl. ind. 1, p. 185; Wight Contr. bot. of Ind. 91: Benth. Fl. Hongkong. 288; Miquel Prol.jap. 74 ;

Var. triceps; Killingia triceps Rottb. Gram. p. 14; Wight (loc. cit.) 91; G. Staunt. Plant. chin. exiscc. (1793).

"Capitulis ovato-subglobosis, 2 vel sæpius 3 sessilibus, inæ" qualibus; involucro 3-4 phyllis, phyllis capitulos multo superan"tibus; foliis linearibus, margine scabridis, culmo longioribus; » culmis erectis, trigonis, 40-45 centim. altis, basi cæspitosis; » spiculis diandris unifloris : squamis ovali-oblongis, acutis, mem» branaceis, hyalinis, glabris, carina subciliatis, quinquenerviis;

» akenio oblongo-compresso; radice fibrosa, annua. »

Hab. : Sables maritimes du cap Chan-tong, sur les rives du Ta-hô (O. Debx.), partie occid. du Chan-tong (G. Staunton.) Septembre.

$A r . g e ́ o g .:$ Asie intertrop. et temp., Indes-orient., Népaul. —Chine (Hong-kông, Koûang-tong, Kianğ-si, Kiang-nan, Chan-tonğ, etc.). - Japon. - Iles de la Malaisie, Timor, Java. - Iles Maurice. Australie. - Afriq. intertrop., Sierra-Leone, Fernando-Pô. Amér. trop., Antilles françaises, etc.

\section{LIPOCARPHA R. Brown.}

216. L. microcephala Steud. Syn. glum. 2, p. 130; Kunth Enum. plant. 2, p. 268 ; Benth. Fl. Honykong. 389; Miq. Prol.jap. 79; Hypcelytrum microcephalum R. Brown Prodr. 220.

Icon. nostra, tab. III, fig. 2 (une fleur grossie).

«Herbacea, annua, humilis, radice dense fibrosa, culmis simpli» cibus, setaceis, triquetris, 6-10 centim altis, lævibus, basi vagi- 
»natis, vaginis foliiferis nudis; foliis anguste linearibus, planis, » culmo brevioribus, subglabris involucro diphyllo, phyllis lon» gissimis; capitulis ad apices culmorum aggregatis, ternis, » rarius binis, subglobosis; squamis internis 2, cuneatis, angus» tis, basim akenii amplectentibus, squama exteriore duplo lon》 giore, apice subulato-aristato.»

Hab. : Sables et champs cultivés du littoral, baie de Ki-tsen-sôo. - Fl. et fr. septembre.

Ar. géog. : Chine bor. et austr. Hong-kông, Chan-tong. Japon. - Java. - Australie.

Obs. 1 : Le Lipocarpha microcephala, dont la présence sur les sables du Tché-foû, est un fait très-intéressant pour l'histoire de la dispersion géographique de cette plante, ressemble à s'y méprendre à l'Isolepis squarrosa Ræm. et Schult. Mais il suffit d'examiner avec soin les deux écailles inférieures de la fleur, qui sont pour ainsi dire soudées à leur base et qui étreignent si bien le fruit, qu'elles semblent faire corps avec lui, pour être certain d'avoir sous les yeux une espèce du genre Lipocarpha, et sans aucun doute le $L$. microcephala (Teste A. Franchet, in lilt.), dont l'habitat a été déjà signalé dans le sud de la Chine et au Japon.

Obs. 2: Je possède plusieurs échantillons de l'Eleocharis acicularis $\mathrm{R}$. Brown (Scirpus acicularis Lin.), récoltés par G. Staunton en 1793 dans les marécages qui avoisinent le canal impérial, au sud de la province du Chan-tong. Cette plante trèscommune dans toute l'Europe, et dont l'area s'étend jusqu'aux limites de l'extrême-Orient dans leś provinces de l'Amour et de l'Ussuri, au Japon et dans l'Amérique boréale, se retrouvera probablement dans les marécages non encore explorés du promontoire du Chan-tong.

\section{ISOLEPIS R. Brown.}

217. I. Micheliana Ræm. et Schult. Syst.veget. 2, p. 114 ; Steud. Syn. glum. 2, p. 98; Maxim. Prim. amur. 300; Regel Tent. A. ussur. 160 ; Miq. Prol.jap. 75 ; Dichostylis Micheliana Nées ab Esemb. in Wight Contr. bot. of Ind. 94; Scirpus Michelianus Lin.

$H a b$. Les sables humides et saumâtres, sur les rives du Ta-hô (grande rivière), près de son embouchure dans la mer, à N'ginhaï-tchéoû, au cap Chan-tong. - Fl. 18 septembre 1860. 
Ar. géog. : Eur. aust. et or., Tauride, Astrakan, mer Caspienne.Asie cent. et bor., Indes orient. - Chine litt. - Prov. de l'Amour et de l'Ussuri. - Japon. - Afriq. bor. et intertrop., Algérie, Senégal, etc.

218. I. capillaris Ræm. et Schult. Syst. vey. 2, p. 118; Kunth Enum. 2, p. 211; Steud. Syn. glum. 2, p. 171; Miquel Prol. jap. 75 ; I. barbata R. Brown var. capillaris Nées ab Esemb. in Wight Cont. bot. of Ind. 109.

Var. indica Kunth (loc. cit.); Scirpus capillaris Lin. Mant. 321; Sc.tenuissimus Don Prodr. Nep. 40.

Hab. : Les sables maritimes, sur la plagee de Ki-tsen-sôo. - Fl. et fr. octobre.

Ar. géog. : Eur. aust., Russie mérid. - Asie intertrop. et temp., Indes-orient., Népaul, Ceylan. - Chine bor., Chan-tong. - Japon. - Afriq. occ., Grand-Bassan, Sénégal, Nigritie. - Amér. bor. et aust.

Obs. 1: L'Isolepis barbata R. Br., auquel Nées ab Esemb. réunit comme variété l'I. capillaris de Ræm. et Schult. croît sur les sables maritimes du sud de la Chine, au Japon, aux Indesorient., etc. (x. Staunton l'a récolté égalementsur les sables marit. du Chan-tong, mais sans indication de localité. Peut-être ce botaniste l'a-t-il rencontré sur les rivages de Ki-tsen-sôo, où l'escadre anglaise avait jeté l'ancre pendant son voyage en Chine. La plante de Staunton est plus élevée que l'I. capillaris, ses capitules sont plus denses, et les épillets plus fournis. M. A. Franchet n'y voit qu'une forme plus robuste de l'I. capillaris.

Obs. 2: J'indique encore comme étant très-probable sur les sables maritimes du Tché-fou, l'habitat de l'Isolepis squarrosa Ræm. et Schult. dont je possède des échantillons recueillis par G. Staunton dans les provinces de Kiang-nan et lu Chan-tong, sur les rives du canal impérial. Voici une courte diagnose de cette espèce.

I. squarrosa Ræm. et Schult. Syst. veget. 2, p. 110 ; Scirpus squarrosus Lin. Mant. 181; Sc. sinensis Osbeck Iter. p. 200.

"Radice fibrosa, annua, culmis minutis, erectis (6-8 centim. »fliformibus, subtetragonis, compressis, cæspitosis, basi foliatis; » foliis linearibus, culmo brevioribus, striatis, subulatis; spiculis » 2-3, sessilibus vel breve pedicellatis, ovatis ; involucro diphyllo, 
》 phyllis inæqualibus, altero capitulum multo superante, basi dila" tatis; squarnis membranaceis, 2 internis linearibus, 3 nerviis, » aristatis, 1 externa longiore, apice subulata, akenia involven»tibus. »

\section{FIMBRISTYLIS Vahl.}

219. F. Sieboldii Miquel in Cat. mus. bot. Lugd. Batav. p. 118 (1870); F. Zeiocarpa Miquel Prol.jap., p. 76 (1867) non Maximowicz, quæ alia species!

«Forma genuina : cæspitosa, annua, culmis erectis, striatis, 30-50 » centim. altis, gracilibus, compressis, basi foliatis; foliis anguste » linearibus, margine scabriusculis, culmo multo brevioribus; invo» lucro monophyllo, brevi; spiculis 1-3 (rarius), uno sessili, alteris » pedicellatis, ovali-oblongis, acutis; squamis ovatis, mucronatis, » ferrugineis; acheniis pedicellatis, lævibus, nitidis, obovoïdeis, » compressis, fusco-castaneis; stylo bifido.»

Hab. : Les dunes maritimes de Foû-chan-yên, et les mares saumâtres ou salées au N.-0. de Yan-taï. - Fl. et fr. 4-14 septembre. Ar. géog.: Chine bor. et aust.; Hong-kông, Kouâng-tong, Chantong. - Japon. - Iles Philippines.

Obs. : Le nom spécifique de leiocarpa donné primitivement par Miquel dans son Prolusio, p. 76, ne peut être conservé à ce Fimbristylis, parce que la même dénomination a été employée huit ans auparavant par M. Maximowicz, in Prim. flora amurensis, pour désigner une autre espèce de l'Amour, du groupe des $F$. dichotoma et annua. Dans son Catalogue du musée botanique de Leyde, Miquel a changé le nom de leiocarpa en celui de Sieboldii que nous adoptons. La plante du Tché-foû est parfaitement varactérisée par ses tiges grêles, dressées, par ses feuilles étroitement linéaires, presque filiformes, par ses épillets 1-3, ovales-oblongs, acuminés, et surtout par ses achaines entièrement lisses, pédicellés, ovoïdescomprimés et d'un brun foncé.

220. F. Buergeri Miquel Prolusio flora japon. p. 76.

«Affinis $F$. subbispicat $a$ Nées ab Esemb., sed differt : foliis latio》 ribus, marginibus ad apicem scabris; spiculis 6-10, umbellam » inæqualem efformantibus, longioribus, conico-subcylindricis; » squamis fuscis vel griseo-fusco variegatis. »

Hab. : Les dunes maritimes et les mares saumâtres ou salées, au N.-0. de Yan-taï - Fl. et fr. 4-14 septembre. 
Ar. géog. : Chine (Chan-tong). - Japon.

Obs. : Le $F$. Buergeri ne diffère du $F$. subbispicata que par ses feuilles beaucoup plus larges, ses épillets beancoup plus allongés, et réunis au nombre de 6-10 en forme d'ombelle.

221. F. squarrosa Valh Enum. 2, p. 289; Kunth Enum. plant. 2, p. 224; Steud. Syn.glum. 2, p. 110; Bent. Fl. Hongkong. 392; Maxim. Prim. fl. amur. 301; Regel Tent. flor. ussur. 163.

« Cæspitosa, radice fibrosa, annua; culmis glabris, 8-12 centim. »altis, basi foliatis; foliis anguste linearibus, planis, subtus » marginibusque scabris, vaginis hirto-pubescentibus; involucro » tetraphyllo, phyllis linearibus, anthela brevioribus, vel eam vix » æquantibus; umbella decomposita, spiculis oblongis squamosis, » centrali sessili, alteris pedicellatis divaricatisque; squamis ova» tis, aristatis, mucronulatis, fusco-brunneis; stylo bifido, basi »villoso. »

Hat. : Champs sablonneux du littoral, à Yan-taï, Ki-tsen-sôo, etc. - Fl. 26 septembre.

Ar. géog.: Asie intertrop. et temp., Indes-orient. - Chine (Hong-kông, Chan-tong). - Prov. de l'Amour et de l'Ussuri. Iles de la Sonde, Java, Sumatra. - Australie. - Afriq. intertrop. Amér. aust.

222. F. tomentosa Valh Enum. 2, p. 200; Nées ab Esemb. in Nov. act. natur. cur. XVIII, suppl. p. 815, et in Wight Cont. bot. of Ind. 100; Steud. Syn. glum. 2, p. 115 ;Kunth Enum. 2, p. 224 pro parte; $F$. diphylla var. tomentosa Benth; Flor. Hongkong. 382; Miquel Prol.jap. 76; G. Staunt. Plant. chin. exsicc. (1793) sub Scirpo.....

« Cæspitosa, humilis, culmis sulcatis, angulosis, 10-12 centim . » altis, glabris; foliis involucrantibus $3-5$, foliolis linearibus, basi » dilatatis, margine pilosis, ciliatis, duobus umbellam duplo » superantibus, alteris brevioribus; foliolis involucelli linearibus, » glabris, setaceis; foliis culmo brevioribus, planis, striatis, mar» gine pilosis; vaginis fuscis, membranaceis, undique pilosis; » umbella decomposita, patenti, radiata; capitulis 4-6-radiis spica" tis; spiculis ovatis, acutis, solitariis: squamis acutis, ovatis, 》 monandris, fuscis, dorso membranaceis ; akeniis obovatis, multi» costatis. » 
$H a b$. : Sables humides ou inondés à haute mer, près de N'gin-haïtchéoû, au cap Chan-tong (18 septembre 1860). Sables des rives du eanal impérial dans la province de Chan-tong ( $G$. Staunton).

Ar.géog.: Asie intertrop. et temp, Indes-orient. - Chine (Hongkông, Kiang-nan, Chan-tongr). - Japon. - Iles Philip. - Java. Afriq. Intertrop.

Obs. : Cette espèce peu répandue sur le littoral de la Chine se distingue par ses chaumes glabres striés-sillonnés, par ses feuilles plus courtes que les chaumes, velues, ciliées sur les bords, à gaines membraneuses très-velues à la base, par son oinbelle composée de 6-8 rayons, dont 2-3 à un seul épillet ovale aigu, et les autres rayons plus ombellulés à leur sommet; par son involucre de 3 à 5 folioles, dont 2 dépassent du double les capitules floraux, les autres folioles beaucoup plus courtes, velues, ciliées sur les bords, par les folioles de l'involucelle courtes, glabres, linéaires et sétacées au sommet, etc.

223. F. miliacea Valh Enum.2, p. 287; Steud. Syn. glum.2, p. 43; Bent. Fl. Hongkong. 293; Miq. Prol.jap. 77, Trichelostylis miliacea Nées in Wight. Contr. bot. of Ind. 103; Scirpus miliaceus Lin.; G. Staunt. Plant. chin. exs. (1793).

Hab. : Sables et prairies rnarécag. du littoral de Yan-taï. - Fl. septembre. - Rives du canal impérial dans l'ouest du Chan-tong (G. Staunt.).

Ar. géog. : Asie intertrop. et temp., Indes-orient., Silhet, Ava, Népaul, Ceylan. - Chine (Hong-kông, Koûang-tong, Chan-tong; Kiang-nan, etc.). - Japon. - Iles Philipp. — Java, Madagascar. Afriq. tropicale.

Obs. : Parmi les Cypéracées provenant des récoltes de sir Georges Staunton dans le nord de la Chine, et dont une partie avait été acquise jadis par mon très-regretté correspondant et ami A. Maille, ils'est trouvé une espèce de Fimbristylis reconnue nouvelle par M. Franchet, et que nous dédions ensemble à l'auteur même de la découverte. M. Franchet a bien voulu me communiquer la description qu'il en a faite, d'après les échantillons de mon herbier.

224. F. Stauntcni O. Debeaux et A. Franchet Mss. (1875); G. Staunton Plant. chin. exsicc. (1793), sub Scirpo....

Icon. nost. tab. III, fig. 1 (une fleur grossie). 
«Fleurs nombreuses (20-40), formant des épis globuleux ou » ovoïdes disposés en ombelles simples, celui du centre sessile, les » autres inégalement pédonculés, solitaires ou quelquefois accom» pagnés de 1-2 épis sessiles placés latéralement, disposition qu'on » observe fréquemment chez les $F$. squarrosa et dichotoma et qui » semble résulter de l'atrophie d'un pédoncule dont la présence » aurait rendu l'ombelle plus ou moins composée. Écailles oblon"gues atténuées au sommet, presque moitié plus longues que le » fruit mûr, blanchâtres ou d'un roux pâle, membraneuses, trans»parentes sur les bords, parfois un peu ponctuées, obscurément » trinerviées avec la nervure dorsale verte ou ferrugineuse, plus » fortes que les latérales, ne dépassant pas le sommet de l'écaille, » ou faisant un peu saillie en forme de mucron glabre, légèrement » déjeté en dehors. Achaines jaunes, cylindriques oblongs, un » peu arqués, très-obtusément trigonés, régulièrement et finement »cancelless, relevés de 12-15 côtes longitudinales, brièvement » atténués à la base, pourvus d'un anneau fugace et supporté par » un stype. Style glałore, renflé à la base en un bulbe qui égale le » tiers de la longueur de l'achaine, du sommet duquel il est séparé » par un col ou étranglement très-marqué. Branches stigmatiques » au nombre de trois, très-papilleuses allongées. Étamines 2, éga» lant environ la longueur du style.

» Plante annuelle, glauque, glabre, à chaumes anguleux, com" primés (?); feuilles plus courtes que les chaumes, linéaires, pla» nes, courbées en faulx, obtuses, serrulées sur les bords et surtout » au sommet; involucre formé de 4-5 bractées, sétacées ou sembla» bles aux feuilles, très-inégales, plus longues ou plus courtes » que les rayons de l'ombelle. » (A. Franchet).

$H a b$. : Sables marécagreux de la province de Chan-tong(G.Staunt.), sans indication de localité.

Ar. géog. : Chine bor. et or.

Obs. : Les épis du F. Stauntoni rappellent beaucoup par leur forme ceux du $F$. miliacea, mais ils sont plus gros, et les deux plantes diffèrent notablement d'ailleurs. C'est plutôt des $F$. tenuis Ræm. et Schult. et $F$. consanguinea Kunth, qu'il faudrait le rapprocher à cause de son style trifide et de ses achaines cancellés. Ce dernier caractère l'éloigne tout à fait du $F^{\prime}$. leiocarpa Maxim. dont l'achaine est entièrement lisse. 


\section{CAREX L.}

225. G. macrocephala Wild. Herb. n ${ }^{0} 1725$ ('Teste Kunth Enum. pl. 2, p. 428); Steud. Syn. glum. 2, p. 190 ; Trev. in Ledeb. Fl. ross. IV, p. 271; Regel Tent. fl. ussur. 184, tab. XII; A. Gray Plant.jap. Perry exped. 328; Boott. in Hnok. Fl. bor. amer. 2, p. 215.

"Planta chinensis speciminibus japonicis omnino simillima, » humilis, non cæspitosa, radice longe repente, pennæ anserinæ » crassitie; caulibus simplicibus, solitariis cum spica 10-12 centim. » altis, erectis, obtuse trigonis, striatis, glabris, basi fibris folio" rum vetustorum indutis; foliis caulium sterilium longissimis » (35 centim. et ultra longis, 5-6 mill. latis), junioribus perspicue » carinatis, fere triangularibus, supra substriatis, subtus nervoso» sulcatis, margine serrulatis, foliolis involucri 2-3, inæquilongis, » spicam duplo superantibus, plano-striatis, ad marginem scabri» dis; spica terminali capitulum macrostachyum ovato-oblongum »densumque efformante; spiculis androgynis (masculis cum femi"neis mixtis;; bracteis inferioribus dimidiam partem capituli » subæquantibus, superioribus plus duplo akenio longioribus, » omnibus ovatis, in acumen argute serrulatum attenuatis; "squamis ovatis, acuminatis, apice serrulatis, ad medium 5-7 » nerviis, glabris, utriculo brevioribus vel eum subæquantibus, " late marginatis, brunneo-virescentibus; utriculis (akeniis) nitidis » fusco-brunneis, ventre planis, dorso convexis, ovatis, in rostrum » acuminatis, bicuspidatis, utrimque plurinerviis, margine cartila" gineo-serrulatis, 8-10 millim. longis, 4 millim. latis ; stylo apice » bifido. »

Hab. : Sables maritimes sur la plage de Ki-tsen-sôo.-Fr. 4-15août. Ar.géog. : Asie bor., or.-Sibérie (Pallas).-Chine, Chan-tong au Tché-foû (O.Debeaux). - Ussuri (Regel).—Japon (A.Gray, Maxim., L. Savatier). - Amér. bor. (Hoo\%.).

Obs. : La forme du Tché-foû, que l'on pourrait nommer longifoliata, est la seule que l'on rencontre sur les sables inaritimes de cette contrée. Cette forme se retrouve également au Japon. Elle se distingue du C. mxcrocephala (forma genuina), par les feuilles des rejets stériles très-allongées (35 à 40 centim.), et celles des tiges fertiles dépassant de beaucoup les capitules fructifères, toutes scabres et dentelées sur les bords. 


\section{GRAMINÉES JUSS.}

PANiCUM L.

226. P. (Digitaria) ciliare Retz Obs. IV, p. 16; Steud. Syn.glum. 1, p. 39; Miquel Prol.jap. 165; Digitaria ciliaris Pers. Syn.I, p. 85; G. Staunt. Plant. chin. exsicc. (1793).

Hab. : Cultures, bords des champs et des chemins, à Yan-taï, Si-nen-kôo, etc. - Fl. septembre-octobre.

Ar. géog.: Eur. - Asie bor., occ. et or.; Asie min.; Indes-orient. - Chine (Chan-tong, Kiang-nan). - Japon. - Iles Philipp., Java, Australie. - Afriq. bor. et aust., cap de Bonne-Esp.

\section{OPLISMENUS P. Beauv.}

227. O. frumentaceus Kunth Enum. 1, p. 146; Miquel Prol. jap. 162; Panicum frumentaceum Roxbg. Flor. ind. 1, p. 307; Steud. Syn. glum. 1, p. 47; G. Staunt. Plant. chin. exsic. (1793); sinice CHOûI-PAï.

Hab.: Cultivé en grand dans les terres sablonneuses de la plaine, et jusque sur les dunes maritimes de Foû-chan-yên. Fr. septembre. - Champs du Chan-tong occid. (G. Staunt.)

Ar.géog.: Asie intertrop., Indes-orient. - Chine (Chan-tong, Kiang-nan, Pé-tché-ly). - Corée. - Japon. - Afriq. cent. et occ., Nigritie (Vogel). - Amér. cent., Guadeloupe. - Nouv. Calédonie.

228. O. crus-galli Kunth Enum. 1, p. 143; Miquel Prol. jap. 162, Panicum crus-galli Lin.; Bunge Enum. chin. bor. n 397; Benth. Flor. Hongkong. 411 ; Echinochloa crus-galli Ledeb. Flor. ross. IV, p. 473; Turczan. Fl. baik.-dahur. 3, p. 299; Maxim. Prim. amur. 331; sinice Paï.

Forma mutica: humilior quam planta europeana, glumis non perspicue aristatis.

Hab. : Sables marécageux du littoral, au N.-O. de Yan-taï. Fl. septembre.

Ar. géog.: Eur. - Afriq. bor.-Asie bor. et or., Sibérie altajque, Baikalie, Daoûrie, Mongolie. - Chine bor. et aust. - Amour et Ussuri.-Japon, Australie.-Amér. bor. et cent., Guadeloupe, etc. 


\section{SETARIA P. Beauv.}

229. S. glauca P. Beauv. Agrost. 51; 0. Debx. Fl. Shang-haï, no 139; Miquel Prol.jap. 163, et Fl. ind. batav. 3, p. 466; Maxim. Prim. amur. 330; Regel Tent. ussur. 172; Panicum glaucum Lin.; Benth. Fl. Hongkong. 411; Steud. Syn. glum. 1, p. 50.

Hab. : Champs sablonneux de la plaine du Tché-foû. - Fl. aoat et septembre.

Ar. géog. : Régions intertrop. des deux mondes. - Eur. - Afriq. bor., occ. etaust. - Nigritie (Vogel). - Asie bor., occ. et or., Indesorient. - Chine bor. et aust. - Amour et Ussuri. - Japon. Java, Australie. - Amér. bor. et cent. - Antilles françaises.

230. S. italica Kunth Enum. plant. 1, p. 153; Ledeb. Fl. ross. IV. p. 471 ; Maxim. Prim. amur. 330; Regel Tent. A. ussur. 172; Miq. Prol.jap. 163; Panicum italicum Lin.

Var. germanica P. Beauv. Agrost. 51; 0. Debx. Shang-hä̈, $\mathrm{n}^{\circ} 140$ Panicum germanicum Bunge Enum. chin. bor. $\mathrm{n}^{\circ} 399$; sinice MEI-TZÉ, Siâo-MEI.

Hab. : Cultivé en plein champ dans les terres sablonneuses et les sables au N.-O. de Yantaï. - Fr. août.

Ar. géog. : Eur. - Asie intertrop. et temp. - Indes-orient. Chine bor., cent. et aust. - Amour et Ussuri. - Japon. - Australie. - Amér. bor.

\section{PANICUM L.}

231. P. miliaceum Lin. Spec. 86; Steud. Syn. glum. 1, p. 77; Ledeb. Fl. alt. 1, p. 77; Bunge Enum. chin. bor. no 400; O. Debx. Fl. Shang-haï, no 141; Maxim. Prim. amur. 330; Regel Tent. fl. ussur. 171; Miq. Prol. jap. 163; sinice СночMEI, Kou-TZÉ.

$H a b$. : Cultivé en grand dans les terres sablon. de la plaine et sur les basses collines micasch. - Fr. en septembre.

Ar.géog. : Eur. - Asie intertrop. et temp. - Indes-orient. - Chine bor. et cent. - Amour et Ussuri. - Japon.

ERIOCHLOA Kunth.

232. E. villosa Kunth Enum. 1, p. 72; 0. Debx. Fll. Shang-hä̈ $\mathrm{n}^{\circ}$ 142; Helopus villosus Nées ab Esemb. in Mart.Brasil, 2, 
p. 17; Miquel Prol.jap. 164; Paspalum villosum Thumbg., Fl. jap. 45.

$H a b$. : Les collines arides micaschit. au-dessus de Si-nen-kôo, falaises de Ki-tsen-sôo. - Fl. 1 août.

Ar. géog. : Chine litt., bor. et cent. (Kiang-sou, Chan-tong). Prov. de l'Amour et de l'Ussuri. - Japon. - Amér. aust., Brésil.

\section{GYMNOTHRYX P. Beauv.}

233. G.japonica Kunth Enum.1, p. 158; Benth. F'l.Hongkong. 415; Miq. Prol. jap. 164; Pennisetum japonicum Trin. Icon. 2, tab. 19; Steud. Syn. glum. 1, p. 102; A. Gray in Plant.jap. Perry expéd. 329 ; Panicum hordeiforme'Thumbg. Fl. jap.46, tab. 9.

Hab. : Les prairies du littoral, où cette graminée est tellement abondante, qu'elle forme pour ainsi dire à elle seule la base du foin du Tché-foû. - Fl. juillet et août.

Ar.géog. : Chine bor., cent. et aust.: Hong-kông, Koûang-tong, Kiang-nan (G. Staunton), Chan-tong. - Japon.

\section{ARUNDINELLA Raddi.}

234. A. anomala Steud. Syn. glum. 1, p. 116; Miquel Prol. jap. 164.

«Herbacea, radice fibrosa, perenni (?). in locis humidis stolo» nifera; culmis erectis simplicibus, 60-90 centim. altis, glabris, »foliis late lanceolatis, acuminatis (15 cent. long., 10 millim. » latis), rigidis, striato-nervosis, margine scabris, ciliatis, basi 》dense pilosis, pilis e glandis quasi tuberculosis nascentibus, » vaginis foliorum glabris, vel ad margines tantum ciliatis; » panicula simplici, brevi, vel composita, elongata (20 centim. et »ultra); spiculis erectis, glumis glabris nervosis, pallidis, infe" riore dimidiam partem flosculi superante, vel rarius eum subæ» quante, (sub lente) apice bidentata, brevi, mucronulata, flosculis » masculis diandris. »

Hab. : Les collines arides micaschist. au-dessus de Si-nen-kôo, (150 à 200 mèt. d'alt)., et les prairies saumâtres du littoral. au N.-0. de Yan-taï, - Fl. 10 août, 6 septembre. $A r . g \hat{o} o g$. : Chine, Chan-tong (O. Debx.) - Japon (Miquel).

Obs. : La présence de cette rare espèce sur le territoire chinois 
n'avait pas encore été signalée, que je sache, par aucun botaniste. L'Arundinella anomala possède deux stations bien différentes au Tché-foû, et varie beaucoup pour la hauteur de ses chaumes, selon la nature du sol. La forme de sa panicule, la villosité plus ou moins grande de toutes ses parties, etc., sont également trèsvariables.

\section{CRYPSIS L.}

235. G. aculeata Ait. Hort. kew. 1, p. 48 ; Steud. Syn.glum. 1, p 151; Ledeb. Fl. alt. 1, p. 75 ; Schanus aculeatus Lin.; G. Staunton Pl.chin. exsicc.(1793), et in Voy. Macartney, 4, p. $152(1805)$.

$H a b$. : Sables et débris micaschist. dans le lit des torrents, au cap Chan-tong. - Champs sabl. qui bordent le canal impérial à l'ouest de cette province (G. Staunton).

Ar. géog. : Eur. aust. - Afriq. bor. et occ., Algérie, Sénégal. Asie bor., Sibérie alt., Désert de Kirghyz. - Chine (Chan-tong, Kiang-nan, Pé-tché-ly).

\section{PHRAGMITES Trin.}

236. Ph. communis Trin. Fund. agrost. 134; Steud. Syn.glum. 1, p. 195; Ledeb. Fl. alt.1, p.28; Turczan. Fl. baik.-dahur.3, p. 315; Maxim. Prim. amur. 321; Miquel Prol.jap. 166; Arundo phragmites Lin.; Thumbg. Fl.jap. 55; G. Staunt. Plant. chin. exsicc. (1793); sinice HoûEI, YÉ-Tsâo.

Forma A gracilis P. Mabille in Rech. sur les plantes de la Corse. Fasc. 2, p. 44 (1869.)

"Panicula depauperata, e nigro fulva, glumis 23 floris. »

Forma B eriopoda P. Mab. (loc. cit.); Arundo rivularis Ledeb. Fl. alt. 1, p. 89.

«Humilis (75-100 cent. altus), panicula compacta fere in thyr» sum brevem; glumis 4-5 floris, fuscis; pedicellis villosis; foliis 》 angustis, acuminatis pungentibus. »

Hab. : La var. gracilis, dans l'intérieur du Chan-tong, au bord des rivières. - Rives du canal impérial (G. Staunton).

La var. B eriopoda abonde dans les marécages saumâtres, et sur les sables maritimes de Foû-chan-yên au N.-O. de Yan-taï. - Fl. 2 septembre, 9 octobre.

Ar.géog. : Eur.—Rég. caucas. - Asie bor., Sibérie alt., Baikalie, 
Daourie, Mongolie. - Chine (Kiang-si, Kiang-nan, Hô-nan, Chantong, Pé-tché-ly, etc.) - Prov. de l'A mour et de l'Ussuri. - Japon. - Australie. - Amér. bor. - Afriq. bor.

Obs. : La forme eriopoda du Ph. communis constitue une variété des plus remarquables. Elle est caractérisée par ses feuilles étroitement linéaires-lancéolées et longuement acuminées. Cette variété se retrouve auprès des lacs salés de la Sibérie altaïque, et en France sur les sables de la Sọlogne (A. Franchet, in litt.). M.P. Mabille l'a retrouvée dans la forêt de Montmorency, près de Paris.

\section{CHLORIS Swartz.}

237. G. caudata Trin. Bunge Enum. plant. chin. bor. n ${ }^{\circ}$ 404: G. Staunt. Plant. chin. exsicc. (1793), sub andropogone...

«Herbacea, cæspitosa, radice perenni repente; culmis prostatis, " dein ad nodos flexuosis, erectis, simplicibus, cumpressis, gla» bris, 30-35 cent. altis; foliis lineari-lanceolatis, acutis, planis, »vaginisque glabris; spicis 7-9 digitatis, radiato-erectis, 50 mil» lim. longis; spiculis imbricatis; glumis 2, aristatis, inæquilongis, »superiore caudata, flosculum hermaphroditum apice ciliato» villosum subæquante, aristis fere æqualibus; flosculis sterilibus »apice setigeris, setis omnibus æqualibus, glumas subduplo » excedentibus. »

Hab. : Prairies, champs cultivés, et collines micaschist. du Tché-fou; abonde également sur les sables maritimes. Fl. d'août à septembre.

Ar. géog. : Chine bor., Chan-tong, Kiang-nan, Pé-tché-ly.

Obs. : Le Chloris caudata, quoique décrit par Bunge en 1831, n'est pas mentionné dans l'Enumeratio plantarum de Kunth, ni dans le Synopsis glumacearum de Steudel. Cette espèce est excessivement répandue au Tché-foû, où elle paraît remplacer (par ses racines longues et rampantes) le Cynodon dactyion L. de nos contrées. Elle est surtout caractérisée par ses tigres noueuses articulées, couchées d'abord à la base, puis redressées, par ses épis floraux au nombre de 7 à 9, dressés, digités, à épillets denses et imbriqués, par ses glumes longuement aristées et ses glumelles velues ciliées à leur partie supérieure.

\section{ELEUSINE Gærtn.}

238. E. indica Gærtn. Fruct. 1, p. 8; Steud. Syn. glum. 1, p. 211; 
Ledeb. Fl. alt. IV, p. 453; Benth. Fl. Hongkong. 429; Miq. Prol.jap. 167, et $\mathrm{Fl}$. ind. bot. 3, p. 385; Cynosurus indicus Lin.; G. Staunt. Plant. chin. exsicc. et in "Liste des plantes du Chan-tong du Voy. Macart. (édit. franc.) IV, p. 153.»

$H a b$. : Les prairies humides et les cultures du littoral, à Yan-taï et Ki-tsen-sôo. - Fr. en septembre.

Ar. géog. : Asie intertrop. et temp., Indes-orient., Amboine. Chine (Hong-kông, Chan-tong, Kiang-nan, Pé-tché-ly). — Japon. - Iles Philipp. - Java. - Iles de la Société. - Afriq. or. et occ.

- Egypte, Sierra-Leone, Nigritie, Fernando-Pô. — Iles Açores. Amér. bor., cent. et aust.; Antilles françaises.

\section{CYNODON Rich.}

239. G. dactylon Pers. Syn. 1, p. 85; Steud. Syn. glum. 1, p. 212 ; Ledeb. Flor.ross. 4, p. 452; Benth. Flor. Hongkong. 428; Miq. Prol.jap. 167, et Flor. ind. batav. 3, p. 382.

$H a b$. : Champs, cultures, et lieux vagues incultes autour de Yan-taï et de Ki-tsen-sôo; assez rare. - Août et septembre.

Ar. géog. : Eur. - Afriq. bor., occ. et or.-Chine (Hong-kông), Kiang-nan (G. Staunt.), Kiang-si, Chan-tong, Pé-tché-ly. Japon. - Iles Philipp. - Java. - Les Marquises. - Australie. Amér. bor. et cent., la Martinique et Guadeloupe, etc.

Obs. : La forme du 'T'ché-foû a les feuilles étroites, linéaires et glabres. Les graines sont également glabres inférieurement.

\section{ERAGROSTIS P. Beauv.}

240. E. pilosa P. Beauv. Agrost. 71 ; Steud. Syn. glum. 1, p. 263 ; Ledeb. Flor. ross.IV, p. 282, et alt.1, p. 95; Turczan. Flor. baik.-dahur. 2, p. 329; Maxim. Prim. amur. 320; Benth. Flor. Hongkong. 432; Miquel Prol. jap. 168; Poa pilosa Lin.; G. Staunt. Plant. chin. exsicc. (1793). *orma A elatior Miquel (loc. cit.)

«Bipedalis, panicula pedali, laxa, radiis longe pedunculatis, » axillis parce longiterque piliferis. »

Forma B humilis O. Debx. in Herb

«Culmis 8-10 centim. altis, ramulis floriferis laxis, tenuiori»bus. »

Hab.: Champs sablonn. et cultures du littoral; lit des torrents, à 
sec pendant l'été, etc. Très-r'épandu dans toute la plaine du Tchéfoû. - Fl. d'août à octobre.

Ar. géog. : Eur. aust. - Asie bor., or. et cent., Sibérie altaïque, Désert de Kirghyz, Baikalie, Mongolie. - Chine (Hong-kông), Kiang-nan etKiang-si (G. Staunt.), Chan-tong, Pé-tché-ly. — Prov. de l'Amour et de l'Ussuri. - Japon. - Afriq. bor. et cent., Algérie, Nubie, - Amér. bor., cent. et aust. - Mexique, Brésil, Chili.

- Antilles françaises.

Obs. : Le botaniste sir G. Staunton a recueilli sur les rives du canal impérial, dans la partie la plus occidentale du Chan-tong, une espèce très-voisine de l'E. pilosa; je veux parler de l'E. verticillata Ræm. et Schult. que l'on retrouvera probablement sur d'autres points de cette province. En voici une courte diagnose et synonymie :

$E$. verticillata Ræm. et Schult. Syst. veget. 2, p. 575; Steud. Syn. glum. 1, p. 263; Poa verticillata Cavan. Icon. 1, p. 63; P. chinensis Link non Roth.

"Culmis e basi ramosis; foliis vaginisque glabris; panicula » patula, radiis capillaribus, patentibus seu flexuoso-erectis, infe» rioribus subverticillatis, in axillis glabris; spiculis linearibus » subulatis, 7-11 floris; glumis glabris, minutis. 》

Plante voisine de l'É. pilosa dont elle se distingue par sa glabrescence, par sa panicule flexueuse élevée, à la fin étalée, et les rameaux florifères inférieurs presque verticillés.

241. E. merastachya Link. Enum. hort. Berol. 1, p. 285; Poa megastachya Kunth Enum. 1, p. 333; Eragrostis pocoïdes, var. A., Trin. in Act. petropol. VI, p. 404; Steud. Syn. glum . 1, p. 263, var. A major; Ledeb. Flor. alt. 1, p. 94; Briza eragrostis Lin.; G. Staunt. Plant. chin. exsicc. (1793).

Hab. : Prairies et champs sablonn. du Tché-foû. Partout autour de Yan-taï, de Ki-tsen-sôo, etc. - Fl. de juillet à octobre.

Ar. géog. : Eur. aust. - Asie bor. et or. - Sibérie altaïque, Mongolie. - Chine : Kiang-nan (G. Staunt.), Chan-tong (O. Debx.). Pé-tché-ly (Maxim.) - Afriq. bor. - Amér. bor. - Antilles francaises.

Obs. : L'E. megastachya est tellement abondant au Tché-foû, qu'il y forme pour ainsi dire des prairies naturelles. Aussi est-il fauché et séché avec soin par les indigènes, pour servir à la nour- 
riture des animaux domestiques herbivores pendant l'hiver. Cette plante est d'ailleurs identique dans tous ses caractères avec l' $E$. megastachya de l'Europe australe.

242. E. ferruz̧inea P. Beaur. Agrost. 71; Steud. Syn. glum. 1, p. 267; Miq. Prol. jap. 168; Poa ferruginea Thumbg. Fl. japon. 50; Kunth Enum. 1, p. 330.

"Facies E. megastachyce, sed differt paniculis patulis, laxis, » longe pedicellatis, pedicellis filiformibus; $]$ spiculis 5-9" floris, »oblongis, glumis acutis, carina scabris, fusco-variegatis vel fer》 rugineis. »

Hab. : Les prairies humides et sablonn. sur la plage de Foûchan-yên, au N.-O. de Yan-taï. - Septembre.

Ar. géog. : Chine, Chan-tong et Kiang.nan (G. Staunt.) Japon.

243. E. tenella P. Beauv. Agrost. 71; Steud. Syn.glum. 1, p. 266; Benth. Fl. Hongkong. 431: Miquel Prol.jap. 168; Poa tenella Lin. Spec. 101; G. Staunt. Plant. chin. exsicc. (1793); P.japonica Thumbg. Fl.jap. 51.

Hab. : Sables et lits desséchés des torrents, au-dessus de Si-nenkôo. - Septembre.

Ar. géog. : Asie intertrop. et temp., Indes-orient. - Chine (Hongkong, Fo-kien, Kiang-nan, Chan-tong). — Japon. - Iles Philipp. - Les Moluques.

\section{ANTHISTIRIA Lin.}

244. A. arguens Wild. Spec. IV, p. 91, non Heyne; Steud. Syyn. glum. 1, p. 401; Kunth Enum. 1, p. 482; Miquel Prol. jap. 178; Stirpa arguens Lin. Andropogon ciliatum Thumbg.: Flor.jap. 45 (ex parte) non Thumbg. Flor.cap. 40.

Hab. : Les collines sèches micasch. et bords des ravins, au-dessus de Si-nen-kôo et de Ki-tsen-sôo. - Fl. août et septembre.

Ar. géog.: Asie intertrop. et temp., Indes-orient. - Chine (Chan-tong). - Corée. - Japon.

Obs. : L'Anthistiria arguens we peut être rapproché que de l'A. cilidia L., espèce propre à l'Afrique australe. Il en diffère par ses chaumes simples et glabres, par ses feuilles longuement linéaires pourvues de quelques poils à la base, ainsi que par les gaines qui sont ciliées tuberculeuses vers leur partie supérieure interne, par 
sa panicule très-étroite à épillets (3-1 sur le même épi) lâches, subconiques, dressés, puis penchés, par ses bractées étroites-acuminées, plus ou moins tuberculeuses, velues sur les bords, par les glumes des épillets fertiles lancéolées, scabres au sommet, et par son arête velue dans la moitié supérieure seulement.

\section{ANDROPOGON L.}

245. A. schænanthus Roxbg. Flor. ind. 1, p. 279, non Lin. (Teste A. Franchet); Niquel Prol. jap. 178; A. iwarankusa Roxbg.; Kunth Enum. 1, p. 493; Steud. Syn. glum. 1, p. 388? A. Martini Nées Plant. Meyen. 189; Benth. Fl. Hongkong. 424 ; sinice Mâo-YAN.

«Perennis, herbaceus, glaber, culmis simplicibus, pennæ anse» rinæ crassitie, erectis, 80 centim. altis, apice compressis; foliis » linearibus, acutis (10 centim. long., 5 mill. latis), ad margines 》 scabriusculis, vaginisque glabris: panicula fasciculari, spiculis » axillaribus geminatis (una terminali) composita; spatha com» muni cymbiformi; rachi articulata pilosaque; flosculis fertilibus »aristatis, masculis muticis univalvibus; gluma inferiori flosculi » fertilis dorso plana, ceteris nervosis, nervis valde proeminulis, » margine membranaceis, superne ciliatis denticulatis. »

Hab. : Les hautes montagnes du Tché-foû, dans la région sup. de 800 à 1,000 mèt. d'alt. - Fl. 6 septembre.

Ar. géog. : Asie intertrop. et temp., Indes-orient., Ceylan. Chine (Hong-kông, Chan-tong). - Japon (Kiû-siû).

Obs. : L'Andropogon schæenanthus Roxburg des montagnes du Chan-tong n'a pas les racines aromatiques à odeur forte et pénétrante de l'A. aromaticus Sieber (A. schcnanthus Lin). ex Trin. Andropog. spec. Mem. acad. Saint-Pétest. VI ${ }^{\mathrm{e}}$ série, p. 283, qui est une plante spéciale à l'Afrique austro-occident. et à l'île Naurice). Dans l'espèce dı Tché-fô̂, les racines sont courtes, fibreuses, sans arôme, ni aucune saveur. Les feuilles radicales très-étroitement linéaires, glabres et scabres sur les bords, atteignent à peine en longueur la moitié du chaume, taudis que dans l'd. inarankusa Roxb. qui est considéré par Nées ab Esemb. comme un synonyme de l'A. schcenanthus Robx., les feuilles sont aussi longues que les tiges florifères. Ce caractère a paru insuffisant à Niquel pour séparer dans son Prolusio flore japonice les A. schcenanthus Roxbg de l'A. ivarankusa Roxbg. Mais à l'exemple de Nées in Linnea VI, 
p. 281, Miquel réunit égralement à l'A . schcnanthus de Roxbg. et à tort, selon nous, l'espèce linnéenne du même nom ainsi que les A. Martini Nées et A. Gueringii Steud. comme n'étant que des formes d'une plante variable selon ses diverses stations.

\section{ISCH AMUM Lin.}

246. I. barbatum Retz Obs. VI. p. 35; Kunth Enum. 1 p. 513; Benth. Fl. Fiongk. 425; Miq. Prol.jap. 179; Andropogon antephoroïdes Steud. Syn.glum. 1, p. 175 ex Miq. (loc. cit.) Affinis A. barbigeri Steud.

Hab.: Les dunes de Foû-chan-yên et les sables des prairies saumâtres, au N.-O. de Yan-taï. - Fl. septembre et octobre.

$A r . g e ́ o g .:$ Asie litt., intertrop. et temp. : Indes-orient. - Chine (Hong-kông, Chan-tong). 一Japon (Kiû-siû). - Java.

247 I. ciliare Retz Obs. VI, p. 36; Kunth Enum. 1, p. 513; Trin. Androp. spec. in Mém. acad. St-Pétersb. VI, sér. 2, p. 393; Andropogon Retzii Steud. Syn. glum. 1, p. 375; Pollinia ciliaris Spreng. Syst. 1, 289; G. Staunt. Plant. chin. exs. (1793) sub Andropogon...

"Culmis nodosis articulatis, nodis superioribus barbatis; foliis » lineari-lanceolatis ; spicis geminis, 5-6 centim. longis, articulis »dorso ciliatis; pedicellis lævibus; spiculis geminis aristatis, »gluma inferiori apice nervoso-striata, marginata, marginibus »membranaceis. »

Hab. : Les mares saumâtres au milieu des dunes de Foû-chanyên, au N.-O. de Yan-taï. -Fl. septembre.

Ar. géog. : Asie intertrop. et temp., Indes-orient. - Chine (O. Debx.), Kiang-nan (G. Staunt.).

248. I. Sieboldii Miquel Prolusio Aore japon. p. 179; Andropogon crassipes Steud. Syn.glum. 1, p. 375; ex Miquel loc.cit.

«Culmis nudis erectis, 45-50 centim. elongatis, interdum nodo» sis, nodis glabris nigrescentibus; foliis linearibus acuminatis, »nervosis, glabris, marginibus serrulato-scabris, vaginis ore » pilosis et antice superne ciliatis; ligulis brevibus glabris; spicis » 2 , inæquilongis, fusco-viridibus, geminatis; gluma exteriore » oblongo lanceolata, dorso depressa, basi non rugosa, marginibus » membranaceis, inferiore carinata compressa, carina serrulato- 
》scabra, altera spicula padicellata, subconformi, glumis exterio» ribus glabris, apice pilosulis; paleis omnibus muticis. (Miquel.

Hab. : Les prairies du littoraal et le lit desséché des torrents andessus de Ki-tsen-sôo. - Fl. 19 aout 1860.

Ar géog. : Chine : Chan-tong (O. Debx.) - Japon (Keiske).

\section{SORGHUM Mænch.}

249. S. vulgare Pers. Syn. 1, p. 101; 0. Debx. Fl. Sllang-hä̈, $n^{\circ}$ 137; Maxim. Prim. amur. 331; Andropogon sorghum Roxbg. Fl. ind. 1, p. 273; Steud. Syn. glum. 1, p. 393; sinice Kâ̂-HIANG.

Forma A genuina.

"Culmo valido arundinaceo, 1-1/2 mèt. alto, nodis pubescenti» bus, foliis vaginisque glabris, margine-serrulato-scabris, cul» misque viridescentibus. »

Forma B rubens Pers. Syn. 1, p. 101; sorghum rubens Wild. Enum. hort. berol. n 1036; Andropogon rubens Kunth Enum. 1, p. 502; Steud. Syn. glum. 1, p. 393.

"Culmo validissimo, 2 met. et ultra elongato, cum foliis glu» misque apice rubro-sanguineo striatis vel maculatis; panicula " ampla, ovata, ramosa. "

Hab. : La forme genuina est cultivée en grand dans la plaine sablonn. de Yan-taï, et la forme rubens sur les basses collines micaschist. au-dessus de Si-nen-kôo. Les tiges servent au chauffage des habitations rurales pendant l'hiver, et les graines à la nourriture des animaux domestiques. - Fl. et fr. d'août à septembre.

Ar. géog. : Asie intertrop. et temp. Indes-orient. - Chine bor., or. et cent. - Prov. de l'Amour et de l'Ussuri. - Japon. - Eur. aust. - Afriq. bor. et subtrop., Guinée. - La variété mubens en Abyssinie.

Obs. : Le Sorghum saccharatum Roxbg. est cultivé dans les provinces méridionales de la Chine, sous les noms de Lôu-chôu et KIN-TSAÔ-TCHÉ.

250. s. halepense Pers. Syn. 1, p. 101; Andropogon halepense Sibth. et Sm. Flor.grac. 1, tab. 68; Steud. Syn. glum. 1, p. 394; Holcus halepensis Lin., G. Staunt. Plant. chin. exsic. (1793)! Hab. : Champs sablonneux et cultures du littoral. - Fr. septembre. 
Ar.géog. : Eur. aust. - Asie bor., cent. et or.; Asie min. : Syrie; - Indes-orient. - Chine (Chan-tong, Kiang-nan, etc.) - Japon. - Australie. - Afriq. bor., occ. et aust., Algér., Cap-Vert, Cap de Bonne-Espér. - Amér. cent. et aust., Cuba, Brésil, etc.

Obs. : Il est à présumer que cette espèce, qui est acclimatée sur quelques points maritimes dans les diverses parties du globe, a été également introduite au Tché-foû avec des graines étrangères, et se sera répandue dans les cultures de cette région.

IMPERATA' Cyrillo.

251. I. arundinacea Cyr. Icon. 2, tab. 11 ; Benth. Fl. Hongkong. 419: Bunge Enum chin.bor. no 417; Miq. Prol.jap. 176 ; et Fl. ind. bat. 3, p. 514 .

Var.pedicellata; I. pedicellata Steud. in Bot. zeit. (1846), p. 22, et Syn.glum.1,p. 405; A. Gray Plant.jap. Perry exped. 329; I. Kanigii et I. Thumbergii Nées Fl. afric. aust. 1, p. 89, ex Hooker;

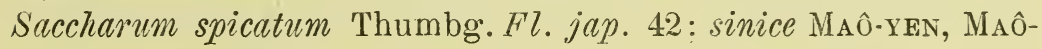
KEN.

"Culmis teretibus, gracilibus, glabris, erectis, cum spicis 60» 80 centim. altis, ad nodos parce pilosis, pilis albidis, in fascicu" lum unicum aggregatis: foliis planis linearibus, glaberrimis, »ad oras vaginarum et secus basim pilusis; panicula spicata, elon»gata, gracili, flexuosa, cylindracea, 12-15 centim. longa; spiculis » omnibus plus minusve pedicellatis. "

Hab. : Les sables maritimes sur la plage de Ki-tsen-sôo. - Fl. 8 octobre 1860 .

Ar. géog. : Eur. aust. - Afriq. bor., occ. et aust., Algérie, Sénégal, Sierra-Leone, Cap de Bonne-Esp. - Asie intertrop. bor. et or., Indes-orient. - Chine (Hong-kông', Koûang-tong', Chan-tong, Pé-tché-ly). - Japon. - Sumatra, Java, Australie. -- Amér. aust., Chili.

Obs. : Kunth dans son L'numeratio plant. 1, p. 477, et Miquel dans le Prolusio (loc. cit.) réunissent à l'Imperata arundinacea les $I$. pedicellata, I. Kanigii et $I$. Thumbergii qui ne sont que des formes de l'arundinacea, quoique Steudel les ait décrites comme espèces distinctes dans son Synopsis. La forme du Tché-foû mérite cependant d'être signalée, sans être séparée toutefois de l'espèce typique. Elle se distingue au premier coup d'œil de la plante de l'Europe australe, par le faciès grêle et délicat de toutes ses 
parties, ainsi que par la longueur des chaumes, des feuilles et de l'épi floral.

Dans la variété pedicellatı, la seule que l'on rencontre au Tchéfoû, on remarque un faisceau de poils soyeux appliqués sur chaque nœud des chaumes; les feuilles sont également velues-soyeuses à l'orifice des gaines, et dans l'intérieur de celles-ci. Enfin tous les épillets sont manifestement pédicellés.

La floraison de cette plante n'a lien que tardivement au Chantong (du $I^{\text {er }}$ au 15 octobre), tandis que dans le sud de la Chine à Hong-kông, la floraison est déjà fort avancée du ler au 15 mai, ce qui s'explique facilement par la différence des latitudes de ces deux points. Il y a lieu d'observer aussi, sur les échantillons de l'I. arundinacea que j'ai récoltés le 12 mai 1860 à Kow-lông (partie du continent chinois qui fait face ì l'île de Hong-kông'), la présence à la base des chaumes de nombre uses gaines à tissu fibreux et résistant, lequel résulte, sans aucun doute, de la décomposition du parenchyme foliaire des années précédentes, et dont les g’aines auront persisté à la base des chaumes. C'est une particularité remarquable à noter, car dans l'I. pedicellata du nord de la Chine, ces gaines fibreuses n'existent pas, celles-ci disparaissant de la surface du sol avec les chaumes florifères, aussitôt la fructification terminée.

ERIANTHUS Rich.

252. E. speciosus O. Debeaux Msc. et in Herb. (1860).

«Perennis, culmis simplicibus, glabris (crassitie pennæ anse» rinæ), erectis-virgatis, 90-100 centim. altis, parce ramosis, nodis » glabris: foliis longe linearibus, acuminatis, margine revolutis " scaberrimisque, intus ore vaginarum ciliatis pilosis; panicula » spicatim digitata, terminali; spicis 8, gracilibus, inæquilongis »(10-12 cent.), spiculis geminis basi articulatis, hermaphroditis, » undique hirsutis; pilis setosis albidis, vel flavidis, glumas » superantibus; glumis fuscis, nitidis, lanceolatis, inferiore dorso » sulcata, apice membranacea, superiore ad marginem carinam»que fusco vel albido ciliata; aristis flosculi superioris flexuoso» contortis, glabris, flavis, inferne barbatis, glumas 5-plo supe》) rantibus.

》Species pulcherrima, ex affinitate Erianthi r’ufipili Steud. » videtur, sed valde distincta culmis glabris, foliis ad vaginas 
》pilosis, spicis digitatis in eodem fasciculo congestis, spiculis » hirto-pilosis, pilis albis nitidis, rarius flavis, aristis contortis, »villis flosculi superioris $3-4$ plo longioribus. 》

Hab. : Les hautes montag'nes du Tehé-foû, de 1,000 à 1,100 mètres d'alt. dans les fissures des rochers. - Fl. 6 septembre 1860.

Ar.géog. : Chine bor. et or., le Chan-tong. (O. Debx.)

Obs. I. : Par les rameaux articulés de sa panicule, notre nouvelle espèce appartient au grenre Erianthus et non au grenre Eulalia, ainsi qu'on pourrait le croire au premier abord. L'E. speciosus vient se placer près des $E$. rufus Nées et $E$.rufipilus Steud., tous deux originaires du Népaul. Il se rapproche surtout de l'E. mufipilus dont il est facile de le distinguer, par les caractères décrits dans la diagnose qui précède. Cette espèce est sans aucun doute, par son port élevé et sa panicule soyeuse, l'une des plus belles espèces de la région montagneuse du Chan-tongr.

Obs. II.: La tribu des Bambusinere ne fonmit aucune espèce qui soit indigène ou acclimatée dans le district du Tché-foû. Comme à Shang-haï, on remarque dans les plaines de Chan-tong le magnifiques cultures de céréales, à l'exception du riz (oriza sativa L.), lequel est cultivé dans la partie la plus occidentale de cette province. Les céréales généralement cultivées au Tché-fou sont : le maïs (Zea mais L., sinice Yu-MI et Kô-LIâNG), le blé (Triticum vulgare L., sinice Siâ̂-MI), l'orge (Hordeum distichum L., sin. Tâ-MAï, et $H$. hexastichum L., sin. Tâo-Yâ), etc.

\section{EQUISÉTACÉES Dec.}

\section{EQUisetum $L$.}

253. E. ramosum Schleich. Cat. p. 27 (1807); Ledeb. F'l. alt. IV, p. 321 ; Miq. Prol. jap. p. 389; E. ramosissimum Desiont. F'. atl. 2 p. 398; G. Staunt. Plant chin. exsic. (1793); sinice Mô-TZÉ.

Hab. : Dunes et mares saumàtres de Foû-clian-yèn et sables marit. au N.-O. de Yan-taï. - Fr. 4 septembre.

Ar.géog. : Lur. - Afriq. bor. - Asie bor., Sibérie altaïque. Chine bor. et moy., Chan-tong, Kiang-nan, Kiang-soû, Chen-si, Pétché-ly. - Japon. - Amér. bor. 


\section{RHYZOCARPEEES DEC.}

\section{Marsilea I.}

254. M. quadrifoliata Lin. Spec.; Miq. Prol.jap. p. $349 ;$ M. tetraphylla Thumbg. Hl.jap. 340.

Hab. : Les mares d'eau douce au milieu des pâturağes maritimes de Foû-chan-yên et des dunes du littoral. - Réc. non fruct. le 4 septembre.

Ar. géog. : Eur. temp.; France, Espagne, Portugal, Italie, Allemagne, Suisse, Basse-Autriche, Oural. - Chine bor., le Chan-tong. - Java (Zollinger). - Japon. - Amér. bor.

\section{LYCOPODIACÉES DeC.}

Selaginelia Dest.

255. S. pulvinata Hook. et Grév. Enum. filicum in Hook. Bot. misc. 2, no 98, sul Lycopodio; Haxim. Prim amur. 335; Regel Tent. ussur ;175; Miquel Prol. jap. $349 ;$ S. involvens Spring Monog. Lycop. 2, p. 63; Lycopodium involvens Swartz Syn. 182; Benth. Fl. Hongkong. 436; Lyc. circinale Thumbg. Fl.jap. 341 ; sinice'Tsian-Mang, T'sé-Mâng, KôUeipé.

Hab. : Région montagn. moy. et boisée, de 400 à 600 mètr. d'alt. au pied des pins (Pinus Massoniana) et des chênes (Quercus serrata). - Fr. 6 septembre.

Ar. géog. : Asie bor. et or., Mongolie. - Chine : Chan-tong, Pétché-ly. - Prov. de l'Amour et de l'Ussuri. - Japon.

Obs. : Cette élégante Sélaginelle se roule en forme de boule par la dessication, et les frondes s'étalent dès qu'elles sont exposées à l'humidité. Les Chinois du Chan-tong' l'emploient dans leur thér'apeutique comme succédanée du ChÉ-HôUei (Nipholozus lingua Kunze) du Japon, dans les maladies des bronches et dı poumon. Aussi trouve-t-on fréquemment le $S$. pulvinata à l'état frais sur le marché des villages. Mes plus beaux échantillons proviennent du marché de Yan-taï, près du camp de Tché-fon̂.

256. S. mongolica Kuprecht Distrib. crypt. vasc. in imper. ross. in Beitr. zur Pflanz des Russ. (1815), fasc. 3, p. 36; Spring Monog. Lycop. p. 262; Milde Filic. Europ. p. 267: Maxim. Prim. amur. in Index flor. Pekin. p. 479 et 486. 
"Folia dimorpha tetrasticha, bifariam extensa lateralia paten» tia, e basi vaginante fissidentoidea, media margine superiore » seu interiore fere recto, margine inferiore seu exteriore magis ") convexo ciliata; rami primarii ramulique patentissimi; bractece » margine late pellucido, undique argute dentatæ, dorso cari» natæ. »

$H a b$. : Sur tous les rochers micaschist. du littoral, les falaises de la pointe de Tché-foû et de la baie de Ki-tsen-sôo; s'élève jusqu'à 300 mètr. d'alt. - Fr. de septembre à décembre.

Ar. géog. : Asie bor., Mongolie. - Chine, Chan-tong (O. Debx.) nov. 1860, Schottmuller août 1861); Pé-tché-ly à Pé-king (Maxim.)

$O b s$. : Le $S$.mongolica tapisse tous les rochers du littoral de ses frondes jaunes-rougeâtres, et disparaît à une altitude de 300 à 400 mètres. Cette espèce est très-voisine du S. sanguinolenta Spring, dont elle diffère par ses feuilles latérales des jeunes rameaux demiembrassantes et non simplement sessiles comme dans le $S$. sanguinolenta, par les tiges primaires et les jeunes rameaux étalés-divariqués, et de couleur jaune-rongeâtre et non dichotomes au sommet et de couleur rouge-foncée, enfin par les bractées à marge pellucide et très-finement dentées.

\section{FOUGËRES I.}

\section{Asplenium L.}

257. A. nipponicum Metten. Annal. mus. botan. Lugdun. batav. 2, p. 240 ; Miquel Prol. fl. jap. 339.

$H a b$. : Région montagn. sup. du T'hé-foû, de 800 à 1,000 mèt. d'alt., dans les fissures des rochers et au fond des ravins abrités.Fruct. 16 septembre 1860.

Ar. géog. : Chine bor., Chan-tong et Pé-tché-ly. - Japon (Nippon).

Obs. : L'A splenium nipponicum, dont la détermination rigoureuse est due à M. A. Franchet, est excessivement voisin des A. macrocarpum Blume, et $A$. Goringianum Metten. in Asplen. p. 198, tab. VI, ainsi que de l'A. thelipteroides Michaux découvert dans la province de l'Amour par M. Maximowicz. Il en diffère par ses pinnulles notablement pétiolées, tandis qu'elles sont sessiles ou presque sessiles dans les $A$. macrocarpum et Goringianum, ce dernier étant considéré par M. Baker comme un synonyme du premier. A 
part cette légère différence des pinnules pétiolées, la plante du Tché-foû est de tout point semblable à l'A. Goringianum du Japon. Notre fougère offre aussi l'aspect de l'A. thetipteroides Mich. dont elle s'éloigne par les lobes des folioles beaucoup plus profonds et comme incisés pour ainsi dire. D'après M. Hance, l'A. nipponicum a été découvert par le R. P. David des missions étrangères, aux environs de Pé-king dans le Pé-tché-ly.

258. A. macrocarpum Blume (non Fée) Enum filic.jaran. p. 162; Hook. Spec.plant. 3, p. 222; Baker Syn. filicum. 227; Athyrium foliolosum Moore Ind. filic. 143; Lastrcea macrocarpa Moore Index,95; A splenium fallax Metten Asplen. 194, tab. VI, fig. 7-8.

« Stipe de 15 à 25 cent. ferme dressé, d'un jaune pâle, écailleux » inférieurement; fronde longue de 35 à 65 , large de 12 à 20 cen》tim. orale, lancéolée avec de nombreuses pinnes de chaque côté, » les inférieures longues de 10 à 15 cent., larges de 3 à 5 , lancéo» lées et partagées jusqu'au rachis en nombreuses pinnules oblon» gues rhomboïdales, incisées, crénelées ou pinnatifides; texture » herbacée à rachis nu; veines latérales des segments fourchues: » sores nombreux larges; involucres oblongs reniformes larges de » 2 à 3 millim. » (Traduit de Baker.)

$H a b$. : Région montagn. sup. du Tché-foû (de 800 à 1,000 mèt. d'alt.), avec l'espèce précédente. - Fr. 6-16 septembre 1860.

Ar.géog. : Asie bor. et cent., Himalaya (2,000 mètr. d'alt.), Ceylan. - Chine : Prov. du Chan-tong (O. Debeaux! et $\mathrm{D}^{\mathrm{r}}$ Maingay ex Baker). - Java.

Obs. : L'Asplenium Goringianum Mett. du Japon est probablement la même plante que l'A. macrocarpum, mais avec les dents des pinnules plus aiguës. Par son port général, il n'est pas éloigné du Nephrodium spinulosum, et à cause de son involucre peu réniforme, M. Moore le considère comme un Lastrcea. La figure de l'A. fallax de Mettenius (loc. cit.) convient exactement à mes spécimens du Tché-foû, èt M. Franchet les rapporte sans aucun doute à l'A. macrocarpum qui en est le nom princeps. 
CHARACÉES L. C. Rich.

Chara L.

259. Gh. fætida Al. Braun Monog. char. in Bot.zeit. (Flora), 1835; Walm. Essai char. in Act. Soc. Lin. Bordx. XXI, p. 63; Chara vulgaris Lin. ex parte;

Var. halophila O. Debeaux in Herb. 1860.

"Valde affinis Chare fetide videtur, sed differt; caulibus » tenuioribus, minutilis, 4-6 centim. elongatis, parum ramulosis, » profunde 5-6 sulcatis, passin articulatis, pallide virentibus, » dein albido-crustaceis, undique rugoso-tuberculosis, verticillis »apice confertis, ramentis contorto-sulcatis, laxis, apiculatis, »nuculis ad verticillos solitariis, ovato-oblongis. »

$H a b$. : Les mares d'eau salée, sur les falaises ou les sables inondés à haute mer, près de N'yrin-haï-tchéoû au cap Chan-tong. Fruct. le 18 septembre.

Ar.géog. : Eaux salées de la Chine bor. - Le type en Europe. Asie bor., Sibérie, Altaï, etc.

Obs. : Le Chara fetida (forma typica) se montre indifféremment dans les eaux douces ou saumâtres et même les eaux thermales. Il semble être positivement cantonné en Europe. Au nord, il ne dépasse pas l'Allemagne, et au midi l'Espagne. La plante de Suède est une des nombreuses formes de ce Chara fatida pour certains auteurs, tandis que pour Walmann, il constitue une espèce distincte, le $C h$. longibracteata, très-commun en Laponie, tandis que le type fatida y est excessivement rare. Ce Ch. longibracteata est aussi très-abondant en Algérie (M. Cosson), et se rait répandu dans toutes les parties du globe.

La forme Halophila se rapproche du Ch. longibracteata par ses verticilles lâches, à rameaux allongés peu ramifiés, mais elle s'en éloigne d'un autre côté par ses tiges profondément 5-6 ang־ulaires sillunnées, un peu contournées, et rugueuses-tuberculeuses. Elle croît par petites touffes isolées, de 4 à 6 centim. de hauteur, sur le lit sableux qui recouvre le fond des mares d'eau salée, au milieu des falaises micaschisteuses du cap Chan-tong.

260. Ch. condensata Walmann (loc. cit).p. 77; Ch. crinita A. Braun Monog. char. (ex parte).

Var. minutula O. Debeaux in Herb. 1860. 
"Monoica, minutula, pallide virens, seu albo crustacea, cauli» bus substriatis, gracilibus, 4-5 centim. elongatis, passim acu» leolatis, aculeis hyalinis, strictis, patentibus; verticillis glome》 rulatis, confertis, subglobosis undique hirto-horridulis: ra》mentis curtis, aculeatis, albidis; nuculis suborvideis, fere glo» bulosis, sub lente parum costulatis.

»Affin is satis formæ typicæ $C h$. condensate Walm., sed tenuior »humiliorque, glomerulis minutissimis, caulibusque aculeato» spinescentibus distincta.»

Hab. : Avec la précédente dans les mares d'eau salée au cap Chan-tong.

$A r . g e ́ o g .:$ Le type : Europe : France, Italie, Hongrie, Allemagne, Danemark, Norwége, Suède, Prov. baltiques de la Russie, Asie bor. - Mongolie chinoise. - La variété : Chine bor., or.

Obs. : Notre variété minutula se distingue facilement de la forme typique par sa taille de beaucoup plus petite 4 à 6 centim. de hauteur), par ses verticilles plus rapprochés et grêles, par ses tiges épineuses, à aiguillons courts et transparents, par ses nucules presque globuleuses, paraissant sillonnées à leur surface, et enfin par sa station remarquable dans l'eau salée. La forme du $C h$. condensata signalée par Walmann dans la Mongolie chinoise pourrait bien appartenir à la variété du cap Chan-tong.

\section{MOUSSES L.}

ARCHIDIUM S'chimp.

261. A. sinense Durieu in Bull. Soc. botan. France, IX, p. 161 (1862).

$H a b$. : Les rochers abrités des vents du Nord, dans la baie de Ki-tsen-sôo et sur les falaises de la pointe du T'ché-foû. - Fr. $1^{\text {er }}$ novembre 1860.

Ar. géog. : Chine bor., or.

Ols. : L'A. sinense est très-distinct de l'A. alternifolium Schimp. dont il a le faciès, par la disposition des feuilles autour de la tige fructifère, par ses feulles périchétiales plus larges à la base, moins longuement subulées, à nervure plus saillante, et surtout par ses capsules latérales.

Il diffère également de l'A. capense Hornsch. par ce dernier caractère qui le rapproche d'autre part des $A$. chinense Schimp. et 
A. laterale Bruch, chez lesquels la capsule est également latérale. L'Archidium sinense paraît voisin d'une espèce d'Algérie, l'A. Duriaanum schimp. dont il a presque les feuilles caulinaires, mais dans celle-ci la capsule est terminée comme dans l'A.alternatifolium (Dur.).

GYMNOSTOMUM Hedwig.

262. G. microstomum Hedw. Syn. musc. 3, p. 7l; Schimp. Šyn. musc. p. 34; Dec. et Duby Bot.gall. 2, p. 580; Hymenostomum microstomum $\mathrm{R}$. Brown.

Hab. : A terre au pied des rochers de la pointe du Tché-fon et du vieux fort chinois. - Fruct. $1^{\text {er }}$ novembre.

Ar. géog. : Eur. - Afr. bor. - Asie bor., or. et occ. - Chine bor., or. - Amér. bor.

\section{PLAGIOTHECIUM Schim.}

263. P. lancifolium 0. Debeaux Msc. et in Herb. (1860); sinice T'sin-TAï.

"Tiges molles, lâchement dressées, simples ou rameuses, rami" fiées à moitié hauteur, longues de 1 à 2 centim. et formant des » touffes compactes, soyeuses, d'un vert-doré brillant, passant au » roux dans les vieilles tiges. Fenilles ovales-lancéolées, longue》 ment acuminées, entières, lisses, pellucides, engainantes, planes » et légèrement enroulées, à nervure atteignant le ̌́ de la lon» gueur de la feuille seulement. Pédicelle pourpre flexueux, très» fragile et d'une délicatesse extrême (difficile à distinguer à » l'œil nu), tordu tantôt à gauche, tantôt à droite et moins long »que les rameaux. Capsule dressée, subcylindrique, petite (moins » de 1 millimètre), légèrement arquée et rugueuse (à l'état sec), "munie d'un col basilaire peu prononcé. Opercule... un large »anneau. Péristome de la même couleur que la capsule. Dents.... » (on distingue la base seulement sur nos échantillons d'herbier.) $H a b$. : Recouvre toutes les roches micaschist. du littoral et les roches des basses collines jusqu'à 250 mètr. d'alt. environ. Baie de Yan-taï, de Ki-tsen-sôo et pointe du Tché-foû. - Fr. très-avancés au $1^{\text {er }}$ avril 1861.

Ar.géog. : Chine bor. or.

Obs. I : Le Plagiothecium lancifolium paraît être très-abondant au Tché-fou, car il y tapisse toutes les roches maritimes de son feuil- 
lage vert-doré, devenant roux-foncé en vieillissant. Cette espèce rappelle par son port et sa structure le $P$. sylvaticum schimp. de nos contrées, et s'en éloigne par la forme de la capsule. Dans le $P$. sylvaticum, celle-ci est beaucoup plus grande, et le pédicelle égale deux fois au moins la longueur des tiges, tandis que dans notre P. lancifolium, la capsule est très-petite, et le pédicelle ne dépasse pas même les tiges.

Obs. II : Dans l'importante famille des champignons, je signalerai la présence au Tché-fô des espèces suivantes, qui sont d'ailleurs fort recherchées des Chinois pour l'usage médical ou alimentaire.

Polyporus lucidus Friès; sinice Tchou-LIN-HÔUA, employé dans la médecine vétérinaire, et fréquent dans la région montagneuse boisée.

Polyporus versicolor Friès; Epicr. 471 (Boletus versicolor L., Thumbg.): sinice HoûA-TCHÉ, abondant au Tché-fô, au Japon, etc., et employé dans les maladies inflammatoires du poumon et des intestins.

Exidia auricula Friès (Tremella auricula L., Thumbg.); sin. Mou-y et Mouv-EûL, c'est-à-dire oreille de bois, champignon gélatineux que les Chinois airnent tant à mêler à la plupart de leurs mets et qui croît sur le bois de chêne (Quercus serrata) en décomposition. M. l'abbé David l'a rencontré en quantité pendant son récent royage en Chine (1872-1874), dans les provinces du Yun-nan, Hou-pé, etc., où ce champignon était l'objet d'une culture très-productive. Cette espèce vit également au Japon.

Dans la tribu des champignons hypogés, nous trouvons aussi, au 'Tché-foû, la célèbre substance médicamenteuse nommé par les Chinois Foû-LIN et Pé-Foû-LIN, attribuée au Pachyma coniferarum par Horaninow, et au $P$. Hoëlen par Friès, et qui vit en parasite sur les racines du Pinus Massoniana et des Cunninghamia sinensis. MM. Hanbury et Carey (Linn. soc. Lond. tom. XXIII, p. 94 (1861), considèrent cette tubéracée comme résultant d'un état maladif des racines, lequel est occasionné très-probablement par la présence d'un champignon, dont le mycélium pénètre de toutes parts la substance ligneuse et en modifie la structure $(L$. Soubeiran).

On rencontrera probablement aussi dans la région mont. du Chantong, le Mylitta lapidescens Horaninow, sinice LoûEI-HoûA, dont j'ai vu des spécimens desséchés dans les pharmacies de Yan- 


$$
-175-
$$

taï, et qui sont employés comme anthelminthiques et dans les diverses maladies des enfants. Ce champignon hypogé croît en abondance au Chen-si, dans le 'Tssé-chûen, le Hoû-pé et autres provinces de la Chine centrale. 

de géographie bolanique des plantes du Tché-foú.

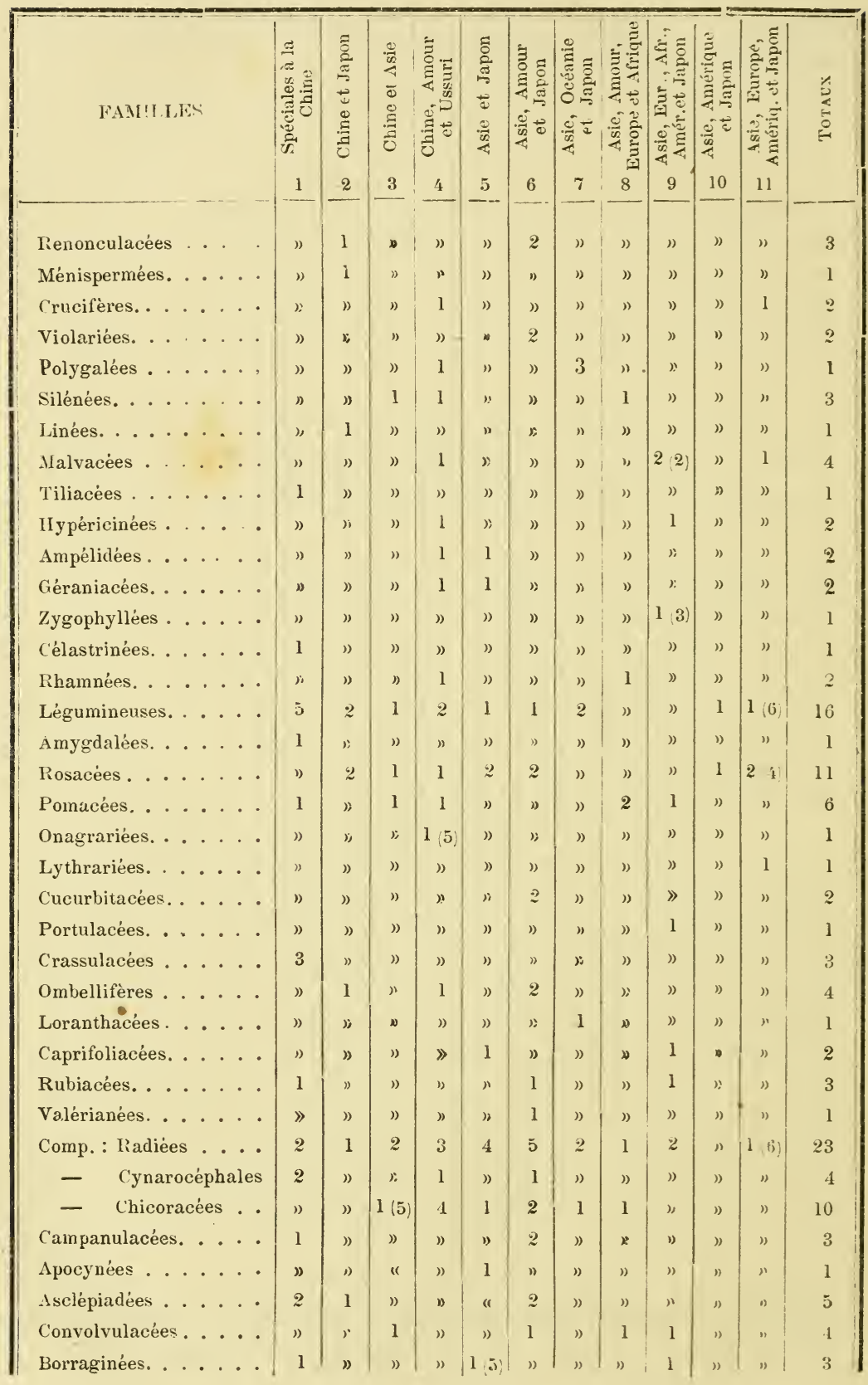




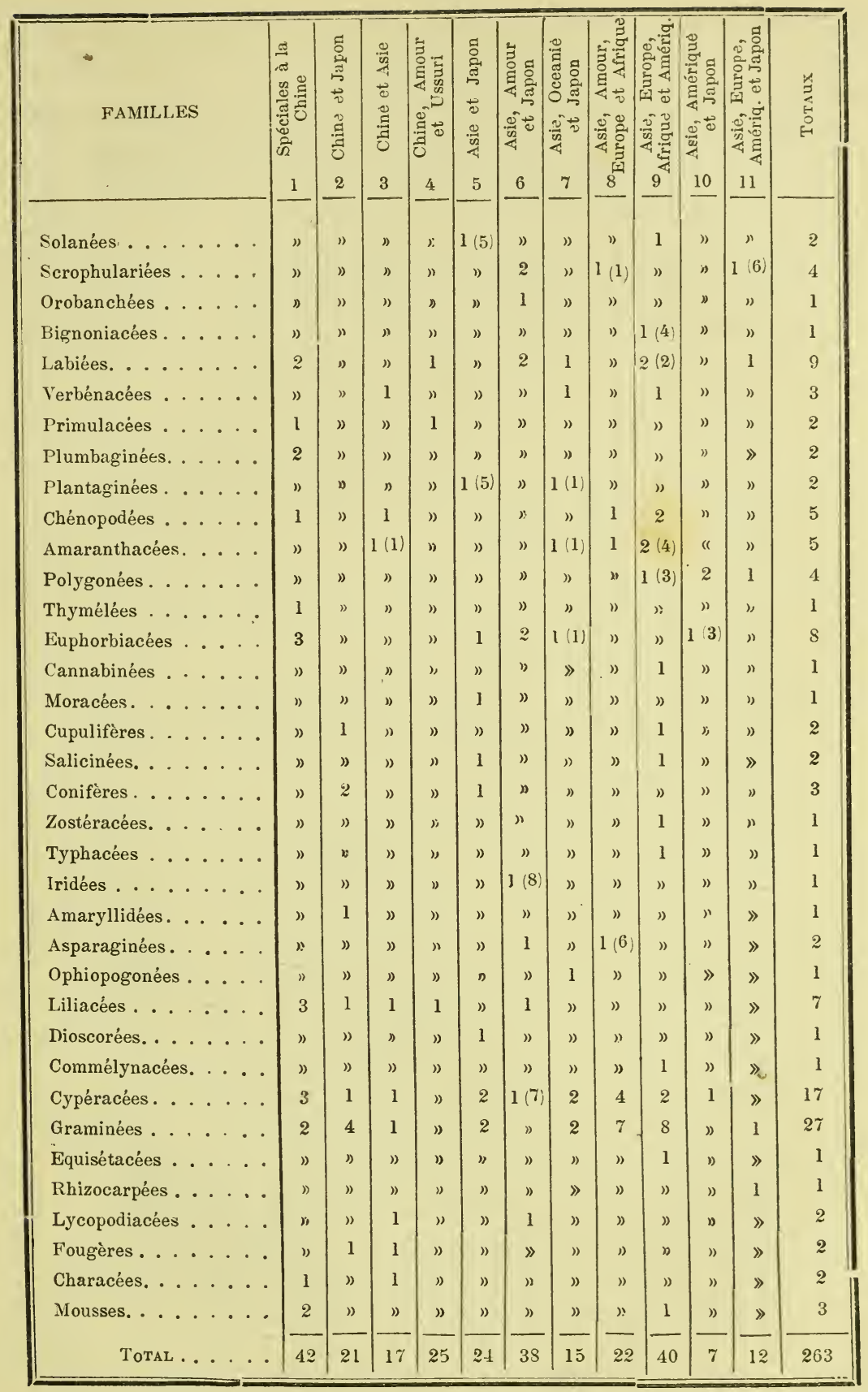

(1) - LAfrique; (2) + l'Amour; (3) + l'Océanie; (4) - l'Europe; (5) + l'Europe ; (6) - l'Amérique; (7) + l'Amérique; (8) - le Japon. 


\section{RÉGAPITULATION}

$1^{\circ}$ Chine. - Plantes spéciales à la Chine.

$2^{\circ}$ Chine et JAPON. - Plantes qui se trouvent à la fois dans une ou plusieurs provinces de la Chine et au Japon seulement.

$3^{\circ}$ Chine et Asie. - Plantes qui croissent dans diverses régions de l'Asie boréale, centrale et austro-orientale, ainsi que dans l'empire chinois..........................

$4^{\circ}$ Chine, A mour et Ussuri. - Plantes spéciales à la fois à la Chine et aux provinces orientales de l'Amour et de l'Ussuri, Ochotsk, etc.

$5^{\circ}$ Chine, Asie et Japon. - Plantes qui se retrouvent au Japon, ainsi que dans quelques régions asiatiques intertropicales ou tempérées.

$6^{\circ}$ Asie, Amour et JAPON. - Plantes dont l'aire de dispersion s'étend dans plusieurs provinces de l'Asie, de l'Amour, de l'Ussuri et au Japon.........................

$7^{\circ}$ Asie, OcÉanie, Japon. - Plantes répandues en Asie, au Japon et dans plusieurs îles de la Malaisie, des Philippines, l'Australie, etc.

$8^{\circ}$ Asie, Europe, Afrique, Japon et Amour. - Plantes qui vivent à la fois, dans diverses régions de l'Asie, l'Afrique, l'Europe, et qui se retrouvent au Japon et dans les provinces de l'Amour et de l'Ussuri....................

$9^{\circ}$ Asie, Afrique, Europe, Amérique et Japon. - Plantes qui se retrouvent dans quelques régions des diverses parties du globe, l'Océanie excepté.......................

$10^{\circ}$ Asie, Amérique et JaPon. - Plantes observées dans l'Asie boréale ou centrale et au Japon, etc., que l'on retrouve dans-l'Amérique boréale ou australe.

$11^{\circ}$ Asie, Europe, Amérique, Japon et Amour.- Plantes déjà signalées en Asie, en Europe, dans l'Amérique boréale ou australe, dans les provinces de l'Amour, de l'Ussuri, au Japon, et qui n'ont pas encore été découvertes en Afrique et dans l'Océanie...

La récapitulation qui précède offre quelques exceptions dont il 
a fallu tenir compte, afin de ne pas augmenter dans notre tableau le nombre des colonnes afférentes aux diverses stations botaniques, stations qui peuvent varier, selon le cas, dans telle colonne et rester invariables dans telle autre. J'ai dû restreindre le nombre des rapports de la répartition géographique des plantes du Tché-foû́ pour rester dans les limites déjà établies dans le tableau final de la, florule de Shang-haï. Mais si nous considérons, d'un autre côté, les rapports numériques d'après l'habitat constaté de chaque espèce dans les diverses régrions du globe, nous trouverons les résultats ci-après :

$1^{\circ}$ Chine borÉale (Province de Pé-tché-ly et environs de Pé-king

20 JAPON et CORÉE 166 esp. sur 263 , soit $3 / 5^{\text {e }}$ envir .

$3^{\circ}$ Aste boréale (Sibérie altaï$158 \gg$ » $\gg 3 / 5$ que, Baikal, Daoûrie, Mongolie.........................

4 AMour, Ussuri, Ochotsk......

5 AsIE CENT. et occ. (Himalaya, Thibet, Cochinch., etc.).... 6。 ChINe MÉRID. (Provinces au Sud du Chan-tong)...........

7. Europe aust., or. et occ ...... $8^{\circ}$ Afrique bor., aust., etc. (Égypte, Algérie, Sénégal, cap de Bonne-Espér.)........ 9o AmÉr. Cent., Bor, et Aust. (États-Unis, Canada, Antilles, Brésil).................

$10^{\circ}$ OCÉAnIe (Iles de la Sonde, de la Malaisie, Les Philipp., Australie, N Nle-Caléd., îles de l'Oc. indien)............... $11^{\circ}$ Spéciales à la Chine (Chine bor., cent. et aust.)......... $120 »$ $114 »$

$111 \gg$ $\gg \gg 2 / 5 \gg$

$82 »$

$80 \gg$

$» \gg 1 / 3$ ॥

$\gg \gg 1 / 3$ »

$68 \gg \gg \geqslant 1 / 4 »$

$60 »$ » $\mathrm{I} / 4$ » $» \gg 1 / 6 \quad$ » 42 » $1 / 6$ »

Il nous est possible d'après ces chiffres, de nous rendre un comṕce assez exact des rapports g’éographiques qui existent pour 
les plantes du Tché-foû. La végétation de cette partie du promontoire de Chan-tong a évidemment la plus grande affinité avec la floredu Pé-tché-ly, puisque sur un total de 263 espèces nous en avons observé 166 ou environ les $35^{\circ}$ dont la présence a été signalée dans la région montagneuse au nord de Pé-king ou autour de cette capitale. Les affinités ne sont pas moindres avec la flore du Japon, celle ci étant représentée au Tché-foû par 158 espèces ou les $3,5^{\mathrm{e}}$ environ de la totalité des plantes récoltées.

Mais ce qui a lieu de nous surprendre au premier abord, c'est la similitude de la végétation du Chan-tong oriental avec celle de l'Asie boréale (Sibérie altaïque et baikalienne, Daoûrie, etc.), laquelle figure dans notre florule dans la proportion de $3 / 7$, et ne comprend pas moins de 120 espèces propres à ces régions boréales. Ces rapports sontégalement les mêmes avec les provinces de l'ExtrêmeOrient (provinces de l'Amour, de l'Ussuri, de l'Ochotsk et Kamtschatka, etc.), car nous trouvons encore au Tché-foû 114 espèces, les $3 / 7$ environ, qui appartiennent à la flore de ces régions.

Enfin nous remarquons avec non moins d'intérêt que les affinités avec la flore de l'Asie centrale (Himalaya, Thibet, Népaul), et de l'Asie intertropicale (Indes-orientales, Ceylan, etc.) sont formulés par un nombre (111 esp.) très-rapproché de celui déjà trouvé pour les provinces du bassin de l'Amour (114 esp.); ce qui nous permettra d'avancer avec quelques probabilités que la flore du massif montagneux du Chan-tong est, à quelques exceptions près, identique à celle de l'Asie boréale-orientale, tandis que celle du littoral offre de son côté la plus grande analogie avec la végrétation des réggions maritimes de l'Așie méridionale, du Japon, de l'Océanie et de l'Afrique intertropicale.

Si nous poursuivons notre rapide examen des affinités botaniques du Tché-foû, nous trouvons en égale proportion les plantes propres à la Chine méridionale (82 esp.), et celles qui ont été déjà signalées dans l'Europe austro-orientale (80 esp.), c'est-à-dire un peu moins du tiers du nombre des plantes observées. Viennent ensuite en moindres proportions les plantes signalées dans l'Afrique boréale et intertropicale, le cap de Bonne-Esp., représentées par 68 espèces, ou environ un peu plus du quart de la flore. Ce chiffre élevé des affinités botaniques du 'Tché-foû avec le continent africain et les îles de l'Océan indien (Madagrascar, Maurice et Bourbon) se retrouve sur d'autres points de la Chine littorale, au 
Kiang-soû par exemple. Il pourrait s'expliquer en partie par l'influence des courants sons-marins, analogues dn Gulf-slream dans l'Océan atlantique, et qui partant des côtes réchauffées de l'Afrique équatoriale et des îles de l'Océan indien intertropical, seraient entraînés vers les régions relativement froides de la Chine boréale, de la Corée et de la Mandchourie, etc.. On sait d'ailleur's que les graines emportées par les courants océuniques peuvent parfaitement résister, dans certaines conditions, à l'action de l'eau salée, et germer ensuite avec vigueur' sur les rivages où elles auront été rejetées.

En ce qui concerne les deux. Amériques qui figurent par 60 espèces au Tché-foû. je dois faire observer que la plupart des plantes dont l'aire de dispersion s'étend jusque dans le NouveauMonde sont en général ubiquistes ou acclimatées dans cette région. Miquel a déjà signalé ces mêmes affinités botaniques de la flore du Japon, avec la végétation de l'Amérique boréale, surtout de la partie située à l'est des montagnes rocheuses (1). Ce botaniste a surtout insisté sur' la possibilité de la migration des végétaux de l'A mérique an Japon, en suivant les chaînes des îles Alentiennes et Kouriles. Enfin les rapports géographiques du Tché-foû avec l'Océanie, la Malaisie, l'Australie, etc., tiennent le dernier rang, et ne sont représentés que par 44 espèces ou un sixième environ dans notre florule.

Jai déjà examiné, au commencement de cette notice, quel est l'aspect de la végétation sur le littoral, dans les basses collines, et dans la région montagneuse du Chan-tong; mais il serait opportun, je crois, de signaler les caractères particuliers qu'offre la flore du Tché-fon. Un fait remarquable se présente à nos observations, c'est l'habitat sur les sables maritimes de Ki-tsen-sôo et de Yan-taï de plusieurs plantes spéciales au sud de la Chine (rivages du Koûang-tong et de Hong-kông), et dont il serait difficile d'expliquer l'origine, si l'on n'admettait point l'influence le lonor des côtes de la Chine des courantssous-marins se dirigeant du Sud vers le Nord. Je citerai les plantes ci-après qui se rencontrent à la fois sur les sables de l'île de Hong-kông et de la presqu'île du Tché-foû, malgré l'énorme distance qui sépare ces deux points:

(1) Archives néerland., vol. 2, p. 153. 
Crotalaria brevipes, Cassia mimosoides, Bidens pilosa, Vitex ovata, Acalypha pauciflora, s'cilla chinensis, Cyperus iria, C. rolundus, et C. sanguinolentus, Killingia monocephala, Gymnothryx japoxica, Elensine indica, etc. La plupart de ces espèces ne s'éloignent pas des rivages de la mer, et l'on peut concevoir leur facile acclimatation dans une contrée relativement froide, lorsqu'on a vu toute la plainedu Tché foû se recouvrir à la fin de novembre d'une épaisse couche de neige, qui suffit pour garantir les plantes annuelles ou vivaces de l'action destructive du froid très-rigoureux dans cette partie de la Chine.

Plusieurs végétaux répandus dans l'Asie subtropicale, les îles de l'Océanie et de la Malaisie, l'Afrique équatoriale ou australe apparaissent sur les sables du Chan-tong, et rendent l'étude de cette flore excessivement intéressante. Je note, en passant, les espèces suivantes: Hibiscus ternatus, Abutilon Avicenne de l'Afrique boréale, Eclipta marginata de Java, Mazus rugosus des Indes-orient., Salvia plebeia de Ceylan et de Hong-kông, Salsola kali de l'Afrique boréale, Securinega obovata de Madagascar et des Indes-orient., etc. Nous y voyons dominer aussi quelques genres qui sont, pour ainsi dire, caractéristiques de la flore maritime du 'T'ché-foû, entr'autres les Lespedeza, Vicia, Potentilla, Artemisia, Vitex, Cyperus, Fimbristylis, Chloris, etc., à l'exclusion toutefois du genre Desmodium, de la famille des légumineuses, qui produit plusieurs espèces au Japon, et qui n'est pas encore représenté dans la province du Chan-tong.

Il me reste à considérer maintenant dans quel ordre numérique nous pouvons ranger les familles naturelles qui offrent le plus grand nombre d'espèces au T'ché-foû.

Je les trouve ainsi classées, d'après le tableau déjà établi, pour les affinités de gẻographie botanique (1).

(1) Dans une uotice fort intéressante, intituléc « Sur le caractìre et l'origine de la flore du Japon » et insérée dans les Annales néerlandaises, rol. 2 (1867), Miquel fait ressortir l'ordre des familles dominantes au Japọn d'après leur importance numérique. Cet ordre est le suivant :

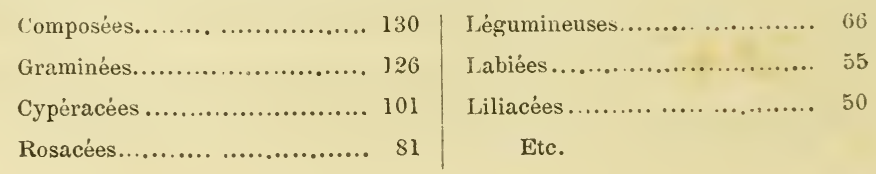




\begin{tabular}{|c|c|}
\hline Composees............... $37 \mathrm{csp}$. & Labies ................... 9 epp \\
\hline (iraminécs............. 27 & Euphorbiacées......... \& » \\
\hline 'yypéracées............ 17 » & Liliacées..... \\
\hline Légumineuses........... 16 》 & Ponacées................6 6 \\
\hline osacées. & Etc. \\
\hline
\end{tabular}

Le nombre relativement considérable des composées, des graminées et des cypéracées ne saurait nous surprendre. Ces plantes résistent en général à l'action destructive du froid, et s'acclimatent facilement dans des milieux identiques quant à leur station, sinon par la latitude de ces mêmes contrées. Je remarque toutefois qu'il n'existe au 'Tché-foû qu'une seule espèce de Carex, le C. macrostachya, tandis que ce genre est largement représenté au Japon, ainsi que dans les provinces orientales de l'Amour et de l'Ussuri. La famille des Fougères ne m'a offert aussi que deux espèces (les A splenium nipponicum du Japon et l'A. macrocarpum de l'Himalaya), ce qui contraste avec la richesse de cette famille dans le sud de la Chine, au Japon et dans les provinces de l'Amour. Cela tient, sans aucun doute, à la sécheresse relative de la presqu'île du Tché-foû et au déboisement de la région montagneuse. L'absence de l'humidité à la surface du sol expliquera pourquoi, malgré mes nombreuses recherches, je n'ai pas pu rencontrer une seule orchidée au Tché-foû, et cependant cette famille produit dans le nord de la Chine, au Japon, dans l'Amour et l'Ussuri, une foule d'espèces des plus intéressantes.

Mes herborisations, poursuivies journellement au Tché-foû, de juillet 1860 jusqu'à l'époque de l'arrivée de la neig'e à la fin de novembre de cette inême année, ont eu pour heureux résultat d'établir l'habitat en Chine de plusieurs plantes qui n'y avaient pas encore été sig'nalées, du moins à ma connaissance. J'ai hâte de inentionner les suivantes, remarquables surtout par leur area géographique :

Cette ressemblance des familles dominantes au Japon et au 'Tché-foù confirme la loi établie par MM. Alph. de Candolle dans sa géographie lotanique, vol. ¿2, p. 1245, et d'après laquelle « daus lia zone tempérés septentrionale, les familles » les plus nombreuses sontles composées, les graminées, les cypéracées et les lé» gumineuses, puis viennent les sruvifères, ombelliferes suivies, mais d'une ma» nière moins constante, par les labiées, les r'osacées, etc. » 
Thalictrum hypoleucum sieb.

Viola Gmeliniana Ræm.

Hypericum perforatum $\mathrm{L}$.

Geranium nepalense Sw.

Spircea betulcefolia Pall.

Sanguisorba canadensis L.

Lythrum virgatum L.

C:ernovia lovigata Ture\%.

Eclipta marginata Hochs.

Artemisia sacrorum Led.

- mongolica D. C.

- capillaris Thumb.

Scorsonera parviflora Jacq.

- macrosperma Turcz.

Botriospermum Kuzsnetzouii.
Agriophilum squarrosum Moq.

Salsola kali L.

Securinega obovata Mull.

Allium tenuissimum L.

Isolepis Micheliana Ræm.

Fimbristylis Sieboldii Miq.

- Buergeri Miq.

Carex macrocephala Wild.

Eriochloa villosa Kunth.

Arunctinella anomala Steud.

Anthistiria arguens Wild.

Ischcemum Sieboldii Miq.

Marsilea quadrifolia L.

Selaginella mongolica Rupr.

Chara foetida L. var.

Avant de terminer ces observations sur les affinités botaniques du Tché-fố, il me reste à comparer la florule de cette contrée avec celle de Shang-haï, afin d'en faire ressortir les points qui les rapprochent ou peuvent les séparer. Ce qui frappera d'abord le botaniste qui aura examiné le catalogue de notre florule, c'est d'y trouver un nombre de plantes spéciales à la Chine du double plus élevé qu'à Shang-haï. Ainsi nous possédons au Tché-foû $1 / 6^{\mathrm{e}}$ environ de plantes spéciales ạ lieu de $1 / 11^{\text {e }}$ qui est la proportion déjà signalée pour Shang-haï. Ce nombre relativement élevé provient évidemment de la nature du sol et de la diversité des sta tions botaniques. Je dois ajouter aussi, que je suis arrivé de primeabord dans une contrée non encore explorée, et qui devait fournir de nombreux éléments pour mes recherches, tandis qu'à Shang'haï, dont le territoire est plat, uniforme, marécageux ou couvert de nombreuses cultures, et de plus exploré par une foule de botanistes, je n'avais à y espérer que des récoltes peu abondantes, et pas ou presque pas d'espèces inédites.

Les rapports de la flore chinoise avec les provinces les plus orientales de l'Asie (Amour, Ussuri, Ochotsk) sont ici d'autant plus considérables, que nous nous rapprochons le plus de ces contrées. Ainsi nous remarquons au Tché-foû la présence de 114 espèces, soit un peu plus des $2 / 5^{\circ}$, tandis que ces rapports ne sont que de $1 / 5^{\mathrm{e}}$ à Shang-haï, et probablement dans la province de Kiang-sou. 
Les affinités avec le Japon ne sont pas tout-à fait identiques au Tché-foû et à Shang-haï. Dans cette dernière localité, j'ai déjà signalé 124 espèces sur 152 comme se retrouvant au Japon, ou les $5 / 6^{\mathrm{e}}$ de la flore; au Chan-tong, le nombre des plantes qui vivent également au Japon, s'élève à 158 esp. seulement sur un total de 263 , ou les $3 / 5^{\text {e }}$ environ. Cette différence peut s'expliquer en partie par la position géographique du Chan-tong, lequel est placé sous une latitude plus boréale, et aussi par son orientation exposée à l'action des vents violents du nord-est, et enfin par la sécheresse de toute la région montagneuse. L'absence au Tché-foû de certaines familles très-nombreuses en espèces au Japon, Éricacées, Cannacées, Mélanthacées, Aroïdées, Orchidées, Palmiers, Fougères, etc., rend très-probable cette assertion de ma part.

Chose à noter en passant, nous observons au Tché-fou les mêmes rapports qu'ì Shang-haï, en ce qui concerne la flore asiatique en général (Asie boréale, centrale et intertropicale). Les affinités botaniques sont représentées dans ces deux contrées par les $2 / 3$ environ. Les rapports numériques sont identiques avec l'Europe austro-orientale à Shang-haï comme au Tché-fou, et sont également formulés par un tiers environ. J'ajoute enfin, pour terminer cette comparaison, que les affinités de la flore du Chan-tong avec l'Afrique boréale subtropicale et australe, ainsi qu'avec les deux Amériques, ont sensiblement diminué comparées surtout avec celles du Kiang-soû. Ainsi au lieu du 1/3 comme proportion numérique déjà observée à Shang-haï, nous ne trouvons plus que $1 / 4$, comme rapports de la végétation du Chan-tong, avec les continents africain et américain.

Il paraît résulter de cette importante question de géographie botanique, qu'au Tché-foû, comme à Shang-haï, les affinités de la végétation sont à peu près identiques avec l'Europe, l'Afrique et l'Océanie. Elles sont moindres au Tché-foû avec le Japon, et d'un autre côté beaucoup plus considérables avec les provinces de l'Amour et de l'Ussuri. En général, la végétation littorale du nord de la Chine se rapproche beaucoup de celle du Japon, par l'identité de quelques familles dominantes sur les sables ou les roches maritimes; mais la flore des basses collines et de la région montagneuse offre beaucoup ou plus de ressemblance avec celle de la Sibérie altaïque et baïkalienne, de la Daoûrie et de la Mongolie.

Si quelques espèces végétales propres au sud de la Chine, et 
appartenant aux genres Crotalaria, Cassia, Scilla, Cyperus, Killingia, Imperata, etc., se trouvent en abondance sur les sables du Tché-foû, il ne s'ensuit pas pour cela que cette contrée soit favorable à l'acclimatation des plantes herbacées ou ligneuses des contrées moyernes ou méridionales de la Chine. Le froid rigoureux qui règne pendant plusieurs mois dans cette partie du Chantong s'oppose, au développement de ces végétaux, qui d'ailleurs prospèrent fort bien à Shang-haï. Je me bornerai à citer les Lagerstramia indica, Eriobotrya japonica, Fontanesia phillyracoides, Chamcrops excelsa, Bambusa vulgaris, etc., qui n'existent point au 'Tché-fố ou n'ont pu s'y acclimater.

Les végétaux de grande culture, tels que les S'esamum indicum, Sirla tiliefolia, Cannabis indica, le tabac, le blé, le sorgho commun et plusieurs autres graminées donnent d'excellents produits au Tché-foû. Je n'ai point vu toutefois des cultures de riz, de coton, de nélumbo et de Corchorus capsularis, plantes qui réussissent très-bien dans les terrains humides de la Chine centrale, et qu'il est à peu près impossible de cultiver dans le promontoire du Chantong.

La floriculture est loin d'être en honneur parmi la population des misérables villages du Tché-foû. Les indigènes ont trop à faire pour subvenir aux besoins de leur alimentation sur un sol relativement ingrat; aussi négligent-ils la culture des plantes d'agrément. J'ai vu cependant chez quelques commerçants aisés de Yantaï, de belles collections de chrysanthèmes doubles et de pivoine moutan, aux couleur's variées. Autour des habitations et des pagodes, le Magnolia yulan et le Grenadier commun, le Sophora du Japon, deux ou trois espèces de Conifères et le Nélumbo sont les seuls végétaux qui soient l'objet de quelque culture de la part des Chinois du promontoire du Chan-tong. 


\title{
ADDEVDA ET EMENDANDA
}

\author{
FLORULE DE SHANG-HAI
}

\section{Artemisia Thumbergiana Max.}

La synonymie de cette plante, décrite antérieurement par M. Hance est fixée par M. Franchet de la manière suivante :

Artemisia apiacea Hance Diagn. plant. nov. chin.bor. aust. ined. 1, p. 9, in Wal. Ann. bot.2, p. 285 (exclus. sect. mentione, teste Maxim. ex Hance ipso in litt. ad Dom. A. Franchet); A Franchet et Savat. Enum. plant.jap. 1, p. 238; A. Thumbergiana Maxim. Mélang. biolog. vol. 8, p. 528; A. abrotanum Thumbg. F'l. jap. p. 309.

\section{Aplotaxis Bungei Benth. et Hook.}

D'après MM. Franchet et Savatier, la synonymie de cette plante doit ètre ainsi établie :

Aplotaxis Bungei Benth. et Hook. Gen. plant. vol. 2, p. 472 : Dec. Prodr. VI, p. 539; Cirsium lyratum Bunge Enum. plant. chin.bor. no 203; Hemisiepta lyrata Bunge Dorpat.jahrbr. 1, p. 221; Maxim. Mélang. biolog. vol. 9, p. 334; Bunge in Fish. et Mey. Ind. semin. horti. Petrop. p. 38 (1835); A. multicaulis Miquel an Dec. ?

MM. Franchet et Savatier observent (loc. cit.) que les échantillons japonais sont identiques à ceux provenant de mes récoltes à Shang-haï. Dans cette espèce, l'aigrette intérieure à poils soudés en anneau à la base est très-caduque, et laisse facilement voir après sa chute la membrane lacérée du côté externe qui couronne l'achaine. De Candolle a contesté l'existence de cette aigrette externe, et Miquel l'a mal observée. Les savants auteurs de l'Enumeratio plantar japon., etc., pensentque l'Aplotaxismulticaulis de Dec. et de Miquel est une espèce voisine de l'A. Bungei, 
mais à laquelle de Candolle attribue une aigrette externe trèscourte, caduque et formée de poils presqu'écailleux, ce qui n'existe pas dans la plante de Shang-haï.

\section{Saussurea (Theodora) glomerata Poir.}

M. de Herder établit ainsi la synonymie de cette espèce dans son ouvrage publié avec la collaboration de $\mathrm{M}$. Regrel, et intitulé "Planta Raddeana monopetale » Pars III, p. 48, du tome III.

Saussurea amara Lin.; Ledeb. Flor. ross. II, p. 664: Icon. tab. 67 et 68; ejusd. Flor. alt., IV, p. 21: Turczan. Flor.baik.-dahur. II, p. 118.

Var. B glomerata Trautr. S.glomerata Poir. Encycl. suppl. 5, p. 71; Dec. Prodr. VI, p. 537; S. amara Auct. ex parte; S. amara var. altaica Fish. in Herb. S. macrocephala Lessing in Linnea VI, p. 87: S. Gmelini Hort. Dorp. in Herb. Schrad.

Obs. : Cette plante fort rare en Chine et dont on rencontre ensemble deux formes, elatior et humilis, sur les bords vaseux du Wam-pôo, à Shang-haï et Wôo-song, paraît être assez répandue dans les régions sibériennes orientales. M. de Herder a signalé sa présence à Jakoutsk, sur les rives de la Léna, à Nertkintsk, à Kiaka, et près de la Sélinga dans la Transbaïkalie, dans les lieux saumâtres du désert d'Orenbourg, sur les bords de l'Angara à Irkoutsk, dans la Daoûrie, la Mongolie et les montagnes du Pétché-ly, au nord de la Chine. Le $S$. glomerata ne dépasse pas la province de Kiang-soû, vers le sud de ce vaste empire.

\section{Diospyros schi-tzé Bunge.}

Chez les individus de cet arbuste qui croissent spontanément au Japon, le diamètre du fruit varie de 3 à 4 centimètres; mais ill est plus considérable lorsque l'arbre qui les produit est soumis à la culture. Ces variations dans la forme et les dimensions du fruit ont donné lieu à la création de plusieurs espèces parmi lesquelles il faut ranger le D. costatı Carrière in Revue horticole (1870).

MM. Franchet et Savatier auxquels j'emprunte cette note pensent devoir établir ainsi la synonymie de l'arbre de Shang-haï.

Diospyras kaki. Lin. fil. Suppl. 439; Hiern. on Ebenac. p. 226; Thumbg. Flor.jap. 157 (ex parte); D. schi-tzé Bunge Enum. chin. 
bor. $\mathrm{n}^{\circ} 237 ;$ D. costata Carrière, Rev. hort. (1870), et D. Roxburgii Carr. Rev. hort. (1872), p. 255.

On doit aussi ajouter le Japon dans l'Aréa géographique du D. schi-tzé.

\section{Fontanesia phillyræoides Lab.}

\section{Var. Chinensis O. Debeaux.}

Dans l'observation qui accompagne la diagnose de ma variété chinensis, j'ai attribué à tort à M. Carrière la création du F'ontanesia Fortunei in "Revue horticole de l'année 1859. " C'est M. Decaisne qui a établi cette espèce (Rev. hort. (1859), p. 43, cum icone). Le nom de l'illustre professeur du Muséum doit donc être lu dans l'observation précitée, à la place de celui de M. Carrière et à côté du F. Fortunei.

Le $F$. phillyræoides var. chinensis de Wôo-sông et de Shanghaï, qui est si remarquable par ses grandes feuilles lancéolées, ne peut être assimilé au $F$. Fortunei Decne, dont la floraison est plus tardive de 5 à 6 mois, et qui est de beaucoup plus petit dans toutes ses dimensions.

C. Koch dans sa dendrologie, assigne pour habitat au F. Fortunei la Chine et le Japon. Il est certain (Teste A. Franchet) que cet arbrisseau n'est point spontané au Japon.

Il existe encore dans les catalogues des horticulteurs ou de quelques jardins botaniques, un Fontanesia chinensis Hortul. sans description connue il est vrai, et qui pourrait bien appartenir soit au F. Fortunei, soit à la variété chinensis du $F$. phillyraoides. C'est un point qui reste à vérifier.

\section{Carex Pierotii Miquel.}

D'après Miquel lui-même, on doit restituer à cette espèce le nom de $C$. suberea antérieurement donné par Booth. Nous aurons ainsi la synonymie ci-après :

Garex suberea Booth Mss. Herb. mus. Lugd. batav. ex Miq. Prol.jup. 143; C. Pierotii Miq. loc. cit., p. 80.

M. Maximowicz qui a étudié avec soin les cypéracées du musée de Leyde, pense (Teste Franchet in litt.) que le C. Pierotii Miq. n'est qu'un synonyme du $C$. suberea Booth. et que dans tous les cas, ce dernier nom doit être appliqué à la forme dont les fruits 
(utricules) sont marqués de côtes épaisses bien distinctes, caractère déjà signalé dans la plante des rives du Wam-pôo à Shanghaï et Wôo-sông.

\section{APPENDIX}

\section{Quelques mots sur la situation du Tché-foû en $18 \% \%$}

Depuis l'évacuation à la fin de février 1861, du territoire du Tché-foû occupé pendant huit mois par une partie de l'armée expéditionnaire française en Chine, cette contrée qui fut si inhospitalière à notre égard, a subi une transformation complète, grâce à la salubrité de son climat, et à sa position admirable à l'entrée du golfe du Pé-tché-ly. Nous lisons en effet, dans le récit des voyages de M. J. Thompson en Chine et l'Indo-Chine (1), des détails fort intéressants sur le district de Tché-foû, et que je ne peux m'empêcher de communiquer à mes lecteurs. Je laisse donc parler M. Thompson :

«Depuis quelques années, Tché-foû (qu'il prunonce Tchi-fô̂) est » devenu la ville d'eau favorite des étrangers qui résident à Pé-kin » et à Shang-haï, car c'est là qu'on pent le mieux jouir, pendant les » mois les plus chauds de l'été, des bains de mer et de l'air vif et » fortifiant que l'on peut respirer au bord de l'Océan. La plage sur " laquelle est bâti l'hôtel européen contourne le pied d'une chaîne » de collines gazonnées, et rappelle par son cours demi-circulaire » et son aspect général la baie de Brodic, sur la côte ouest d'É» cusse. Je garde un sourenir très-distinct de la baie de Tché-fố, » de sa longueur qui, à l'époque, me paraissait interminable, de » son sable doux et fin..... L'hôtel est une maison sans prétentions, » mais charmante, et où, grâce aux soins intelligents de son pro» priétaire, les étrangers trouvent toute espèce de confort.

» Le quartier étranger de Tché-foû se trouve sur le bord opposé » de la baie (la rade de Yan-taï); c'est, ou peu s'en faut, l'endroit le

(1) Dix ans de voyages en Chine et l'Indo-Chine, par J. Thompson; Paris, 1 vol. grand in-8, 1877, à la librairie Hachette. 
» moins engageant que l'on puisse trouver sur la côte. Toutefois, »nous ne devons pas oublier qu'il jouit de l'honneur d'être situé » sur le sol le plus classique de l'Empire, dans les lieux mêmes où » les travaux d'art du célèbre Yû furent en partie accomplis. Con» fucius naquit aussi dans la province de Chan-tong, aussi bien »que Mencius son successeur. »

Aujourd'hui que la célébrité est doublement acquise à la plage de Tché-foû, les touristes européens ne manqueront pas d'y affluer chaque année. Aussi ai-je l'espoir que la florule du Tché-foû, surtout si elle a l'honneur d'être connue des étrangers résidant en Chine, pourra rendre quelques services à ceux qui voudront se livrer à l'étude de la végétation de cette contrée si curieuse à visiter à plus d'un titre.

o. D.

\section{EXPLICATION DES PLANCHES}

de la florule du Tché-foû

\section{PLANCHE I}

FIG. I. - Statice Franchetii 0. Debx. (Échantillon de grandeur naturelle, dont on a coupé la tige florifère principale.)

Fig. II. - Une fleur grossie.

\section{PLANCHE II}

Fig. I. - Cyperus sinensis 0. Debx., de grandeur naturelle.

Fig. II. - $a$ Épillet grossi, $b$ achaine couronné par le stigmate et grossi, $c$ et $d$ glumes externes grossies.

\section{PLANCHE III}

Fig. I. - $a$ Fleur du Fimbristylis Stauntoni O. Debx., et A. Franchet, grossie à 25 diamètres ; $b$ écaille interne, $c$ écaille externe, $d$ coupe transversale de l'achaine, à un grossissement de 25 diam.

Fig. IJ. - Fleur du Lipccarpha microcephala Steud. grossie; $a$ écaille externe, $b$ et $d$ écailles internes, $c$ achaine.

FIg. III. - Cyperus subfuscus. O. Debx.; $a$ plante de grandeur naturelle, $\downarrow$ un épillet grossi; $c$ achaine grossi, à 25 diam., et derrière lequel on a figuré les étamines pour en indiquer la forme et la position. 



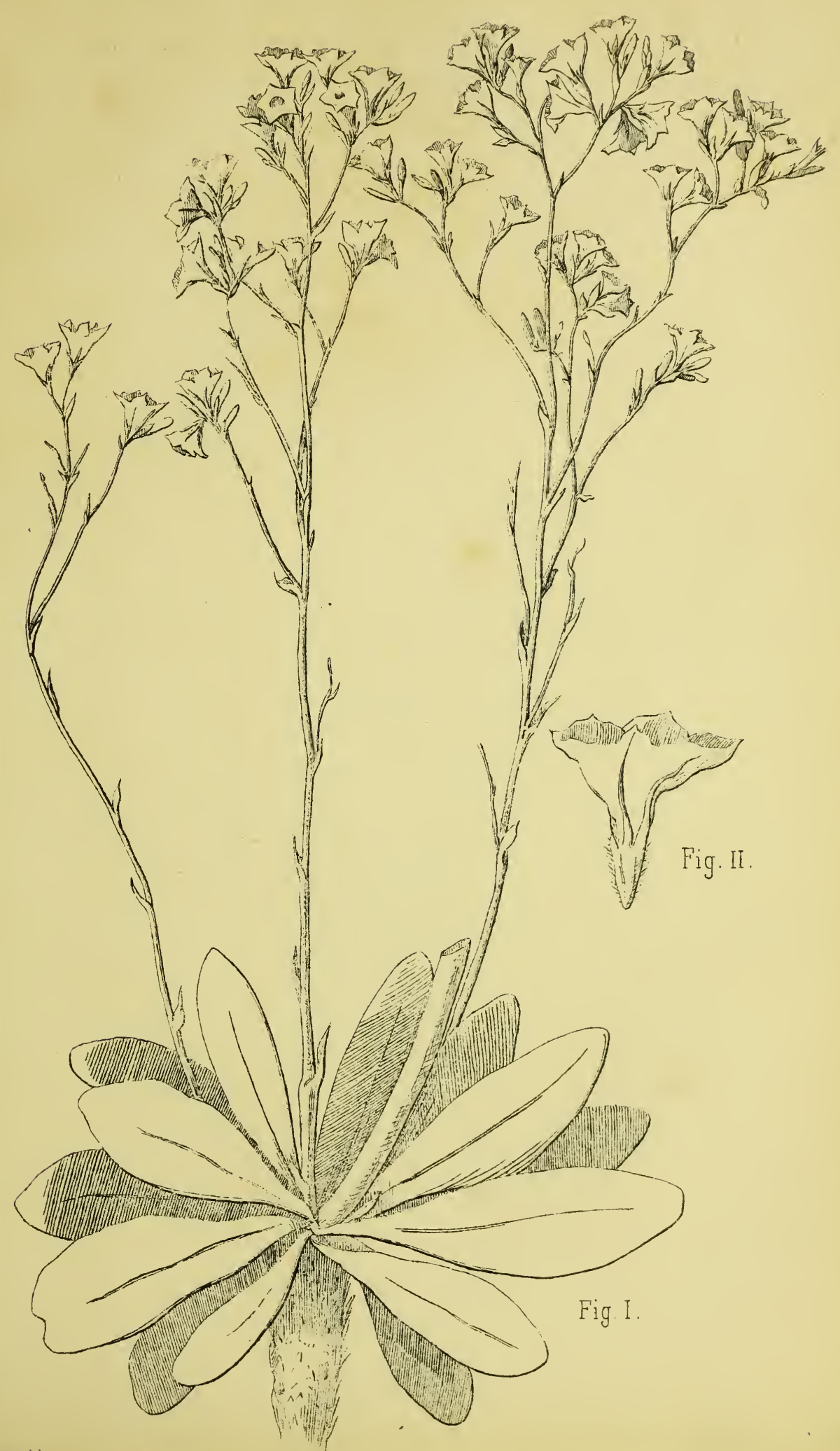





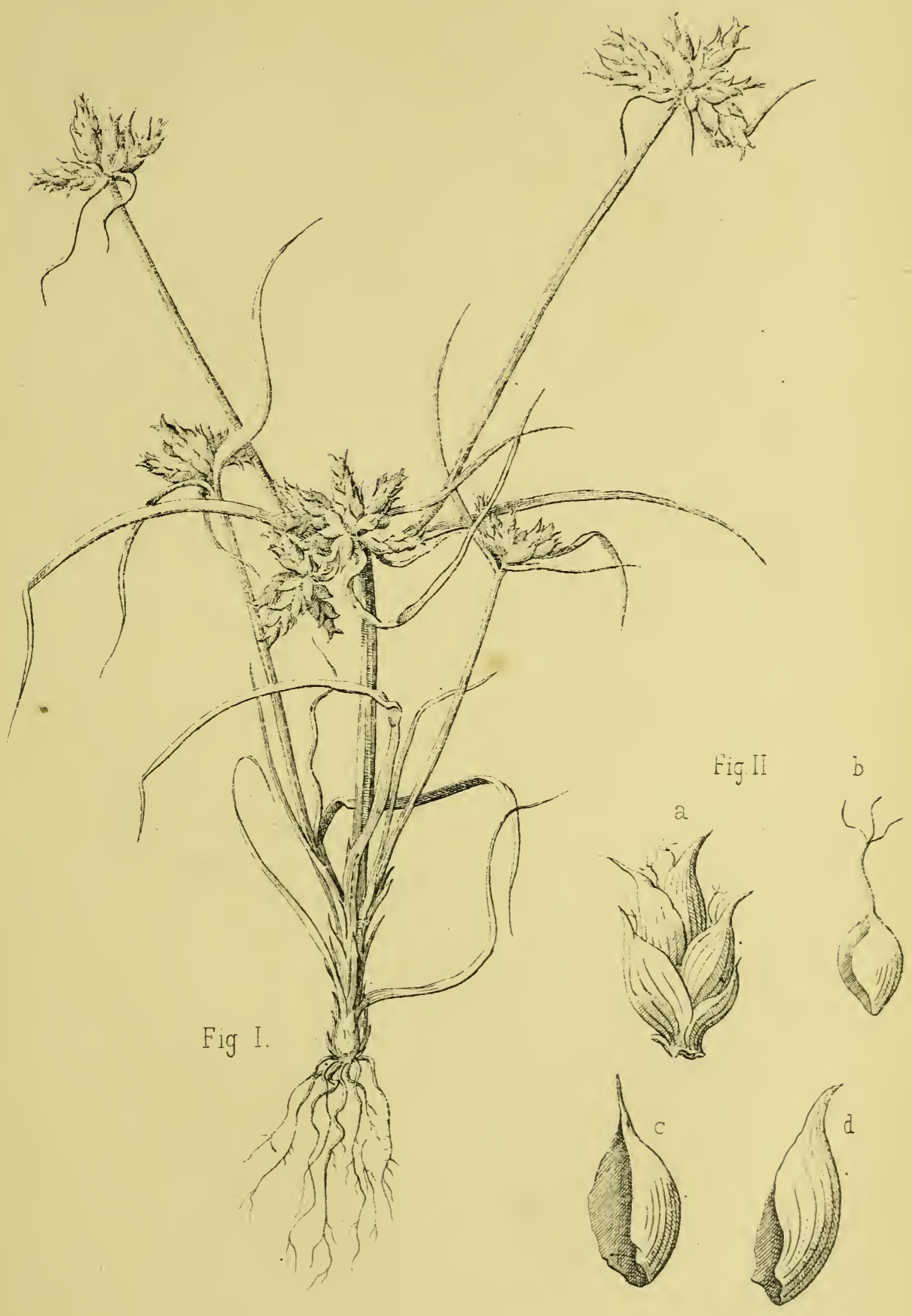

Bucguoy ad nat. del. 

FLOR. DU TCHÉ-FOU.

PL III.
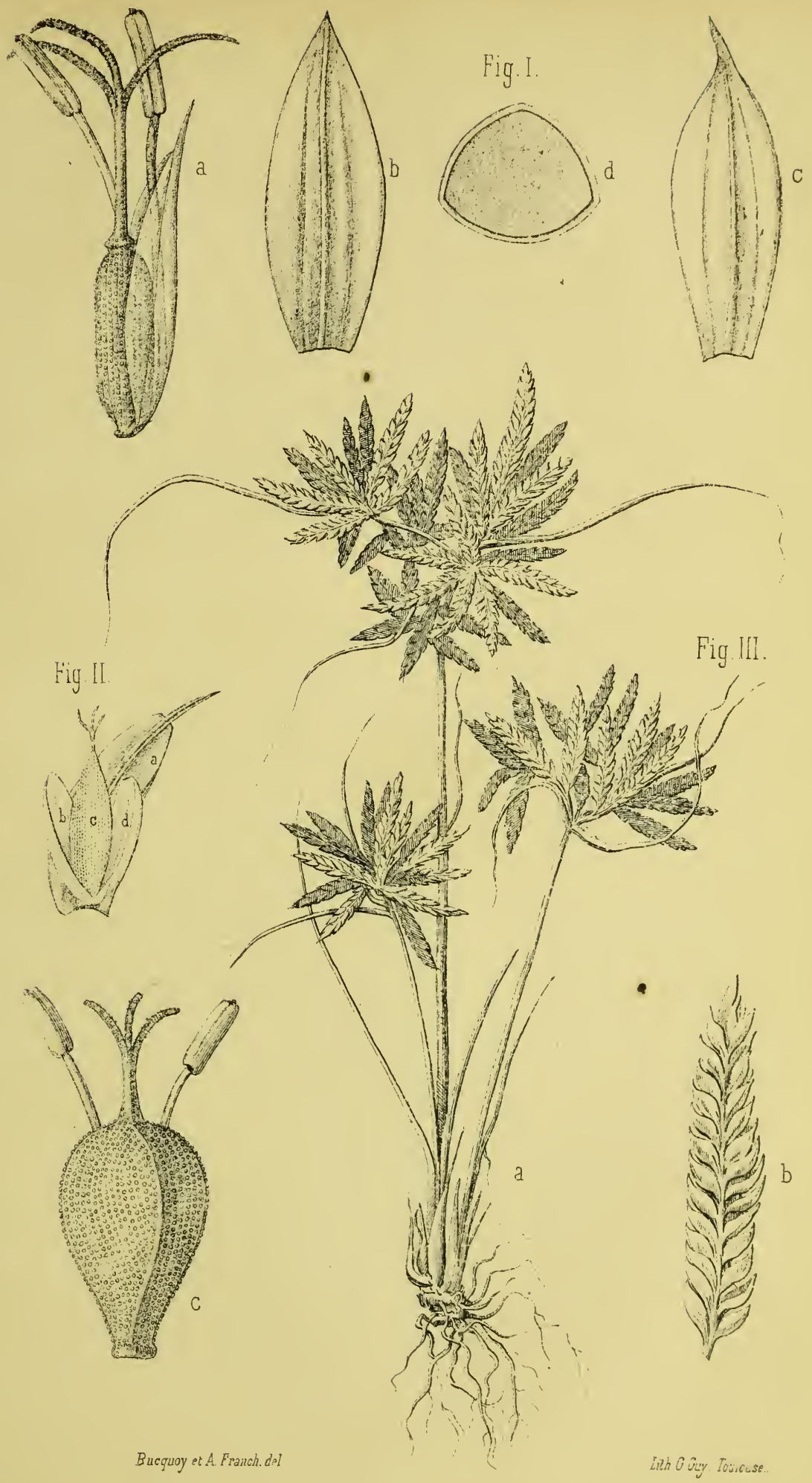

I Fimbristylis Stauntoni O. Debx etA.Franch.

II. Lipocarpha microcephala Steud. III. Cyperus subfuscus O. Debeaux. (Grand. naz.) 



\section{CONTRIBUTIONS A LA FLORE DE LA CHINE}

FASCICULE IV

\section{FLORULE DE TIEN-TSIN (PROVINCE DE PÉ-TCHÉ-LY)}

\section{Par M. O. DEBEAUX}

PHARMACIEN-MAJOR DE 1 re CLASSE

CHEVALIER DE LA LÉGION L'HONNEUR

Membre de la Société botanique de France, correspondant de la Société Linnéenne de Bordeaux et de plusieurs autres Sociétés savantes.

Extrait des Actes de la Société Linnéenne de Bordeaux.

Tome XXXIII, lre et $2^{e}$ LIVRaisons 1879.

\section{PARIS}

F. SAVY, LIBRAIRE-ÉDITEUR, BovleVARD SAINT-Germain, 77. 
BordeaUX. - TYP. J. DURAND, RUE VITAL-CARLeS, 24. 
CONTRIBUTiONS A LA FLORE DE LA CHINE

\title{
FLORULE DE TIEN-TSIN
}

\section{(PROVINGE DE PÉ-TGHÉ-LY)}

\author{
Par M. O. DEBEAUX,
}

Pharmacien-major de première classe,

Membre correspondant.

\section{AVANT-PROPOS}

La ville chinoise de Tien-tsin, dont le nom indigène a pour signification littérale "cité céleste », est située dans la province de Pé-tché-ly, à l'extrême nord de la Chine, sur la rive droite du fleuve Pei-hô, et dans l'angle que forme le canal impérial ou Yü-kiang-hô, c'est-à-dire "la rivière qui porte les grains », à sa jonction avec le Pei-hô. Celui-ci, que l'on peut aussi écrire et prononcer Pé-hó, signifie pour les Chinois le fleuve du Nord. Tien-tsin se trouve par $113^{\circ} 94$ environ de longitude Est, et $39^{\circ} 10$ de latitude Nord, à 48 kilomètres de l'embouchure du Pei-hô près des forts de Tâ-kòu, et à 95 kilomètres au sud de Pé-king capitale de l'empire. On parvient facilement à Tien-tsin par le golfe de Pé-tché-ly, et le fleuve Pei-hô qui est navigable, l'hiver excepté, même pour les steamers d'un fort tonnage, ou bien encore par une bonne route qui relie les forts de Tâ-kôu aux faubourgs de Tien-tsin, sur la rive gauche du Pei-hô. Mais les Chinois préfèrent se servir pour leurs transactions commerciales de la voie du canal impérial, lequel traverse, du Sud au Nord, une 
grande partie de la Chine centrale, depuis Hâng-tchéoû dans le Tché-kiâng̊, jusqu'à Tien-tsin dans le Pé-tché-ly.

Arrivé à cette dernière destination à la fin d'arril '1861, aussitôt après la débâcle du Pei-hô, pour remplir les fonctions de pharmacien en chef à l'hôpital temporaire établi dans cette localité, j’y ai séjourné jusqu'à la fin de son occupation par l'armée française en. Chine, survenue au commencement de novembre de cette même année. Il m'a donc été possible d'étudier, pendant près de six mois, la végétation spontanée et de grande culture de cette contrée qu'aucun botaniste n'avait encore parcourue ou visitée, depuis le passage de sir Georges Staunton, attaché à l'ambassade de lord Macartney en 1793.

Au premier abord, j’ai été frappé, dès mon arrivée dans le Pé-tché-ly, de la pauvreté de la flore des environs de Tien-tsin. Cette pauvreté me paraît provenir, d'abord de la nature sablonneuse du sol, lequel est entièrement composé de terrains d'alluvion, mais surtout aussi des progrès de l'agriculture dans cette contrée. Toutes les terres qui bordent le canal impérial et le Pei-hô pouvant être arrosées facilement par des norias ou des canaux d'irrigation, sont transformées chaque année en belles cultures de céréales et de légumineuses diverses, entretenues arec les plus grands soins, et dont toutes les plantes étrangères sont rigoureusement extirpées par les indigènes. Au delà des terres arrosables et propres à la culture, principalement sur la rive gauche $d u$ Pei-hô, on trouve d'immenses plaines sablonneuses, couvertes ca et là de marécages saumâtres d'une certaine étendue. Ceux-ci se dessèchent en partie pendant l'été sous l'action du soleil, et se recouvrent d'une couche épaisse de Kien, substance saline composée d'un mélange de chlorure de sodium, de carbonate et de sulfate de soude. Je ne dois pas oublier d'ajouter que les salines de l'État sont situées à Tien-tsin, sur la rire gauche du Pei-hô, et sur une étendue de plusieurss kilomètres le long de la rivière. C'est là que sont apportées chaque année, de tous les points maritimes et commerciaux de l'empire, des quantités considérables de sel marin, dont le gouvernement chinois a seul le monopole. Aussi toute la plaine qui aroisine les salines impériales, est-elle aride et impropre à aucune culture, tant le sol est imprégné de matières salées, qui font efflorescence à sa surface, aussitôt que la saison des pluies est terminée. Au bord 
des mares d'une certaine étendue et dont l'eau saumâtre ou salée ne s'évapore pas en totalité, j'ai pu récolter un petit nombre de plantes halophytes, et que l'on retrouve sur les rivages du golfe de Léa-tong.

J'ai déjì dit qu'une chaussée assez bien entretenue reliait, sur la rive gauche du Pei-hô, les forts de Tâ-koû à l'un des faubourgs de Tien-tsin. Sur le parcours de cette route, qui se continue jusqu’à Pé-king, et à dix kilomètres environ au sud de Tien-tsin, se trouve un très-beau yamoun (pagode entourée d'un grand parc) abandonné par les bonzes qui l'habitaient lors du passage de l'armée française, et qui a été plusieurs fois le but de mes herborisations. En avant de ce yamoun, les Chinois ont construit une fortification en terre de grandes dimensions, et à laquelle était adossé un vaste camp retranché. Sur les talus de cet important ouvrage défensif nommé Fort de Tien-kô, et que l'armée chinoise n'a même pas songé à occuper lors de notre marche sur Pé-king, j'ai récolté, ainsi que dans la plaine inculte qui l'entoure, quelques plantes spéciales à l'extrême nord de la Chine, et que je n'ai pas rencontrées ailleurs dans un grand rayon autour de Tien-tsin. Je citerai principalement les Orychophragmus sonchifolius, Rubia cordifolia, Calimeris altaica, Artemisia mongolica, Scorzonera parviflora var., Ixeris versicolor, Rehmania glutinosa, Bothryospermum chinense, Erytrichium pedunculare, Callistegia acetosellxfolia, Marrubium incisum, etc., qui sont propres à cette localité. La végétation de cette partie du territoire de Tien-tsin, est en général très-pauvre en espèces, mais celles-ci offrent au botaniste le plus grand intérêt, soit par leur rareté, soit encore par leur habitat ou leur dispersion géographique.

Le Pei-hô forme au-dessus de Tien-tsin, après sa jonction avec le canal impérial, une grande courbe nommée vulgairement dans ce pays «la boucle du Pei-hó ». Sur les sables secs ou marécageux qui constituent le sol à peu près inculte decette presqu'île, on remarque une pagrode entourée d'un grand cimetière chinois. J'ai dirigé fort souvent mes excursions dans la boucle du Pei-hô, en remontant le cour's de ce fleuve, et j'en ai rapporté un petit nombre d'espèces fort intéressantes, et dont quelques-unes sont nouvelles pour la flore du Pé-tché-ly. Je nommerai surtout les suivantes, qui m'ont paru devoir caracté- 
riser la végétation des sables d'alluvion du Pei-hô; Ranunculus chinensis et $\boldsymbol{R}$. orizetorum, Lepidium micranthum, Bunias tcheliensis (Spec. nov.), Astragalus glanduliferus (Spec. nov.), Potentilla amurensis, Tamarix chinensis, Iris uniflora, Carex Bungeana, Eluropus littoralis var. sinensis, etc.

Enfin les terrains vagues ou incultes au pied des murailles de la ville fortifiée de Tien-tsin, et les jardins qui entourent les pagodes dans les faubourgs, m'ont encore fourni quelques plantes indigènes, en petit nombre il est vrai, mais que l'on retrouve dans des stations analogues, dans presque toutes les provinces de l'empire.

Afin de donner une idée exacte au botaniste, de la végétation de Tien-tsin, j'ai pensé qu'il était utile d'énumérer dans cette florule, les plantes de grande culture que l'on retrouve parfois à l'état subspontané, et qui occupent pour les besoins de l'alimentation toutes les terres arrosables du territoire de ce pays. Je mentionne en passant, comme végétaux de grande culture dans le Pé-tché-ly, les espèces ci-après : Brassica sinensis, Raphanus sativus, Cucumis melo, Fagopym esculentum, Zea mays, Hordeum distichum, Sorghum vulgare, etc.

Les nombreux villages situés sur les deux rives du Pei-hô, en descendant le fleuve vers son embouchure, sont entourés de jardins oì règnent pendant l'été une fraîcheur et une richesse de végétation vraiment remarquables. De vigoui eux arbres fruitiers, dont les fruits savoureux rappellent par leur gout ceux de l'Europe australe, se font admirer dans ces jardins, d'où s'élèvent parfois des massifs de verdure, dus à la présence d'arbres résineux parfaitement acclimatés dans cette contrée. La plupart de ces arbres de la famille des conifères, et dont la présence a été déjà signalée au Tché-foû et à s'hang-haï, se montrent assez nombreux dans les parcs des yamouns, des temples bouddhiques et autour des innombrables tumulus disséminés dans toute la plaine du Pei-hô, et ils résistent très-bien an froid rigoureux qui règne chaque année. de décembre à mars, dans la province du Pé-tché-ly. On ne doit pas oublier en effet, qu’à Tien-tsin, le thermomètre centigrade descend sourent jusqu'à $17^{\circ}$ et $19^{\circ}$ au-dessous de zéro, pendant les mois de décembre et de janvier qui sont les plus rigoureux de l'hiver dans cette contrée. Pendant l'été, au contraire, le thermomètre s'élève 
jusqu’à 38 et même à 40 degrés centigrades au-dessus de zéro, et cette haute température a été observée quelquefois penclant plusieurs jours consécutifs à Tien-tsin et à Pé-king, quoique cependant cette dernière cité soit distante de la première d'environ cent kilomètres plus au nord. La persistance du froid et sa rigueur dans une contrée relativement éloignée des régions arctiques, expliquent l'impossibilité pour certains végétaux de la Chine moyenne et littorale, de pouvoir s'acclimater à Tien-tsin et dans toute l'étendue de la province de Pé-tché-ly. Je citerai entre autres les Camellia japonica, Pronia moutan, Nandina domestica, Nelumbium speciosum, Eriobotrya japonica, Chamxrops exselsa, Stillingia sebifera, etc., qui prospèrent très bien en pleine terre à Shang-haï, et qui, résistant à des froids annuels de 12 à 13 degrés centigrades au-deśsous de zéro, ne peuvent végéter qu'en serre chaude, sous le climat de Tien-tsin et de Pé-king.

D'un autre côté, j'ai observé, non sans quelque surprise, dans plusieurs jardins de Tien-tsin, un petit nombre d'arbustes propres aux régions tempérées de l'Asie, ou aux contrées les plus chaudes de l'Europe australe, et qui sont parfaitement acclimatés dans le nord du Pé-tché-ly, malgré les rigueurs du froid dans ce pays. J'ai remarqué surtout les suivants à cause de leur vulgarité à Tien-tsin : Zizyphus vulgaris var. sativus, Sophora japonica, Ailanthus glandulosa, Punica granatum, Forsythia suspensa, Syringa chinensis, etc.

En résumé, le territoire de Tien-tsin possède une flore très voisine de celle de Pé-king, mais non aussi riche que celle-ci. La végétation du nord du Pé-tché-ly et des abords de la capitale de l'empire nous étant bien connue, par suite des recherches et des publications déjà anciennes de Bunge, Georges Staunton, Tatarinow, Turczaninow, et celles beaucoup plus récentes de MM. l'abbé David, Hance, Maximowicz, Dr Wels Williams, etc., il m'a été facile d'arriver sûrement à la détermination rigoureuse des plantes récoltées par moi-même autour de Tien-tsin. Quelques rares espèces toutefois ont échappé aux investigations des habiles explorateurs qui m’ont précédé dans le Pé-tché-ly, et j'ai la bonne fortune de pouvoir les signaler pour la première fois à l'attention des botanistes, soit comme espèces nourelles, soit comme formes ou variétés remarquables. Il no faut pas 
d'ailleurs perdre de vue, que le Pei-hô prend sa source dans les montagnes situées au nord de Pé-king, et que, dans sa course vers la mer du Léa-tong où il se déverse, il a pu déposer sur ses rives, des graines de plantes provenant des confins de la Mongolie chinoise, et qui se sont ensuite parfaitement acclimatées dans ce nouvel habitat.

La plaine du Pei-hô est soumise, ainsi que je l'ai avancé dans la florule de Shang-haï, à l'influence des grandes inondations. Cette influence qui est si manifeste dans le great-plain, c'est-à-dire dans les vastes plaines arrosées par les fleuves Hiang-tssé-kiang et Hôang-hô, est encore plus facile à constater sur le parcours aussi restreint et aussi uniforme que celui du Pei-hô. C'est donc sur les sables et les abords de ce fleuve, que j'engagerais les botanistes-voyageurs à faire de nombreuses recherches, d'avril a septembre, persuadé qu'ils en rapporteront une foule d'espèces inconnues jusqu'à présent. 


\section{FLORULE DE TIEN-TSIN}

RENONCULACÉES Juss.

\section{Ranunculus chinensis Bunge Enumeratio plantarum chinx}

borealis (1831), no 10, page 3 du tirage à part; 0 . Debeaux Florule de Shang-haï, nu 2; Maximowicz Primit. flor. amur. p. 22; Régel Tentam. flor. ussur. p. 8.

Habitat : Les sables marécageux dans la boucle du Pei-hô près de Tien-tsin, et en remontant ce fleuve vers Tong-chéoû. Fl. du 15 au 30 mai.

Aréa géographique: Chine bor. et moy. sur les alluvions du great-plain dans le Kiang-soû, le Pé-tché-ly à Pé-king, Tientsin, etc.; Amour et Ussuri. - Japon.

2. Ranunculus oryzetorum Bunge Enum. chin. bor., no 9 ;

O. Debx. Flor. Shang-haï, n ${ }^{0} 3$; Sinice tzÉ-yùEn-tsâo, TchyLAN-TSâO.

Hab. : Les petites mares, aux bords du Pei-hô, où il est abondant. Fl. du 20 au 30 mai, mêlé à l'espèce précédente.

Ar. géog. : Chine bor. et moy., Kiang-soû à shang-haï; Pé-tchély (Bunge).

Obs. Bunge signale avec doute (loc. cit.), le R. orizetorum comme étant annuel. Je dois ajouter que cette espèce est réellement annuelle. Dans le nord de la Chine, elle est manifestement plus grêle et surtout beaucoup plus glabre que dans les spécimens récoltés à Woô-Song et à Shang-haï, dans la province de Kiang-soû.

3. Pæonia albiflora Pallas Flor. ross. 2, tab, 84; D. C. in Prodr. 1, p. 66; Ledeb. Flor. ross. 1, p. 74; Turczan. Flor. baik. dahur., 1, p. 89; Maxim. Prim. amur. p. 89; Franchet et Savatier Enum. plant. in Japon. crescent., 1, p. 24, n 63; P. officinalis Thunberg Fl. Japon., p. 230; Sinice Сно-гô, Ре́-тсно-уо̂. 
Hab. : Dans tous les parcs et jardins, autour des yamouns, et des habitations rurales, où cette plante est généralement cultivée pour la beauté de ses fleurs. - Fl. du ler au 1j juin.

Ar. géog. Asie bor., Daoûrie, Mongolie chinoise; Pé-tché-ly à Pé-king (Bunge); Amour et Ussuri. - Japon.

Obs. Cette magnifique espèce qui produit par la culture, de nombreuses variétés à fleur's simples, semi-pleines ou doubles, n'est que subspontanée à Tien-tsin. Elle se trouverait toutefois à l'état spontané, dans la région montagneuse des provinces de Hô-nan et de N'gan-Hoêy. Les Chinois cultivent aussi, mais seulement en serre tempérée sous le climat de Tien-tsin, la Piroine moutan dont ils obtiennent également de nombreuses variétés.

\section{CRUCIFÈRES Juss.}

4. Nasturtium palustre D. C. Syst. veget. 2, p. 19; Bunge Enum. chin. bor. no 28; Turczan. Fl. baik. dahur. 1, p. 108; Maxim. in Index floræ pekin. (in Prim. amur. p. 469); Franch. et Savat. Enum. plant. jap., 1, n ${ }^{0} 130 ; N$. amphibium Thunbg. Fl. jap., p. 260.

Forma dissecta Franch. et Savat. (loc. cit.) : foliis omnibus profunde pinnatifidis.

$H a b$. : Les sables humides ou marécageux, dans la boucle du Pei-hô, au-dessus de Tien-tsin. - Fl. 20-30 mai.

Ar. géog. : Eur., Asie bor., occ. et or.; Tauride, Perse, sibérie alt., Baikalie, Kantskatka, Mongolie, Chine bor., Pé-king. Amour et Ussuri. - Japon. - Amér. bor.

Obs. Dans tous les échantillons récoltés à Tien-tsin, les feuilles, même les inférieures, sont profondément pinnatifides. La plante du Pei-hô correspond très-exactement à la forme dissecta de. MI. Franchet et Savatier, laquelle provient du Japon.

5. Brassica chinensis Lin. Amxn. 4, p. 280; O. Debx. Filor. Shang-haï, no 15; Maxim. Prim. amur. p. 47; Régel Tent. ussur. p. 23; Franch. et Savat. Enum. jap. 1, no 155; B. orientalis Thunbg. Fl. jap., p. 261, non Lin.; sinice PÉ-TsAï. 
Hab. : Cultivé en grand dans tous les champs de la plaine du Pei-hô et des rives du canal impérial.

Ar. géog.: Chine bor. et cent. Kiang-soû, Tché-kiang; Pé-tché-ly, Amour et Ussuri. - Japon.

6. Lepidium micranthum Ledeb. Flor. ross: 1, p. 205 et $\mathrm{Fl}$. alt. 3, p. 193; O. Debx. Flor. de Shang-haï, $\mathrm{n}^{0} 18 ; L$. incisum M. Bieb. Fl. taur. cauc. 2, p. 98; L. muderale \Bunge Enum. chin. bor. no 36 , non Lin.

$H a b$. : Les sables secs ou humides dans la boucle du Pei-hô, où il est très-abondant. - Fl. du 20 au 30 mai.

Ar. géog. : Asie bor. occ. et orient., Baikalie, Mongolie.-Chine bor. et 'moy., Kiang-soû à Shang-haï (O. Deb.); Pé-tché-ly à Pé-king (Bunge).

\section{Lepidium latifolium Lin.}

Var. pubescens Ledeb. Flor. ross. 1, p. 207; L. latifolium var. angustifolium C. A. Mey. in Ledeb. Flor. alt. 3, p. 139; L. affine Bunge Enum. chin. bor. no 39, non Ledeb.

Plante beaucoup plus petite dans toutes ses parties que la forme typique de l'Europe australe; feuilles ovales, oblongues, pubescentes en dessous, glauques en dessus, entières ou finement dentées en scie, à dents rares et peu profondes.

Hab. : Les sables du Pei-hô, au-dessus du pont de pierre, sur la route de Pé-king, près de Tien-tsin.

Ar. géog. : Le type : Europe, Asie bor., Caucase, Sibérie baikal., Daoûrie, etc. La variété : Sibérie baikal., Daoûrie, Désert des Kirghys, Mongolie, Chine bor., Pé-tchély à Pé-king (Bunge), Tien-tsin $(O . D e b$.

8. Orychophragmus sonchifolius Bunge Enum.plant. chin. bor. $\mathrm{n}^{\circ} 40$ in Mém. des savants étrang. de Saint-Pétersb., II., p. 81.

Plante herbacée annuelle, entièrement glauque; tiges simples ou peu rameuses, grêles, pauciflores, de 20 à 30 centim. de hauteur; feuilles radicales pinnati-lyrées, sinuées-dentées à lobes plus ou moins profonds, le terminal très-grand, arrondi, les caulinaires semi-amplexicaules avec deux oreillettes à leur base, relles-ci un peu dentées, glabres et glaucescentes; fleurs assez 
grandes (semblables par leur forme à celles de l'Hesperis matronalis L.), d'un rose violacé, plus pâles vers l'onglet; pétales longuement onguiculés à limbe plan, obovale arrondi; calice renflé, formant deux cavités vers sa base, à divisions inégales lancéolées; étamines exsertes; siliques allongées, subtétragones, à valves carénées et terminées par le style également tétragone, comprimé et persistant. - Fleur. du $1^{\text {er }}$ au 30 juin.

$H a b$. : Les sables frais ou humides au bord des mares, près du yamoun de Tien-kô sur la route de Tâ-koû.

Ar. géog. : Chine bor., Pé-tché-ly à Pé-king (Bunge); Tien-tsin (O. Deb.).

Obs. Le genre Orychophragmus vient se placer à côté des Moricandia. Il a été créé par Bunge (loc. cit.) en 1831, et ne renferme qu'une seule espèce, l'O. sonchifolius, dont l'aréa ne dépasse pas Pé-king au nord, et Tien-tsin dans le sud de la province de Pé-tché-ly.

9. Bunias (Lelia) Tcheliensis O. Debeaux (Spec. nov.) in Herb., 1861.

Icon nostra tab. I.

"Planta herbacea, annua, caulibus gracilibus, striatis, simpli» cibus vel ramosis, nunc erectis (15-20 centim. altis), nunc pros» tratis, intertexte divaricatis; foliis inferioribus sinuato-runci»natis, in petiolum breve attenuatis, caulinis sessilibus auricu» latis, subsagittatis, supra hispidulis, subtus glabris, omnibus » margine parce hispidis; floribus minutis, albidis, apice ramo»rum confertis, dein spicam elongatam laxamque efformantibus; » siliculis apteris, longe pedicellatis (4 millim. latis, $5-6$ mill. »altis), subtetragonis, bilocularibus, rugoso-sulcatis, subito » acuminatis, pedicellis 8-10 millim. longis, patulis, alternis, » æqualiter e basi caulium distantibus. »

Hab. : Les sables humides ou incultes des rives du Pei-hô, dans la boucle au-dessus de Tien-tsin. - Fl. du 20 au 30 mai.

Ar. géog. : Chine bor., Pé-tchély à Tien-tsin (O.Debx.)

Obs. Cette curieuse espèce du genre Bumias, vient se ra'ıger par ses silicules biloculaires, dans la section Lxlia à côté des $B$. orientalis et $B$. cochlearioides.

Le $B$. tchelierssis se distingue du premier, par sa végétation annuelle et mon bisannuelle, par ses silicules à quatre angles 
pyramidaux profondément sillonnés, et non simplement verruqueuses, tuberculeuses et arrondies. Il diffère du B. cochlearioides espèce propre à la Soôngarie et au désert de Kirghyz, par ses silicules portées sur des pédicelles allongés et non très-courts, ceux-ci espacés régulièrement sur les tiges, et alternant entre eux de manière à former un long épi non interrompu, écartés horizontalement à angle droit, et non dressés.

Le B. tcheiiensis, dont M. le docteur Bucquoy m'a remis un dessin très exact et qui est figuré dans cette florule, est le seul représentant de ce genre, qui soit connu jusqu'à présent dans l'empire chinois. Il est fort abondant sur les sables des rives du Pei-hô, et varie aussi beducoup par son port, la forme des feuilles, la longueur des épis floraux, etc. Mais ses silicules tétragones, dressées à l'extrémité de long’s pédicelles étalés horizontalement sur les tiges, suffisent pour le distinguer au premier coup d'œil, des espèces d'ailleurs peu nombreuses que renferme le genre Bunias.

10. Raphanus sativus Lin. Spec. 935; Ledeb. Flor. ross., 1, p. 225; Maxim. Prim. amur. p. 47; Régel Tent. ussur. p. 23; Franch. et Savat. Enum. japon., 1, no 163; Sinice Lô-pô, Ta-lô-pô.

Hab. : Cultivé en plein champ dans tout le territoire de Tientsin, et subspontané au bord des chemins, autour des habitations rurales, etc.

Ar. géog. : Originaire de l'Asie bor. et occid.; Chine bor. moy. et aust., Pé-tché-ly à Pé-king (Maxim.) - Amour et Ussuri. Japon.

Obs. Outre les nombreuses variétés du raifort commun, les habitants du Pé-tché-ly cultivent, pour servir à l'alimentation, les Brassica oleracea L., Sinice PÉ-Tsä̈, et $B$. rapa L., Sinice, TA-Lô-Pô, plantes qui servent aussi à l'ornement des jardins.

STERCULIACÉES R. Br.

11. Sterculia platanifolia Lin. Suppl. 423; D. C. Prod. 1, 483; O. Deb. Fl. Shang-haï, nº 22; Franch. et SSav. Enum. plant. 
jap. 1, no 267; Firmiana platanifolia R. Brown in Plant. javan. rar. 235; Sinice OQ-TONG-chû.

Hab. : Planté dans les parcs et les jardins des yamouns, des temples bouddhiques, etc, et les cours intérieures des habitations.

Ar. géog. : Asie cent., Indes-Orient. - Chine bor. moy. et aust.; Pé-tché-ly à Pé-king (G. Staunton).

Obs. I. Bunge signale dans son Enumeratio plant. chinæ bor. $\mathrm{n}^{\circ} 56$, une autre espèce de Sterculia, le $S$. pyriformis Bunge, et qui serait rare dans les jardins de Pé-king. Je n'ai vu d'autre arbre de la famille des Sterculiacées à Tien-tsin, que le $S$. platanifolia, qui est distinct du $S$. pyriformis par ses feuilles glabres sur les deux faces et non velues, tomenteuses en dessous.

Obs. II. La vigne (Vitis vinifera L.), Sinice Poû-TAô, est cultivée de temps immémorial à Tien-tsin et à Pé-king. Les raisins du Pé-tché-ly ont la réputation, d'ailleurs bien méritée, d'être les plus beaux et les meilleurs de tous ceux qui sont produits par les diverses variétés de la vigne en Chine. Pour conserver les cépages pendant les froids rigoureux de l'hiver (17 à 19 degrés centig. au-dessous de zéro), les Chinois prennent le suin de coucher au mois de novembre le tronc principal de chaque vigne, et sans l'arracher, dans des fosses de 50 à 60 centimètres de profondeur et creusées au pied de l'arbuste. Les vignes dépouillées des sarments sont placées au fond des fossés dans le sens de la longueur, et sont recouvertes ensuite de terre bien tassée. Vers la fin d'avril, les fosses sont ouvertes à nouveau, et les tiges sarmenteuses de la vigne étant redressées, celles-ci sont fixées sur des tuteurs ou contre des treillages, et ne tardent pas à se couvrir de bourgeons. Les raisins ne sont employés qu'à l'usage alimentaire, et non pour en obtenir du vin et des liqueurs alcooliques. Les Chinois n'emploient pour cette dernière fabrication, que les semences du riz cultivé ou d'autres graminées.

\section{ZYGOPHYLLÉES R. Brown.}

12. Tribulus terrestris Lin. Spec. 544; Bunge Enum. chin. bor. no r6; O. Deb. Fl. du T'ché-foù, no 3丂; Sinice Ki-ly. 
Hab. : Les sables secs, sur la rive gauche du Pei-hô, près des forts de Tien-kô, etc.

Ar. géog.. : Eur. cent. et aust. - Afriq. bor. - Asie bor. cent. et or. - Mongolie, Chine bor. et marit.; Chan-tong au Tché-fou (O. Deb.); Pé-tché-ly à Pé-king - - Japon.

\section{XANTHOXYLÉES JusS.}

13. Allantus glandulosa Desf. Act. acad. paris. p. 263 (1786)'; D. C. Prod. 2, p. 39; Bunge Enum. chin. bor. no 87; Maxim. Index fl. Pekin. p. 470; Sinice Тоноù, Тснш-тсной.

Hab. : Dans les parcs, les jardins, autour des habitations rurales, etc., où il atteint des proportions considérables.

Ar. géog. : Chine bor. à Pé-king (Bunge et Maxim.). Tien-tsin (O. Deb.).

Obs. Je dois faire observer en passant, combien est impropre le nom de Vernis du.Japon, donné vulgairemert en Europe à cet arbre d'origine chinoise. La présence au Japon de l'A. glandulosa n'est mentionnée dans aucun ouvrage spécial à la flore japonnaise, et tous les botanistes-voyageurs s'accordent à le considérer comme étant originaire des régions boréales de la Chine, d'où il aurait été importé au Japon comme arbre d'ornement. On le trouve aujourd'hui parfaitement àcclimaté dans le midi de la France, les Pyrénées-Orientales, la Corse, l'Espagne, l'Italie, etc.

J'ai rencontré dans quelques jardins à Tien-tsin, le Koelreuteriapaniculata Lin., élégant arbuste de la famille des Sapindacées, ainsi que l'Esculus chinensis Bunge (Hippocastanées), tous les deux signalés dans la région montagneuse et boréale du Pé-tché-ly, et nullement spontanés à Tien-tsin .

\section{RHAMNÉES R. BROWN.}

14. Zizyphus vulgaris Lam. Ill. gen. tab. 185; Bunge Enum. chin. bor. no 21; O. Deb. Fl. de Shang-hä̈, no 29, var. B. inermis; Z. chinensis Lam.; Sinice KIN-TSAÔ. 
"Arboreus, inermis, baccis magnitudine pruni, sapore dulci.»

Mab.: Répandu dans tous les jardins de la ville et des faubourgs, autour des pagodes, etc.

Ar. géog. : Asie temp. bor. cent. et aust. - Mongolie, Chine bor. et cent., Pé-tché-ly à Pé-king (Bunge); Kiang-soû à Shlanghaï (O. Deb.). - Japon. - Europe aust. - Lfriq. bor., etc.

\section{LÉGUMINEUSES JusS.}

15 Sophora japonica Lin. Mant. 68; D. C. Prodr. 2, p. 95; Maxim. Index fl. Pekin. in Prim. amur. p. 470; O. Debx. Fl. Shang-haï, no 31; Franch. et Savat. Enum. plant. jap. 1, no 467; Sinice Hôar-HôA.

Hab. : Abondant autour de Tien-tsin, dans les jardins, les cours intérieures des habitations rurales, au bord des champs, etc., où il est cultivé pour ses fleurs tinctoriales.

Ar. géog. : Chine bor., moy. et aust., Pé-tché-ly à Pé-king (Bunge), Tien-tsin (O. Deb.); Kiang-soû, Tché-kiang, etc. Importé de la Chine au Japon dont il n'est pas origoinaire, mais où il est très bien acclimaté.

16. Astragalus (Galegiformes) glanduliferus 0. Debeaux (Spec. nova), in Herb. 1861.

Planta herbacea perennis, multicaulis; caulibus 25-30 centim. * altis, erectis seu prostratis, striati-sulcatis, undique glandulosis, 》hirsuto-puberulis, e basi ramosis, ramis patulis dein erectis, »brevibus; foliis 5-6 jugis, foliolis ramorum inferiorum ovato» ellipticis, superioribus ovato-lanceolatis, parum mucronulatis, »breviter petiolulatis, margine scabris, supra glanduloso-his* pidis, subtus scabris incanescentibus; petiolo communi glan»duloso setosoque; inflorescentia spicata, terminali axillarique; » spicis densis, confertis, longe petiolatis; floribus erecto-patulis, »breve petiolulatis, albidis vel luteo-pallescentibus; petalis »(carina, vexillo alisque) calicem duplo superantıbus; calicis »laciniis lanceolatis, glanduloso-pubescentibus; leguminibus » minutis, subcylindricis, nutantibus, apice rostrato-attenuatis, 
» styloque longe exserto terminatis. Semina matura non vidi. *

$H a b$. : Les sables et les pelouses sèclies des rives du Pei-hô, autour du fort détaché placé à l'entrée de la boucle de cette rivière, au dessus de Tien-tsin. - Fl. du 20 au 30 mai.

Ar. géog. : Cline bor., Pé-tché-ly.

Obs. Le genre Astragalus présente les plus grandes difficultés, pour la détermination des espèces propres à l'extrême Orient. Aussi ai-je prié M. A. Franchet, de vouloir bien soumettre ì l'examen de M. Maximowicz, la forme singulière rencontrée aux environs de Tien-tsin. Le savant botaniste de Saint-Pétersbourg' a fait savoir, dans sa réponse à M. Franchet, que l'Astragalus des rives du Pei-hô, est voisin il est vrai des $A$. chinensis Lin. fil. Decades 1, p. 6 et A. melilotoides Pallas, mais qu'il constitue une espèce bien distincte de celles-ci, par la pubescence glanduleuse qui recouvre toute la plante. Ces glandes pellucides sont si abondantes sur les feuilles, les pédicelles et les calices, que l'on douterait presque d'avoir sous les yeux, úne espèce lu genre Astragalus. M Franchet m'a assuré avoir lu avec attention la description de près de huit cents espèces du groupe Astragalus, un roi des genres, sans avoir pu trouver nulle part, la mention de la présence des glandes chez aucune d'entre elles. Aussi penset-il avec raison, que la plante singulière qui nous occupe, doit être inconnue jusqu'à piésent.

Notre A. glanduliferus ne saurait d'ailleurs ètre confondi avec l'A. chinensis qui a les tiges subglabres, les feuilles à 10-13 paires de folioles, les fleurs pendantes et les légumes ovales-renflés, à peine mucronulés. On ne saurait non plus le prendre pour l'A. melilotoides dont les tiges sont égalernent glabres, les rameaux floriféres trois fois plus longs que la feuille, et dont les légumes sont ovales-dressés, caractères qui n'existent nullement dans notre $A$. glanduliferus..

Obs. II. On trouve cultivés dans une foule de jardins, comme plantes d'ornement, le Wistaria chinensis D. C. originaire des montagnes au nord du Pé-tché-ly, et l'Albizzia julibrissin Benth., Sinice Hô-Koûan, ce dernier répandu surtout dans les parcs et autour des pagodes.

Les Chinois cultivent à Tien-tsin, quelques légumineuses pour servir à l'usage alimentaire et principalement, la fève (Fabr vulgaris Mrnch), Sinice Tsin-Hò̀. le pois commun (Pisum sati- 
vum L.), Sinice Hon̂ng-Tao, diverses variétés de haricots (Phaseolus vulgaris L.), Sinice Tsin-TÉou-TzÉ, le Lablab vulgaris L., Sinice Pren-téou, le Soja hispida L., Sinice Hong-TÉouTZÉ, etc.

\section{AMYGDALÉEŚS Juss.}

17. Amygdalus communis Lin. Spec. 676; Bunge Enum. chin. bor. no 125; Sinice Hang, YôH-HaNG.

$H a b$. : Les haies des jardins et les cultures autour de Tien-tsin. Ar. géog. : Asie occid. - Afriq. bor. - Chine bor., Pé-tché-ly à Pé-king (Kunge).

Obs. L'amandier est cultivé en grand dans la province de Pé-tché-ly pour ses fruits (amandes), Sinice Trex-mra, dont il se fait un commerce considérable, surtout pour les besoins de la confiserie. Il existe en effet à Tien-tsin, plusieurs maisons renommées pour la confection des pralines, et ce produit très-recherché des Chinois, s'expédie par milliers de caisses dans toutes les provinces de l'empire. La variété de l'amandier à fruits amers, Sinice HrN-GIn, HIN-Kô-GIN, se rencontre aussi à Tien-tsin, mais les amandes amères trouvent leur principal emploi dans la thérapeutique.

18. Persica vulgaris Mill. Dict. III, p. 465; Amygdalus persica Lin.; Bunge Enum. chin. bor. no 128; Sinice Tâ̂-GIN-TZÉ.

Hab. : Même habitat que le précédent.

Ar. géog. : Asie occid., Tauride, Caucase, Perse, etc. - Cultivé dans les provinces moy. et boréales de la Chine, à Pé-king (Bunge) - Japon.

19. Prunus armeniaca Lin. Spec. 679; Bunge Enum. chin. bor. $n^{\circ} 129$; Armeniaca vulgaris D. C. Prodr. 2, p. 532; Sinice HANG-GIN, Kin-Gin.

Hab. : Dans toutes les cultures de la vallée du Pei-hô, etc.

Ar. géog.: Asie occid., Arménie, Caucase. - Chine bor., Pétché-ly à Pé-king (Bunge), etc. - Nat. dans l'Eur. aust., l'Afr. bor. 
20. Prunus cerasus Lin. Spec. 679; Ledeb. Flor. russ. 2.. p. 6 ;

Maxim. in Index flor. pekin., p. 471; Sinice YU-LY, TSIẤ-MeI. $H a b$. : Les haies des jardins et des propriétés rurales où il esst subspontané.

Ar. géog. : Europe orient. - Asie occid., Caucase. - Chine bor. et moy. Pé-tché-ly à Péling (Maxim.), etc.

Obs. Dans cette espèce, les fruits sont aigres et très-petits.

ROSACÉES JusS.

21. Potentilla (Boreales) amurensis Naxim. Prim. flor. amur., p. 98; Régel Tent. fl. ussur. p. 56, tab. IV, fig. I.

"Planta herbacea, annua, ramosissima, undique molliter "pilosa; caulibus erectis (30-40 centim. et ultra), dein deflexis " prostrastive, subdichotomis; foliis inferioribus longe petiolatis, 》 caulinis ternatis petiolum requantibus vel eo brerioribus; »foliolis utrinque puberulis, subtus margineque pilis albidis 》vestitis, petiolulatis, late rhomboideis, floralibus profunde » tripartitis, basi obliquis, omnibus oblongo-obovatis, ad medium 》 usque inciso-serratis, serraturis ovatis obtusiusculis; stipulis 》ovato-lanceolatis, integris; sepalis subæqualibus, externis 》 lineari-oblongis, internis ovatis, acutiusculis; petalis longe »disjunctis, minutis, ovato-oblongis, integris, calice plus duplo »longioribus, flavis; carpellis numerosis, minutissimis, longitu》 dinaliter subrugosis.

Hab. : Les sables et les fossés humides dans la boucle du Pei-hô, où il est très abondant. - Fl. du 2 J ou 30 mai,

Ar. géog.: Chine bor., le Pè-tché-ly à Tien-tsin (O. Deb.); Amour (Maxim.); Ussuri (Régel).

Obs. Cette rare espèce, dont la présence n'était signalée seulement que dans les provinces de l'Amour et de l'Ussuri, n'a de rapports qu'avec le $P$. millegrana Engelm. plante de l'Amérique boréale. Elle s'en distingue par un facies tout différent, par sa taille plus grêle, par ses folioles profondément divisées, les terminales longuement pétiolulées, par ses rameaux raccoureis au sommet et surtout par les pétales beaucoup plus petits que dans toutes les espèces du groupe du $P$. supina. 
On rencontre souvent dans les haies et les clotures des jardins les Rosa indica L., Sinice N.ıN-tsiax-Hoâ, "et Kerria japonica D.C., Sinice Tana-ti-Hoâ, ce dernier à fleurs pleines le plus souvent.

\section{POMACÉES Lindl.}

22. Pyrus malus Lin. Spec. 686; Bunge Enum. chin. bor. $\mathrm{n}^{\circ}$ 158; O. Deb. Fl. du Tchéfou, no 58; Sinice Prs-Koño.

Hab. : Les cultures et les jardins dans la plaine du Pei-hô.

Ar. géog. : Europe, Afrique bor. - Asie bor. et occid. - Chine bor. et cent., Pé-tché-ly à Pé-king. — Japon.

23. Pyrus (Pyrophorum) sinensis Lindl. Bot. reg. tab. 1248; P. communis var. sinensis Lindl.; Franch. et Savat. Enum. plant. jap. 1, p. 138; Miquel Prol. jap. p. 228; P. communis L.; Bunge Enum. chin. bor. no 159 ex parte; Sinice Li-Tzé, Paì-Li, Kaoûo-tsang.

Hab. : Cultivé partout à Tien-tsin.

Ar. géog. : Chine bor. Chan-tong et Pé-tché-ly; - Mongolie chin. - Amour et Ussuri. - Japon.

Obs. D'après M. Maximowicz, le $P$. sinensis Lindl. serait spécial à l'extrême Orient, et à l'exclusion du $P$. communis et de ses variétés. L'espèce du Pé-tché-ly serait identiquement la même que le P. ussuriensis de Maxim. in Prim. flor. amur. p. 102, mais décrit postérieurement.

24. Pyrus cydonia Lin Spec. 687; O. Deb. Fl. du Tchéfou', $\mathrm{n}^{\circ}$ 62; Cydonia chinensis Bunge Einum chin. bor. $\mathrm{n}^{\circ} 162$; Sinice Mào, Mou-KồA.

Hab. : Les haies et les clôtures des jardins et des propriétés rurales.

Ar. géog. : Asie occ. et orient. - Caucase, Chine bor. et moy., Chan-tong, Pé-tché-ly à Pé-king (Bunge); - Natur. en Europe, Afriq. bor., etc.

Obs: J'ai trouvé cultivé, mais rarement autour des pagrodes, le Cratægus pinnatifida Bunge (0. Deb. Fl. du Tché-fou $n^{\circ}$ 57), Sinice Shon-ché-тzÉ, ainsi que le Pmunus spectabilis Ait. Sinice 
HAI-TANG-Irô i, dont les fruits rouges, de saveur acide et de petite dimension, sont recherchés par les enfants.

GRANATÉES D.C.

25. Punica granatum Lin. Spec. 675; var. A. mbrum D. C. Prodr. 3, p. 3; Bunge Enum. chin. bor., n 28; O. Deb. Fl. de Shang-hä̈, no 43; Sinice TsÉ-TIEN-PI, H.1N-LIÉOU-PI.

$\| a b$. : Arbuste très répandu autour des yamouns, des bonzeries, etc., et dans tous les jardins de Tien-tsin.

Ar. géog. : Afriq. bor. - Europe aust. - Asie occid. et bor. Chine bor. et cent., Kiang-son. Pé-tché-ly. - Japon,

\section{TAMARISCINÉES Desv.}

25. Tamarix chinensis Loureiro Flor. cochinch. 1, p. 228; D. C. Prodr. 3, p. 96; Bunge Enum chin. bor. no 172; Turczan. Enum. plánt. chin. bor. $\mathrm{n}^{\circ}$ 79; Siebolt et Zuccar. Fam. nat. japon. 1, p. 161 et $\mathrm{Fl}$. jap. 1, p. 132; Sinice SAN-TCHÉôU-LIÉÔU, TCHÉ-Yang, TChoû-NAN-LIÉÔU.

"Suffrutex erectus, glaber, ramis nutantibus, 1 mèt. 75 cent. » -2 mèt. elongatis; foliis minutis, imbricatis, acutis; spicis » paniculatis tenuibus; floribus rubescentibus, petalis linearibus, » erectis. - Floret junio. »

Hab. : Les bords des marécages et des salines près de Tientsin. - Sables incultes dans la boucle du Pei-hô.

Ar. géog. : Chine aust. et bor. - Konâng-tòng, Pé-tché-ly à Pé-king (Bunge). - Japon.

Obs. Cette espèce est très voisine du Tamarix articulata Pallas, par ses rameaux paraissant articulés à la base, mais elle s'en éloigne par ses épis floraux beaucoup plus allongés, formant par leur ensemble des panicules très-fournies.

CUCURBITACÉES JUSS.

27. Gucumis melo Lin. Spec. 1436; Seringe in D. C. Prodr. 3, 
p. 300 ; Bunge Emum. chin. bor. no 175; O. Deb. Fl. du Tchéfoù, no 65 ; Sinice SiEx-KónA, TiEx-KoûA.

Hab. : Cultivé en plein champ, dans tous les terrains sablonneux des rives du Pei-hồ et du canal impérial.

Ar. géog. : Asie cent., Indes-Orient. - Chine bor., Chan-tong et Pé-tché-ly. - Amour et I'ssuri. — Japon, etc.

Obs. Les melons de Tien-tsin sont loin d'aroir l'arôme et la saveur de ceux de l'Europe moyenne et australe. Ils sont en général d'une grosseur médiocre et lisses à la surface. Leur chair est blanche, d'un goût fade et comme farineux. Néanmoins il s'en fait un commerce incroyable pour l'alimentation du peuple.

28. Momordica charantia Lin. spec. 1433; Seringe in D. C. Prodr. 3, p. 311; Benth. Fl. Hong-kong. p. 12.5, Bunge Enum. chin. bor. $n^{0}$ 178; Franch. et Savat. Enum. plant. jap. 1, no667; Sinice LaI-Koûa, Kor-Kioûs.

Hab. : Les haies des jardins, et fréquemment cultivé dans les cours des habitations de la ville et des faubourgs.

Ar. géog. : Asie cent., Indes-Orient. - Chine aust. moy. et bor., Pé-tché-ly à Pé-king (Bunge). — Japon.

Obs. Le 1 . charantia, que I. Savatier a rencontré spontané au Japon, sert à former dans le nord de la Chine, des treilles élégantes pour se garantir de l'ardeur du soleil. Dès le mois de juillet, ces treilles sont parsemées de fruits rougres pendants, et de l'efiet le plus gracieux.

Plusieurs autres espèces de Cucurbitacées sont cultivées dans le Pé-tché-ly pour l'usag'e alimentaire, ou les besoins de l'économie domestique. Je citerai surtout les suivantes :

- Lagenaria vulgaris Ser.; Sinice Koû-Loû, Poû-Loù, dont on trouve de nombreuses variétés dans les jardins du Pei-ho.

- Cucumis satius Lin.; Sinice Sax-Koùa, dont le fruit est très employé pour l'alimentation,

- Cucumis citrullus Ser. (Citrullus edulis Spach.); Sinice siLoûa, Hax-Koûs, cultivé en pleins champs pour ses fruits (pastèques), dont toute la population est très friande pendant l'été.

- Cucumis flexuosus Lin.; Sinice Yùb-Koû., sisn-Koûs, espèce très curieuse par ses fruits allongés, cylindriques, sillonnés et Hexueux-recourbés.

- Cucurbitu pepo Lin.; rinice Mà-Kò̀s, T'IEs-Koùd. etc. 
CAPRIFOLIACEES JUSS.

29. Lonicera chinensis Wats, Dendrol. brit. tab. 117; Bunge Enum. chin. bor. no 195 ; O. Debx. Fl. de Shang-haï, no 52; Sinice KIN-Ŷ̂N-HôA.

Hab. : Les haies des jardins et des propriétés, parc du yamoun de Tien-Kô, etc.

Ar. géog. : Asie cent. Népaul. - Chine moy. et bor., Pé-tché-ly à Pé-king (Bunge). - Japon.

Obs. La famille si importante des Ombellifères ne produit aucune espèce qui soit spontanée dans le territoire de Tien-tsin. On cultive seulement dans cette localité, pour l'usage culinaire, la carotte (Daucus carota L.), Sinice Hông-Lô-Pôv, le persil (Petroselinum sativum Hotfm., Sin. Cha I-tsai, le cerfeuil (Anthriscus cerefolium L.), la coriandre (Coriandrum sativum L.), Sin. TzÉ-Lô etc.

\section{RUBIACÉES JusS.}

30. Rubia cordifolia Lin. Mant. p. 197; Ledeb. Fl. ross. 2, p. 405; Bunge Enum. chin. bor. no 200; Turczan. Fl. baik.dahur. 1, p. 528; 0. Debx. Fl. du Tché-foù, no 78; Sinice TienTSâo.

Hab. : Bords des champs, des chemins, les sables incultes près des forts de Tien-kô, etc.

Ar. géog. : Asie bor. et intertrop.; Sibérie transbaikal., Daoûrie, Mongolie. - Chine bor., Chan-tông (O. Deb.), Pé-tché-ly à Pé-king (Bunge). - Amour et Ussuri. - Japon. - Iles de la Sonde, Sava, etc.

\section{DIPSACÉES D. C.}

31. Scabiosa Fisheri D. C. in Prod. II, p. 658; Ledeb. Fl. ross. 2, p. 456; Turcz. Fl. baik.-dahur., 2, p. 342; liaxim. Prim. 
amur. p. 142; Régrel et de Herd. Plantix Raddeanx 2 Heft, 1, p. 44; S. comosa Rxin. et Schult.; S. dahurica Pallas.

Var. alba Régel (loc. cit.)

Plante herbacée bisannuelle; tiges de 40 à 60 centim. de hauteur, rameuses dans leur moitié supérieure, giabres ou légèrement pubescentes; feuilles pinnatipartites, les inférieures à divisions linéaires ou linéaires-oblongues, entières ou incisées pinnatifides, les supérieures linéaires très entières; capitules floraux subovales-hémisphériques; corolles plus longues que l'involucre, celui-ci égalant ou plus long que le disque; couronne de l'involucelle membraneuse, deux à trois fois plus courte que le tube; disque du calice subsessile; divisions calicinales terminées par cinq soies d'un jaune-pâle et denx fois plus longues que la couronne; fleurs d'un blanc un peu jaunâtre.

$H a b$. : Les sables incultes de la plaine du Pei-hô, près des forts de Tien-kô - Fl. en juillet.

Ar. géog. : Sibérie altaïque, Baikalie, Kiatka, Daoûrie, Mongolie. - Chine bor., Pé-tché-ly à Pé-king (Maxim. et Kirilow). - Amour et Ussuri.

COMPOSÉES Adans.

32. Callimeris altaica Nées ab Esemb. Aster. p. 228; Ledeb. Fl. ross. 2, p. 432; Turczan. Fl. baik.-dahur. 2, p. 16; Régel et de Herder Plantæ liaddeanx, 3 Heft, 2, p. 18;

Var. B. subincana Lallem. Inder semin. hort. Petropol. VIII (1841), p. 52; Calimeris canescens Nées (loc. cit.) p. 229: D. C. in Prodr. V, p. $2 ; 9$.

Planté entièrement pubescente-blanchâtre, rivace, à tiges courtes. dressées de 20 à 40 centim. de hauteur, rameuses daus leur moitié inférieure, irréğulièrement corymbiformes, et feuillées inférieurement; feuilles lancéolées-linéaires, obtuses, entières: ciliées-scabres sur les bords, incanescentes en dessous, scabreshispides en dessus; rameaux florifères allongés, uniflores, feuillés dans toute leur étendue, à feuilles étroitement linéaires-lancéolées, blanches-incanescentes sur les deux faces; écailles de l’inrolure glabriuscule, lancéolées-acuminées, à marge blanchâtre: 
réceptacle muni de soies d'un brun roussâtre, de moitié plus courtes que les ligules, celles-ci larges, membraneuses et blanchâtres.

Hab. : Bords des chemins et des champs sablonneux près des salines et des forts de Tien-kô. - Fl. en.juin.

Ar. géog. : Sibérie aitaïque sur les rives de la Sélinga (Pallas), et près de Nerchintsk, Baikalie, Daoûrie sur les sables de l'Argun, Kiatka; Mongolie. - Chine bor., Pé-tché-ly à Pé-king (Bunge, Maxim. et Kirilow).

Obs. Le $C$. altaica est une plante très polymorphe, et dont la forme subincana décrite comme espèce par Nées d'Esembeck, a été réunie avec raison au $C$. altaica (forma typica), par Ledebour et MM. Régel et de Herder.

33. Tripolium vulgare Nées ab Lisemb.; Ledeb. Flor. ross. 2, p. 477; Turczan. Fl. baik. dahur. 2, p. 16; Maxim. in Indflor. pekin. (Prim. flor. amur.), p. 472; O. Debx. Fl. du Tchéfoù, $\mathrm{n}^{\circ} 84$.

Var. salina D. C. Prodr. V, p. 253; Sinice Fô-nın.

$H a b$. : Les sables marécageux et les mares saumâtres près des salines de Tien-tsin. - Fl. août-septembre.

Ar. géog. : Europe marit. - Afriq. bor. - Asie bor., occ. et or. - Chine bor. et marit., Chan-tông, Pé-tché-ly à Pé-king, Tientsin et Tâ-koû; - Amour. - Japon.

\section{Artemisia vulgaris Lin. Spec. 1188.}

Var. mongolica Besser Dissert. de Abrot. p. 53; D. C. Prodr. 6 , p. 113; O. Debx. Fl. du Tché-foù, no 97; A. vulgaris var. temuifolia Ledeb. Fl. ross. 2, p. 535̆; Régel et de Herder Plantæx Raddeanx reisen, III, p. 75; Sinice N'GAI-Ỳे.

Hab. : Les sables marécageux près des salines de Tien-kô.

Ar. géog. : Asie bor., Sibérie altaïque, Baikalie, Daôurie, Soôngarie, Mongolie. - Chine bor., Chan-tong, Pé-tché-ly à Pé-king (Staunton), etc.

35. Cirsium segetum Bunge Encm. chin. bor. no202; 0. Debx. Fl. de Shang-haï, $n^{\circ} 35$ et du Tché-fori. no 106: Sinire YüEN-тCHÉ. T'̆-KY. 
Hab. : Les champs cultivés et les sables du Pei-hô près de Tien-tsin - Fl. juillet.

Ar. géog. : Chine bor. et moy. litt., Kiang-sô̂, Chan-tong. Pé-tché-ly à Pé-king (Bunge) et Tien-tsin (O. Deb.)

36. Scorzonera parviflora Jacq. Aust. 4, tab. 105; U. C. Prodr. 7, p. 121 ; Ledeb. Fl. altaica, 4, p. 151; 0. Deb. $1 \%$ du Tché-foù, no 110 (Teste A. Franchet).

Var. Tcheliensis O. Debx. Mss. (1861).

»Souche bisannuelle à racine pivotante, de la grosseur du " petit doigt, pourvue au collet des débris des anciennes feuilles; " tiges nombreuses, rameuses dès la base, décombantes, puis »flexueuses-dressées, de 10 à 15 centim. de hauteur, glabres, 》 uniflores (par suite de l'avortement des autres capitules), et rare» ment biflores; péricline entièrement glabre à écailles ovales, "lancéolées, un peu plus courtes que les fleurons, scarieuses»membraneuses sur les bords, les plus extérieures embrassant le » pédoncule à sa base, celıi-ci glabre, allongé, presque filiforme; »feuilles radicales lancéolées-linéaires, atténuées en pétiole. » courbées en faux, mucronulées au sommet, les caulinaires beau» coup plus courtes, demi-embrassantes; glabres sur les deux »faces. »

$H a b$. : Les sables incultes, au bord des champs et des chemins, près des saliness et des forts de Tien-kô. - Fl. du $1^{\text {er }}$ au 15 mai.

Ar. géog.: Le type : Eur. aust., France mérid., Hongrie. Asie bor., Sibérie altaïque, Soôngarie, Désert de Noryn et salines d'Ilesky (Pallas), Désert de Kirghyz - Chine bor. le Chan-tong (O. Deb.) - La variété : Chine bor., le Pé-tché-ly.

Obs. La variété Tcheliensis du S. parviflolia paraît devoir se rapprocher du S. caricifoliu Pallas, par son facies et l'ensemble de ses caractères; mais elle s'en éloigne par ses feuilles plus courtes, de 5 à 6 centim. de longueur, et non de 20 à 25 centim., par ses tiges courtes, décombantes, flexueuses, puis redressées uniflores, rarement à deux capitules et non dressées-élerées, et enfin par la glaucescence de toutes ses parties.

Le $S$. parviftora n'avait encore été rencontré dans la province de Pé-tché-ly par aucun botaniste-voyageur. J'ai déjà signalé dans les prairies du Tché-fon près de Yan-taï, la présence de la forme elatior de cette même espèce. 
37. Ixeris versicolor D. C. Prodr. VII, p. 150; Benth. Flora Hong-kong., p. 193; Franch. et Savat. Émum. plant. jap. 1, $\mathrm{n}^{\circ} 269$; Barkausia versicolor Spreng. Syst. veget. III, p. 661; Régel Tent. ussur. p. 98; Maxim. Prim. amur. p. 180; Prxnanthes versicolor Bunge Enum. chin. bor. $\mathrm{n}^{\circ}$ 227; Lactuca versicolor Benth. et Hook. Genera plant. 2, p. 526.

Plante herbacée, polymorphe, à racine perennante; tiges de 1 . à 20 centim. de hauteur, tantôt simples pauciflores, tantôt très rameuses dès la base, à cymes subdichotomes, fournies et formant un corymbe plus ou moins dense; feuilles inférieures étroites lancéolées, entières, simplement dentées ou profondément roncinées-pinnatifides, les caulinaires demi-embrassantes, obtuses, ou brièvement sagittées-auriculées; fleurs jaunes, roses ou blanches; akènes marqués de dix côtes longitudinales, aiguës sur les arêttes.

$H a b$. : Les sables sur les terres incultes des rives du Pei-hô, audessus de Tien-.tsin, en remontant la rivière vers Pé-king. - Fl. du 20 au 30 mai.

Ar. géog. : Daoûrie (Turczan.), Mongolie (Maxim.) - Chine bor., le Pé-tché-ly à Pé-king (Bunge), Chine mérid. à Hong-kông (Benth.). - Amour et Ussuri. - Japon.

Obs. Le polymorphisme des feuilles et la variation de la couleur des fleurs, ont été constatés également dans la province de l'Amour par M. Maximowicz, et au Japon par MM. Franchet et savatier.

3). Sonchus brachyotus D. C.Prodr. VII, p. 186; O. Debeaux El. de Shang-hä, $\mathrm{n}^{\circ} 66$ et du Tché-foù, $\mathrm{n}^{\circ} 117$; Maxim. Prim. amur. in Index flor. pekin. p. 474.

$H a b .:$ Les marécages saumâtres près des salines de Tien-kô, et au bord des fossés d'irrigation. - Fl. aont-septembre.

Ar. géog. : Asie bor., Sibérie altaïque, Chine bor. et moy. littor., Kiang-Soû, Chan-tong, Pé-tché-ly à Pé-king (Bunge).

39. Xanthium strumarium Lin. Spec. 1400; O. Debx. Fl. du Tché-fou, no 118; Bunge Enum. chin. bor. no 339; Maxim. Prim. amur. in Index flor. pelirr. p. 474; Franchet et Savatier Enum. plant. japon. 1, p. 231 Sinice TSAN-EûL-TZÉ.

Var. indicum; X. indicum Roxburg (al. pl. horti Colcut. 67. 
Hab. : Les sables, les champs cultivés sur les rives du Pei-hô, surtout sur les bords de la route de T'â-Koû. - Fl. août et septembre.

Ar. géog. : Asie bor., occ. et or., Indes-Orient. - Chine aust., moy. et bor., Hong-kông', Kiang-soû, Chan-tong, Pé-tché-ly à Pé-king (Bunge). - Amour. - Japon.

Obs. On cultive dans presque tous les jardins de Tien-tsin, plusieurs plantes de la famille des composées, soit comme plantes d'ornement, soit pour servir à l'alimentation.

J'ai remarqué les espèces suivantes :

- Tajetes patula L. et T. erecta L., Sinice Tsir-tsiar-hòे;

- Callistephus sinensis Cass. (Aster sinensis L.) Sinice PÉ-KIngHoâ, dont les Chinois cultivent avec passion de nombreuses et belles variétés;

- Chrysanthemum indicum L., Sinice Hoâa-Kioû-Hò.., et aussi Tâ-Kıôu-HôA, plante très recherchée pour l'ornement des habitations;

- Helianthus ammuus L., Sinice HoûEI-KoûAN-Hoâ;

- Calendula officinalis L., Sin. Kin-Tsin-Hoâ;

- Artemisia dracunculus L., Sin. Tsin-hoâ;

- Matricaria parthenium L., Sin. KaN-kiN-HOâ;

- Carthamus tinctorius L., Sin. Hoâng-LaN-Hoâ;

- Cichorium endivia L., Sin. Koû-TSAI;

- Lactuca sativa et ses variétés horticules, Sin. PaI-Kôu, et TsarTSAÏ.

\section{EBÉNACÉES Vent.}

40. Diospyros schi-tzé Bunge Enum. chin. bor. $n^{\circ}$ 237; O. Debeaux Fl. de Shang-haï, no 70 et Fl. du Tché-foù in Addenda; D. Kaki Lin. Suppl. 439; Franch. et Savat. Enum. plant. jap. 1, no $1119 ;$ D. costata et $D$. Roxburgin Carrière in Revue horticole 1870-1872.

Hab. : Les parcs et les jardins, autour des pagrorles et des temples bouddhiques.

Ar. géog. : Asie intertrop. temp. et bor.; Indes-Orient., Cochinchine. - Chine: Kiang-soù, Tché-kiang. Chan-tong, Pé-tché-ly à Pé-king, etc. - Japon. 
Obs. J'ai rencontré fréquemment dans les cultures et les jardins autour de Tien-tsin, les deux arbustes ci-après de la famille des oléacées :

$1^{\circ}$ Syringa dubia Pers. Syn. plant. 1, p. 9; D. C. Prodr. VIII, p. 282; S. chinensis Wild. Spec. 1, p. 48; Bunge Enum. chin.bor. $\mathrm{n}^{\circ} 241$, espèce „également répandue dans les parcs et les jardins de l'Europe australe, et dont l'origine n'est pas encore bien connue;

$2^{\circ}$ Forsythia suspensa Wahl Enum. hort. berol. 1, P. 39; Bunge Enum. chin. bor. no 242, Franch. et Savat. Enum. jap. 1, no 1139. Sinice Linv-koâ. Ce dernier arbuste serait importé de la Chine au Japon (Franch. et Sav.). D'après de Candolle, il serait spontané en chine, mais on ne posséderait aucune indication sur son habitat.

\section{CYRTANDRACÉES Lindl.}

41. Rehmannia glutinosa Libosch in Hort. Petropol. (1835); D. C. Prodr. IX, p. 2\%; Miquel Prol. fl. japon. p. 55; Gerardia glutinosa Bunge Enum. chin. bor. no 280; Maxim. in Mélanges biologiques IX, p. 371; Melittis japonica Thunbg. Icon. plant. japon. Decas III, tab. 8; Digitalis glutinosa Gærtn.; Sinice Tr-koûang, Sen-ti-koûang.

Plante herbacée, vivace, velue-bubescente, à racines simples, pivotantes, de la grosseur du doigt; tiges simples (16-20 cent. de hauteur), nues à la base, dressées, velues-hérissées et couvertes de longs poils laineux, portant à leur sommet quatre à six fleurs disposées en cymes subdichotomes; feuilles radicales opposées, atténuées en pétiole, longuement ovales-lancéolées, sinuées-dentées et ciliées aux bords, pubescentes en dessous, d'un vert foncé en dessus, de moitié moins longues que les tiges, les caulinaires et les supérieures étroites, à sinus beaucoup plus nombreux et plus profonds; fleurs les plus inférieures toujours pédicellées, les supérieures subsessiles, à corolle tubuleuse, velue-laineuse en dehors; tube de 3 à 4 centim. de longueur, trois fois plus long que les divisions calicinales, celles-ci ovales, lancéolées et très velues-glanduleuses en dessous, ainsi que les 
pédicelles; limbe de la corolle oblique, marqué de cinq divisions irrégulières, d'un pourpre-noir en dedans; capsule ovoïde, presque globuleuse à la maturité et surmontée par le style persistant et très allongé; graines noires, très petites, ovalesréticulées.

Hab. : Bords des sentiers, au pied des vieux murs et autour des étangs marécageux, près du yamoun de Tien-kô. - Fleur du $1^{\text {er }}$ au 15 mai.

Ar. géog. : Mongolie chin. - Chine bor. et moy., Kiang-si, Hô-nân, Pé-tché-ly à Pé-king (Bunge) et à Tien-tsin (O. Deb.). Japon.

Obs. I. Cette espèce des plus rares, et qui m'a paru être réellement spontanée dans la localité indiquée près de Tien-tsin, est très remarquable par ses fleurs semblables à celles de la digitale pourprée, mais un peu plus petites, velues-glanduleuses en dehors, et d'un pourpre noirâtre au dedans. D'après MM. Franchet et Savatier, le $R$. glutinosa serait cultivé au Japon comme plante d'ornement.

Obs. 11. On rencontre aussi fréquemment autour des pagodes, et des habitations de la campagne et des faubourgs, le Catalpa Bungei C. A. Méyer Dissert. p. 3 (1837); D. C. Prodr. IX, p. 226; O. Debx. Fl. de Shang-haï, no 74; C. syringæfolia Bunge, Sinice Kô-tsien, arbre également répandu dans la plaine autour de Pé-king. Le $C$. Bungei, qui est probablement originaire des montagnes au nord de Pé-tché-ly, forme un arbre assez élevé, à feuilles ovales-acuminées ou sinuées-sublobées, membraneuses, subglabres en dessous. Ses fleurs sont semblables pour leur forme à celles du C. Kempferi D. C., avec cette différence notable qu'elles sont pointillées de petites taches d'un pourpre vif, et non ponctuées de jaune et de pourpre. Les Chinois lui donnent le nom d'arbre fétide, à cause de la mauvaise odeur que répandent les feuilles, lorsqu'on les froisse entre les doigts.

CONVOLVULACÉES JUSS.

42. Convolvulus arvensis Lin.; Bunge Enum. chin. bor. no 260; O. Debx. Fl. du Tché-foù, no 129; Sinice PÉ-tsâx, Tsoû-TSAN. 
Hab. : Les champs sablonneux et les cultures dans la plaine du Pei-hô.

Ar. géog. : Europe. - Asie bor., occ. et or. - Afrique bor. - Amér. bor., etc.

43. Calystegia acetosellæfolia Turczan. Fl. baik.-dahur. 2, p. 289, in nota; Naxim. Prim. amur. p. 200; Régel. Tent. ussur. p. 107; Convolvulus acetosellxfolius Turczan. in Bull. Soc. nat. Mosc. (1840) p. 73, non Vahl.; Sinice Srûax-hoâ.

Var. gracilis O. Debeaux Misc. in Herb. 1861.

"Tiges grêles peu ou point volubiles, rameuses dès la base, » à rameaux couchés-étalés, puis dressés, de 15 à 20 cent. de lon» gueur; feuilles glabres, hastées-sagittées, les inférieures à limbe 》 arrondi au sommet, les caulinaires à partition médiane allongée» lancéolée, acuminée et mucronulée à son extrémité; oreillettes » larges bilobées, souvent munies d'un troisième lobule acces» soire; corolle semblable à celle du Convolvulus arvensis, à fond » blanc et à limbe et nervures carnés. "

Hab. : Les champs sablonneux, les bords des chemins et les sables incultes près des forts de Tien-kô; se retrouve aussi près du cimetière indigène dans la boucle du Pei-hô, etc. - Fl. 2530 mai .

Ar. géog. : Sibérie, Baikalie, Daoûrie, Mongolie. - Chine bor., le Pé-tché-ly à Pé-king (Turczan.), Tien-tsin (O. Deb.), etc. Amour et Ussuri.

Obs. On cultive dans une foule de jardins l'Ipomea quamoclit Lin. (Quamoclit vulgaris Choisy), Sinice Tsıex-hoâ et le Pharbitis nil Choisy, Sin. Tsien-Neôu-Lrôu.

BORRAGINÉES Desv.

44. Tournefortia arguzia Ræm. et Schult. Syst. veget. IV, p. 540; D. C. Prodr. IX, p. 512; Bunge Enum. chin. bor. no 268; O. Debx. El. du Tché-foù, no 132; Sinice Tsoû-TSAÔ. Tsé-Tan, Ti-hiûen.

Var.B. angustifolia D. C. (loc. cit.) O. Debx. (loc. cit.)

$H a b$. : Les sables incultes, sur toutes les fortifications de Tien-kô; salines de Tien-tsin, etc. - Fl. du 20 au 30 mai. 
Ar. géog. : Europe austro-orient. - Asie bor., occ. et or., s'ibérie altaïque, Daoûrie, Mongolie. Chine bor., Chan-tong (O. Deb.), Pé-tché-ly à Pé-king (Bunge). - Japon.

Obs. La forme angustifolia du $T$. arguzia à feuilles étroiteslancéolées, est la seule que l'on rencontre à Tien-tsin. Je fais aussi observer que sa floraison est d'un mois et demi plus précoce que dans le Chan-tong au Tché-fô̂, quoique cette contrée soit située beaucoup plus au sud, et dans une région relativement plus chaude.

45. Bothriospermum chinense Bunge Enum plant. chin. bor. no 266; D. C. Prodr. X. p. 116; B. bicarunculatum Fish. et Mey. Index sem. hort. Petrop. 1835. - Icon. nost. tab. II.

Plante herbacée, bisannuelle, entièrement couverte de poils blancs, sétacés; tig’es simples, ou rarement peu rameuses, dressées, de 15 à 20 centim. de hauteur; feuilles radicales longuement pétiolées, entières, oblongues-lancéolées ou ovales-oblongues (8-9 centim. de long. avec le pétiole, 14-16 millim. de larg.), les caulinaires sessiles, ovales-oblongues, obtusiuscules, toutes velues, crliées en dessous, hispides-hérissées et tuberculeuses en dessus; pédicelles dressés, très courts, velus-ciliés ainsi que les divisions extérieures du calice, celui-ci dressé-étalé plus long que les pédicelles; capsule trois fois plus grosse que les nucules; nucules petites, longues de 2 à 3 mill., d'un blanc pâle, finement granuleuses à leur surface, et ridées près de l'orifice central; fleurs d'un beau bleu, rarement blanches, dépassant à peine le calice.

$H a b$. : Les sables incultes, au bord des champs et des chemins, près des salines et des forts de Tien-kô - Fl. 10-20 mai.

Ar. géog. : Chine bor., Pé-tché-ly à Pé-king (Bunge), Tien-tsin (O. Deb.).

Obs. Depuis l'époque où fut publiée par Bunge son Emumeratio plantarum chinx borealis, le Bothriospermum chinense n'avait pas été observé en Chine ailleurs qu'à Pé-king', J'ai été assez heureux pour retrouver cette rare espèce à Tien-tsin, et de pouvoir la faire connaître par un dessin très exact, que je dois à l'obligreance de M. le docteur Bucquoy. Le $B$. chinense se distingue des autres espèces de ce grenre, par ses nucules orales transversalement, granuleuses-rugueuses à leur surface et caré- 
nées sur le dos. Elles sont munies vers la base d'un petit orifice rugueux et marquées de chaque côté d'une dépression demi-circulaire qui contourne l'ombilic pour ainsi dire.

46. Eritrychium pedunculare Alph. D. C. Prodr. X, p. 128; O. Deb. Fl. de Shang-haï no 78; Franch, et Savat. Enum. plant. japon. 1, p. 335; Myosotis peduncularis Bunge Enum. chin. bor. $\mathrm{n}^{\circ} 26.5$ ex parte; M. chinensis D. C. Prodr. (loc. cit.) ex Miquel in Prolusio fl. japon. p. 28, Teste Maximowicz.

$H a b$. : Les bords des marécages et des fossés d'irrigation, près du yamoun et des forts de Tien-kô, dans la plaine du Pei-hô. Fl. et fr. du 15 au 30 mai.

Ar. géog. : Asie bor., Daoûrie, Mongolie, Astrakan; - Chine bor. et moy., Kiang-soû, Pé-tché-ly à Pé-king (Bunge.) - Amour. - Japon.

Obs. L'E. pedunculare est une plante polymorphe, à tiges tantôt dressées et tantôt étalées rampantes sur le sol. Les feuilles varient également et sont ovales-lancéolées longuement atténuées en pétiole, on encore largement ovales-oblongues et brusquement rétrécies en un long pétiole. Les spécimens provenant de Tien-tsin ne diffèrent en rien, de ceux que j'avais antérieurement récoltés à Shang-haï et à Woô-sông en juin 1860.

SOLANÉES JUSS.

47. Lycium sinense Mill. Dict. no 5 ; Bunge Enum. chin. bor. no $276 ; 0$. Deb. Fl. de Shang-haï, no 70 et Fl. du Tché-foui, no 135 ; L. Barbarum var. sinense Ait.; Sinice Koû-KI-тZÉ, TI-Kô̂-TZÉ.

Hab. : Les haies des jardins, au pied des vieux murs, dans les faubourgs de Tien-tsin; parc du yamoun de Tien-kô, etc. .

Ar. géog. : Chine aust., moy. et bor. Kiang-sô, Chan-tông, Pé-tché-ly à Pé-king (Bunge). - Natur. dans l'Europe australe, Pyrén. Crient. à Perpignan (O. Deb.). - Japon.

48. Solanum nigrum Lin. Spec. 266; Turczan. Fl. baik.-cletuı'. 2, p. 324; Bunge Enum. chin. bor. no 271; Naxim. Prim. fl. 
umur. p. 204; Régel. Tent. fl. ussur. p. 109; Franch. et Savat. Enum. jup. 1, no 1129; Sinice Trex-хâo-TSâo, LôxgKoûEI.

Hab. : Les terres incultes au pied des murailles de Tien-tsin, et cultures des rives du Pei-hô.

Ar. géog. : Europe. - Asie bor., occ. et or.; Sibérie altaïque, Daoûrie, Baikalie; - Chine bor., Pé-tché-ly à Pé-king. - Amour et Ussuri, - Japon. - Amér. bor. et aust. - Iles Canaries. Afriq. bor. : Arabie, Algérie, Egypte, etc.

49. Physalis alkekengi Lin. Spec. 262; D. C. Prodr. XIII, p, 438; Bunge Enum. chin. bor. no 275; Miquel Prol. fl. japon. p. 234; Franch. et Sarat. Enum. pl. jap. 1, p. 340; Sinice Tsoâng-tsiang, Koû-Niang.

Hab. : Les cultures dans la boucle du Pei-hô, autour de la pagode de cette localité.

Ar. géog. : Eur. moy. et aust. - Asie occ., Caucase; - Chine (Herb. Dec.) sans indication d'habitat; Pé-tché-ly à Pé-king (Bunge), Tien-tsin (O. Deb.). - Japon. - Amér. bor.

Obs. La plante du Pei-hô est tout à fait identique à mes échantillons provenant du midi de la France, Agen, Toulouse, etc. Son habitat, doublement fconstaté dans le Pé-tché-ly par Bunge et par moi-même, constitue un fait de géographie botanique des plus intéressants.

Plusieurs autres espèces de la famille des solanées, sont cultivées à Tien-tsin pour l'usage alimentaire,ou les besoins de l'économie domestique. Je ne signalerai ici que les plus importantes :

- Solanum tuberosum L. Sin. Hô-LAN-choû, ShaN-Yé-Choû, plante introduite en Chine, et cultivée seulement autour des villes ouvertes au commerce étranger.

- Capsicum annuum L. Sin. La-tsiaÒ, TÔ-Hô-TSIâ̂;

- Capsicum longum D. C. Sin. Tsin-tsaô;

- Lycopersicum esculentum Mill. Sin. Fìv-Krâ;

- Melongena esculenta Tourn. Sin. Kin-tzé, Nix-tsin-kiâ;

- Nicotiana rustica L., Sin. Yèn-tsâo;

- Nicotiana tabacum L., Sin. YèN-Ỳ̀, GrN-Tsaô, et par corruption aujourd'hui TAN-Pấ-Kò. 
SCROPHULARIÉES R. Brown.

50. Mazus rugosus Loureiro Fl. coch. 1, p. 385; Maxim. Ind. fl. pekin. in Prim. fl. amur. p. 475; 0. Deb. Fl. de Shang-hai, $\mathrm{n}^{\circ} 80$ et $\mathrm{Fl}$. de Tché-foú, $\mathrm{n}^{\circ}$ 138; Lindernia japonica Thunbg.

Hab. : Les champs sablonneux des rives du Pei-hö; sables marécageux près des salines de Tien-kô, etc. - Fl. aoûtseptembre.

Ar. géog. : Asie subtrop., cent. et bor., Indes-Orient., Cochinch., Malaisie. - Chine littor., cent. et bor., Kiang-soû, Chan-tong, ?é-tché-ly à Pé-king (Maxim.). - Japon. - Amour et Ussuri.

\section{LABIÉES JusS.}

51. Marrubium incisum Bentham Labiat. gener. et spec p. 586, et in Prodr. XII, p. 447; Leomumes supinus Wild. Spec. plant. III, p. 116; Bunge Enum. chin. bor., no 286.

Plante herbacée, vivace, velue-pubescente, à tiges couchées ou flexueuses-dressées, de 15 ì 20 centimèt. de longueur; feuilles suborbiculaires, vertes en dessous, beaucoup plus pâles en dessous, palmées-lobées à lobes profondément incisés; verticilles floraux de six à dix fleurs; bractées sétacées; divisions calicinales glabres, au nombre de cinq, à peine tomenteuses et terminées par un acumen sétacé; corolle d'un blanc pâle, velue extérieurement, du double plus longue que le calice, à lèvre supérieure oblongue, très entière.

$H a b$. : Les sables incultes au bord des sentiers, des fossés, etc., entre les salines et les forts de Tien-kô, sur la rive gauche du Pei-hô. - Fl. 1er au 10 mai

Ar. géog. : Sibérie orient., Daon̂rie, Mongolie - Chine bor., Pé-tché-ly à Pé-king"(Bunge), Tien-tsin (O. Deb.).

52. Leonurus [sibiricus Lin. Spec. 818; Benth. in D. C. Prodr. XII, p. 501; Maxim. Index fl. pekin. (loc. cit.), p. 47i; 
O. Debx. Fl. de Shang-hai, no 87 et Fl. du Tché-foù, n 150 ;

Franch et Savat. Enum pl. japon. 1, p. 380; L. altissimus Bunge Nov. gen. et spec. chin. bor. ined.; Sinice Ké-KoûEr, YA-Moû-N'GAI, 'TCHôNG-KoûEI-TCHÉ.

Hab. : Les fossés d'irrigations dans la plaine sablonneuse du Pei-hô. - Fl. juillet et août.

Ar. géog. : Asie, Afrique et Amér. subtrop. - Mongolie. Chine bor., moy. et aust.; Pé-tché-ly à Pé-king, Chan-tong, Kiang-soû, etc. - Japon. - Iles Philipp. - Brésil. - Iles SaintThomas et de la Trinité, Jamaïque, Cuba, etc.

Obs. : Quelques espèces de la famille des labiées, sont cultivées dans les jardins de Tien-tsin. Les plus répandues sont les suivantes : la menthe poivrée (Mentha piperita L.), Sinice Pô-нô et PÉ-Tché, le basilic commun (Ocymum basilicum L.), Sin. Hrâng-HôA-tzé, l'Esholtzia cristata L., Sin. HrânG-Jông, etc.

\section{VERBĖNACÉES JusS.}

53. Vitex incisa Lamark Dict. enc. 2, p. 605; Bunge Enum. bor. $\mathrm{n}^{\circ} 295$; O. Deb. Fl. du Tché-foû, $\mathrm{n}^{\circ} 154 ; V$. negundo Lin. ex Bentham; Sinice Man-king, Hoûang-kin, Tsin-tsâo.

Hab. : Cà et là autour des tumulus dans la plaine du Pei-hô; yamoun de Tien-kô. - Fl. août.

Ar. géog. :Mongolie. - Chine bor., cent. et aust., Hôngkông, Chan-tong, Pé-tché-ly à Pé-king (Bunge), etc.

Obs. Le Clerodendron frtidum, Bunge Enum. plant. chin. bor. $\mathrm{n}^{0} 229$, élégant sous-arbrisseau à fleurs odorantes d'un pourpre vif et disposées en un corymbe terminal serré, se rencontre fréquemment dans les jardins de Tien-tsin et autour des pagodes.

On trouve également cultivé partout, comme plante d'ornement, le Mirabilis dichotoma L., Bunge Enum. chin. bor. no 306, Sinice YEN-TCHÉ-Hoâ, Lô-KoûEI, à fleur's roses, purpurines, blanches, jaunes ou panachées et très odorantes le soir.

PLANTAGINÉES Juss,

54. Plantago major Lin. Spec. 163; Bunge Enum chen. bor. no 304; 0. Deb. Fl. de Shang-haï, no 93; Sinice LiÉoll-kia, TCHÉ-TSIEN. 
Hab. : Les cultures, les fossés d'irrigation dans la plaine du Pei-hô etc.

Ar. géog. : Europe. - Afriq. bor. - Asie bor., occid. et orient. - Chine bor., cent. et aust.; Pé-tché-ly à Pé-king (Bunge). Amour et Ussuri. - Japon.

\section{AMARAN'THACÉES R. Brown.}

55. Amaranthus blitum Lin. Spec. 140.5; Moq. in D. C. Prodr. XIII, 2, p. 263; Bunge Enum. chin. bor. $\mathrm{n}^{0} 307$; O. Deb. Fl. du Tché-foù, no 170.

Var. sylvestris Moq. (loc. cit.); A. sylvestris Desfont.

Hab. : Les champs sablonneux, les cultures au bord des fossés, etc., autour de Tien-tsin.

Ar. géog. : Eur. - Afr. bor. - Asie bor., occ. et cent. - Chine bor., Chan-tong, Pé-tché-ly à Pé-king (Bunge), etc.

Obs. Plusieurs espèces d'Amaranthus sont généralement cultivées dans les jardins de Tien-tsin, d'où elles s'échappent parfois pour végeter dans les terrans vagues ou sur les sables des rives du Pei-hô. J'ai remarqué par leur fréquence dans les cultures les A. caudatus L., Sin. MA-TCHà-Ŷ̂N; A. paniculatus Moq. var. sanguineus (A. sanguineus L.), Sin. Tian-Min-sin; les Celosia cristata et $C$. argentea L., Sin. KI-KoûAng-Hoâ; le Gomphrena globosa L., Sin. Tsin-Tsièn-TZÉ, etc.

\section{CHÉNOPODÉES Vent.}

56. Chenopodina maritima Moq. in D. C. Prodr. XIII, 2 p. 161, var. A. vulyaris Moq.; O. Debx. Fl. de Shang-huï, $\mathrm{n}^{\circ} 97$ et $\mathrm{Fl}$. clu Tché foù, $\mathrm{n}^{\circ}$ 164; Bunge Enum. chin. bor. $\mathrm{n}^{0} 311$.

Hab. : Les marécages saumâtres et les fossés aquatiques près des salines de Tien-kô. - Août et septembre.

Ar. géog. : Eur. marit. - Asie bor., occ. et or. - Chine moy. littor. et bor. Kiang-soû, Chan-tong, Pé-tché-ly à Pé-king, etc. -Afriq. bor. - Japon. 
5\%. Chenopodium album Lin. Sipec. 119; Moq. in 1). C. Prodr. XIII, p. r0; Bunge Enum. plant. chin. hor. no 313; O. Deb, $\mathrm{Fl}$. de Shanr-ílai, no 98.

Var. A. commune Moq. (loc. cit.); Sinice Hrex-tsaï, Tchú-yèx. Hab. : Les champs sablonneux et les cultures diverses dans la plaine du Pei-hô. - Juin-juillet.

Ar. géog. : Régions intertrop. des deux mondes. - Asie bor., occ. et or. - Chine bor., moy. et aust, Hông-kông, Kiang-soû, Pé-tché-ly à Pé-king. - Japon. - Afriq. bor., etc.

Obs. I.e Spinacia sleracea L., Sinice Pé-Lixg, Pí-tsaï et le Beta vulgaris var. rapacea Koch (Bette-rave), Sinice HôxG-Lô-Pôc, sont fréquemment cultivés pour l'usage alimentaire.

\section{POLYGONÉES JUSS.}

58. Polygonum aviculare Lin. Spec. 519; Bunge Enum. chin. bor. no 32́ं ; O. Deb. Fl. de Shang-haï, no 99 et Fl. du Tché-foù, no 177; Sinice 'Tché-choûI-Tsâo.

Hab. : Les champs, les cultures et les sables humides, dans la plaine du Pei-hô.

Ar.géog. : Eur. - Asie bor., occ. et or. - Mongolie. - Chine bor., moy., Kiang-soù, Chân-tông, Pé-tché-ly à Pé-king, etc. Afriq. bor. - Amér. bor. - Japon.

Obs. Les Polygonum barbatum L., Sin. Mâo-Liıò, et P. orientale L., Sin. Tâ-TSIN-Yê, se trouvent dans tous les jardins, et souvent subspontanés.

59. Fagopyrum esculentum Mœnch. Meth. 290; Meissn. in D. C. Prodr. XIV, p. 143; O. Deb. Fl. du Tché-jou, no 174; Maxim. in Ind. fl.pekin. (Prim. fl. amur.) p. 476 ; Sinice KrıôMEI, Nin-Fô-TZḱ.

Hab.: Cultivé en grand dans les terres sablonneuses de la plaine de Pei-hô.

Ar. géog. : Orig. de l'Asie cent. — sibérie altaique, Mongolie. - Chine bor., Pé-tché-ly à Pé-king (Maxim.), Chan-tông (O.Deb.) Japon. - Cult. en Eur.,•Amér. bor., etc. 
EUPHORBIA CÉES JUSS.

60. Euphorbia humifusa Wild. Enum. hort. berol. Suppl. p. 13; Bunge Enum. chin. bor. no 329; O. Deb. Fl. de Shanghaï, no 102 et Fl. due Tché-fou, $n^{\circ} 17 j ; E$. pseudo-chamxsyce Fish. et Mey.; Sinice Tâ-ky, Ti-kin.

Hab. : Les sables dans la boucle du Pei-hô, et autour des salines de Tien-kô. - Juillet.

Ar. yéor. : Asie bor., Sibérie altaïque, Baikal, Oural, Mongolie; - Chine bor., moy., litt. et aust. Kiang-soû, Chan-tong, Pé-tché-ly à Pé-king (Bunge), etc. - Corée. - Japon.

61. Ricinus communis Lin. Spec. 1430; Mull. in D. C. Prodr. XVI, p. 1016; Bunge Enum. chin. bor. no 323; O. Deb. Fl. de Shang-haï, n ${ }^{\circ}$ 104; Franch. et Savat. Emum. plant. japon. 1, p. 424; Sinice PI-Mâ, T'IEN-Mâ, Tâ-Mâ-TzÉ.

Var. inermis Muller (loc. cit.).

$M a b$. : Fréquent sur les sables d'alluvion dans la boucle du Pei-hô, au-dessus de Tien-tsin; cultivé dans une foule de jardins.

Ar. géog.: Régions intertrop. des deux mondes. - Chine aust., moy. et bor., Hòng-kông, Koûan-tông, Tché-kiangr, Kiang-soû, Pé-tché-ly à Pé-king (Bunge), etc. - Japon . - Afriq. bor.

CANNABINÉES L. Mey.

62. Cannabis sativa Lin. Spec. 1457; D :C. Prodr. XVI, p. 30; Bnnge Eimum. chin. bor., no 338; O. Deb. Fl. de Shung-haï, $\mathrm{n}^{\circ}$ 106, et $\mathrm{Fl}$. de Tché-foù, no 184; C. chinensis Delile.

Var. A. vulgaris D. C. (loc. cit.); Sinice Mâ, Tâ-»râ, Hô-Mà. Tsoû-TSAÒ.

Hab. : Cultivé en pleins champs dans la plaine de 'Tien-tsin, et souvent subspont. sur les sables du Pei-hô.

Ar. géog. : Asie cent. et bor., Sibérie alt. et baikal., Soôngarie, Désert de Kirghyz, Iongolie. - Chine bor., moy. et aust. - Pé-tché-ly à Pé-king, etc. - Japon. - Afriq. bor. 


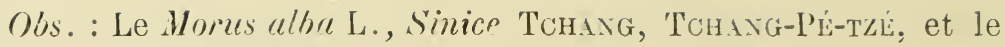
Broussonetia papyrifera Tent., Sinice Tchoû, PI-kioû, se rencontrent rarement autour des habitations et des temples bouddhiques.

\section{JUGLANDÉES D. C.}

63. Juglans regia Lin. Spec. 1485; D. C. in Prodr. XVI, 1, p. 135; Bunge Enum. chin. bor. no 350 .

Var. sinensis D. C. in Ann. scienc. natur. Série 1V, vol. XVIII, p. 33 et in Prudr. (loc. cit.) p. 137; Franch. et Savat. Enum. japon. 1, p. 455; Sinice Tché-Tâo, Hoû-Tâ̂, Tâ-chang-Hic.

Hab. : Arbre répandu partout autour de Tien-tsin, dans les haies, au bord des champs, les jardins, etc.

Ar. géog. : Asie bor., occ. et or., Transcauc., Géorgie., Asie Mineure, Arménie, Beloutchistan, Indes-Orient., Birmanie. Chine bor., Pé-tché-ly à Pé-king (Bunge) et Tien-tsin (O. Deb.). - Japon, Amour. - Afriq. bor., Algérie (Cosson). - Amér. austr. Chili. - Natur. dans toute l'Europe cent. et aust.

Obs. Les fruits verts de la variété sinensis, la seule qui soit cultivée dans le nord de la Chine sont petits, subovales et marqués de deux côtes bien apparentes. La coque intérieure (noix) est fort dure, réticulée-striée à sa surface externe.

\section{CUPULIFÈRES Rich.}

64. Quercus obovata Bunge Enum. chin. bor. no 348; D. C. in Prodr. XXI, 1, p. 13; Qu. dentata Thunbg. ex Miquel Ann. mus. Lugdun.-batav. 1, p. 105; Sinice Tchoû-sHû, Hon̂.

"Arbor mediæ magnitudinis, ramis erectis-patulis, junioribus »subtomentosis; foliis obovatis, subsessilibus, grosse sinuatis, »lobis rotundatis, integerrimis, supra scabriusculis, subtus »tomentosis; fructibus (glandibus) agrgregatis, sessilibus termi》nalibusque; cupulæ squamis externis linearibus, acutis, glan»dem subgløbosam superantibus (Bunge). 
I/nh. : Ca et là danis la plaine du Pei-hô, autour des tumulus et des pagodes; parcs des yamouns de Tien-kô.

Ar. géog. : Chine bor., Pé-tché-ly à Pé-king; Mongolie, Mandchourie mérid. à Talienwan (Caruthers).

65. Castanea vulgaris Lamck. Dict. 1, p. 708; D. C., Prodr. XV1, 1, p. 11/4; O. Deb. Fl. du Tché-foù, n० 187; Castanea vesca Gœrtn. Fruct. tab. 3; Bunge Enum. chin. bor. no 349;

C. Bungeana Blume Ann. mus. Lugdun.-batav. 1, p. 245;

Sinice PAN-Li-Li, Lien-tZÉ.

Hab. : Les cultures dans toute la plaine du Pei-hô.

Ar. géog. : Europe mérid. - Asie bor., occ. et or. - Chine bor., Chan-tong (O. Deb.), Pé-tché-ly à Pé-king (Bunge). Japon. - Natur. dans l'Amér. bor.

\section{SALICINÉES' Rich.}

6). Salix babylonica Lin. Spec. 1473; Bunge Enum. chin. bor. n 34 ;; O. Deb. Fl. de Shang.hä̈, no 110 ; Sinice LrâoTrào, LiÉoù-pê.

Hal. : Abondant autour de Tien-tsin, les jardins, les sables du Pei-hô, etc.

Ar.géog. : Asie or. et bor. - Chine moy. et bor. Kiang-soû, Tché-kiang, Pé-tché-ly à Pé-king (Bunge) et Tien-tsin (O.Deb.). Natur. dans tnute l'Europe.

67. Salix amygdalina Lin. Spec. 1443; Ledeb. Fl. ross. III, p. 598; Turczan. Fl. baik.-dahur. III, p. 99; Trautv. in Maxim. Prim. amur. p. 241; Régrel Tent. ussur. p. 131; Sinice Yang-Lí́on, Mon-Yang, PÉ-Yang;

Forma subdiscolor O. Debeaux Msc. in Herb. (1861).

"Suffrutex erectus, 1 mèt. 75 cent. -2 mèt. et ultra altus, "parce ramosus, ramis erectis; foliis superioribus anguste lan》 ceolatis, subabrupte acuminatis, argute serratis, seu in eorlem » arbusculo integerrimis, supra intense viridibus, subtus glau» cescentibus, eis ramorum inferiorum sat parvis, ovato-lanceo» latis, discoloribusque. Flores fructusque non vidi. » 
$\| a b$. : Les rives du P’ei-hô et aux bords des fossés d'irrigation. - Capsules fructifères ouvertes le $1^{\text {er }}$ mai.

Ar. géog.: Eur. - Asie bor. et occ. Régions caspiennes, Caucase, Sibérie altaïque, Baikalie. - Chine bor., Pé-tché-ly. Amour.

Obs. La forme subdiscolor du nord de la Chine, ne diffère de la forme typique de l'Europe australe, que par les feuilles de l'extrémité des rameaux lancéolées-entières, ou flnement dentées, glabres en dessus, un peu pubescentes en dessous, celles du milieu des rameaux beaucoup plus petites, ovales-lancéolées, glabres en dessus, blanchâtres en dessous. Le port et le facies sont d'ailleurs semblables à ceux de la variété discolor du $\mathrm{b}$. amygdalina du midi de l'Europe, quoique ses dimensions soient beaucoup plus réduites.

\section{CONIFÈRES Jus's.}

68. Pinus Thunbergii Parlatore in D. C. Prodr. XVI, p. 388; Franch. et Savat. Enum. plant. japon. 1, p. 464; P. Massonianu Sieb. et Zucc. Fl. jup. 2, p. 24; Bunge Enum. chin. bor. $\mathrm{n}^{\circ} 354$, ex parte, non Lambert; P. syleestris Thunbg. Fl. jap. p. 274, non Lin; Sinice Sông, Tâ-sora.

Ilab. : La plaine du Pei-hô, autour des pagodes, des tumulus, etc. Les jardins du yamoun à Tien-kô.

Ar. géog. : Chine bor., le Pé-tché-ly à Pé-king (Bunge). Japon.

Obs. Cet arbre dont le port est très élevé, offre de loin l'aspect du Pinus sylvestris de nos contrées. On remarque quelques individus de cette espèce groupés ça et là, au-dessous de Tien-tsin, sur la rive droite du Pei-hô. Il est très probable qu'ils ont été importés du nord du Pé-tché-ly, dont le $\boldsymbol{P}$. Thunbergii est originaire.

69. Biotia orientalis Endlich. Syn. conif. p. 47; Bunge Enum. chin. bor. no 3i2; 0. Deb. Fl. de Shang-hai, no 114 et Fl. du Tché-foui, no 192; Franch. et Savat. Enum. plant. jap. 1, no 1677; Sinice Pí-sôxG, PÉ-choû.

Hab. : Même localités que le précédent, mais beaucoup plus répandu. 
Ar. géog. : Chine bor. et cent., Kiang-son, Tché-kiang, Chantong, Pé-tché-ly à Pé-king (Bunge). - Japon.

70. Juniperus chinensis Lin. Mant. p. 127; Parlat. in D. C. Prodr. XVI, p. 487; Bunge Enum. chin. bor. no 353; Sieb. et Zucc. Fl. japon. II, p. 58; Franch. et Savat. Enum. jap. 1, p. 472; J. barbadensis Thunbg. Fl. jap. p. 2:1; J. Thunbergii Hoock. et Arn. Voy. cap. Beckey, p. 271; Sinice JoûYânG, JonG-KtANG-SÔxG.

Hab. : Dans tous les jardins des temples bouddhiques à Tientsin, et dans la plaine du Pei-ho, autour des habitations, des yamouns, etc.

Ar. géog. : Asie cent. et bor., Himalaya, Kamon, Thibet, Indes-Orient., Cachemyr, Népaul, Boutan, etc. - Mongolie, Chine aust. et bor., Hong-kông, Koûang-tong; Pé-tché-ly à Pé-king (Bunge), etc. — Corée. - Japon.

71. Gingko biloba Lin. Mant. 2 p. 313; Parlat. in D. C. Prodr. YVI, p. 506; Kæmpfer Amæn. exot. p. 811; Thunbg. Fl. jap., p. 358; Franch. et Savat. Enum. jup. 1, p. 474; O. Deb. Fl. Shang-haï, n ${ }^{0}$ 117; Salisburia adianthifolia Smith; Bunge Enum. chin. bor. no 3íl; Sinice PÉ-Kovô, Grn-Kô, Lâ-Kô, YûNG-HâNG.

$/ / a b$. : Mèmes localités, et en société avec les arbres conifères qui précèdent.

Ar. géog. : Chin. bor. et moy., 'Tché-kiang, Kiang-soù, Pétché-ly à Pé-king, etc. - Japon.

IRIDÉES Juss.

72. Iris uniflora Pallas in Wild. Herb. no 995; Ledeb. Fl. ross. IV, p. 94 Maxim. Prim. fl. amur. p. 270; Tucrzan. Fl. baik.-tahur, p. 194; Régel Tent. /l. ussur. p. 147; sinice Mâ-Lâx-Hoâ.

"Planta humilis, crespitosa, glabra, caulibus erectis, sim》plicibus, unifloris, basi fibroso-tunicatis, ' 8 -10 centimèt. altis, 》gracilibus; foliis angustis, lineari-lanceolatis, acutis, scapo »duplo longrioribus, striato--sulcatis, ad marginem scariosis, » subfalcatis, strictis; sjathis duobus subscariosis, corollae tubo » paulo minoribus vel æqualibus; petalis imberbibus, cæruleo- 
"violaceis longe unguiculatis, limbo integro plano, ovali»oblongo; antheris dimidium corollæ fere aquantibus. Flore ${ }^{t}$ » ineunte maio. »

Hab. : Les sables secs au bord des champs et des chemins, près du fort détaché à l'entrée de la boucle du Pei-hô.

Ar. géog. : Asie bor. et or., Sibérie, altaïque, Daoûrie. Chine bor. Pé-tché-ly (O. De').). - Amour et Ussuri.

Obs. L'Iris uniflora du nord de la Chine se rapporte trèsexactement à la description et à la figure de Pallas. Cette espèce offre quelques ressemblances arec l'I. humilis M. Bieb. plante spéciale à la région caucasique, mais elle s'en éloigne par son port plus élevé, ses feuilles plus larges, marquées de stries profondes et plus nombreuses, par sa spathe beaucoup plus longue que le tube et non plus courte que lui, par ses pétales à limbé entier, plane, et non frangé-plissé et obscurément dentelé.

L'I. uniflora n'avait pas encore été signalé sur le territoire clinois, et sa présence dans le Pé-tché-ly, ne fait que démontrer une fois de plus, les affinités botaniques de cette province arec la Sibérie altaïque et la Daoûrie.

On cultive :omme plante d'ornement dans les jardins de Tientsin et de Pé-king, le Pardanthus chincnsis Kern. (P. dichotomus Ledeb.), Sinice Chu-kian-hôA, dont jai déjà signalé l'habitat dans les basses montagnes du Tché-fôt. La patate douce (Diascorea sativa L.) est aussi cultivée pour ses racines alimentaires, dans les jardins de la! plaine du Pei-hô.

\section{LILIACÉES D. C.}

73. Allium Bouddhæ O. Debeaux in Flor. du Tché-fon, $\mathrm{n}^{\circ} 202$ : Sinice Kiä̈-pÉ.

$H a b$. : Cultivé en grand dans tout le territoire du Tien-tsin, et usité comme condiment dans la cuisine chinoise. - Fl. ió30 août.

Ar. géog. : Chine bor., Chan-tông et Pé-tché-ly.

Obs. Cette espèce, dont je ne connais pas encore l'orişine et que je crois être spéciale à la région littorale du Chan-tòng, est l'objet d'une culture considérable pour les besoins de l'alimen- 
tation. On cultive aussi dans les champs sablonneux diverses variétés de l'oignon commun (Allium cepa L.), Sin. Thoûna; le porreau (A. porrum, L.) Sin. Kini; l'ail cultivé (A. salicum, L.), Sin. Ta-tsoung, etc.

CYPÉRACÉES JUSS.

74. Gyperus (Eucyperus) Iria Lin. Spec. 67; Steud. Syn. glumac. 2, p. 23; O. Deb. Fl. du Tché-foù, no 213; Franch. et Sav. Enum. jap. II, p. 103. G. Staunton Plantæ Tchelienses exsicc. (1793).

Hab. : Les sables humides ou marécageux du Pei-hô, près des forts de Tien-kô; littoral de Tâ-kôu. - Juillet.

Ar. géog. : Asie intertrop. et temp. - Chine litt. bor. moy. et aust., Hong-kong, Chan-tong, Pé-tché-ly. — Iles Philipp. Japon.

75. Gyperus (Eucyperus) rotundus Lin. Syst. 98; Steud. Syn. glum. 2, p. 32, ex parte; O. Deb. Fl. du Tché-foû, $n^{\circ}$ 214; Franch. et Savat. Enum. II, p, 106; C. tuberosus, Walr.; G. Staunt. Plant. Tchel. exsic.; Sinice Tsân-Lin, HroTsâô, Kin-tsan-Lin.

$H a b$. : Les sables humides ou marécageux des rives du Pei-hô. Abondant sur le littoral à Tâ-koû.

Ar. géog. : Régions intertrop. et temp. des deux mondes. Asie subtrop. - Chine aust. moy. et bor., Hong-kong, TchéKiang, Kiang-Soù, Chan-tong, Pé-tché-ly. - Japon. - Afriq. subtrop. - Amér. cent. et aust.

76. Carex Bungeana O. Debeaux Mss. (1861); C. Heterostachya Bunge Enum. chin. bor. $\mathrm{n}^{0} 387$, non Torrey nec Desvaux; Sinice Tché-тsaô.

"Planta humilis, perennis, cæspitosa, culmis gracilibus, 》filiformibus, folio brevioribus vel subæqualibus, triquetris, "nudis, basi foliatis, superne scabris, 20-25 centim. altis; foliis » linearibus, glaucis, vix scabridis; spica mascula terminali, »elongata, solitaria; spicis femineis inferioribus binis, vel 
》ternis, approximatis, sessilibus, culmo adpressis; bracteis 》 amplexicaulibus, spica longioribus; squamis masculis ovatis, " acuminatis, glabris, femineis latis, ovatis, navicularibus, "apice mucronatis, margineque scariosis, akenia subrquan"tibus; akeniis (utriculis) fusco-brunneis, oblongis, utrinque » attenuatis, obscure triquetris, apice scabriusculis, bicuspidatis; » stigmatibus tribus.

IIab. : Les sables secs et incultes, près du fort isolé dans la boucle du Pei-hô, où il forme un gazon bas et serré. Fl.15-20 mai.

Ar. géog. : Chine bor., le Pé-tché-ly à Pé-king (Bunge), Tien$\operatorname{tsin}(O$. Deb.)

Obs. Il m'a paru absolument nécessaire de changer le nom primitif donné par Bunge à cette plante, par la raison majeure qu'il existait déjà deux espèces différentes du g’enre C'arex de l'Amérique boréale, qui portent la même dénomination de C. heterostachya, et qui n'ont aucune affinité avec celle du Pei-hô. Le C. Bungeana, délié naturellement à l'auteur de sa découverte autour de Pé-king en 182:, rappelle, par son port, les $C$. hirta et $C$. ericetorum de nos contrées. L'espèce chinoise est parfaitement caractérisée par ses écailles persistantes, creusées et longuement mucronulées qui entourent les utricules à la maturité, et par la forme remarquable de celles-ci. Les utricules, dans notre $C$. Bungeana, sont en effet, obiongues, atténciées aux deux extrémités, obscurément triquêtres, acuminées et bicus pidées au sommet.

\section{GRAMINÉES' Juss.}

77. Zea mays Lin. Spec. 1378; Steud. Syn. glum., 1, p. 9; Maxim. Prim. amur. p. 326; Régel Tent. ussur. p. 1i1; Miquel Prol. jap., p. 161 ; Snice Kô-Lrâx, Yû̀-Mi, Pàô-Kô.

Mab. : Cultivé dans tous les champs sablon. de la plaine du Pei-hô et des rives du canal impérial.

Ar. géog. : Origin. du Paraguay, d’où il a été importé en Europe, en Asie, en Chine, au Japon, dans l'Afrique bor. et cent., etc.

78. Oplismenus crus-galli Kunth Enum. plant. 1, p. 143; O. Deb. Fl. clu Tché-foù, no 228; Miquel Prol. jap. p 162; 
Panicum crus-galli Lin.; Benth Fl. Hong-kong. p. 41l; Bunge Enum. Chin. bor., no 397; Franch. et Savat. Enum. jap. 11. p. 160; Sinice Pai, Chovei-Pai.

Hab. : Les terres sablonneuses humides ou marécageuses des rives du Pei-hô; forts de Tien-kô. Fruct. septembre.

Ar. géog. : Europe, Afrique bor., Asie bor. et or. - Chine bor. et aust., Hong-kông, Chan-tong, Pé-tché-ly. - Japon. - Cochinchine. - Java. - Amér. bor.

Obs. Cette espèce, qui croît à l'état spontané dans quelques provinces de la Chine, serait également cultivée dans le Pé-tchély (Teste Macartney), pour servir à la nourriture des animaux domestiques. Les habitants de cette contrée auraient l'habitude de semer l'O. crus-galli en sillons, au milieu des rangs du Zea mays, afin que cette plante soit abritée, par ce dernier, des rayons du soleil pendant l'été. Il ne resterait plus ensuite qu'à les faucher l'une après l'autre à leur maturité.

79. Setaria glauca P. Beauv. Agr. p. .51; O. Deb. Fl. du Tchéfou, $\mathrm{n}^{\circ} 229$; Miq. Prol. jap. p. 163; Panicum glaucum Lin.; Benth. Fl. Hong-kong. p. 411; Franch. et Savat. Enum. jap. 11, p. 161.

Hab. : Les champs sablon., les jardins et les cultures autour de Tien-tsin.

Ar. géog. : Régions intertrop. des deux mondes. - Asıe bor., occ. et or. - Chine bor. et aust., Hong-kông, Pé-tché-ly à Péling (Maxim.). - Japon. - Ussuri. - Java. - Europe. - Afriq. bor., occ. et or., - Amér. bor. et cent., etc.

80. Setaria viridis P. Beauv. Agrost. p. 1ว̆l; Steud. Syn.glum 1, p. 51; Turczan. El. baik-dahur. II, p. 298; Maxim. Prim. amur. p. 83; Panicum viride Lin.; Bunge Enum. chin. bor., no 398; Franch. et Savat. Enum. jap. II, p. 162.

Hab. : Avec l'espèce précédente, mais beaucoup plus rare.

Ar. géog. : Europe, Caucase. - Asie bor., occ. et or. - Sibérie alt., Daoûrie, Mongolie. - Chine bor., Pé-tché-ly. - Amour et Ussuri. - Japon. - Amér. bor., etc. - Afriq. bor., etc.

81. Setaria italica Kunth. Enum. plant. 1, p. 153; Miq. Prol. fl. jap. p. 163; Franch. et Savat. Enum. jap. II, p. 162. 
Var. germanica P. Beauv. Agrost. p. 51; 0. Deb. Fl. de Shanyhaï, $n^{\circ} 140$ et du Tché-foù, no 230; Panicum gernanicum Vild.: Bunge Emum. chin. bor., no 399; Sinice Mei-Tzé, Sia o-Mrî.

Hab. : Cult. dans toutes les terres maigres et sabl. de la plaine du Pei-hô.

Ar géog. : Eur. - Asie intertrop. et temp. - Chine bor., et aust., Pé-tché-ly à Pé-king. - Amour et Ussuri. - Japon. Amér. bor.

82. Panicum miliaceum Lin. Spec. 86; Steud. Syn. glum. 1, p. 77; Bunge Enum. chin. bor., no 400; O. Deb. Fl. du Tchéfoû, no 231 ; Miquel Prol. jap., 163; Sinice Choû-мег, Kô-тzé. Hab. : Cultivé, comme l'espèce précédente, dans tout le territoire de Tien-tsin.

Ar. géog. : Eur. - Asie intertrop. et temp. - Chine bor. et moy., Tché-kiang, Kiang-soû, Chan-tong et Pé-tché-ly.-Amour et Ussuri. - Japon.

Obs. Les graines des Oplismenus crus-galli, Setaria italica et Panicum miliaceum sont généralement employées dans le Pétché-ly, à l'alimentation des habitants, à cause de leur prix moins élevé que celui du riz. Les voyageurs modernes qui ont parcouru cette contrée et qui ont pu admirer ses belles cultures du Panic millet, supposent avec raison que cette plante est connue à la Chine de temps immémorial, et n'a pu y être introduite par importation. La paille de cette graminée sert également à faire des nattes grossières, ou à chauffer lès habitations pendant l'hiver.

83. Tragus tcheliensis O. Debeaux Mss. (1861); Lappayo racemosa Bunge Enum. chin. bor. $n^{\circ}$ 401, ex parte; G. Staunton Plant. tchel. exsicc. (1793) sub Cenchro racemoso.

"Plante annuelle, pluricaule non gazonnante; tiges simples »ou peu rameuses dès la base, striées, dressées, hautes de 1.5 à \20 centimètres avec l'épi terminal et glabres; feuilles linéaires » lancéolées, planes, scabriuscules, bordées de cils blancs sétacés: » gaines florales lâches, un peu renflées, glabres, atteignant la » moitié environ de l'épi terminal, celui-ci dense, subcylindri» que de 7 à 8 centimètres de long., d'un blanc-pâle et à raclıs, »hispide; pedicelles sessiles, uniflores; épillets très-petits, 
》ovales-oblongs, acuminés; glumes extérieures munies de "quatre côtes hérissées de pointes recourbées en crochet. » tuberculeuses à la base, inégales et irrégulièrement espacées. »

Hab. : Les sables secs et incultes dans la plaine du Pei-hô, près des salines de Tien-kô.

Ar. géog.: Chine bor., Pé-tché-ly à Pé-king (Bunge, Staumt.), et à Tien-tsin.

Obs. : La plante du nord de la chine qui a été confondue par Bunge avec le Tragus racemosus, ne peut-être rapprochée que de cette dernière espèce. Elle en diffère par ses tiges dressées, peu rameuses, entièrement glabres, par ses feuilles plus larges. bordées de cils raides et sétacés, par son épi cylindrique-allongé, compacte, par ses épillets uniflores, sessiles, par ses glumes plus courtes et plus larges, marquées de quatre arètes longitudinales, dont chacune est couverte d'aspérités subtuberculeuses à la base et recourbées à leur sommet en forme de crochet. Cette graminée est une des plus intéressa!ntes de la province du Pé-tché-ly, et tout à fait différente du $T$. racemosus, par son port, la forme de son épi, des épillets et des glumes externes. Je la considère donc comme une bonne espèce, et je crois devoir lui donner le nom de la contrée, où elle a été rencontrée seulement jusqu'à ce jour.

84. Phragmites communis Trin. Fund. agrost. 134, Steud. Syn. glum. 1, p. 195; Ledeb. Fl. ross. IV, p. 193; Turczan. Fl. baik-dahur. III, p. 315; Maxim. Index fl. pekin. p. 478; Regel Tent. ussur. p. 168; O. Deb. Fl. du Tché-foù, no 236; Franch. et Savat. Emum. jap. II, p. 170; Sinice HoûEI, YÉ-TSAÔ.

Forma gracilis O. Debeaux (loc. cit.)

"Planta humilis, panicula depauperata glumis 2-3 floris, paleis » paulo longioribus quam in forma typica. »

Hab. : Les rives du Pei-hô, depuis Tien-tsin jusqu’á Tà-koû. Septembre-octobre.

Ar. géog. : Eur. cauc. - Asie bor., Sibérie altaïque, Baikalie, Daoûrie, Mongolie. - Chine bor. et moy. litt. Chan-tong, Pé-tché-ly. - Amour, Ussuri. - Japon. - Amér. bor. - Af. bor.

8: Phragmites longivalvis Steudel Syn. ghmac. 1, p. 195; 
Ph. communis var. longivalvis Miq. Prol. jap. p. 166. Franch. et Sav. Enum. jap. II. p. 170; Staunt. Plent.tchel. exsicc., sub Arundine phraymites (Teste A. Franchet).

"Culmo valido, erecto, glabro, foliato; foliis lanceolatis, »glabris, lævibus, acuminatis, punctulatis margineque scabrius» culis; ligula brevissime ciliata; panicula ampla, ovatd, densa, 》25-30 centim. et ultra longa, 10 cent. lata; spiculis plerumque "trifloris; etiam 5-; floris; glumis inæqualibus, cum valvulis »flosculorum inferiorum distincte nervosis, his acuminatis, » elongatis; pilis flosculos excedentibus (Steud.) »

1lab. : Les rives du Pei-hô, avec l'espèce précédente, mais plus rare. - Septembre.

Ar. géog. : Chine bor., Pé-tché-ly à Pé-king (Staunt.) - Japon. Obs. : D'après les observations de MM. Franchet et Savatier et celles de Steudel, qui a toutefois maintenu comme espèce, le Phragmites longivalvis, celui-ci serait à peine une variété du Ph. communis. Les caractères tirés des feuilles lisses sur les bords et non scabres, et ceux de leur ponctuation, me paraissent bien fugaces. Il ne reste plus, pour légitime le maintien de cette forme spécifique, que la longueur relative des glumelles et le nombre des épillets (le plus souvent trois au lieu de cinq).

Le port élevé du Ph. longivalvis et l'ampleur de sa panicule, le font également distinguer du $P h$. communis, avec lequel il croit d'ailleurs sur les rives du Pei-hô.

86. Aluropus littoralis Trin. Fund. agrost. tab. 3; Ledeb. Flor. ross. IV, p. 369; Steud. Syn. glum. 1, p. 297; Dactylis littoralis Wild. Spec. 1, p. 408; Kunth. Enum. plant. 1, p. 324; Agrostis pungens Pallas.

Var. sinensis O. Debeaux Mss. in Herb. (1861).

"Planta robustior, spicis densioribus $(25$ millim. longis, » 8-10 millim. latis in diametro), quam in forma europeana; » pedunculis erectis, magis elongatis; nodis culmorum dense »villosis. »

Hab. : Les sables secs et incultes autour des tumulus, et près du fort isolé dans la boucle du Pei-hô. - Fl. 20-30 mai.

Ar.géog. : Eur. aust. - Afriq. bor. - Asie bor., Sibérie transbaik. - Chine bor., Pé-tché-ly à Tien-tsin (O. Deb.).

Obs. La présence sur les sables du Pei-hô de l'Elluropus 
littoralis est un fait de géographie botanique des plus intéressants. La plante de Tien-tsin ne diffère à peine de la forme typique de l'Europe australe, que par ses nouds très velus à la base, par son port plus robuste, ses pédoncules florifères dressés, et son épi plus gros, plus dense et plurifore. L' $\boldsymbol{E}$. littoralis du nord de l'Afrique, a également les nœuds des chaumes un peu velus à leur base, mais ses pédoncules sont grêles et flexueux. L'épi floral, dans ce dernier, est beaucoup plus court et plus réduit dans ses dimensions (10 millim. long., 5 millim. larg.) que dans notre variété de Tien-tsin. Les feuilles de la forme sinensis sont aussi du double plus allongées; mais ces divers caractères, dus à l'habitat de cette plante dans une région relativement froide, ne me paraissent pas suffisants pour la séparer de la forme typique de l'Europe méridionale.

87. Hordeum distichum Lin. Spec. 125; Steud. Syn. glum; 1, p. 351; Kunth Enum. plant. 1, p. 455; Zeocriton distichum P. Beauv. Agrost. p. 125; Sinice MaI, Tâ-мâr.

Hab. : Cultivé dans les terres sablonneuses du Pei-hô, et des rives du canal impérial.

Ar. géog.: Orig. de la Tartarie mongole, et cultivé depuis un temps immémorial dans les provinces boréales de la Chine, pour la nourriture de l'homme et des animaux domestiques.

Obs. On cultive également l'H. vulgare Lin., Sinice TAô-rì, et l'H. hexastichum L. Sinice Tầ-MaI, pour les mêmes usages, mais plus rarement.

88. Andropogon tropicus Spreng. Syst. 1, p. 207; Franch. et Savat, Enum . jap. II, p. 192; Benth. Fl. Hong-Kong. p. 423; Miquel Prol. fl. jap. 178; Holcus fulvus R. Br. Prodr. 200; A. dichroanthus, Steud. Syn. glum. 1, p. 393; Andropogon Ischœmum Bunge Enum. chin. bor. no 409 ex parte; G. Staunt. Plant. tchel. exsicc. (1793).

"Planta chinæ borealis humilior quam in forma tropicali, » paniculaque magis contorta ac depauperata; culmis .50-60 cent. » elongatis; ramis spicifloris verticillatis; panicula effusa; spicis " 8-13 floris, rachi pedunculisque plumosis, barbatis; paleis » ovatis, acutis; glumis villosis muticis; arista flosculi fertilis » imberbi, valvulis duplo longiore; foliis scaberrimis, striati- 
》 sulcatis (30-40 centim. longis, in millim. latis), acutis, nodis » hirsuto-pilosis (Steud.)

Hab. : Les sables secs et salés, dans la plaine inculte du Pei-ho (G. Staunt.)

Ar. géog: : Asie subtrop. Ceylan, Indes-Or., Malaisie, Timor, Molıques, Philippines, Australie. - Japon. - Chine bor. et aust. Pé-tché-ly à Pé-king (Bungr), Tien-tsin (Staunt.), Hong-Kông (Benth.)

Obs. : Cette espèce, dont la panicule est beaucoup plus appauvrie dans le nord de la Chine, que dans la forme des régions subtropicales, était signalée depuis longtemps par Bunge dans la province de Pé-tché-ly. G. Staunton l'a récoltée le premier sur les sables du Pei-ho en 1793, et l'a également confondue avec l'Andropogon ischœmum L. On retrouve en abondance l'A. tropicus au Japon, près de Nangasaki dans l'île de Kioù-sioû (A. Franchet).

89. Sorghum vulgare Pers. Syn. plant. 1, p. 101; 0. Deb. Fl. de Shang-haï, n 13 et Fl. du Tché-foù, n² 249; Andropogon sorghum Steud. Syn. glum 1, p. 393; Sinice Kâo-GâxG. Var. A genuina O. Deb. Fl. du Tché-fou (loc. cit.)

Var. B rubens Pers.; O. Deb. (loc. cit.); Sorghum rubens Wild; Andropogon rubens Kunth.

$H a b$. : Les deuix variétés A et B sont cultivées en grand dans toute la plaine du Pei-ho et sur les rives du canal impérial. - Fl. et Fr. août et septembre.

Ar. géog. : Asie intertrop. et temp., Indes.-orient.; Chine bor., moy. et aust., Kiang-Soû, Tché-kiang, Chan-tong, Pé-tché-ly. Amour et Ussuri. - Japon. - Afriq. bor. et aust. - Eur. aust., etc.

Obs. Le Sorgho commun est l'une des graminées les plus utiles dans le nord de la Chine. Ses graines servent à la nourriture de l'homme et à la préparation d'une boisson alcoolique nommée San-choù, analogue à celle que l'on obtient du riz dans plusieurs autres provinces de l'empire. Les feuilles du Sorgho sont utilisées pour la litière des animaux domestiques, et les tiges desséchées sont pour ainsi dire arec ceiles du maïs, le seul combustible employé pendant l'hiver, pour le chauffage intérieur des habitations rurales.

Plusieurs autres espèces de la famille des graminées sont cul- 
tivées à Tien-tsin pour les besoins de l'alimentation. Je citerai principalement le riz Oryza sativa L.), Sinice TAô, Toû, Nô, Y'́TzÉ, etc., cultivé dans les terres basses et marécageuses qui avoisinent le canal impérial dans l'ouest du Pé-tché-ly; l'avoine (Avena sativa), Sinice Liû-tan-mei, et Hiang-Mei; le blé Trilicum vulgare L.), Sinice Siâ̂-MeI, Tâ-MEI, dont la farine, quoique grossière, est beaucoup plus estimée que le riz dans le Pé-tché-ly, pour la nourriture de ses habitants.

Dans les jardins, j'ai remarqué le Coyx lacryma L. Sinice Y-GIN-MI, Y-YN-GIN, dont les graines, nommées vulgairement en France larmes de Job, sont employées dans la fabrication des chapelets, etc.

EQUISÉTACÉEŚ D. C.

90. Equisetum ramosum Schleich, Cat. p. 27; O. Deb. Fl. du Tché-fou, $\mathrm{n}^{0} 253$; Maxim. Ind. fl. pekin. in Prim. amur. p. 479; Miq. Prol. jap. $\mathrm{n}^{\circ} 389$; G. Staunt. Plant. tchel. essic. (1793); Sinice Mo-TzÉ, KoûAng-KIng.

Hab. : Les sables marécag'eux et salés des rives de Pei-ho. Salines et forts de Tien-kô près de Tien-tsin. - Août et sept.

Ar. géog. : Eur. - Afriq. bor. - Asie bor., Sibérie altaïque, Mongolie. - Chine bor. et litt. Chan-tong (O. Deb.), Pé-tché-ly à Pé-king (Maxim.) et Tien-tsin. - Japon. - Amér. bor., etc.

\section{GÉOGRAPHIE BOTANIQUE}

\section{1. - Aperçu des principales affinités de géographie botanique du territoire de Tien-tsin.}

Il ne me reste plus, pour arriver au terme de mes recherches sur la lavégétation de Tien-tsin, qu’à examiner quelles sont les principales affinités de la Géographie botanique de cette contrée. J'aurai ensuite à comparer, dans un deuxième chapitre, les résul- 
tats obtenus, au sujet des affinités botaniques des trois florules de Shang-haï, du Tché-fou ot de Tien-tsin.

J'ai déjà dit, dans l'avant-propos de cette florule, que la pauvreté de la flore de Tien-tsin, étonne surtout le botaniste au premier abord. L'aridité du sol situé en dehors de la zone cultivée et arrosable de la plaine du Pei-hô, contraste singulièrement avec les belles cultures de cette même zone, et ne permet pas d'y faire de fructueuses herborisations. Étant donné le petit nombre d'espèces que j'ai recueillies autour de Tien-tsin, il ne m'est pas pas possible de dresser par families le tableau des affinités botaniques des plantes mentionnées dans cette florule. J'ai dû me borner à prendre une note exacte pour chaque espèce des diverses régions où celle-ci a été signalée jusqu’à ce jour, et à résumer ensuite l'ensemble des stations géographiques énumérées pour chacune d'entr'elles. Nous arrivons ainsi aux résultats ci-après :

$1^{\circ}$ Spéciales a la Chine. - Plantes dont la présence n'a été signalée juspu’à présent que dans l'empire chinois.... 13 espèces

$2^{\circ}$ Chine et Japon - Plantes spéciales à la fois à la

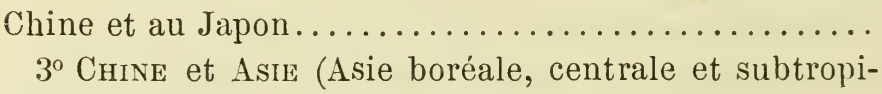
cale). - Plantes qui se trouvent en Chine et dans diverses régions de l'Asie continentale...............

$4^{\circ}$ Chine, Amour et Ussuri. - Plantes qui se rencontrent aussi dans les provinces limitrophes de l'Amour et de l'Ussuri............................

$5^{\circ}$ Chine, Asie 'et Japon. - Plantes observées dans diverses régions asiatiques et au Japon..............

$6^{\circ}$ Asie, Amour, Ussuri et Japon. - Plantes dont la présence a été constatée en Asie, les provinces de l'Amour et de l'Ussuri et aussi au Japon............. $6 \gg$

70 Asie, OcÉanie, Japòn. - Plantes dont l'aire de dispersion s'étend en Asie, au Japon et dans plusieurs îles de laMalaisie, de l'océan Indien, du Pacifique.....

$8^{\circ}$ Asie, Europe, Afrique, Japon. - Plantes que l'on retrouve en Asie, en Europe, dans quelques contrées de l'Afrique littorale, boréale ou centrale et au Japon. : $11 »$

$9^{\circ}$ Asie et Japon, Europe, Afrique et AMt́rique. -

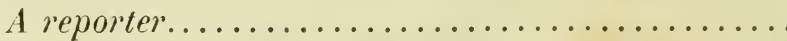




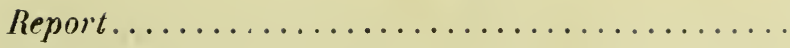

Plantes trouvées à la fois en Asie et au Japon, en Europe, dans l'Afrique boréale ou centrale, en Améri-

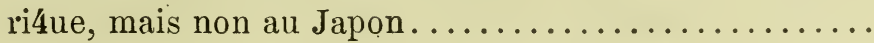

$10^{\circ}$ AmÉrique et JAPon. - Plantes spéciales à la fois

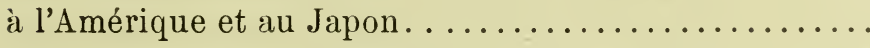

$11^{\circ}$ Asie, Europe, Amérique, Amour, Ussuri et Japon. - Plantes observées en Asie, en Europe, en Amérique, dans les provinces de l'Amour et de l'Ussuri et au Japon .............................. 8

Total....................

$11\left({ }^{1}\right) \gg$

$\gg \quad \gg$

90 espèces

Les affinités botaniques de ces quatre-vingt-dix plantes composant la florule de Tien-tsin avec les diverses contrées du globe, se classent dans l'ordre ci-après, suivant leur importance numérique :

$11^{\circ}$ PÉ-TChÉ-Ly (Environs de Pé-king). . . . . 81 esp. $=90 \%$

$2^{\circ}$ Asie boréale, centrale ou australe $\ldots 64 »=71 \%$

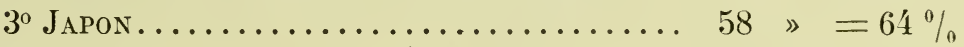

$4^{\circ}$ Europe..................... $42, "=46 \%$

5 AFrique bor. et cent. litt.......... 30 » $=33 \%$

$6^{\circ}$ Amour et Ussuri.................. $26{ }^{\star}=30 \%$

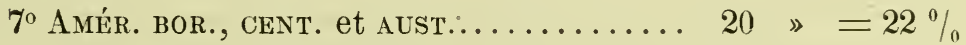

8 Spéciales a la Chine............... $13 \gg=14 \%$

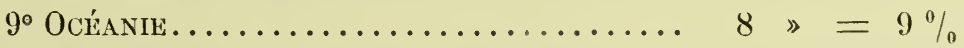

Il devient facile, d'après ces chiffres, qui sont d'ailleurs relevés avec un soin minutieux, de se rendre un compte exact des rapports botaniques de la florule de Tien-tsin. Quatre-vingt-une espèces sur quatre-vingt-dix, ou les neuf dixièmes, se retouvent dans la même province et aux environs immédiats de Pé-king. Cette intimité de la végétation de Tien-tsin s'explique par le peu de distance qui sépare ce dernier point de la capitale de l'empire chinois, et par l'uniformité de la plaine et du sol traversés du Nord au Sud par le fleuve Pei-hô.

(1) La nécessité de grouper les stations géographiques d'après l'ordre établi dans les florules précédentes de Shang-haï et du Tché-foî, rend les chiffres des nos 8 et 9 un peu inexacts en ce qui concerne le Japon. On doit exclure quatre espèces pour le Japon au no 8 et deux seulement pour le no 9 . 
Les rapports botaniques avec l'Asie boréale, centrale et intertropicale tiennent ici le second rang, et sont beaucoup plus considérables dans le Pé-tché-ly que dans les provinces de Kiang-soû et de Chan-tong, où nous les trouvons classés au troisième rang avec le Japon. Les affinités avec le Japon sont encore les mêmes ì Tien-tsin eț au Tché-foû (3/ŏ environ), mais moins élevées qu'à shang-haï (5/6). Viennent ensuite, dans un ordre à peu près identique, l'Europe, l'Afrique, l'Amérique, l'Amour et l'Ussuri, l'Océanie. J'observe toutefois en passant que les rapports de la flore de Tien-tsin avec celle des provinces de l'Amour et de l'Ussuri sont moindres qu'au Tché-foû, mais un peu plus élevés que ceux déjà exprimés (pour ces mêmes provinces) dans la florule de Shang-haï. En ce qui concerne les plantes spéciales à la Chine, la proportion numérique des espèces récoltées à Tien-tsin $14 \%$, soit $1 / 7$ environ de la flore, est un peu plus élevée dans cette province qu'à Shang-haï, $(1 / 11)$, et à peu près identique avec celle que j'ai fait connaitre dans la florule du Tché-foû (1/6), pour les espèces de cette province qui sont spéciales à la Chine.

\section{SII. - Examen comparatif des affinités botaniques des territoires de Shang-haï, Tché-foû et Tien-tsin}

Le territoire de Tien-tsin n'étant éloigné des rivages du Léatong ou mer du Pé-tché-ly, que par une disţance de 'quarantehuit kilomètres environ, doit être placé, au point de vue de sa végétation, dans la région littorale intérieure de la Chine. J'ajoute que la plaine sablonneuse et uniforme que parcourt le fleuve Pei-hô du Nord-Est au Sud-Ouest, est soumise chaque jour aux influences de la marée, qui atteint parfois à Tien-Tsin une hauteur considérable. Il faut aussi tenir compte, d'un autre côté, de la faible altitude du sol au-dessus du niveau de la mer et des éléments minéralogiques qui sont répandus à sa surface, et qui n'entrầnent pas une différence bien sensible entre les terres marécagreuses du Pé-tchè-ly et les sables inondés des rivagres du Léatong.

Il est donc rationnel d'admettre, que la flore de la rég'ion littorale est dominante à Tien-tsin, aussi bien que sur les rives du 
Wam-poô, à shang-haï, et sur les sables du T'ché-foû. Nons pourrons déduire de cette considération, à quelques rares exceptions, que les mêmes influences sur la distribution géographique des plantes: agissent dans les trois portions des territoires que j'ai pu explorer dans le Kiang-soû, le Chan-tong et le Pé-tché-ly, eu égard à la latitude de ces provinces.

J'ai consigné dans le tableau suivant $n^{\circ} 1$, le résultat numérique comparatif de la distribution géographique des plantes observées à Shang-haï, au Tché-foû et à Tien-tsin, d'après les bases adoptées dans chacune de ces trois florules.

Tableau no 1 récapitulatif des affinités botaniques des trois florules littorales de Shang-haï, du Tché-foa et de Tien-tsin.

\begin{tabular}{|c|c|c|c|c|c|}
\hline STATIONS BOTANIQUES" & Shang-hai & ithe-100 & Tien-ls' $\mathrm{n}$ & TOTAUX & 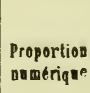 \\
\hline lo Chine (spéciales)... & 14 & 42 & 13 & 69 & $14 \%$ \\
\hline $2^{\circ}$ Chine et Japon............. & 28 & 21 & 6 & $5 \check{5}$ & $11 \%$ \\
\hline $3^{\circ}$ Chine et Asie continentale....... & 11 & 17 & 15 & 43 & $9 \%$ \\
\hline $4^{\circ}$ Chine et prov. d'Amour, Ussuri.. & 3 & 25 & 5 & 33 & $7 \%$ \\
\hline $5^{\circ}$ Chine, Asie et Japon.......... & 18 & 24 & 8 & 50 & $10 \%$ \\
\hline $6^{\circ}$ Amour et Ussuri, Japon.. & 6 & 38 & 9 & 53 & $11 \%$ \\
\hline 7o Asie, Océanie, Japon........ & 14 & 15 & 4 & 33 & $7 \%$ \\
\hline $8^{\circ}$ Asie, Europe, Afrique, Japon.... & 12 & 22 & 11 & 45 & $9 \%$ \\
\hline $\begin{array}{l}9^{\circ} \text { Asie, Europe, Afrique, Amour, Us- } \\
\text { suri et Japon . . . . . . . . . }\end{array}$ & 40 & 40 & 11 & 91 & $18 \%$ \\
\hline $10^{3}$ Amérique et Japon............ & 3 & 7 & $"$ & 10 & $\begin{aligned} 10 \% \\
2 \%\end{aligned}$ \\
\hline $\begin{array}{l}110 \text { Asie, Europe, Amérique, Amour, } \\
\text { Ussuri et Japon.............. }\end{array}$ & 3 & 12 & 8 & 23 & $5 \%$ \\
\hline Totaux..... & 152 & 263 & 90 & 505 & \\
\hline
\end{tabular}

Il résulte avec évidence de ce tableau que, sur un chiffre moyen de cinq cents espèces observées dans les trois provinces de Kiang-soû, Chan-tong et Pé-tché-ly, la proportion numérique la plus élevée, $18 \%$, se rapporte aux plantes qui se trouvent à la fois en Asie, en Europe, en Afrique, en Amérique et au Japon. 
ll doit en ètre ainsi, en effet, à cause du nombre relativement élevé des plantes ubiquistes et des végétaux de grande culture ou simplement cultivés dans les jardins, et que nous avons énumérés dans nos trois florules. Viennent ensuite, au deuxième rang, les plantes spéciales à la Chine $14 \%$, puis, dans une égale proportion, les espèces signalées seulement à la Chine et au Japon, et celles observées dans diverses régions asiatiques en Océanie et au Japon, soit environ $11 \%$. Nous trouvons ensuite, dans un ordre à peu près égal $(10 \%)$, les plantes propres à la fois à l'Asie continentale et au Japon, puis celles qui ont été observées en Asie, en Europe, en Afrique et au Japon. Nous constatons, enfin, que les végétaux spontanés, dont la présence n'a été signalée seulement que dans l'Amour et l'Ussuri $(7 \%)$, sont dans le même rapport que ceux dont l'aire de dispersion s'étend jusque dans les îles de la Malaisie, de l'océan Pacifique et en Australie.

Les affinités botaniques avec l'Amérique et le Japon, à l'exclusion des autres contrées du globe, ont la plus faible proportion numérique, soit $2 \%$ seulement.

Avant de terminer ces observations, j'ai lieu de penser qu'il serait intéressant d'indiquer ici dans quel ordre geéographique viennent se ranger les cinq cents plantes formant l'ensemble des florules de Shang-haï, du Tché-foû et de Tien-tsin. On pourra juger beaucoup plus facilement, par le deuxième tableau comparatif ci-après, quels sont les résultats généraux auxquels ont abouti mes recherches, relativement à la dispersion géographique des plantes composant la végétation de la Chine maritime moyenne et boréale 
Tableau $n^{\circ} 2$, indiquant la répartition géographique des plantes observées à Shang-haï, au Tché-foú et à Tien-tsin.

\begin{tabular}{|c|c|c|c|c|c|}
\hline REGIONS DU GLOBE & $\underset{1: \text { Esp }}{\text { Shagg-hai }}$ & $\begin{array}{l}\text { Tchŕ-fou } \\
203 \text { Esp. }\end{array}$ & $\begin{array}{l}\text { Tien- tsin } \\
90 \text { Bsp. }\end{array}$ & $\begin{array}{c}\text { Totaux } \\
- \\
505\end{array}$ & $\begin{array}{l}\text { Proportion } \\
\text { aumértque }\end{array}$ \\
\hline Japon................. & 124 & 165 & 58 & 347 & $69 \%$ \\
\hline Asie boréale, cent. et aust..... & 95 & 174 & 64 & 333 & $66 \%$ \\
\hline Chine boréale, env. de Pé-king: . & 70 & 166 & 81 & 317 & $64 \%$ \\
\hline Europe.............. & 54 & 80 & 42 & 176 & $35 \%$ \\
\hline Ainour et Ussuri............ & 27 & 114 & 26 & 167 & $31 \%$ \\
\hline Afrique bor., cent., litt. et aust. . & 55 & 68 & 30 & 153 & $31 \%$ \\
\hline Amérique bor. et aust... & 46 & 60 & 20 & 126 & $23 \%$ \\
\hline Océanie. . & 34 & 45 & 8 & 87 & $17 \%$ \\
\hline Chine (spéciales). & 14 & 42 & 13 & 69 & $14 \%$ \\
\hline
\end{tabular}

Ce tableau établi d'après les divers habitats constatés pour chaque espèce, dans les diverses régions du globe, vient corroborer l'opinion que j'ai émise dans mes précẹdentes florules, sur les affinités géographiques de la flore du littoral chinois. Il confirme, en second lieu, les remarques faites sur ces mêmes affinités, par les botanistes voyageurs qui ont exploré après moi les contrées boréales et maritimes de l'empire chinois. Les rapports géographiques formulés par le chiffre le plus élevé, soit trois cent quarante-sept espèces sur cinq cents ou $69 \%$ du total observé. sont en parfait accord avec tout ce qui a été déjà écrit sur la géographie botanique de la Chine maritime, et démontrent l'intimité de sa flore avec celle du Japon. Cette intimité n'est pas moins grande avec la flore asiatique (Asie boréale centrale et subtropicale), puisque nous polivons constater la présence de trois cent trente-trois espèces (soit $66 \%$ ) sur le sol chinois et qui se retrouvent dans diverses provinces du vaste continent asiatique. Mais je tiens ì insister principalement, sur les rapports vraiment remarquables de la végétation de Shang-haï, du Tché-foû et de Tien-tsin, avec ceux de la flore des environs de Pé-king au nord du Pé-tché-ly. La proportion numérique 
$(64 \%)$ des plantes qui croissent également dans l'extrème nord du Pé-tché-ly, malgré la position géographique de cette contrée, aurait lieu de nous surprendre, si l'on ne savait d'un autre côté, que les environs immédiats de Pé-king ont été explorés par plusieurs botanistes, à l'investigation desquels presque aucune plante n'a pu échapper. J'ajou terai aussi que, par suite des rapports journaliers du commerce général de la Chine avec la capitale de l'empire, une foule de végétaux originaires des provinces moyennes et littorales, ont fini par s'acclimater autour de Pé-king, et augmenter ainsi la richesse dé sa végétation.

Je n'insisterai pas sur les autres affinités géographiques, qui sont d'ailleurs consignées dans le tableau précédent, et nous dévoilent un des coins si mystérieux et inconnus jusqu'à ce jour de la flore chinoise. 


\section{ADDENDA ET CORRIGENDA}

\section{FLORULE DU TGHÉ-FOU}

3 bis. Aconitum Carmichaeli 0. Debeaux Mss. (1879); Aconitum (nov. spec.) by W. B. Hemsley, Notes on some chinese plants, with descriptions, etc., in Trimens journal of Botany, vol. XIV, p. 205 (187i).

"Herba perennis erecta; folia radicalia ignota, caulinia petio"lata, coriacea, sparse et minute pubescentia, tripartita, lobis "lateralibus profunde bifidis, centrali obovato-spathulato, omni" bus basi valde attenuatis, lobis secundariis brevibus, latis, 》 rotundatis, mucronatis vel lanceolatis-acutis; floribus magnis, 》 albo-cæruleis, leviter pilosis, longe pedicellatis, sepalis anticis 》obliquiter lineari-oblongis, lateralibus obovato-rotundatis, " postico elongato-cucullato, filamentis ad medium late scarioso"alatis; folliculis quinque erectis, reticulato-nervosis, stylo "persistente coronatis (Hemsley). "

$H a b$. : La région montagneuse du Tché-fou, près de Bamboú Temple (très-pobablement la Pagode neuve), où il a été découvert par le $\mathrm{D}^{\mathrm{r}}$ Carmichael en 187 ;

Obs. Cette espèce, que je n'ai pas rencontrée au Tché-foû, et dont M. Hemsley n'a fait connaître que la description, serait voisine de l'Aconitum sinense Siebold (A. Fisheri Reichb.) Je la désigne provisoirement du nom de l'auteur de sa découverte.

5. Dontostemon dentatus Bunge.-Ajouter à la synonymie : Franchet et Savatier, Enum. plant. in japonia cresc. 1, p. 37; et dans l'aréa géographique : Japon, dans la région moy. du Nippon (L. Savatier). 
8. Viola Gmeliniana Ræm. et Schult.

Etablir ainsi la synonymie de cette espèce :

Viola Patrinii var. B. Gmeliniana Miquel in Prol. floræ japon. p. 84., non Rrem. et Schult.; Franch. et Savat. Enum. plant. japon. 1, p. 41; V. Patrinii var. chinensis Ging. in I). C. Prodr. 1, p. 293. ex parte, sensu clar. Maximowiczii in Mélanges biologiques, IX, p. 722; V. Grmeliniana var. hispida O. Debeaux Flor. Tché-foù olim.

Obs. C'est d'après l'avis de M. A. Franchet, que je rapporte aujourd'hui le Viola Gmeliniana de la florule du Tché-foû, au V. Patrinii var. Gmeliniana de Miquel (loc. cit.). M. MIaximowicz. réserve le nom princeps de $V$. Gmeliniana Ræm. et Schult. ̀̀ une espèce d'un autre type, dont les feuilles sontplus larges au milieu qu'à la base. Le I. Patrinii, dont notre plante a tout à fait l'aspect, est entièrement glabre, et a les pétales imberbes à leur base, tandis que ceux de la forme du Tché-fôn sont velus-hispides ainsi que les divisions calicinales.

\section{Sílene aprica Turczan.}

Obs. Cette espèce appartient à la section des Melundriun Rorhb. et non à celle des Rupifraga. Sa synonymie doit donc être rectifiée de la manière suivante :

SILEnf (Melandrium) APRICA Turcz. Cat. plant. Baik.-dahur. n० 221; Ledeb. Flor. ross. 1, p. 317; Fisch. et Meyer Ind. sem. hort. petrop. p. 38 (1835); Bunge Enum. chin. bor. no 50; Naxim. Prim. flor. amur. p. 55; Melandrium apricum Rohrbach Mono!/. gatt. Silen. p. 231 ; Franch. et Savat. Enum. plant. jap. 1, p. 48.

On doit aussi ajouter le Japon (Kỉoû-siô̂), dans la distribution géographique de cette plante.

\section{Geranium nepalense Swet.}

Ols. Dans tous les échantillons récoltés au Tché-foû, les poils glanduleux qui recouvrent les pédoncules, les divisions calicinales et les capsules, sont rares, ou manquent même sur quelques individus. MIM. Franchet et Savatier pensent (Enum. plant. japon.) qu'il n'y a pas lieu de séparer cette dernière forme de l'espèce typique, même à titre de variété.

41. Vicia unijuga A. Braun.

Ajouter à la synonymie : Franch. et Savat. Enum. plant. jap. 
2, p. 324 (1876); Lathymus Messerschmidii Franch. et Savat, loc. cit. 1, p. 106.

Obs. Cette plante décrite par Linné sous le nom d'Orobus lathyroides, et qui a été considérée par MM. Franchet et Savatier en 1874 dans le premier volume de leur Enumeratio, comme appartenant au genre Lathyrus, a été réunie plus tard, en 1876 , par ces mêmes botanistes, au Vicia unijuga. Celui-ci est assez répandụ au Japon (Kiou-sioû, Nippon, Yéso, etc.); aussi doit-on ajouter cette contrée dans la distribution géogaphique établie pour le $V$. unijuga, dans la florule du Tché-fon.

\section{Potentilla chinensis Ser.}

Ajouter à la synonymie : Franch. et Savat. Enum. plant.jap. 2, p. 3 i8, var. A. micrantha.

Obs. MM. Franchet et Savatier rapportent (loc. cit.) la plante du Tché-foû, à la forme 2 de leur variété micrantha, qu'ils n'ont vue d'aillenrs que de cette localité et de la Soôngarie. Ils la caractérisent ainsi :

"Caules' erecti, subsimplices, in parte superiori tantum ramosi, "ramis erectis fastigiatis; corymbi multiflori in paniculam am. " plam subfastigiatam dispositi; calices parvi, sepalis vix 3 milli》met. longis; flores explanati, circiter 8-10 millim. in diametro; » folia supra atroviridia, glabrata, subtus incana. »

55. Sanguisorba canadensis var. B. latifolia Ledeb. $=\mathrm{S}$. Media D. C. Prodr. 2, p. 394 (Species propria); S, canadensis var. B. media Franch. et Savat. Enum. 2, p. 345, sub Poterio.

Obs. J'ai déjà fait connaître les différences qui séparent le Sanguisorba media D. C. du S. tenuifolia de la florule du Tchéfoû, et j'avais rapporté la première de ces espèces à la var. latifolia du S. canadensis. Mais M. Franchet élève quelques doutes sur la valeur de cette détermination, et il pense avec raison que la plante du Tché-fô est plutôt la forme media du S. canadensis, ou le $S$. media D. C. qui doit figurer au $n^{0} 55$ de la florule du 'Tché-foû. Cette espèce se trouve également au Japon avec des fleurs rosées comme dans les échantillons provenant des marécages de Yan-taï. Dans le $S$. media, les feuilles sont dépourvues de stipelles, et les filets staminaux sont quatre à cinq fois plus 
longs que le calice. Le $S$. media de Regel et Till. in Tont. florid ussuriensis, n'est qu'une forme ì fleurs purpurines du S. tenuifolia.

60. Pyrus communis var. A. pyraster. $=P$. communis var. sinensis Lindley Botan. reg. tab. 1248; Franch. et Savat. Enum. plant. jup. 1, p. 138; Miquel Prol. jap. p. 228; P. ussuriensis Maxim. Prim. fl. amur., p. 102, ipso teste in Mélanges biolog. vol. IX, p. 168.

Obs. M. Decaisne maintient comme espèce légitime (Compt. rend. Acad. scienc. tome 75) le $P$. sinensis Lindl., bien facile it distinguer à ses feuilles bordées de dents très serrées et très aiguës. Mais le nom de $P$. sinensis, qui est le plus ancien, ne peut être maintenu, qu'ì la condition de conserver dans le genre Cydonia, le Pyrus chinensis de Poiret (Cydonia chinensis Bunge et auct. orient.). Le $P$. sinensis, que j'avais rapporté à tort à la variété pyraster du $P$. communis, existe aussi au Japon et dans toute l'Asie orientale, à l'exclusion du $P$. communis et de ses variétés. Les fruits du $P$. sinensis sont comestibles; aussi, doit-on attribuer à cette espèce les poires savoureuses du rhan-tong.

\section{GROSSULARIÉES}

70 bis. Ribes chifuensis Hance On some mountains plants from Northern China, in The Journal of botany. - London, février 1875, p. 33.

"Ramis subteretibus ramulisque tenuibus pubescentibus; 》 foliis opacis, petiolo basi dilatato, laminam æquante fultis, basi 》 sinu lato cordatis, æquelongis ac latis, ad medium usque trilo》bis, lobis late ovatis, grosse et obtuse inciso-serratis, supra "breviter pilosis, subtus cum petiolis pedunculisque dense " molliter tomentosis; floribus....; fructibus (ob racemum bre》vissimum subnullum) quasi 3-4 fasciculatis, pedunculo 3, perli》cello 1-1/2 lineali, bracteis....? Baccis ovoideis pedunculo cum " pedicello par umbrevioribus, rubris, limbo calicino coronatis, "lobis calicinis breviter oblongis, obtusissimis, arcte reflexis »tubi longitudine, petala spathulato-orbicularia plus duplo »excedentibus; antheris vix apicem tubi attingentibus.» (Hance.) $H a b$. : Cette espèce, de la section Ribesia et voisine du $R$. fas- 
ciculatis, a été récoltée dans le territoire du Tché-fon, en 1873, par M. Swinhoë.

\section{Artemisia integrifolia L.}

Ar. géog. : Ajouter le Japon dans la distribution géographique de cette plante.

\section{Adenophora coronopifolia Fischer.}

Ajouter à la synonymie : Franch. et Savat. Enum. plant. jap. 2, p. 425; A. polymorpha var. coronopifolia Trautv. et de Herder in Plantx Radd. reisen, IV, Heft. 1, p. 24.

Obs. D'après MM. Franchet et Savatier (loc. cit.), l'Adenophora coronopifolia, dont la présence a été récemment signalée au Japon par M. le docteur Vidal, est une plante très glabre, à feuilles assez rapprochées sur la tige, étroitement lancéoléeslinéaires, longuement acuminées, atténuées en pétiole et bordées de petites dents calleuses. Les fleurs, peu nombreuses et disposées en un épi simple, ont leur calice à divisions lancéolées, linéaires, entières, et les capsules elliptiques penchées. Cette espèce diffère de l'A. polymorpha Ledeb. par ses feuilles très étroites, ses pedoncules allongés, ses fleurs penchées, toujour's solitaires. Dans l'A. polymorpha, lorsque l'inflorescence est très réduite, les fleurs sont fasciculées par 2 et par 3 , et brièvement pédonculées.

L'A. coronopifolia, tel que je l'ai déjà décrit dans la florule du 'Tché-foû, renfermerait donc deux espèces, et l'on doit considérer' comme-appartenant à l'A. polymorpha, les formes à feuilles larges, ovales ou obcordées, à inflorescence plus ou moins rameuse, et à fleurs disposées en petits glomérules de deux à trois, formant un épi court et terminal.

120 bis. Adenophora polymorpha Ledebour Flor. ross. 2,. p. 893; Franch. et Savat. Enum.plant. jap. 2, p. 422; T'rautv. et de Herd. in Plantx Radd. reisen, IV, Heft I, ex parte.

$H a b$. : Les roches micaschisteuses et les falaises escarpées de la pointe du Tché-foû et de la baie de Ki-tsen-sôo, en société avec les A. coronopifolia et trachelioüdes.

Ar. géog. : Sibérie altaïque, Daoûrie; - Chine bor. Chan-tong; - Japon. 


\section{Adenophora trachelioides Maxim.}

Ajouter à la synonymie : A. remotiflora Miq. Prol. fl. jap. p. 12.; Franch. et Savat. Enum. plant. jap. 1, p. 279, et 2, p. 424 (teste ipso clar. Maximowicz). - Ajouter aussi le Japon dans sa distribution géographique.

\section{1 bis. Adenophora Isabellæ Hemsley Notes on some} chinese plants, in Trimens journal of botany, vol. XIV (1876); A. trachelioüdes Maxim. var. cordatifolia O. Debeaux $\mathrm{Fl}$. du Tché-foù, no 121.

"Herba perennis. ramosa, 2-3 pedalis; foliis radicalibus......., 》 caulinis cordatis, ovato-cordatisve, acutis, grosse duplicato» serratis, parce hispido-pilosis, 2 poll. longis latisque; petiolo 》 circiter sesquipoll. longo; floribus racemoso-paniculatis, bre» viter pedicellatis, bracteatis, brasteis parvis, lanceolatis, acutis ; »calicis lobis glaucis, oblongo-lanceolatis, per anthesin ad 4 li»neas longis, persistentibus; lobis corollæ rotundatis, subacutis; 》 ovario triloculari, polyspermo (Hemsley). »

$H a b$. : Les collines (région moyenne), au voisinage du Tchéfoû (Forbes, 1874); falaises du cap de Tché-foû (O. Debeaux, 1860).

Obs. L'A. Isabellæx me paraît n'être, d'après la description de M. Hemsley, que la forme cordatifolia de l'A. trachelivides Maxim. que j’ai signalée dans la florule du Tché-foû avec cette courte diagnose : "Foliis radicalibus angustis, superioribus ovatis cordatis acutis. »M. Hemsley trouve, dans la forme des feuilles, la longueur des pétioles, etc., des caractères suffisants pour considérer, comme espèce distincte, la variété cordatifolia à feuilles cordées ou obcordées, presque aussi larges que longues de l'A. trachelicides. Je ne trouve aucune difficulté, à adopter comme espèce, l'A. Isabellæ de M. Hemsley.

\section{OLÉACÉES}

121 bis. Ligustrum ibota Sieb. et Zuccar. Verh. batar. Genostsch. 12, p. 36, et in Fam. nat. flor.japon. 2. p. 167; Franch. et Savat. Enum. plant. jap. 1, p. 313; Miquel Prol. jap. p. 1.51; Hance Plants from North. China, in the Joumal of botany, London (1878) p. 110 ; L. vulgare Thunbg. Fl. Jap. 
p. 17, teste Miquel; L: obtusifolium Sieb. et Zucc. Fam. nat. no 567; Miquel Prol. jap. p. 151; Ilutta Kœmpf. Amon. exot. p. 896.

Hab. : Les environs de Yan-taï, oì il a été trouvé, en 1873, par II. C. Stuhlman.

Obs. Pendant mon séjour au Tché-foû, j’ai remarqué, dans un jardin attenant à la pagode du village de Ki-tsen-sôo, près de Yan-taï, un Ligustrum (peut-être le L. ibota?), mais que je n'ai point récolté, le rapportant alors à une espèce d'origine japonaise et cultivée dans cette station. Si le $L$. ibota du Tché-fô est spontané dans cette contrée, sa distribution géographique s'étendrait de la Chine boréale au Japon.

D'après MM. Franchet et Savatier (loc. cit.), le L. ibota varie à feuilles très-obtuses ou non, peu aiguës, un peu hispides en dessous ou très glabres sur les deux faces. L'inflorescsnce peut aussi varier et se trouver glabre ou velue-hispide, mais elle est nettement caractérisée par le tube de la corolle très allongé, dépassant longuement le calice, pàr ses anthères linéaires et par ses fleurs disposées en grappes ovales très courtes.

\section{Plectranthus pekinensis Maxim.}

Obs. Dans sa monographie des Plectranthus de l'Asie orientale, M. Maximowicz a réuni comme variété au $P$. amethystoides, son $P$. pekinensis antérieurement décrit dans le Primitix flor: amurensis, et il ne différencie ce dernier que par son calice pubescent, celui du $P$. amethystoides type étant glabre. Il y a donc lieu de modifier ainsi la synonymie des deux formes observées par moi au Tché-foû, et que j'ai réunies au $P$. pekinensis :

Forma A. floribunda $=P$. amethystoides var. pekinensis Maxim.; P. pekinensis Maxim. olim : "Caulibus ad medium " ramosis; calicibus pedicellisque dense incanescentibus. »

Furma $B$. paniculata $=P$. amethystoides f. typica "Calicibus pedicellisque subglabris. »

Ces deux formes du P. amethystoides se distinguent nettement des espèces voisines $P$. serra, $P$. glancocalix, par leurs feuilles, qui sont crénelées et non dentées.

149 bis. Scutellaria (Stachymacris) sciaphila s. Moore, in Trimen's journal botany, vol. XIII, p. 228 (18\%5). 
"Caule erecto, glabrato; foliis petiolatis oblongis, obtusis, basi » subrotundo-truncatis, grosse sparseque crenato-dentatis, tenui» ter pubescentibus; inflorescentiæ partibus hirsuto-tomentosis; 》bracteis deltoideo-ovatis calicem vix superantibus; corolla 4-plo » longiore, pubescenti, 18 millimet. longa. Folia 30-3j millim. » longa, 10-15 mill. lata; petiolus 3-5 mill. longus. Racemi termi»nales erecti, 80 mill. et ultraa longi. »

Hab.: Les collines (région moyenne) du Tché-foû (S. Moore 1874).

Obs. Espèce voisine du $S$. macrantha Fish., mais bien distincte par ses tiges glabres et non pubescentes, par ses feuilles pétiolées, oblongues-obtuses, grossièrement dentées et non sessiles, étroitement lancéolées, aiguës et entières, par ses épis floraux moins denses et moins alløngrés, par ses fleurs de moitié plus petites, etc.

15.5. Lysimachia Klattiana Hance in The journal of botany, London (1878), p. 246; L. cuspidata Klatt Der Gatt. Lysim. p. 35, tab. 20, non Blume; Hance in The joumal of botany, London (1877), p. 357.

"Perennis, simplex, ascendens vix pedalis; caulis sat dense »villosus, pilis hyalinis pluricellularibus; folia caulinia $3-4$ verti» ciliata vel conferta, subalternia, superiora sub floribus approxi» mata quasi involucrata, omnia (præter infima oblonga sparsa) » lanceolata-acuta, in petiolum brevem attenuata, pilis sparsis » præsertim subtus villosula, punctis pellucidis haud crebre » punctata, vel etiam impunctata; flores ad apicem caulis (racemo » valde abbreviato) congesti, bracteis foliaceis suffulti; pedicelli " pilis subulatis hirtelli, florem vix æquantes; calix ad basin fere »usque partitus, laciniis e basi lanceolata longe subulatis, silia» tis, corolla paulo brevioribus; corolla lutea profonde partita, " lobis obtusis; stamina corolla duplo breviora, antheris oblongis, »filamentis alte coadunatis (circiter ad $1 / 2$ longitud.); stylus » stamina vix æquans, stigmate punctiformi. Capsulam non vidi»mus, ex icone citata anguste ovata, basi styli acuminata evadit ». Franchet in litt.). »

Hab. : Le territoire de Tché-foû (Swinhoë, Stulhman); les environs de Shang-haï (De Poli), Hong-kong (Fortune d'après Klatt).

Obs. Le L. Llattiana appartient à la section des Cilicina (Sensu Klatt loc. cit.). Le L. Alfredi Hance de Foù-chân, en est très voisin 
et s'en distingue surtout par les poils glanduleux qui recouvrent les pédicelles et les calices, ainsi que par les ponctuations noires des feuilles (Hance, loc. cit.). La villosité du L. Klattiana est entièrement formée de poils subulés, constitués par 5-10 cellules.

\section{ARTOCARPÉES.}

185 bis. Cudraria triloba Hance Plantas quatuor novas Hong-kongenses exhibit, in the journal of botany of Lomdon, $\mathrm{n}^{0}$ de décembre 1874.

Obs. Cette espèce, antérieurement décrite par M. Hance, est seulement mentionnée dans le journal de botanique de Londres (loc. cit). M. Hance ne fait connaitre qu'un nouvel habitat « Specimina quxdam Cudrarix trilobx (Mihi) in agro chifuensi, ab amico Swinhö̈ lecta, folia rhombea vel trapezoideo-ovata, haud lobata ostendunt. Variatio compar in nonnullis Fici speciebus occurrit."

Dans tous les échantillons du $C$. triloba, qui sont conservés dans l'herbier de M. Franchet et qui proviennent du Kiang-soù, les feuilles sont obliquement ovales.

\section{ORCHIDÉES.}

194 bis Habenaria sagittifera Reichb. fil. Botan. zeity. (1845), p. 334; Hance in Journ. of botany of London (1878) p. 238; Franch. et Savat. Emum. plant. jup. 2, p. 33; H. linecrifotia Maxim. Prim. fl. amur. p. 269; Regel Tent. fl. ussur. p. 143, tab. 10; Miquel Prol. fl. j(m). p. 140.

"Glabra, caule folioso, foliis anguste-linearibus, lanceolatis, 》 racemo laxo multifloro; bracteis quam flores brevioribus; sepalis »lateralibus falcato-ovatis deflexis; petalis e basi dimidiato-bas» tata labello adnata deltoideo-ovatis, labello unguiculato cru» ciato-tripartito, lobis lateralibus linearibus, antice cuspidato »dentatis, lobo intermedio lineari integro; calcari basi inflato, » circiter germinis longitudine.

》 Radix e tuberibus duobus oblongis integris, radicibusque »simplicibus e basi caulis egredientibus composita. Caulis »foliosus, 40-60 centimet. altus, foliis decrescentibıs, omnibus » anguste lineari-lanceolatis (Regel). » 
Hab.: La région montagneuse moyenne et boisée du Tchéfoû, 500 à 600 mèt. d'altit.), où cette orchidée a été découverte en 1873, par M. C. Stulliman.

Ar. géog.: Chine bor.; Amour et Ussuri; Japon (Kiòu-sioû, Nippon et Yéso).

294 bis. Allium nereideum Hance, in Annal. des scienc. natur. (Botanique) 1866, p. 245; Allii macrostemonis Bunge affine.

"Bulbo ovato albo, scapo tereti solido, striato, superne nudo, » inferne 3-4 foliato; foliis angustissimis, semiteretibus canali» culatis; spatha brevi acuminata; umbella 2-8 flora bulbifera; "floribus pedicello duplo triplove brevioribus, perigonii laci» niis æqualibus, oblongis, obtusiusculis, dilutissime lilacino» albidis, linea carinali viridulo-purpurea percursis; staminibus » simplicibus æqualibus, perigonium superantibus, filamentis »albis, basi dilatatis, antheris violaceis; ovario trigastro papil"loso, albo, loculis dorso superne maculis binis viridibus notatis: 》stylo staminibus paulo longiore, stigmate inconspicuo, capitato, » integro (Hance).

Hab. : Le littoral du Tché-foû (MM. Gilbert et L. King, 1861) ex Hance loc. cit.

\section{Gyperus sanguinolentus Vahl.}

Ajouter le Japon, dans l'aréa géographique de cette espèce.

\section{Fimbristylis Buergeri Miquel; Franch. et Savat. Enum.} plant. jap. 2, p. 119.

Obs. La description de Miquel dans le Prolusio fl. japon. p. 76, convient de tout point à la plante du Tché-foù. Les chaumes s'élèvent jusqu'à 70 centim. de hauteur, et ses feuilles raidescanaliculées n'atteignent guère que le quaart de cette hauteur. Les épis, au nombre de dix à quinze, sont cylindriques, aigus à leur extrémité et longs de 10 à 12 millim., disposés en ombelle, avec un épi sessile au centre de celle-ci et des rayons, lesquels sont plus ou moins dépassés par la bractée involucrable. Les écailles sont ovales, arrondies, coriaces avec ou sans mucron, et les akènes lenticulaires, bruns, creusés de scrobicules régulièrement disposées en lignes longitudinales, et formant des côtes par leurs bords saillants (A. Franchet). 


\section{Fimbristylis squarrosa Vahl.}

Aux synonymes cités il faut ajouter le suivant: Franch. et Savat. Enum. plant. jap. 2, p. 118, et placer le Japon, dans son aréa géographique.

\section{Arundinella anomala steudel.}

Ajouter les synonymes suivants: Franch, et savat. Enum. plant. jap. 2. p. 165 et p. 597; Paspalum mandshuricum Maxim. Prim. fl. amur. p. 328; Chalynochlamis anomala A. Franchet (1879).

Obs. Plante polymorphe, à panicule très développée dans les échantillons chinois ou japonais. Celle-ci mesure jusqu'à 50 à 80 centim. en longueur (A. Franchet). Les feuilles sont linéaires ou linéaires-lancéolées, et les rameaux de la panicule tantôt simples, dressés, tantôt divisés plus ou moins étalés. La glumelle supérieure de la fleur mâle est presque toujours ciliée-laineuse sur les bords, et dans la fleur hermaphrodite celle-ci est scabre et granuleuse sur le dos. La glumelle inférieure est toujours plus ou moins longuement mucronulée.

M. Maximowicz a donné de cette plante (loc. cit.) une excellente description sous le nom de Paspalum; mandshuricum, tandis que, de leur côté, MM. Franchet et Savatier ont fait une étude très-approfondie des organes de floraison de cette rare espèce, pour laquelle ils se proposent de créer un genre nouveau, celui de Chalinochlamys, encore inédit.

La dispersion géographique de l'A. anomala s'étend, de la province de Chan-tong en Chine, à ia Mandchourie et à l'Ussuri, ainsi qu'au Japon (région inontagn. et moyenne du Nippon et de Yéso. (L. Savatier.)

\section{Eragrostis pilosa Pal. de Beauv.}

Ajouter à la synonymie : Franch. et Savat. Enum. plant. jap. 2, p. $176 ;$ E. multicaulis Steud. Syn. glum. 2, p. 423 (excl. synon. E. tenellulx); Glyceria airoides Steud. Syn. glum. 2, p. 287 (teste A. Franchet).

Obs. La plupart des spécimens de l'E. pilosa récoltés au Tchéfoû et appartenant à la forme A elatior, sont munis, à l'aisselle des rameaux, de quelques poils assez longs, mais en petit nombre, tandis que ceux-ci n'existent pas dans la forme B humilis 
(var. nima Miquel, loc. cit.). Les rameaux de la panicule, d'abord dressés et presque apprimés, s'étalent ensuite à angle droit après la floraison. Les épillets sont formés de huit à dix fleurs dans les individus robustes, et de quatre à six dans la forme naine. Les variations elatior et nana passent si bien de l'une à l'autre, que MM. Franchet et Savatier n'ont pas cru devoir les conserver comme distinctes dans leur Enumeratio.

\section{Eragrostis megastachya Link.}

Ajouter le Japon dans l'aréa géographique de cette espèce.

243 bis. Bromus japonicus Thunbg. Flora japonica, p. 52, tab. 12; Hance Journ. of botany of London (1878), p. 234; Franch. et Savat. Enum. plant. jap. 2, p. 181; Miquel Prot. jap. p. 171 ; B. villiferus Steud. Syn. glum. 2, p. 326 pro parte.

"Culmus cum panicula usque bipedalis, vayinx dense et » patentissime pubescentes; ligula brevis, truncata, inæqualiter »dentata, basi pilosa; folia 20-32 centimet. longa, anguste linea» ria, præsertim inferiora pubescentia, cum vaginis glabres» centia, striato-nervosa, nervis in vaginam parallele continuatis; » panicula radii inferiores $5-6$, pauce spiculati (1-7); spiculx 5 -11 »floræ, glumæ inæquales, 3.7 nerviæ; palea inferior apice »obtusa, vel in superioribus bidentata, nervis 3 validioribus; 》aristæ paleam æquantes nec breviores in apice spiculæ vix » pollicares, divergenti-fasciculatæ, flosculorum. inferiorum ad» pressæ. »

Hab. : Les terres sèches et incultes du Tché-foû, près de Yantaï (Swinhö̈, 1873).

Ar. géog.: Chine bor., le Chan-tong. - Japon (Kioû-sion, Nippon).

\section{Asplenium nipponicum Metten.}

Ajouter à la synonymie: Franch. et Savat. Enum. plant. japon. 2, p. 224; Baker Syn. filic. ed. 2, p. 227; Hance On some mountain plants from northern China, in The journ. of the Linnean Society, Londres (1872), vol. XIII, p. 66.

Obs. Cette fougère est bien voisine de l'A. Goringianum, mais elle s'en distingue par ses sores linéaires, droits, ou seulement recourbés en fer à cheval, par son indusion érodé, denticulé sur 
les bords, etc. L'A, nipponicum varie beaucoup par la forme des pinnules, mais dans toutes ses variations, les sores sont relevés de crètes aiguës.

Ar. géoq. : Chine bor., le Pé-tch-ly, à Pé-king (R.-P. David); Chan-tong au Tché-fou (O. Deb.), Chine méridionale (Hance); Mandchourie. - Japon au Nippon (L. Savatier).

\section{7 bis. Aspidium Forbesii Hance in The journal of botany of}

London (1877), p. 198. - A. xmuli Swartz affine ex Hance.

« Stipite 3-4 pollicari, stramineo, supra cum rachi sulcato paleis " parvis ovato-lanceolatis integris, acuminatis, medio saturatius 》 coloratis, facile decirluis, lamina 67 pollicari, deltoideo-trian»gulata, acuminata, basi tri, superne bi-pinnatisecta, viridi-gla》 berrima, subtus pallidiori glandulis nitentibus jilisque septatis » vix conspicuis sparsim consita, rachi viridula parce paleacea, 》segmentis primariis præter superiora petiolatis, infimis ovato》acuminatis, superioribus lanceolatis, segmentis secundariis 》 inferioribus petiolatis, ultimis vel laciniis oblongis, plerumque 》obtusis, acutiuscule incisis vel serratis, venam immersam » inconspicuam ramis semel furcatis excipientibus; laciniis "superioribus tantum soriferis, soris mediocribus, apices lacinia » rum fere inter costulam et marginem occupantibus, indusio . 》 reniformi plano, cinnamomeo, glandulis omnino destituto, » paraphysibus nullis (Hance).

$H a b$. : La région montagneuse boisée du Tché-foû, à 600 mèt. d'altitude. Découvert en octobre 1874 par M. F.-B. Forbes.

Dans la classe des champignons qui est si pauvrement représentée au Tché-fou, il me reste à signaler, dans la région montagneuse de cette contrée, la présence du Schyzophyllum commune Friès, agaricinée bien commune en Europe, et dont la dispersion jusque dans l'extrême Orient constitue un fait de géographie botanique des plus intéressants.

Schyzophyllum commune Friès, Epicrisis p. 403; Hiquel Prol. jap. p. 350; Agaricus alneus Lin.

Var. palmatum O. Debeaux Mss. in Herb. (1860).

"Réceptacle plus ou moins convexe, coriace, tomenteux, mar\qué ęn dessus de nombreuses divisions inégales, qui rendent »le bord de celui-ci frangé ou comme palmé; pédicule latéral 
$\downarrow$ très court, lamelles rayonnantes se dirigeant du centre à la ఎ circonférence, bifides ou ramifiées et très inégales. »

Hab.: Sur les vieilles écorces du Quercus serrata, dans la région mont. du Tché-foû, à 500 mèt. d'altitude.

Ar. géog. : Europe bor., cent. et aust. - Chine bor. (O. Deb.) - Japon (Siebold).

Obs. Cette variété à bords frangrés ou palmés, se rencontre également dans l'Europe centrale ou australe (Teste clar. C. Roume. gruère). L'inégalité des lamelles inférieures, qui sont le plus souvent bifurquées ou ramifiées, donnent à la var. palmatum du Schyzophyllum commune, l'aspect d'un Panmus; mais on ne saurait la séparer comme espèce de la forme typique, malgré le caractère peu important d'ailleurs, des lobes plus ou moins pro. fonds et nombreux de son réceptacle. 


\section{INDEX BIBLIOGRAPHIQUE}

BANks. Icones selectæ plantarum; Londini, in-folio, cum 59 tabul. (1791),

- Benthay. Flora hongkongensiss; London, 8 (1861).

- Ejusdem operis supplementum auctore Hance, in the journ. Soc. Linn. Lond. vol. XIII.

Besser: Enumer. plantar. Baikal.; Extrait du Flora (1834).

Blume. Museum botanicum Lugduni-Batav.; Lugduni-Batav. 2 vol. in-fo $(1849-1856)$.

Boissier. Flora orientalis; Basileæ, vol. I-IV, in-80 (1867-1875).

Bunge. Decades plantarum Mongolico-chinensium, Casan; in- ${ }^{\circ}$ (1835).

- Enumeratio plantarum quas in China boreali collegit; Petropoli, in-4 (183丂) Extr. des Mém. des savants étrang.. vol. $2^{\mathrm{e}}$.

David (Rév. P.). Journal d'un voyage en Mongolie; parties I-III (Extrait des nouv. archiv. du Muséum d'hist. nat. de Paris) (186\%-1869).

- Journal de mon troisième voyage d'exploration dans l'empire chinois. - Paris, 2 vol. in-18 (1875).

Decandolle (A.P.). Botanique de la Chine septentrionale. Extrait de la Biblioth. univers. de Genève, $\mathrm{n}^{\circ}$ de janvier (1834).

Fortune (Rob.). Voyage horticole en Chine in Mém. Société centrale d'horticulture; Paris, in- $8^{\circ}$, (1853).

Franchet et Savatier. Enumeratio plantarum in Japonia crescentium; Parisiis, 2 vol. grand in-8 $8^{\circ}$ (1875-1879).

Grielin. Flora sibirica; Petropoli, 4 vol. in- $4^{\circ}$, cum 288 tab. (1747). Gray. List of dried plants collected in Japan by sir Wels Wil liams. - Boston (18.59). Mem. of the american acad. of sciences and arts, vol. VI. 
Haxce. Symbolæ ad floram sinicam, in Ann. scienc. natur. Paris (1860) in-8

- Decades plant. novar. Chinæ borealis, in the journal of the linnean Society, London, 1873-I878.

KæmpFer. Amenitates exoticæ; Fasc. V; Lemgoviæ, in- $4^{0}$, cum mult. tab. (1712).

Ledebour. Flora altaica, adjutoribus C.-A. Meyer et Bunge; Berolini, 4 vol. in- $8^{\circ}$ (1829-183:3).

- Flora rossica, sive enum. plant. in totius imperii rossici prov., etc.; Stuttgard, 4 vol. in- $8^{\circ}$ (1841).

- Icones plant. nov. floram rossicam illustrantes; 4 vol. in-fo $(1829)$.

Loureiro. Flora cochinchinensis; Berolini, 2 vol. in- $8^{\circ}$ (179i).

Maxımowicz. Primitiæ floræ amurensis; Petropoli, vol. in-4. Extrait des IIém. de l'Acad. des sciences de saint-Pétersbourg, vol. IX (1859).

- Rhamnæ orientales-asiaticæ, in Mém. Acad. scienc. de Saint-Pétersbourg. Année 1866, in- $4^{\circ}$.

Diagnoses breves plantarum Japoniæ et Mandshuriæ; Decades I-XI, in Mélanges biolog. du. Bullet. de l'Académie des sciences de Saint-Pétersbourg (1866-1874).

Ophiopogonis Species, in Mélanges biolog. Saint-Pétersbourg, vol. VIII, in-8 .

Mrquel. Annales musei botanici Lugduno-Batavi; Lugd.-Batar. 2 vol. in-f $\mathrm{f}^{0}(1863-1866)$.

- Prolusio floræ japonica; Amstelodami, in-fo, cum 3 tab. (1866-1867).

- Catalogus musei botanici Lugduno-Batavi, Pars prima : Flora japonica; La Haye, in-8 $8^{0}(1870)$.

- Sur le caractère et l'origine de la Flore du Japon, in Archives néerlandaises, vol. 2, la Haye (1867).

Pallas. Iter (édit. française avec notes de Lamark), 8 vol. in- $8^{\circ}$ et un atlas in- $\mathrm{f}^{\circ}$.

- Flora rossica; Petropoli, 2 vol. in-8 (1784 et 1788).

- Illustrationes plantarum imperfecte vel nondum cognit.; Lipsiæ, 4 fasc. in- $\mathrm{f}^{0}$ (i803-1806). 
Regel. Tentamen floræ ussuriensis; Petropoli, in- $4^{\circ}$, in Mém. acad. scienc. Saint-Pétersbourg, 7e série, vol. IV (1861).

- Reisen in der suden von Ost-Siberien in der jahren 185.51859, durch S. Radde : Dicotyledonx-Polypetalx; vol. I, Pars 1 et 2 , in Bull. soc. des natur. de Moscou (1861-1862).

- et de Herder. Ejusdem operis Monopetalx. vol. III, Pars 1, 2, 3 et 4, Mosccu, in-8* (1864-1870).

Savatier (L.). Livres Kwa-wi, par Youan-Si, traduits du japonais; Paris, un vol. graud in- $8^{\circ}$ (1873).

Sснмidт (Fred.). Reisen in Amur-lande und auf der insel Sachalin, in Mém. acad. scienc. de Saint-Pétersbourg, $\boldsymbol{7}^{\mathrm{e}}$ série, vol. XII; un vol. in-4 $4^{0}$ (1858). .

Siebold et Zuccarini. Flora japonica; Centuria prima; Lugd.Batav., in-fo (1835). - Cent. secunda, 1 vol. in-fo (continué par Miquel avec $127 \mathrm{pl}$.).

- Plantarum quas in japonia collegit de siebold genera nova, notis charact., etc., illustrata; Munich, in- $4^{\mathrm{N}}$, cum 5tab. (1845).

- Floræ japonicæ familiæ naturales, adjectis gen. et specier., etc. Sectio I et II; Munich, in- $4^{0}$ (1843).

Staunton. Voyage dans l'intérieur de la Chine et en Tartarie de lord Macartney, pendant les années 1793 à 1794, avec nombreuses listes de plantes. Trad. de Castéra. Paris, 4 vol. in $-8^{\circ}$ (1804).

Suringar: Algæ japonicæ musei Lugd.-Batavi; in-40 cum 24 tab. Harlem (1870).

Thunberg $\left({ }^{1}\right)$. Flora japonica; Lipsiæ, in-80 (1874).

- Icones plant. japonic. Decades I-V; Upsalæ, in-fo cum 24 tab. (1799).

- Voyages de Thunberg au Japon. Trad. par Langlès. Paris, 2 vol. in $-4^{\circ}(1796)$.

Trautvetter et Meyer. Florula ochotensis, in Middendorfs sibirishe Reisen. - Bandt I et II.

(1) Lire le nom de Thunberg (Thunba., Thunbergi), dans les florules qui précèdent, au lieu de Thumberg (Thumbg., Thumbergi) qui a été imprimé ainsi et non corrigé pendant l'impression. 
Turczaninow. Catalogus plantarum in regionibus baikalensibus et in Dahuria, etc., in Bull. de la Société des natur. de Mos cou (1838).

- Decades I-III plantar. Mongolico-chinensium, in Bull. soc. nat. de Moscou, vol. V.

- Enumeratio plantarum Chinæ borealis quas collegit Kirilow, in Bull. Soc. natur. Moscou, vol. X (1833).

- Flora Baikalensi-dahurica; Moscou, 2 vol. in-8 et supplément (1842-1846).

Wight. Contributions to the botany of India; London, in- $8^{\circ}(1834)$.

Zoldinger. Systematiches verzeichnig der in indishen archipel. in der jahren 1842-1\&48. - Zurich, ir.-8 (18.54). 


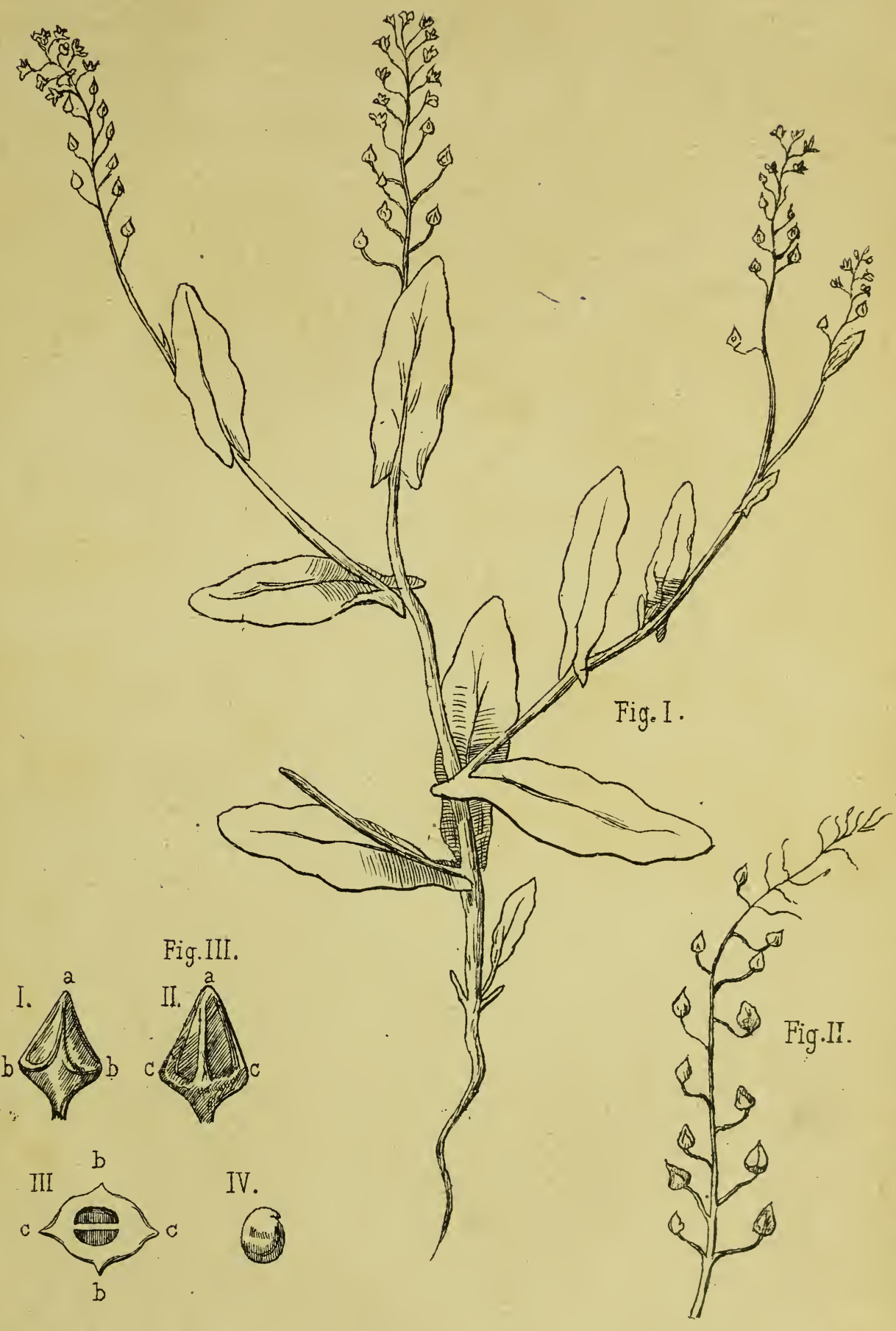

Bunias T'Cheliensis. O Debeaux. 



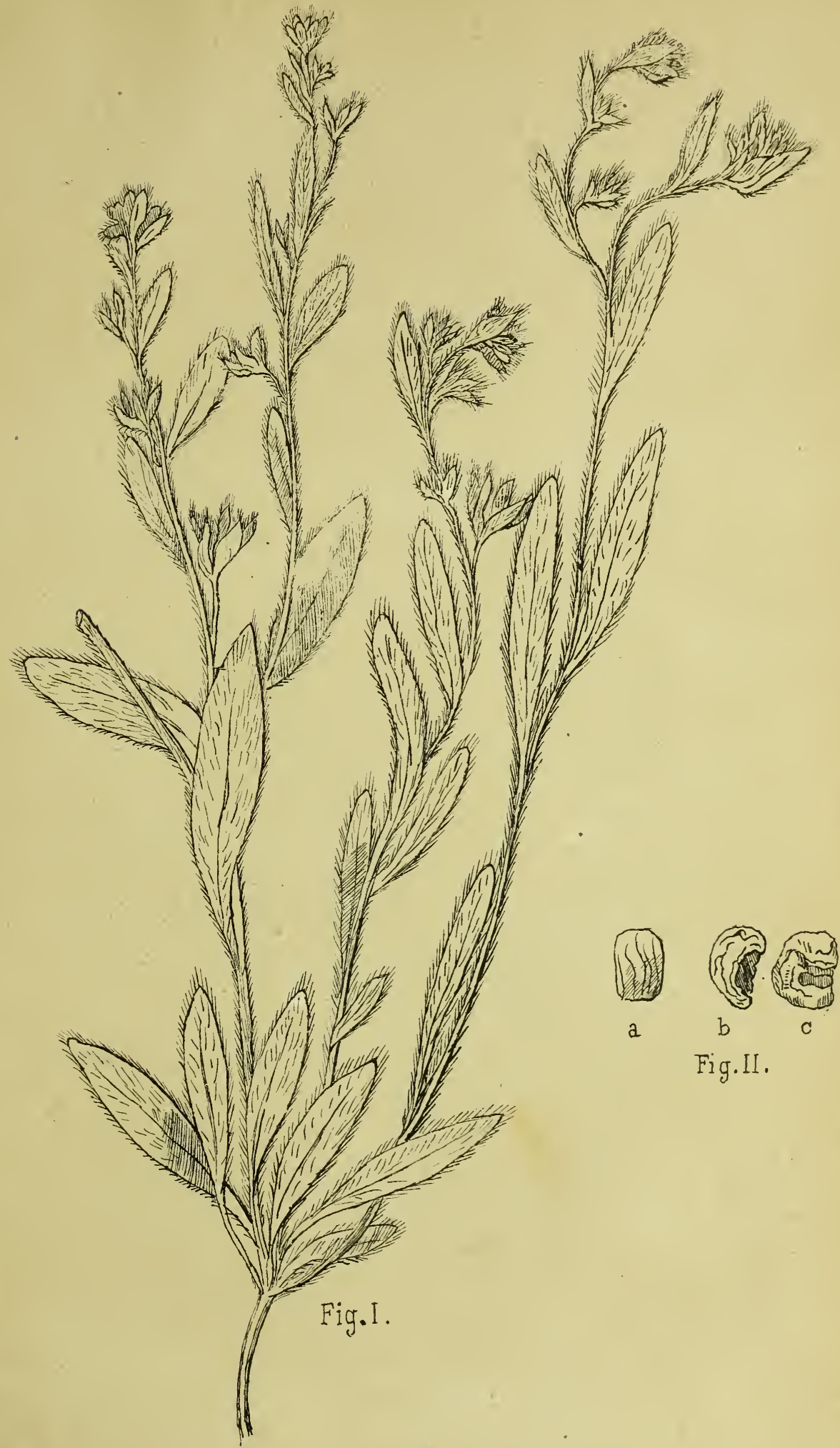





\section{OBSERVATION FINALE}

Après un intervalle de dix-sept années qui nous sépare, au moment où j'écris ces lignes, de l'époque à laquelle la ville. de Tien-tsin fut évacuée par l'armée française expéditionnaire en Chine, au mois d'octobre 1861, il m'est, enfin, permis de terminer un travail dont je m'étais seulement borné à recueillir les matériaux, pendant mon séjour de près de trois années à Shang-haï, au Tché-foû et à Tien-tsin. Je dois cet heureux résultat au concours obligeant de mon collaborateur et ami, M. A. Franchet, dont les remarquables publications sur la flore japonaise, sont justement appréciées aujourd'hui par tous les botanistes. Je lui renouvelle ici mes plus sincères et mes plus vifs remerciements. Je n'oublierai pas de témoigner aussi toute ma reconnaissance à la Société Linnéenne de Bordeaux, qui a ouvert si généreusement ses Actes à mes modestes recherches sur la flore chinoise.

Perpignan, 31 octobre 1878.

O. D.

Explication des planches de la florule de Tien-tsin.

PLANCHE I

Fig. 1. Bunias tcheliensis 0 . Debeaux (échantillon en fleur, de grandeur naturelle).

Fig. II. - Un rameau avec fruits murs, de grandeur naturelle.

FIg. III. - I $a, b$; II $a, c c$; Silicule grossie vue de côté et de face; III $b, c$, coupe de la silicule par $b, c$ et grossie; IV une des graines montrant la forme et la position de l'embryon et grossie

\section{PLANCHE II}

Fig. I. - Bothriospermum chinense Bunge (de grandeur naturelle).

Fig. II. - $a, b, c$; nucule grossie, vue de face, de côté et en dessous. 



\section{$\because x^{1}$}

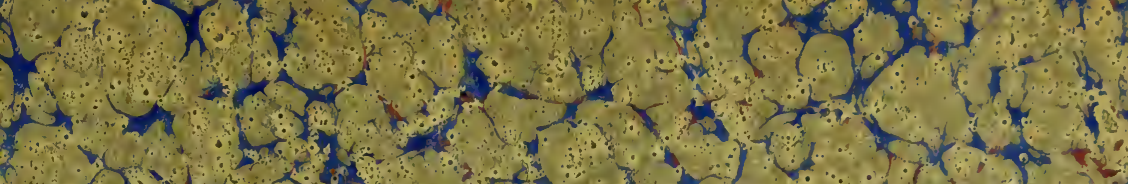

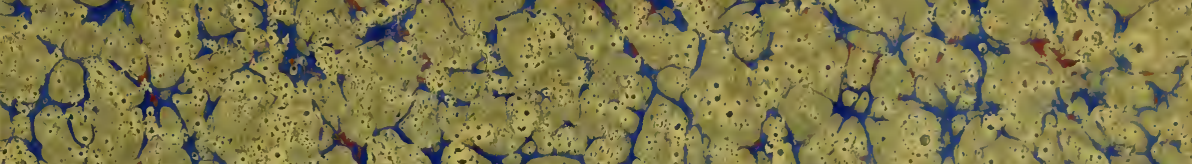

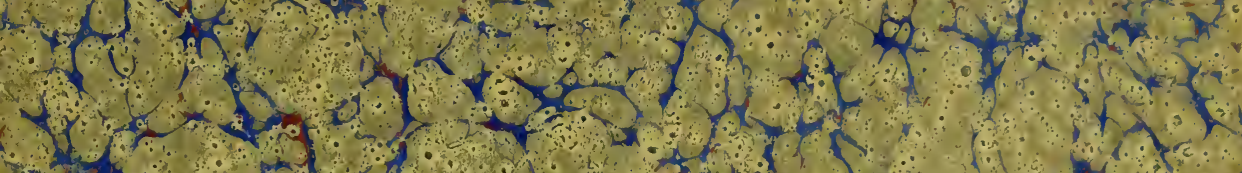

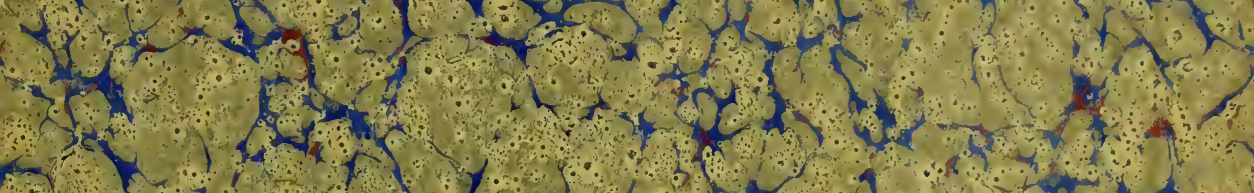

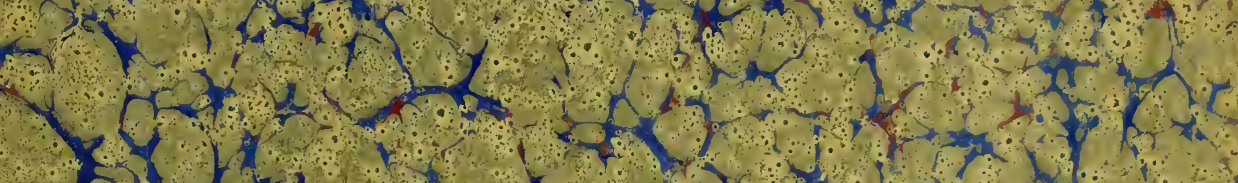

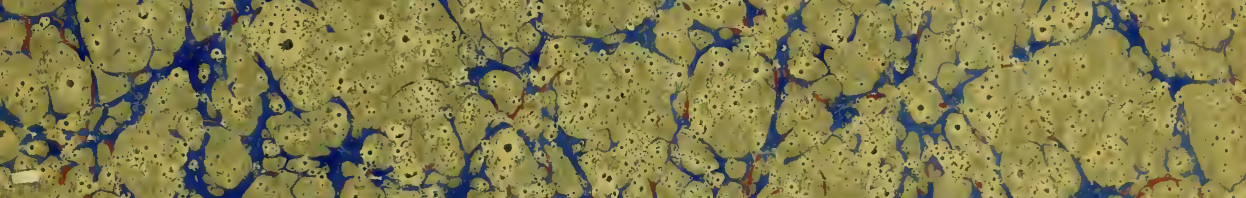

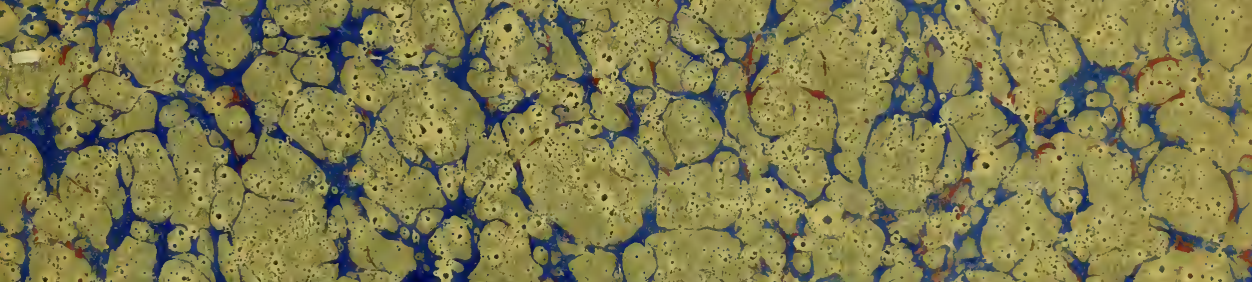

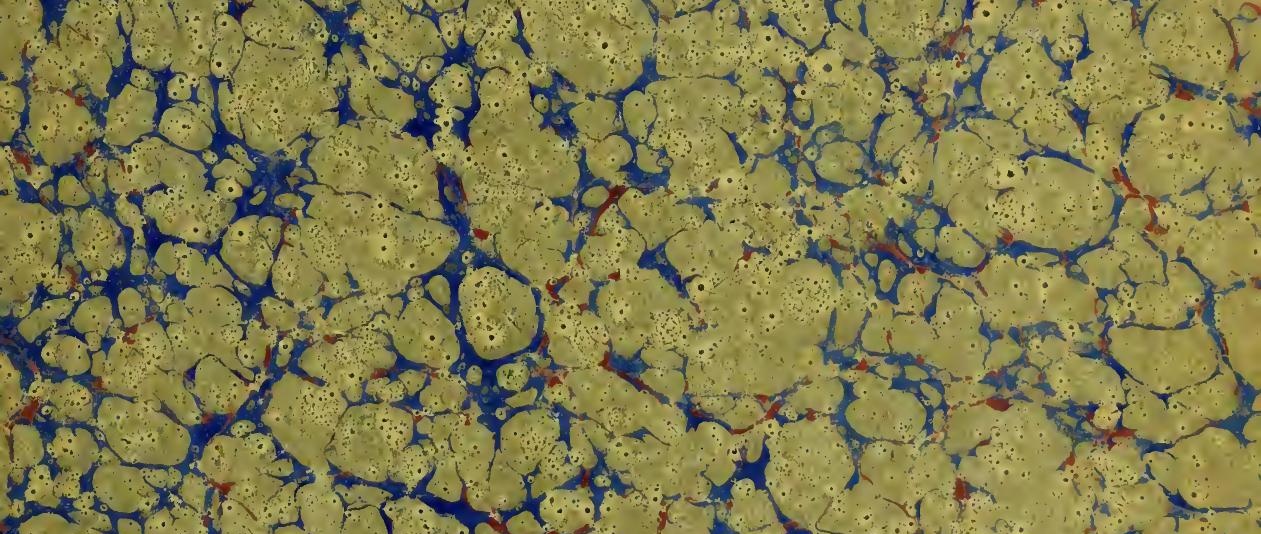

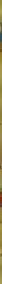

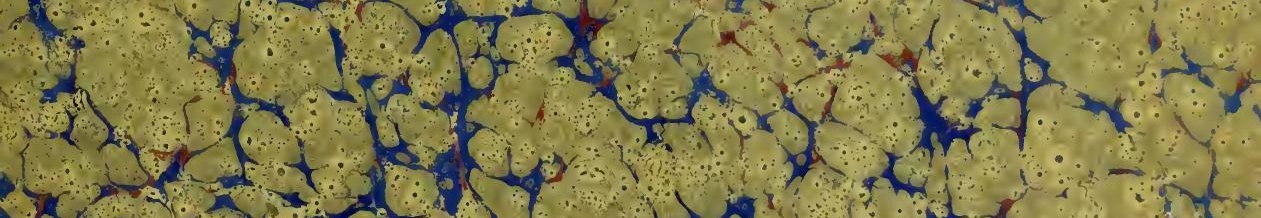
$x\left(x^{2}+x^{2}+0^{2}\right.$

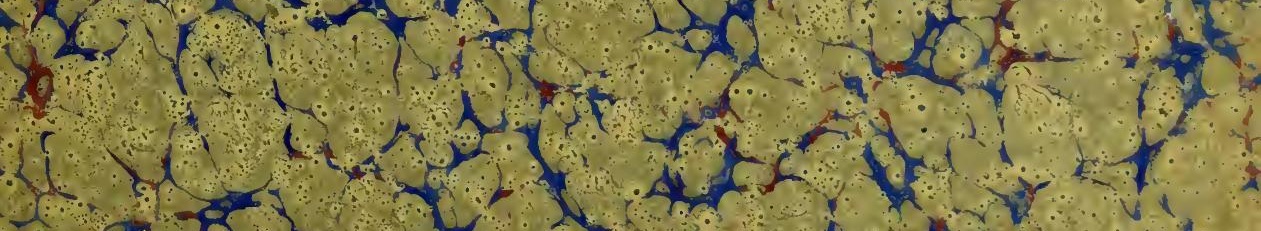


